\title{
SuperCDMS Prototype Detector Design and Testing
}

\author{
A THESIS \\ SUBMITTED TO THE FACULTY OF THE GRADUATE SCHOOL \\ OF THE UNIVERSITY OF MINNESOTA \\ BY
}

Allison Blair Kennedy

IN PARTIAL FULFILLMENT OF THE REQUIREMENTS

FOR THE DEGREE OF

Doctor of Philosophy

Vuk Mandic

October, 2017 
(C) Allison Blair Kennedy 2017 ALL RIGHTS RESERVED 


\section{Acknowledgements}

Although this thesis is a presentation of my personal efforts throughout graduate school, in truth it is a reflection of the communal support and guidance provided to me during my whole education. Foremost I want to acknowledge the love and encouragement of my parents, for they fostered in me the curious spirit and perseverance required for this endeavor. I cannot fathom all that you have done for me, and no amount of gratitude will be enough. A million thank you's to you both. And, of course, I thank my brothers, Bruce and Peter, for being role models of intellectual excellence.

I would like to thank the teachers and professors that ignited my love of math and science, starting with my high school calculus and physics teachers, Anastasia Eldredge and Jenny Steiger. Thank you for introducing me to these topics with a devotion I would soon come to share. I would also like to thank the Math and Physics departments at Macalester College for their engaging lectures and personal dedication their students' growth. In particular, thanks to my advisors, Tonnis ter Veldhuis and Tom Halverson for their patient guidance throughout my undergraduate career.

My graduate school career has been filled with too many helpful individuals to count. I could not have survived my first year without the community of fellow first years; behind every completed assignment is endless hours of baffled frustration, false leads, and a few joyous moments of clarity with Bern Youngblood, Melanie Galloway, Peter Martin, Quentin Ebner, Andy Julin, and others. I am also grateful for the guidance and friendship extended to me by the senior graduate students, especially Jan Zirnstein, Dima Spivak, Terry Bretz-Sullivan, Adam Peterson, Alex Gude, Charles McEachern, Dominick Rocco, and Ilana Percher, to name only a few. I'd also like to thank the friends I have made throughout my time in grad school, including Pat Meyers, Zach Lesko, Jon Garamella, Ryan Marshall, Yilikal Ayino, Mark Rustad, and Justin Willmert, among 
others. And finally, to all the past members of the Big Bangers, thank you for being the best softball team the grad school rec league has ever seen.

My work with CDMS is only possible thanks to the knowledge and experience of my fellow collaboration members. In particular I would like to thank Nader Mirabolfathi, Sten Hansen, Rupak Mahapatra, Andrew Jastram, Rusty Harris, Mark Platt, Amy Roberts, Matt Pyle, and Noah Kurinsky for their roles in keeping the UMN test facility operational and pursuing relevant studies, such as those mentioned in this thesis. I am exceedingly grateful to Nader Mirabolfathi, without whom the K100 dilution unit might still be oil-logged and stubbornly room-temperature.

The UMN CDMS group would not be possible without the leadership of Prisca Cushman and Vuk Mandic; I am grateful to them for sharing their insight and expertise with me, helping me to grow into professional physicist. Additionally, I am thankful to Vuk for fulfilling his role as my advisor with understanding and kindness. I would also like to acknowledge the postdocs and fellow graduate students of the UMN CDMS group for all that they have done for me. Thank you to senior grad students Jianjie Zhang and Roxanne Radpour, and postdocs Hassan Chagani and Matt Fritts for instilling in me the foundational knowledge for fridge operation and detector characterization. And of course, none of these studies would be possible without the efforts of undergraduate research assistants; thank you to Chris Phenicie, Alex Codoreanu, Dan Codoluto, and Gina Ristani for all of the time put into keeping the lab running.

Thank you to Mark Pepin, Tommy Hofer, Scott Fallows, Hannah Rogers, and D'Ann Barker for sharing their own expertise with me, whenever I had a question or needed a hand. My infinite gratitude to grad student Nick Mast and lab technician David Strandberg; you made the worst days in lab bearable and the best days extraordinary, with your perseverance, your know-how, your humor, and (of course) your riddles. And a huge thank you to Anthony Villano for all of the time and effort spent aiding my research. The expertise you have shared with me is invaluable, and your friendship will always be cherished.

Finally, thank you to Ty, my soul mate, for your patience, unconditional support, and love these past twelve years. This wouldn't have been possible without you by my side. I love you dearly.

Thank you, I am forever indebted to to you all. 


\section{Dedication}

To Brutus, for being a survivor. 


\begin{abstract}
A substantial amount of astrophysical evidence indicates that approximately a quarter of all energy in the universe is composed of a nonluminous, and nonbaryonic "dark" matter. Of the potential dark matter particle candidates, Weakly Interacting Massive Particles, or WIMPs, is particularly well motivated. As a means to directly detect WIMP interactions with baryonic matter, the Cryogenic Dark Matter Search (CDMS) project was established, operating at the Soudan Underground Laboratory from 2003 - 2015, under the CDMS II and SuperCDMS Soudan experiments. CDMS detectors simultaneously measure the ionization and phonon energies of recoil events in $\mathrm{Si}$ and $\mathrm{Ge}$ crystals kept at cryogenic temperatures in a low-background environment. The ratio of ionization energy to recoil energy serves as a discrimination parameter to separate nuclear recoil events from the electron-recoil background.

The next installation, SuperCDMS SNOLAB, is preparing for future operation, with an initial payload of eighteen $\mathrm{Ge}$ and six $\mathrm{Si}, 100 \mathrm{~mm}$ diameter, $33 \mathrm{~mm}$ thick detectors. Of this initial payload, eight Ge and four Si detectors will operate in a high-voltage $(\sim 100$ V) mode, which have an increased sensitivity to low-mass WIMPs due to decreased energy thresholds.

The SuperCDMS test facility at University of Minnesota aids in the detector R\&D and characterization of prototype detectors, as part of the scale-up effort for SuperCDMS SNOLAB. This thesis presents the first full ionization and phonon characterization study of a $100 \mathrm{~mm}$ diameter, $33 \mathrm{~mm}$ thick prototype Ge detector with interleaved phonon and ionization channels. Measurements include ionization collection efficiency, surface event rejection capabilities, and successful demonstration of nuclear recoil event discrimination. Results indicate that $100 \mathrm{~mm}$ diameter, interleaved Ge detectors show potential for use in SuperCDMS SNOLAB.

As part of detector $R \& D$, the Minnesota test facility also looks beyond the next stage of SuperCDMS, investigating larger individual detectors as a means to easily scale up the sensitive mass of future searches. This thesis presents the design and initial testing results of a prototype $150 \mathrm{~mm}$ diameter, $33 \mathrm{~mm}$ thick silicon ionization detector, which is 5.2 times larger than those used in SuperCDMS at Soudan and 2.25 times larger than
\end{abstract}


those planned for use at SuperCDMS SNOLAB. In addition, the detector was operated with contact-free ionization electrodes to minimize bias leakage currents, which can limit operation at high bias voltages. The results show promise for the operation of both large volume silicon detectors and contact-free ionization electrodes for scaling up detector mass and bias. 


\section{Table of Contents}

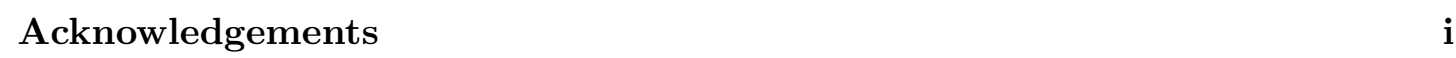

Dedication iii

Abstract iv

List of Tables $\quad$ xi

List of Figures $\quad$ xii

1 Dark Matter 1

1.1 Introduction . . . . . . . . . . . . . . . . . . . . . . . . . . 1

1.2 Dark Matter Evidence $\ldots \ldots \ldots \ldots$. . . . . . . . . . . . . . 1

1.2 .1 Evidence from Observations of Clusters and Galaxies. . . . . . . 2

$1.2 .2 \quad$ Evidence from Cosmological Observations . . . . . . . . . . . . 3

1.2 .3 Evidence from Gravitational Lensing . . . . . . . . . . . . . . . . 10

1.3 Dark Matter Characteristics and Candidates . . . . . . . . . . . . . 12

$1.3 .1 \quad$ Non-particulate Solutions . . . . . . . . . . . . . . . . . . 12

1.3 .2 Axions . . . . . . . . . . . . . . . . . . . . . . 13

1.3 .3 Sterile Neutrinos $\ldots \ldots \ldots \ldots$. . . . . . . . . . . . . 14

1.3 .4 WIMPs . . . . . . . . . . . . . . . . . 14

1.4 Dark Matter Detection . . . . . . . . . . . . . . . . . . . . . . . . . . . . 19

$1.4 .1 \quad$ Collision Experiments . . . . . . . . . . . . . . . . . . . . . 19

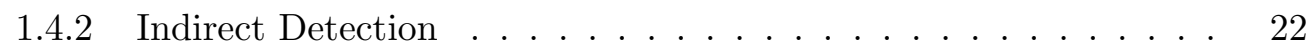

1.4 .3 Direct Detection . . . . . . . . . . . . . . . . . . . 25 
2 Cryogenic Dark Matter Search Experiment $\quad 41$

$2.1 \quad$ A Brief History of CDMS . . . . . . . . . . . . . . . . . . . . . . . 41

$2.1 .1 \quad$ CDMS I . . . . . . . . . . . . . . . . . . . . . 42

2.1 .2 CDMS II $\ldots \ldots \ldots \ldots \ldots$

$2.1 .3 \quad$ SuperCDMS Soudan $\ldots \ldots \ldots$. . . . . . . . . . . . 50

2.1 .4 CDMSlite . . . . . . . . . . . . . . . . . 50

2.2 CDMS Detectors and Signals $\ldots \ldots \ldots \ldots \ldots \ldots$

$2.2 .1 \quad$ ZIP technology . . . . . . . . . . . . . . . . . . . 53

2.2 .2 Detector Types . . . . . . . . . . . . . . . . . . . . 54

$2.2 .3 \quad$ Ionization Measurement . . . . . . . . . . . . . . . . . . . 59

2.2 .4 Phonon Measurement . . . . . . . . . . . . . . . . . . . . 62

$2.2 .5 \quad$ Ionization Yield . . . . . . . . . . . . . . . . . 70

2.3 SuperCDMS SNOLAB and Prototype $100 \mathrm{~mm}$ iZips . . . . . . . . . 73

$2.3 .1 \quad$ Facility and Experimental Setup . . . . . . . . . . . . . . . 73

$2.3 .2100 \mathrm{~mm}$ SuperCDMS Detectors . . . . . . . . . . . . . . . 73

$2.3 .3 \quad$ SuperCDMS SNOLAB Projected Sensitivity $\ldots \ldots \ldots$. . . . . 75

3 Cryogenic Detector Testing and Characterization Facility at Univer$\begin{array}{ll}\text { sity of Minnesota } & \mathbf{7 7}\end{array}$

$3.1 \quad$ Dilution Refrigerator . . . . . . . . . . . . . . . . . . . . . . . 78

$3.1 .1 \quad$ Dilution Refrigeration . . . . . . . . . . . . . . . . . 78

3.1 .2 Testing Facility Cryostat Setup . . . . . . . . . . . . 82

3.2 Cold Hardware . . . . . . . . . . . . . . . . . . . . . . . . . . . 86

3.3 Warm Hardware and Data Acquisition Software . . . . . . . . . . . . . . 93

3.4 Data Processing . . . . . . . . . . . . . . . . . . . . . . . . . . . . . . . 98

$3.4 .1 \quad$ Optimal Filter $\ldots \ldots \ldots$. . . . . . . . . . . . . . . . . . . . 98

$3.4 .2 \quad$ Non-Stationary Optimal Filter . . . . . . . . . . . . . . . 105

3.5 Radioactive Background in the Cryogenic Laboratory $\ldots . . . . .106$

$3.6 \quad 100 \mathrm{~mm}$ iZIP Detectors $\ldots \ldots \ldots \ldots$. . . . . . . . . . . . 123

3.6 .1 Overview of Prototype $100 \mathrm{~mm}$ Detectors . . . . . . . . . . . . . 124

$3.6 .2 \mathrm{G106 \textrm {a }} \ldots \ldots \ldots \ldots \ldots \ldots \ldots$ 
\begin{tabular}{|lll}
4 & $100 \mathrm{~mm}$ Detector Ionization Characterization & 129
\end{tabular}

4.1 Charge Quality Cuts . . . . . . . . . . . . . . . . . . 130

$4.1 .1 \quad$ Charge chisq Cut . . . . . . . . . . . . . . . . . 130

$4.1 .2 \quad$ Charge Threshold Cut . . . . . . . . . . . . . . . . . . 132

$4.1 .3 \quad$ Charge Radial Partition Cut . . . . . . . . . . . . . . . . 133

4.1 .4 Charge Baseline Cut . . . . . . . . . . . . . . . . . 135

4.2 Calibration and Charge Energy Spectra . . . . . . . . . . . . . . . 136

$4.2 .1 \quad$ Charge Resolution . . . . . . . . . . . . . . . . . . . . 143

$4.3 \quad$ Cross Talk . . . . . . . . . . . . . . . . . . . . . . . . . . . . . . 144

4.4 Neutralization and Timing Cut . . . . . . . . . . . . . . . . . 147

4.5 Charge Collection Efficiency . . . . . . . . . . . . . . . . . . . . 151

4.5 .1 Charge Breakdown . . . . . . . . . . . . . . . . . 153

$4.5 .2 \quad$ Charge Collection Efficiency Measurements . . . . . . . . . . . 153

$5 \quad 100 \mathrm{~mm}$ Detector Phonon Characterization 159

5.1 Phonon Channel Setup . . . . . . . . . . . . . . . . . . . . . . 160

5.1 .1 Tuning the SQUIDs . . . . . . . . . . . . . . . . 160

5.1 .2 Ib-Is and Transition Temperatures . . . . . . . . . . . . . . 162

5.2 Raw Phonon Pulse Studies ． . . . . . . . . . . . . . . . . . . . . . . . 168

$5.2 .1 \quad$ Constructing Relative Calibration . . . . . . . . . . . . . . 171

$5.2 .2 \quad$ Stability of the Relative Calibration . . . . . . . . . . . . 175

5.2 .3 Effects of the Relative Calibration . . . . . . . . . . . . . 179

$5.2 .4 \quad$ Constructing Non-Stationary Optimal Filter . . . . . . . . . . . 180

5.3 Reprocessing with Relative Calibration and NSOF . . . . . . . . . . . . 183

$5.3 .1 \quad$ Improvement to Raw Pulse Fitting . . . . . . . . . . . . . . . . . 184

5.3 .2 Phonon Quality Cuts . . . . . . . . . . . . . . . 187

$5.3 .3 \quad$ Phonon Spectra . . . . . . . . . . . . . . . . . . . . . . . . 189

$5.3 .4 \quad$ Ionization Yield with Surface Event Rejection . . . . . . . . . . . 193

$5.3 .5 \quad$ Fitting Yield Bands . . . . . . . . . . . . . . . . . 206

$5.3 .6 \quad$ Surface Event Rejection . . . . . . . . . . . . . . . . . . 207

$\begin{array}{|lll|}6 & 150 \mathrm{~mm} \text { Si Contact-Free Detector } & 214\end{array}$

$6.1 \quad$ Project Motivation . . . . . . . . . . . . . . . . . . . . . . . . . . . . 214 


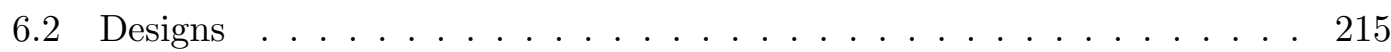

6.2 .1 Detector Designs $\ldots \ldots \ldots \ldots \ldots$

6.2 .2 Contact-Free Electrodes $\ldots \ldots \ldots \ldots$

6.2 .3 LEDs . . . . . . . . . . . . . . . . . . . 217

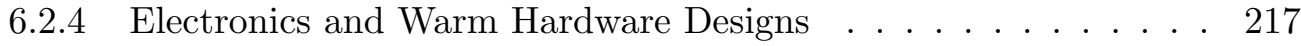

6.2 .5 Modifications to Inner Vacuum Chamber . . . . . . . . . . . 222

$6.2 .6 \quad$ Cryogenics and Data Run . . . . . . . . . . . . . . . . . 222

6.3 Results . . . . . . . . . . . . . . . . . . . . . . . . . . . 224

6.3 .1 Time Domain Fit. . . . . . . . . . . . . . . . . . . . . . . 224

$6.3 .2 \quad 17 \mathrm{kHz}$ Ringdown $\ldots \ldots \ldots \ldots \ldots \ldots \ldots \ldots \ldots$

6.3 .3 Quality Cuts . . . . . . . . . . . . . . . . . 228

6.3 .4 Leakage Current . . . . . . . . . . . . . . . . . . . 228

6.3 .5 Ionization Spectra $\ldots \ldots \ldots \ldots \ldots \ldots$

$6.3 .6 \quad$ Charge Collection Efficiency and Energy Resolution . . . . . . . 232

6.3 .7 Neutralization . . . . . . . . . . . . . . . . . . . 237

$6.4150 \mathrm{~mm}$ Contact-Free Initial Run Conclusions and Next Steps . . . . . . 240

$6.4 .1 \quad 150 \mathrm{~mm}$ Si 5Q Device . . . . . . . . . . . . . . . . . . . 240

6.5 Projected Sensitivity of $150 \mathrm{~mm}$ Si Detectors use at SuperCDMS SNOLAB 241 6.5 .1 Calculating Maximum Likelihood . . . . . . . . . . . . . . 241

6.5 .2 Assumptions . . . . . . . . . . . . . . . . . . . 244

6.5 .3 Results . . . . . . . . . . . . . . . . . . . . 249

7 Conclusion and Future Outlook 255

$\begin{array}{ll}\text { References } & 258\end{array}$

\begin{tabular}{|l} 
Appendix A. Acronyms, Variables, and Data Quantities \\
276
\end{tabular}

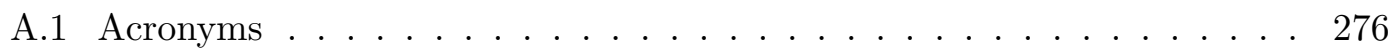

A.2 Variables . . . . . . . . . . . . . . . . . . . . . . 279

A.3 Data Quantities . . . . . . . . . . . . . . . . . . 286

Appendix B. CDMS Cross Talk Correction with OF Fitting Algorithm 289 


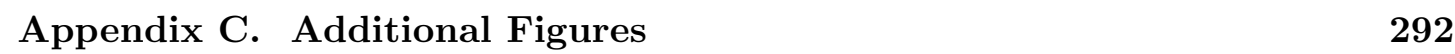

C.1 $I_{b}-I_{s}$ Figures for All Phonon Channels . . . . . . . . . . . . . . . . . . 292

C.2 Stability of Relative Calibration for Phonon Channels . . . . . . . . . . 301 


\section{List of Tables}

$1.1 \quad$ EFT Interaction Operators $\ldots \ldots \ldots$

3.1 Identified sources with peaks in the facility background spectra [189] . . 108

$3.2 \quad$ Elastic Scatter Legendre Polynomial Coefficients [mb/sr] . . . . . . . . . 112

3.3 Polyethylene Cross Section and Attenuation Coefficients . . . . . . . . . 116

3.4 Table of $100 \mathrm{~mm}$ devices tested at UMN . . . . . . . . . . . . . . . 128

$4.1 \quad$ G106a Charge Energy Resolutions for $\mp 4$ V Applied Bias . . . . . . . . 144

4.2 G106a $60 \mathrm{keV}$ Peak Decay $\ldots \ldots \ldots$. . . . . . . . . . . 150

$4.3 \quad$ G106a Charge Breakdown Study [207] . . . . . . . . . . . . . . . . . . 158

5.1 G106a Transition Temperatures [207] . . . . . . . . . . . . . . . . . . . 164

$5.2 \quad$ G106a Total Phonon Energy Resolutions for $\mp 4$ V Applied Bias . . . . . 193

5.3 Surface Event Rejection Efficiency for 10 - $100 \mathrm{keV}$ Events . . . . . . . . 213

A.1 Acronyms . . . . . . . . . . . . . . . . . . . 276

A.2 Variables . . . . . . . . . . . . . . . . . . . . . . . . 279

A.3 Data Quantities . . . . . . . . . . . . . . . . 286 


\section{List of Figures}

1.1 Superposition of 21 Sc galaxy rotation curves. Figure courtesy of [2]. . . 3

1.2 Planck 2015 band power spectrum. In upper panel, the best-fit base theoretical spectrum (red) is fitted to the Planck data (blue, $\pm 1 \sigma$ error bars), while lower panel shows residuals with respect to the model. Figure courtesy of $9 . \ldots \ldots \ldots \ldots \ldots$

1.3 (a) Optical image of bullet cluster with weak lensing mass contours. (b) X-ray image with overlaid mass contours, with the gas lagging behind negligibly interacting galaxies and dark matter. Figure courtesy of [16]. 11

1.4 Selected exclusion limits on axion masses $\left(m_{A}\right)$ and couplings to two photons $\left(g_{A \gamma}\right)$, from a variety of sources. Dark-matter axions lie between the two classes of axion models, KSVZ (blue dotted) and DFSZ (magenta) in the mass range $\sim 1-100 \mu \mathrm{eV}$. Figure courtesy of [29]. . . . . . . . 14

1.5 Sectors of the WIMPless model, with gauge-interaction mediated SUSY breaking to the MSSM and hidden (dark) sector, containing dark matter particle X. Additional hidden sectors and dark matter components could also be present. Optional connecting sector containing particle(s) Y, which is (are) charged under both the MSSM and the dark sector. Figure courtesy of $[44] . \ldots \ldots \ldots 18 \ldots \ldots$ 
1.6 The $\left\{m_{1 / 2}, m_{0}\right\}$ parameter space for two cases: (LEFT) $\tan \beta=10$, $\mu>0, A_{0}=0$ and (RIGHT) $\tan \beta=30, \mu>0$, assuming $A_{0}=2.3 m_{0}$.

Contours include constant $m_{h}$ (dot-dashed red) and $M_{\chi^{ \pm}}=104 \mathrm{GeV}$ (dashed black). Shaded exclusion regions include stau LSP (bottom brick red) and stop LSP (RIGHT plot, top brick red) with neutralino LSP in between, $b \rightarrow s \gamma$ (dark green), $B \rightarrow \mu^{+} \mu^{-}$(light green line), which also excludes everything to its left. Preferred regions of $\left(g_{\mu}-2\right)$ (pink shaded $2 \sigma$, dashed back line $1 \sigma)$, and LSP density determined by Planck [9] (blue). The regions to the left of the 95\% C.L. LHC curves (dark pink), are excluded by LHC data. Figure courtesy of [53]. . . . . . . . . . 20

1.7 Post-LHC Run I Likelihood contours for spin-independent cross section, $\sigma_{p}^{\text {SI }}$ in mSUGRA paradigm. Best fit (green star), and $1 \sigma$ (red) and $2 \sigma$ (blue) contours shown, with shaded region color indicating dominate annihilation mechanism for obtaining correct relic density: stau coannihilation (pink), A-funnel (blue), focus point (cyan), stau co-annihilation/Afunnel hybrid (purple) and chargino co-annihilations (green). Current sensitivities of XENON100 [57] and LUX [58] experiments shown in black and green lines, and projected 95\% C.L. sensitivities of LUX-Zepelin (LZ) experiment [141] shown in purple line. Neutrino floor shown by dashed orange line, indicating the region below which astrophysical neutrino backgrounds dominate (shaded yellow). Figure courtesy of [56].

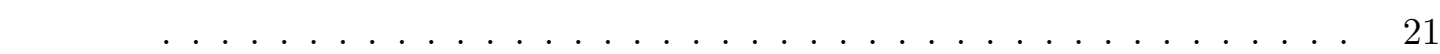

1.8 H.E.S.S. velocity-averaged annihilation cross section, $\langle\sigma v\rangle$, limits at $95 \%$ C.L. (red points) for the line scan between $100 \mathrm{GeV}$ and $2 \mathrm{TeV}$, with MC estimations (red solid, red dashed, light blue). Former limits from H.E.S.S. I [67] (blue points), and Fermi LAT [68] (black) also shown. The $130 \mathrm{GeV}$ line feature $\langle\sigma v\rangle$ value (green) is from Fermi LAT [66]. Figure courtesy of $[65] . \ldots \ldots \ldots \ldots \ldots$ 
1.9 Exclusion limits from IceCube [70] (solid line), Super-Kamiokande [75] (dashed), and ANTARES [74] (dotted) neutrino telescopes, for $b b$ (blue), $W^{+} W^{-}$(green), and $\tau^{+} \tau^{-}$annihilation channels. Also included are direct detection limits from XENON100 [57, PandaX-II [78, and LUX

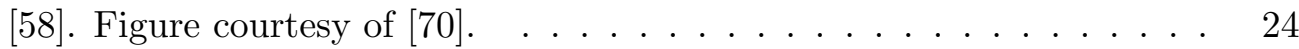

1.10 Schematics of the PICO-60 and PICO-2L bubble chamber experiments. Figures courtesy of $[113]$ and [111]. . . . . . . . . . . . . 35

1.11 Cartoon demonstrating the working principle of a dual-phase LXe TPC. Top and bottom PMTs measure prompt scintillation light (S1) generated in LXe, and delayed secondary signal (S2) induced by ionization electrons in the gas phase. Figure courtesy of [118]. . . . . . . . . . . . . . 36

1.12 Cartoon demonstrating the working principle of CCD dark matter direct detection, with WIMPs coherently scattering off of silicon nuclei in the bulk of the CCD, producing ionization from the nuclear recoil which drifts along the z-direction and is collected at the CCD gates. Figure courtesy

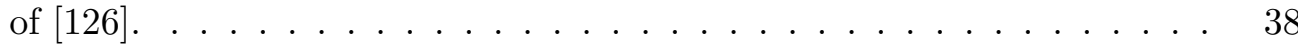

1.13 Current experimental limits (solid lines), projected sensitivities (dashed lines), and candidate regions (shaded) on spin-independent cross sections as a function of WIMP mass for various experiments. Current limits include second CDMSlite run [136, SuperCDMS low threshold [125], SuperCDMS high threshold [137], SIMPLE [109], PICO-2L [112], PICO-60 [114], XENON100 [117], PandaX-II [78], LUX [138], XENON1T [120], CRESST-II [139], DAMIC [140], and EDELWEIS-III [130]. Projected sensitivities include XENON1T [119], DAMIC100 [127], LZ [141], and SuperCDMS SNOLAB Si iZIP, Si HV, Ge iZIP, and Ge HV [135]. Closed contours shown include CRESST-II [142] (pink), and DAMA/LIBRA [143] (tan) and CDMS II Si [144] (blue). The dark matter discovery limit [145] (dotted orange line over yellow region) represents the cross section at which the interaction rate from dark matter particles becomes comparable to the solar neutrino coherent elastic scattering rate. . . . . 40

2.1 Layout of the CDMS I experimental setup at the Stanford Underground Facility. Figure from [149]. . . . . . . . . . . . . . . . . . . . . 43 
2.2 Four BLIP detectors next to a CDMS tower. . . . . . . . . . . . . . . . 44

2.3 A cross sectional view seen from the side (TOP) and top-down (BOTTOM) of the CDMS experiment layout at Soudan Underground Laboratory. Cryostat, electronic and cold stems shown in brown/copper polyethylene shown in green, (ancient) lead shown in (light) grey, and muon veto shield shown in light blue. Figure courtesy of A. Villano and ‥Sanders . . . . . . . . . . . . . . . . . . . . . . 46

2.4 CDMS II towers in Soudan cryostat. Cold hardware (discussed in Section 3.1) is installed on the upper right tower. . . . . . . . . . . . . . 48

2.5 The SuperCDMS low threshold 90\% confidence upper limit (solid black) shown with 95\% C.L. systematic uncertainty band [125] the second CDMSlite run (gray solid). The expected sensitivity in the absence of a signal is shown as 68\% (dark green) and 95\% (light green) C.L. bands. Closed contours shown are CDMS II Si 144 (dotted blue), CoGeNT [163 (yellow), CRESST-II [142] (dashed pink), and DAMA/LIBRA [143] (dash-dotted tan) preferred parameter space. The 90\% C.L. exclusion limits shown are CDMS II Ge [132] (dotted dark red), CDMS II Ge low threshold [164 (dashed-dotted red), CDMSlite [134] (solid dark red), LUX [58] (solid green), XENON10 S2 only [165] (dashed dark green), and EDELWEISS low threshold [129] (dashed orange). Figure from [125]. . . . . . . . . . 51

2.6 Median (90\% C.L.) and 95\% interval of the WIMP limit from the second CDMSlite run [136] (black thick solid curve surrounded by salmonshaded band) compared to other selected results. Other $90 \%$ upper limits shown are from the first CDMSlite run [134] (red thin solid curve), SuperCDMS [125] (red thin dashed curve), EDELWEISS-II [129] (red thin dotted curve), LUX [58 (dark-yellow thick dot dashed curve), CRESST [139] (magenta thick dashed curve), and DAMIC [140] (purple thick dotted curve). Closed regions are CDMS II Si 90\% C.L. [144] (blue dashed shaded region), and CoGeNT 90\% C.L. [163] (dark-green shaded region).

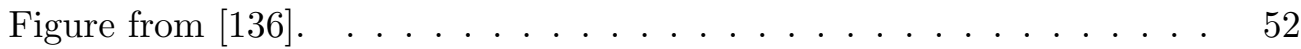

2.7 CDMS II oZIP (LEFT) and SuperCDMS iZIP (RIGHT) detectors, shown in copper housings. . . . . . . . . . . . . . . . . . . . . . . 54 
2.8 Phonon and ionization channel layout for oZIP (LEFT) and iZIP (CENTER and RIGHT) detectors. Four quadrant phonon channels are deposited on to one oZIP detector face, and the other detector face comprises of the inner disk and outer ring ionization electrodes. The iZIP detector features four phonon sensors, and inner disk and outer ring ionization electrodes per face, with a $60^{\circ}$ offset between iZIP phonon layout on top and bottom faces, to aid in position reconstruction. Figure modified from A. Phipps [167] . . . . . . . . . . . . . . . . . . 55

2.9 LEFT: Interleaved ZIP design, with alternating phonon TESs held at 0 $\mathrm{V}$ and ionization electrodes biased at $\pm 2 \mathrm{~V}$. RIGHT: Electric field lines across the crystal due to interleaved sensor layout. Electron/hole pairs created in the bulk of the detector are collected on both sides of the detector, whereas electron/hole pairs produced near the detector surface $(\sim$ $1 \mathrm{~mm}$ ) are collected only on one side. Holes will propagate along field lines, whereas electrons will propagate obliquely due to the electrons having an anisotropic effective mass, expressed as a tensor. Figures courtesy of [162] and S. Hertel [169]. . . . . . . . . . . . . . . . . . . 57

2.10 TOP: iZIP sensor schematic with zoom of charge electrodes interleaved with phonon QETs. BOTTOM: Schematic of QET chain, showing portions of two QETs, each with eight $300 \mu \mathrm{m} \times 55 \mu \mathrm{m}$ aluminum fins (blue), amorphous silicon (green), and $250 \mu \mathrm{m} \times 1 \mu \mathrm{m}$ tungsten strip (red), connected via Al bias rail (pink). . . . . . . . . . . . . 58

2.11 Simplified ionization channel read-out circuit. Voltage bias $V_{B}$ is applied to the detector with capacitance $C_{D} \approx 100 \mathrm{pF}$, after passing through biasing resistors with $R_{B}=40 \mathrm{M} \Omega$. A coupling capacitor $C_{C}=300$ $\mathrm{pF}$ blocks the biasing voltage from the feedback loop, comprised of a JFET amplifier kept at $150 \mathrm{~K}$, a feedback resistor $R_{F}=40 \mathrm{M} \Omega$, and parasitic feedback capacitor $C_{f}=1 \mathrm{pF} . C_{p}$ represents any stray parasitic capacitance. . . . . . . . . . . . . . . . . 6 60 
2.12 Cartoon depicting the process of quasiparticle trapping in a QET. Phonons (dark purple) enter the superconducting $\mathrm{Al}$ (green) fins containing Cooper pairs (depicted as blue circles enclosed in yellow ovals). If the phonons have sufficient energy $\left(>2 \Delta_{A l}\right)$, they can break up Cooper pairs into quasiparticles (dark orange circles). Quasiparticles will diffuse through the Al traveling from high to low energy regions (depicted in top graph), entering the $\mathrm{Al} / \mathrm{W}$ overlap region, and eventually the W TES (red). As the quasiparticles diffuse they will lose energy, depicted as the gradient transition from dark orange to yellow. During these processes new phonons may be created with energy $<2 \Delta_{A l}$ (light purple), which will not break up Cooper pairs and are considered lost energy. . . . . . . . . 66

2.13 Simplified single phonon channel read-out circuit. Shunt resistor $R_{s h} \approx$ $20 \mathrm{~m} \Omega$, and TES with variable resistance $R_{T E S} \approx 100-200 \mathrm{~m} \Omega$ draws stable current $I_{b}$ from applied voltage bias $V_{b}$. When an event occurs, the TES temperature and thus $R_{T E S}$ increases, which changes the value of current, $I_{s}$, passing through $R_{T E S}$ and the 10-loop input coil with $L_{i}=250 \mathrm{nH}$. The input coil is coupled to the one-loop feedback coil with $L_{f b}=25 \mathrm{nH}$ through the SQUID array, yielding feedback current $I_{f b}=10 * I_{s}$, due to the 10:1 loop ratio between coils. The current through $L_{f b}$ is then read out as an output voltage $V_{o}$ after passing through a feedback resistor $R_{f b}=1 \mathrm{k} \Omega$ and undergoing amplification. . . . . . . . 69

2.14 A plot of ionization yield versus recoil energy for oZIP detector ${ }^{252} \mathrm{Cf}$ calibration data (yellow points). Data is mostly divided into the electron recoil band (red) with an ionization yield around 1, and nuclear recoil band (blue) with a reduced ionization yield around 0.3. A typical charge threshold band (green) and recoil energy threshold (dotted black) are also included. Figure courtesy of A. Villano. . . . . . . . . . . . . . 71

2.15 Muon flux versus depth (mwe) for various underground facilities, including the Stanford Underground Facility (SUF), Soudan Underground Laboratory, and SNOLAB (Sudbury). Figure courtesy of J. Cooley. . . . . . 74

2.16 Schematic of SuperCDMS SNOLAB experiment setup, including shielding. Figure from [135]. . . . . . . . . . . . . . . . . 74 
2.17 Projected sensitivity for the SuperCDMS SNOLAB experiment. Sensitivity projections for Si iZIP (dashed light blue), Si HV (dashed dark blue), Ge iZIP (dashed light red) and Ge HV (dashed dark red) are de-

termined using the optimum interval method [181][182]. Solid lines are

the current experimental exclusion limits in the low-mass WIMP region,

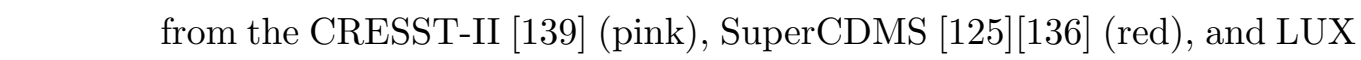
[138] (green) experiments. The dark matter discovery limit [145] (dotted orange line over yellow region) represents the cross section at which the interaction rate from dark matter particles becomes comparable to the solar neutrino coherent elastic scattering rate. Figure from [135]. . . . . 76

$3.1 \quad$ A cartoon depicting the dilution refrigeration process. . . . . . . . . . 79

3.2 A cartoon depiction of the current UMN test facility lab setup. In the upper left corner (SW corner of lab) the K100 dilution fridge is supported by a roughly $1.35 \times 2.16 \times 2.18 \mathrm{~m} \mathrm{Al}$ infrastructure. To the left is the Intelligent Gas Handling (IGH) unit, used to control the circulation of mixture. Copper and steel lines connect the fridge and IGH to circulation $\left({ }^{3} \mathrm{He}\right)$ and auxiliary $\left({ }^{4} \mathrm{He}\right)$ pumps in the pump room. Turbo and rough pumps in front of the fridge are used to pump out the Inner Vacuum Can (IVC) during cooldown. Detector interface and readout is done at the DAQ station, in front of the fridge. The Class 10,000 clean room resides in the bottom right corner (NE corner of lab). It contains a Class 1000 clean bench for electrical checks and tower installation. The clean room also contains two N2 purge cabinets, housing detectors, towers, and other various cold hardware. Figure modified from H. Rogers. . . . . . . . . . 83

3.3 Various layers of the UMN detector testing facility K100 dilution refrigerator. (LEFT) The inside of the fridge, with inner vacuum cannisters removed. (CENTER) Inside of dilution fridge with inner vacuum cannisters sealed and inner polyethylene shielding. (RIGHT) Main bath dewar raised around cryostat. . . . . . . . . . . . . . 85

$3.4 \quad$ G106a $100 \mathrm{~mm}$ iZIP detector inside copper housing, with top lid removed to show iZIP mask, cirlex clamps, and wirebonds connecting channels to Al bonding pads on DIB. . . . . . . . . . . . . . . . 87 
3.5 A $100 \mathrm{~mm}$ detector with source holder installed on a CDMS II tower. Three vacuum side coaxes connect the detector housing DIBs to the base of the tower, using extender plugs. The tower thermometer wire emerges from the source holder and is wound about the tower spool before passing through a hole on the adapter plate. The adapter plate will secure the tower to the base of the $30 \mathrm{mK}$ can. . . . . . . . . . . . . . . . . . . . 89

$3.6 \quad$ A photo and circuit diagram of a CDMS SQUET card. . . . . . . . . . . 91

3.7 (LEFT) A bottom-up view of a single SQUET card and stripline attached to the CDMS II tower at the $4 \mathrm{~K}$ stage. (RIGHT) A side view of three SQUET cards and striplines installed at the $4 \mathrm{~K}$ stage inside the opened IVC. . . . . . . . . . . . . . . . . . . . 92

3.8 Three sample pulses of each channel on a single DIB, demonstrating the RevC DCRC pulse shape (TOP ROW) without a low-pass pi-filter between the DCRC and Ebox, (MIDDLE ROW) with a low-pass pi-filter \begin{tabular}{|c|}
\hline between the DCRC and Ebox, (BOTTOM ROW) with a low-pass pi- \\
\hline
\end{tabular} filter and a cable between horizontally resting DCRC and Ebox. Images taken with Labview data acquisition software. . . . . . . . . . . . . . . 95

3.9 A comparison of Soudan (green and yellow) and UMN facility (orange and purple) noise PSDs for (TOP) charge and (BOTTOM) phonon channels. The PSDs represent the noise level at the feedback capacitor of charge readout circuit (see Fig. 2.11) and the input coil of the phonon readout circuit (see Fig. 2.13t. . . . . . . . . . . . . . . . . . . . . . . . . . . . 97

3.10 A total phonon raw pulse (blue, TOP) from UMN test facility data undergoes the basic optimal filter algorithm (reproduced here in Matlab for clarity). The resulting $\hat{a}\left(t_{0}\right)$ amplitude function (red, BOTTOM) is searched within a designated window region (highlighted in yellow). The maximum amplitude (blue circle), is selected as the amplitude RQ, with corresponding bin shift. In this particular example, this bin shift of 4053 bins will be converted into a negative shift time before it is saved as the PTOFdelay RQ value. . . . . . . . . . . . . . . . . . . 102 
3.11 (TOP) Gamma-ray spectra results from A. Emerick and K. Weir, with various direction-specific lead shields. This figure does not take detector geometry into account. Once geometry is accounted for, no directional component to the gamma-ray background was found. Figure from [189]. (BOTTOM) A comparison of the gamma-ray spectrum from Tate Labs (blue) and from the Physics and Nanotechnology building (red). . . . . 109

3.12 Incoming neutron energy spectrum for the Soudan cavern housing SuperCDMS. Monte Carlo simulation results by A. Reisetter. Figure modified from R. Radpour. . . . . . . . . . . . . . . . . . . . . . . . . 110

3.13 Differential Neutron Flux Measurements for Neutron energy spanning 1 $-10^{4}$ MeV. Differential flux is normalized to New York, NY at sea level. Measured values (black dots) are fit with two models (black solid and dashed lines). Figure courtesy of [192]. . . . . . . . . . . . . 110

3.14 Neutron differential elastic scattering cross sections of Ge in the lab frame. Dotted blue curve is the best Legendre polynomial fit to the experimental points (red). Cross section data from [195]. . . . . . . . . . . . . . . 112

3.15 Differential neutron interaction rate $d \eta_{n} / d E_{n}$ for a $100 \mathrm{~mm}$ Ge iZIP when a full range of recoil energies is considered (salmon), compared to $d \eta_{n} / d E_{n}$ constrained to nuclear recoils in the $10-100 \mathrm{keV}$ energy range (teal). . . . . . . . . . . . . . . . . . . . . . . . . . . 114

3.16 Comparison between unattenuated differential neutron interaction rates, and neutron rates when attenuated by a $20 \mathrm{~cm}$ thick polyethylene shield. Unattenuated $d \eta_{n} / d E_{n}$ for all $E_{R}$ (salmon) and for $E_{R} \in[10 \mathrm{keV}, 100$ $\mathrm{keV}$ ] (teal) continue on for higher neutron energies, whereas attenuated $d \eta_{n} / d E_{n}$ for all $E_{R}$ (orange) and for $E_{R} \in[10 \mathrm{keV}, 100 \mathrm{keV}]$ (navy) are effectively null for $E_{n}>5 \mathrm{MeV} . \ldots \ldots \ldots 117$ 
3.17 (LEFT) Incident photon energy spectra with various thicknesses of pure lead shielding. No shielding spectrum (black) recorded with HPGe detector. Lead shielding spectra (blue, red, and magenta) predicted with Monte Carlo simulation, assuming full solid angle coverage. (RIGHT) Cross-sections for gamma interactions in lead, used to predict the lead shield attenuation. For the $0-0.6 \mathrm{MeV}$ incident phonon energy region, photoelectric absorption is the dominant process, with sharp edges at the K-shell, L-shell, and M-shell binding energies. In the 0.6 - $5 \mathrm{MeV}$ region Compton scattering becomes the dominant process. Figures courtesy of H. Chagani. . . . . . . . . . . . . . . . . . . . . . . . . 118

3.18 (LEFT) High-purity germanium (HPGe) detector in partially-constructed lead and polyethylene shielding mounted on aluminum frame on rolling platform. HPGe detector was used to study the attenuation factor of the lead gamma-ray shielding. (RIGHT) Fully constructed lead and polyethylene shielding, before gamma-ray shielding modifications. . . . . 120

3.19 Gamma-ray background spectra taken with HPGe detector, demonstrating the effectiveness of the lead shield with various modifications. . . . . 121

3.20 (LEFT) Nine inner layers of 12" diameter, 1" thick polyethylene shielding supported by baffles. At the top, additional polyethylene supports four layers of 4" diameter, 1/8" thick lead. (BOTTOM) Neutron and gamma shield erected around main bath, including 8 " of polyethylene and 0.5 " lead top shielding between main bath and shield walls. Lead top shielding stacked atop polyethylene not shown. . . . . . . . . . . . . . . . . . 122

3.21 G106a side 1 and side 2 phonon channel layout overlaid on $100 \mathrm{~mm}$ iZIP mask. A zoom in on the phonon mask shows $100 \mathrm{~mm}$ iZIP QET design, with tungsten (green) TES strip overlapping aluminum fins (blue), all deposited on a layer of amorphous silicon (pink). . . . . . . . . . . 127 
4.1 (LEFT) A charge template constructed by N. Mast from a thousand well \begin{tabular}{|c|c|c|}
\hline behaved charge pulses. Template has typical $400 \mu \mathrm{s}$ (100 bins) pre-trigger \\
\hline
\end{tabular} region, a roughly $1 \mu$ s rise time and $40 \mu$ s fall time. Figure courtesy of N. Mast [201]. (RIGHT) A raw pileup QIS1 event (blue) is fit with a QIS1 template (red) using the OF fitting algorithm. Although the first pulse is reconstructed well by the fit, the second event is completely neglected. As a result, the $\chi^{2}$ of this fit will be high, and the event will be excluded. 131

4.2 Charge $\chi^{2}$ versus Side 1 (LEFT) and Side 2 (RIGHT) calibrated ionization energy for G106a with $\mp 4 \mathrm{~V}$ applied bias. Events which pass the $\chi^{2}$ cut (red) fall below the parabolic cut line (cyan), and events which fail the cut (blue) fall above the line. An event must pass the cut on both detector sides to be considered a well constructed event. . . . . . . . . 132

4.3 Distributions (blue) of Side 1 (LEFT) and Side 2 (RIGHT) inner charge amplitude distributions of beginning-of-run random events. A Gaussian (green) is fit to the positive portion of the distribution, to estimate the mean and width. A threshold cut (red) is made $5 \sigma$ beyond the mean. . 133

4.4 A COMSOL [202] simulation showing the electric field lines (black) and potential (color scale) of a Ge detector bias $\pm 50 \mathrm{~V}$ with a 30 mil gap

between the crystal and the copper housing. Note the nonuniform electric
field lines at the crystal's edge. Figure courtesy of N. Kurinsky [203]. . . 134

4.5 Charge outer amplitude (with zero time shift) versus inner amplitude for Side 1 (LEFT) and Side 2 (RIGHT). The mean and standard deviation of the outer amplitude are found for $10 \mathrm{keV}$ bands in inner charge amplitude, and a third-order polynomial function is fit for the mean outer channel \pm twice the standard deviation (cyan). Events within the polynomial fits (red) pass the cut, while events that fail (blue) are removed. . . . . . . 135

4.6 Distribution of mean-subtracted baseline values for (LEFT) Qi Side 1 and (RIGHT) Qi Side 2 after $\chi^{2}$ cut is applied. Distributions are fit with a Gaussian (green), and baseline thresholds are set to $\pm 3 \sigma$ (black). Baselines within the thresholds are kept (red), while events with baselines outside the thresholds are removed (blue). . . . . . . . . . . . . . 136 
4.7 (LEFT) The $60 \mathrm{keV}$ peak of a Qsum1 spectrum (blue), fitted with a Gaussian (to fit peak) plus an exponential decay (to fit low energy background) (red). This fitting method is used for calibration and energy resolution studies. (RIGHT) Qsum2 versus Qsum1 demonstrates the incomplete charge collection of surface events. An ${ }^{241} \mathrm{Am}$ source held near Side 1 of the detector produces a $60 \mathrm{keV}$ peak in Qsum1. Due to incomplete charge collection, Qsum2 sees a source peak smudged out from 60 $\mathrm{keV}$ down to $0 \mathrm{keV} .60 \mathrm{keV}$ peak smearing in Qsum1 is due to gammas that escape after depositing a portion of their energy. Gammas are more likely to scatter and escape near the surface, which explains why $60 \mathrm{keV}$ peak smearing in Qsum1 is more apparent for the smallest Qsum2 values. Events which occur in the bulk of the detector should deposit equal \begin{tabular}{|c|c|}
\hline energy onto side 1 and side 2 ionization channels. These bulk events can \\
\hline
\end{tabular}

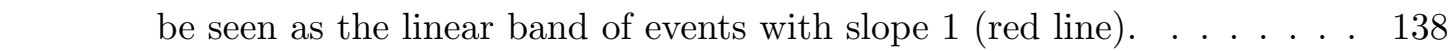

4.8 (LEFT) The nonlinearity of Pt with respect to the mean of QiS1 and QiS2, is made apparent by comparing Pt versus Qimean (red) to a line of slope one (blue). Fitting a fourth order polynomial (black) to the mean Pt values for slices of Qimean energy (green circles) yields a nonlinear correction. (RIGHT) Applying the nonlinear correction to the original \begin{tabular}{|c|}
\hline data (red) yields data with a linear relationship between Pt and Qimean \\
\hline
\end{tabular} (blue), which follows a linear trend with slope of one (green). . . . . . . 141

4.9 Qsum1 and Qsum2 spectra for (TOP) $\mp 2 \mathrm{~V},($ TOP MIDDLE) $\mp 4 \mathrm{~V}$, (Bottom MIDDLE) $\pm 2 \mathrm{~V}$ (Bottom ) $\pm 4 \mathrm{~V}$ applied bias, calibrated using $60 \mathrm{keV}$ peak. Visible peaks include the $10.36 \mathrm{keV}$ peak from activation with ${ }^{252} \mathrm{Cf}$, the ${ }^{241} \mathrm{Am} 60 \mathrm{keV}$ peak, and the ${ }^{133} \mathrm{Ba} 356 \mathrm{keV}$ peak. Each spectrum is comprised of $2-5$ data series, with $\chi^{2}$ cut applied to remove poorly fit events. . . . . . . . . . . . . . . . . . . . . . 142 4.10 Qimean $10.36 \mathrm{keV}$ peak width $(1 \sigma)$ versus applied bias. . . . . . . . . 145 
4.11 (LEFT) Cross talk is demonstrated here in the outer channel signal amplitude's near-linear dependence on the signal amplitude of the inner channel, for both (TOP) Side 1 and (BOTTOM) Side 2. A rotation is applied to correct for cross talk, with the angle of rotation determined by fitting a line (green) to the linear trend in Qo versus Qi (blue). (RIGHT) Qo versus Qi for (TOP) Side 1 and (BOTTOM) Side 2, with the cross talk correction rotation applied. . . . . . . . . . . . . . 146

4.12 Side 1 Charge amplitude versus time for $\pm 1 \mathrm{~V}, \mp 1 \mathrm{~V}, \pm 2 \mathrm{~V}, \mp 2 \mathrm{~V}, \pm 4 \mathrm{~V}$, and $\mp 4 \mathrm{~V}$ data. Plots are comprised of several series, typically of length 15 or 30 minutes, with standard ionization quality cuts applied. The 60 keV peak can be seen as a distinct line, which begins to droop, indicating a loss of crystal neutralization. . . . . . . . . . . . . . . . . . . . 148

4.13 Two $\pm 1 \mathrm{~V}$ data series are shown in this qsum1 versus time plot. Loss of neutralization, indicated by the dropping $60 \mathrm{keV}$ event line, is apparent within $<10$ minutes of each series. Fortunately, grounding, flashing and cooling the detector, which occurs in the blank, event-less spaces between data series, neutralizes the crystal. As a result, the $60 \mathrm{keV}$ peak is returned to its original amplitude at the start of each series. . . . . . 149

4.14 (TOP) A clean signal observed on Side 2 channel D with a $-11.5 \mathrm{~V}$ bias applied. (BOTTOM) Distortions typical of charge breakdown on Side 2 channel $\mathrm{D}$, once the bias has been increased to $-12.0 \mathrm{~V}$. . . . . . . . 153

4.15 Charge collection efficiency for (TOP) Side 1 and (BOTTOM) Side 2 of G106a, using Run 54 bias sweep data. Efficiency measurements were taken with the $60 \mathrm{keV}$ peak, with (blue) and without (Qsum1 only, green) a charge symmetry cut applied, and with the $356 \mathrm{keV}$ peak (red). Due to events mainly occurring in the bulk, the $356 \mathrm{keV}$ measurements show a generally symmetric results between bias polarity. . . . . . . . . . . . 155 
4.16 Previous charge collection efficiency studies, from G106a UMN Run 39 (cyan) and $76 \mathrm{~mm}$ diameter, $2.54 \mathrm{~cm}$ thick Ge ZIP G3D (black), are compared to Run 54 results (60 keV peak in blue, $356 \mathrm{keV}$ peak in red). \begin{tabular}{|c|}
\hline Run 39 and 54 studies using the $60 \mathrm{keV}$ peak match nicely for fields \\
\hline
\end{tabular} greater than $\pm 0.5 \mathrm{~V} / \mathrm{cm}$, indicating repeatable results. When source location is taken into account, the larger detector volume of G106a does not demonstrate ill effects on charge collection efficiency when compared to G3D results, for fields greater than $\sim \pm 1 \mathrm{~V} / \mathrm{cm} . \ldots . . . . .156$

$5.1 \quad$ SQUID tuning setup schematic. Modified from D. Seitz. . . . . . . . . . 161

$5.2 \quad I_{b}-I_{s}$ measurement for channel PE Side 1 (D3) of G106a, with super\begin{tabular}{|c|}
\hline conducting region highlighted in red, and normal regions highlighted in \\
\hline
\end{tabular} \begin{tabular}{l}
\hline blue (negative $I_{b}$ ) and magenta (positive $I_{b}$ ). Fitting the linear super- \\
\hline conducting and normal regions allows for estimation of the parasitic and \\
\hline normal resistances of the sensor. The fits and estimated resistance values \\
\hline
\end{tabular}

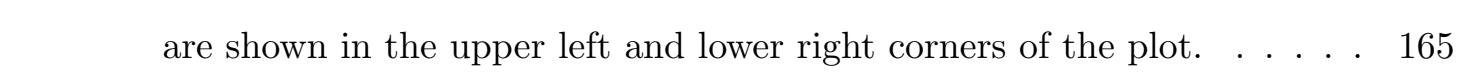

$5.3 \quad R_{s}$ plotted versus $I_{b}$ for G106a phonon channel PE Side 1 (D3). Plot \begin{tabular}{|c|}
\hline shows sensor resistance increasing from zero in the superconducting re- \\
\hline gion and plateauing towards the positive and negative QET bias $R_{n}$ val- \\
\hline
\end{tabular} ues. Positive and negative QET bias curves are fit to the data, shown in

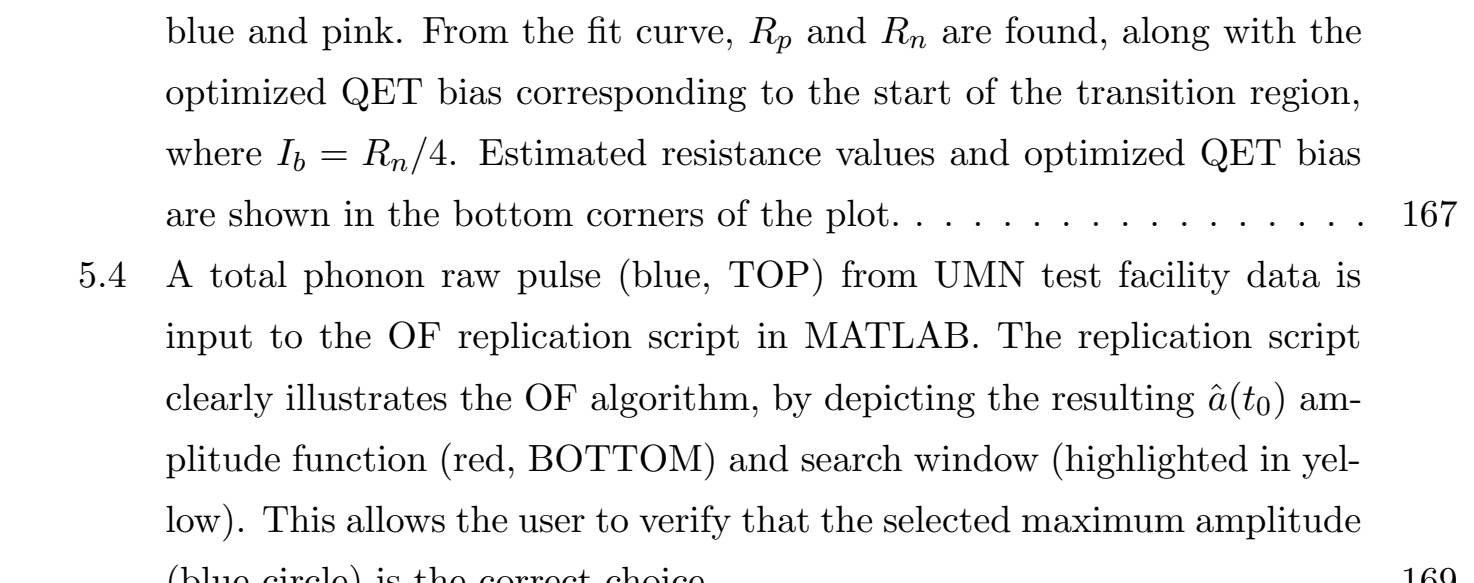
(blue circle) is the correct choice. . . . . . . . . . . . . . 169 
5.5 Total phonon templates used for UMN test facility OF processing. The previous template (red) did not accurately represent raw pulses, and was thus replaced by a new template (black), created from averaging a thousand well-behaved raw events. . . . . . . . . . . . . . . . . . . . . . . . 170

$5.6 \quad$ A reconstructed event, using the old and newly constructed total phonon template, to the raw pulse (purple). The fit constructed using the RQ value calculated in CDMSBats is shown in solid maroon and navy lines for the old and new template, respectively. The fit constructed using the MATLAB algorithm is shown in dotted pink and light blue lines for the old and new template, respectively. . . . . . . . . . . . . . . . . 171

5.7 The twelve phonon channel pulses from an example event without relative calibrations applied. Peak tails $(\sim 800 \mu$ s after rising edge $)$ containing signal from collected athermal phonons are not aligned. . . . . . . . . . 172

5.8 The phonon (TOP) and charge (BOTTOM) channel traces for a postmuon event. Charge channels have already recovered from the muon recoil event, and have no noticeable disfigurations. The phonon channels are still collecting thermalized phonons from the muon event when the post-muon event occurs, resulting in a downward sloping prepulse region typical of post-muon phonon traces. . . . . . . . . . . . . . . . . 174

5.9 (TOP) Calibration values over time for all channels with respect to base channel PBS1. (BOTTOM) Calibration value deviation from mean over time for all channels with respect to base channel PBS1. Series used include those of a 15 hour data taking period in Run 54. . . . . . . . . 176

5.10 (TOP) Calibration values and (BOTTOM) values deviation from mean over time for all channels with respect to base channel PBS1. Includes data taken over the whole, 1-month, Run 54 data taking period. . . . . 178

5.11 Spectra of Psum (blue), PsumC calibrated using the individual series factors (red), and PsumC calibrated the mean of the series factors (green) for seventy Run 54 data series. Both $10.36 \mathrm{keV}$ activation peak and 60 $\mathrm{keV}$ source peak are visible. Absolute calibration applied to align 10.36 keV peak. . . . . . . . . . . . . . . . . . . . 179 
5.12 Fitting the (LEFT) $10.36 \mathrm{keV}$ activation peak and (RIGHT) $60 \mathrm{keV}$ source peak for uncalibrated Psum (blue), PsumC calibrated using the

\begin{tabular}{|c|}
\hline individual series factors (red), and PsumC calibrated the mean of the \\
\hline
\end{tabular}
series factors (green). . . . . . . . . . . . . . . . . 180

5.13 A sample of raw pulses (LEFT) before and (RIGHT) after they are shifted over by their time shift RQ value (converted to ADC bins). With the pulses all correctly aligned to start at the 500th bin, the pulses can be used to construct the covariance matrix. . . . . . . . . . . . . . . . . . 181

5.14 (LEFT) A sampling of a hundred raw, total phonon events, normalized to a maximum amplitude of one. Although they appear similar in shape, a zoom-in near the peak shows the subtle differences in the rising edge of each event. The mean of each event is shown in black. (RIGHT) The discrepancies in each peak's rising edge are made more apparent in the trace residuals, the remainder once the mean has been subtracted from the pulse. These residuals are a result of position dependence in the event. They are treated as noise and de-weighted in the Non-Stationary Optimal Filter processing algorithm. ～. . . . . . . . . . . . . . . 182

5.15 From the initial randoms taken at the start of each series, a PSD is found on pulse-less (only noise) traces. As input into covariance constructing script, the stationary noise PSD (green) is subtracted from the total pulse variations including noise (black). What remains is the pulse variations only (blue), without the presence of stationary noise. . . . . . . . . . 183

5.16 Comparing the NSOF (dotted pink) and standard OF (solid green) template fits for two typical raw total phonon pulses of energy $14 \mathrm{keV}$, and $73 \mathrm{keV}$, respectively. Comparing fits by eye indicates a superior fit by the NSOF algorithm. . . . . . . . . . . . . . . . . . . . . . 185 
5.17 The ratio of the template fit integration over the raw pulse integration value, for both the NSOF (pink solid line) and the OF (green solid line) total phonon RQ's. Gaussian fits to PTNFamp distribution (pink dotted $)$ has mean of $1.0360( \pm 0.001)$ and resolution of $4.576 \mathrm{e}-2( \pm 0.076 \mathrm{e}-$ 2). This is a significant improvement in pulse reconstruction accuracy and precision when compared to the PTOFamp distribution Gaussian fit (green dotted), with a mean of $1.071( \pm 0.001)$ and resolution of $6.670 \mathrm{e}-$ $2( \pm 0.142 \mathrm{e}-2) . \ldots \ldots \ldots \ldots \ldots$. . . . . . . . . . . . . . . . . . . . . . .

5.18 A post-muon, total phonon pulse (black), if fit with the standard OF (green solid), and the NSOF (pink dotted) algorithms, using the same template. Neither algorithm provides a close fit to the raw pulse, due to the pulse deformations caused by the thermal phonons produced in an earlier muon interaction. . . . . . . . . . . . . . . . . . . . . . . . 186

5.19 Total Phonon $\chi^{2}$ calibrated ionization energy for G106a with \pm 4 V applied bias. Events which pass the $\chi^{2}$ cut (red) fall below the parabolic cut line (cyan), and events which fail the cut (blue) fall above the line. An event passing this cut must also pass the Side 1 and Side 2 charge $\chi^{2}$ cuts to be considered a well constructed event. . . . . . . . . . . . . . . 187

5.20 A raw total phonon pileup event (black) is fit with a total phonon template, using the NSOF (dotted pink) and OF (solid green) fitting algorithms. Although the first pulse is reconstructed well by the fits (particularly OF, which does not deweight the rising edge "noise"), the second pulse is completely neglected. As a result, the $\chi^{2}$ of this fit is high, and the event will be removed. . . . . . . . . . . . . . . . . . . . . 188

5.21 Distribution of mean-subtracted baseline values for (LEFT) PF Side 1 and (RIGHT) PF Side 2 with the $\chi^{2}$ cut applied. Distributions are fit with a Gaussian (green), and baseline threshold are set to $\pm 3 \sigma$ (black). Baselines within the thresholds are kept (red), while events with baselines outside the thresholds are removed (blue). . . . . . . . . . . . . . . 189 
5.22 (TOP) Total phonon energy spectra, comparing the NSOF (blue) fitting to the OF (green) fitting. (MIDDLE) The Qimean energy spectrum. (BOTTOM) Recoil energy spectra, derived from the total phonon energy and Qimean. As with total energy, the NSOF (blue) fitting and standard OF (green) fitting are compared. All charge and phonon RQ quality cuts applied. . . . . . . . . . . . . . . . . . . . . 191

5.23 PtNF $10.36 \mathrm{keV}_{e e}$ peak resolution versus applied bias, before the Luke gain contribution is included. . . . . . . . . . . . . . . . . . . . . 194

5.24 Yield versus recoil energy for (TOP) OF and (BOTTOM) NSOF total phonon processing. Previously discussed quality cuts have been applied, including charge and phonon $\chi^{2}$, charge threshold, charge radial partition, and charge and phonon baseline cuts. Run 54 and Run 56 data included. 196

5.25 Side 2 charge signal (QiS2) versus Side 1 charge signal (QiS1), showing a charge-symmetric data region with slope of one, and non-symmetric data, such as surface events. Events from the ${ }^{241} \mathrm{Am}$ source $60 \mathrm{keV} \gamma$ 's created a vertical blob of events extending from the symmetric region downward to a null QiS2 signal. A charge symmetry cut is applied (cyan lines), removing surface events (blue) while keeping symmetric bulk events (red). 197

5.26 (TOP) Total phonon energy spectra, comparing PtNF with (red) and without (blue) surface cut applied to the PtOF with (black) and without (green) surface cut. (MIDDLE) The Qimean energy spectrum, with (blue) and without (red) surface cut applied. (BOTTOM) Recoil Energy energy spectra, comparing PrNF with (red) and without (blue) surface cut applied to the PrOF with (black) and without (green) surface cut. All charge and phonon RQ quality cuts applied. . . . . . . . . . . . . . 199

5.27 Yield versus recoil energy for (TOP) OF and (BOTTOM) NSOF total phonon processing. Previously discussed quality cuts have been applied, including charge and phonon $\chi^{2}$, charge threshold, charge radial partition, and charge and phonon baseline cuts. The surface cut has also been applied.200 
5.28 Zero-energy events, $10.36 \mathrm{keV}, 60 \mathrm{keV}$, and $356 \mathrm{keV}$ source peaks are fit with a Gaussian plus exponential decay background on QiS1, QiS2, Qimean, PTNF, and PTOF. The resulting peak mean and resolution values are used in a weighted fit to determine the functional form parameters in Eq. 5.20 , Phonon energy values are in units $\mathrm{keV}_{t} . \quad \ldots \ldots$. . . . . . 201

5.29 Comparing the (LEFT) ionization and (RIGHT) total phonon energy resolution functional forms of G106a (black) to the SuperCDMS Soudan $76 \mathrm{~mm}$ iZIPs (colored lines). UMN functional forms are fit to $\mp 4 \mathrm{~V}$, Qimean and PtNF peaks. Soudan functional forms are fit to Run $133 \pm 2$ V data, using maximum Qsum and PtNF. . . . . . . . . . . . . . . . . 202

5.30 (LEFT) Ionization yield versus recoil energy for typical $76 \mathrm{~mm}$ iZIP detector (T1Z2) ${ }^{252} \mathrm{Cf}$ calibration data from Soudan R123 (black), with a $\pm 2 \mathrm{~V}$ applied bias. Data is predominately divided into ER band (red) and NR band (blue). Figure courtesy of J. Filippini [215]. (RIGHT) Modeled Soudan data with an applied bias difference of $4 \mathrm{~V}$, and energy resolutions of a selected Soudan detector T1Z2. . . . . . . . . . . . . . . 204

5.31 (LEFT) Ionization yield versus recoil energy for G106a ${ }^{252} \mathrm{Cf}$ calibration data, with a $\mp 4 \mathrm{~V}$ applied bias. Only events which pass quality cuts and surface cut are shown. (RIGHT) Modeled UMN data with an applied bias difference of $8 \mathrm{~V}$, and energy resolutions of G106a at $74 \mathrm{~V} . \quad$. . . 205

5.32 Predicted PrNF $10.36 \mathrm{keV}$ peak width versus applied bias. Predicted peak width is derived using Eq. 5.29 , from measured values of the 10.36 \begin{tabular}{ll|l|l|l}
$\mathrm{keV}$ peak width in Qimean and PtNF spectra, shown in Figs. & 4.10 & and
\end{tabular} 5.23, respectively. . . . . . . . . . . . . . . . . 205

5.33 Predicted thickness of yield (LEFT) ER and (RIGHT) NR bands at a recoil energy of $10.36 \mathrm{keV}_{e e}$ versus applied bias. Yield band thickness is derived using Eq. 5.30 , from measured values of the $10.36 \mathrm{keV}$ peak width in Qimean and PtNF spectra, shown in Figs. $4.10 \mid$ and $\mid 5.23$, respectively. 206 
5.34 Yield versus recoil energy for (TOP) OF and (BOTTOM) NSOF total phonon processing, with quality cuts and surface event cut applied. ER (blue) and NR (cyan) bands are fit to the mean plus standard deviation (pink) of the yield distributions for a set of twelve logarithmically spaced energy bins. . . . . . . . . . . . . . . . . . . . . . . . 208

5.35 Bulk events (red) comprise the ER and NR yield bands. Events which fail the charge symmetry cut and are thus surface events (blue), tend to bleed out of the ER band, into the NR band region. . . . . . . . . . . 210

5.36 The surface event rejection efficiency versus recoil energy for all events (solid black), ER events (solid blue), NR events (solid red, ${ }^{252} \mathrm{Cf}$ dotted red, Non- ${ }^{252} \mathrm{Cf}$ dashed red), Leakage Events (solid green, ${ }^{252} \mathrm{Cf}$ dotted green, Non- ${ }^{252}$ Cf dashed green $)$. . . . . . . . . . . . . . . . . . . 211

5.37 (LEFT) Yield distributions demonstrating surface event rejection efficiency of the charge symmetry cut in the $10-100 \mathrm{keV}$ recoil energy range. Distribution of all quality data (blue) is compared to distribution with surface event cut applied (red). (RIGHT) Surface event rejection efficiency versus yield. Both plots are presented for (TOP) Run $54{ }^{252} \mathrm{Cf}$ data (TOP MIDDLE) Run 54 non- ${ }^{252}$ Cf data (BOTTOM MIDDLE) Run 56 data (BOTTOM) Run 56 and Run 54 data. . . . . . . . . . . . . . . 212 
6.1 (TOP) Contact-free detector design, with two aluminum electrodes (grey) surrounding a $150 \mathrm{~mm}$ Si crystal (red) with a $<0.5 \mathrm{~mm}$ gap between faces, all secured within copper housing. Both electrodes feature two circuit boards with LEDs on their face side (shown in green on readout side), and are connected to DIBs (green) mounted on their housing. Additionally, the readout electrode features a collimated ${ }^{241} \mathrm{Am}$ source (purple) in a copper source holder at the center of the electrode. Wire (not shown) extending from the DIB to the small screw (brass) secured in each electrode will provide applied bias and electrode readout. (BOTTOM) Fabricated electrodes in housings. Top side of readout electrode (on left) shows ${ }^{241} \mathrm{Am}$ copper source holder, charge signal readout and LED wiring leading to housing-mounted DIB. Face side of bias electrode (on right) shows UV and IR LEDs each mounted on two small circuit boards. . . . . . . . . . . . . . . . . . . . . . 218

6.2 Stages of mounting crystal and electrodes into housings. (TOP LEFT) In preparation for crystal mounting, cirlex clamps are installed on the bottom of the crystal housing, which is then set on the clean bench. Polished crystal is set on stand (with protective kim wipe) in the center of the housing. (TOP RIGHT) Housing is slowly raised until bottom clamps catch on the crystal, raising it above the stand. (MIDDLE LEFT) Housing and crystal are set on the clean bench, and top clamps are carefully installed to secure the crystal in its housing. (MIDDLE RIGHT) DIBs are installed onto both electrode housings before electrodes are added. (BOTTOM LEFT) After electrodes have been secured in place with clamps, wires are soldered, connecting the DIB channels to LED boards and the bias/readout screw secured to the electrode face with additional copper tape for good contact. (BOTTOM RIGHT) The ${ }^{241} \mathrm{Am}$ source is added to the readout electrode, and kapton tape is places around the wires for additional protection and insulation. . . . . . . . . . . . . . . . . 219

6.3 UV and IR LEDs mounted to a custom-made PCB board are attached to the contract-free electrode. Boards are wired to LED and phonon return lines on the DIB. . . . . . . . . . . . . . . . . . . . . . . . . . 220 
6.4 (LEFT) Band structure of Ge and (RIGHT) Si at $300 \mathrm{mK}$. Typical CDMS $940 \mathrm{~nm}(1.3 \mathrm{eV})$ LED's have sufficient energy to excite electrons through the direct band gap of $\mathrm{Ge}, E_{\Gamma 1}=0.8 \mathrm{eV}$, and the indirect band gap of Si, $E_{g}=1.12 \mathrm{eV}$. Newly-added $310 \mathrm{~nm}$ UV LEDs (4.0 eV) have sufficient energy to excite electrons through the direct band gap of Si, $E_{\Gamma 1}=3.4$ eV. Figures courtesy of Ioffe Physico-Technical Institute [222]. . . . . . 220

6.5 Schematic of the Run 60 contact-free electronics setup. Readout electrode DIB passes through typical CDMS ionization readout circuit, comprised of coaxial cables, SQUET card, stripline, and MIDAS-controlled DCRC. 221

6.6 (LEFT) Assembled electrodes and crystal housings are stacked with top and bottom lids. Stack is secured with threaded rods which pass through the top lid and stack to secure into the bottom lid. Tower thermometer is secured to the top lid using one of the threaded rods. (MIDDLE) Brass screw secure bottom lid of stack to tower. Flexible side coax is attached to electrode DIB and tower SCUHBA. One half of the new adapter plate is installed on the tower. (RIGHT) Installation of flexible side coaxes and adapter plate complete. Coax wires are secured around tower spool with floss and kapton tape. Finished tower is ready for installation in the fridge.223

6.7 Tower successfully installed in new $30 \mathrm{mK}$ can, with sufficient space to avoid contact between can walls and outward facing flexible side coax SCAB. Additional kapton tape was used to cover the flexible side coax components to provide additional protection. . . . . . . . . . . . 223

6.8 An example of the split regimes that occur in charge amplitude. Figure shows uncalibrated charge amplitude versus time. After $\sim 600 \mathrm{~s}$ of data taking, the amplitude enters a new regime, with the uncalibrated $60 \mathrm{keV}$ peak increasing from $\sim 0.002$ a.u. to $\sim 0.006$ a.u. for the remainder of the data series. . . . . . . . . . . . . . . . . . 225

6.9 (LEFT) Typical ionization pulse signal with $17 \mathrm{kHz}$ ringdown in pulse tail shown in black, overlaid with fitted pulse template in magenta. Fitted template amplitude, time delay, and $\chi^{2}$ quantities are used for data analysis.(RIGHT) Zoom in of raw pulse comparing time-domain fit (magenta) to standard CDMS OF fit (yellow). . . . . . . . . . . . 226 
6.10 Example pulse shapes observed during $17 \mathrm{kHz}$ ringdown studies, using (LEFT) the standard vacuum side coax and (RIGHT) the flexible side coax. The simulated input pulse is shown in light blue, and the response measured from the DCRC is shown in yellow. The vacuum side coax shows no oscillations and decays with $250 \mu$ s decay time. On the rising and falling edges of the square wave, the flexible side coax has a ringdown of $\sim 4 \mathrm{kHz}$ with a decay of $1.5 \mathrm{~ms}$, whether or not the coax is connected to the detector. . . . . . . . . . . . . . . . . . . . . 227

6.11 Charge $\chi^{2}$ versus calibrated ionization energy for Si $150 \mathrm{~mm}$ contact-free detector series with $+20 /-10 \mathrm{~V}$ applied bias. Events which pass the $\chi^{2}$ cut (red) fall below the parabolic cut line (cyan), and events which fail the cut (blue) fall above the line. Due to changing noise levels, $\chi^{2}$ cuts are applied on a series-by-series basis. . . . . . . . . . . . . . . . . 229

6.12 PSDs of 1000 initial random triggers taken at the start of $0 \mathrm{~V}, 30 \mathrm{~V}, 50 \mathrm{~V}$, $70 \mathrm{~V}, 90 \mathrm{~V}$, and $110 \mathrm{~V}$ biased data series. Results indicate similar noise across all voltage biases, with some deviation between biases in the low frequency $\left(<10^{4} \mathrm{~Hz}\right)$ range. Changes in noise do not follow the expected patterns due to leakage current through the crystal, which would increase with bias, possibly indicating that variations in noise are due to the state of readout electronics during data taking, more so than applied bias. . 229

$6.13 Q_{T D F}$ spectrum with (red dashed) and without (blue solid) the $\chi^{2}$ cut applied, for (LEFT) the whole $10 \mathrm{~V}$ net bias data series and (RIGHT) the first five minutes of the same data series. The $\chi^{2}$ cut removes poorlyfit noise from the low energy region $(\sim 0-40 \mathrm{keV})$, while the $60 \mathrm{keV}$ ${ }^{241} \mathrm{Am}$ peak is unaffected. Loss of neutralization results in the ${ }^{241} \mathrm{Am}$ peak falling into the low energy region after the first five minutes of data taking, making it difficult to distinguish from noise. . . . . . . . . . . 232 
6.14 Energy spectra taken with an ${ }^{241} \mathrm{Am}$ source mounted near the detector and with (red) and without (blue) a ${ }^{57}$ Co source exterior to the cryostat. The $60 \mathrm{keV}$ peak of ${ }^{241} \mathrm{Am}$ and the $122 \mathrm{keV}$ edge of ${ }^{57} \mathrm{Co}$ as well as their Compton edges can be seen. Note that the ${ }^{57} \mathrm{Co}$ spectrum is smeared to low energies due to the interaction with intervening cryostat material. Figure courtesy of N. Mast. . . . . . . . . . . . . . . . . . . . 233

6.15 Individually calibrated $Q_{T D F}$ spectra constructed from the (LEFT) 0 5 minute region and (RIGHT) 5 - 10 minute region of a $10 \mathrm{~V}$ biased (blue), $50 \mathrm{~V}$ biased (green) and $90 \mathrm{~V}$ biased (red) data series. The 0 - 5 minute region spectra share similar features, with slight differences in the $60 \mathrm{keV}$ peak resolution. The $50 \mathrm{~V}$ and $90 \mathrm{~V}$ spectra constructed from the 5 - 10 minute region also share features from the first 5 minute spectra, showing no signs of loss of neutralization for these series. The $10 \mathrm{~V}$ spectrum demonstrates loss of neutralization in the $5-10$ minute region, with the ${ }^{241} \mathrm{Am}$ peak falling to $\sim 30 \mathrm{keV}$. . . . . . . . . . . 234

6.16 Charge collection efficiency as a function of applied bias. Results of broken down into categories of LED type used for most recent flashing or baking (IR flash in magenta, UV flash in cyan, IR bake in yellow, UV bake in green). Suspect data is highlighted in red. Contact-Free detector results are compared to a 2007 measurement of the $2.54 \mathrm{~cm}$ thick $\mathrm{Si}$ detector, S10C (black). . . . . . . . . . . . . . . 235

6.17 Charge energy resolution as a function of applied bias. Suspect data is highlighted in red. Figure courtesy of N. Mast. . . . . . . . . . . . . 236

6.18 Charge neutralization after IR (TOP) and UV (BOTTOM) flashing. Both show successful neutralization of the crystal after flashing. Data from IR flashing shows ${ }^{241} \mathrm{Am} 60 \mathrm{keV}$ peak hold time consistent around 810 seconds, whereas UV flashing data has a mean peak hold time of 435 seconds. . . . . . . . . . . . . . . . . 238

$6.1960 \mathrm{keV}$ peak hold time measurements for bias voltages up to $\approx \pm 70$ Volts. Peak hold time increases linearly with bias, with some variation favoring IR LED flashing over UV LED flashing for increased hold time. . . . . 239 
6.20 Prototype Si $150 \mathrm{~mm} 5 \mathrm{Q}$ device, to be tested at the UMN testing facility during Fall 2017. Although faint in this image, the 5Q mask can be seen on the crystal surface. The charge channels are read out through 3 DIBs on the $\mathrm{HPCu}$ housing. . . . . . . . . . . . . . . . . . . . . . . . . . . 240

6.21 Drawing of a side and top view of the 36.25" inner diameter, 16.3125" tall SNOLAB mixing chamber can (grey), filled with a 21 across by 12 deep arrangement of $150 \mathrm{~mm}$ diameter Si detector (light grey), in OFHC Cu housings (copper). . . . . . . . . . . . . . . . . . 245

6.22 Background spectra, before (LEFT) and after (RIGHT) analysis cuts for Si iZIP, shown as a function of nuclear recoil energy $\left(\mathrm{keV}_{n r}\right)$. Thick black lines represent the total background rates. Electron recoils from Compton gamma rays, ${ }^{3} \mathrm{H}$, and ${ }^{32} \mathrm{Si}$ are grouped together (red), and other components include surface betas (green), surface ${ }^{206} \mathrm{~Pb}$ recoils (orange), neutrons (blue), and CE $\nu$ NS (cyan). Figure courtesy of [135]. . . . . . . 248

6.23 Expected background spectrum (purple solid), energy threshold (dotted black), and simulated events (green) for $150 \mathrm{~mm}$ Si iZIP likelihood calculation results. . . . . . . . . . . . . . . . . . . . . . . . 249

6.24 2D marginalized likelihoods for $150 \mathrm{~mm}$ Si iZIP detectors with $2 \sigma$ contours, for Operators $1,3,4$, and 5. Continued on next page. . . . . . . . 251

6.24 Continued from previous page. 2D marginalized likelihoods for $150 \mathrm{~mm}$ Si iZIP detectors with $2 \sigma$ contours, for Operators 6-9. Continued on next page. . . . . . . . . . . . . . . . . . . . 252

6.24 Continued from previous page. 2D marginalized likelihoods for $150 \mathrm{~mm}$ Si iZIP detectors with $2 \sigma$ contours, for Operators 10 and $11 . \quad$. . . . . . 253

6.25 EFT-calculated projected sensitivities for SuperCDMS SNOLAB cryostat filled with $150 \mathrm{~mm}$ diameter Si detectors (black dotted), compared to current experimental limits (solid lines), projected sensitivities (dashed lines), and candidate regions (shaded) on spin-independent cross sections as a function of WIMP mass for various experiments. For full list of limits and projected sensitivity sources, see Fig. 1.13 . . . . . . . . . . . . . . 254 
B.1 Cross talk templates constructed from a thousand well behaved pulses. Templates represent the typical signal seen in an inner (outer) charge channel due to an outer (inner) event. For example, template named QIS1X represents typical signal in QIS1 from QOS1 event. Only the QOS1X template has the form typically seen in a cross talk template, raising suspicions that the other templates do not properly isolate cross talk contribution from true event signals. Figure courtesy of N. Mast. . 290

C.1 $I_{b}-I_{s}$ measurement for G106a. Continued on next page. . . . . . . . . 292

C.1 Continued from previous page. $I_{b}-I_{s}$ measurement for G106a. Continued on next page. . . . . . . . . . . . . . . . . . . . . . . 293

C.1 Continued from previous page. $I_{b}-I_{s}$ measurement for G106a. Continued on next page. . . . . . . . . . . . . . . . . . . . . 294

C.1 Continued from previous page. $I_{b}-I_{s}$ measurement for G106a. Continued on next page. . . . . . . . . . . . . . . . . . . 295

C.1 Continued from previous page. $I_{b}-I_{s}$ measurement for $100 \mathrm{~mm}$ iZIP G106a, with superconducting region highlighted in red, and normal regions highlighted in blue (negative $I_{b}$ ) and magenta (positive $I_{b}$ ). Fitting the linear superconducting and normal regions allows for estimation of the parasitic and normal resistances of the sensors. The fits and estimated resistance values are shown in the upper left and lower right corners of each channel's plot. Figures courtesy of H. Chagani. . . . . . . . . . . . 296

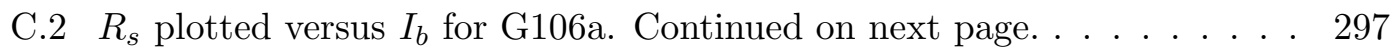

C.2 Continued from previous page. $R_{s}$ plotted versus $I_{b}$ for G106a. Continued on next page. . . . . . . . . . . . . . . . . . . 298

C.2 Continued from previous page. $R_{s}$ plotted versus $I_{b}$ for G106a. Continued on next page. . . . . . . . . . . . . . . . . . . . . . . . 299 
C.2 Continued from previous page. $R_{s}$ plotted versus $I_{b}$ for each G106a phonon channel, excluding channel $\mathrm{C} 2$, which had inconsistent results. Plots show sensor resistance increasing from zero in the superconducting region and plateauing towards the positive and negative QET bias $R_{n}$ values. Positive and negative QET bias curves are fit to the data, shown in blue and pink. From the fit curve, $R_{p}$ and $R_{n}$ are found, along with the optimized QET bias corresponding to the start of the transition region, where $I_{b}=R_{n} / 4$. Estimated resistance values and optimized QET bias are shown in corners of each channel's plot. Figures courtesy of H. Chagani. . . . . . . . . . . . . . . . . . . 300

C.3 (LEFT) Calibration values and (RIGHT) calibration value deviation from mean over time for all channels with respect to the given base channel. Continued on next page. . . . . . . . . . . . . . . . . . . . . . 301

C.3 Continued from previous page. (LEFT) Calibration values and (RIGHT) calibration value deviation from mean over time for all channels with respect to the given base channel. Continued on next page. . . . . . . . 302

C.3 Continued from previous page. (LEFT) Calibration values and (RIGHT) calibration value deviation from mean over time for all channels with respect to the given base channel. Continued on next page. . . . . . . . 303

C.3 Continued from previous page. (LEFT) Calibration values and (RIGHT) calibration value deviation from mean over time for all channels with respect to the given base channel. Continued on next page. . . . . . . . 304

C.3 Continued from previous page. (LEFT) Calibration values and (RIGHT) calibration value deviation from mean over time for all channels with respect to the given base channel. . . . . . . . . . . . . . . . . . . . . . 305 


\section{Chapter 1}

\section{Dark Matter}

\section{$1.1 \quad$ Introduction}

Dark matter is one of the most important problems in cosmology today. Understanding its origin would clarify many astronomical observations as well as strengthen the current understanding of particle physics. This thesis will focus on searching for dark matter in a laboratory setting using cryogenic direct detection methods. As an introduction to the work put forth in this thesis, Chapter 1 will outline the evidence, characteristics, and detection methods of dark matter. Section 1.2 of this chapter will cover evidence supporting the existence of dark matter including evidence from galaxy and cluster observations, cosmological observations such as the Cosmic Microwave Background, and gravitational lensing. This section will also provide an overview of dark matter characteristics and potential dark matter candidates. Section 1.3 will discuss current experimental methods for dark matter detection, specifically from the direct and indirect detection categories, as well as provide some current experimental results. For clarity, all variables, acronyms, and data quantities referenced throughout this text are defined in Appendix A.

\subsection{Dark Matter Evidence}

There exist copious amounts of observational and theoretical evidence supporting the presence of dark matter. Dark matter is often inferred to exist through observations of 
galaxies, clusters and other astronomical phenomena. Studies on large scale structure and the evolution of the universe also suggest the existence of dark matter. This section will provide an overview of a few pieces of evidence for the existence of dark matter.

\subsubsection{Evidence from Observations of Clusters and Galaxies}

The evidence for the existence of dark matter was first observed in the orbital velocities of galaxies in clusters. Results of a 1933 study performed by Fritz Zwicky determined evidence of unseen mass affecting velocities of eight galaxies in the Coma cluster. Using the virial theorem, Zwicky calculated the dispersion velocity of galaxies based on the luminous material, which he compared to the actual galaxy velocities. The results showed the presence of roughly 400 times more mass than what was visible [1]. Zwicky proposed the existence of unseen mass he called dark matter to account for the discrepancy.

On a smaller scale dark matter also affects the rotational curves of individual spiral galaxies. Applying simple Newtonian mechanics to the circular rotation of a galaxy gives an expression for $v$, the rotational velocity,

$$
v^{2}(r)=\frac{G M(r)}{r}
$$

where $G$ is the gravitational constant, $r$ is the distance from the center of rotation, and $M(r)$ is the spherically distributed galactic mass within a sphere of radius $r$. Define $r_{L}$ to be the radius of the sphere encompassing a majority of the luminous matter. Beyond this radius there is little luminous material contributing to the mass, and thus it is expected that $M\left(r_{L}\right)$ remains roughly constant for $r>r_{L}$. With a constant mass, it is

expected by Eq. 1.1 that the rotational velocity would fall as $\propto \sqrt{\frac{1}{r}}$ for $r>r_{L}$. However, multiple studies starting in the 1970's found that the rotational velocity would remain constant, or even increase, beyond the luminous disk, as shown in Fig. 1.1, suggesting that mass continues to increase linearly beyond what can be visibly seen [2] [3]. This gave rise to the well accepted view that a sphere of dark matter resides in the halo surrounding the disk of visible stars and gas. 


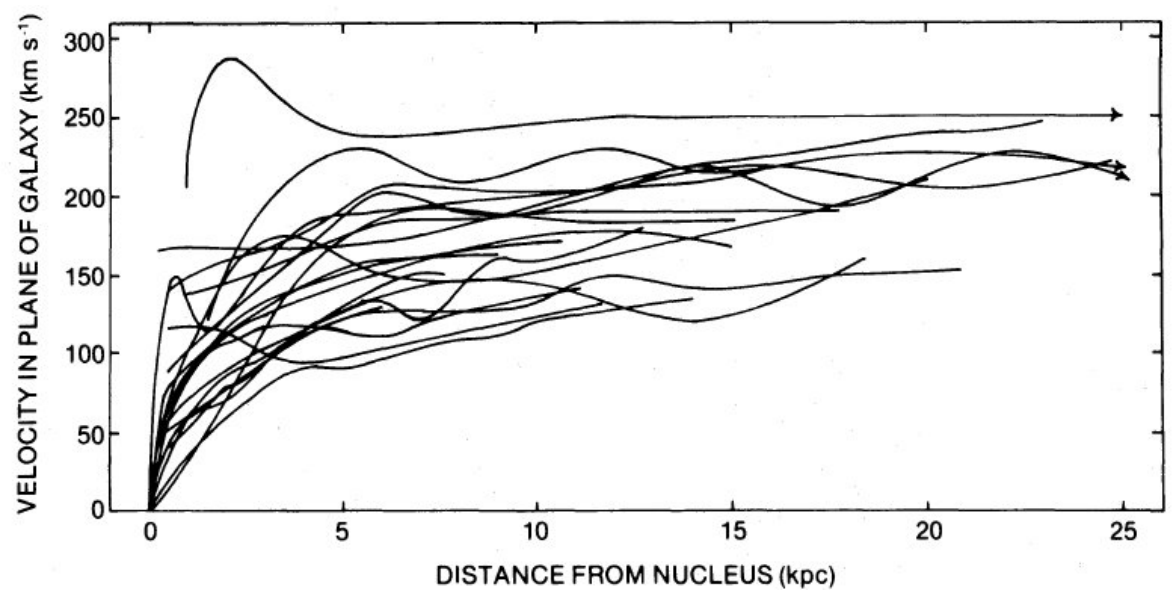

Figure 1.1: Superposition of $21 \mathrm{Sc}$ galaxy rotation curves. Figure courtesy of [2].

\subsubsection{Evidence from Cosmological Observations}

Unlike the evidence presented in the previous section, cosmological observations focus on exploring much earlier time frames in the chronology of the universe. These observations are paired with models on the evolution of the universe to infer the existence of additional, non-baryonic mass.

\section{Friedmann Equation}

To understand the significance of cosmological dark matter evidence one must first understand the underlying equations used to describe the evolution of the universe. Under the assumption of a spherically symmetric, homogeneous and isotropic universe, the metric used to describe spacetime is the Robertson-Walker metric for polar coordinates given by,

$$
d s^{2}=-d t^{2}+a^{2}(t)\left(\frac{d r^{2}}{1-k r^{2}}+r^{2} d \Omega\right)
$$

where $a(t)$ is a time dependent scale factor that describes the expansion of spacetime and $k$ is $+1,-1$, or 0 for a closed, open, or flat universe respectively. From this metric and the assumption of universe homogeneity and isotropy, the energy-momentum tensor describing the entire universe takes the form [4,

$$
T^{00}=\varrho(t), \quad T^{0 i}=0, \quad T^{i j}=g^{i j} a^{-2}(t) p(t),
$$


where $\varrho(t)$ is the proper energy density, $p(t)$ is the pressure, and $g^{i j}$ is the spatial portion of the Robertson-Walker metric, given in Cartesian coordinates $\left(x^{i}, i=1,2,3\right)$ as,

$$
g^{i j}=\delta^{i j}+k \frac{x^{i} x^{j}}{1-k \mathbf{x}^{2}} .
$$

Applying conservation of energy leads to the following equation,

$$
\frac{d \varrho}{d t}+\frac{3 \dot{a}}{a}(p+\varrho)=0 .
$$

If the commonly used $p=w \varrho$ for a time independent $w$ is given as an equation of state of the universe, then this expression is solved to be,

$$
\varrho=\sum_{w} \varrho_{0, w} a^{-3(1+w)} .
$$

Here $w$ can be specified for certain common situations. For non-relativistic matter $p=0$ and thus $w=0$ and $\varrho_{m} \propto a^{-3}$. Relativistic matter such as radiation has $w=\frac{1}{3}$ and thus $\varrho_{r} \propto a^{-4}$. Vacuum energy has the interesting characteristic $\varrho_{\Lambda}=-p$ and thus has a constant proper energy density. Thus an expression for the total energy density is,

$$
\varrho=\varrho_{m, 0} a^{-3}+\varrho_{r, 0} a^{-4}+\varrho_{\Lambda, 0} .
$$

To understand how the scale factor $a(t)$ changes with time, consider the Einstein field equation [4],

$$
R_{\mu \nu}=-8 \pi G\left(T_{\mu \nu}-\frac{1}{2} g_{\mu \nu} T_{\lambda}^{\lambda}\right),
$$

where $R_{\mu \nu}$ is the Ricci tensor. Applying the energy-momentum tensor given in Eq. 1.3 yields,

$$
\begin{aligned}
\frac{-2 k}{a^{2}}-\frac{2 \dot{a}^{2}}{a^{2}}-\frac{\ddot{a}}{a} & =-4 \pi G(\varrho-p), \\
\frac{3 \ddot{a}}{a} & =-4 \pi G(3 p+\varrho),
\end{aligned}
$$

which can be reduced to Eq. 1.5 and,

$$
\dot{a}^{2}+k=\frac{8 \pi G \varrho a^{2}}{3} .
$$

This is one form of the Friedmann equation which describes the expansion of the space via the time-dependence of the scale factor $a(t)$. Here it is useful to introduce the Hubble parameter $H(t)=\frac{\dot{a}(t)}{a(t)}$. Then Eq. 1.11 can be rewritten as,

$$
\frac{8 \pi G}{3 H^{2}}\left(\varrho_{m}+\varrho_{r}+\varrho_{\Lambda}\right)-\frac{k}{a^{2} H^{2}}=1
$$


This can be simplified further by introducing the density parameters for the given constituents: matter, radiation, and vacuum energy,

$$
\begin{gathered}
\Omega_{m}=\frac{8 \pi G \varrho_{m}}{3 H^{2}}, \quad \Omega_{r}=\frac{8 \pi G \varrho_{r}}{3 H^{2}}, \quad \Omega_{\Lambda}=\frac{8 \pi G \varrho_{\Lambda}}{3 H^{2}}, \quad \Omega_{k}=-\frac{k}{a^{2} H^{2}}, \\
\Omega_{m}+\Omega_{r}+\Omega_{\Lambda}+\Omega_{k}=1 .
\end{gathered}
$$

The value of $\Omega_{k}$ determines whether the universe is open, closed or flat. A similar expression as those in Eq. 1.13 for the baryonic matter density with nucleon mass $m_{N}$ and nucleon number density $n_{N}$ is,

$$
\Omega_{b}=\frac{8 \pi G}{3 H^{2}} m_{N} n_{N}
$$

For slow baryons this is equivalent to the baryonic energy density. Comparing the $\Omega_{b}$ to the total matter density $\Omega_{m}$, as will be outlined in the next section, shows a discrepancy that suggests the existence of extra, non-baryonic matter filling the universe.

\section{Big Bang Nucleosynthesis}

Big bang nucleosynthesis began with temperatures $T>10^{10} \mathrm{~K}$, when protons and neutrons interacted with electrons, positrons, and neutrinos. Conversion between protons and neutrons occurred via the following weak processes:

$$
n+\nu \rightleftharpoons p+e^{-}, \quad n+e^{+} \rightleftharpoons p+\bar{\nu}, \quad n \rightleftharpoons p+e^{+}+\bar{\nu} .
$$

For the high temperatures above $\sim 10^{10} \mathrm{~K}$ these interactions are in equilibrium and the ratio of neutron to proton number density is given by $n_{n} / n_{p}=e^{\left(m_{n}-m_{p}\right) / k_{b} T}$. As the universe cools the conversion rate between protons and neutrons $\lambda(p \leftrightarrow n)$ falls, and the system quickly departs from chemical equilibrium. As temperatures drop below $k_{b} T \simeq m_{e}$ the expansion rate $H$ becomes larger than $\lambda(p \leftrightarrow n)$ and particles effectively become too dispersed to meet, leaving multi-body interactions negligible and only neutron decay remaining. When the system first leaves chemical equilibrium the proton-neutron number density ratio is $n_{n} / n_{p} \simeq 1 / 6$, but this drops to $n_{n} / n_{p} \simeq 1 / 7$ before remaining protons and neutrons begin forming stable nuclei [5].

The first nucleosynthesis process to occur is the production of deuterium via $p+$ $n \rightarrow d+\gamma$. Due to deuterium's low binding energy and photodissociation it remains 
rare and further nucleosynthesis is delayed. Once temperatures drop below $T \approx 0.7 \times$ $10^{9} \mathrm{~K}$ deuterium begins to form more complex nuclei, predominately through two-body processes such as,

$$
d+d \rightarrow{ }^{3} \mathrm{H}+p, \quad d+d \rightarrow{ }^{3} \mathrm{He}+n, \quad d+{ }^{3} \mathrm{H} \rightarrow{ }^{4} \mathrm{He}+n, \quad d+{ }^{3} \mathrm{He} \rightarrow{ }^{4} \mathrm{He}+p .
$$

With most remaining neutrons bound in ${ }^{4} \mathrm{He}$ nuclei, nucleosythesis is once again halted by the absence of stable nuclei with an atomic weight of 5 or 8 . Considering the atomic masses of $\mathrm{He}$ and $\mathrm{H}$, the fractional weight abundance of ${ }^{4} \mathrm{He}, Y_{p}$, is approximately,

$$
Y_{p}=\frac{4 n_{H e}}{4 n_{H e}+n_{H}}
$$

where $n_{X}$ is the number density of element $\mathrm{X}$ nuclei. Additionally, $n_{n}$ and $n_{p}$ are related to the number density of $\mathrm{He}$ and $\mathrm{H}$ by $n_{p}=2 n_{H e}+n_{H}$ and $n_{n}=2 n_{H e}$.

Thus the primordial weight fraction of the ${ }^{4} \mathrm{He}$ formed at this time is simply twice the ratio of all neutrons to all nucleons,

$$
Y_{p}=\frac{2 n_{n}}{n_{n}+n_{p}} .
$$

As shown in Eqs. 1.18 and 1.15 , there is a direct link between $Y_{p}$ and the baryonic matter density $\Omega_{b}$. Spectroscopic studies of regions of ionized hydrogen (HII regions) with low metallicity, particularly dwarf galaxies that have not yet formed many stars, provide a value for the ${ }^{4} \mathrm{He}$ weight fraction 17 ,

$$
Y_{p}=0.245 \pm 0.004[7]
$$

From this and similar studies on light element abundances, a range of baryonic matter density was measured to be,

$$
0.021 \leq \Omega_{b} h^{2} \leq 0.024
$$

at $95 \%$ C.L., for $h \equiv H\left(t_{0}\right) / 100 \mathrm{~km} \mathrm{~s}^{-1} \mathrm{Mpc}^{-1}$ the Hubble parameter for the present time $t_{0}$ [7]. This is much greater than the density of luminous material which is found to be $\Omega_{\text {lum }} \simeq 0.0024 h^{-1}$ [8], suggesting that most baryonic material is optically dark. Virial

\footnotetext{
${ }^{1}$ Introducing additional constraints from other cosmological observations, such as the Cosmic Microwave Background (described in Section 1.2.2, yields $Y_{p}=0.24709 \pm 0.00025$ [6].
} 
studies of galaxy cluster masses, such as those calculated by Zwicky, give a mass density value of $\Omega_{m} \simeq 0.21$ [4, much larger than the density provided by baryonic matter. This strongly suggests the existence of a non-baryonic dark matter that contributes a significant portion to the total matter density. Studies on the Cosmic Microwave Background (CMB) confirm these results with stricter constraints on $\Omega_{m}$ and $\Omega_{b}$, as will be discussed in the next section.

\section{Cosmic Microwave Background}

Long after the era of nucleosynthesis the universe was still filled with nuclei, and collisions between photons and electrons kept radiation and matter in thermal equilibrium. The number density of the photons in thermal equilibrium with matter at a temperature $\mathrm{T}$ can be described by the black-body spectrum,

$$
n_{T}(\nu) d \nu=\frac{8 \pi \nu^{2} d \nu}{e^{h \nu / k_{b} T}-1},
$$

for photon frequency between $\nu$ and $\nu+d \nu$. During this era electrons would bond with nuclei to form stable atoms, only to be ejected via scattering by photons of sufficiently high energy. As the universe continues to expand and cool the number of photons with sufficient energy to expel electrons diminishes. Eventually the universe cools sufficiently for expulsion-inducing photon-electron interactions to effectively cease, neutral atoms to form, and photons to begin free expansion. At this time $t_{L}$, referred to as the time of last scattering, the radiation spectrum is unchanged from the original black body spectrum. As the radiation expands freely it is subject to the cosmic expansion $a(t)$. At some time $t>t_{L}$ the number density of photons with frequency between $\nu$ and $\nu+d \nu$ and redshifted temperature $T(t)=T\left(t_{L}\right) a\left(t_{L}\right) / a(t)$ is given by,

$$
n_{T(t)}(\nu) d \nu=\frac{8 \pi \nu^{2} d \nu}{e^{h \nu / k_{b} T(t)}-1},
$$

Although the radiation temperature at the time of last scattering was $\sim 3000 \mathrm{~K}$, the current redshifted temperature of the CMB is $T=2.7255 \mathrm{~K}$.

An interesting and informative characteristic of the CMB is the temperature anisotropies within the mostly uniform spectrum. These anisotropies are usually defined in terms of 
a standard spherical harmonic expansion of the sky,

$$
T(\theta, \phi)=\sum_{\ell, m} a_{\ell, m} Y_{\ell, m}(\theta, \phi)
$$

with angular power spectrum,

$$
\mathcal{C}_{\ell}=\frac{1}{2 \ell+m} \sum_{m=-\ell}^{\ell}\left|a_{\ell, m}\right|^{2},
$$

and band power, given by $\mathcal{D}_{\ell}^{T T}=\ell(\ell+1) \mathcal{C}_{\ell} / 2 \pi$. Here, the TT superscript distinguishes this power map as temperature-temperature, as opposed to polarization modes.

The average background temperature $T=2.7255 \mathrm{~K}$ is the monopole $(\ell=0)$ of the expansion, and the dipole $(\ell=1)$ is caused by a Doppler shift induced by the motion of the observer compared to the blackbody field [10]. Anisotropies on the order of $100 \lesssim \ell \lesssim 1000$ are pressure and gravity-induced sound waves referred to as the acoustic peaks. Before electrons bonded with protons to form an overall neutral universe, baryons and photons are coupled in a baryon-photon plasma. This plasma undergoes harmonic oscillations driven by small perturbations in the gravitational potential. These perturbations cannot be due to the baryons within the plasma and thus suggest a noncoupled dark matter undergoing fluctuations in density [12]. Acoustic waves due to pressure from photon-baryon interactions in dense anisotropies push photon-baryon plasma outwards from the potential well, but do not interact with the dark matter, which stays at the center of the well. Once free electrons become bound into $\mathrm{H}$ and $\mathrm{He}$ during recombination, photons and baryons decouple and photons begin free expansion. The phases of the baryon-photon plasma at the time of last scattering are frozen into the photons' black body spectrum, as shown by the acoustic peaks in Fig. (1.2). Once photon free expansion begins photon-baryon interactions cease and pressure subsides, leaving a shell of baryonic matter at a fixed radius from the dark matter filled center of each anisotropy. These baryonic density fluctuations are known as the Baryon Acoustic Oscillations (BAO). As time continues baryonic and dark matter gravitationally interact to form configurations that now make up the large scale structure of the universe.

If dark matter had been relativistic when it decoupled from ordinary matter, known as hot dark matter, then it would "free-streaming" from high density areas to low density areas, "smoothing" matter density fluctuations in the process, and affecting current large 
scale structure [13]. Studies of the large scale structure provide limits on the amount of hot dark matter in the universe [10] [14, determining that some portion of dark matter must be non-relativistic at the time of decoupling, known as cold dark matter.

Fitting theoretical cosmological models to recent Planck satellite observations of temperature and CMB anisotropies, shown in Fig. 1.2 has produced a measurement of the parameters that dictate the amplitude and positions of the anisotropy peaks, including the baryon density and total mass density. Planck 2015 results found that $\Omega_{m} h^{2}=0.1415 \pm 0.0019$ and $\Omega_{b} h^{2}=0.02226 \pm 0.00023$, for $h=0.6781 \pm 0.0092(68 \%$ C.L.) [9]. These results agree with the density values found by nucleosynthesis and cluster velocity studies and serve as further evidence for the existence of non-baryonic dark matter.

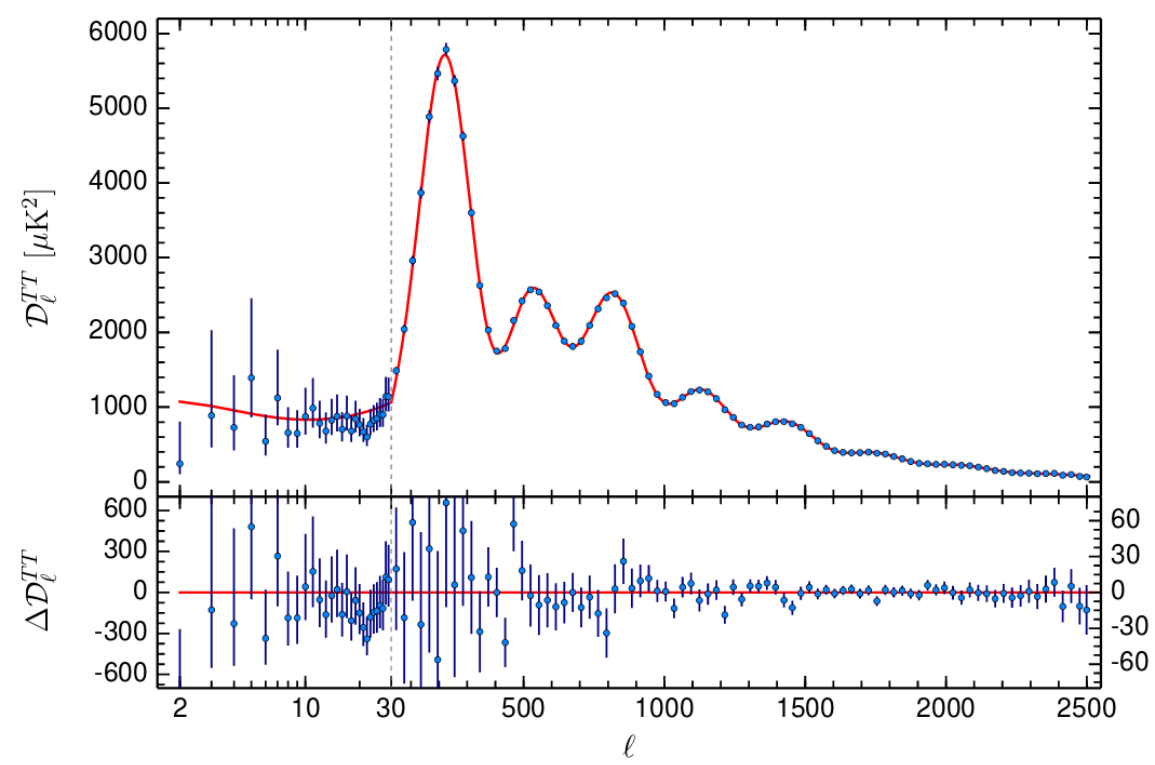

Figure 1.2: Planck 2015 band power spectrum. In upper panel, the best-fit base theoretical spectrum (red) is fitted to the Planck data (blue, $\pm 1 \sigma$ error bars), while lower panel shows residuals with respect to the model. Figure courtesy of [9]. 


\subsubsection{Evidence from Gravitational Lensing}

As with galactic rotation evidence, gravitational lensing provides evidence to dark matter by comparing observable mass to the mass inferred to exist by gravitational arguments. Gravitational lensing is the phenomenon in which light is deflected due to the curvature of spacetime in the presence of massive objects (referred to as the lens), resulting in a distorted, magnified, and brightened image of the original light source. From general relativity, the angle of deflection, $\gamma$, of a light ray due to a lens (in the frame of the lens) is given as,

$$
\gamma=\frac{4 M G}{r},
$$

where $M$ is the mass of the lensing object, $G$ is the gravitational constant, and $r$ distance from the lens to the closest approach of the path between the observer and the image of the source [4]. Strong gravitational lensing applies to massive galaxies and clusters that result in easily visible distortions of the source such as multiple images, radial arcs and, in the case where the source lies directly behind the lens on the line of sight, Einstein rings.

Similar to strong lensing, microlensing occurs when a physically small object passing in front of a source object temporarily brightens the source. Oftentimes this occurs when a planet-sized object passes in front of a star, focusing normally outward light towards Earth and thus brightening the star until the planet has passed by [15]. Conversely, weak gravitational lensing refers to a deflection of source light that is so slight that the shear distortion can be treated as locally linear, changing the shape of galaxies such that the major to minor axis ratio is adjusted by roughly $2 \%$ [15]. Since this distortion is smaller than the variation in galactic shape it cannot be detected through the observation of individual galaxies. However, without a preferred direction to the universe the shape of galaxies should average to be circular. Weak gravitational lensing can thus be measured statistically by studying the shear distortion of adjacent galaxies along the same line of sight. Therefore weak lensing is a preferred method for studying large structure.

Gravitational lensing in all of these forms can be used to demonstrate the effects of dark matters presence. Surveys such as the SLOAN Lens ACS Survey use both strong and weak lensing to determine the distribution of mass within galaxies or galaxy clusters, which can then be compared to the distribution of luminous mass found through infrared 

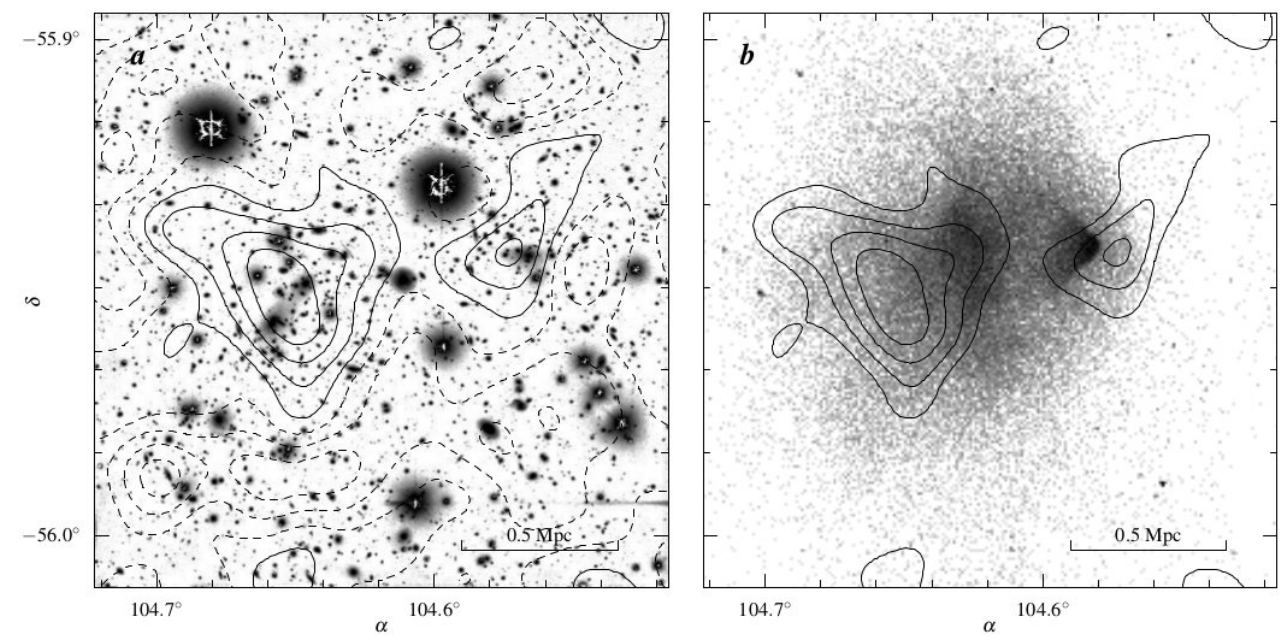

Figure 1.3: (a) Optical image of bullet cluster with weak lensing mass contours. (b) Xray image with overlaid mass contours, with the gas lagging behind negligibly interacting galaxies and dark matter. Figure courtesy of [16].

emission and X-ray luminosity [14]. Microlensing experiments such as EROS-2 ruled out objects within the mass range $0.6 \times 10^{-7} M_{\odot}<M<15 M_{\odot}$ as the primary source of mass within the dark matter filled Milky Way halo [20].

Gravitational lensing reveals a particularly interesting example of dark matter with the Galaxy Cluster 1E0657-558, more commonly known as the Bullet Cluster. The Bullet Cluster contains remnants of two subclusters that previously collided with one another. During the merger some galaxies passed through without much interaction with material in the oncoming subcluster. However, X-ray emissions show that much of the visible matter is baryonic gas focused at the center of the collision, believed to be hot gas from both subclusters that interacted electromagnetically during the collision. A weak gravitational lensing map shows additional non-visible matter clumps surrounding the collisionless galaxies, suggesting that this matter was also able to pass through the merger without any appreciable interaction [16]. The position of the invisible matter relative to the interacting baryonic gas and collisionless galaxies provides limits on the self-interaction cross section of dark matter. The ratio of hot gas to all mass within the cluster was determined to be roughly $\frac{1}{6}[4]$. 


\subsection{Dark Matter Characteristics and Candidates}

The observations described above only reflect a portion of the abundant examples of observed and theoretical dark matter effects. It is important to note that a majority of proof is based on gravitational evidence. At this point in time there is only speculation on whether dark matter interacts via the other forces or even how many "types" of dark matter exist. Though there are promising dark matter candidates, more experimental data is needed to determine which of the candidate(s) is (are) correct. Currently dark matter is known to be comprised of mostly cold, non-baryonic material that has mass, is stable on cosmological times scales, and does not significantly interact with radiation or ordinary matter.

\subsubsection{Non-particulate Solutions}

Several possible solutions exist for the missing mass problem, that don't require particle dark matter ${ }^{2}$. Evidence has already been presented from surveys of nuclear abundances to suggest that baryonic dark matter does account for some portion of non-luminous matter. The most common form of baryonic dark matter is the MAssive Compact Halo Object (MACHO). As the name suggests, these are large objects that reside in the galactic halo and emit negligible amounts of radiation, such as black holes, neutron stars and brown dwarfs. MACHO searches such as EROS-2 report with 95\% C.L. that bayronic MACHOs within the mass range $0.6 \times 10^{-7} M_{\odot}<M<15 M_{\odot}$ are not the primary source of mass within the Milky Way halo [20]. This, combined with the evidence for additional, non-baryonic matter, is sufficient to suggest that baryonic MACHOs cannot be the predominant form of dark matter.

Primordial blacks holes (PBH), a subclass of MACHOs, offer a non-particulate solution to dark matter that isn't comprised of baryonic matter. PBHs are formed in the first $\sim 1$ s of the universe ${ }^{3}$, a radiation-dominated era, and thus are not constrained by

\footnotetext{
2 An additional solution to the dark matter problem that does not involve particle dark matter is Modified Newtonian Dynamics. The idea postulates that the acceleration a due to a Newtonian potential, $\boldsymbol{\Phi}$ is modified as $\tilde{\mu}\left(|\mathbf{a}| / a_{0}\right) \mathbf{a}=-\nabla \boldsymbol{\Phi}$, for acceleration scale $a_{0}$, and monotonic function $\tilde{\mu}(x)$ that acts as $\tilde{\mu}(x \gg 1) \approx 1$ and $\tilde{\mu}(x \ll 1) \approx x$ [17. Although Modified Newtonian Dynamics offer an explanation for flat rotation curves [18, the theory cannot account for the invisible matter of the Bullet Cluster [19. Regardless, further consideration of Modified Newtonian Dynamics is outside the scope of this thesis.

3 Formation of PBHs in a perfect fluid universe is caused by fluctuations in density, such that the
} 
limits of baryonic density set forth by BBN and the CMB. Although PBH solutions to dark matter have existed for over 30 years, the model has been pursued with new excitement after LIGO announced the observation of merging two $\sim 30 M_{\odot}$ blacks holes $[22$. Recent studies indicate PBHs in the intermediate-mas range $\left(1 M_{\odot}<M<10^{3} M_{\odot}\right)$, and sublunar black holes $\left(10^{17}-10^{21} \mathrm{~kg}\right)$ can still account for all dark matter, although this result is highly dependent on astrophysical parameters included in the applied constraints 23 .

\subsubsection{Axions}

Originally introduced as part of a solution to the strong CP invariance problem, the hypothetical axion is a potential dark matter candidate. Axions are spinless, electrically neutral, and very light. The axion mass, $m_{A}$, which depends on it's decay constant, $f_{A}$, is given by [24],

$$
m_{A} \simeq 6 \mu \mathrm{eV} \frac{10^{12} \mathrm{GeV}}{f_{A}}
$$

and is limited to first order by stellar evolution such that $m_{A}<10^{-2} \mathrm{eV} / \mathrm{c}^{2}[25]$. The energy density of cold axions is typically given as,

$$
\Omega_{A} h^{2} \sim\left(\frac{f_{A}}{10^{12} \mathrm{GeV}}\right)^{7 / 6}
$$

and thus axions could account for the cold dark matter, if $m_{A} \sim \mathcal{O}\left(10 \mu \mathrm{eV} / \mathrm{c}^{2}\right)$.

Axions differ from other dark matter candidates since they never decoupled from thermal equilibrium and are thus considered a nonthermal relic candidate. Experiments such as the Axion Dark Matter eXperiment (ADMX) are working to detect axions that have converted to photons in a microwave cavity permeated by a magnetic field [26]. ADMX results have already excluded axions masses within the range $1.9 \mu \mathrm{eV}<$ $m_{A}<3.69 \mu \mathrm{eV}$ [27]. ADMX-HF, secondary ADMX platform meant to focus on highmass and thus high-frequency searches, has set strong limits on the effective coupling of the axion to two photons, $g_{A \gamma}$, in the mass range $23.55 \mu \mathrm{eV}<m_{A}<24.0 \mu \mathrm{eV}[28$ ]. Selected exclusion limits on the axion mass-coupling parameter space are shown in Fig. 1.4. with ADMX results included in the "Microwave Cavity" limit.

amplitude of perturbations in density, $\delta \rho / \rho$ is at least $\delta \rho / \rho=[3(1+w) /(5+3 w)] \sin ^{2}[\sqrt{w} /(1+3 w)]$. For radiation fluid, $\delta \rho / \rho \approx 0.4135[21$. 


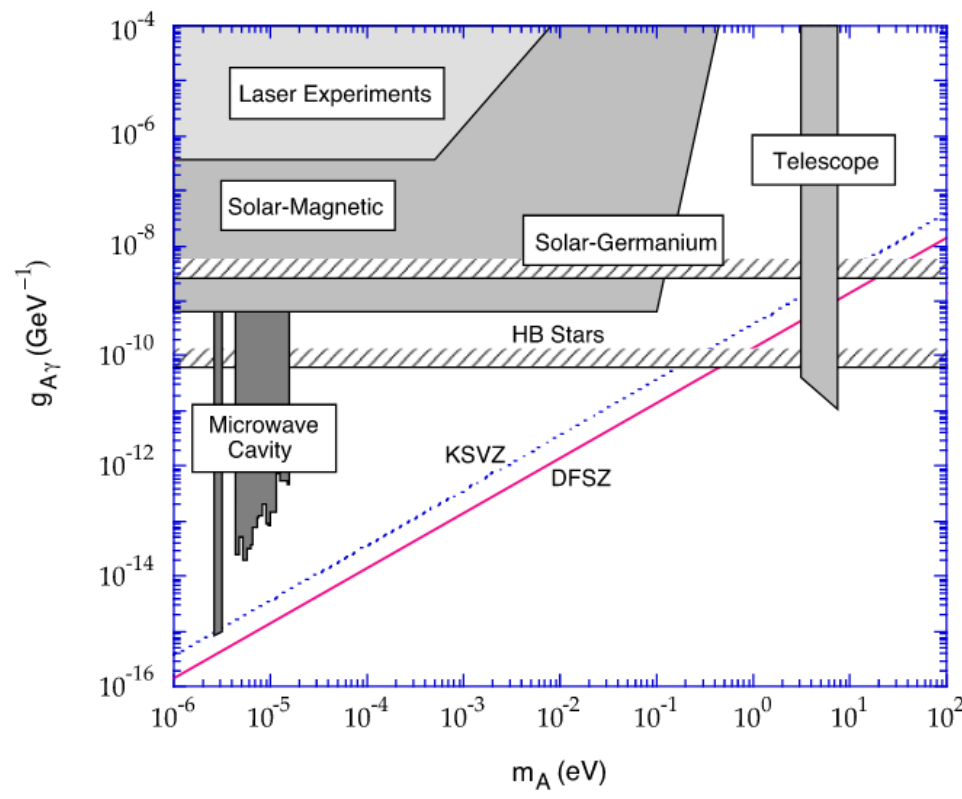

Figure 1.4: Selected exclusion limits on axion masses $\left(m_{A}\right)$ and couplings to two photons $\left(g_{A \gamma}\right)$, from a variety of sources. Dark-matter axions lie between the two classes of axion models, KSVZ (blue dotted) and DFSZ (magenta) in the mass range $\sim 1-100 \mu \mathrm{eV}$. Figure courtesy of [29].

\subsubsection{Sterile Neutrinos}

Sterile Neutrinos, a hypothetical addition to the Standard Model that complements lefthanded neutrinos, have right-handed chirality and do not interact via the weak force. Sterile neutrinos, $\mathcal{N}$, are not completely stable, but instead decay on the scale of the age of the universe. They can decay through the dominate channel, $\mathcal{N} \rightarrow \nu+\nu+\tilde{\nu}$, and the subdominate channel, $\mathcal{N} \rightarrow \nu+\gamma$. The decay products of the subdominate channel are not dark, but actually observable, making it possible to constrain sterile neutrino mass and lifetime based on astrophysical observations and models of sterile neutrino and Standard Model neutrino mixing. Fermi Large Area Telescope results set the sterile neutrino lifetime as $\lesssim 10^{19}$ yrs, for $20<M_{\mathcal{N}}<50 \mathrm{keV}$ [30]. Additional observational data, such as X-ray measurements, further constrain the sterile neutrino mass to $1<M_{\mathcal{N}} \lesssim 50 \mathrm{keV}[31$.

\subsubsection{WIMPs}

The most prominent group of dark matter candidates is arguably the Weakly Interacting Massive Particle, or WIMP. WIMPs are expected to have mass between $1 \mathrm{GeV}$ and 10 
$\mathrm{TeV}$, and cross sections of roughly weak strength [32. WIMPs are proposed to have decoupled from thermal and chemical equilibrium with Standard Model particles once the rate of conversion between WIMPs and other particles became less than the expansion rate of the universe, and are thus considered a thermal relic candidate. Neutrinos were originally proposed as a potential hot WIMP candidate, but the three currently known neutrinos have insufficient mass to account for the present density of dark matter (sum of neutrino masses is constrained to $<0.23 \mathrm{eV}[9]$ ). Furthermore, neutrino contribution to cosmic density has been limited to $\Omega_{\nu} h^{2}<0.0025$ [7] by studies of BAO and large scale structure, far less than the total mass density.

\section{SUSY Candidates}

Currently the most plausible WIMP candidate is a hypothetical particle required by supersymmetric theories. Supersymmetry (SUSY) requires a boson (fermion) supersymmetric partner, or superpartner, for each fermion (boson) in the SM. The Minimal Supersymmetric Standard Model (MSSM) offers the minimal extension to the Standard Model required to be consistent with the phenomenology. To remain consistent with previous experimental results of lepton and baryon number conservation, MSSM also proposes a symmetry called R-Parity, which adds a quantum number of +1 for all SM particles and -1 for their superpartners that must be conserved during interactions [4. This conservation requires a Lightest Supersymmetric Particle (LSP), that cannot decay, and is thus a stable dark matter candidate. Which superpartner qualifies as the LSP depends on the type of supersymmetry breaking. If the breaking of SUSY occurs via the electroweak and strong forces then the gravitino, the spin $3 / 2$ superpartner of the graviton, is a strong LSP candidate. If breakdown occurs via gravity, then the LSP is likely either a sneutrino (the spin 0 superpartner of the neutrino), or the neutralino (the spin 1/2 mixture of wino, bino and Higgsinos [13]) [4]. Due to its large coupling with the Z boson, the standard left-handed sneutrinos of MSSM either have insufficient relic abundance due to quick annihilation, or have a scattering cross section within a region of parameter space already excluded by direct dark matter detection searches 33. Extensions to MSSM do allow for sneutrino dark matter, particularly right-handed sneutrinos. 
One popular paradigm for the study of neutralino dark matter is minimal SUper GRAvity (mSUGRA), a supergravity framework and constrained MSSM were SUSY is broken by gravity. The mSUGRA parameter space is comprised of: $m_{0}$, the common mass of the scalars at Grand Unified Theory (GUT) scale; $m_{1 / 2}$, the GUT scale gaugino mass; $A_{0}$, the GUT scale trilinear soft breaking term; $\tan \beta$, the weak scale ratio of Higgs field vacuum expectation values; $\mu$, the superpotential Higgs (Higgsino) mass term whose magnitude is determined by the conditions of appropriate electroweak symmetry breaking [34].

Within the whole of mSUGRA parameter space, cold dark matter is restrained to only a few distinct regions which possess an enhanced neutralino annihilation cross section:

1. The stau-neutralino co-annihilation region $\left(\tilde{\tau}-\tilde{\chi}_{1}^{0}\right)$, where neutralinos co-annihilate with staus that are thermally present in early cosmic plasma [35. This region requires low $m_{0}$ where stau mass, $m_{\tilde{\tau}} \simeq m_{\tilde{\chi}_{1}^{0}}$, such that the difference in stau and neutralino mass is $\sim 5-15 \mathrm{GeV}[36]$.

2. The A-annihilation funnel region (for CP-odd Higgs boson, A) at high $\tan \beta(\sim 50)$, where $2 m_{\tilde{\chi}_{1}^{0}} \simeq m_{A}$. In this region $\chi_{1}^{0}$ pair annihilation is enhanced by broad schannel A-resonance [37.

3. The h-resonance region (for CP-even, neutral Higgs boson, $h$ ) at low $m_{1 / 2}$ values, where $2 m_{\tilde{\chi}_{1}^{0}} \simeq m_{h}$. In this region $\chi_{1}^{0}$ pair annihilation is enhanced by narrow s-channel $h$-resonance [38].

4. The hyperbolic branch/focus point region at large $m_{0}$ and small $|\mu|$, where the neutralino has a larger Higgsino component 4 , allowing for enhanced neutralino annihilation into WW, ZZ, or Zh [39].

5. The stop-neutralino co-annihilation region $\left(\tilde{t}-\tilde{\chi}_{1}^{0}\right)$, where neutralinos co-annihilate with light stops. This region requires $A_{0}$ values such that $m_{\tilde{t}} \simeq m_{\tilde{\chi}_{1}^{0}}[40$.

\footnotetext{
${ }^{4}$ In the bulk of the parameter space $\tilde{\chi}_{1}^{0}$ is nearly pure-bino. In the focus point region it is more mixed bino-Higgsino.
} 
In the remaining parameter space the neutrino relic density, $\Omega_{\tilde{\chi}_{1}^{0}}$, is roughly $10-1000$ times larger than the measured dark matter abundance, and is thus excluded from consideration in the case of thermally-produced, neutralino-only cold dark matter. More relaxed MSSM models have additional parameters, providing new, more flexible parameter space regions with acceptable relic density and cross section.

\section{Dark Sector Candidates}

Dark sector models, also called hidden sector models, rely on dark matter being a component of a "dark sector" which does not interact via Standard Model gauge fields. Dark sector theories propose an additional force, often called a dark electromagnetism, which is mediated by a long-range $\mathrm{U}(1)$ gauge field, and couples only to dark matter [41. The hypothetical dark electromagnetic force carrier, called a dark phonon $(\hat{\gamma})$, would be lighter in mass than the WIMP, with dark matter annihilation/creation, $\chi \bar{\chi} \rightarrow \hat{\gamma} \hat{\gamma}$, to occur predominantly through t-channel exchange. Kinetic mixing between dark photons and ordinary photons would then provide the leading interaction with the Standard Model. The degree of kinetic mixing and the dark phonon mass would determine the cross section describing the elastic scattering of this WIMPs with protons [42].

Models utilizing SUSY and the dark sector can remove the tight experimental constraints typical of LSP dark matter while still achieving the desired relic density. These models, which are WIMPless when the dark matter mass and coupling parameters are not on the weak scale, propose supersymmetry breaking mediated by gauge interactions to the MSSM and the dark sector, which contains the dark matter particle. WIMPless models benefit from automatically having the approximately correct relic density to match cosmological measurements, regardless of the dark matter candidate mass (within a $10 \mathrm{MeV} \lesssim M_{\chi} \lesssim 10 \mathrm{TeV}$ range) or whether the candidate is a boson or fermion [43]. If no direct coupling exists between the dark sector and the Standard Model, then the dark matter will only interact with known particles very weakly. However, connecting sectors could be present, containing particles charged under both the MSSM and the dark sector [44]; Fig. 1.5 illustrates the connecting sector containing such particles, Y. In this case, both MSSM and dark sector gauge groups would contribute to the Y superpartner mass, resulting in $M_{Y} \sim \operatorname{Max}\left(m_{\text {weak }}, M_{\chi}\right)$, where $m_{\text {weak }}$ is the characteristic weak scale mass. 


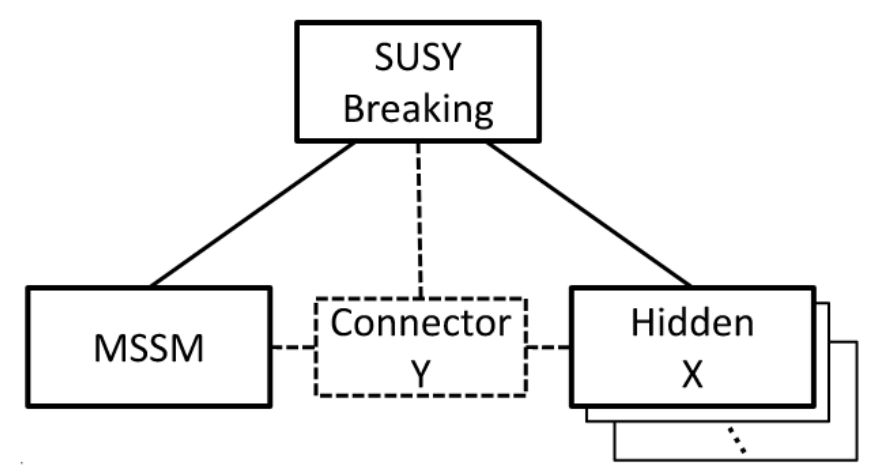

Figure 1.5: Sectors of the WIMPless model, with gauge-interaction mediated SUSY breaking to the MSSM and hidden (dark) sector, containing dark matter particle X. Additional hidden sectors and dark matter components could also be present. Optional connecting sector containing particle(s) Y, which is (are) charged under both the MSSM and the dark sector. Figure courtesy of [44].

Asymmetric dark matter (ADM) models propose an asymmetry between dark matter and its antiparticle. Drawing upon observations that,

$$
\Omega_{\chi} \approx 5 \Omega_{b}
$$

ADM proposes that the present-day abundance of dark matter is due to a dark matter asymmetry that is related to the measured baryon/anti-baryon asymmetry, which in turn results in the current baryon abundance [45]. The relation between asymmetries, due to certain processes that occur during the early universe but freeze out at temperatures above $M_{\chi}$, gives rise to a relation between densities, such that $n_{\chi} \sim n_{b}$, for baryonic density, $n_{b}$ [46]. Comparing this relation with that of Eq. 1.28 yields,

$$
\Omega_{\chi} \sim \frac{M_{\chi}}{m_{b}} \Omega_{b}
$$

Taking proton mass for $m_{b}$ gives $M_{\chi} \sim 5-15 \mathrm{GeV} / \mathrm{c}^{2}$. ADM tends to be an umbrella model, which can pair with various other models, yielding a broad range of potential dark matter characteristics [47] [48]. ADM-proton interactions are possible, but not required; in the case of a spin-independent interaction, the cross section ranges from $10^{-41}$ to $10^{-45} \mathrm{~cm}^{2}$ [49] [46]. 
Overall, WIMPs have become a prevailing dark matter candidate, and shall be the focus of this thesis.

\subsection{Dark Matter Detection}

With such an abundance of evidence it is not surprising that many experimental collaborations employ various methods to discern more of the WIMP's characteristics.

\subsubsection{Collision Experiments}

WIMP production might be a possibility for high energy collision experiments such as CERN's Large Hadron Collider (LHC), which could produce supersymmetric particles from proton-proton collisions [50]. These dark matter particles could not be directly observed in the LHC, but instead must be deduced from nonconservation of observed momentum. Recent results from ATLAS [51] and CMS [52], the two general-purpose particle physics detectors built on the LHC, saw no significant excess above the Standard Model expectation in the 2012 data taken with the LHC operating at a center of mass (COM) energy of $8 \mathrm{TeV}$. By not observing supersymmetry, these results pushed the supersymmetric scale towards $\mathcal{O}(\mathrm{TeV})$ or greater [53].

In terms of the mSUGRA model, the negative search results of earlier LHC studies [54] 55] excluded all of the $h$-resonance region, the preferred (least fine-tuned) portion of the stau and stop co-annihilation regions, and the preferred (lowest $m_{0}$ and $m_{1 / 2}$ ) regions of the A-resonance annihilation region [34]. Additionally, a SM-like Higgs boson with mass $m_{h} \sim 125 \mathrm{GeV}$ provides further constraints, including $m_{0} \gtrsim 0.8 \mathrm{TeV}$, and $m_{A} \gtrsim 1 \mathrm{TeV}$ among others. 95\% C.L. exclusions limits from 2012 data are shown in Fig. 1.6 along with some addition constrains from the theory and preferred regions, much of which is now excluded.

Given the ATLAS and CMS results, the best chance for direct LSP dark matter detection is shown in the likelihood contours of Fig. 1.7, in which the best-fit point has a spin-independent cross section of $\sim 10^{-47} \mathrm{~cm}^{2}[56]$. 

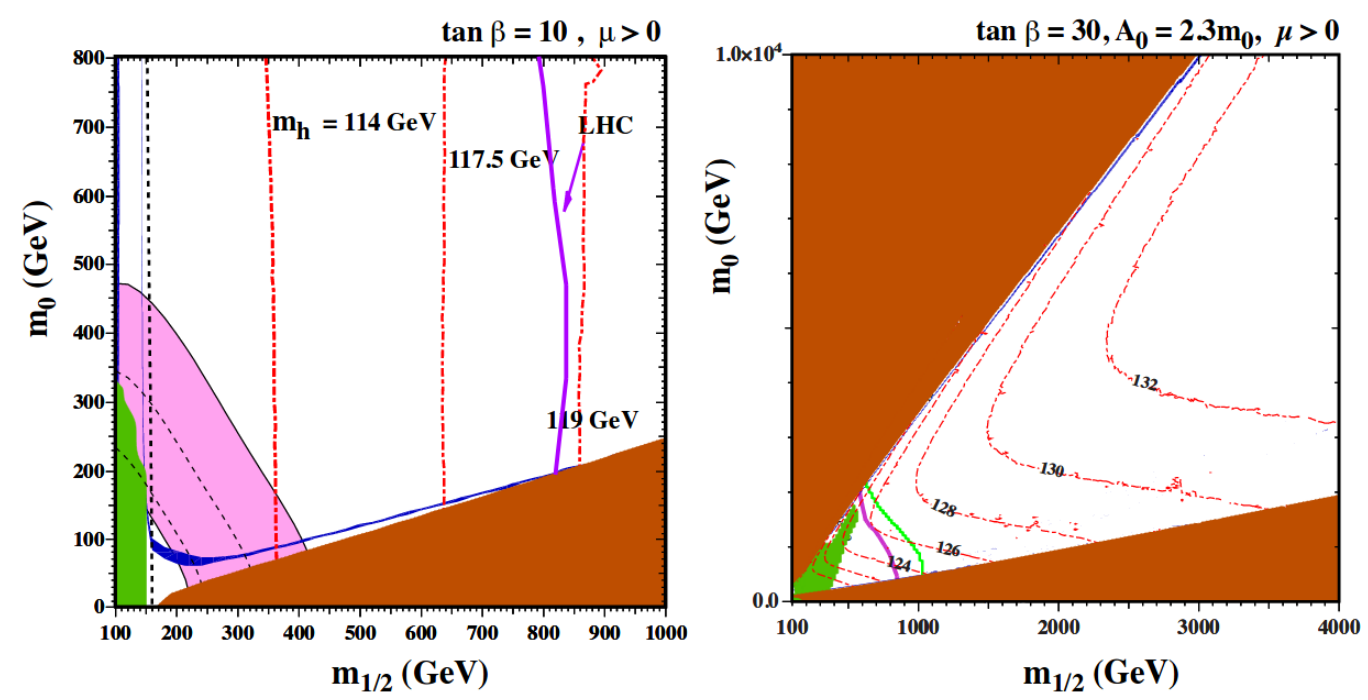

Figure 1.6: The $\left\{m_{1 / 2}, m_{0}\right\}$ parameter space for two cases: (LEFT) $\tan \beta=10, \mu>0$, $A_{0}=0$ and (RIGHT) $\tan \beta=30, \mu>0$, assuming $A_{0}=2.3 m_{0}$. Contours include constant $m_{h}$ (dot-dashed red) and $M_{\chi^{ \pm}}=104 \mathrm{GeV}$ (dashed black). Shaded exclusion regions include stau LSP (bottom brick red) and stop LSP (RIGHT plot, top brick red) with neutralino LSP in between, $b \rightarrow s \gamma$ (dark green), $B \rightarrow \mu^{+} \mu^{-}$(light green line), which also excludes everything to its left. Preferred regions of $\left(g_{\mu^{-}}-2\right)$ (pink shaded $2 \sigma$, dashed back line $1 \sigma$ ), and LSP density determined by Planck [9] (blue). The regions to the left of the $95 \%$ C.L. LHC curves (dark pink), are excluded by LHC data. Figure courtesy of [53]. 


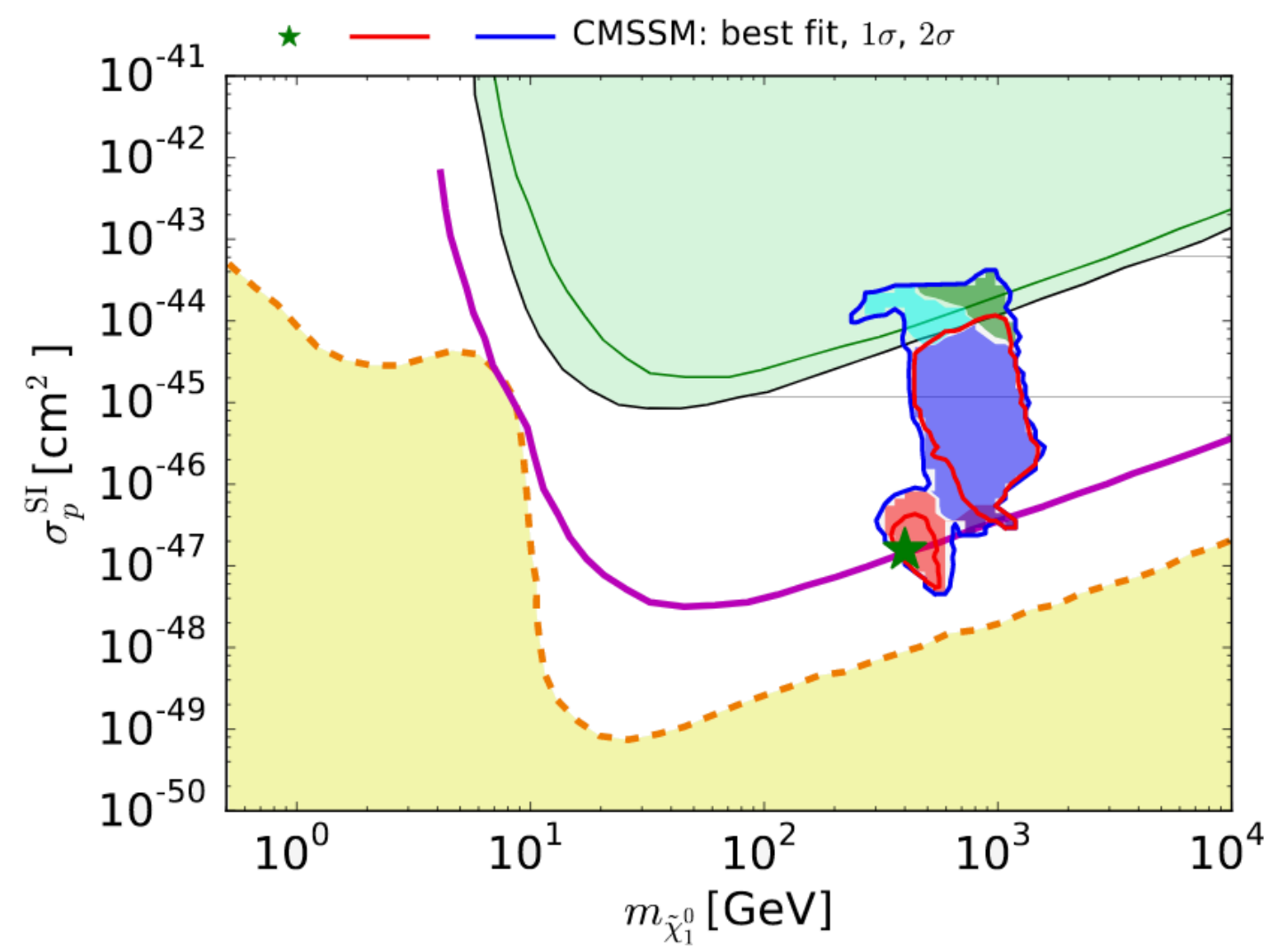

Figure 1.7: Post-LHC Run I Likelihood contours for spin-independent cross section, $\sigma_{p}^{\mathrm{SI}}$ in mSUGRA paradigm. Best fit (green star), and $1 \sigma$ (red) and $2 \sigma$ (blue) contours shown, with shaded region color indicating dominate annihilation mechanism for obtaining correct relic density: stau co-annihilation (pink), A-funnel (blue), focus point (cyan), stau co-annihilation/A-funnel hybrid (purple) and chargino co-annihilations (green). Current sensitivities of XENON100 [57] and LUX [58] experiments shown in black and green lines, and projected 95\% C.L. sensitivities of LUX-Zepelin (LZ) experiment [141] shown in purple line. Neutrino floor shown by dashed orange line, indicating the region below which astrophysical neutrino backgrounds dominate (shaded yellow). Figure courtesy of [56]. 


\subsubsection{Indirect Detection}

Indirect detection is the experimental method of searching for the remnants of WIMPWIMP annihilations as evidence of dark matter. The rate of these annihilations depends on the dark matter density, and thus is more likely to occur in regions of high dark matter accumulation. The galactic center of the Milky Way is just such a dense, dark matter filled region. Additionally, dark matter particles can become trapped in large objects such as the Earth or sun after loosing energy via scattering with nucleons inside these objects.

Dark matter annihilations could result in several types of detectable products including continuous spectrum and mono-energetic gamma-rays, neutrinos, and various types of antimatter. Gamma-rays produced by dark matter annihilations cannot cleanly pass through Earth's atmosphere and thus are best detected above the atmosphere via satellites or balloon-borne telescopes, or by studying the resulting particle showers [4]. Since gamma-rays cannot escape the dense matter in objects like the Earth and sun, gamma-ray emissions from the galactic center are the focus of many studies. The EGRET experiment found a region of the galactic center emitting gamma-rays in the energy range of $\sim 1-10 \mathrm{GeV}$ at an excess to what would be expected from interactions between primary cosmic rays and interstellar medium [60. The FERMI Large Area Telescope (LAT) 4-year measurement of the isotropic gamma-ray background 5 and 6 year measurement of Milky Way satellite galaxies reported no evidence for a dark matter signal, setting competitive limits on the dark matter-induced isotropic gamma-ray signal for masses up to tens of $\mathrm{TeV} / \mathrm{c}^{2}[61$, and increasing the annihilation cross section by a factor of $\sim 2$ for large dark matter masses $\left(M_{\chi, 5} \geq 1 \mathrm{TeV} / \mathrm{c}^{2}\right.$ and $M_{\chi, \tau^{+} \tau^{-}} \geq 70$ $\mathrm{GeV} / \mathrm{c}^{2}$ ) 62. Similar results are reported by the H.E.S.S. Cherenkov telescope, such as the H.E.S.S. inner galactic halo 10-year study [63], and observations of dwarf spheroidal galaxies 64]. A H.E.S.S. search for dark matter line-like signals performed in the vicinity of the Galactic Center, ruled out at 95\% C.L. the presence of a $130 \mathrm{GeV}$ line 65] previously reported in Fermi LAT data [66, as shown in Fig. 1.8

\footnotetext{
${ }^{5}$ The isotropic gamma-ray background is the remainder of the extragalactic gamma-ray background once the contribution from survey-detected extragalactic sources has been subtracted.
} 


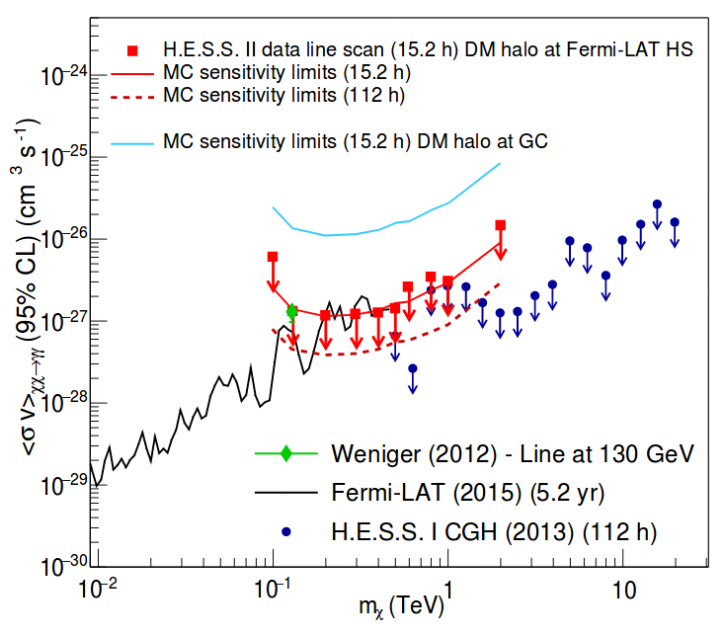

Figure 1.8: H.E.S.S. velocity-averaged annihilation cross section, $\langle\sigma v\rangle$, limits at $95 \%$ C.L. (red points) for the line scan between $100 \mathrm{GeV}$ and $2 \mathrm{TeV}$, with MC estimations (red solid, red dashed, light blue). Former limits from H.E.S.S. I 67] (blue points), and Fermi LAT [68] (black) also shown. The $130 \mathrm{GeV}$ line feature $\langle\sigma v\rangle$ value (green) is from Fermi LAT [66]. Figure courtesy of [65].

Unlike gamma radiation, neutrinos are able to escape the dense inner regions of celestial objects, inspiring many collaborations to explore the sun and Earth as sources of increased neutrino production via accumulating WIMP annihilations. Neutrino telescopes can detect the upward through-going muons from muon neutrino interactions with the Earth. Results from IceCube/DeepCore, ANTARES, and Super-Kamiokande neutrino telescopes, located at the South Pole, Mediterranean Sea, and Japan, respectively, found no significant excess over background of neutrinos from dark matter self-annihilations in the Milky Way [69] [70] 71] [72, nor from dark matter annihilation in the Sun [73] [74] [75], or the center of the earth [76] [77]. Exclusion limits on spin-independent WIMP cross section from these neutrino telescope results are shown in Fig. 1.9.

Antimatter can be produced in several varieties from various dark matter annihilation modes. The most common forms of antimatter utilized in indirect detection are positrons, antiprotons, and antideuteron. As with gamma-rays, antimatter would not be able to escape nuclei-dense regions such as Earth's core. Instead the antimatter of interest is produced in WIMP annihilations in the galactic halo. Antimatter particles then propagate through the halo in non-straight paths, diffusing and losing energy via Compton scattering and synchrotron effects of galactic magnetic fields. Indirect detection experiments search for antimatter signals in excess of the background produced by standard cosmic ray processes. In 1995 the High Energy Antimatter Telescope (HEAT) 


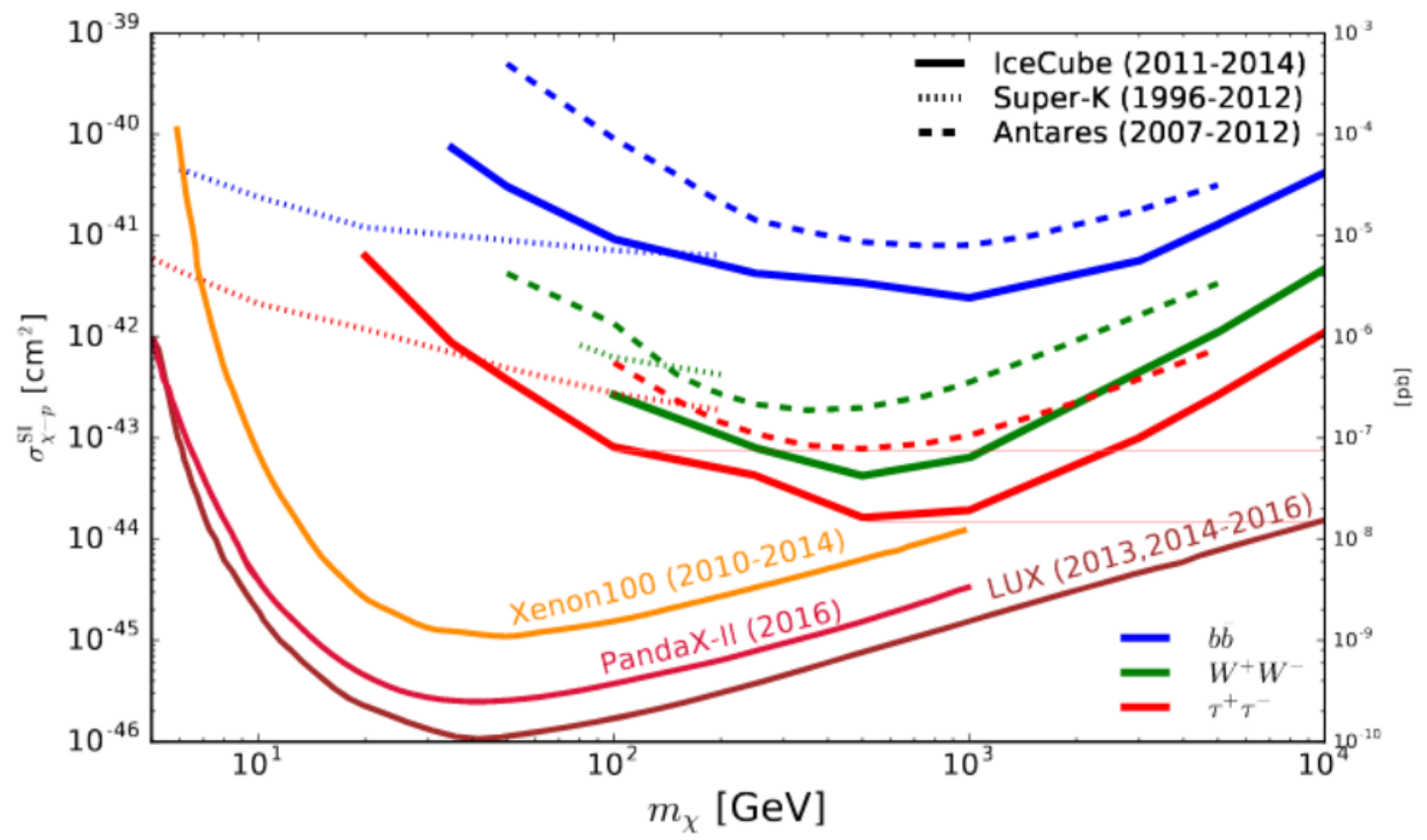

Figure 1.9: Exclusion limits from IceCube [70] (solid line), Super-Kamiokande 75] (dashed), and ANTARES [74] (dotted) neutrino telescopes, for $b b$ (blue), $W^{+} W^{-}$ (green), and $\tau^{+} \tau^{-}$annihilation channels. Also included are direct detection limits from XENON100 [57], PandaX-II [78], and LUX [58]. Figure courtesy of [70]. 
detected an excess in the flux of positrons peaking around 7-20 GeV suggesting a potential exotic component to the usual background [79]. This measurement repeated in 2000 with new version of the HEAT instrument, HEAT-pbar, with similar results [80].

PAMELA [81] and AMS02 [82] have some of the best measurements of both antiproton and positron flux, finding an excess in positron flux that might require an exotic or astrophysical explanation in the $1.5-300 \mathrm{GeV}$ range, and determining antiproton energy spectrum consistent with expectations. Though these measurements could be explained through WIMP annihilation, it is difficult to reconcile them with electron spectrum measurements of PAMELA [83], and this would require cross sections above expectation for a thermal WIMP, except under certain models [7].

\subsubsection{Direct Detection}

Whereas indirect detection experiments search for dark matter through its annihilation products, direct detection experiments search for nuclear recoils produced in WIMP interactions with ordinary matter. As the focus of direct detection experiments, it is extremely important that the recoil energy and WIMP-Nucleus event rate is well understood, even if little is known about the properties of WIMPs. The expected differential event rate depends on astrophysical models of dark matter, properties of elastic dark matter-nucleon interactions, and detector target material properties.

\section{Astrophysical Input and the Standard Halo Model}

The expected event rate depends on the number of target particles, the number of incident particles, the relative velocity between the two, and the interaction cross section. For a target with $N_{T}$ nuclei per unit mass, the differential event rate per mass, $d R$, is given by,

$$
d R=N_{T} v d \sigma d n
$$

for a WIMP velocity $\mathbf{v}(v=|\mathbf{v}|)$, a differential WIMP-nucleus cross section $d \sigma$, and a local differential WIMP number density, $d n$. The velocity-dependent WIMP number density is given by,

$$
d n(v)=\frac{n_{0}}{k} f\left(\mathbf{v}, \mathbf{v}_{\mathbf{E}}\right) d^{3} \mathbf{v}
$$


where $n_{0}$ is the mean WIMP number density, which depends on the local WIMP mass density, $\rho_{0}$, and WIMP mass, $M_{\chi} ; v_{E}$ is the Earth's velocity relative to the dark matter halo; $f\left(\mathbf{v}, \mathbf{v}_{\mathbf{E}}\right)$ is the WIMP velocity distribution function of the halo, with respect to Earth; and $k$ is normalization such that $n_{0}=\int_{0}^{v_{e s c}} d n$ for local galactic escape velocity, $v_{\text {esc }}$ [84].

Parameters $n_{0}$ (or more particularly, $\rho_{0}$ ), $v_{E}$, and $v_{e s c}$, as well as the velocity distribution $f\left(\mathbf{v}, \mathbf{v}_{\mathbf{E}}\right)$, are estimates based on astronomical measurements that combine to form the Standard Halo Model (SHM) for dark matter. The SHM assumes an isotropic and isothermal spherical dark matter distribution with a density that goes as $\rho(r) \propto r^{-2}$ where $r$ is the distance from the galactic center [85]. The relationship between density and radial distance can be deduced, beginning with the collisionless Boltzmann equation for a $6 \mathrm{D}$ phase space density $f(\mathbf{x}, \mathbf{v})$,

$$
\frac{\partial f}{\partial t}+\mathbf{v} \cdot \vec{\nabla} f-\vec{\nabla} \Phi \cdot \frac{\partial f}{\partial \mathbf{v}}=0
$$

where $\Phi$ is the Newtonian potential. In the SHM case, the solution is found to be,

$$
f(\mathbf{x}, \mathbf{v})=\mathcal{C} e^{-E \chi / k_{B} T}
$$

where $E_{\chi}=\frac{1}{2} M \chi v^{2}+M_{\chi} \Phi(\mathbf{x})$ is the dark matter energy, and $\mathcal{C}$ is a normalization constant. Taking the zeroth velocity moment of Eq. 1.33 gives the position-dependent probability density 6

$$
\begin{aligned}
\nu(\mathbf{x}) & =\int f(\mathbf{x}, \mathbf{v}) d^{3} \mathbf{v}, \\
& =\mathcal{C} e^{-M_{\chi} \Phi(\mathbf{x}) / k_{B} T} \int e^{-\frac{1}{2} M \chi v^{2} / k_{B} T} d^{3} \mathbf{v}, \\
& =\mathcal{C}^{\prime} e^{-M_{\chi} \Phi(\mathbf{x}) / k_{B} T},
\end{aligned}
$$

where the integral and other constants have been absorbed into factor $\mathcal{C}^{\prime}$. From this it can be seen that the dark matter density goes as $\rho \propto e^{-M_{\chi} \Phi(\mathbf{x}) / k_{B} T}$. Substituting the natural $\log$ of this relation into the Poisson equation for the system,

$$
\frac{1}{r^{2}} \frac{d}{d r}\left(r^{2} \frac{d \Phi}{d r}\right)=-4 \pi G \rho
$$

\footnotetext{
6 A technical definition of $\nu(\mathbf{x})$ is the probability per unit volume of finding a particular particle at $\mathbf{x}$. From this the number density is given by $n(\mathbf{x})=N \nu(\mathbf{x})$ for a system of $N$ total particles.
} 
yields the relationship,

$$
\frac{1}{r^{2}} \frac{d}{d r}\left(r^{2} \frac{d \ln (\rho)}{d r}\right)=-\frac{4 \pi G k_{B} T}{M_{\chi}} \rho
$$

This solves to,

$$
\rho(r)=\frac{M_{\chi}}{2 \pi G k_{B} T r^{2}} .
$$

This solution to the Poisson equation gives the $\rho \propto r^{-2}$ behavior needed for flat rotation curves. This is the singular isothermal sphere (SIS) profile [86], an unphysical profile due to the singularity at the origin and non-converging integration behavior 7 . A coordinate change can avoid the singularity, using dimensionless variables,

$$
\tilde{\rho} \equiv \frac{\rho}{\rho_{c}}, \quad \tilde{r} \equiv \frac{r}{r_{0}}, \quad \text { where } \quad r_{0} \equiv \sqrt{\frac{9 k_{B} T}{4 \pi M_{\chi} G \rho_{c}}} .
$$

Here $\rho_{c}$ is the galactic central density, and $r_{0}$, referred to as the King radius, is the radius at which the isothermal sphere's density has reduced to roughly half of $\rho_{c}$ [89]. In terms of the dimensionless variables, Eq. 1.38 becomes,

$$
\frac{1}{\tilde{r}^{2}} \frac{d}{d \tilde{r}}\left(\tilde{r}^{2} \frac{d \ln (\tilde{\rho})}{d \tilde{r}}\right)=-9 \tilde{\rho}
$$

For $r \gg r_{0}$, the solution to Eq. 1.41 (found through numerical integration with defined boundary conditions $\tilde{\rho}(0)=1$ and $d \tilde{\rho} / d \tilde{r}=0$ ) approaches $\tilde{\rho} \propto \tilde{r}^{-2}$, maintaining the behavior of the SIS solution in Eq. 1.39. Approaching the galactic center, the dimensionless density plateaus to $\tilde{\rho}=1$, avoiding the SIS singularity at $r=0$.

The canonical value for the local dark matter in the $\mathrm{SHM}$ is $\rho_{0}=0.3 \mathrm{GeV} / \mathrm{c}^{2} \mathrm{~cm}^{3}$ [90]. However, recent measurements give a wide range of local density values that fall beyond corresponding statistical errors [90, 91] 92, 93]. This indicates model-dependent, systematic differences in measurement methods that are not yet understood [94], but outside the scope of this thesis.

\footnotetext{
${ }^{7}$ Alternative density profiles have been developed which more accurately model galactic mass distribution at large radii. The truncated isothermal sphere, developed by Brainerd, Blandford, and Smail, has a density given by $\rho_{B B S} \propto \frac{1}{r^{2}} \frac{s^{2}}{r^{2}+s^{2}}$, for truncation radius $s$ [87]. The universal density profile, also called the Navarro, Frenk, and White profile, has a density given by $\rho_{N F W} \propto\left[\left(r / r_{s}\right)\left(1+r / r_{s}\right)^{2}\right]^{-1}$, for a scale radius, $r_{s}$ [88. For simplicity, the SIS profile will be used in this thesis.
} 
The velocity distribution function is found by integrating Eq. 1.33 over spatial coordinates,

$$
\begin{aligned}
f(v) & =\int f(\mathbf{x}, \mathbf{v}) d^{3} \mathbf{x} \\
& =\mathcal{C} e^{-\frac{1}{2} M \chi v^{2} / k_{B} T} \int e^{-M_{\chi} \Phi(\mathbf{x}) / k_{B} T} d^{3} \mathbf{x} \\
& =\mathcal{C}^{\prime \prime} e^{-\frac{1}{2} M \chi v^{2} / k_{B} T}
\end{aligned}
$$

where again the integral and other constants have been absorbed into factor $\mathcal{C}^{\prime} 8$. Allowing the $1 \mathrm{D}$ radial velocity dispersion to be defined as, $\sigma_{v} \equiv \sqrt{k_{B} T / M_{\chi}}$, the velocity distribution can be rewritten as $f(v) \propto e^{-v^{2} / 2 \sigma_{v}^{2}}$. The temperature of the dark matter halo cannot be measured, but $\sigma_{v}$ can be determined from other measurable quantities. In the singular isothermal sphere profile, the dispersion relates to the galactic circular velocity as $v_{c}=\sqrt{2} \sigma_{v}$. In the King radius solution $v_{c}(\tilde{r})$ is not constant, but at large distances from the galactic center, where the galactic rotation curve has become asymptotic (such as in Earth's galactic neighborhood), the characteristic (most probable) dark matter velocity, $v_{0}$ is the circular velocity. Thus the velocity dispersion can be related to a measurable quantity via $v_{0}=v_{c}(\tilde{r} \rightarrow \infty)=\sqrt{2} \sigma_{v}$.

The galactic rotation velocity at Earth's distance from the galactic center is referred to as the local standard of rest (LSR), $\Theta_{0}$. Since Earth is sufficiently far from the galactic center $(\approx 8.5 \mathrm{Kpc})$, and thus resides in a near-flat region of the galactic rotation curve, it holds true that $\Theta_{0}=v_{c}(r \rightarrow \infty)$, and thus the characteristic velocity of dark matter in the SHM is the LSR. Canonical SHM calculations draw upon a 1986 measurement of LSR [95], setting $v_{0}=220( \pm 20) \mathrm{km} / \mathrm{s}$. Recent measurements span a $196-270 \mathrm{~km} / \mathrm{s}$ region, but remain close to this value [96] 97] 98].

Thus far the velocity distribution of Eq. 1.44 has been expressed in terms of the halo reference frame. To determine the distribution in the lab (i.e. Earth) frame, the dark matter velocity must be boosted such that,

$$
\mathbf{v} \rightarrow \mathbf{v}+\mathbf{v}_{\mathbf{E}}
$$

where $\mathbf{v}_{\mathbf{E}}$ is Earth's velocity relative to the halo frame. This velocity is the culmination

\footnotetext{
${ }^{8}$ These integration constants will be take the form $1 / k$ in the final expression.
} 
of several astrophysical velocities, given by,

$$
\mathbf{v}_{\mathbf{E}}=\Theta_{0}+\mathbf{v}_{\odot}+\mathbf{v}_{\oplus} .
$$

As previously discussed, $\boldsymbol{\Theta}_{\mathbf{0}}$ is the local standard of rest, and also the value of characteristic dark matter velocity, $v_{0}$. The standard Solar velocity with respect to neighboring stars, referred to as the peculiar velocity, is represented by $\mathbf{v}_{\odot}$. Typically only the component of the Solar peculiar velocity in the direction of galactic rotation is considered in the SHM, such that $v_{\odot}=12.2 \mathrm{~km} / \mathrm{s}$, while inward and upward components are ignored [99]. Earth's orbit around the Sun, inclined at $\sim 60^{\circ}$ relative to the plane of the galactic disk, also contributes to $v_{E}$ [100], with Earth's orbital velocity given by $v_{\oplus}=29.8 \mathrm{~km} / \mathrm{s}$. However, with the exception of annular modulation-focused studies, Earth's orbital velocity is assumed to average out over a year, and is ignored. Therefore, Earth's velocity with respect to the WIMP halo is taken to be $\mathbf{v}_{\mathbf{E}}=\mathbf{v}_{\mathbf{0}}+\mathbf{v}_{\odot}$.

Incorporating the boost gives the final velocity distribution form for a Maxwellian distribution 9 about $v_{0}$,

$$
f\left(\mathbf{v}, \mathbf{v}_{\mathbf{E}}\right)=e^{-\left(\mathbf{v}+\mathbf{v}_{\mathbf{E}}\right)^{2} / v_{0}^{2}},
$$

where all the integration constants, neglected here, have been absorbed into the $1 / k$ factor included in Eq. 1.31. With this distribution the normalization factor $\mathrm{k}$ is given by,

$$
\begin{aligned}
k & =\int_{0}^{2 \pi} d \phi \int_{-1}^{1} d(\cos \theta) \int_{0}^{v_{e s c}} f\left(v, v_{E}\right) v^{2} d v \\
& =\left(\pi v_{0}^{2}\right)^{3 / 2}\left[\operatorname{erf}\left(\frac{v_{e s c}}{v_{0}}\right)-\frac{2 v_{e s c}}{\sqrt{\pi} v_{0}} e^{-v_{e s c}^{2} / v_{0}^{2}}\right] .
\end{aligned}
$$

where $v_{\text {esc }}$ limits the WIMP distribution. The galactic escape velocity is set to 544 $\mathrm{km} / \mathrm{s}$ in the SHM, taken from a 2007 RAVE survey measurement [102]. A more recent RAVE study from 2014 gave $90 \%$ uncertainty of $492 \leq v_{e s c} \leq 587 \mathrm{~km} / \mathrm{s}$, with a mean velocity of $533 \mathrm{~km} / \mathrm{s}$ [103].

Since the calculations presented here are in the lab frame, care must used to properly limit the maximum velocity possible for a elastically scattering WIMP, $v_{\max }$. The

\footnotetext{
${ }^{9}$ Empirical model distributions have been constructed from simulations to more accurately describe the halo, particularly at velocities approaching $v_{e s c}$. One such example is Mao et al.'s empirical function $f(v) \propto e^{v / v_{a}}\left(v_{e s c}^{2}-v^{2}\right)^{p}$ 101] with model parameters, $p$, and $v_{a}$, fit to a Milky Way-like galaxy simulation such that $p=2.7$ and $v_{a} / v_{\text {esc }}=0.6875$ [106]. For simplicity, this thesis will use the commonly accepted Maxwellian distribution.
} 
velocity must be bounded such that $\left|\mathbf{v}+\mathbf{v}_{E}\right| \leq v_{\text {esc }}$. For a scattering angle of $\theta$ in the galactic rest frame, the maximum velocity is given by,

$$
v_{\max }(\theta)=\sqrt{v_{e s c}^{2}-v_{E}^{2}\left(1-\cos ^{2}(\theta)\right)}-v_{E} \cos (\theta) .
$$

\section{Target Material}

Given a target with nuclear mass $M_{T}\left(\mathcal{O}\left(\mathrm{GeV} / \mathrm{c}^{2}\right)\right)$, and a WIMP of mass $M_{\chi}\left(\mathcal{O}\left(\mathrm{GeV} / \mathrm{c}^{2}\right)\right)$, and kinetic energy $E_{\chi}=\frac{1}{2} M_{\chi} v^{2}$, the nuclear recoil energy $E_{R}$ for a collision with COMframe scattering angle $\theta$ is,

$$
E_{R}=\frac{\mu_{T}^{2} v^{2}}{M_{T}}(1-\cos \theta)
$$

where $\mu_{T} \equiv M_{\chi} M_{T} /\left(M_{\chi}+M_{T}\right)$ is the reduced mass of the WIMP-target nucleus system. Notice that the average recoil energy is $\left\langle E_{R}\right\rangle=\mu_{T}^{2} v^{2} / M_{T}$. One can justify the direct detection focus on WIMP nuclear recoils by comparing the average nuclear energy of a WIMP scattering off of a Germanium nuclei $\left(\sim 67 \mathrm{GeV} / \mathrm{c}^{2}\right)$ to the energy of a WIMP scattering off of an electron $\left(511 \mathrm{keV} / \mathrm{c}^{2}\right)$. For a $50 \mathrm{GeV} / \mathrm{c}^{2}$ WIMP with velocity $v=$ $10^{-3} c$, the nuclear scatter yields an average energy of $\left\langle E_{R}\right\rangle=12.2 \mathrm{keV}$. The same WIMP scattering with an electron yields an average of $\left\langle E_{R}\right\rangle=0.5 \mathrm{eV}$, below the energy thresholds of current detector technology.

Combining Eq. 1.51 with Eqs. 1.30 and 1.31 gives the differential event rate with respect to recoil energy,

$$
\frac{d R}{d E_{R}}=n_{0} N_{T} \int_{v_{\text {min }}}^{v_{\max }} \frac{d \sigma}{d E_{R}} \frac{v f\left(v, v_{E}\right)}{k} d^{3} v,
$$

where the integration bounds are set by the maximum and minimum velocities, $v_{\max }$, and $v_{\min }$ which can result in a nuclear recoil of $E_{R}$. The upper velocity limit has already been set in Eq. 1.50, based on astrophysical measurements. The minimum velocity is determined from Eq. 1.51,

$$
v_{\min }=\sqrt{\frac{M_{T} E_{R}}{2 \mu_{T}^{2}}} .
$$

\section{Effective Field Theory of Dark Matter Direct Detection}

The differential cross section contained in Eq. 1.52 depends on the WIMP-nuclei interaction model, and the underlying effective field theory operators that mediate the 
interaction. A pragmatic approach to dark matter interaction is model-independent, and goes beyond leading operators selected for simplicity, considering the full set of non-relativistic operators which describe dark matter interaction with a nucleon.

The set of possible interactions is constrained by certain symmetries. In momentum space, let incoming (outgoing) momentum of the WIMP be $\vec{p}\left(\vec{p}^{\prime}\right)$ and let incoming (outgoing) momentum of the nucleon be $\vec{k}\left(\vec{k}^{\prime}\right)$. Galilean invariance then requires that possible momentum combinations satisfy momentum transfer invariance, $\vec{q}=\vec{p}^{\prime}-\vec{p}=$ $\vec{k}^{\prime}-\vec{k}$ where $|\vec{q}|=\sqrt{2 M_{T} E_{R}}$, and relative incoming velocity invariance, $\vec{v} \equiv \vec{v}_{\chi i n}-\vec{v}_{N i n}$, where $\vec{v}$ is the velocity of the WIMP in the nucleon rest frame. Energy conservation is most easily imposed in the center of mass frame, where total kinetic energy depends on the relative velocity between the WIMP and the nucleon in the target material, $v_{r e l}=v_{\chi}-v_{N}$, such that $E=\frac{1}{2} \mu_{N} v_{r e l}^{2}$ for a WIMP-nucleon reduced mass of $\mu_{N}=$ $\frac{M_{\chi} m_{N}}{M_{\chi}+m_{N}}$. In this frame the incoming energy is $E_{i n}=\frac{1}{2} \mu_{N} v^{2}$, and the final energy state is $E_{\text {out }}=\frac{1}{2} \mu_{N}\left(v+\vec{q} / \mu_{N}\right)^{2}$. Imposing energy conservation is thus equivalent to setting,

$$
\vec{v} \cdot \vec{q}=-\frac{q^{2}}{2 \mu_{N}}
$$

Hermiticity of interaction, essentially an equivalent of crossing symmetry since Hermitian conjugation imposes an exchange of incoming and outgoing particles, further constrains the interactions. With this constraint it is useful to work in Hermitian quantities, such as $i \vec{q}$, since $\vec{q}$ is effectively anti-Hermitian. Similarly, $\vec{v}$ is not Hermitian because it does not have definite parity under an exchange of ingoing and outgoing particles,

$$
\vec{v}^{\dagger} \rightarrow \vec{v}_{\chi \text { out }}-\vec{v}_{N \text { out }}=\vec{v}+\frac{\vec{q}}{\mu_{N}} \neq \pm \vec{v} .
$$

Taking advantage of the energy conservation constraint imposed in Eq. 1.54, a quantity similar to $\vec{v}$ that is Hermitian can be constructed,

$$
\vec{v}^{\perp} \equiv \vec{v}+\frac{\vec{q}}{2 \mu_{N}}
$$

such that both terms are linearly independent from each other, since $\vec{v}^{\perp} \cdot \vec{q}=0^{10}$

\footnotetext{
${ }^{10}$ The quantity $\vec{v}^{\perp}$ can be separated into $\vec{v}_{T}^{\perp}$ term, that acts on the coherent center-of-mass velocity of the whole target nucleus, and a $\vec{v}_{N}^{\perp}$ term, which acts on the relative distances of the nucleon within the target nucleus. Thus, $\vec{v}^{\perp}=\vec{v}_{T}^{\perp}+\vec{v}_{N}^{\perp}$, where $\vec{v}_{T}^{\perp}=\vec{v}_{T}+\vec{q} /\left(2 \mu_{T}\right)$ for $\vec{v}_{T}=\vec{v}_{\chi i n}-\vec{v}_{T i n}$, and $\vec{v}_{N}^{\perp}=-1 / 2\left(\vec{v}_{N \text { in }}+\vec{v}_{N \text { out }}\right)[104$
} 
The set of Hermitian quantities thus include,

$$
i \vec{q}, \quad \vec{v}^{\perp}, \quad \vec{S}_{\chi}, \quad \vec{S}_{N},
$$

where $\vec{S}_{\chi}$ and $\vec{S}_{N}$ are the intrinsic spin of dark matter, and the nucleon spin, respectively. Since the spin of dark matter is unknown, this allows for a unified treatment of the spin possibilities. For scalar dark matter, the spin operators will not appear. For spin 1/2 dark matter, the spin operators will be $\frac{1}{2} \vec{\sigma}$, where $\sigma^{i}$ are Pauli sigma matrices, acting on both $\chi$ and $N$ spinors. In the case of vector dark matter, the spin operators are spin 1 representations of the angular momentum generator, $J^{i}$, acting on the $\chi$ vector [104]. These spin operators are already invariant under Hermitian conjugation, so new spin operators needn't be constructed.

The final constraint to be applied is on violations of CP symmetry, or equivalently T symmetry, since any Lorentz invariant local quantum field theory with a Hermitian Hamiltonian must have CPT symmetry. Spin, and velocities change sign under time reversal, and thus $\vec{v}^{\perp}$ and both spins invariants are $\mathrm{T}$ violating, whereas $i \vec{q}$ is $\mathrm{T}$ conserving. For the sake of categorizing operators, it should be noted that spins have even partity, whereas $\vec{q}$ and $\vec{v}^{\perp}$ have odd parity.

For simplicity only elastic scattering is considered, such that all operators are of the form,

$$
\mathcal{L}_{i n t}=\bar{\chi} \mathcal{O}_{\chi} \chi \bar{N} \mathcal{O}_{N} N \equiv \mathcal{O} \bar{\chi} \chi \bar{N} N
$$

for nonrelativistic fields $\chi$ and $N$, and candidate interaction operators, $\mathcal{O}$, formed from WIMP and nucleon operators, $\mathcal{O}_{\chi}$ and $\mathcal{O}_{N}$, respectively [105]. Operators are constructed from the Hermitian quantities listed in Eq. 1.57, with the exception of the simplest interaction possible, the spin-independent operator labeled $\mathcal{O}_{1}$. The scale factor $1 / m_{N}$ has been applied to the momentum transfer quantity to render all operators dimensionless.

The set of operators $\left(\mathcal{O}_{i}\right)$, consisting of four-fermion operators of elastic scattering between a dark matter particle $(\chi)$ and a target material nucleon $(N)$, are listed in Table 1.1, separated into categories based on parity, WIMP spin dependence, and Tconservation ${ }^{11}$. Operators from different categories will not interfere with one another.

\footnotetext{
${ }^{11}$ Four additional operators have been excluded from this list, as they are products of other operators: $\mathcal{O}_{10} \mathcal{O}_{5}, \mathcal{O}_{10} \mathcal{O}_{8}, \mathcal{O}_{11} \mathcal{O}_{3}$, and $\mathcal{O}_{11} \mathcal{O}_{7}$. Additional operators are linearly dependent on others.
} 
Table 1.1: EFT Interaction Operators

\begin{tabular}{c|c}
\hline \hline \multicolumn{2}{c}{ P-even, $\mathrm{S}_{\chi}$-independent, T-conserving } \\
\hline $\mathcal{O}_{1}$ & 1 \\
$\mathcal{O}_{2}$ & $\left(v^{\perp}\right)^{2}$ \\
$\mathcal{O}_{3}$ & $i \vec{S}_{N} \cdot\left(\vec{q} / m_{N} \times \vec{v}^{\perp}\right)$ \\
\hline \hline \multicolumn{2}{c}{ P-even, $\mathrm{S}_{\chi}$-dependent, T-conserving } \\
\hline $\mathcal{O}_{4}$ & $\vec{S}_{\chi} \cdot \vec{S}_{N}$ \\
$\mathcal{O}_{5}$ & $i \vec{S}_{\chi} \cdot\left(\vec{q} / m_{N} \times \vec{v}^{\perp}\right)$ \\
$\mathcal{O}_{6}$ & $\left(\vec{S}_{\chi} \cdot \vec{q} / m_{N}\right)\left(\vec{S}_{N} \cdot \vec{q} / m_{N}\right)$ \\
\hline \hline P-odd, $\mathrm{S}_{\chi}$-independent, T-conserving \\
\hline $\mathcal{O}_{7}$ & $\vec{S}_{N} \cdot \vec{v}^{\perp}$ \\
\hline \hline \multicolumn{2}{c}{ P-odd, $\mathrm{S}_{\chi}$-dependent, T-conserving } \\
\hline $\mathcal{O}_{8}$ & $\vec{S}_{\chi} \cdot \vec{v}^{\perp}$ \\
$\mathcal{O}_{9}$ & $i \vec{S}_{\chi} \cdot\left(\vec{S}_{N} \times \vec{q} / m_{N}\right)$ \\
\hline \hline \multicolumn{2}{c|}{ P-odd, $\mathrm{S}_{\chi}$-independent, T-violating } \\
\hline $\mathcal{O}_{10}$ & $i \vec{S}_{N} \cdot \vec{q} / m_{N}$ \\
\hline \hline
\end{tabular}

Along with spin-independent operator, $\mathcal{O}_{1}$, particularly noteworthy operators include the standard spin-dependent operator $\mathcal{O}_{4}$ and momentum-transfer dependent operators, $\mathcal{O}_{3}$ and $\mathcal{O}_{5}$, which have characteristically different shapes in recoil energy spectra. Operator $\mathcal{O}_{2}$ is often discarded from this list, since it is the only operator that cannot be obtained from leading order terms in the nonrelativistic reduction of a relativistic operator with a spin-0 or spin-1 mediator.

Given the set of operators, the effective interaction Lagrangian is of the form,

$$
\mathcal{L}_{i n t}=\sum_{\tau} \sum_{i} c_{i}^{\tau} \mathcal{O}_{i} \bar{\chi} \chi \bar{\tau} \tau
$$


where $c_{i}^{\tau}$ is the coupling coefficient, $\tau$ is a sum over isoscalar and isovector operators ${ }^{12}$, and $i$ is a sum over all interactions. From this, the differential cross section is calculated as,

$$
\frac{d \sigma}{d \cos \theta}=\frac{\mu_{T}}{32 \pi M_{\chi}^{2} m_{N}^{2}} \sum_{i, j=1}^{11} \sum_{\tau, \tau^{\prime}} c_{i}^{\tau} c_{j}^{\tau^{\prime}} F_{i, j}^{\tau, \tau^{\prime}}\left(v^{2}, q^{2}\right),
$$

where $F_{i, j}^{\tau, \tau^{\prime}}$ are the $\vec{q}$ and $\vec{v}$-dependent form factors, which parameterize the loss of interaction coherence as the WIMP probes the nucleus' inner structure [104]. Absorbing this term into the differential rate described in Eq. 1.52 gives the final expression for differential event rate per detector mass,

$$
\frac{d R}{d E_{R}}=\frac{n_{0} N_{T} M_{T}}{32 \pi M_{N}^{2} M_{\chi}^{2}} \int_{v_{\min }}^{v_{\max }} \frac{1}{k} \frac{f\left(v, v_{E}\right)}{v} d^{3} v \sum_{i, j=1}^{11} \sum_{\tau, \tau^{\prime}} c_{i}^{\tau} c_{j}^{\tau^{\prime}} F_{i, j}^{\tau, \tau^{\prime}}\left(v^{2}, q^{2}\right) .
$$

Given the differential rate, the total number of events detected, $N_{\text {det }}$ in a given energy range between $E_{1}$ and $E_{2}$ is,

$$
N_{\text {det }}=\int_{E_{1}}^{E_{2}} \frac{d R}{d E_{R}} \mathcal{M T} \varepsilon\left(E_{R}\right) d E_{R}
$$

where $\mathcal{M}$ is the total exposed mass of the detector, $\mathcal{T}$ is the total time of exposure, and $\varepsilon$ is the recoil energy dependent counting efficiency, which accounts for the difference in the apparent observed number of recoils and the true number.

\section{Direct Detection Experiments}

With estimations of WIMP mass $\left(\sim 100 \mathrm{GeV} / \mathrm{c}^{2}\right)$, WIMP density $\left(\sim 0.3 \mathrm{GeV}^{-2} \mathrm{~cm}^{-3}\right)$, and target cross section $\left(\sim 10^{-46} \mathrm{~cm}^{2}\right)$, Eq. 1.51) and Eq. 1.62 determine detection sensitivity to recoil energies should be as low as $\sim 1 \mathrm{keV}$ and total event rate sensitivity of $\sim 1 \mathrm{~kg}^{-1} \mathrm{day}^{-1}$ or lower [108. Signals must be distinguished from backgrounds due to cosmic rays, contamination and detector impurities. There are several experimental setups to achieve such levels of sensitivity, and current direct detection experiments have surpassed these sensitivities.

Bubble detector experiments, such as SIMPLE [109] and PICO, suspend droplets of superheated liquid in a gel matrix. Charged particles passing through droplets can

\footnotetext{
${ }^{12}$ It is also possible for $i$ to be the sum over proton and neutron interactions. Converting coupling constants between isoscalar/isovector $\left(c_{i}^{0}, c_{i}^{1}\right)$ and proton/neutron $\left(c_{i}^{p}, c_{i}^{n}\right)$ bases can be accomplished by using $c_{i}^{0}=1 / 2\left(c_{i}^{p}+c_{i}^{n}\right)$ and $c_{i}^{1}=1 / 2\left(c_{i}^{p}-c_{i}^{n}\right)$.
} 
induce a phase transition if they deposit enough energy within a minimum length inside the droplet; roughly $\sim 150 \mathrm{keV} / \mu \mathrm{m}$ [110. PICO bubble chamber runs at SNOLAB, including PICO-2L 2-liter chamber $\mathrm{C}_{3} \mathrm{~F}_{8}$ run [111] [112, PICO-60 $\mathrm{CF}_{3} \mathrm{I}$ run [113], and PICO-60 $\mathrm{C}_{3} \mathrm{~F}_{8}$ run [114 saw no events consistent with a dark matter signal, with energy thresholds reaching as low as $3.2 \mathrm{keV}$. Schematics of the PICO-60 and PICO-2L bubble chambers are shown in Fig. 1.10 .
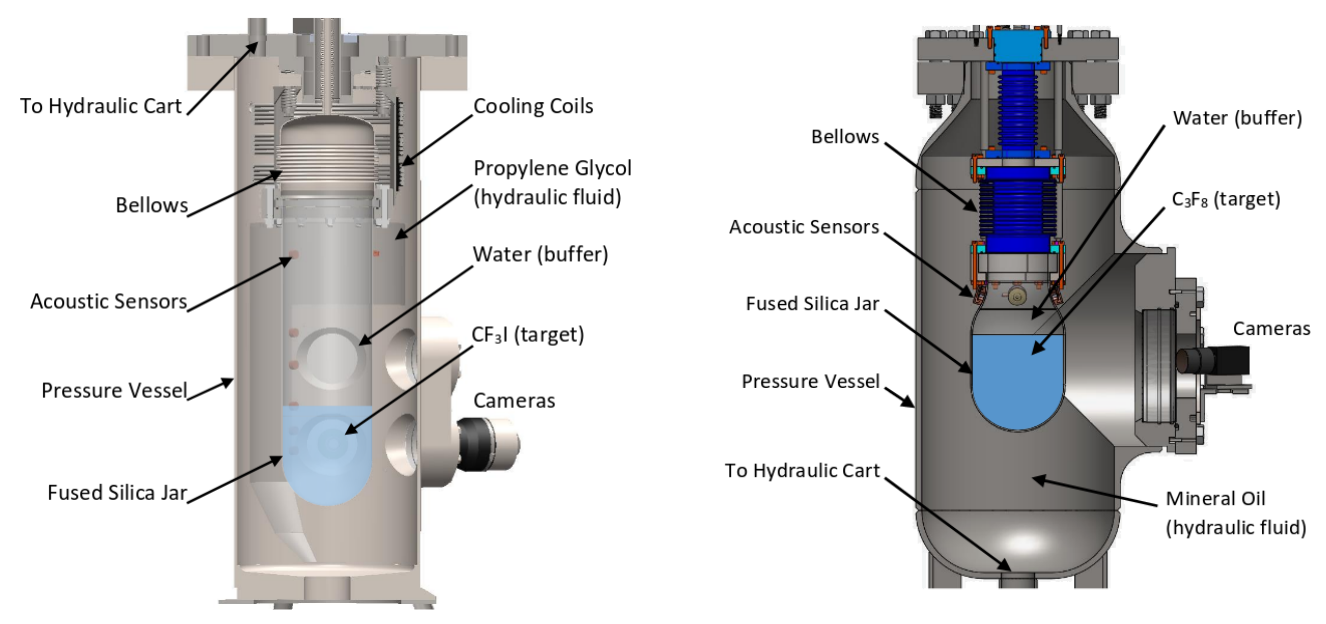

Figure 1.10: Schematics of the PICO-60 and PICO-2L bubble chamber experiments. Figures courtesy of [113] and [11].

Noble liquid detectors use photomultiplier tubes to detect scintillation light produced when particles interact with a liquefied noble gas. DEAP-3600 is a LAr detector based in SNOLAB, which uses the characteristic scintillation pulse shape of Ar to discriminate against background events [115]. Recent DEAP-3600 results observed no signal events, providing the current best spin-independent WIMP-nucleon cross section limit measured in LAr of $<1.2 \times 10^{44} \mathrm{~cm}^{2}$ for a WIMP mass of $100 \mathrm{GeV} / \mathrm{c}^{2}$ (90\% C.L.) [116].

Several prominent Xe detectors exist within the direct detection field. XENON100 at the Laboratori Nazionali del Gran Sasso uses Xe in a dual phase time-projection chamber (TPC) with an additional LXe active scintillator veto [117. Electrons in the target material are extracted from LXe to gas phase by a large extraction field, producing another scintillation light source in addition to the light generated by deexcitation. This allows for the distinction between electron and nuclear recoil. This 
process is demonstrated in Fig. 1.11. The successor of XENON100, XENON1T, is the first WIMP dark matter detector operating with a liquid xenon target mass above the ton-scale [118] [119], and an initial 34 live-day run demonstrated that it is the most sensitive dark matter search experiment for spin-independent WIMP-nucleon scattering for $>10 \mathrm{GeV} / \mathrm{c}^{2}$ WIMPs [120]. Other Xe detectors of note include the LUX dual-phase xenon TPC, operating at the Sanford Underground Research Facility [58], and PandaXII with a half-ton scale dual-phase TPC, at the China Jinping Underground Laboratory 78 .
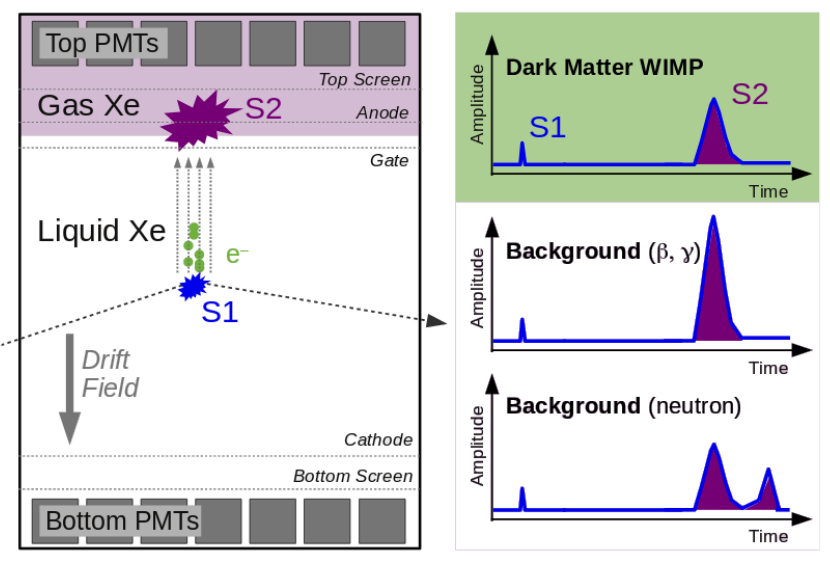

Figure 1.11: Cartoon demonstrating the working principle of a dual-phase LXe TPC. Top and bottom PMTs measure prompt scintillation light (S1) generated in LXe, and delayed secondary signal (S2) induced by ionization electrons in the gas phase. Figure courtesy of [118.

Directional detectors, such as DRIFT and DMTPC, hope to reduce the ambiguity of dark matter detection by determining the angular signature of dark matter wind [121. This signature would be distinct from other sources and could indicate the incoming direction of WIMPs. Directional detectors also use TPCs, though these experiments are filled with low pressure gas such that interactions result in an ionization track of measurable length. DRIFT uses $\mathrm{CS}_{2}$ as the target gas, and has detected nuclear recoil tracks on the order of a few mm long. However, limited spatial resolution and charge diffusion in the detector volume make it difficult to reconstruct distributions along the track for low energies [122], and a competitive measurement of the WIMP signal has yet to be found. DMTPC is a directional detector comprised of two optically isolated TPCs filled with $\mathrm{CF}_{4}$ which began taking data in 2010 [121]. First results of the 10-L DMTPC focuses on a recoil energy range of $80-200 \mathrm{keV}$, and sets a $90 \%$ C.L. upper 
limit on the spin-dependent WIMP-proton cross section of $2.0 \times 10^{-33} \mathrm{~cm}^{2}$ for a 115 $\mathrm{GeV} / \mathrm{c}^{2}$ WIMP [123].

A noteworthy experiment is DAMA/LIBRA, in which thallium-doped NaI crystals attached to low background photomultipliers search for evidence of the seasonal modulation of dark matter wind [124]. This experiment is based on the expectation that the rotation of the Earth around the Sun will alter Earth's velocity with respect to the dark matter halo, producing increased relative speeds and excess in WIMP flux around June 2nd, and a similar decrease in speeds and dearth of flux around December 2nd. With 13 annual cycles of data, DAMA has found evidence to suggest the existence of WIMPs in the galactic halo, assuming the standard WIMP dark matter model [124]. However, this experiment cannot distinguish between electron and nuclear recoils and the WIMP-nucleon cross section required to explain the signal has been excluded by limits set in other experiments such as LUX [58], PandaX-II [78], and CDMS [125]. DAMA candidate regions, as well as the limits and candidate regions of other experiments, are shown in Fig. 1.13, which plots spin-independent cross sections as a function of WIMP mass.

Charge-coupled devices (CCDs) make ideal detectors for lower mass WIMPs $(<10$ $\left.\mathrm{GeV} / \mathrm{c}^{2}\right)$ and low energy recoils $(\mathcal{O}(\mathrm{keV}))$, due to low readout noise and relatively low mass of the device's silicon nucleus. The working principle of CCDs as dark matter detectors is illustrated in Fig. 1.12. The DAMIC experiment at SNOLAB uses the bulk silicon of scientific-grade CCDs as the target for coherent WIMP-nucleus elastic scattering [126]. A $0.6 \mathrm{~kg} \cdot$ day exposure of DAMIC already places constraints on the spin-independent WIMP-nucleus cross section for the $<10 \mathrm{GeV} / \mathrm{c}^{2}$ WIMP mass region, with plans to further probe this region with a $100 \mathrm{~g}$ detector, DAMIC100 [127].

Cryogenic Ge and Si detection will be the focus of this thesis. Cryogenic experiments use semiconductor detectors at $\mathrm{mK}$ temperatures to distinguish between nuclear and electronic recoils on an event by event basis for energy recoils as low as $5-10 \mathrm{keV}$ [32]. The EDELWEISS-II experiment utilized ten $400 \mathrm{~g}$ Ge crystal detectors capable of reading thermal phonon and ionization signals, setting exclusion limits for the $7<$ $M_{\chi}<30 \mathrm{GeV}$ WIMP mass range [128] [129]. EDELWEISS-III intends to focus on the low-mass WIMP region [130], with a projected sensitivity on the spin-independent WIMP-nucleon cross-section of $7 \times 10^{-42} \mathrm{~cm}^{2}$ for a WIMP mass in the range $25 \mathrm{GeV} / \mathrm{c}^{2}$ 


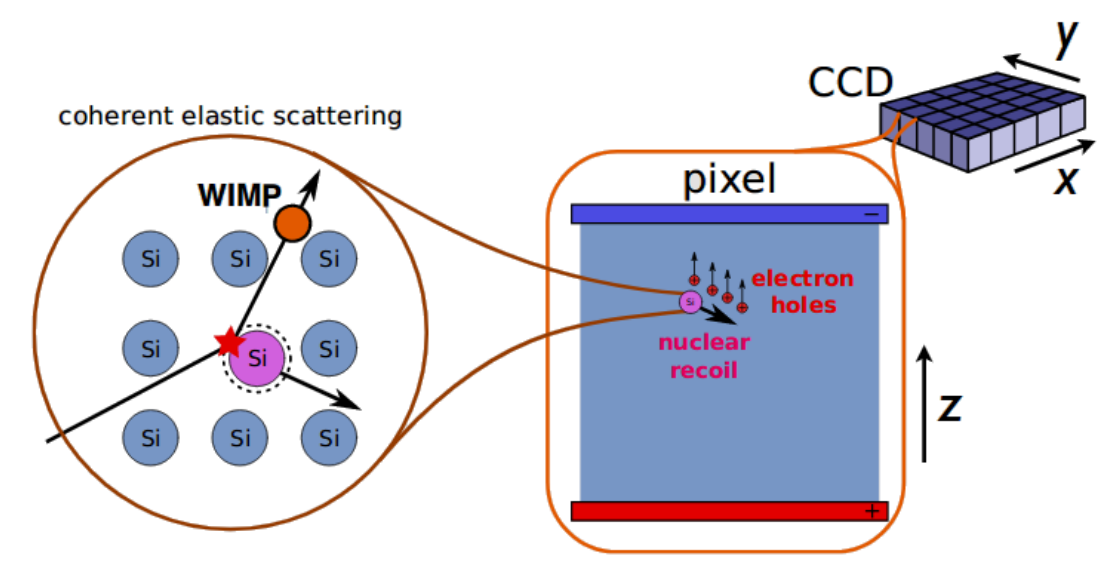

Figure 1.12: Cartoon demonstrating the working principle of CCD dark matter direct detection, with WIMPs coherently scattering off of silicon nuclei in the bulk of the CCD, producing ionization from the nuclear recoil which drifts along the z-direction and is collected at the CCD gates. Figure courtesy of [126].

[130].

The Cryogenic Dark Matter Search (CDMS) released results from its CDMS-II phase, which used nineteen $230 \mathrm{~g}$ Ge detectors and eleven $105 \mathrm{~g}$ Si detectors. CDMS-II Ge detector results found two WIMP-candidate events over a $612 \mathrm{~kg}$-day total exposure [132]. Results of Si detectors found three WIMP-candidate events with an expected total background of 0.7 events over $140.2 \mathrm{~kg}$-days of exposure [133]; these events were consistent with the expected background levels, and not significant enough to claim a WIMP detection. More recent SuperCDMS results have excluded candidate event regions [134]. Operation of CDMS detectors at relatively high voltage, called CDMSlite, has increased detector sensitivities down to $\mathcal{O}(10 \mathrm{eV})$ recoils, resulting in the leading low mass exclusion limit, down to $1.6 \mathrm{GeV} / \mathrm{c}^{2}$ WIMP masses [125]. The next stage of SuperCDMS at SNOLAB aims to combine traditional CDMS detectors with high voltage detectors for both Ge and Si detectors, to continue probing the low-mass WIMP region [135]. The specifics of the CDMS detectors, experimental setup, and exclusion limits will be discussed in more detail in Chapter 2 .

Fig. 1.13 shows the current state of the direct detection field for spin independent 
scattering. In addition to current experimental limits (shown in solid lines), sensitivity projections for upcoming experiments are also shown, in dashed lines. The dotted orange line in Fig. 1.13 represents the cross section at which the interaction rate from dark matter particles becomes comparable to the solar neutrino coherent elastic scattering rate. 


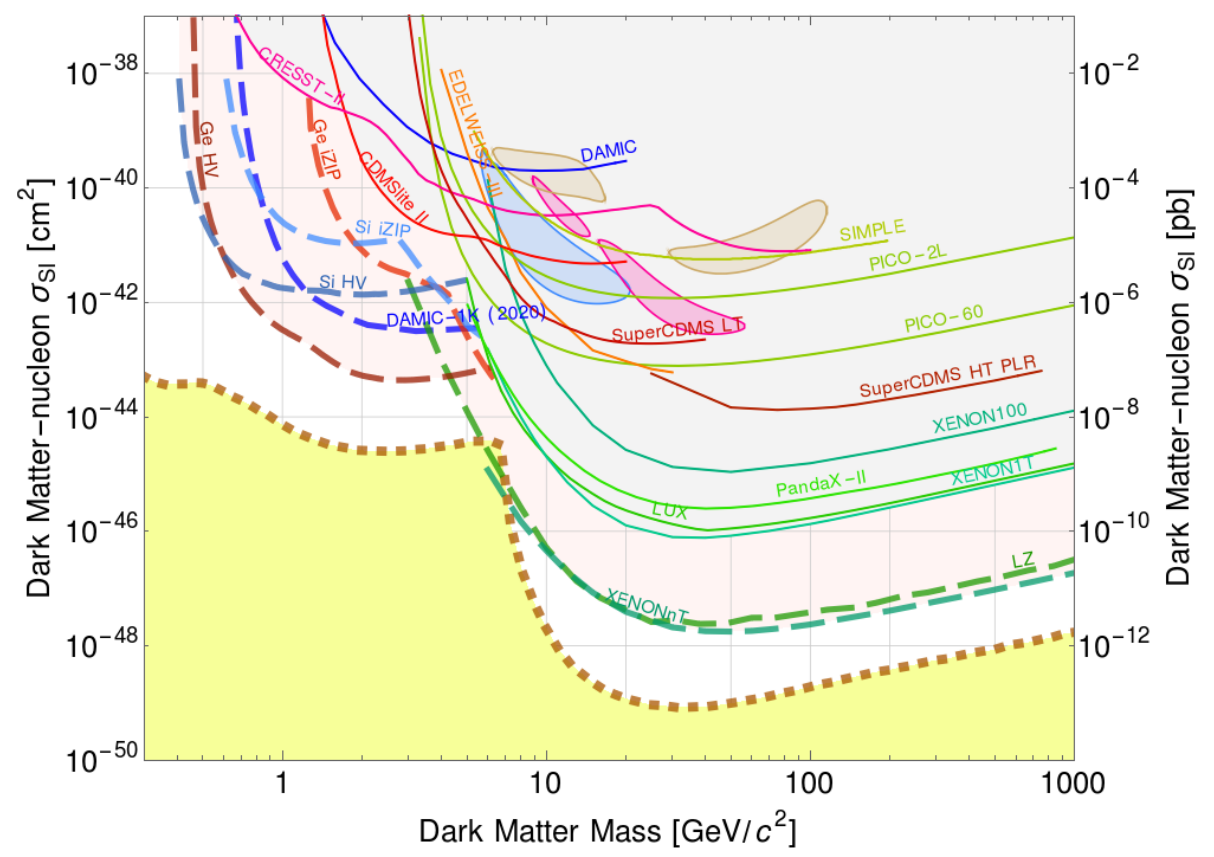

Figure 1.13: Current experimental limits (solid lines), projected sensitivities (dashed lines), and candidate regions (shaded) on spin-independent cross sections as a function of WIMP mass for various experiments. Current limits include second CDMSlite run [136], SuperCDMS low threshold [125], SuperCDMS high threshold [137], SIMPLE [109], PICO-2L [112, PICO-60 [114, XENON100 [117], PandaX-II [78], LUX [138, XENON1T [120], CRESST-II [139], DAMIC [140], and EDELWEIS-III [130]. Projected sensitivities include XENON1T [119], DAMIC100 [127], LZ [141], and SuperCDMS SNOLAB Si iZIP, Si HV, Ge iZIP, and Ge HV [135]. Closed contours shown include CRESST-II [142] (pink), and DAMA/LIBRA [143] (tan) and CDMS II Si [144] (blue). The dark matter discovery limit [145] (dotted orange line over yellow region) represents the cross section at which the interaction rate from dark matter particles becomes comparable to the solar neutrino coherent elastic scattering rate. 


\section{Chapter 2}

\section{Cryogenic Dark Matter Search Experiment}

The purpose of this chapter is to discuss the evolution of the Cryogenic Dark Matter Search as an experiment and a scientific collaboration, focusing on the continual development of direct detection technologies. It will begin with a historic overview of the CDMS experiment and results, followed by an exploration of CDMS detector design and function. This chapter concludes with a look at the next phase of CDMS, SuperCDMS SNOLAB.

\subsection{A Brief History of CDMS}

The inception of SuperCDMS can be traced back to the mid 1980's with the development of a method by which one could measure small $(<0.41 \mathrm{MeV})$ ionization and vibrational energies of neutrinos scattering off of electrons in a crystalline silicon kept at $1-10 \mathrm{mK}$ [146]. The Cabrera group at Stanford University proposed that vibrational energy could be measured by depositing a superconducting thin film on to the Si. By keeping the superconductor at its transitional temperature through an applied voltage bias, a small change in energy $(>10 \mathrm{keV})$ would result in a measurable change in resistance. This transition edge sensor, or TES, garnered interest for use in a cryogenic semiconductor dark matter detector. Simultaneous pursuits by Stanford University, Lawrence Berkeley National Laboratory and University of California Santa Barbara groups to design and 
fabricate dark matter detectors using various phonon sensor types persisted into the 1990's [147]. A notable result for the Sadoulet group at Berkeley during this time is the successful simultaneous measurement of phonon and ionization signals, through the use of a neutron-transmutation-doped (NTD) Ge phonon sensor on a Ge crystal [148.

\subsubsection{CDMS I}

The Cryogenic Dark Matter Search (CDMS) collaboration was formed around 1990 between Berkeley, Stanford and other primarily California-based collaborators to construct and operate a direct dark matter experiment. Due to small expected WIMP event rate $\left(<0.1\right.$ event $\left./ \mathrm{kg}^{-1} \mathrm{day}^{-1}\right)$ background events from unwanted particles interacting in the detector were of particular concern when designing the experimental setup. These particles can originate from cosmic rays, contaminated equipment within the experiment, radioactivity of the experiment facility, and impurities in the detectors themselves. Due to their shared characteristics both neutrons and WIMPs are expected to interact with the target material via nuclear recoil, therefore shielding to reduce neutrons and a well understood neutron background are particularly significant. For a summary of the radiogenic backgrounds of CDMS see Sections 3.5 and 6.5.2. To reduce the presence of unwanted particles in the detector several precautions have been taken.

A cryostat was constructed at the shallow-site of the Stanford Underground Facility (SUF) approximately $10.6 \mathrm{~m}$ or 16 mwe (meters water equivalent) below the surface, reducing the muon background by a factor of 5 , and absorbing the hadronic component of cosmic-ray showers that would otherwise increase background rate and activate materials near the detectors. Unfortunately a vertical muon flux of $29 \mathrm{~m}^{-2} \mathrm{~s}^{-1} \mathrm{sr}^{-1}$ was still present, running the risk of muon spallation and radioactive isotope production from the resulting nuclear capture [149]. To account for this, the experiment included an active muon-veto shield. Roughly $40 \%$ of high energy, externally-created neutrons and $99.7 \%$ of neutrons created inside the shield are identified by the shield through neutron-scintillator interactions, and coincident events in the detector that occur $<50$ $\mu$ s afterwards are vetoed. Additionally, $15 \mathrm{~cm}$ lead, $20 \mathrm{~cm}$ polyethylene, and a final layer of $1 \mathrm{~cm}$ depleted lead within the experiment volume, called the cryostat, were used as passive shielding [150]. Lead effectively blocks gammas by providing densely packed electrons for Compton scattering, and polyethylene shields against neutrons via elastic 


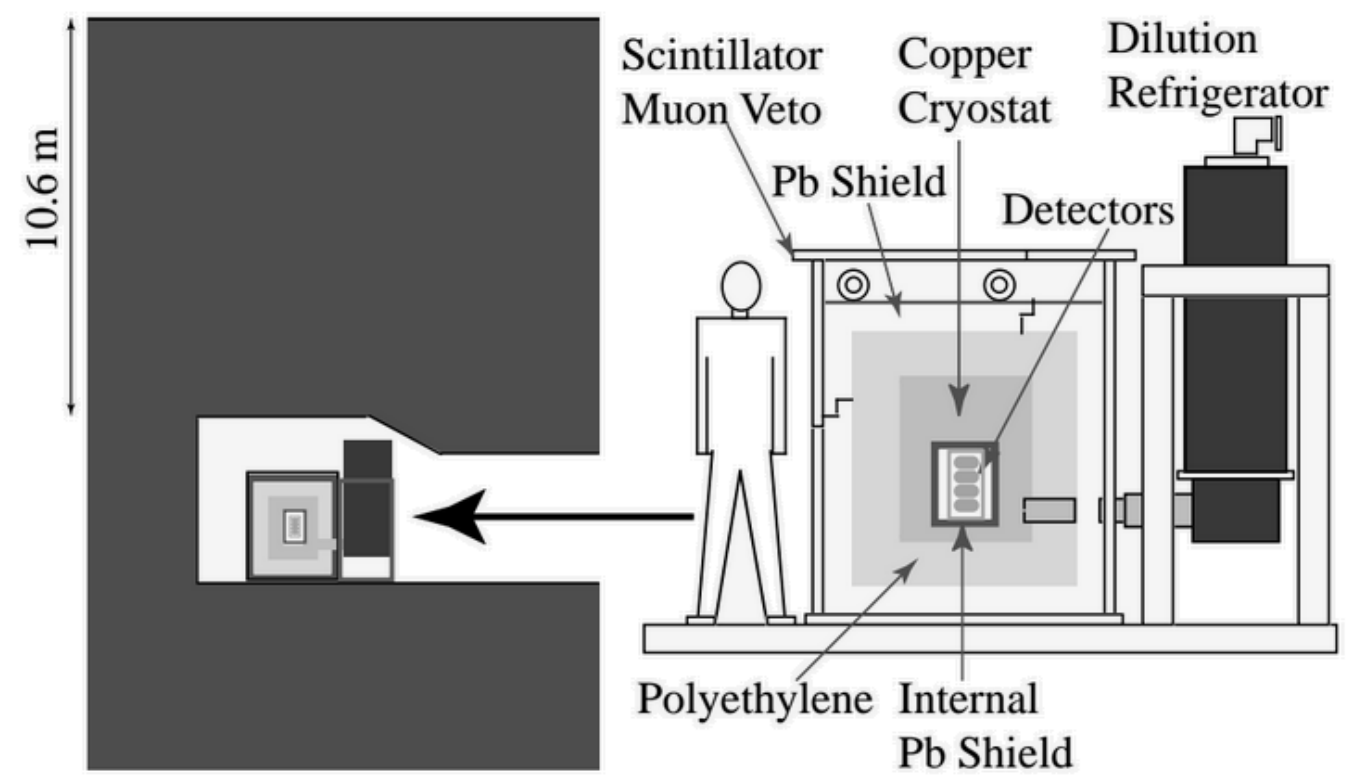

Figure 2.1: Layout of the CDMS I experimental setup at the Stanford Underground Facility. Figure from [149].

nuclear scattering. Cooling power was provided to a radiopure cryostat extension by an Oxford Instruments $400 \mu W$ dilution refrigerator, capable of reaching a $10-20 \mathrm{mK}$ base temperature. The CDMS experimental setup at SUF is shown in Fig. 2.1.

Detector testing at SUF began mid-1996, and in 1998 initial results were acquired with a 4-detector array of Berkeley Large Ionization and Phonon mediated (BLIP) detectors with one $100 \mathrm{~g}$ Z-dependent Ionization and Phonon (ZIP) detector 11 used to measure the neutron background. Shown in Fig. 2.2, BLIP detectors were comprised of $1.2 \mathrm{~cm}$ thick, $6 \mathrm{~cm}$ diameter cylindrical germanium crystals with a mass of $165 \mathrm{~g}$. Two (NTD) germanium thermistors were attached on one crystal face, while radiallysegmented electrodes were deposited on both sides to apply a voltage of $6 \mathrm{~V}$ [151] [149]. Although BLIP detectors did demonstrate event-by-event discrimination between electron and nuclear recoils for events within the bulk of the crystal (99\% rejection efficiency for $>10 \mathrm{keV}$ events), they misidentified $5 \%$ of events above $10 \mathrm{keV}$ that occur on the

\footnotetext{
${ }^{1}$ ZIPs were originally named Fast Large Ionization and Phonon mediated detectors, or FLIPs, though particular designs were sometimes referred simply by first names, such as Alex [150].
} 


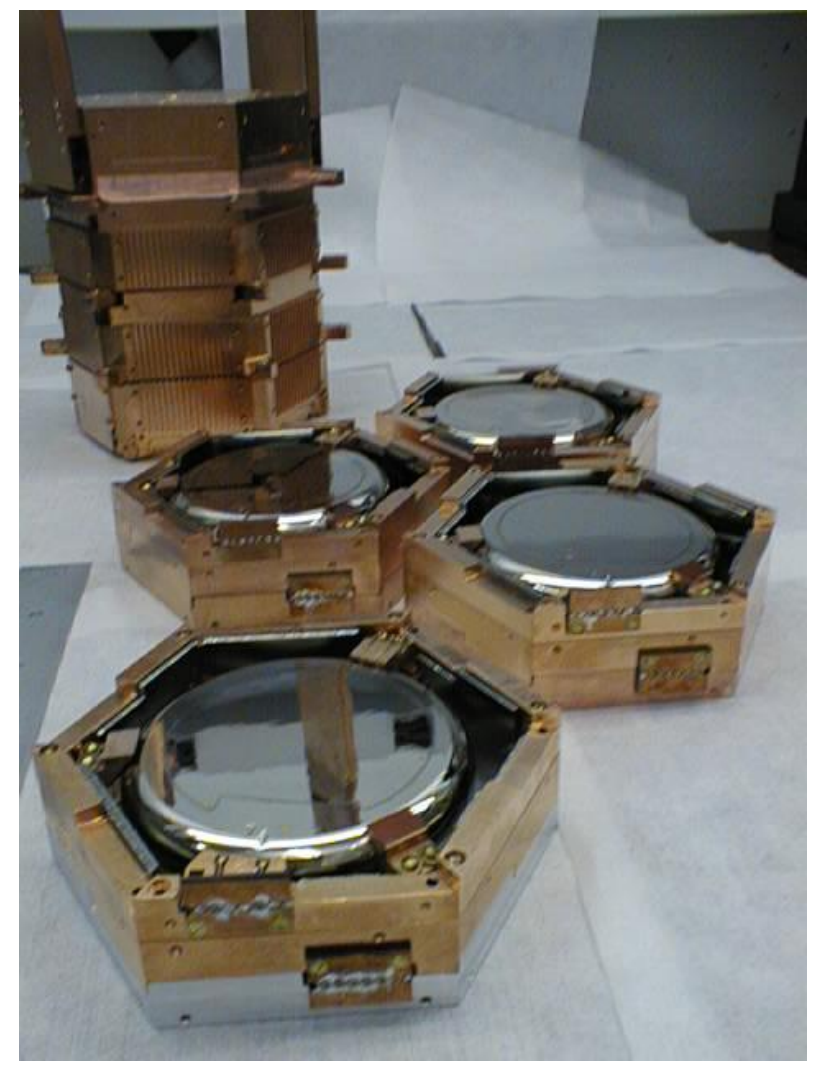

Figure 2.2: Four BLIP detectors next to a CDMS tower.

crystal surface, primarily caused by low-energy electrons [152. Despite this, these initial CDMS results set limits on the spin-independent WIMP-nucleon elastic scatter cross section to exclude previously unexplored parameter space above a $10 \mathrm{GeVc}^{-2}$ WIMP mass [149], and excluded the full $3 \sigma$ WIMP signal region reported by the DAMA experiment [153], with a $>84 \%$ confidence level.

Results from a 2001 experiment run demonstrated the capability of ZIP detectors, which had only been used to measure background in the preceding results. These detectors utilized tungsten transition edge sensors originally proposed for neutrino scatter detection, along with electrodes similar to those deposited on BLIP detectors to act as ionization sensors. Four $250 \mathrm{~g}$ Ge and two $100 \mathrm{~g}$ Si ZIPs were arranged in a stack, each with a thickness of $1 \mathrm{~cm}$, and a diameter of $7.62 \mathrm{~cm}$ [154]. Due to recoil position information present in ZIP phonon signals, electron recoil rejection increased, reaching 
$>99.98 \%$ for photon rejection (within $5-100 \mathrm{keV}$ ), and > 99\% for electron rejection above $10 \mathrm{keV}$ for the four centrally-positioned detectors. These results set the limit on spin-independent WIMP-nucleon elastic scatter cross section over two times lower than previous CDMS results, and excluded a new parameter space for a $8-20 \mathrm{GeVc}^{-2}$ WIMP mass [155.

\subsubsection{CDMS II}

Although experiment runs at SUF had been successful at demonstrating the sensitivity of CDMS detectors, the shallow depth made neutrons produced by cosmic ray muons a limiting background. To reduce this background and expand CDMS experiment capabilities, the construction of a deep underground WIMP search facility at Soudan Underground Laboratory, Minnesota, began in 2001. Designs and construction of the CDMS infrastructure (shown in Fig. 2.3) within one of Soudan's two main caverns was completed by CDMS collaboration members at Fermilab and the University of Minnesota. Due to its $780 \mathrm{~m}$ rock overburden providing 2090 mwe shielding, Soudan reduced the surface muon background by a factor of $5 \times 10^{4}$, and thus the cosmic muon-produced neutron background is reduced by roughly 300 [156].

The CDMS experiment at the new facility utilized the same sized cryostat as was used at SUF. The detectors were operated within a cryostat comprised of six nested cylindrical cans made of OFHC, low-radioactivity copper, the innermost can having a diameter of $30 \mathrm{~cm}$ and a height of $30 \mathrm{~cm}$. This space provided room to accommodate up to seven stacks of six ZIP detectors each, and the required hardware for detector interface.

As with the Stanford Underground Facility, an Oxford Instruments Kelvinox 400$\mathrm{S}$ dilution refrigerator was used to cool the detector volume to $\sim 50 \mathrm{mK}$ via a solid copper finger. Each additional can was thermally coupled to other temperature stages of the dilution unit via horizontal, nested, copper tubes. This collection of thermal connections between the cryostat and the dilution fridge, referred to as the "cold stem", kept the detectors separated from any radioactivity present in the dilution unit. Most of the cryogenic system was continually monitored by a Moore APACS industrial control unit. This includes monitoring and recording temperatures, pressures, and flows in the dilution unit, as well as automatic liquid helium and liquid nitrogen transfers to 

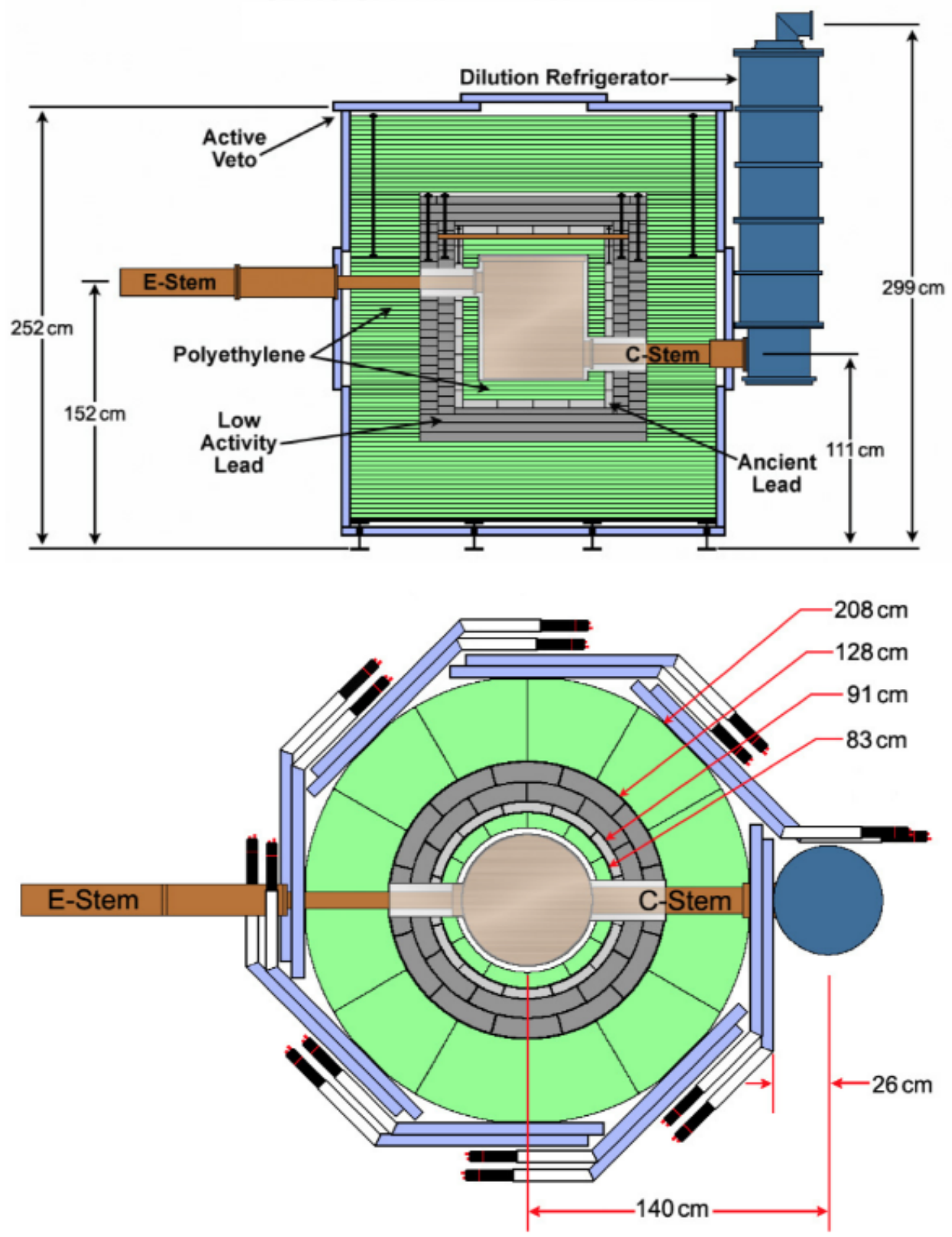

Figure 2.3: A cross sectional view seen from the side (TOP) and top-down (BOTTOM) of the CDMS experiment layout at Soudan Underground Laboratory. Cryostat, electronic and cold stems shown in brown/copper, polyethylene shown in green, (ancient) lead shown in (light) grey, and muon veto shield shown in light blue. Figure courtesy of A. Villano and J. Sanders 
maintain temperature.

Each detector stack was mounted on a "tower" such that each tower was secured within the cryostat with the detectors suspended below, and with a $2 \mathrm{~mm}$ separation between adjacent detectors, shown in Fig. 2.4. Each detector was connected to its own cold electronics card, mounted on the top of the tower, through coaxial wires within the tower. These cards monitored and read out phonon and ionization signals, passing them to room temperature through flexible copper-kapton striplines. These $2.5 \mathrm{~cm}$ wide, 3 $\mathrm{m}$ long striplines exited the cryostat through a horizontal "electronics stem". At room temperature, the striplines connected to Front-end Electronics Boards, or FEBs.

The cryostat acted as a shield that is $99 \%$ hermetic, with only the electronic and cold stems entering into the sealed copper cannisters. Immediately surrounding the cryostat was a $2 \mathrm{~mm}$ thick mu-metal shield, to shield detectors and cold electronics from ambient magnetic fields, which can interfere with phonon sensors and read out. In addition to the roughly $3 \mathrm{~cm}$ of copper shielding provided by cryostat cans, $10 \mathrm{~cm}$ of polyethylene, $4.5 \mathrm{~cm}$ of ancient lead, $18 \mathrm{~cm}$ additional lead, and $40 \mathrm{~cm}$ of additional polyethylene comprised the passive shielding, in order from innermost to outermost. An active muon veto shield, comprised of forty scintillator panels, surrounded the passive shielding. The cryostat, shielding, and dilution unit were enclosed in an RF-shielded room, measured to be a class 10,000 clean room during working hours, and better than a class 1000 clean room ${ }^{2}$ when unoccupied.

CDMS II data was taken from 2003 until 2008, with numerous published results. The first Soudan data run took place from October 2003 to January 2004, and used the same four Ge and two Si ZIP detectors that had been used previously at SUF, in the same stack order. Background rejection efficiency was comparable to SUF runs; for recoil energies $>10 \mathrm{keV}$, photons and surface events were rejected with $>99.99 \%$ and $>96 \%$ efficiency, respectively. Initial Soudan blind analysis results set the exclusion limit on spin-independent WIMP-nucleon elastic scatter cross section for a $60 \mathrm{GeVc}^{-2}$ WIMP mass at $4 \times 10^{-43} \mathrm{~cm}^{2}$, roughly eight times lower than previous SUF CDMS results, and four times lower than the best limit set by EDELWEISS at the time [157]. Additionally, the spin-dependent cross section upper limit was set to $2 \times 10^{-37} \mathrm{~cm}^{2}$ for

\footnotetext{
${ }^{2}$ A class 10,000 clean room has a maximum particulate density of 10,000 particles of size $\geq 0.5 \mu \mathrm{m}$ per cubic foot, and has an ISO equivalent of ISO 7. A class 1000 clean room has a maximum particulate density of 1000 particles of size $\geq 0.5 \mu \mathrm{m}$ per cubic foot, and has an ISO equivalent of ISO 6 .
} 
a WIMP mass of $50 \mathrm{GeVc}^{-2}[158$.

The subsequent data run utilized two towers containing six Ge and six Si ZIP detectors, lasting from March to August of 2004. Primary experiment improvements, besides an increase in total detector mass, included a reduction of electrical noise and improvements to detector operation methods. Analysis was done separately for germanium and silicon detectors, with Ge analysis also using the previous Soudan run data. Ge and Si blind analysis results set the exclusion limit on spin-independent WIMP-nucleon elastic scatter cross section for a $60 \mathrm{GeVc}^{-2}$ WIMP mass at $<1.6 \times 10^{-43} \mathrm{~cm}^{2}$, and $<3 \times 10^{-42}$ $\mathrm{cm}^{2}$ at $90 \% \mathrm{CL}$, respectively [159].

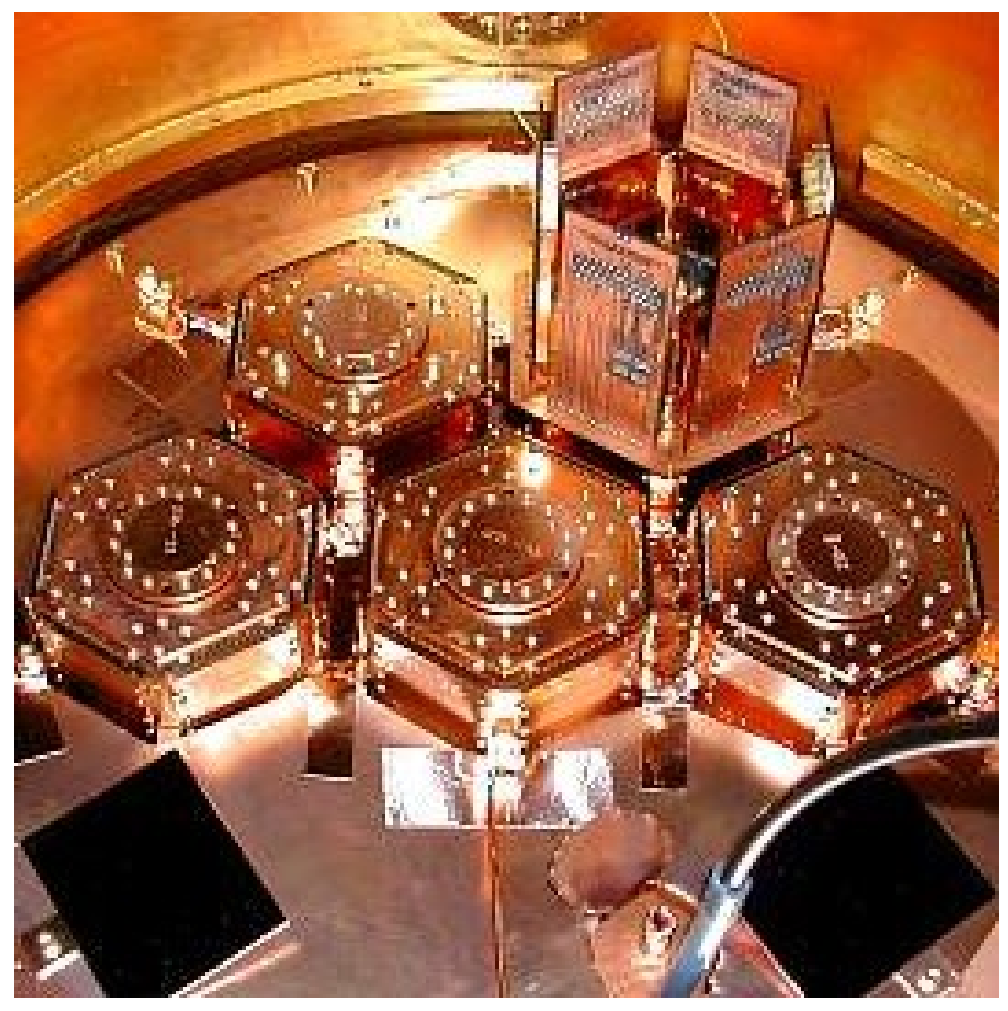

Figure 2.4: CDMS II towers in Soudan cryostat. Cold hardware (discussed in Section 3.1 is installed on the upper right tower.

The CDMS detector payload continued to increase, up to 19 Ge and 11 Si ZIP detectors in five towers. Germanium results from two data runs, between October 2006 and July 2007 were combined with previous run data and published in January 2009. 
A blind analysis on combined Ge results set the exclusion limit on spin-independent, and spin-dependent WIMP-nucleon elastic scatter cross section for a $60 \mathrm{GeVc}^{-2}$ WIMP mass at $<4.6 \times 10^{-44} \mathrm{~cm}^{2}$, and $<1.8 \times 10^{-38} \mathrm{~cm}^{2}$ at $90 \% \mathrm{CL}$, respectively [160]. In 2015 improved surface-event rejection techniques and reduced analysis energy threshold further reduced the Ge spin-independent upper limit to $<1.8 \times 10^{-44} \mathrm{~cm}^{2}$ for a 60 $\mathrm{GeVc}^{-2}$ WIMP mass at $90 \% \mathrm{CL}$ [161]. This reanalysis also significantly improved the limit within the low WIMP-mass region $\left(<15 \mathrm{GeVc}^{-2}\right)$, setting the spin-dependent WIMP-nucleon elastic scatter cross section limit for a $8.6 \mathrm{GeVc}^{-2}$ WIMP mass at $<$ $1.18 \times 10^{-41} \mathrm{~cm}^{2}[161$ ].

Silicon results from 2006 - 2007 data runs, published in 2013, set an upper exclusion limit of $<1.7 \times 10^{-41} \mathrm{~cm}^{2}$ for a $10 \mathrm{GeVc}^{-2}$ WIMP mass at $90 \% \mathrm{CL}$ [133]. As can be inferred from the results summarized here, around this time interest had begun shifting from the $\sim 50 \mathrm{GeVc}^{-2}$ WIMP mass region to the lower mass region of $<15 \mathrm{GeVc}^{-2}$. This is partially due to the CDMS experiment being more apt than other dark matter detection experiments to set new limits in the low mass parameter space (liquid noble gas experiments are better suited for probing high mass regions). It is also due to the unexpected dearth of LHC results supporting supersymmetric models that predict $10 \mathrm{GeVc}^{-2}-1 \mathrm{TeVc}^{-2}$ WIMP masses. Without supporting evidence for the original models, new models were gaining new ground, such as the asymmetric dark matter model that favors a $5-10 \mathrm{GeVc}^{-2}$ WIMP mass [46].

In 2013 CDMS published results of Si data taken between July 2007 and September 2008, which revealed three WIMP-candidate events. The highest likelihood for the candidate events occurred for a WIMP mass of $8.6 \mathrm{GeVc}^{-2}$ and a WIMP-nucleon cross section of $1.9 \times 10^{-41} \mathrm{~cm}^{2}$ [144]. However, there was a $5.4 \%$ probability that the known backgrounds could produce three or more events within this signal region, and profile likelihood studies comparing a WIMP-and-background hypothesis to a backgroundonly hypothesis did not favor the WIMP-and-background hypothesis strongly enough to indicate a dark matter discovery. As discussed in the next section, these results were quickly disputed [125]. 


\subsubsection{SuperCDMS Soudan}

The Super Cryogenic Dark Matter Search, or SuperCDMS was the third and most recent incarnation of the CDMS experiment. The experimental setup remained at the Soudan Underground Laboratory, but the detector payload was upgraded to fifteen Ge interleaved Z-dependent Ionization and Phonon (iZIP) detectors, with three iZIPs mounted into each of the five towers. As will be detailed in Section 2.2.2, the new interleaved design of iZIP detectors improved surface event rejection capabilities, increasing the overall sensitivity of the detectors [162]. Each detector has a $76 \mathrm{~mm}$ (3") diameter and is $25 \mathrm{~mm}$ (1") thick (amounting to $630 \mathrm{~g}$ ), such that the total target mass of the experiment is roughly $9 \mathrm{~kg}$.

Although design, fabrication, and testing for SuperCDMS detector hardware began much earlier, SuperCDMS operation at SUL began in March of 2012. Initial SuperCDMS results were published in 2014, using data taken from October 2012 to June 2013. After a low-threshold analysis and unblinding, eleven WIMP-candidate events were observed, a number mostly consistent with expected background event:3. A new, spin-independent, WIMP-nucleon elastic scatter cross section exclusion limit was set at $<1.2 \times 10^{-42} \mathrm{~cm}^{2}$ for an $8 \mathrm{GeVc}^{-2}$ WIMP mass at $90 \%$ C.L.. This not only disagrees with the CDMS II silicon results [144, but also several other WIMP detection claims observed by CoGeNT [163], CRESST-II [142] and DAMA/LIBRA [143]. Fig. 2.5 shows the closed contours of WIMP detection hints from various experiments, with the exclusion limit of the SuperCDMS low threshold analysis. SuperCDMS high threshold results, shown in Fig. 1.13, set an exclusion limit for WIMP-nucleon spin-independent cross section of $1.32 \times 10^{-44} \mathrm{~cm}^{2}$ at a WIMP mass of $75 \mathrm{GeV} / \mathrm{c}^{2}$ at $90 \%$ C.L. [137], and are pending publication.

\subsubsection{CDMSlite}

CDMSlite is an experiment developed to utilize SuperCDMS iZIP detectors with relatively high applied bias voltages. For reasons discussed in Section 2.2.2, operating iZIP detectors at these biases sacrifices ionization signal in order to amplify the phonon

\footnotetext{
3 Three events with energies of $7.0,7.8$, and $9.4 \mathrm{keV}_{n r}$ were observed within the same detector; a probability of this being an observed background is $4 \times 10^{-4}$. However, a shorted channel to ground might have resulted in an inaccurate background estimate. A follow-up investigation is ongoing.
} 


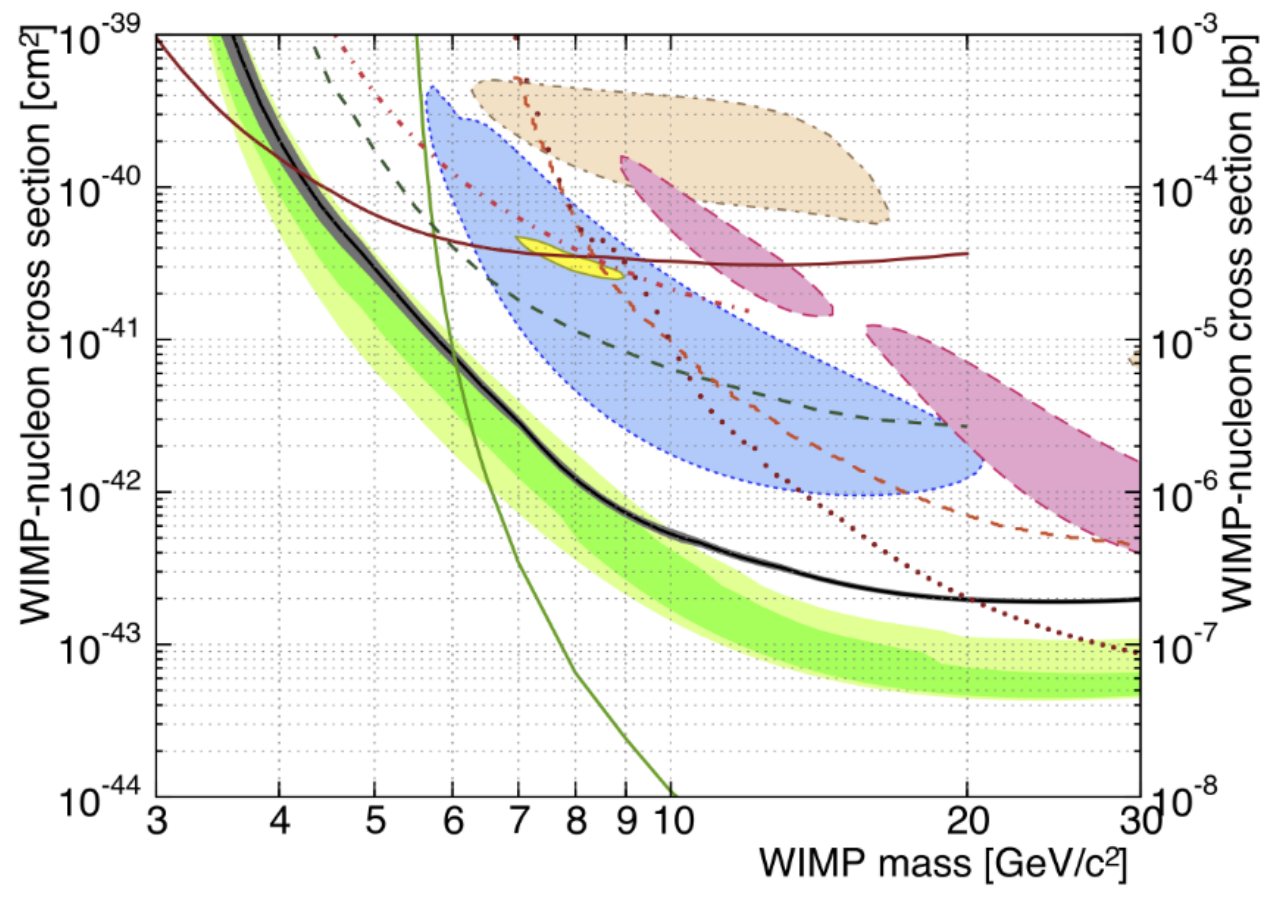

Figure 2.5: The SuperCDMS low threshold 90\% confidence upper limit (solid black) shown with $95 \%$ C.L. systematic uncertainty band [125] the second CDMSlite run (gray solid). The expected sensitivity in the absence of a signal is shown as $68 \%$ (dark green) and 95\% (light green) C.L. bands. Closed contours shown are CDMS II Si [144] (dotted blue), CoGeNT [163] (yellow), CRESST-II [142] (dashed pink), and DAMA/LIBRA 143] (dash-dotted tan) preferred parameter space. The 90\% C.L. exclusion limits shown are CDMS II Ge [132] (dotted dark red), CDMS II Ge low threshold [164] (dashed-dotted red), CDMSlite [134] (solid dark red), LUX [58] (solid green), XENON10 S2 only [165] (dashed dark green), and EDELWEISS low threshold [129] (dashed orange). Figure from [125].

signal and increase its sensitivity by reducing its energy threshold and increasing its energy resolution [166]. Since SuperCDMS electronics were not designed for use with bias voltages larger than $10 \mathrm{~V}$, custom electronics were utilized to safely hold one face of the detector at the desired voltage while the other detector side, kept at $0 \mathrm{~V}$, was used for phonon sensor read out. The first CDMS Low Ionization Threshold Experiment, or 
CDMSlite, data run used a single Ge iZIP detector with an operating bias of $69 \mathrm{~V}$ for a total of 15.7 live days or $6.3 \mathrm{~kg}$.days. This initial 2013 run set a low ionization threshold of $170 \mathrm{eV}$ electron-equivalent, or $170 \mathrm{eV}_{e e}$, and excluded a new spin-independent parameter space for WIMP masses below $6 \mathrm{GeVc}^{-2}$ [134].

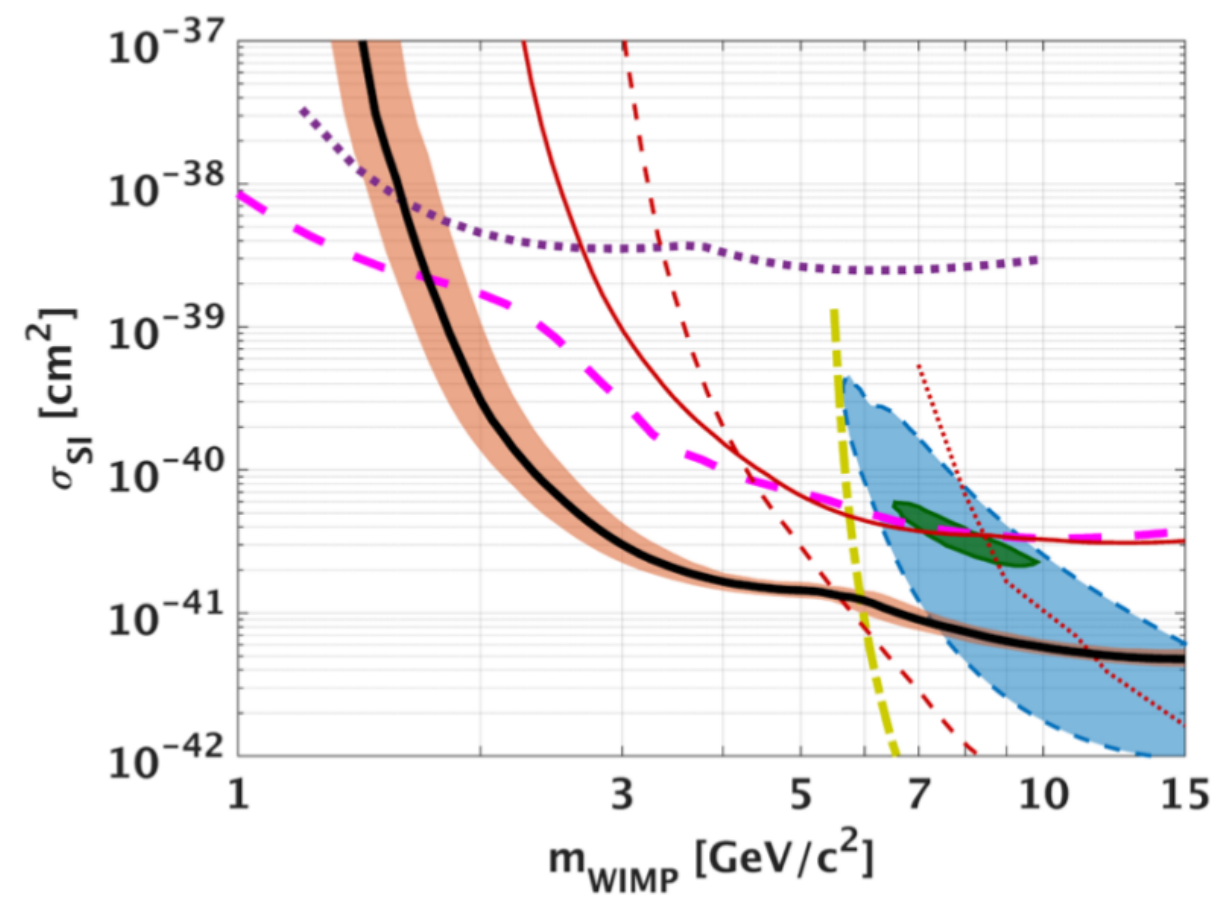

Figure 2.6: Median (90\% C.L.) and 95\% interval of the WIMP limit from the second CDMSlite run [136] (black thick solid curve surrounded by salmon-shaded band) compared to other selected results. Other $90 \%$ upper limits shown are from the first CDMSlite run [134] (red thin solid curve), SuperCDMS [125] (red thin dashed curve), EDELWEISS-II [129] (red thin dotted curve), LUX [58] (dark-yellow thick dot dashed curve), CRESST [139] (magenta thick dashed curve), and DAMIC [140] (purple thick dotted curve). Closed regions are CDMS II Si 90\% C.L. 144] (blue dashed shaded region), and CoGeNT 90\% C.L. [163] (dark-green shaded region). Figure from [136].

With the CDMSlite method proven viable, a second CDMSlite run was conducted using the same iZIP detector from February to November of 2014, divided into two running periods. Hardware and experiment operation improvements were implemented 
to reduce noise, and a fiducialization data cut reduced backgrounds from those reported in the previous CDMSlite results. Second run results were published in 2016 with a $70.10 \mathrm{~kg}$.day exposure, setting ionization thresholds of 75 and $56 \mathrm{eV}_{e e}$ for the first and second running periods, respectively [136]. A new limit was set on WIMP-nucleon spinindependent cross section parameter space for 1.6 - 5.5 $\mathrm{GeVc}^{-2}$ WIMP mass, shown in Fig. 2.6. A third CDMSlite run has completed, with analysis ongoing.

\subsection{CDMS Detectors and Signals}

For a majority of CDMS operation some variation of Z-dependent Ionization and Phonon (ZIP) detector has been used. Despite upgrades in size and channel layout, the core components of ZIP technology have remained unchanged. For more information on BLIP detectors, which will not be discussed here, see [152], [151, [149].

\subsubsection{ZIP technology}

As the name suggests, ZIPs have both phonon sensors and charge electrodes, allowing them to distinguish between electron recoils and nuclear recoils. Faces of the diskshaped high purity Ge or Si crystals are covered with photolithographically-defined Transition-Edge Sensors (TES), distributed into distinct athermal phonon read out channels. The channels have hundreds Quasiparticle assisted Electrothermal feedback TES (QETs), each with a strip of tungsten kept at its transition edge and connected to eight superconducting $\mathrm{Al}$ films [167]. Ionization collecting electrodes are also deposited onto the crystal face, forming concentric inner disk and outer ring charge sensors.

ZIP detectors are operated at temperatures of $\sim 40 \mathrm{mK}$, purposefully cooler than the 60 - $80 \mathrm{mK}$ transitional TES temperatures. An optimal voltage bias can be applied to each phonon channel to bring its sensors to their transitional temperature region. Additionally, cooling the detector eliminates, or "freezes out", a majority of thermal phonons and charge carriers within the crystal bulk.

When an incident particle interacts with atoms in the crystal via electron or nuclear recoil, charge carriers (electron/hole pairs) and athermal phonons are produced. The applied bias produces an electric field in the crystal to inhibit recombination of the charge carries, and instead electrons and holes drift in opposite directions through the 
crystal creating a measurable drift current. Phonons propagate throughout the detector until they are absorbed by the phonon QETs; the resulting energy is converted to a current through TESs and recorded.

\subsubsection{Detector Types}
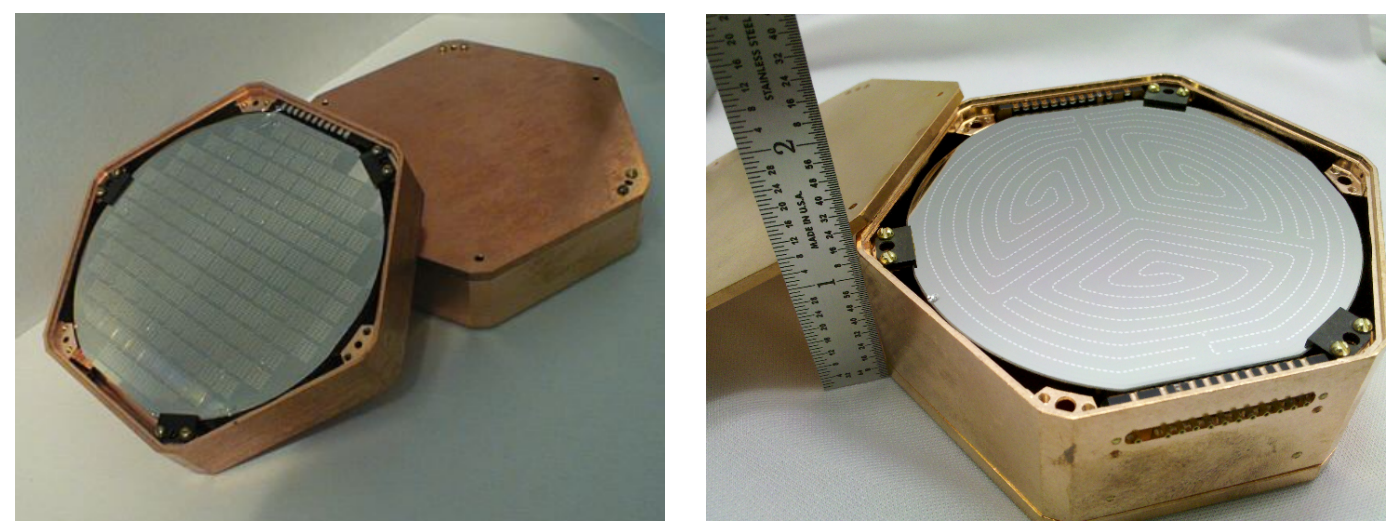

Figure 2.7: CDMS II oZIP (LEFT) and SuperCDMS iZIP (RIGHT) detectors, shown in copper housings.

\section{oZIP}

ZIP detectors used for CDMS I and CDMS II are now referred to as original ZIPs, or oZIPs. oZIPs, shown in the left image of Fig. 2.7, are $10 \mathrm{~mm}$ thick, $76 \mathrm{~mm}$ in diameter and have a mass of $250 \mathrm{~g}$ for Ge and $100 \mathrm{~g}$ for Si. Four quadrant phonon channels are deposited on to one detector face, and the other face is comprised of the inner disk and outer ring ionization electrodes. Phonon channels are labeled A, B, C, and D, and charge channels are labeled Qi and Qo, for the inner and outer charge channels, respectively. Typically, these detectors were operated with a $\sim 3 \mathrm{~V}$ bias applied to both electrode channels, and the phonon channels kept at $0 \mathrm{~V}$.

\section{iZIP}

The most significant change from original ZIP technology to the interleaved ZIP, or iZIP, design is the relative positioning of the charge and phonon sensors. Previously phonon 

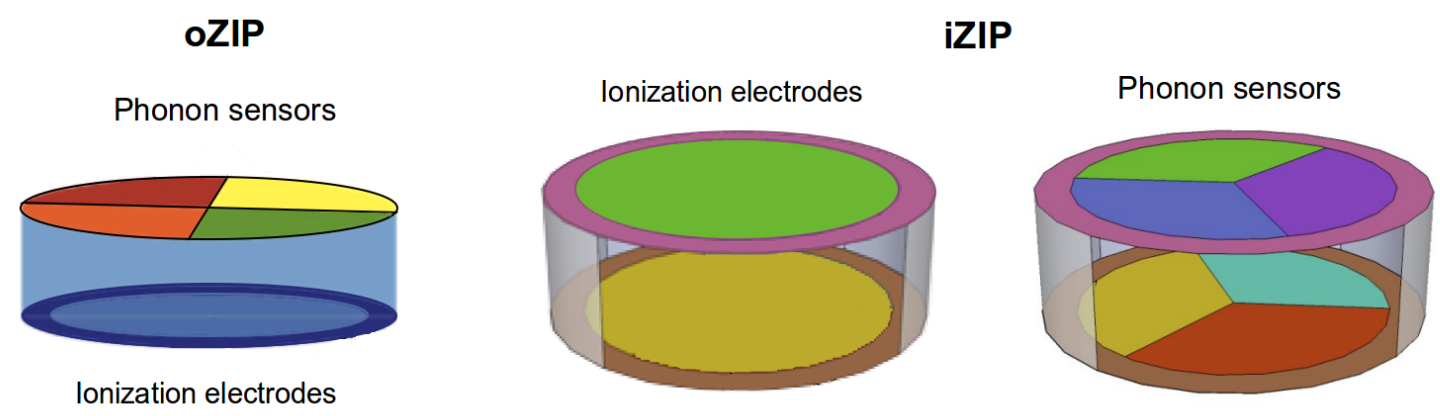

Figure 2.8: Phonon and ionization channel layout for oZIP (LEFT) and iZIP (CENTER and RIGHT) detectors. Four quadrant phonon channels are deposited on to one oZIP detector face, and the other detector face comprises of the inner disk and outer ring ionization electrodes. The iZIP detector features four phonon sensors, and inner disk and outer ring ionization electrodes per face, with a $60^{\circ}$ offset between iZIP phonon layout on top and bottom faces, to aid in position reconstruction. Figure modified from A. Phipps [167] 
and charge channels were on opposite detector faces, but iZIP sensors are deposited on both faces, alternating between ionization and phonon. This change allows for the use of symmetry cuts, which remove surface events (a dominant oZIP background), by cutting on signal asymmetries between detector faces. In addition, the Al fin surface coverage was increased from oZIPs to iZIPs, improving the phonon signal-to-noise ratio. As with oZIPs operation, a bias of $\sim 3 \mathrm{~V}$ is applied to all electrode channels, and phonon channels are kept at $0 \mathrm{~V}$.

iZIP detectors have four phonon channels per face, with a $60^{\circ}$ rotational offset between faces to better provide event position information. Instead of four quadrant channels, iZIPs have an outer ring channel A surrounding three inner channels B, C, and D. In addition to the phonon sensors, each face of an iZIP has two charge electrodes; the inner Qi electrode covering the central bulk of the detector, and the outer Qo electrode which forms a ring shape around Qi [125]. SuperCDMS Soudan iZIPs were exclusively Ge detectors, measuring $76 \mathrm{~mm}$ (3") diameter and is $25 \mathrm{~mm}$ (1") thick, with a mass of $630 \mathrm{~g}$. An example iZIP is shown in the right image of Fig. 2.7, and a comparison of oZIP and iZIP channel layouts is shown in Fig. 2.8. Fig. 2.9, shows the interleaved ZIP design in the left image, and illustrates how the resulting electric field might affect bulk and surface events in the right image.

\section{Sensor Design and Fabrication}

As previously mentioned, ionization electrode and QET materials are deposited onto the crystal substrate photolithographically. Material deposition for charge and phonon iZIPS sensors begins with a $40 \mathrm{~nm}$ thick layer of amorphous silicon, followed by 300 $\mathrm{nm}$ thick $\mathrm{Al}$ and $30 \mathrm{~nm}$ thick $\mathrm{W}$ layers, respectively. After the initial deposition, a photoresist is used to etch away layers and define the sensor components. Each QET consists of one $220 \mu \mathrm{m} \times 1.8 \mu \mathrm{m} \mathrm{W}$ strip connected to eight $327 \mu \mathrm{m} \times 41 \mu \mathrm{m} \mathrm{Al}$ fins $(4.5 \mu \mathrm{m} \text { of } \mathrm{Al} / \mathrm{W} \text { overlap })^{4}$. An $8 \mu \mathrm{m}$ wide $\mathrm{Al}$ bias rail passes through the QETs in a channel, connecting them in parallel. Charge electrode lines were originally also 8 $\mu \mathrm{m}$ wide, but their width was increased to $40 \mu \mathrm{m}$ in later iZIPs in order to reduce the

\footnotetext{
${ }^{4}$ In the original ZIP design material deposition for phonon (charge) sensors used 300 (20) nm thick $\mathrm{Al}$ and 35 (20) nm thick W layers. Approximately $82 \%$ of the detector phonon face was covered with 4144 QETs, 1036 QETs were per quadrant channel. Each QET consisted of a $250 \mu \mathrm{m} \times 1 \mu \mathrm{m}$ W strip connected to eight $300 \mu \mathrm{m} \times 55 \mu \mathrm{m} \mathrm{Al}$ fins [168.
} 

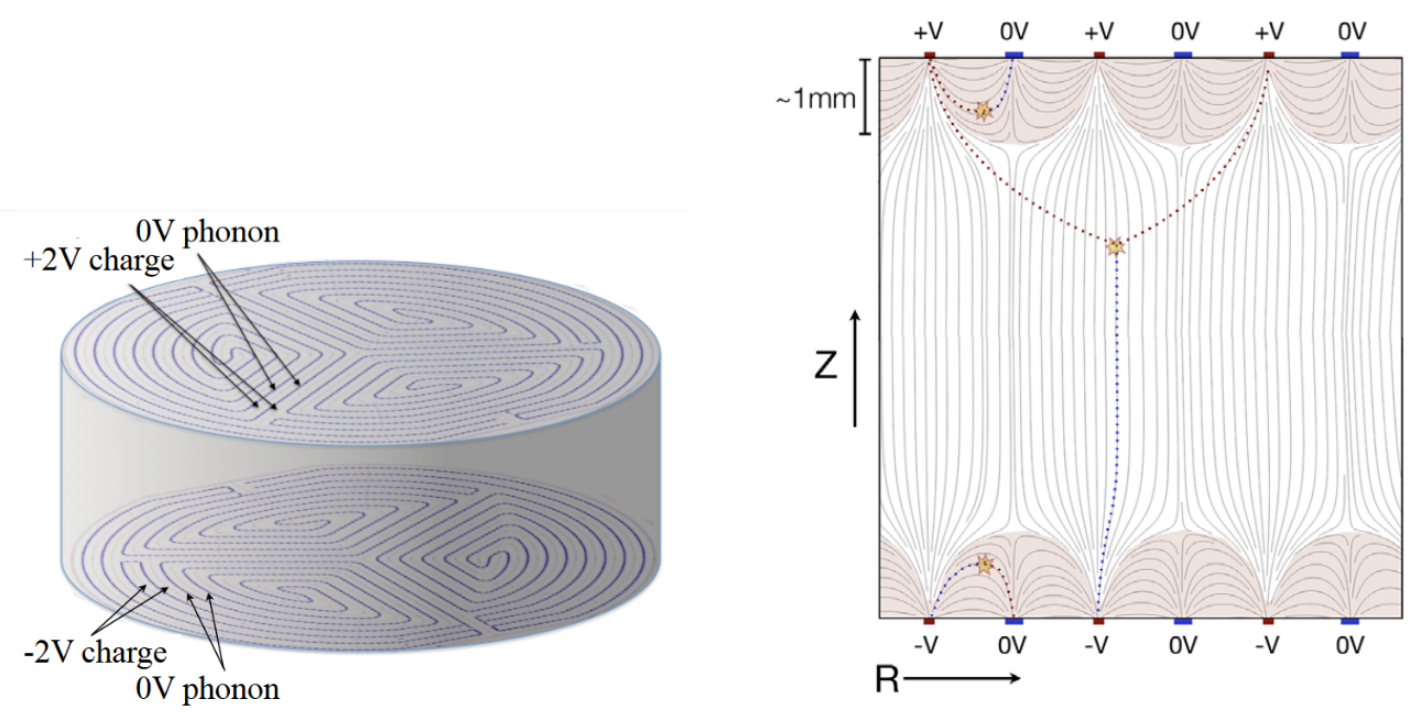

Figure 2.9: LEFT: Interleaved ZIP design, with alternating phonon TESs held at $0 \mathrm{~V}$ and ionization electrodes biased at $\pm 2 \mathrm{~V}$. RIGHT: Electric field lines across the crystal due to interleaved sensor layout. Electron/hole pairs created in the bulk of the detector are collected on both sides of the detector, whereas electron/hole pairs produced near the detector surface $(\sim 1 \mathrm{~mm})$ are collected only on one side. Holes will propagate along field lines, whereas electrons will propagate obliquely due to the electrons having an anisotropic effective mass, expressed as a tensor. Figures courtesy of [162] and S. Hertel [169]. 
maximum field on the detector surface. Adjacent sensors are separated by a $1.07-1.22$ mm gap from the center of the charge line to the center of the QET.

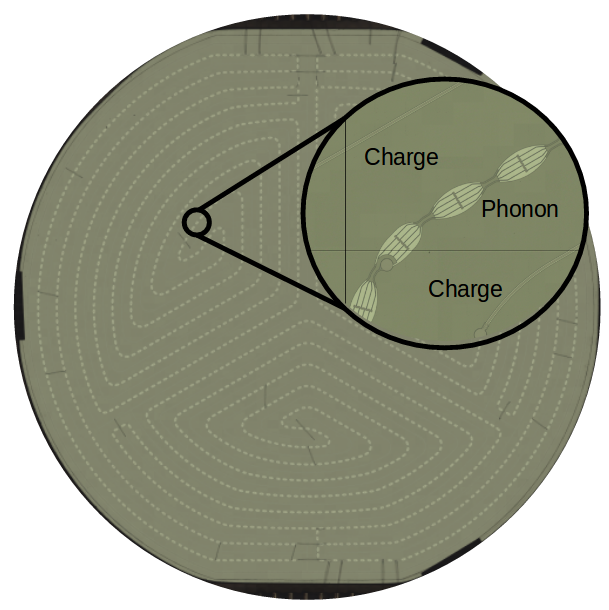

Figure 2.10: TOP: iZIP sensor schematic with zoom of charge electrodes interleaved with phonon QETs. BOTTOM: Schematic of QET chain, showing portions of two QETs, each with eight $300 \mu \mathrm{m} \times 55 \mu \mathrm{m}$ aluminum fins (blue), amorphous silicon (green), and $250 \mu \mathrm{m} \times 1 \mu \mathrm{m}$ tungsten strip (red), connected via $\mathrm{Al}$ bias rail (pink).

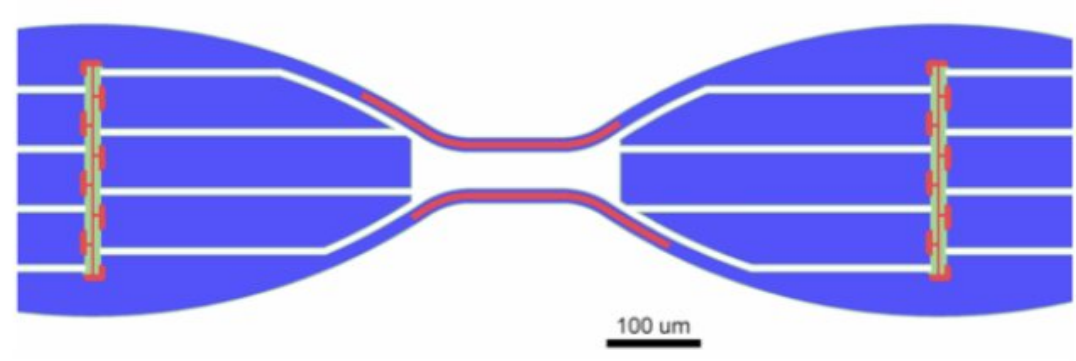

Green : amorphous Silicon Blue : Aluminum Red : Tungsten

In iZIP designs, 458 QETs are used per channel, with a total of 1820 QETs per detector. In total, about $5.2 \%$ of the crystal surface is covered with Al. The number of QETs per channel and the size of the QET components are optimized for phonon collection (which increases with phonon sensor coverage, since charge sensor coverage is effectively fixed), quasiparticle collection (which increases with more TES's and short Al fin lengths), and TES sensitivity (which depends on W strip size and phonon circuit parameters). For more information, see Section 2.2.4. The iZIP sensor and QET schematics are shown in Fig. 2.10. 


\subsubsection{Ionization Measurement}

Cooling a $\mathrm{Ge}(\mathrm{Si})$ detector to $<4 \mathrm{~K}(<10 \mathrm{~K})$ reduces the thermal energy to below what is typically required to excite an electron through the $0.74 \mathrm{eV}(1.17 \mathrm{eV})$ bandgap to populate the conduction band [168]. Essentially, the detector is "frozen out", as there are no free charge carriers. If an incident particle deposits sufficient energy $(\gtrsim 3.0 \mathrm{eV}$ for $\mathrm{Ge}, 3.8 \mathrm{eV}$ for $\mathrm{Si})^{5}$, an electron will be excited into the conduction band. If the electron has sufficient momentum, it will continue to free nearby electrons from their bound state in the valence band, creating a cascade effect until a "cloud" of electrons with low momentum has formed near the initial interaction site. Each liberated electron will leave a vacant state in the valence band, referred to as a "hole", that will act like a positively charged particle also within this cloud.

If there is no external field across the crystal, then the electron and hole pairs will recombine. However, if a bias is applied through the ionization electrodes deposited on the detector faces, then the resulting field will pull the charge carriers towards electrodes of opposite polarity, inhibiting recombination and creating a drift current. Holes tend to propagate along the field lines, whereas electrons will propagate obliquely, as demonstrated in Fig. 2.9. This is due to the electrons having an anisotropic effective mass, expressed as a tensor. The rate of change of electron group velocity is determined by the inverse mass tensor, which has off-diagonal elements in the standard detector coordinate system. These off-diagonal elements contribute an acceleration component that is non-parallel to the force of the applied electric field on the electrons [167].

The drift current produced by the charge carriers moving in the crystal will create a corresponding image charge current in the conducting electrodes [170]. As ShockleyRamo theory postulates, the electrodes will feel a current even if they do not collect the charge carriers, as long as the charge carrier movement creates an instantaneous change of the electrostatic flux lines that end on the electrode. The image current, $i$, felt by an electrode for an electron moving at speed $v$ is given by,

$$
i \propto e v E_{v}
$$

where $E_{v}$ is the component of the electric field in the direction of $v$ which would exist

\footnotetext{
${ }^{5}$ Electron/hole production requires deposited energy be greater than the band gap energy because some portion must go towards optical phonon production, in order to conserve momentum [166].
} 
at the electron's instantaneous position, if the electron were removed from the system, the electrode were given a unit potential, and all other conductors were grounded [171. Essentially, the image current seen by the electrode is proportional to the charge carrier's velocity and charge, and depends on the carrier's distance from the electrode.

\section{Ionization Signal Read-out}

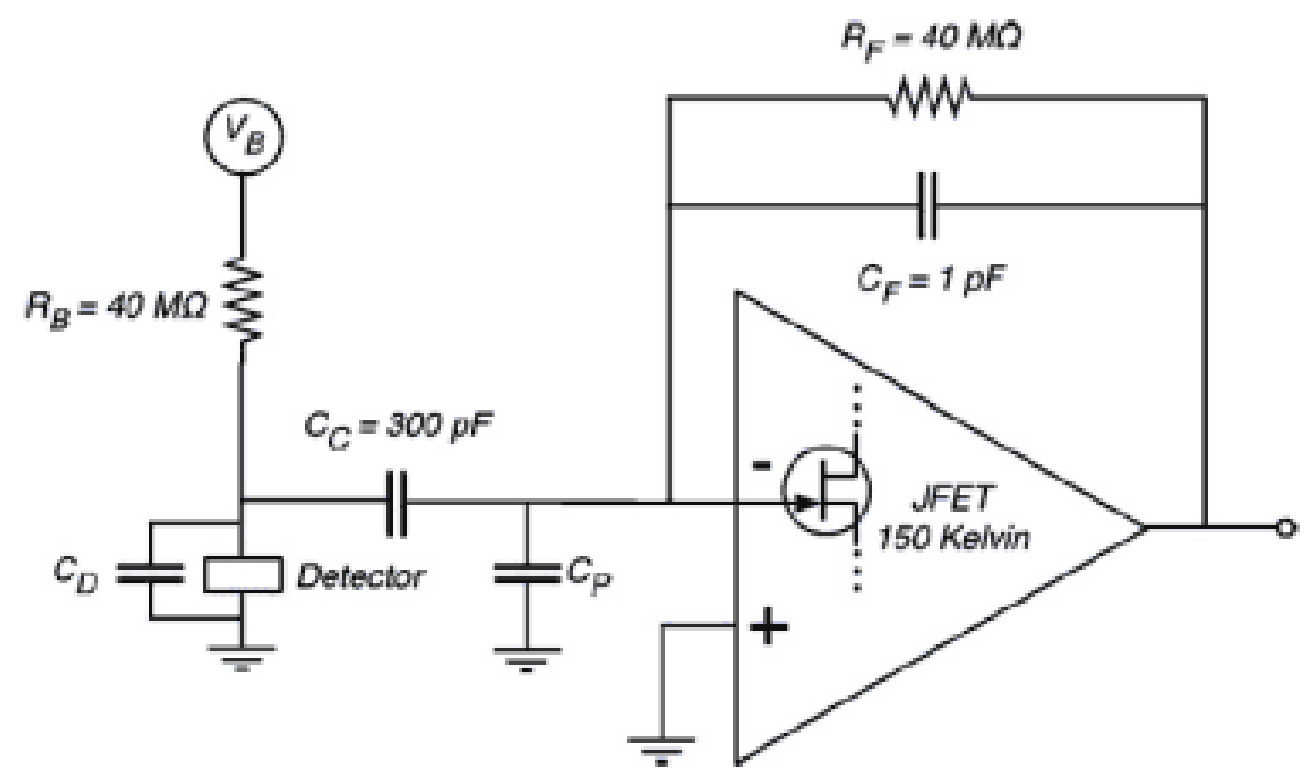

Figure 2.11: Simplified ionization channel read-out circuit. Voltage bias $V_{B}$ is applied to the detector with capacitance $C_{D} \approx 100 \mathrm{pF}$, after passing through biasing resistors with $R_{B}=40 \mathrm{M} \Omega$. A coupling capacitor $C_{C}=300 \mathrm{pF}$ blocks the biasing voltage from the feedback loop, comprised of a JFET amplifier kept at $150 \mathrm{~K}$, a feedback resistor $R_{F}=40 \mathrm{M} \Omega$, and parasitic feedback capacitor $C_{f}=1 \mathrm{pF} . C_{p}$ represents any stray parasitic capacitance.

The current induced on the electrodes passes through an amplifier circuit so as to produce a usable output signal. A schematic for the ionization signal readout of a single channel is shown in Fig. 2.11. As mentioned previously, a voltage bias $V_{B}$ is applied to the detector electrodes in order to drift charge carriers towards the electrodes. Bias resistors, totaling $R_{B}=40 \mathrm{M} \Omega$, are placed between the detector and bias source in 
order to prevent the induced signal from shorting immediately [172]. A large value is selected for the bias resistance in order to reduce Johnson noise, which can be treated as a parallel current noise with a single-sided power spectral density (in units $\mathrm{A} / \sqrt{\mathrm{Hz}}$ ) of,

$$
i_{B}=\sqrt{\left(\frac{4 k_{B} T_{B}}{R_{B}}\right)},
$$

where $T_{B}$ is the temperature of the resistor [167]. The detector itself can be treated as the Ramo current signal in parallel with a $\sim 100 \mathrm{pF}$ capacitor. This detector capacitance is determined by the geometrical configuration of the electrodes in reference to each other and electrical ground on the detector housings. The smaller the capacitance, the more sensitive the detector will be to charge.

A coupling capacitor $C_{C}=300 \mathrm{pF}$ blocks the biasing voltage from the feedback loop, where the current signal will be amplified. The amplifier efficiency of the coupling capacitor (how much of the signal isn't lost) depends on the complex impedances of the coupling capacitor, the detector side of the circuit, and the amplifier side, but in general a larger coupling capacitance will increase the efficiency. $C_{p}$ represents any stray parasitic capacitance.

The input voltage is then amplified before digitization. The high impedance detector requires an amplifier with a higher input impedance to keep the signal from being shorted. Although room temperature operational amplifiers can have high input impedance, the cabling required to bring the signal to room temperature would also have a capacitance in parallel to the amplifier input, and would degrade the sensitivity to charge. Thus, the first-stage amplifier used is a Junction gate Field-Effect Transistor (JFET) amplifier kept at $150 \mathrm{~K}$, which converts input voltage to current. The current signal then travels to the room temperature amplification stages for additional gains, without the cabling capacitance affecting the amplifier input.

In order to achieve stable overall gain throughout long data runs, a closed loop amplifier system is used, with feedback resistor $R_{F}=40 \mathrm{M} \Omega$, and parasitic feedback capacitor $C_{f}=1 \mathrm{pF}$. Although the initial signal from the Ramo current has a varied shape which depends on (among other things) the trajectories of the charge carriers, the final ionization output has a defined pulse shape with an amplitude given by $q / C_{f}$, where $q$ is induced charge in the circuit, and a decay time given by $R_{f} C_{f}$. Any affects on the 
rising edge of the pulse due to charge propagation in the crystal will be lost due to the digitizer rate of $1.25 \mathrm{MHz}(0.8 \mu$ s per sample), which is not sensitive enough to detect these $\mathcal{O}(\mathrm{ns})$ fluctuations. Because the pulse decay time is dependent only on feedback circuit components, the pulse shape is constant, with an amplitude proportional to the induced Ramo current, and thus proportional to the number of electron/hole pairs produced in the original detector recoil.

As will be discussed in more detail in Chapter 4, this amplitude will be used to calculate the ionization energy of an event, or $E_{Q}$, which is defined as,

$$
E_{Q}=\epsilon_{\gamma} N_{e / h}
$$

the recoil energy of an event that produces $\mathrm{N}_{e / h}$ electron/hole pairs in a material that requires $\epsilon_{\gamma}$ energy to produce each pair $\left(\epsilon_{\gamma}=3.0 \mathrm{eV}\right.$ for $\mathrm{Ge}, 3.8 \mathrm{eV}$ for $\left.\mathrm{Si}\right)$, assuming the event is an electron recoil with $100 \%$ charge collection efficiency.

\subsubsection{Phonon Measurement}

During an incident particle interaction some portion of the deposited energy will go into the production of quantized vibrational modes of the crystal lattice, known as athermal phonons. Athermal phonons are out-of-equilibrium phonons, which means they are not thermal phonons caused by the lattice having nonzero temperature. The thermal average number of phonons for a given angular frequency mode $\omega$ in the crystal at temperature $\mathrm{T}$ is given by the Bose-Einstein distribution [166],

$$
\langle n\rangle=\frac{1}{e^{\hbar \omega / k_{B} T}-1} .
$$

Thus the thermal phonon distribution depends on the temperature of the crystal, and for operating CDMS temperatures of $\sim 50 \mathrm{mK}$, the distribution will peak around $\hbar \omega \approx 12 \mu \mathrm{eV}$. This is well below the sensitivities of CDMS phonon sensors, and thus only athermal phonons well out of equilibrium will be detected.

\section{Primary Phonons}

Primary phonons are created during the initial scattering at the Debye energy $(\sim 10 \mathrm{THz}$ in $\mathrm{Ge}$ and $\mathrm{Si}$ ), and they immediately begin a process of quasi-diffusion. Quasi-diffusion 
is dominated by isotropic scattering and anharmonic decay, in which a single phonon decays into two phonons of smaller energy. The rate of anharmonic decay goes as $\tau_{d} \propto(1$ $\mathrm{THz} / \nu)^{5}$ for phonon frequency $\nu$. Comparatively, the elastic isotropic scattering has a time constant of $\tau_{s} \propto(1 \mathrm{THz} / \nu)^{4}$, representing the time between scatters.

Calculating these two time constants for a phonon frequency of $\nu=1 \mathrm{THz}$, gives $\tau_{d} \approx 10 \mu$ s and $\tau_{s} \approx 0.4 \mu \mathrm{s}$. The small $\tau_{s}$ value means that these high energy phonons have a small mean free path and scatter often, but $\tau_{d}$ determines that the phonons will undergo decay within microseconds, reducing their frequency and therefore increasing their mean free path. Once phonon frequency drops below $\sim 1 \mathrm{THz}$ for $\mathrm{Si}$ or $0.6 \mathrm{THz}$ for Ge, the mean free path of the phonon becomes comparable to the detector dimensions [173]; such phonons are referred to as ballistic phonons.

Ballistic phonons can travel freely to the surface of the crystal which will either be bare, or covered with an $\mathrm{Al}$ superconducting film. If they hit a bare surface they will reflect and continue scattering; after enough time $(\sim 800 \mu \mathrm{s})$ in the detector, such as after multiple ballistic scatters, the phonons are said to no longer contain position information about the initial interaction. However, these phonons still have use in CDMS phonon data analysis, as will be discussed in Chapter 5. If they hit an aluminum fin, they will be absorbed into the TES, eventually contributing their energy to the total phonon measurement. If the total energy of an initial electron recoil is given by $E_{R}$, then the energy of the primary phonons produced is,

$$
E_{P}=E_{R}-E_{g a p} N_{e / h}
$$

were $E_{g a p} N_{e / h}$ represents the energy used to excite the $N_{e / h}$ initial electron/hole pairs across the bandgap of energy $E_{g a p}$.

\section{Neganov-Trofimov-Luke Phonons}

In addition to primary phonons created by the initial scattering, accompanying phonons, known as Neganov-Trofimov-Luke (NTL) phonons, are created when charge carriers drift across the crystal. As charge carriers are accelerated through crystal by the applied electric field, they will interact with atoms in the lattice and transfer energy to the lattice, creating NTL phonons [174][175]. These interactions will restrict the charge carriers to a terminal drift velocity comparable to the speed of sound and dependent on 
the magnitude of the electric field; for a $\sim 30 \mathrm{mK}$ germanium crystal with an applied field of $1-5 \mathrm{~V} / \mathrm{cm}$, the drift velocity is roughly $10-30 \mathrm{~km} / \mathrm{s}$ for holes and $15-50 \mathrm{~km} / \mathrm{s}$ for electrons [176].

All of the work done by the electric field on the charge carriers traveling at the terminal drift velocity is transferred to the lattice for phonon production. Thus, the NTL contribution to total phonon energy of a given event with a voltage difference of $V$ between detector faces is,

$$
E_{N T L}=e N_{e / h} V-E_{K E},
$$

where $N_{e / h}$ is the number of electron/hole pairs created, and $E_{K E}$ is the kinetic energy of the charge carriers at the terminal drift velocity.

\section{Relaxation Phonons}

A final source of phonons can be found on the detector surface, at the end of the charge carriers' trajectories. Electrons and holes will recombine with their corresponding image charges once they reach their respective electrodes, creating a stationary neutral site, and depositing their energy into the phonon system. At the electrodes, charge carriers downscatter and relax to the Fermi level. The energy $E_{g a p} N_{e / h}$ that had originally been spent to liberate the charge carriers across the band gap, and any additional kinetic energy $E_{K E}$, will thus be returned to the system through in the form of relaxation phonons. The total energy $E_{r e l}$ of relaxation phonons is,

$$
E_{\text {rel }}=E_{\text {gap }} N_{e / h}+E_{K E} .
$$

The total phonon energy, $E_{T}$, is therefore given by,

$$
E_{T}=E_{P}+E_{N T L}+E_{r e l}=E_{r}+e N_{e / h} V
$$

\section{Quasiparticle Trapping}

In order to turn phonons into a measurable signal via the TES, the phonon energy must first be collected from the crystal surface. This is accomplished by depositing aluminum quasiparticle traps on each detector face, a method first proposed by N. Booth [177]. 
The complete system of traps and TES's are referred to as Quasiparticle-trap assisted Electrothermal-feedback Transition-edge-sensors, or QETs.

At CDMS operational temperatures, the deposited Al fins are superconducting, and thus contain Cooper pairs. As depicted in Fig. 2.12, phonons entering the traps with an energy larger than twice the superconducting Al energy gan $6 \Delta_{A l}=340 \mu \mathrm{eV}$, have sufficient energy to break the Cooper pairs, creating quasiparticles. This energy threshold corresponds to $\nu \approx 82 \mathrm{GHz}$, less than the $\sim 1 \mathrm{THz}$ frequency minimum at which ballistic phonons travel freely towards the detector surfaces; phonons reaching the surface are expected to have sufficient energy for quasiparticle production. Those with insufficient energy will not break Cooper pairs, instead their energy will return to the detector, not contributing towards the final measured phonon signal, and resulting in a loss of about $5-10 \%$ of the initial energy $]^{7}[173$. The pair breaking process can create lower energy phonons, which will repeat the process if they are energetic enough. Created phonons of energy $<2 \Delta_{A l}$ will not create quasiparticles and instead will return to the detector as lost energy.

Quasiparticles will diffuse through the Al, losing energy via electron-electron scattering and shedding phonons; created phonons could create more quasiparticles if their energy is sufficient, but an additional $\sim 50 \%$ of the initial energy is lost through low energy shed phonons [150] 173]. On the timescale of $1-2 \mu \mathrm{s}^{8}$, the quasiparticles will diffuse towards a region where tungsten overlaps the aluminum, known as the "bi-layer". The band gap energy of a Type I superconductor is proportional to the material's transition temperature $T_{C}$, and thus the tungsten TES has a much smaller energy threshold for breaking Cooper pairs, about $2 \Delta_{W} \sim 20 \mu \mathrm{eV}$. Because the bi-layer region is comprised of both tungsten and aluminum, it has a energy gap threshold between the two materials, such that $2 \Delta_{A l}>2 \Delta_{B i}>2 \Delta_{W}$. If diffusing quasiparticles enter the bi-layer and lose sufficient energy, they cannot reenter the Al-only region and are considered "trapped". Quasiparticles continue scattering and producing phonons (produced phonons will be

\footnotetext{
${ }^{6}$ The superconductor energy gap for quasiparticle excitations is analogous to the semiconductor bandgap for excited electron-hole pairs [173].

7 This is only one source of energy loss in the phonon measurement. Accounting for all sources of energy loss (of which only a few significant examples are discussed in this thesis) yields a total energy loss of $\sim 85 \%$, depending on detector type [178].

8 The quasiparticle diffusion time depends on the mean free path of the quasiparticle, and the thickness of the $\mathrm{Al}$ fin, which is set to optimize quasiparticle collection efficiency.
} 

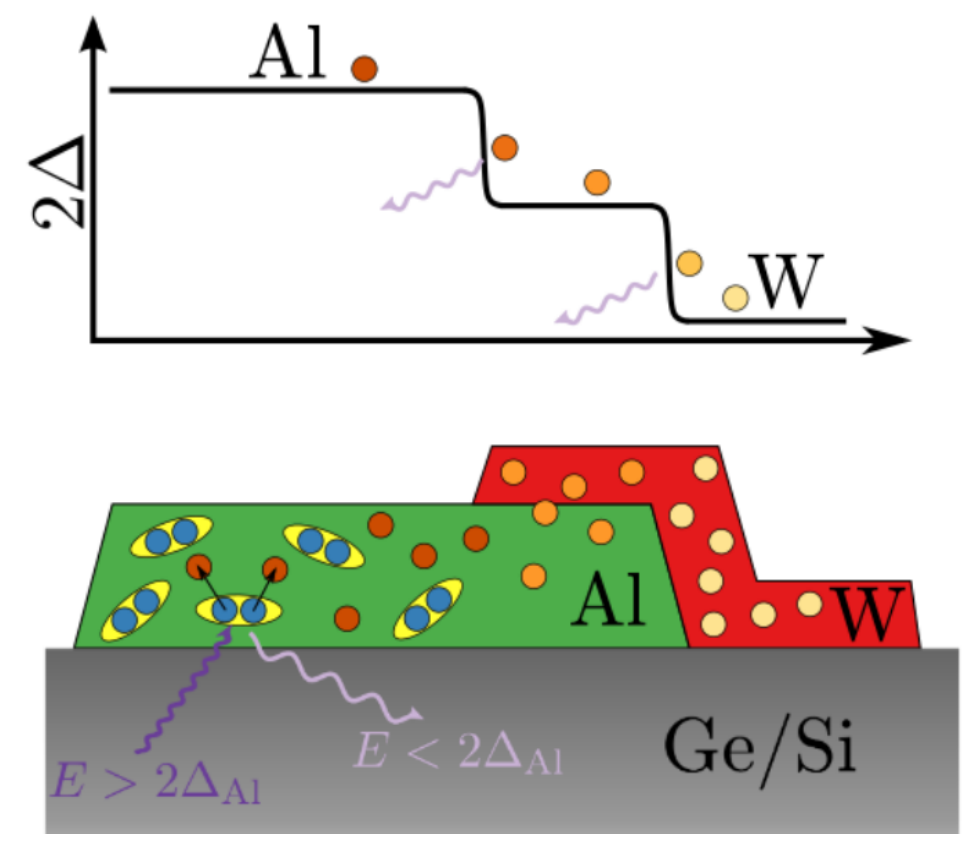

Figure 2.12: Cartoon depicting the process of quasiparticle trapping in a QET. Phonons (dark purple) enter the superconducting Al (green) fins containing Cooper pairs (depicted as blue circles enclosed in yellow ovals). If the phonons have sufficient energy $\left(>2 \Delta_{A l}\right)$, they can break up Cooper pairs into quasiparticles (dark orange circles). Quasiparticles will diffuse through the $\mathrm{Al}$ traveling from high to low energy regions (depicted in top graph), entering the $\mathrm{Al} / \mathrm{W}$ overlap region, and eventually the $\mathrm{W}$ TES (red). As the quasiparticles diffuse they will lose energy, depicted as the gradient transition from dark orange to yellow. During these processes new phonons may be created with energy $<2 \Delta_{A l}$ (light purple), which will not break up Cooper pairs and are considered lost energy. 
energy lost) until they become trapped within the tungsten layer. Electron-phonon scattering is roughly 100 times less frequent than electron-electron scatter in the tungsten, and energy removed from the quasiparticles through electron scatters will remain in the tungsten and not be lost.

\section{Electrothermal Feedback TES}

Once the phonon energy has been collected in the tungsten portion of the QET, it can be converted into an electrical signal. As previously mentioned, Transition Edge Sensors are kept at their transition temperature between superconducting and normally conducting via Joule heating in a method known as electrothermal feedback (ETF).

Considering the thermal model of the TES/fridge system, electrons in the TES are thermally coupled to phonons in the TES, which are thermally coupled to phonons in the crystal, which in turn are thermally coupled to the cryostat. Due to large conductivities, the cryostat, crystal, and $\mathrm{W}$ phonon systems are at the same temperature. Therefore the primary thermal impedance to consider comes from the coupling of phonons and electrons in the TES. Given the heat capacity of electron system $c_{v}$, thermal conductivity coefficient, $\kappa$, the respective temperatures of the sensor and the substrate (crystal and cryostat), $T$ and $T_{s}$, the constant voltage bias across the TES, $V_{b}$, and its temperature dependent resistance, $R(T)$, then the heat flow equation of the system is given by,

$$
c_{v} \frac{d T}{d t}=\frac{V_{b}^{2}}{R(T)}-\kappa\left(T^{5}-T_{s}^{5}\right) .
$$

Note that the conductivity exponent was empirically found to be $n=5$ for this system 179 .

When the system is in equilibrium $(d T / d t=0)$, the Joule heating from the voltage bias is balanced by the cooling of the cryostat. When quasiparticles from the Al fins deposit energy into the electron system the temperature changes. This small change in temperature results in a large change in TES resistance (due to $\mathrm{W}$ being on the transition edge between normal and superconducting), which is measured by the phonon read-out circuit. The increased resistance reduces the Joule heating component, allowing the TES to return to the original equilibrium temperature.

The small change in quasiparticle energy can be modeled as a perturbation $\delta T$ 
around the equilibrium temperature. In this case Eq. 2.9 yields,

$$
c_{v} \frac{d \delta T}{d t}=-\left(\frac{V_{b}^{2}}{R^{2}(T)} \frac{d R(T)}{d T}+5 \kappa T^{4}\right) \delta T,
$$

which can be solved to,

$$
\delta T(t) \sim e^{-t / \tau_{E T F}}
$$

with the assumption that the TES is operated at a temperature reasonably removed from $T_{s}$, and thus $V_{b} / R(T)=\kappa T^{5}$. Here $\tau_{E T F}$ is the sensor cool-down time constant given by,

$$
\tau_{E T F}=\frac{\frac{c_{v}}{5 \kappa T^{4}}}{1+\frac{1}{5} \frac{T}{R(T)} \frac{d R(T)}{d T}} .
$$

Its helpful to note that the thermal conductance of the electron system is given by $g=5 \kappa T^{4}$, and thus the standard return to equilibrium time constant, $\tau_{0}=c_{v} / g$, is included in $\tau_{E T F}$. Allowing $\alpha=\frac{T d R(T)}{R(T) d R}$ (a dimensionless constant describing the transition sharpness) reveals the classic speed up equation in $\tau_{E T F}$,

$$
\tau_{E T F}=\frac{\tau_{0}}{1+\frac{\alpha}{n}}
$$

From this calculation it is seen that a temperature perturbation introduced in the TES is met with an exponential cool-down with time constant $\tau_{E T F}$. This is the fundamental notion behind electrothermal feedback, which allows the TES to be kept at a stable operating temperature as phonon events are read-out.

The time constant $\tau_{E T F}$ was empirically found to be $\sim 50 \mu \mathrm{s}$, although this value is dependent on fabrication details and where the TES is operated along its transition edge. Recall that ballistic phonons within the detector are not expected to become thermalized until $\sim 800 \mu s$ after the initial event. Therefore the time scale of the phonon signal, or pulse, is limited by the phonon timescale, and not the TES timescale.

\section{Phonon Signal Read-out}

A simplified phonon read-out circuit for a single channel is shown in Fig. 2.13. Along with the previously discussed TES with variable resistance $R_{T E S} \approx 100-200 \mathrm{~m} \Omega$, a shunt resistor $R_{s h} \approx 20 \mathrm{~m} \Omega$ draws current $I_{b}$ from applied voltage bias $V_{b}$. In equilibrium, $V_{b}$ provides enough power to the TES to balance cooling from the cryostat, 


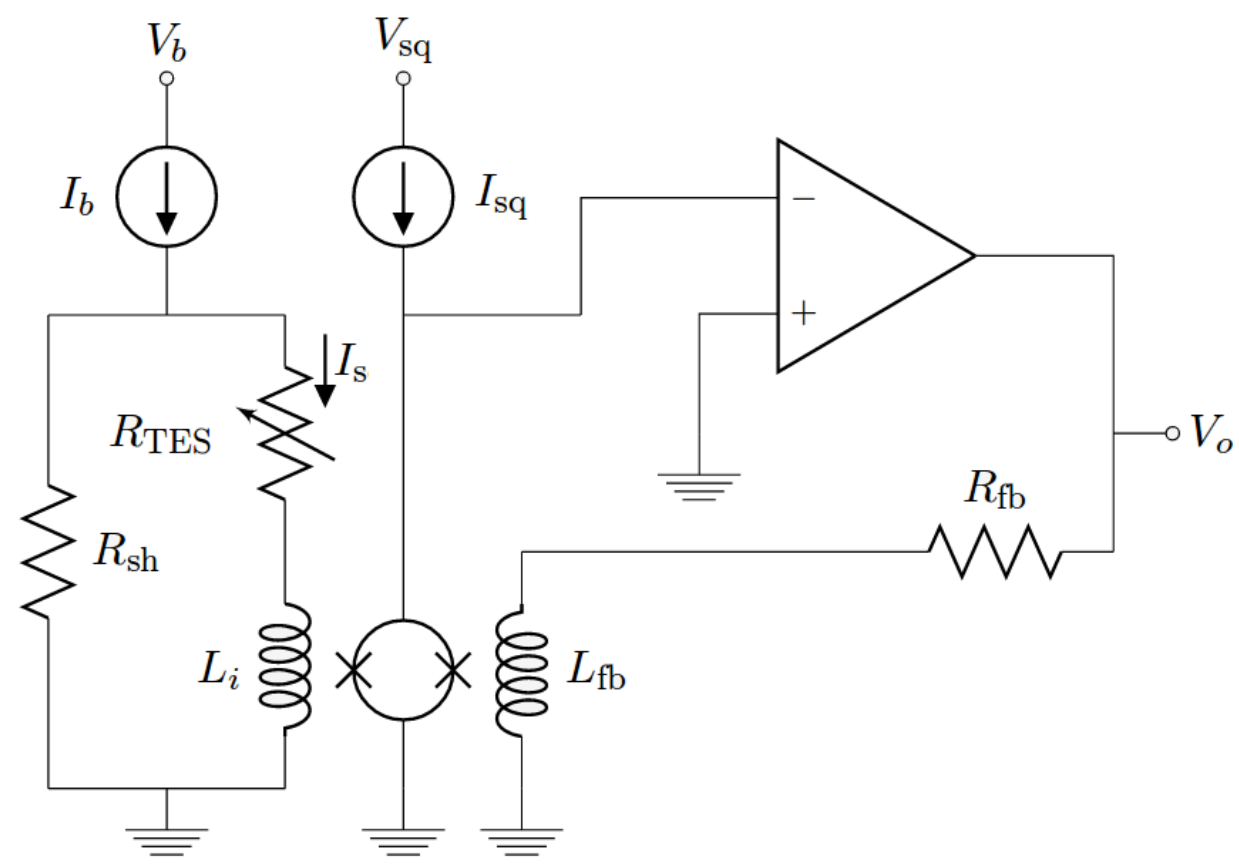

Figure 2.13: Simplified single phonon channel read-out circuit. Shunt resistor $R_{s h} \approx 20$ $\mathrm{m} \Omega$, and TES with variable resistance $R_{T E S} \approx 100-200 \mathrm{~m} \Omega$ draws stable current $I_{b}$ from applied voltage bias $V_{b}$. When an event occurs, the TES temperature and thus $R_{T E S}$ increases, which changes the value of current, $I_{s}$, passing through $R_{T E S}$ and the 10-loop input coil with $L_{i}=250 \mathrm{nH}$. The input coil is coupled to the one-loop feedback coil with $L_{f b}=25 \mathrm{nH}$ through the SQUID array, yielding feedback current $I_{f b}=10 * I_{s}$, due to the 10:1 loop ratio between coils. The current through $L_{f b}$ is then read out as an output voltage $V_{o}$ after passing through a feedback resistor $R_{f b}=1 \mathrm{k} \Omega$ and undergoing amplification. 
maintaining a stable operating temperature and a stable $I_{b}$. When an event occurs, the TES temperature and thus $R_{T E S}$ increases, which changes the value of the current through the TES, $I_{s}$.

The current is also passing through the 10-loop input coil $\left(L_{i}=250 \mathrm{nH}\right)$ of a Superconducting Quantum Interference Device (SQUID) array, changing its magnetic flux. The CDMS SQUID array is comprised of 100 individual SQUIDs surrounded by planar input and feedback coils [180. SQUIDs are ideal for use with TESs because they are high bandwidth but have low impedance and low noise. The SQUIDs used have a modulation depth (peak-to-peak amplitude of the $\mathrm{V}-\Phi$ curve) of $5 \mathrm{mV}$, a $\sim 1.2$ $\mathrm{nV} / \sqrt{\mathrm{Hz}}$ voltage noise across the SQUID [168], and a current-per-flux quantum of 25 $\mu \mathrm{A}$, referenced relative to the input coil [173].

The change in magnetic flux in the SQUID results in a change in voltage across its terminals. An amplifier driven by this voltage feeds a current $I_{f b}=10 * I_{s}$ back into the one-loop feedback coil with $L_{f b}=25 \mathrm{nH}$, in order to cancel the change in magnetic flux through the SQUID. The resulting current through $L_{f b}$ is then read out as an output voltage $V_{o}$ after passing through a feedback resistor $R_{f b}=1 \mathrm{k} \Omega$,

$$
V_{o}=10 I_{s} R_{f b}
$$

This value depends on current $I_{s}$ which relates directly to the energy deposited by the quasiparticles, and thus is proportional to the energy deposited by phonons, $E_{T}$. As with the ionization signal output, the measured phonon voltage output is then digitized, this time at a rate of $0.625 \mathrm{MHz}(1.6 \mu$ s per sample) for iZIP detectors?

\subsubsection{Ionization Yield}

To summarize, the relevant energies measured by the iZIP detector phonon and charge sensors are given by,

$$
\begin{array}{r}
E_{T}=E_{P}+E_{N T L}+E_{r e l}=E_{R}+e N_{e / h} V, \\
E_{Q}=\epsilon_{\gamma} N_{e / h},
\end{array}
$$

respectively. Recall that the recoil energy, $E_{R}$, represents the total energy deposited in the initial interaction. Assuming an electron recoil with $100 \%$ charge collection

\footnotetext{
${ }^{9}$ Due to smaller TES surface coverage, trace lengths were $3.28 \mathrm{~ms}$ for oZIP digitization
} 
efficiency, $E_{Q}$ would also represent the total energy deposited by the initial interaction, since the smaller-mass incident particle cannot transfer sufficient energy to displace the surrounding nuclei from their crystalline locations; instead this energy goes into electron/hole pair production. Therefore, in the case of electron recoils, or ER's, $E_{Q}=$ $E_{R}$.

Now consider a case where the initial event was a nuclear recoil. In this instance the incident particle can deposit significant energy into crystal nuclei as well as electron/hole production. Although excited nuclei can spend energy on electron/hole pair production, they can also lose energy though phonon emission or displacing additional nuclei from their crystalline locations. By the end of an NR event, the total recoil energy has been collected as phonons, just as was described in Section 2.2.4 however fewer electron/hole pairs were produced since energy was spent via interactions with nuclei; this is known as quenching. It results in a reduced ionization signal and a smaller $E_{Q}$ when compared to an ER event of the same recoil energy.

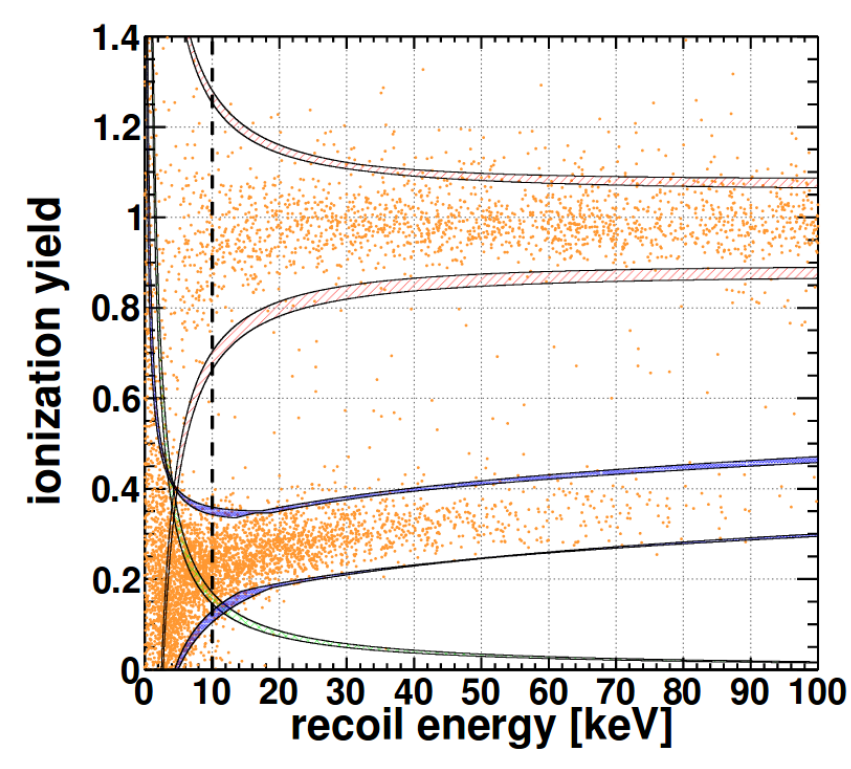

Figure 2.14: A plot of ionization yield versus recoil energy for oZIP detector ${ }^{252} \mathrm{Cf}$ calibration data (yellow points). Data is mostly divided into the electron recoil band (red) with an ionization yield around 1, and nuclear recoil band (blue) with a reduced ionization yield around 0.3. A typical charge threshold band (green) and recoil energy threshold (dotted black) are also included. Figure courtesy of A. Villano.

Using this distinction in $E_{Q}$, a quantity referred to as the ionization yield, $Y$, is used 
to distinguish electron and neutron recoil events,

$$
Y=\frac{E_{Q}}{E_{R}}
$$

This value is normalized to one for electron recoil events. In terms of the charge and total phonon energy (i.e. the measured signals),

$$
Y=\frac{E_{Q}}{E_{T}-\frac{e V}{\epsilon_{\gamma}} E_{Q}} .
$$

Fig. 2.14 shows a typical ionization versus recoil energy plot where electron and nuclear recoils are included. The different recoils form distinct yield bands. As is standard for SuperCDMS analysis, the yield has been normalized such that an electron recoil has a value of $Y \approx 1$, whereas nuclear recoils will result in $Y \approx 0.3$. The widening of the bands at low recoil energies is due to decreased signal-to-noise in the measurements. Separation of the bands dissolves rapidly for $E_{r}<10 \mathrm{keV}$, and by $E_{r} \approx 5 \mathrm{keV}$ they overlap at the $3 \sigma$ level. Essentially this means the discrimination power offered by ionization yield diminishes for low recoil energies. Typically a recoil energy threshold of $E_{r} \gtrsim 10 \mathrm{keV}$ is used by CDMS to avoid this region, at the sacrifice of low WIMP mass sensitivity. A charge threshold is also included, following $1 / E_{r}$ behavior determined by Eq. 2.18.

It should be noted that some events in Fig. 2.14 fall between the ER and NR bands; these are referred to as surface events. If an incident particle (typically a $\beta$ particle, in this case) scatters within $\sim 10 \mu \mathrm{m}$ of the surface (referred to as the "dead layer"), the initial charge carrier cloud can self-shield against the applied field and will be collected immediately by the nearest electrode. These events have a suppressed ionization signal and thus a reduced yield for their given $E_{r}$, making an electron recoil event easy to mistake for nuclear recoil events. As discussed in Section 2.1.1. BLIP detectors misidentified $5 \%$ of surface events above $10 \mathrm{keV}$.

Fortunately, a ZIP phonon pulse timing parameter allows one to distinguish surface events from true nuclear recoil events, as surface events tend to have faster phonon pulses. Additionally, the interleaved electrode design of the iZIP detector creates a lateral electric field near the surface such that surface events result in ionization signals on only one side of the crystal while bulk events result in signals on both sides. Therefore 
side asymmetry is a strong indicator of surface events. Yield discrimination and surface event rejection will be discussed further in Section 5.3.4.

\subsection{SuperCDMS SNOLAB and Prototype $100 \mathrm{~mm}$ iZips}

SuperCDMS Soudan data taking concluded in November 2015, but preparations for the next generation, SuperCDMS at the SNOLAB facility in Sudbury, Canada, have been ongoing. This future experiment aims to probe smaller WIMP-nucleon cross sections using increased shielding, larger, more sensitive iZIP detectors, and improved read-out hardware. Initial SuperCDMS SNOLAB experiment runs are expected to begin in 2020.

\subsubsection{Facility and Experimental Setup}

Construction of the SuperCDMS SNOLAB experiment has already begun in the "ladder lab" drift within SNOLAB, the home of the Sudbury Neutrino Observatory (SNO) experiment. SNOLAB is located approximately $2 \mathrm{~km}$ (1.5 miles) underground, providing 6010 m.w.e of overburden shielding against cosmic-ray secondaries, such as muons. Comparisons of muon flux verses depth for SUF, Soudan, SNOLAB, and various other underground facilities is shown in Fig. 2.15

CDMS detectors will be cooled to $\sim 15-30 \mathrm{mK}$ in a "dry" dilution refrigerator (one that uses cyrocoolers instead of a ${ }^{4} \mathrm{He}$ bath). The experiment cryostat, called the "SNOBOX", will be surrounded by $40 \mathrm{~cm}$ of polyethylene for neutron shielding, and $23 \mathrm{~cm}$ of low-activity lead for gamma shielding. A thin metal shield blocks radon diffusion into the inner shielding. Polyethylene and water tanks provide additional neutron shielding as the outermost layer. As with previous CDMS experiments, an electronics stem and a cryogenics stem will be the only entries into the cryostat. A schematic of the SuperCDMS SNOLAB experiment setup is shown in Fig. 2.16.

\subsection{2 $100 \mathrm{~mm}$ SuperCDMS Detectors}

For initial data runs, SuperCDMS SNOLAB aims to achieve a $36 \mathrm{~kg}$ target mass using larger individual detectors; $100 \mathrm{~mm}(\sim 4 ")$ diameter and $35 \mathrm{~mm}(\sim 4 / 3 ")$ thick Ge (Si) detectors would weigh 1.39 (0.61) kg each, with an initial payload of 10 Ge iZIPs, $2 \mathrm{Si}$ 


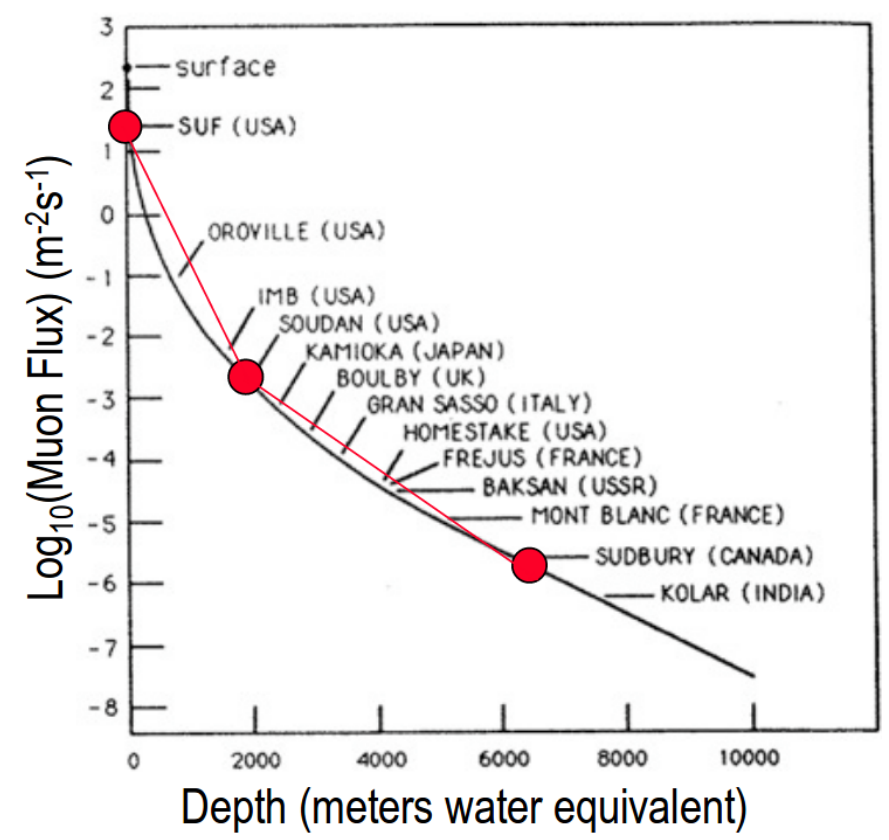

Figure 2.15: Muon flux versus depth (mwe) for various underground facilities, including the Stanford Underground Facility (SUF), Soudan Underground Laboratory, and SNOLAB (Sudbury). Figure courtesy of J. Cooley.

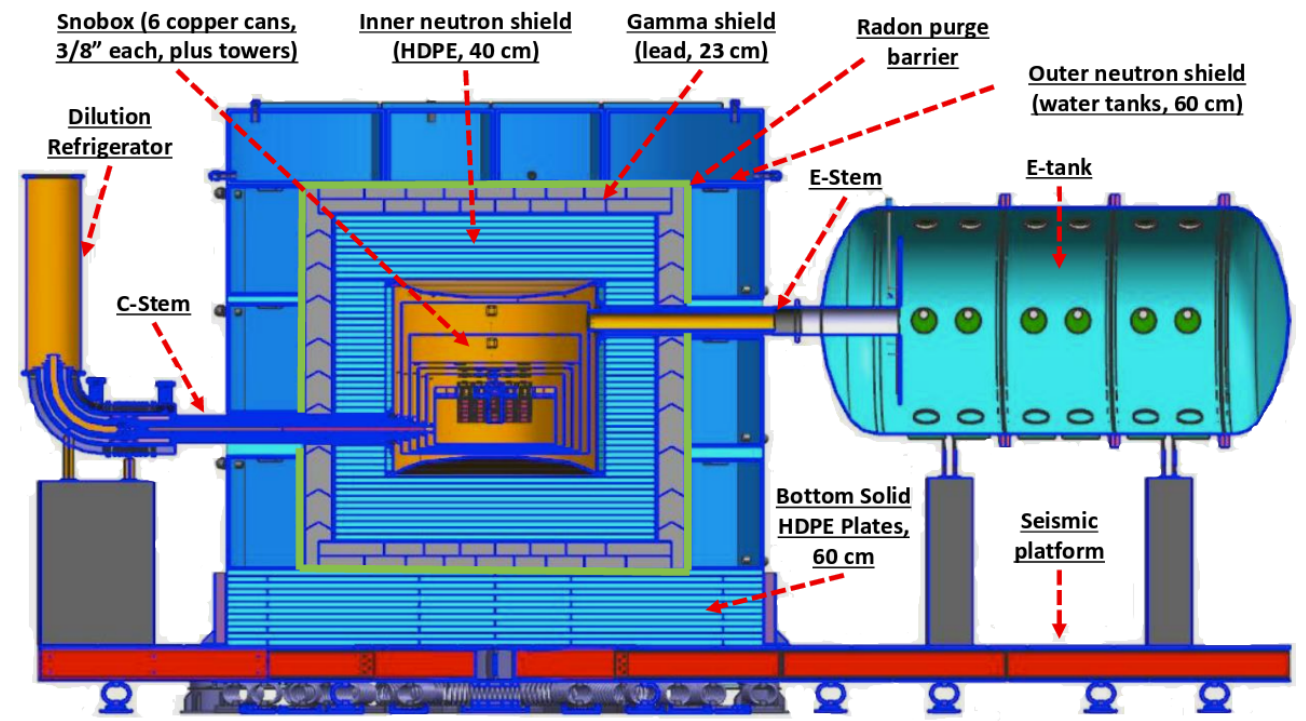

Figure 2.16: Schematic of SuperCDMS SNOLAB experiment setup, including shielding. Figure from [135]. 
iZIPs, 8 Ge high voltage (HV) detectors, and 4 Si HV detectors. Although only four 6detector towers will be used at first, the cryostat was designed with a 31 tower capacity. HV detectors, designed after the success of CDMSlite, will have six phonon sensors on each detector face (with $45^{\circ}$ offset between faces), and no ionization readout. They will be operated at $\sim 100 \mathrm{~V}$, amplifying the NTL phonon signal to reach projective NR energy threshold as low as $40 \mathrm{eV}$ [135].

$100 \mathrm{~mm}$ iZip detectors will also be comprised of six phonon sensors per face, along with the standard inner disk and outer ring charge channels. A voltage bias of $\sim 4-10$ $\mathrm{V}$ is applied to the ionization electrodes, and the phonon sensors are grounded. Ge ( $\mathrm{Si}$ ) $100 \mathrm{~mm}$ iZIPs are expected to reach a projected NR energy threshold of $166(272) \mathrm{eV}$ [135]. An in-depth study of prototype $100 \mathrm{~mm}$ iZIP detectors will be done in Chapters 4 and 5 .

\subsubsection{SuperCDMS SNOLAB Projected Sensitivity}

HV and iZIP detectors will be used in tandem to probe the low-mass WIMP region $\left(1-10 \mathrm{GeV} / \mathrm{c}^{2}\right)$; taking advantage of NTL phonon amplification, HV detectors are designed to have better sensitivity to $\leq 5 \mathrm{GeV} / \mathrm{c}^{2}$, while ER/NR discrimination results in better iZIP sensitivity above $\sim 5 \mathrm{GeV} / \mathrm{c}^{2}$.

As shown in Fig. 2.17, the SuperCDMS SNOLAB experiment is expected to have an initial sensitivity to nuclear recoil cross sections $\sim 1 \times 10^{-43} \mathrm{~cm}^{2}$ for a WIMP mass of $1 \mathrm{GeV} / \mathrm{c}^{2}$, and probe WIMP mass regions down to sub-GeV $/ \mathrm{c}^{2}$. As the SuperCDMS SNOLAB experiment payload is increased, it is expected to continue reducing the cross section sensitivity, approaching the cross section at which the interaction rate from dark matter particles becomes comparable to the solar neutrino coherent elastic scattering rate; this is referred to as the dark matter discovery limit [145]. Sensitivity projections for a full SNOLAB cryostat payload, using $150 \mathrm{~mm}$ diameter Si detectors, is discussed in Section 6.5 of this thesis. 


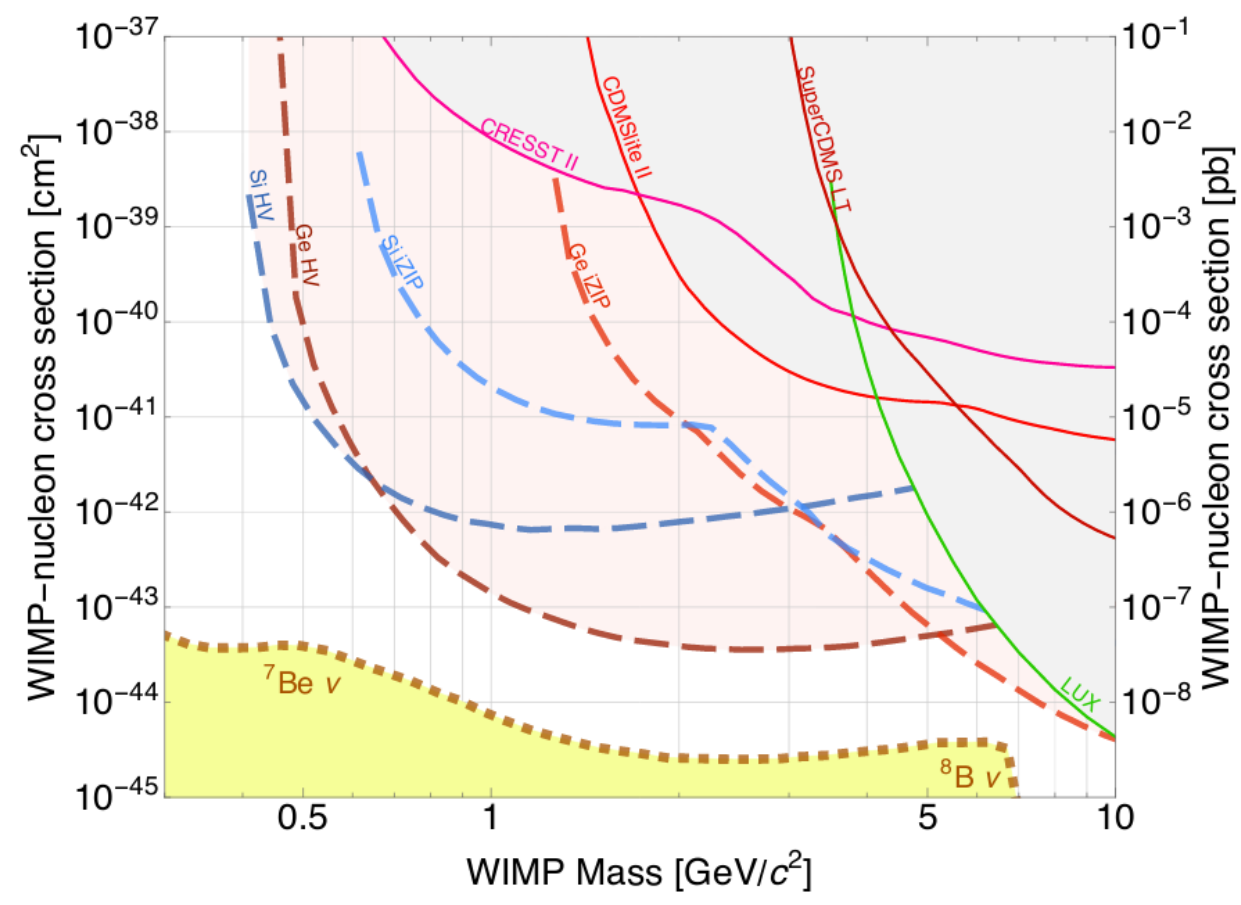

Figure 2.17: Projected sensitivity for the SuperCDMS SNOLAB experiment. Sensitivity projections for Si iZIP (dashed light blue), Si HV (dashed dark blue), Ge iZIP (dashed light red) and Ge HV (dashed dark red) are determined using the optimum interval method [181] 182. Solid lines are the current experimental exclusion limits in the lowmass WIMP region, from the CRESST-II [139] (pink), SuperCDMS [125] [136] (red), and LUX [138] (green) experiments. The dark matter discovery limit [145] (dotted orange line over yellow region) represents the cross section at which the interaction rate from dark matter particles becomes comparable to the solar neutrino coherent elastic scattering rate. Figure from [135]. 


\section{Chapter 3}

\section{Cryogenic Detector Testing and Characterization Facility at University of Minnesota}

Efforts to create a new CDMS facility for R\&D and detector testing and characterization at the University of Minnesota Twin Cities campus began in 2008. Not only would this facility aid in the pursuit of next generation CDMS technologies, but it would also function as an independent research lab. In particular, the facility aims to characterize established CDMS detectors before they are utilized in the SuperCDMS experiment underground, as well as characterize prototype detectors and other test devices as a means to recognize problems and develop solutions during scaling-up.

The facility, detailed in this section, was originally housed in the subbasement of the Tate Laboratory of Physics, at the University of Minnesota East Bank campus. Lab setup, infrastructure design and construction, dilution fridge reengineering and installation, and the integration of CDMS hardware and DAQ were tasks primarily completed by V. Mandic and J. Zhang; a detailed account of establishing the facility can be found in J. Zhang's thesis [183]. By October 2009 the facility was in operation, with a $76 \mathrm{~mm}$ CDMS II iZIP detector serving as the first full-scale test detector. After some initial runs, the fridge was upgraded to house $100 \mathrm{~mm}$ prototype detectors. The first $100 \mathrm{~mm}$ detector run began in February of 2011, with an ionization-only, four 
channel device.

In summer 2014 the facility was moved $\sim 300 \mathrm{~m}$ away to the newly constructed UMN Physics and Nanotechnology building basement, where it currently resides. After several months of reconstruction and testing, the first successful fridge run at the new location began April of 2015.

\subsection{Dilution Refrigerator}

Due to the significant role it plays in CDMS detector testing, this section will begin with an overview of dilution refrigeration, before covering the specifics of the UMN facility dilution unit.

\subsubsection{Dilution Refrigeration}

Dilution refrigeration uses an endothermic dilution process between helium isotopes

${ }^{3} \mathrm{He}$ and ${ }^{4} \mathrm{He}$ to provide continuous cooling power, illustrated in Fig. 3.1. Initial cooling is provided by filling the bath surrounding the dilution unit first with liquid nitrogen (LN2) (to reach $77 \mathrm{~K}$ ), and then liquid ${ }^{4} \mathrm{He}(\mathrm{LHe})$ (to $\sim 4 \mathrm{~K}$ ). This LHe bath will need to be maintained while the fridge is kept in operation. Further cooling to roughly $1 \mathrm{~K}$ is provided by the $1 \mathrm{~K} \mathrm{Pot}$, a small vacuum-pumped bath of liquid ${ }^{4} \mathrm{He}$, supplied by the larger surrounding LHe bath.

\section{Condenser Line}

To reach $\mathcal{O}(\mathrm{mK})$ temperatures, a mixture of ${ }^{3} \mathrm{He}$ and ${ }^{4} \mathrm{He}$ gases must be cycled through the fridge lines via a room temperature rotary pump. Before entering the fridge, ${ }^{3} \mathrm{He}$ gas is cleaned of any contaminants (mainly air, water, or oil) and precooled by passing through LN2 and LHe cold traps. Cold traps utilize charcoal (or similar material) cooled by the cryogenic liquid; as the ${ }^{3} \mathrm{He}$ passes through the trap contaminates in the gas freeze to the charcoal and are left behind, to be removed from the system later.

Once inside the fridge through the condenser line, ${ }^{3} \mathrm{He}$ gas comes into thermal contact with the $1 \mathrm{~K}$ Pot, and is liquefied to a temperature of $\sim 1.5 \mathrm{~K}$. The now liquid ${ }^{3} \mathrm{He}$ next enters a capillary with restricted flow, known as the main impedance, which provides sufficient pressure (30 - 200 mbar) to ensure that the ${ }^{3} \mathrm{He}$ will condense at $1.5 \mathrm{~K}$. Beyond 
$1 \mathrm{~K}$ Pot comprised of vacuum-pumped ${ }^{4} \mathrm{He}$ bath.

$1 \mathrm{~K}$ Pot liquefies incoming ${ }^{3} \mathrm{He}$ gas.

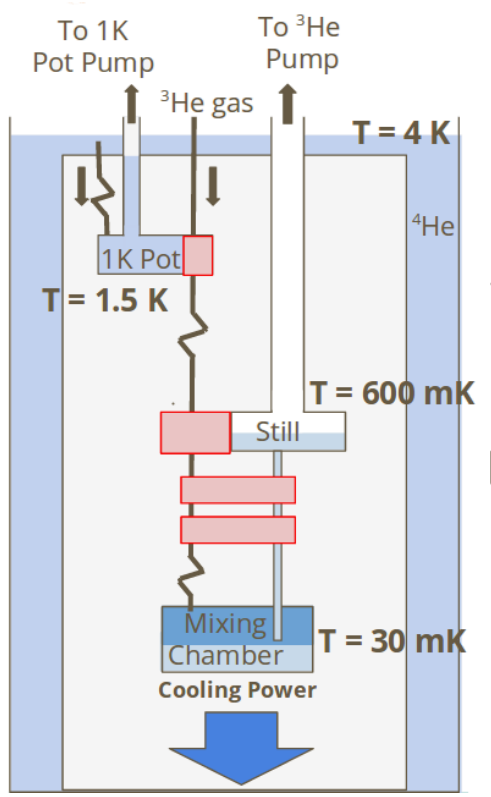

Room temperature ${ }^{3} \mathrm{He}$ pump pulls ${ }^{3} \mathrm{He}$ through superfluid ${ }^{4} \mathrm{He}$ (at rest) in Still and into the cold traps.

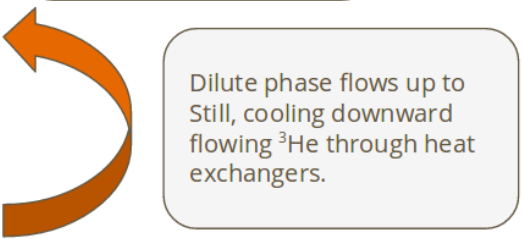

Mixture of ${ }^{3} \mathrm{He}$ and ${ }^{4} \mathrm{He}$ undergoes spontaneous phase separation into ${ }^{3} \mathrm{He}$-rich (concentrate) and ${ }^{3} \mathrm{He}$-poor (dilute) phase.

Figure 3.1: A cartoon depicting the dilution refrigeration process.

the main impedance the ${ }^{3} \mathrm{He}$ is cooled to $\sim 500$ - $700 \mathrm{mK}$ by heat exchangers in contact with the fridge still (discussed below). The liquid continues to pass through a second impedance (to avoid ${ }^{3} \mathrm{He}$ reevaporation) and more heat exchangers (cold plate), cooling as it reaches the mixing chamber.

\section{Mixing Chamber}

The mixing chamber contains two phases of the ${ }^{3} \mathrm{He}-{ }^{4} \mathrm{He}$ mixture in equilibrium: a dilute phase (roughly $6.6 \%{ }^{3} \mathrm{He}, 93.4 \%{ }^{4} \mathrm{He}$ ), and a ${ }^{3} \mathrm{He}$ concentrated phase (essentially just ${ }^{3} \mathrm{He}$ ). A separation between these phases will spontaneously occur when the mixture is cooled below approximately $870 \mathrm{mK}$, because the phases are quantum liquids. Classically one would expect the liquids would separate into two phases of pure ${ }^{3} \mathrm{He}$ and

${ }^{4} \mathrm{He}$ in order for the entropy of mixing to reach zero as $T \rightarrow 0$. However, the isotopes comprising the dilute phase are both at their respective ground states, allowing the mixing as $T \rightarrow 0$. With a nuclear spin of 1 , liquid ${ }^{4}$ He obeys Bose statistics, condensing to a superfluid state at $2.177 \mathrm{~K}$, and reaching a quantum mechanical ground state around $\mathrm{T}<0.5 \mathrm{~K}[179]$. In this state the ${ }^{4} \mathrm{He}$ acts as an inert background, contributing to the 
liquid volume and effective mass, but negligible heat capacity. On the other hand, ${ }^{3} \mathrm{He}$ has a nuclear spin of $1 / 2$, and thus obeys Fermi statistics. Since the ${ }^{3} \mathrm{He}$ has been diluted with inert ${ }^{4} \mathrm{He}$, its effective mass and Fermi temperature will be altered.

The limiting concentration of ${ }^{3} \mathrm{He}$ in the dilute phase is determined by comparing the binding energy of a ${ }^{3} \mathrm{He}$ atom in pure ${ }^{3} \mathrm{He}$ (such as in the concentrated phase at $\mathrm{T} \rightarrow 0)$ to a ${ }^{3} \mathrm{He}$ atom entering the dilute phase with ${ }^{3} \mathrm{He}$ concentration $x$. In pure ${ }^{3} \mathrm{He}$ (and $\mathrm{T} \rightarrow 0$ ) the binding energy, $\epsilon_{3, c}$ depends on the latent heat of evaporation, $L_{3}$, such that,

$$
\left|\epsilon_{3, c}\right|=\frac{\left|L_{3}\right|}{N_{0}},
$$

where $N_{0}$ is Avogadro's number. Things become more complicated when we consider the binding energy of ${ }^{3} \mathrm{He}$ in the dilute phase. Because both helium isotopes have the same electronic structure, they have identical van der Waals forces. However, the larger mass of the ${ }^{4} \mathrm{He}$ atom results in a smaller zero-point energy, $E_{0}=h^{2} / 8 m a^{2}$, and thus a smaller occupying volume than a ${ }^{3} \mathrm{He}$ atom. Since ${ }^{4} \mathrm{He}$ atoms take up less space, a single ${ }^{3} \mathrm{He}$ atom could get closer to ${ }^{4} \mathrm{He}$ atoms and thus be more strongly bound, compared to a single ${ }^{3} \mathrm{He}$ in pure ${ }^{3} \mathrm{He}$. In terms of binding energies of the single ${ }^{3} \mathrm{He}$ atom,

$$
\begin{gathered}
\left|\epsilon_{3, d}(x=0)\right|>\left|\epsilon_{3, c}\right|, \\
-\epsilon_{3, d}(x=0)<-\frac{L_{3}}{N_{0}} .
\end{gathered}
$$

As more ${ }^{3} \mathrm{He}$ atoms are added to the dilute phase the binding energy $\epsilon_{3, d}(x>0)$ will continue to increase, both due to magnetic interactions and an effect of density. Essentially, this means $\left|\epsilon_{3, d}(x>0)\right|>\left|\epsilon_{3, d}(x=0)\right|$. However, since ${ }^{3} \mathrm{He}$ atoms are Fermi particles they must abide by the Pauli Exclusion Principle; only two ${ }^{3} \mathrm{He}$ atoms of opposite nuclear spin can fill a given energy state, and additional ${ }^{3} \mathrm{He}$ atoms must fill successively higher states up to the Fermi energy, $E_{F}=k_{B} T_{F}$. This decreases the binding energy of the ${ }^{3} \mathrm{He}$ atoms by $k_{B} T_{F}$, where the Fermi Temperature $T_{F} \propto x^{2 / 3}$.

As more ${ }^{3} \mathrm{He}$ atoms are added to the dilute phase, the binding energy will decrease until it reaches the binding energy in pure ${ }^{3} \mathrm{He}$. This provides the equilibrium concentration, found to be when $x=6.6 \%$, 


$$
-\epsilon_{3, d}(6.6 \%)+k_{B} T_{F}(6.6 \%)=-\frac{L_{3}}{N_{0}} .
$$

Although this calculation uses $\mathrm{T}=0$, it essentially holds true for low, nonzero temperatures. For $\mathrm{T}<0.1 \mathrm{~K}$ the limiting concentration of ${ }^{3} \mathrm{He}$ in the dilute phase is given by [179],

$$
x=0.066\left(1+8.3 T^{2}\right) .
$$

Due to its lower density, the concentrated phase will float on top of the dilute phase. Incoming liquid ${ }^{3} \mathrm{He}$ from the condenser line will enter the concentrated phase. A lower, wider fridge line will pull mixture from the dilute phase out of the mixing chamber.

\section{Still Line}

The dilute phase reaches upwards, out of the mixing chamber and towards the still. As mixture flows, it passes through heat exchangers, and cools the downward flowing ${ }^{3} \mathrm{He}$ in the condenser line. The still is kept at $\sim 600 \mathrm{mK}$, a temperature ideal for keeping the ${ }^{3} \mathrm{He}$ vapor pressure large, but the ${ }^{4} \mathrm{He}$ vapor pressure low. At this temperature the liquid dilute phase in the still has a ${ }^{3} \mathrm{He}$ concentration of $<1 \%$, whereas the vapor is comprised of $90 \%{ }^{3} \mathrm{He}$. A still heater supplies any additional heat needed to keep the still around this optimal temperature.

The room temperature pump keeps the pressure inside still at $\sim 10 \mathrm{~Pa}$, pulling ${ }^{3} \mathrm{He}$ vapor while superfluid ${ }^{4} \mathrm{He}$ remains unperturbed in the still's liquid dilute phase. This creates an osmotic pressure difference between the mixing chamber and the still. The osmotic pressure difference, $\Delta \pi$, is given by [179],

$$
\Delta \pi \approx \frac{\left(0.4 x_{m c} T_{F}-x_{s t} T_{s t}\right) R}{V_{m, 4}}
$$

where $x_{m c}$ and $x_{s t}$ are the ${ }^{3} \mathrm{He}$ concentrations in the mixing chamber and still respectively, $T_{F}$ is the Fermi temperature (since ${ }^{3} \mathrm{He}$ in the mixing chamber is in the Fermi degenerate state), $T_{s t}$ is the still temperature, and $V_{m, 4}$ is the molar volume of ${ }^{4} \mathrm{He}$. This pressure difference will maximize to $\Delta \pi \approx 20$ mbar as the ${ }^{3} \mathrm{He}$ concentration in the still approaches zero, assuming the mixing chamber contains $6.6 \%{ }^{3} \mathrm{He}$ concentration and is at $T \leq 10 \mathrm{mK}$. The pressure difference is sufficient to drive ${ }^{3} \mathrm{He}$ from the 
concentrated phase of the mixing chamber to enter the dilute phase, flowing up into the still to replace the evaporating ${ }^{3} \mathrm{He}$. Evaporated ${ }^{3} \mathrm{He}$ from the still passes through the room temperature pump and enters the cold traps, beginning the cycle anew.

The process of ${ }^{3} \mathrm{He}$ passing through the phase boundary between dilute and concentrated phases is endothermic and removes heat from the mixing chamber. This provides cooling power to anything in thermal contact with the mixing chamber, including warm, incoming ${ }^{3} \mathrm{He}$ in the heat exchangers as well as an experiment, such as a CDMS detector. The cooling power, $\dot{Q}$ can be found by balancing the enthalpy, as $\dot{n}_{3}$ moles of ${ }^{3} \mathrm{He}$ transfer from the concentrated to the dilute phase,

$$
\dot{n}_{3}\left(H_{3, d}\left(T_{m c}\right)-H_{3}\left(T_{m c}\right)\right)=\dot{n}_{3}\left(H_{3}\left(T_{e x}\right)-H_{3}\left(T_{m c}\right)\right)+\dot{Q}
$$

where $H_{3}$ and $H_{3, d}$ are the enthalpy of pure ${ }^{3} \mathrm{He}$ and ${ }^{3} \mathrm{He}$ in the dilute phase respectively, and $T_{m c}$ and $T_{e x}$ are the temperatures of the mixing chamber and the heat exchangers respectively. By using experimental results for specific heat, and taking advantage of the thermal equilibrium between the two phases in the mixing chamber (which requires their chemical potentials to equate), the enthalpies are given by,

$$
\begin{array}{r}
H_{3}(T)=H_{3}(0)+11 T^{2} \quad[\mathrm{~J} / \mathrm{mol}], \\
H_{3, d}(T)=H_{3}(0)+95 T^{2} \quad\left[\mathrm{~J} / \mathrm{mol} \text { of }{ }^{3} \mathrm{He}\right] .
\end{array}
$$

Therefore the cooling power is found to be,

$$
\dot{Q}=\dot{n}_{3}\left(95 T_{m c}^{2}-11 T_{e x}^{2}\right) \quad[W]
$$

If the heat exchangers are at the mixing chamber temperature (or no warm ${ }^{3} \mathrm{He}$ is being added), a maximum cooling power can be found,

$$
\dot{Q}_{\max }=84 \dot{n}_{3} T_{m c}^{2}
$$

So for a typical circulation rate of $100 \mu \mathrm{mol}{ }^{3} \mathrm{He} \mathrm{s}^{-1}$, and a minimum mixing chamber temperature of $30 \mathrm{mK}$, the maximum cooling power is $\sim 8 \mu \mathrm{W}$.

\subsubsection{Testing Facility Cryostat Setup}

The testing facility at UMN, shown in Fig. 3.2, is equipped with an Oxford Instruments Kelvinox 100 dilution refrigerator, modified for use with CDMS detector. Modifications 


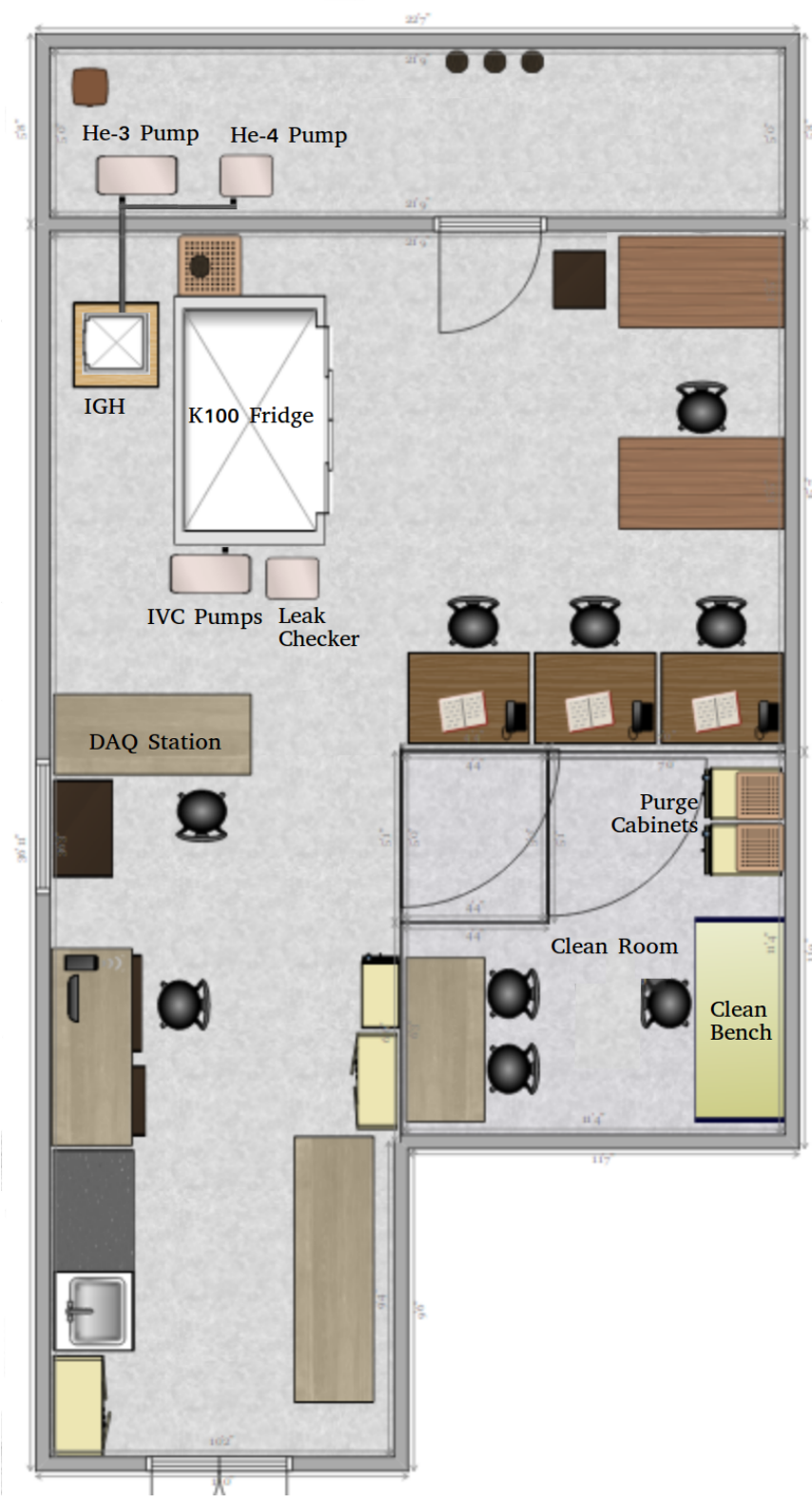

Figure 3.2: A cartoon depiction of the current UMN test facility lab setup. In the upper left corner (SW corner of lab) the K100 dilution fridge is supported by a roughly $1.35 \times 2.16 \times 2.18 \mathrm{~m}$ Al infrastructure. To the left is the Intelligent Gas Handling (IGH) unit, used to control the circulation of mixture. Copper and steel lines connect the fridge and IGH to circulation $\left({ }^{3} \mathrm{He}\right)$ and auxiliary $\left({ }^{4} \mathrm{He}\right)$ pumps in the pump room. Turbo and rough pumps in front of the fridge are used to pump out the Inner Vacuum Can (IVC) during cooldown. Detector interface and readout is done at the DAQ station, in front of the fridge. The Class 10,000 clean room resides in the bottom right corner (NE corner of lab). It contains a Class 1000 clean bench for electrical checks and tower installation. The clean room also contains two N2 purge cabinets, housing detectors, towers, and other various cold hardware. Figure modified from H. Rogers. 
include removal of the sliding seal and superconducting magnet; enlarged Inner Vacuum Can (IVC) to accommodate CDMS detectors and hardware; additional inner structures for shielding and electronic readout; and flanged collar to connect dilution unit with surrounding dewar.

The dilution unit, named "K100", is capable of cooling the mixing chamber to temperatures of $30 \mathrm{mK}$. The fridge is supported by a $205 \times 117 \times 14 \mathrm{~cm}$ aluminum platform on four $25 \mathrm{~cm}$ diameter, sand-filled pillars, as shown in Fig. 3.3 . A roughly 1.2 $\times 1.2 \times 2.0 \mathrm{~m}$ pit lies directly below fridge, for storage of the LHe dewar when not in use. Removable doors and a pulley system secured to dewar "ears" allow the dewar to be raised and lowered easily. The fridge IVC can be treated as two distinct chambers. The upper can, roughly $9.7 \mathrm{~cm}$ in diameter and $43.2 \mathrm{~cm}$ tall, houses the dilution components such as the still and mixing chamber. The lower can has been enlarged to roughly 22.5 $\mathrm{cm}$ in diameter and $47.5 \mathrm{~cm}$ tall in order to contain the necessary CDMS cold hardware, including the potential to house up to $150 \mathrm{~mm}$ (6") detectors with necessary hardware and shielding.

Oxford Instruments includes a Kelvinox Intelligent Gas Handling (IGH) system to monitor pressures and control mechanical valves for the circulation (condenser and still) lines as well as the auxiliary lines, such as lines connecting to the $1 \mathrm{~K}$ Pot, and the main bath dewar. Below the IGH lies the ${ }^{3} \mathrm{He}-{ }^{4} \mathrm{He}$ mixture storage keg, where mixture is contained when not in circulation. Condenser, still, and auxiliary lines pass through the West wall of the lab and enter the pumping room, shown in Fig. 3.2. The ${ }^{3} \mathrm{He}$ circulation pump is a Leybold D65B rotary vacuum pump, with a pumping speed of $65 \mathrm{~m}^{3} \mathrm{~h}^{-1}$. A Leybold S16B rotary vacuum pump is used as ${ }^{4}$ He auxiliary pump. The auxiliary lines are also connected to ${ }^{4} \mathrm{He}$ and nitrogen gas lines, used to flush the dewar during cooldown procedures.

The IVC is connected to a pumping manifold comprised of a scroll pump, turbo pump, leak checker, and a connection to ${ }^{4} \mathrm{He}$ and nitrogen gas lines. Nitrogen and helium gas is used to pressurize the IVC before it is opened to keep air and other contaminates from entering the IVC, and used as exchange gas when cryogenic liquids are added to the dewar during initial cool down stages. The scroll pump is used to pump the IVC down to $\sim 1 \times 10^{-2}$ mbar, after which the turbo pump is used to reach a vacuum level of $\sim 1 \times 10^{-6}$ mbar. The leak checker is also connected to the IVC, and 

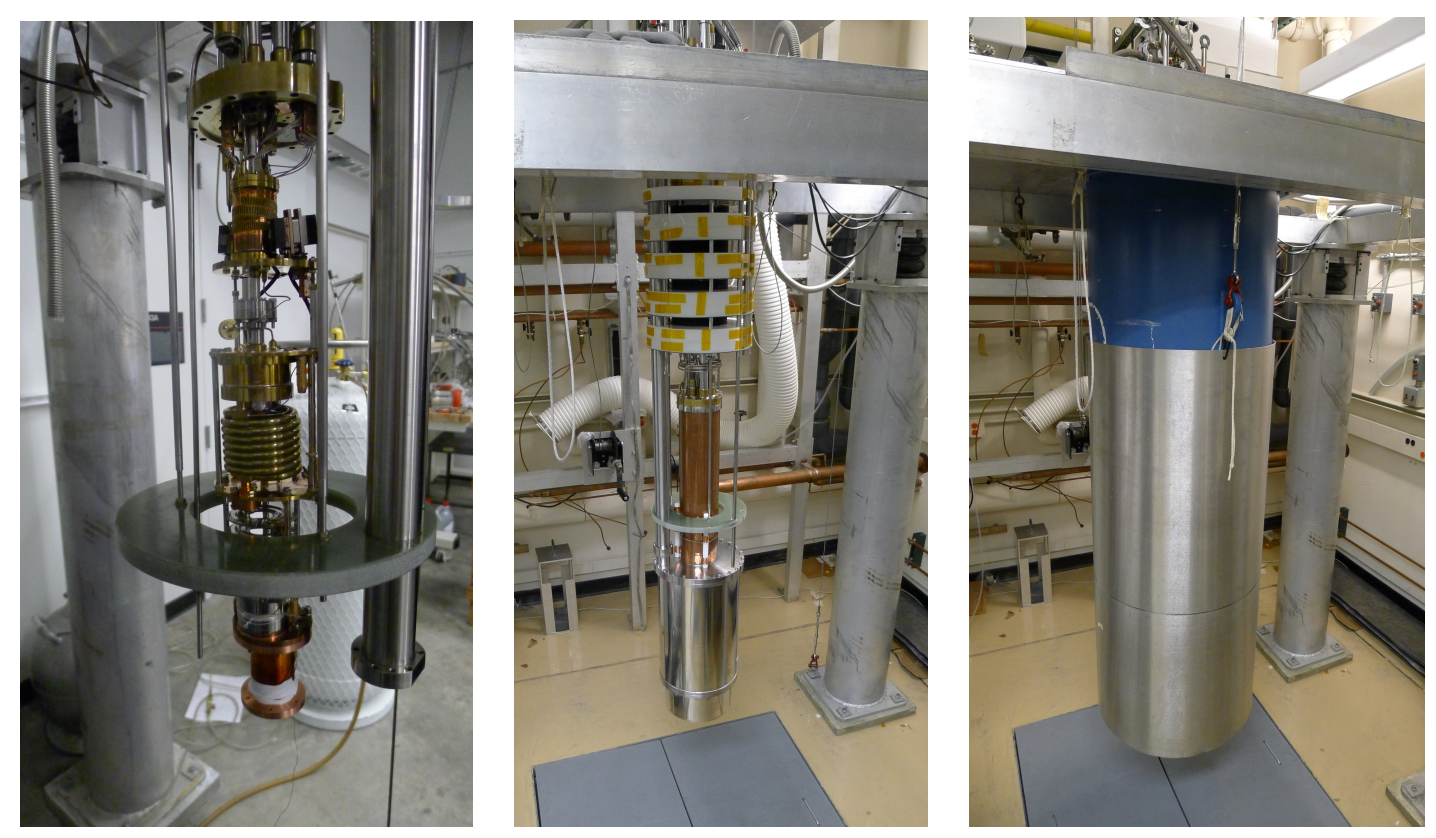

Figure 3.3: Various layers of the UMN detector testing facility K100 dilution refrigerator. (LEFT) The inside of the fridge, with inner vacuum cannisters removed. (CENTER) Inside of dilution fridge with inner vacuum cannisters sealed and inner polyethylene shielding. (RIGHT) Main bath dewar raised around cryostat. 
used during various stages of cooldown to check for leaks between the main bath and the IVC, as well as between the circulation lines and the IVC. Although it is incredibly situational, a leak rate of $<1 \times 10^{-6} \mathrm{mbar} \mathrm{L} \mathrm{s}^{-1}$ at room temperature without spikes in rate is generally considered acceptable.

\subsection{Cold Hardware}

This section will highlight some key CDMS hardware utilized by the UMN facility inside K100 and thus called "cold hardware". Due to low background requirements set by the experiment, radiopure materials are used whenever possible. Thus all circuit boards are comprised of multi-layer Kapton laminates, and low-activity solder was used for all solder connections.

Performance at low temperature must also be considered during material selection. For example, stainless steel screws, which become somewhat magnetic at low temperatures due to the iron phase transition, should not be used near the SQUIDs. Due to its thermal properties, oxygen-free high thermal conductivity (OFHC) copper is used for radiation shields (such as temperature stage cans), and thermal links; $\mathrm{Cu}$ components are polished regularly to remove oxidation and maintain good thermal conductivity. For insulating components G10 fiberglass is used.

\section{CDMS II Tower and Detector Housing}

The UMN test facility uses two CDMS II style detector towers. Towers are installed upside down in K100, relative to installation at Soudan; the following tower description will refer to the orientation of K100-installed towers. The hexagonal tower is roughly $14 \mathrm{~cm}$ tall, and $8 \mathrm{~cm}$ wide. A cylindrical, $9 \mathrm{~cm}$ long $\mathrm{Cu}$ spool at the top of the tower connects the detectors to the rest of the tower, providing adequate length to keep the detectors away from the material contaminants in the tower, minimizing the solid angle of exposure and reducing contamination. Up to six ZIP detectors can be installed on a single CDMS II tower, however only single-detector tests have been performed with the tower at the UMN facility.

The tower base is comprised of four hexagonal copper sections, heat-sunk to progressively higher temperature stages: $30 \mathrm{mK}$ (mixing chamber), $100 \mathrm{mK}$ (cold plate), 
$600 \mathrm{mK}$ (still), and $4 \mathrm{~K}\left(\mathrm{~L}^{4} \mathrm{He}\right)$. The copper sections are connected via three, internal, low thermal conductivity graphite tubes, in order to keep the heat-load between temperature stages sufficiently low for fridge operation. Coaxial NbTi wiring runs from the $30 \mathrm{mK}$ temperature stage to the $4 \mathrm{~K}$ stage, connecting detector readout through the side coaxial cables to the SQUET cards (see Section 3.2).

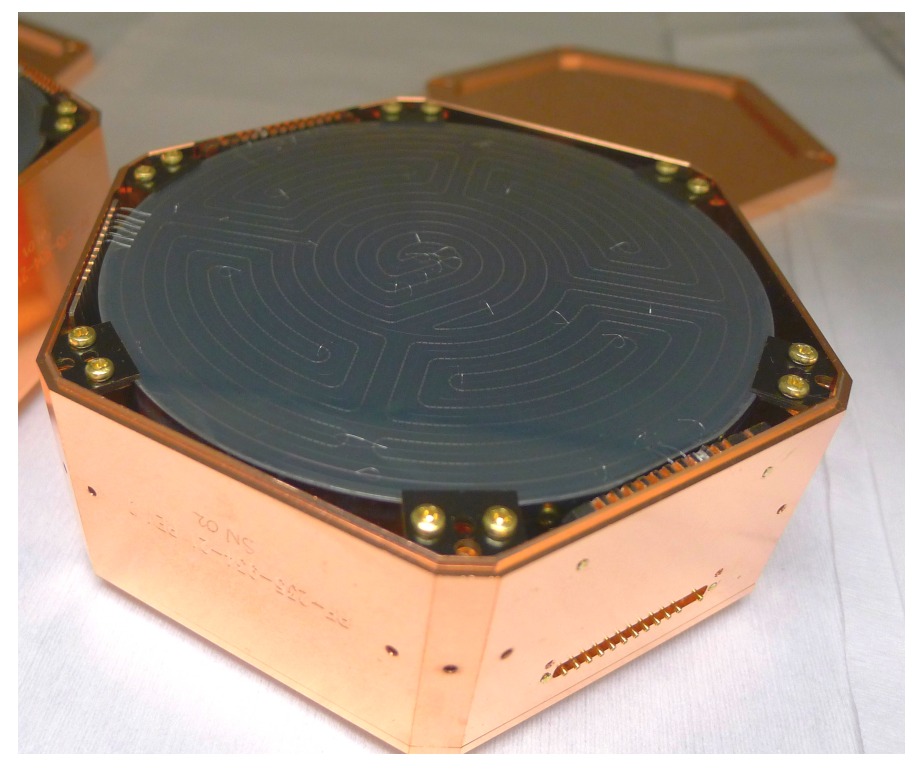

Figure 3.4: G106a $100 \mathrm{~mm}$ iZIP detector inside copper housing, with top lid removed to show iZIP mask, cirlex clamps, and wirebonds connecting channels to Al bonding pads on DIB.

Detectors inside hexagonal, OFHC copper housing (shown in Fig. 3.4 are installed on top of the tower spool, secured with threaded brass rods into a copper base plate. Detector housing is made thin, about $2 \mathrm{~mm}$, to minimize the presence of material near the detector. Detector Interface Boards (DIBs) are mounted on the inner wall of the housing, their $\sim 1.5 \mathrm{~mm}$ thickness allowing them to fit without touching the detector. One DIB can be seen towards the lower right of Fig. 3.4. The standard CDMS DIB is a roughly rectangular, 3.3 by $2.9 \mathrm{~cm}$ board composed of cirlex, with twelve solder-coated copper traces on each side. The traces connect a MillMax socket array connector, which protrudes through a $3 \mathrm{~mm}$ wide slit in the housing, to aluminum bonding pads on the top and bottom of the DIB. From the Al pads, wire bonds connect to the surface of 
the detector. In order to neutralize the detector when cold 1 , each horizontal surface of the DIB also possesses two infrared (940 nm) LEDs (one connected for use, one as backup), mounted with Ag conductive epoxy. Although the housing designs vary for detector type, the detectors tested at UMN typically have twelve cirlex clamps (6 per side), screwed into the housing, to secure the detector to the housing while keeping it electronically isolated.

If desired, calibration sources, such as ${ }^{241} \mathrm{Am}$ and ${ }^{210} \mathrm{~Pb}$, can be installed in a separate source holder of similar shape to the detector housing, stacked either above or below the detector. Up to four ${ }^{241} \mathrm{Am}$ sources of activity $1 \mu \mathrm{Ci}$ are available, blocked by 1.6 $\mathrm{mm}$ thick lead disks with a $0.2 \mathrm{~mm}$ diameter collimating hole, for an approximate 20 $\mathrm{Hz}$ event rate. ${ }^{241} \mathrm{Am}$ sources provide a $60 \mathrm{keV}$ gammas in the detector bulk, useful for electron recoil energy calibration. The ${ }^{210} \mathrm{~Pb}$ source provides $17.0 \mathrm{keV}$ and $63.5 \mathrm{keV}$ betas, useful for demonstrating surface event rejection. The source holder also typically contains a tower thermometer since it is in good thermal contact with the detector housing.

\section{Vacuum Side Coaxial Cables}

Vacuum side coaxial cables, often shortened to vacuum side coaxes, pass signals from the DIB to the base of the tower. The vacuum coax is comprised of sixteen NbTi superconducting wires encased in individual copper channels, and covered by a copper shielding plate. This is done to avoid the charge-buildup that would occur between the conductor and insulator in a typical coaxial cable, caused by the triboelectic effect.

On the detector side of the coax, the wires connect to a Side Coax Assembly Board (SCAB), which mates to the DIB with twelve MillMax pins. On the tower side, the coax wires connect to the Side Coax Upper Heatsink Board Assembly (SCUHBA). Non-ionization traces (such as phonon and LED channels) are simply soldered to the assembly board and connect directly to the outgoing MillMax pins. The inner and outer charge channels are broken up into bias gate and feedback lines, with the bias line passing through an equivalent $40 \mathrm{M} \Omega$ bias resistor, the gate and feedback lines passing through the $300 \mathrm{pF}$ coupling capacitor (see Section 2.2.3), and the feedback line passing

\footnotetext{
${ }^{1}$ The process of neutralizing the detectors with LEDs, called flashing, will be discuss in more detail in Section 4.4
} 


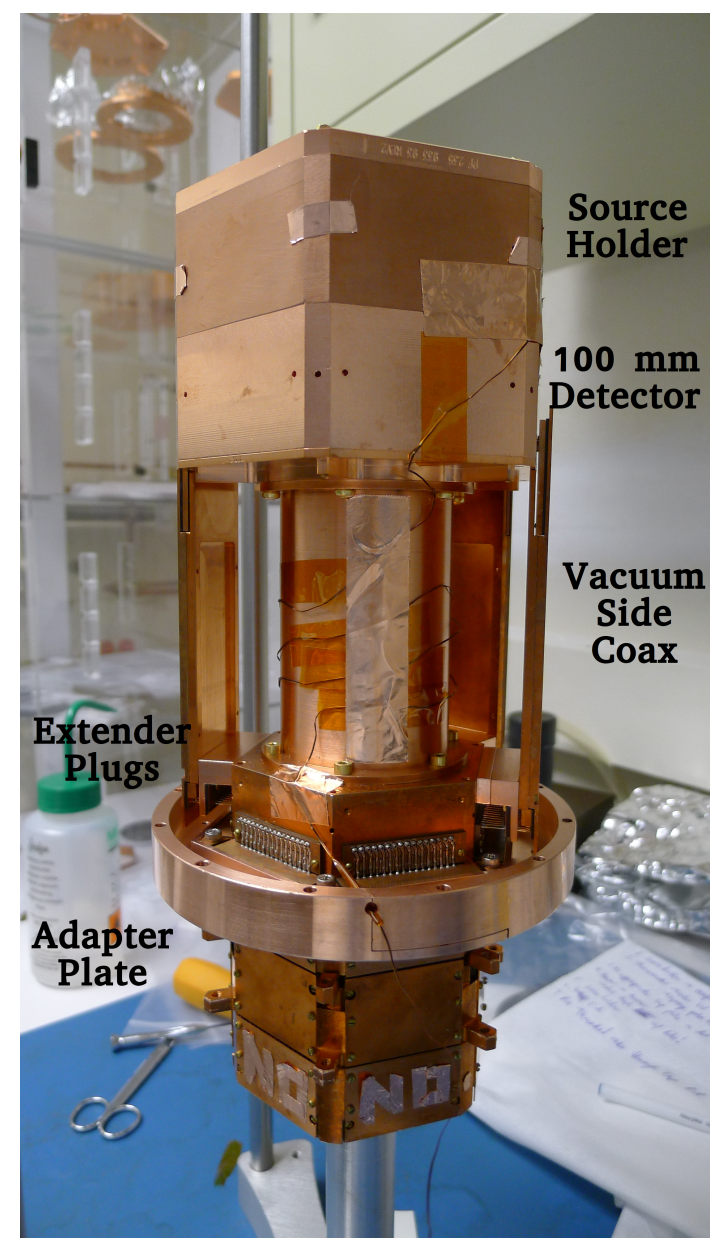

Figure 3.5: A $100 \mathrm{~mm}$ detector with source holder installed on a CDMS II tower. Three vacuum side coaxes connect the detector housing DIBs to the base of the tower, using extender plugs. The tower thermometer wire emerges from the source holder and is wound about the tower spool before passing through a hole on the adapter plate. The adapter plate will secure the tower to the base of the $30 \mathrm{mK}$ can. 
an equivalent $40 \mathrm{M} \Omega$ feedback resistor before connecting to the MillMax pins.

Since the testing facility uses a CDMS II based detector tower, traditionally used for holding $76 \mathrm{~mm}$ detectors, SCUHBA extender plugs were used with $100 \mathrm{~mm}$ diameter detectors to bridge the $25 \mathrm{~mm}$ gap between the tower and the vacuum coaxial cables connected to the detector housing. Detector, source, and coax installation is done in the test facility clean room, discussed in Section 3.5. After detectors and coaxes have been mounted on the tower, an $\mathrm{OFHC} \mathrm{Cu}$ adapter plate is attached at the tower 30 mK stage. Figure 3.5 shows a completed tower, with $100 \mathrm{~mm}$ iZIP, source holder, three vacuum side coaxes (each connecting one DIB to the tower base), and the adapter plate. Before the tower leaves the clean room, and electrical continuity check is performed, and the tower is double bagged in clear polyethylene bags to minimize contamination during the transfer to the cryostat.

The adapter plate secures the tower to the innermost vacuum shielding can, called the $30 \mathrm{mK}$ can. The $30 \mathrm{mK}$ can is in good thermal contact with the mixing chamber and the detectors; typically a mixing chamber around $30 \mathrm{mK}$ cools the detectors to $\sim 40$ $\mathrm{mK}$. Once the tower has been installed in the IVC (and adapter plate secured to the 30 $\mathrm{mK}$ can), the $100 \mathrm{mK}$ and $600 \mathrm{mK}$ stages are thermally heat sunk to the fridge. Thin copper plates and heat sinks connect the $100 \mathrm{mK}$ tower stage to the $100 \mathrm{mK}$ shielding can, which encompasses the $30 \mathrm{mK}$ can and is in contact with the fridge coldplate. The $600 \mathrm{mK}$ is thermally connected to the still via a long copper rod which passes between the $30 \mathrm{mK}$ and $100 \mathrm{mK}$ cans. The $600 \mathrm{mK}$ stage protrudes at the base of the tower, surrounded by (and thermally isolated from) the bottom $4 \mathrm{~K}$ stage. The $4 \mathrm{~K}$ stage is in thermal contact with the IVC through copper heat sinks.

\section{SQUET cards}

As discussed in Sections 2.2.3 and 2.2.4, ionization and phonon signal readout depends on a JFET amplifier and SQUID array, respectively. Shown in Figure 3.6, boards containing FET and SQUID electronics are combined into a single card, called the SQUET card, which connects to the base of the tower. The FET and SQUID cards are connected via CuNi-coated, NbTi flyover wires covered in kapton, since the FET is operated at $150 \mathrm{~K}$ while the SQUID is operated at $600 \mathrm{mK}$, to reduce Johnson noise.

Niobium-titanium is superconducting at these temperatures, and thus the two stages are 
thermally isolated. The CuNi coating makes soldering possible without causing thermal shorts. The flyover wires are twisted with a ground wire in order to reduce cross-talk between channels.

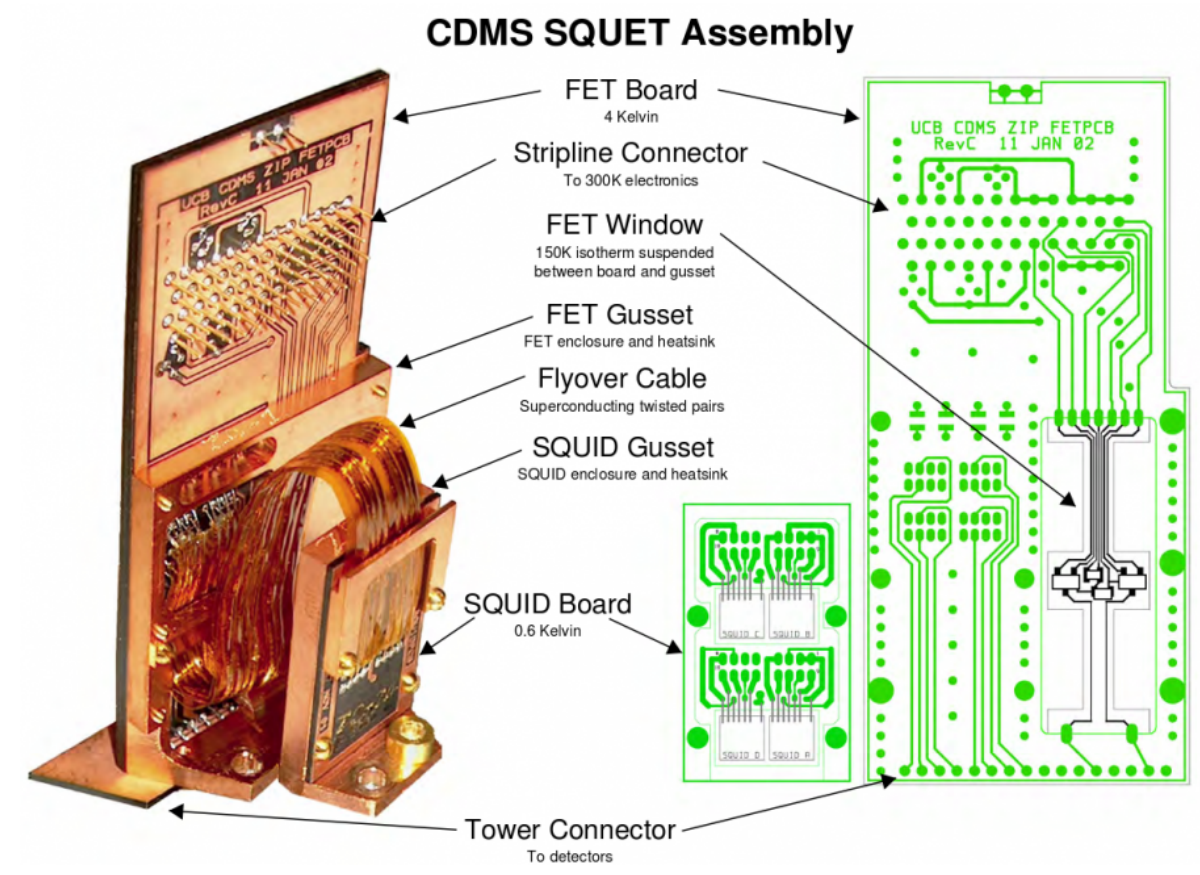

Figure 3.6: A photo and circuit diagram of a CDMS SQUET card.

In addition to housing the JFET amplifier needed for ionization signal readout, the FET card also hosts the components required to interface with the SQUID card, and to connect to the lines which pass information to room temperature electronics. The FET board is made of $1.62 \mathrm{~mm}$ thick cirlex with $3 \mu \mathrm{m}$ thick electrical connections patterned out of copper. A $\mathrm{Cu}$ bracket at the base of the card, which includes a sixteen MillMax pin array, electronically and thermally connects to the tower $4 \mathrm{~K}$ stage.

Above the tower connection lies the flyover (discussed above), and the FET window. Inside the $0.15 \mathrm{~mm}$ thick kapton window, two JFETs (one for inner and outer charge channels) are operated at $150 \mathrm{~K}$. The surrounding cavity is coated in an infrared absorber, and the kapton window size is selected such that the power dissipated by the FETs, $\sim 4.5 \mathrm{~mW}$ each, self heats them to their optimal temperature. To initially start 
the FETs, a $1 \mathrm{k} \Omega$ resistor serves as a FET heater. All FET components in the window are connected to $\mathrm{Cu}$ pads using $\mathrm{Ag}$ epoxy. A $\mathrm{Cu}$ gusset covers the FET window. The top portion of the FET holds a 50 MillMax pin array, which connects to striplines running to room temperature electronics, as shown in Figure 3.7.

The SQUID board is similarly made from $0.81 \mathrm{~mm}$ thick cirlex, patterned with copper. The board is thermally connected to the inner base of the tower at $600 \mathrm{mK}$, with brass screws. Copper traces from the soldered flyover wires lead to $\mathrm{Al}$ spuddered pads, which are wirebonded to the SQUID chips. The SQUIDs are epoxied to $\sim 0.05$ $\mathrm{mm}$ tick niobium foil (providing some magnetic shielding) which is glued to the surface of the board.

\section{Striplines}
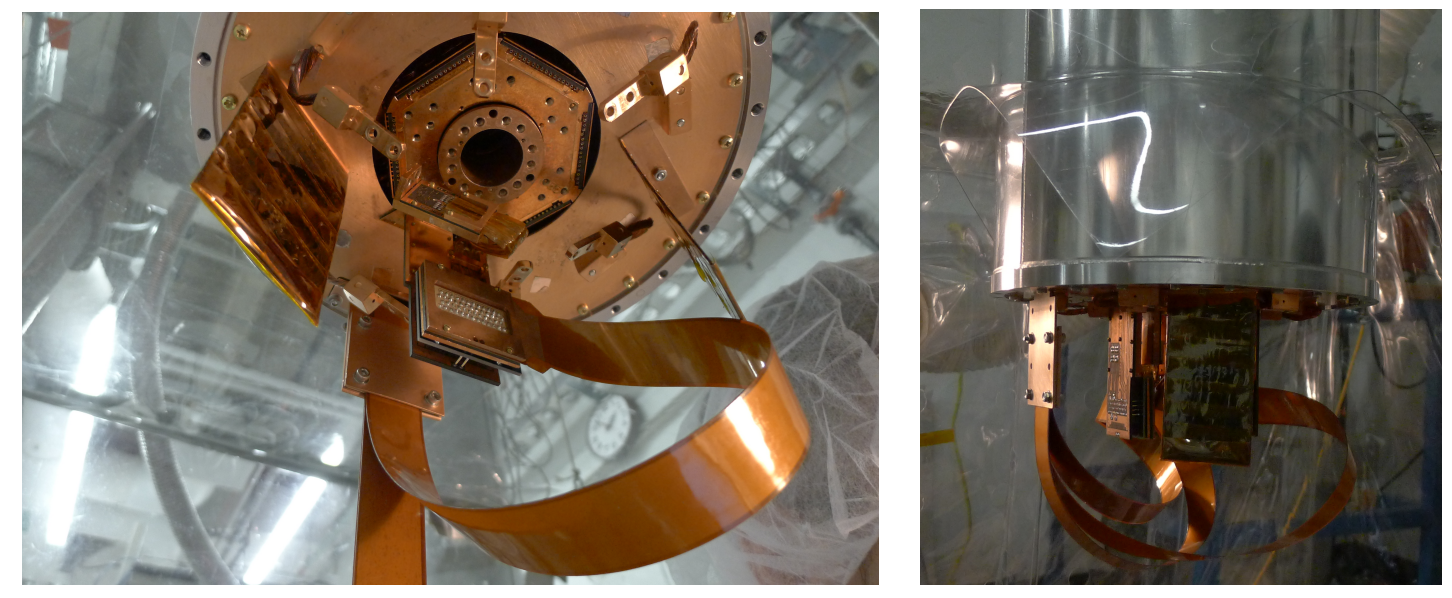

Figure 3.7: (LEFT) A bottom-up view of a single SQUET card and stripline attached to the CDMS II tower at the $4 \mathrm{~K}$ stage. (RIGHT) A side view of three SQUET cards and striplines installed at the $4 \mathrm{~K}$ stage inside the opened IVC.

Striplines are 1" wide, 10' long multi-layer shielded flex circuits that connect to the SQUET cards and ascend up to the room temperature electronics box. Striplines are composed of fifty $0.127 \mathrm{~mm}$ wide copper traces sandwiched between two $0.127 \mathrm{~mm}$ kapton layers, two sputtered $\mathrm{Cu}$ ground planes, and a final two $0.0254 \mathrm{~mm}$ kapton layers. A 50 MillMax socket array on the cold end of the stripline mates with the FET board. 
On the warm end the stripline mates with the electronics box (see Section 3.3) with a 50-pin D-connector.

Due to a arduous installation processes, three striplines are always installed at the UMN test facility, even if only one or two striplines are connected to FET boards. Unused striplines are gently coiled at the bottom of the IVC, to avoid damage from strain. As shown in Figure 3.7, the three striplines are pressed together and heat sunk at the $4 \mathrm{~K}$ stage before passing upward, out of the IVC via the 1.5" stripline port. To reduce thermal loads from room temperature radiation in the port, the striplines are installed with seven $\mathrm{Cu}$ radiation baffles; additional $\mathrm{Al}$ foil is added at the base of the stripline port to further block radiation.

\subsection{Warm Hardware and Data Acquisition Software}

Although focus tends to be given to prototype detectors when discussing UMN facility studies, detectors only represent a fraction of the next-generation technology studied. Nowhere is this more evident than warm hardware and data acquisition at UMN. Warm hardware refers to any hardware that resides outside of the cryostat; this hardware mainly serves to interface with the cold-end electronics and pass along signals to detector control and readout software.

\section{E-box and DCRCs}

After passing through the stripline port and a few room-temperature steel bellows, the striplines enter the $20 \mathrm{~cm}$ diameter, $33 \mathrm{~cm}$ long steel electronics box, or Ebox. The Ebox is openly connected to the IVC, and thus is also pumped down to the IVC operating pressure of $\sim 10^{-6}$ mbar; at room temperature connections, rubber o-rings between the bellows and Ebox provide suitable seals to maintain this vacuum level. Opposite the stripline entry port, the Ebox hosts six 50-pin male-to-male D-connectors welded into the steel. Thus the Ebox serves to pass signals from the striplines to electronics outside of the fridge vacuum. The Ebox also serves as the fridge's only connection to electrical ground, to avoid signal interference due to ground loops; ground is maintained through electronic chassis, as well a grounding strap connecting the Ebox to the Al fridge frame.

Detector readout and control is performed via SuperCDMS Detector Control and 
Readout Cards (DCRCs). The DCRCs control all of the cold electronics, including biasing the detector charge electrodes, biasing the SQUIDs, heating the FETs, flashing the LED's etc., as well as the triggering and digitization of phonon and charge signals.

DCRCs contain a roughly $12 \mathrm{~cm}$ by $25 \mathrm{~cm}$ circuit board, sandwiched between two copper RF shields. A D-sub 50 connector on one end of the board connects to the Ebox, while the other end hosts three ethernet ports; power is supplied by Power Over Ethernet (POE) to one port, while the other two ports allow a time-syncing link between separate boards, since each board interfaces with a single DIB. DCRCs have been in development for over a decade, and currently the UMN test facility uses a collection of third-generation boards, referred to as RevC and RevC.1 boards. A RevD board is currently being tested at other facilities, for use in SuperCDMS SNOLAB.

Originally up to three RevC or RevC.1 DCRCs were plugged in directly to the Ebox, such that they were vertically side by side, one for each DIB. However, it was determined in August of 2015 that phonon channel noise improves when a D-sub low-pass pi-filter (3.2 MHz cutoff) is placed between the DCRC and the Ebox. It was also noted that noise was reduced when DCRCs are resting side by side, horizontally, connected by Dsub cables and a gender swapper. Figure 3.8 demonstrated the improvement in phonon pulse shape with the introduction of the pi-filter and the horizontal DCRC positioning. After this discovery, a Cu-plate shielded pi-filter, D-sub cable, and gender swapper became the standard operational setup between the Ebox and each DCRC.

Fig. 3.9 compares the noise PSDs of typical UMN facility $100 \mathrm{~mm}$ iZIP data (with the D-sub cable and pi-filter setup) to PSDs of a Soudan $76 \mathrm{~mm}$ iZIP during operation. Inner charge channels and phonon B channels are selected as a good representation of charge and phonon channel noise, respectively. Noise PSDs are created for each channel of each data series, as part of data processing (see Section 3.4), constructed from randomly triggered events taken at the start of the series. The PSDs are constructed to represent the noise level at the feedback capacitor of charge readout circuit (see Fig. 2.11) and the input coil of the phonon readout circuit (see Fig. 2.13). The PSDs of Fig, 3.9 indicate that the UMN facility has similar noise levels as Soudan, although UMN PSDs exhibit higher noise in the $>200 \mathrm{kHz}$ region. The UMN test facility also has slightly elevated signal noise in the low frequency region, due to a higher event rate resulting in more pulses slipping into the randomly triggered events, used for PSD 

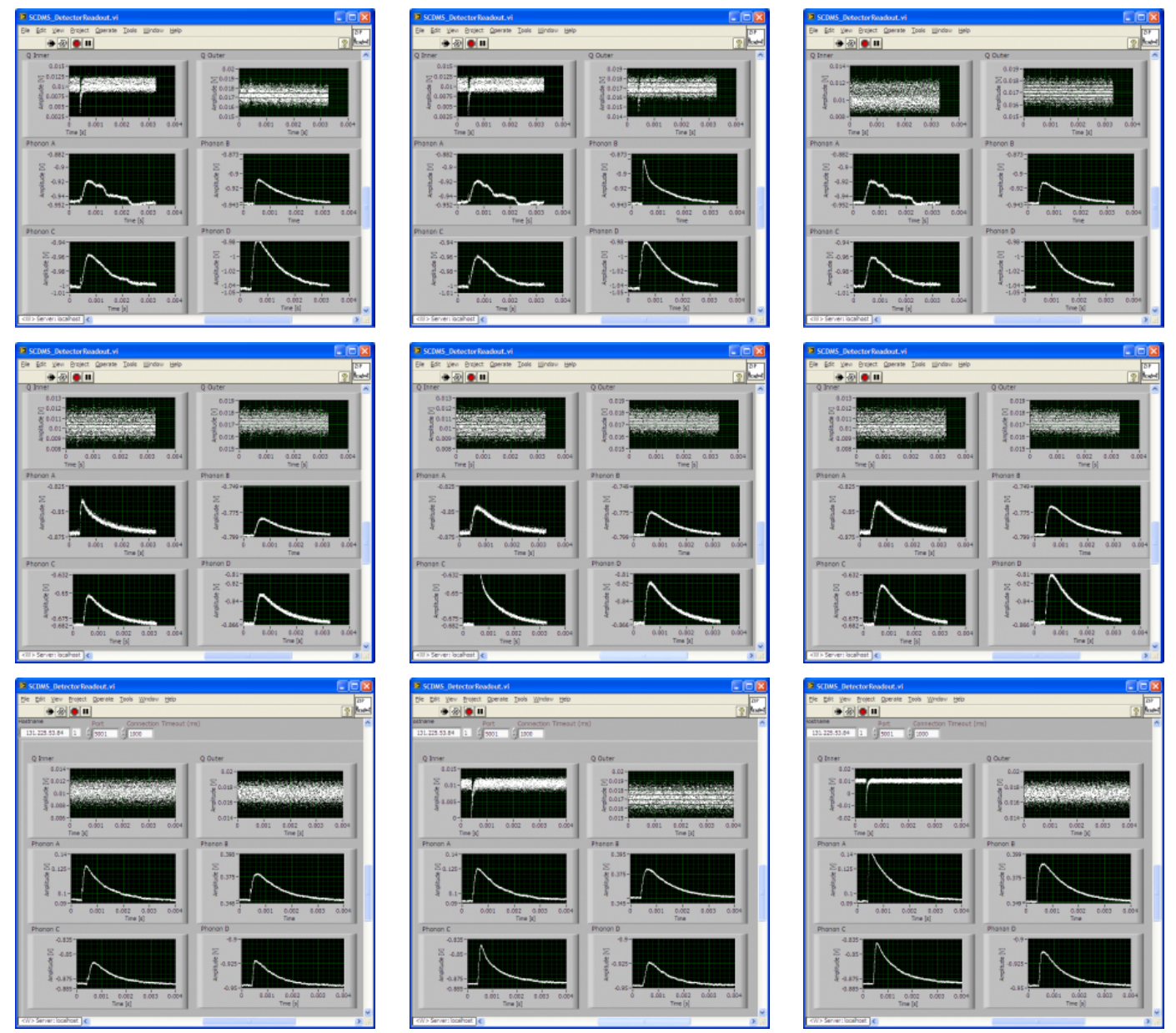

Figure 3.8: Three sample pulses of each channel on a single DIB, demonstrating the RevC DCRC pulse shape (TOP ROW) without a low-pass pi-filter between the DCRC and Ebox, (MIDDLE ROW) with a low-pass pi-filter between the DCRC and Ebox, (BOTTOM ROW) with a low-pass pi-filter and a cable between horizontally resting DCRC and Ebox. Images taken with Labview data acquisition software. 
construction.

\section{Data Acquisition Software}

Data Acquisition (DAQ) software communicates with the DCRCs, over an ethernet switch, to set electronic parameters (such as voltage bias, LED flashing current etc.) as well as to pass along trigger information. Previously the test facility used a LABview software package on a Windows computer, but in 2015 the facility upgraded ${ }^{2}$ to the Maximum Integrated Data Acquisition System (MIDAS), a general-purpose software package for event-based data acquisition [184]. MIDAS software purposed for SuperCDMS is still in development, though functional for UMN facility data acquisition needs; MIDAS is tuned to operate $100 \mathrm{~mm}$ detectors with twelve phonon channels and four charge channels, in regular iZIP mode, and a higher voltage mode, similar to CDMSlite operation.

DAQ software includes a GUI to display the detector's twelve channel outputs, allowing the user to monitor the effects of altering the detector parameter 3 . This general method is used to find the transition temperature of each phonon channel, as well as determine the optimal SQUID settings for data taking (a process discussed in detail in Section 5.1.1.

At the start of data taking the DAQ is used to set parameters such as data series length, trigger settings, trace length (including sample length of pre-trigger and posttrigger), and random events, in addition to the SQUID and voltage bias settings already discussed. During data taking, the analog signals from the detector are continuously digitized by the DCRC and stored in a circular buffer. This buffer can hold up to two seconds of signal data, many times larger than the $3.27 \mu$ s standard trace length used at the test facility. A trigger queue stores the addresses of triggers in the buffer, to be queried by the DAQ software. Based on the trigger address and the pre- and posttrigger sample lengths set before data taking, the DAQ requests the data surrounding the trigger address from the buffer. The DAQ stores each readout pulse, referred to as a raw event, in compressed binary files, along with header information indicating the

\footnotetext{
${ }^{2}$ A LABview program is still used to monitor the dilution unit temperatures and pressures, and to interface with the IGH.

${ }^{3}$ MIDAS is accessible via web browser, however using the channel display GUI requires a VNC session to a facility Linux computer.
} 

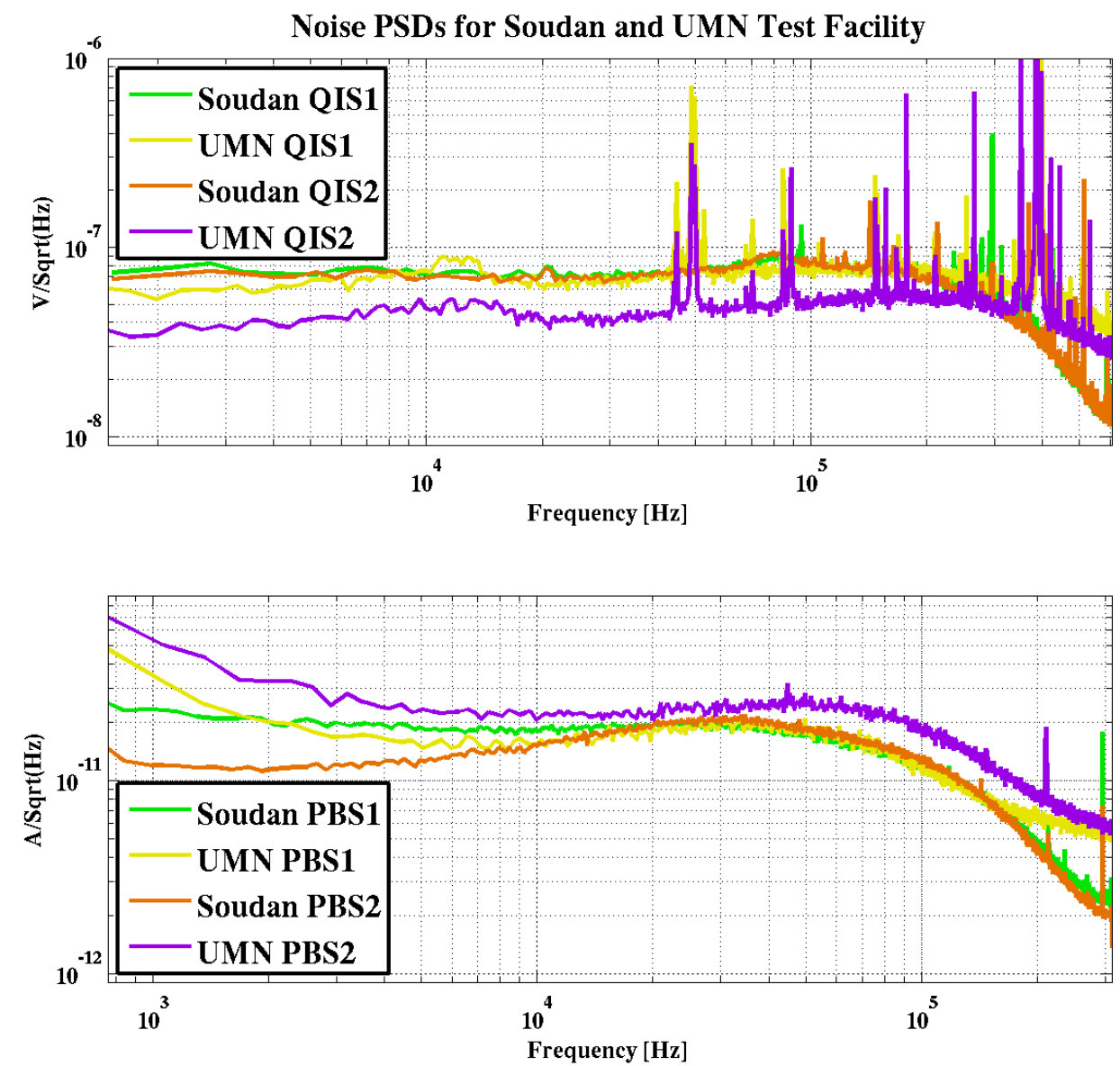

Figure 3.9: A comparison of Soudan (green and yellow) and UMN facility (orange and purple) noise PSDs for (TOP) charge and (BOTTOM) phonon channels. The PSDs represent the noise level at the feedback capacitor of charge readout circuit (see Fig. 2.11) and the input coil of the phonon readout circuit (see Fig. 2.13). 
channel, time stamp, etc., to be processed.

\subsection{Data Processing}

In order to pursue various detector characterization studies, the raw pulse data must be reduced to usable quantities such as pulse amplitude, rise time, etc.. Raw data is processed by the standard CDMS data processing package, called CDMSBats. Because some modes of processing can take upwards of an hour per file, parallel processing is performed using the HTCondor system. Essentially all processing begins with a processing of the random events taken at the beginning and sometimes end of a data series; random events are events taken at a fixed rate and not because the event satisfies trigger conditions. Thus, randoms events provide information on the noise of the system, when pulses are not present. Random event processing outputs, referred to as Filter files, contain raw noise events, Fourier transforms, and power spectral densities (PSDs) of the raw events, with some cuts applied to remove events which accidentally contain pulses. Filter files also contain pulse templates and modified templates, used for fitting raw pulses in subsequent processing algorithms.

Depending on the goals of the user, various modular processing procedures can be utilized, although typical data analysis depends on some form of the Optimal Filter (OF) pulse fitting algorithm, described in detail in Section 3.4.1. Other common processing algorithms include a basic pulse characterization algorithm, which finds pulse baselines and extrema (among other quantities), and walk algorithms, which use constant or variable frequencies on individual phonon pulses to determine the pulse rising and falling time constant. Processing algorithms utilize detector and hardware configuration settings, and thus can generate their output, called reduced quantities (RQ's), in relevant units such as seconds, Amperes, and Volts.

\subsubsection{Optimal Filter}

Optimal filtering is a process for extracting the relevant signal from data where the signal shape is known, and the background consists of Gaussian random noise with a known power spectral density. Fortunately these conditions are met within the CDMS 
experiment, since both charge and phonon pulses follow a well understood shape (outlined in Section 2.2.3 and 2.2.4 for which templates can be constructed, and random events provide a noise PSD for each data series. The optimal filter estimates the signal amplitude by maximizing the signal-to-noise ratio.

\section{Basic Optimal Filter Theory}

In general, one can treat a digitized pulse, $S_{i}$, spanning time $T$ with $N$ bins, as the sum of some expected pulse shape or template, $A_{i}$, multiplied by an amplification factor, $a$, and random noise, $n_{i}$, with a known PSD, $J$, such that,

$$
S_{i}=a A_{i}+n_{i}, \quad i=0,1, \ldots, N-1 .
$$

From this, one can define a scalar discriminant, $\chi^{2}$, which quantifies goodness-of-fit for a particular amplitude [185],

$$
\chi^{2}(a)=\sum_{n=0}^{N-1} \frac{\left|\tilde{S}_{n}-a \tilde{A}_{n}\right|^{2}}{J_{n}}
$$

where $\tilde{S}_{n}$ and $\tilde{A}_{n}$ are the discrete Fourier transforms (DFT) of $S_{i}$ and $A_{i}$, respectively, such that,

$$
\tilde{S}_{n}=\frac{1}{N} \sum_{k=0}^{N-1} S_{k} e^{\frac{2 \pi i n k}{N}}, \quad \tilde{A}_{n}=\frac{1}{N} \sum_{k=0}^{N-1} A_{k} e^{\frac{2 \pi i n k}{N}} .
$$

The goodness-of-fit is calculated in frequency space to avoid correlations in non-white noise that are present in the time-domain, but independent in frequency domain. The "best" fit amplitude, $\hat{a}$, can be found by minimizing $\chi^{2}$,

$$
\left.\frac{\partial \chi^{2}}{\partial a}\right|_{a=\hat{a}}=0 \quad \Longrightarrow \hat{a}=\frac{\sum_{n=0}^{N-1} \frac{\tilde{A}_{n}^{*} \tilde{S}_{n}}{J_{n}}}{\sum_{n=0}^{N-1} \frac{\left|\tilde{A}_{n}\right|^{2}}{J_{n}}} .
$$

The expression for $\hat{a}$ is analogous to the application of a non-causal filter, which filters out unwanted noise, the information of which is stored in $J_{n}$. This fit does not yet take into consideration any time shift that may exist between the template and the 
actual pulse. Assuming that the start of template, $A_{i}$ is shifted by $t_{0}$ with respect to signal $S_{i}$, Eq. 3.13 becomes 166 ,

$$
\chi^{2}\left(a, t_{0}\right)=\sum_{n=0}^{n=N-1} \frac{\left|\tilde{S}_{n}-a e^{-2 \pi i t_{0} f_{n}} \tilde{A}_{n}\right|^{2}}{J_{n}},
$$

where $f_{n}=n / T$. For a given $t_{0}$, the amplitude value $\hat{a}$ which minimizes $\chi^{2}$ is,

$$
\hat{a}\left(t_{0}\right)=\frac{\sum_{n=0}^{N-1} e^{2 \pi i t_{0} f_{n}} \frac{\tilde{A}_{n}^{*} \tilde{S}_{n}}{J_{n}}}{\sum_{n=0}^{N-1} \frac{\left|\tilde{A}_{n}\right|^{2}}{J_{n}}} .
$$

The numerator of this expression is simply the inverse DFT of $\frac{\tilde{A}_{n}^{*} \tilde{S}_{n}}{J_{n}}$, making it computationally faster for processing algorithms to calculate $\hat{a}\left(t_{0}\right)$ than to minimize $\chi^{2}\left(a, t_{0}\right)$, when fast Fourier transform (FFT) algorithms are used [186].

It would be expected that a similar method of setting $\partial \chi^{2} / \partial t_{0}=0$ could be used to determine the best estimation for $t_{0}$. However,

$$
\frac{\partial \chi^{2}}{\partial t_{0}}=-2 a \sum_{n=0}^{N-1} 2 \pi i f_{n} e^{2 \pi i t_{0} f_{n}} \frac{\tilde{A}_{n}^{*} \tilde{S}_{n}}{J_{n}}=0,
$$

is nonlinear and computationally intensive to solve analytically. As an alternative, consider that,

$$
\frac{\partial \hat{a}\left(t_{0}\right)}{\partial t_{0}}=\frac{\sum_{n=0}^{N-1} 2 \pi i f_{n} e^{2 \pi i t_{0} f_{n}} \frac{\tilde{A}_{n}^{*} \tilde{S}_{n}}{J_{n}}}{\sum_{n=0}^{N-1} \frac{\left|\tilde{A}_{n}\right|^{2}}{J_{n}}} .
$$

Since $\partial \hat{a}\left(t_{0}\right) / \partial t_{0} \propto \partial \chi^{2} / \partial t_{0}$, the value of $t_{0}$ which maximizes $\hat{a}$ will also minimize $\chi^{2}$ (assuming second order derivatives check out).

\section{Optimal Filter Processing Algorithm}

Using this underlying theory, the OF processing algorithm will take in the raw charge or phonon event, and apply a FFT to convert it into the frequency domain. The frequencydomain pulse is then multiplied by the complex conjugate of a pulse template that has 
already undergone a FFT, and been deweighted by the noise PSD, read in from the filter files. An inverse FFT of the resulting product is the maximum amplitude $\hat{a}\left(t_{0}\right)$ for every possible time shift $t_{0}{ }^{4}$.

An optimization algorithm then searches for the time shift value that corresponds to the maximum fitted template amplitude (also finding the minimal $\chi^{2}$ in the process). A For loop searches $\hat{a}\left(t_{0}\right)$ for the largest $\hat{a}$ value, looking in a range from the zeroth bin (corresponding to no time shift between the pulse and template) and increasing to a maximum window limit, $w_{\max }$, and then from a minimum window limit, $w_{\min }$ to the final $(N-1)$ bin. Finding a maximum $\hat{a}$ within the $\left[0, w_{\max }\right]$ would mean that a positive time shift optimizes the maximum amplitude, whereas a maximum $\hat{a}$ within the $\left[w_{\min }, N-1\right]$ would correspond to a negative time shift, since shifting the template by $n$ bins is equivalent to shifting the template by $n-N$ bins.

The window maximum and minimum values are used to restrict where the maximum amplitude of the filtered pulse is searched for, saving processing time and avoiding fits on undesired additional (pileup) pulses that could occur far from the trigger. The window depends on the length of the pulse pre-trigger (set at the start of data taking), and the positive and negative window ranges, with all values being read out from a processing configuration file. An example search window is highlighted in yellow in Fig. 3.10.

The largest amplitude found within these windows is the optimal filter amplitude $R Q$ value. For a phonon pulse, this $\mathrm{RQ}$ value is labeled $\mathrm{P}^{*}$ OFamps, where ${ }^{*}$ is the placeholder for a single channel (for example, AS1 or BS2), or for a summed pulse quantity, such as PS1 for all phonon channels on side 1 or PT for the total phonon amplitude. The suffix amps is included because the phonon readout circuit gains and values are applied to convert the original ADC output into the maximum current passing through the TES, in Amps. For a charge pulse, the RQ value is labeled $\mathrm{Q}^{*}$ OFvolts, since the output is converted to voltage. In the case of charge quantities, ${ }^{*}$ can again refer to individual channels, such as IS1 (charge inner on side 1). A full list of data quantities used in this thesis is provided in Appendix A.1.

For both charge and phonon channels, the bin which corresponds to this maximum amplitude is the bin shift value. As a final step to finding the time shift, the inverse

\footnotetext{
${ }^{4}$ Recall that each digitized pulse is $N$ bins long, so a shift $n$ bins is the same as shifting by $n \pm N$ bins, and therefore the number of possible shifts is merely $\mathrm{N}$.
} 

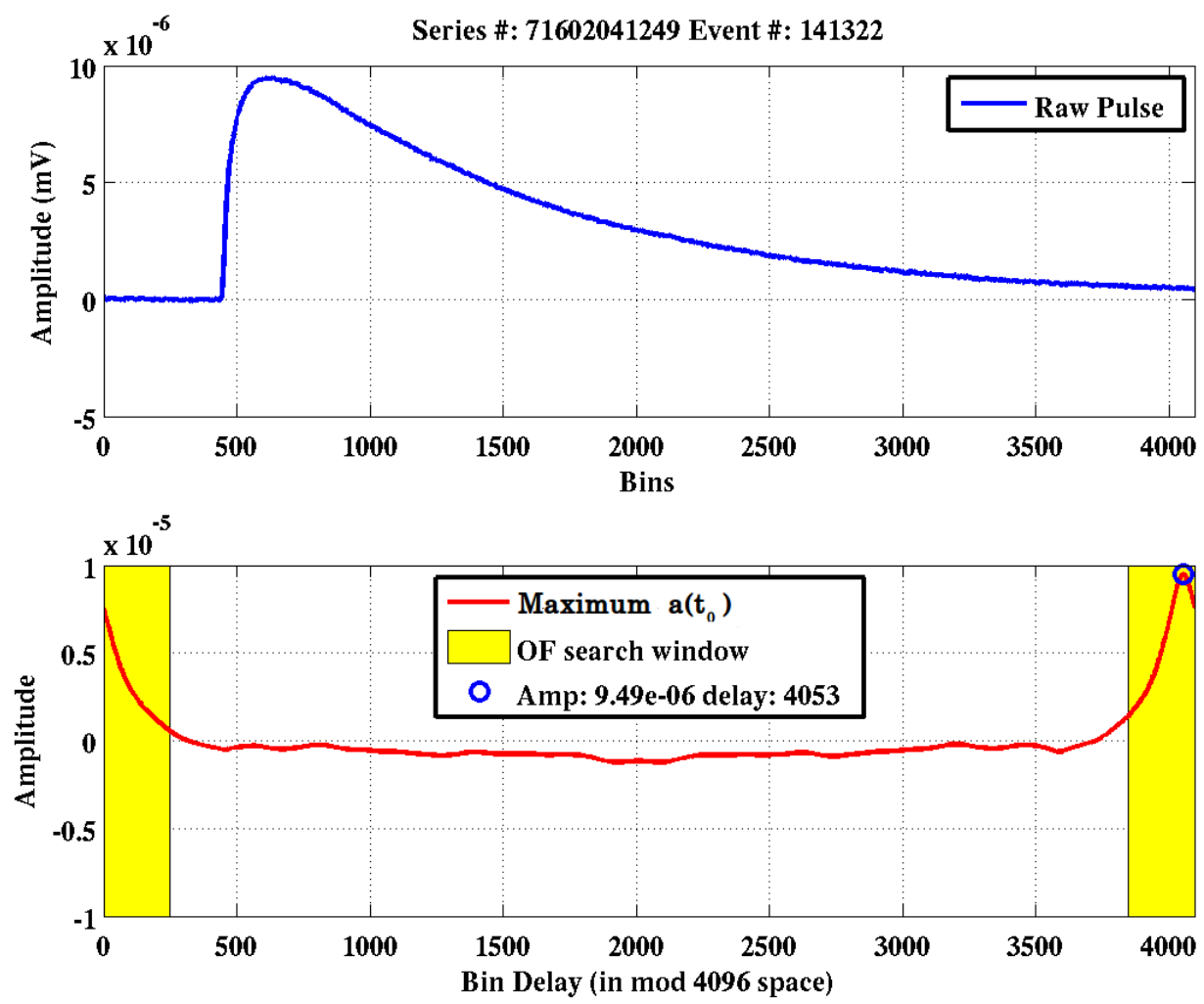

Figure 3.10: A total phonon raw pulse (blue, TOP) from UMN test facility data undergoes the basic optimal filter algorithm (reproduced here in Matlab for clarity). The resulting $\hat{a}\left(t_{0}\right)$ amplitude function (red, BOTTOM) is searched within a designated window region (highlighted in yellow). The maximum amplitude (blue circle), is selected as the amplitude RQ, with corresponding bin shift. In this particular example, this bin shift of 4053 bins will be converted into a negative shift time before it is saved as the PTOFdelay RQ value. 
$\bmod N$ of the bin shift is taken (such that values in the $\left[w_{\min }, N-1\right]$ range are now negative), and the resulting value is multiplied by time per ADC bin to give the time shift, in seconds. This value will be written as the *OFdelay RQ value (for example, QIS1OFdelay or PTOFdelay), since the time shift value can also be thought of as the time delay between the actual pulse and the template start time.

To reconstruct the estimate of the raw pulse, the template is multiplied by the amplitude RQ value and shifted by the proper number of bins. The $\chi^{2} \mathrm{RQ}$ value is calculated by summing the square of the absolute value of the difference between the raw pulse FFT and the reconstructed pulse FFT over all bins, normalized by the noise FFT squared RQ read in from the filter files; this is effectively Eq. 3.16 for the best fit amplitude and time shift. The OF processing algorithm will write out the chisq RQ as *OFchisq, such as PAS1OFchisq or QS1OFchisq.

A final optimal filter RQ worth mentioning is the zero time shift amplitude value. This value refers to the maximum amplitude if the time delay is fixed at zero. It is particularly useful when dealing with noise data, since it gives a more accurate view of the noise environment; the typical OF fit will output the largest amplitude within the window. This value is output at the typical amplitude output with an zero added to the end, such as QIS1OFvolts0.

\section{Extensions to the Basic Optimal Filter}

The method described in the previous sections outlines the simplest scenario of fitting, in which a single template fits a single pulse; this is known as $1 \times 1 \mathrm{OF}$. It is commonly used for fitting individual and total phonon pulses, but it can also be expanded upon to "fit" 5 different needs of the user.

Extending the basic optimal filter principals to simultaneously fit $M$ templates to $N$ channels, is referred to as $N \times M$ OF. CDMS utilizes this more general form to perform two-template fits and remove cross talk between channels, which require $1 \times 2$ OF and $2 \times 2$ OF algorithms, respectively. The relationship between signal, template, and noise described in Eq. 3.12, must now be expressed using matrices, such that,

$$
S=\left[\begin{array}{ll}
A_{1} & A_{2}
\end{array}\right]\left[\begin{array}{l}
a_{1} \\
a_{2}
\end{array}\right]+n,
$$

\footnotetext{
${ }^{5}$ This is hopefully the only intentional pun included in this thesis.
} 
for $1 \times 2 \mathrm{OF}$, and,

$$
\left[\begin{array}{l}
S_{1} \\
S_{2}
\end{array}\right]=\left[\begin{array}{cc}
A_{1} & A_{2 x} \\
A_{1 x} & A_{2}
\end{array}\right]\left[\begin{array}{l}
a_{1} \\
a_{2}
\end{array}\right]+\left[\begin{array}{l}
n_{1} \\
n_{2}
\end{array}\right],
$$

for $2 \times 2 \mathrm{OF}^{6}$. The general $\chi^{2}$ for the $N \times M$ OF can be written as [186],

$$
\chi^{2}=\left[\tilde{S}^{* \alpha}-e^{2 \pi i t_{0} f_{n}} a^{j} \tilde{A}_{j}^{* \alpha}\right]\left\{\tilde{\mathbf{V}}_{\alpha \beta}^{-1}\right\}\left[\tilde{S}^{\beta}-e^{-2 \pi i t_{0} f_{n}} a^{k} \tilde{A}_{k}^{\beta}\right],
$$

where the $N$ events are represented by Greek indices, the $M$ templates are represented by Latin indices, and contracted indices are summed over implicitly. Here, $\tilde{\mathbf{V}}_{\alpha \beta}=$ $\tilde{\sigma}_{\alpha} \tilde{\rho}_{\alpha \beta} \tilde{\sigma}_{\beta}$ represents the covariance matrix between the noise of different events with individual variances given by $\tilde{\sigma}_{\alpha}^{2}$ and correlation $\tilde{\rho}_{\alpha \beta}$. If the noise between events is uncorrelated, the covariance matrix becomes a diagonal matrix such that $\tilde{\mathbf{V}}_{\alpha \alpha}=\tilde{J}_{\alpha}$.

The amplitude for which $\chi^{2}$ is minimized in this case is given by,

$$
\hat{\mathbf{a}}=\mathbf{P}^{-1} \cdot \mathbf{q}\left(t_{0}\right)
$$

where matrices $\mathbf{P}$ and $\mathbf{q}$ are defined as,

$$
\begin{array}{r}
P_{j k}=\sum_{n=0}^{N-1} \tilde{A}_{j}^{* \alpha}\left\{\tilde{\mathbf{V}}_{\alpha \beta}^{-1}\right\} \tilde{A}_{k}^{\beta}, \\
q_{j}=\sum_{n=0}^{N-1} \tilde{A}_{j}^{* \alpha}\left\{\tilde{\mathbf{V}}_{\alpha \beta}^{-1}\right\} \tilde{S}^{\beta} e^{2 \pi i t_{0} f_{n}} .
\end{array}
$$

From these, the most reduced $\chi^{2}$ as parameterized by time shift $t_{0}$, can be simplified by expanding Eq. 3.22 ,

$$
\chi^{2}\left(t_{0}\right)=\tilde{S}^{* \alpha}\left\{\mathbf{V}_{\alpha \beta}^{-1}\right\} \tilde{S}^{\beta}-\left[\mathbf{P}^{-1} \cdot \mathbf{q}\left(t_{0}\right)\right]^{j} \tilde{A}_{j}^{* \alpha}\left\{\mathbf{V}_{\alpha \beta}^{-1}\right\} \tilde{A}_{k}^{\beta}\left[\mathbf{P}^{-1} \cdot \mathbf{q}\left(t_{0}\right)\right]^{k} .
$$

For more information on extended OF theory and utilizing extended OF algorithms in CDMS processing see the theses of R. Basu Thakur's [185] and J. P. Filippini's [186]. A brief discussion of the $2 \times 2$ OF use in cross talk correction is included in Appendix B.

\footnotetext{
${ }^{6}$ Here bin indices have been omitted for clarity.
} 


\subsubsection{Non-Stationary Optimal Filter}

Up until this point it has been assumed that the noise present in the system had no time correlation (i.e. stationary noise), and thus the frequency-domain modes were independent. However, time-dependent noise is commonplace in SuperCDMS data, and must be effectively dealt with.

Although phonon pulses tend to follow a particular pulse shape, position-dependence of the phonons produces fluctuations in the first $\sim 100 \mu$ s of the signal, causing deviations in the peak shape of the phonon signal. The Non-Stationary Optimal Filter (NSOF) aims to deweight this portion in the $\chi^{2}$, by treating the deviations as noise. This is done by creating an auto-covariance matrix, $\tilde{\mathbf{V}}$, from the residual differences of hundreds of total phonon pulses and a template. The details of creating this matrix will be discussed in Section 5.2 .4

The NSOF is applied to only the total phonon pulse, PT, using a single template. Thus $\chi^{2}$ for NSOF is given as,

$$
\chi^{2}=\sum_{n n^{\prime}}\left[\tilde{S}_{n}^{*} e^{2 \pi i t_{0} f_{n}}-a \tilde{A}_{n}^{*}\right]\left\{\tilde{\mathbf{V}}_{n n^{\prime}}^{-1}\right\}\left[\tilde{S}_{n^{\prime}} e^{-2 \pi i t_{0} f_{n}^{\prime}}-a \tilde{A}_{n^{\prime}}\right]
$$

where $n$ and $n^{\prime}$ are bins which correspond to frequencies $\nu$ and $\nu^{\prime}$, respectively. Notice that the exponential factor is now applied to the signal instead of the template. This is to address the need of phase-locking the noise to the template, since the noise change corresponds to the start of a pulse. Therefore, the data is time shifted to match the template, not the other way around as was seen previously.

If $\nu$ and $\nu^{\prime}$ have a strong correlation, then the term $\tilde{\mathbf{V}}_{n n^{\prime}}$ will be large. Because $\chi^{2}$ depends on the inverse of $\tilde{\mathbf{V}}_{n n^{\prime}}$, frequencies with strong correlation will be deweighted in the $\chi^{2}$ calculation. In the event that no correlations exist between different frequencies, $\tilde{\mathbf{V}}_{n n^{\prime}}$ would have only diagonal components, and Eq. 3.27 would be analogous to Eq. 3.16 .

Setting $\partial \chi^{2} / \partial a=0$ can again be used to determine the best amplitude estimate, $\hat{a}$ for a given time shift, $t_{0}$,

$$
\hat{a}\left(t_{0}\right)=\frac{\sum_{n n^{\prime}}{\tilde{S_{n}}}^{*} e^{2 \pi i t_{0} f_{n}}\left\{\tilde{\mathbf{V}}_{n n^{\prime}}^{-1}\right\} \tilde{A}_{n^{\prime}}}{\sum_{n n^{\prime}}{\tilde{A_{n}}}^{*}\left\{\tilde{\mathbf{V}}_{n n^{\prime}}^{-1}\right\} \tilde{A}_{n^{\prime}}} .
$$


As with the $1 \times 1$ OF algorithm, the NSOF CDMS algorithm scans over $t_{0}$ values in a designated region to find the maximum amplitude $\hat{a}\left(t_{0}\right)$, and then calculates the minimized $\chi^{2}$ using Eq. 3.27 .

\subsection{Radioactive Background in the Cryogenic Laboratory}

Characterizing detector performance has always been a forefront goal of the testing facility, and achieving this goal depends on understanding the interactions occurring within each detector. Maintaining a reasonable trigger rate to avoid pulse pileup, identifying source features within spectra, and confidently distinguishing nuclear recoil events from electron recoil events all depend on understanding the radiation background, and effectively reducing the background to a manageable level. This section discusses the test facility incident gamma-ray and neutron background, and overviews the methods by which the background is reduced. These methods include internal and external shielding, and clean, nitrogen-purged environments to reduce detector contamination.

\section{Gamma-Ray Background}

Numerous studies have been conducted to understand the incident gamma-ray background in the previous test facility at Tate Laboratories. Initial studies sought to identify sources of background radiation, using a p-type, high purity germanium (HPGe) detector from Princeton Gamma-Tech, cooled to $\sim 77 \mathrm{~K}$. The detector has an energy range of $40 \mathrm{keV}-10 \mathrm{MeV}$ and an established resolution of $820 \mathrm{eV}-2.3 \mathrm{keV}$ at 1.33 $\mathrm{MeV}$ [187]. The study identified a majority of the background peaks as the daughter products of ${ }^{232} \mathrm{Th},{ }^{238} \mathrm{U}$, and ${ }^{235} \mathrm{U}$ decays, and ${ }^{40} \mathrm{~K}$. These isotopes are long-lived (half lives of 14.1, 4.5, 0.7, and 1.3 Gyrs, respectively), primordial sources that are found in the Earth's crust, mined ores, and thus frequently appear in building materials; they are likely present in the walls and floor of the lab [188].

Undergraduate students A. Emerick and K. Weir sought to elaborate on the study by using lead brick shielding to observe any potentially non-homogeneous characteristics to the natural background. When factoring for the HPGe geometry, and unavoidable deviations in collimation by the lead enclosure, they determined that there was no directional component to the gamma-ray background [189]. The peaks they identified 
are listed in Table 3.1, and their spectra (before accounting for detector geometry) are included in the top plot of Fig. 3.11.

A final background study was performed in 2012 in the Tate lab in conjuncture with the construction of lead gamma-ray shielding. The purpose of the study was to calculate the attenuation of the background due to the shielding. Results of that study will be presented below in Section 3.5 and Fig. 3.19. In general the results confirmed the gamma ray energy spectrum was comprised of ${ }^{232} \mathrm{Th},{ }^{238} \mathrm{U}$, and ${ }^{235} \mathrm{U}$ daughter decays, and ${ }^{40} \mathrm{~K}$.

After moving to the new lab in the Physics and Nanotechnology building, an additional background study was conducted in 2017 to determine any alterations in background. Unfortunately, the HPGe detector setup underwent some unknown change that resulted in the degradation of spectra resolution. In general, the PAN lab background is reduced by a factor of $\sim 2$, compared to that of the Tate lab. Both labs appear to be exposed to the same background sources (see Table 3.1), but the degradation of the PAN lab spectra makes it difficult to fully access individual sources. The Tate and PAN lab gamma-ray spectra are compared in the bottom plot of Fig. 3.11 .

\section{Neutron Background}

Unlike the gamma-ray background, the neutron background energy spectrum at the UMN facility has never been directly measured. Original approximations were made based off of the Monte Carlo modeled cosmogenic neutron energy spectrum at the SuperCDMS Soudan site [191], shown in Fig. 3.12. However, this spectrum was developed specifically for the Soudan cavern, and was not a good approximation for the expected spectrum on the surface. A more accurate estimate for neutron activity in the lab is based on differential neutron flux measurements [192, shown in Fig. 3.13, normalized to New York, NY at sea level, a historical reference point for neutron flux measurements. Monte Carlo simulations included in [193] parameterize the neutron environment, including dependencies on altitude, magnetic latitude and solar activity. The resulting dependence equations in [193] give an $\sim 1.12$ for the overall relative flux between the New York reference point and Minneapolis.

Both the modeled Soudan spectrum and the differential flux measurements only consider neutrons from cosmogenic sources, excluding ambient neutron products from 
Table 3.1: Identified sources with peaks in the facility background spectra [189]

\begin{tabular}{|c|c|c|c|}
\hline Mean Peak Energy $[\mathrm{keV}]$ & Source & Source Energy Value [keV] [190] & Parent \\
\hline 240.45 & ${ }^{214} \mathrm{~Pb}$ & 241.99 & ${ }^{238} \mathrm{U}$ \\
\hline 296.70 & ${ }^{214} \mathrm{~Pb}$ & 295.22 & ${ }^{238} \mathrm{U}$ \\
\hline 340.01 & ${ }^{228} \mathrm{Ac}$ & 338.32 & ${ }^{232} \mathrm{Th}$ \\
\hline 352.69 & ${ }^{214} \mathrm{~Pb}$ & 351.93 & ${ }^{238} \mathrm{U}$ \\
\hline 512.27 & ${ }^{208} \mathrm{Tl}$ & 510.77 & ${ }^{232} \mathrm{Th}$ \\
\hline 584.36 & ${ }^{208} \mathrm{Tl}$ & 583.19 & ${ }^{232} \mathrm{Th}$ \\
\hline 610.35 & ${ }^{214} \mathrm{Bi}$ & 609.31 & ${ }^{238} \mathrm{U}$ \\
\hline 912.14 & ${ }^{228} \mathrm{Ac}$ & 911.20 & ${ }^{232} \mathrm{Th}$ \\
\hline 968.55 & ${ }^{228} \mathrm{Ac}$ & 968.97 & ${ }^{232} \mathrm{Th}$ \\
\hline 1120.71 & ${ }^{214} \mathrm{Bi}$ & 1120.29 & ${ }^{238} \mathrm{U}$ \\
\hline 1238.87 & ${ }^{214} \mathrm{Bi}$ & 1238.11 & ${ }^{238} \mathrm{U}$ \\
\hline 1377.71 & ${ }^{214} \mathrm{Bi}$ & 1377.67 & ${ }^{238} \mathrm{U}$ \\
\hline 1460.83 & ${ }^{40} \mathrm{~K}$ & 1460.83 & $\mathrm{~N} / \mathrm{A}$ \\
\hline 1518.55 & ${ }^{212 g} \mathrm{Bi}$ & 1512.80 & ${ }^{232} \mathrm{Th}$ \\
\hline 1591.42 & ${ }^{228} \mathrm{Ac}$ & 1588.19 & ${ }^{232} \mathrm{Th}$ \\
\hline 1729.53 & ${ }^{214} \mathrm{Bi}$ & 1729.60 & ${ }^{238} \mathrm{U}$ \\
\hline 1764.45 & ${ }^{214} \mathrm{Bi}$ & 1764.50 & ${ }^{238} \mathrm{U}$ \\
\hline 1847.30 & ${ }^{214} \mathrm{Bi}$ & 1847.42 & ${ }^{238} \mathrm{U}$ \\
\hline 2103.63 & Unknown & $\mathrm{N} / \mathrm{A}$ & $\mathrm{N} / \mathrm{A}$ \\
\hline 2118.03 & ${ }^{214} \mathrm{Bi}$ & 2118.55 & ${ }^{238} \mathrm{U}$ \\
\hline 2204.04 & ${ }^{214} \mathrm{Bi}$ & 2204.21 & ${ }^{238} \mathrm{U}$ \\
\hline 2447.65 & ${ }^{214} \mathrm{Bi}$ & 2447.86 & ${ }^{238} \mathrm{U}$ \\
\hline 2614.53 & ${ }^{208} \mathrm{Tl}$ & 2614.53 & ${ }^{232} \mathrm{Th}$ \\
\hline
\end{tabular}



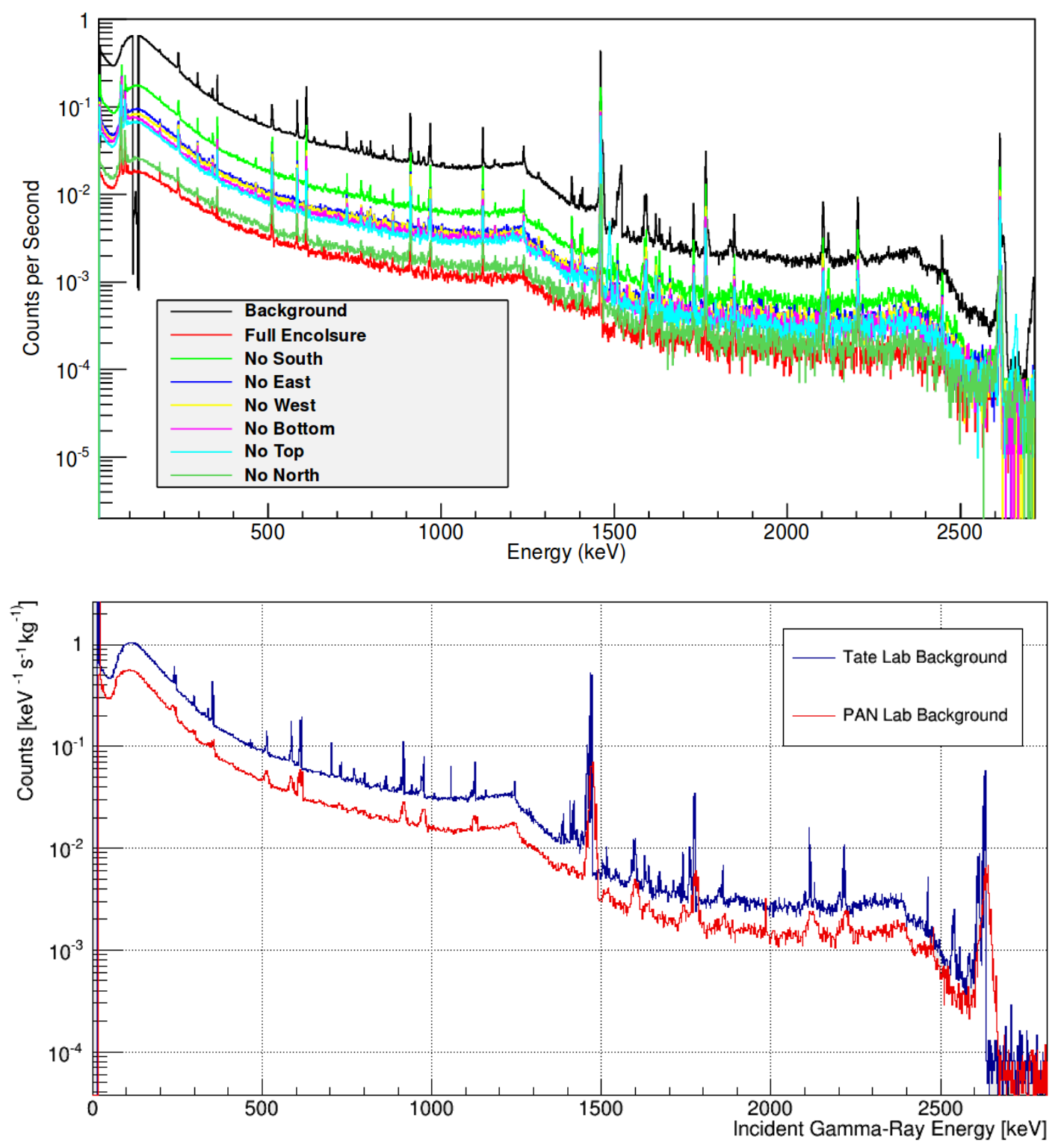

Figure 3.11: (TOP) Gamma-ray spectra results from A. Emerick and K. Weir, with various direction-specific lead shields. This figure does not take detector geometry into account. Once geometry is accounted for, no directional component to the gamma-ray background was found. Figure from [189]. (BOTTOM) A comparison of the gammaray spectrum from Tate Labs (blue) and from the Physics and Nanotechnology building (red). 


\section{Incoming Neutron Energy Spectrum}

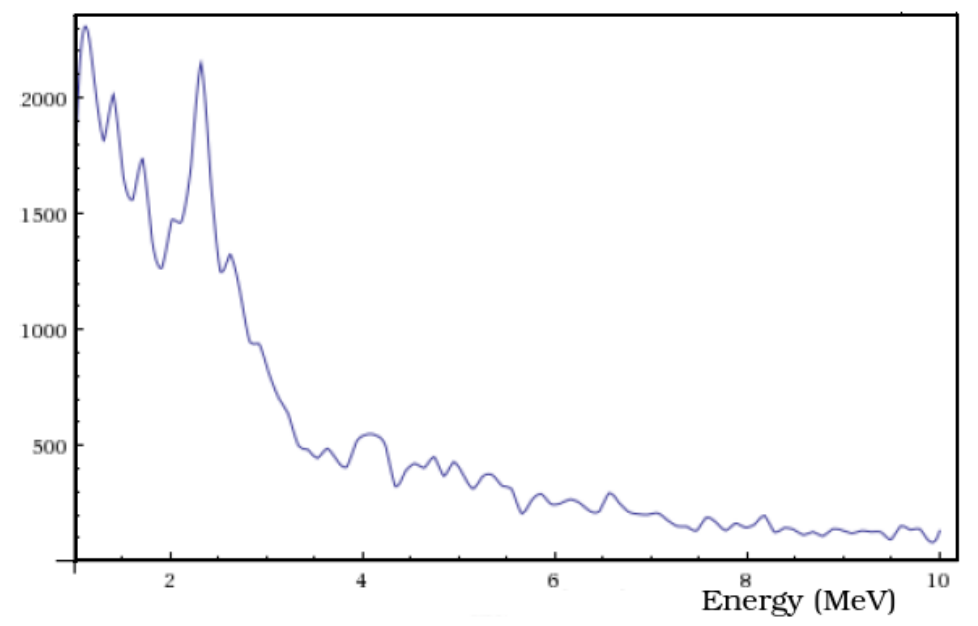

Figure 3.12: Incoming neutron energy spectrum for the Soudan cavern housing SuperCDMS. Monte Carlo simulation results by A. Reisetter. Figure modified from R. Radpour.

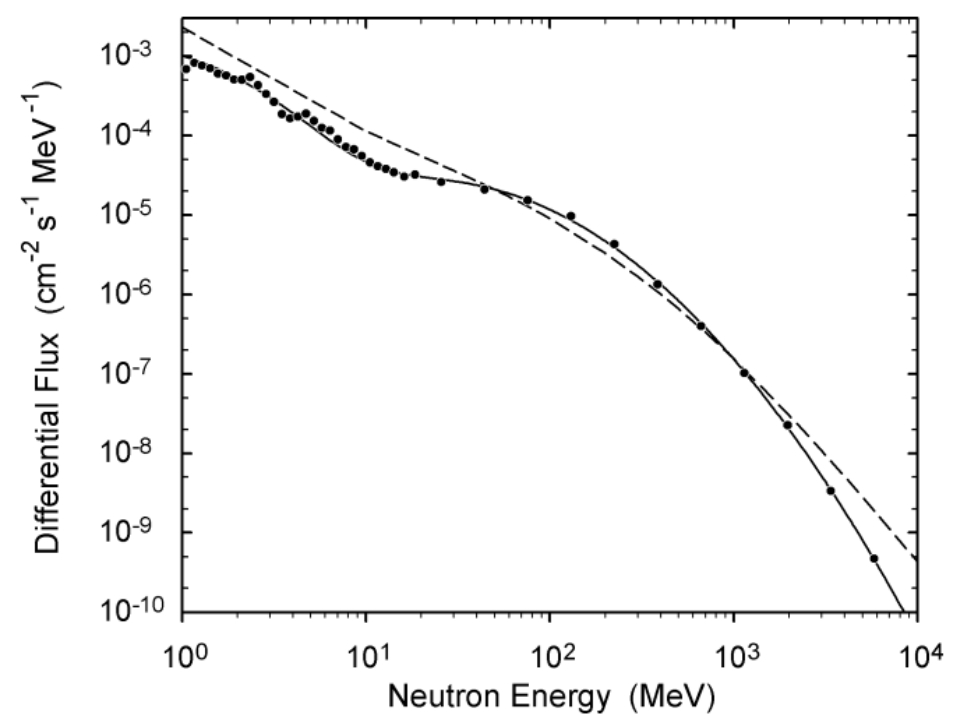

Figure 3.13: Differential Neutron Flux Measurements for Neutron energy spanning 1 $10^{4} \mathrm{MeV}$. Differential flux is normalized to New York, NY at sea level. Measured values (black dots) are fit with two models (black solid and dashed lines). Figure courtesy of 192. 
radioactive decay in the surrounding environment. Radiogenic neutrons are the products of $(\alpha, n)$ reactions and spontaneous fission in the ${ }^{238} \mathrm{U}$ and ${ }^{232} \mathrm{Th}$ decay chains 194 . Neutron-producing reactions are rare, and thus the cosmogenic neutron contribution is several orders of magnitude larger than radiogenic contributions 7 .

\section{Expected Neutron Rate}

An estimate for the neutron events/hour in the $10-100 \mathrm{keV}$ range can be made for a UMN iZIP detector, based on the differential neutron flux measurements of [192], and the $\sim 1.12$ approximation for the relative flux between the New York reference point and Minneapolis. Determining the expected neutron rate also depends on the cross section between neutrons and the target material, in this case natural abundance Ge. The differential cross section (in the lab reference frame) for elastic neutrons scattering off of germanium was measured for $1.2 \mathrm{MeV}$ incoming neutrons, at eight different scattering angles between $20^{\circ}-160$, with a $10 \%$ reported error [195]. The results can be modeled as Legendre polynomial expansions of the form,

$$
\frac{d \sigma}{d \Omega}\left(\theta^{\prime}\right)=\sum_{L} B_{L} P_{L} \cos \left(\theta^{\prime}\right)
$$

where $\theta^{\prime}$ is the lab-frame scattering angle and $B_{L}$ are the polynomial coefficients which represent the best fit to data. The expansion fits were limited to seven terms. Coefficients and estimated errors for the elastic scatters are shown in Table 3.2, Measured differential cross sections and Legendre Polynomial fit is shown in Fig. 3.14. From Eq. 3.29 , the total cross section across all scattering angles is given by,

$$
\sigma_{G e}=10^{-27} \times \int_{0}^{2 \pi} d \phi \int_{0}^{1} \sum_{L} B_{L} P_{L} \cos \left(\theta^{\prime}\right) d \cos \left(\theta^{\prime}\right),
$$

where the factor $10^{-27}$ simply converts the units from $\mathrm{mb}$ to $\mathrm{cm}^{2}$.

Given the differential flux, $j\left(E_{n}\right)$, converted to units of $\left[\mathrm{cm}^{-2} \mathrm{~s}^{-1} \mathrm{keV}^{-1}\right]$, and cross section, $\sigma_{G e}$, the expected differential neutron interaction rate, $\frac{d \eta_{n}}{d E_{N}}$, in units $\left[s^{-1} \mathrm{keV}^{-1}\right]$

\footnotetext{
7 This is only true for surface facilities. SuperCDMS SNOLAB background models predict bulk material contamination to contribute $510(530) \times 10^{6}$ counts $/ \mathrm{kg} / \mathrm{keV} /$ year in a $\mathrm{Ge}(\mathrm{Si})$ iZip. Cosmogenic neutrons are expected to contribute $73(77) \times 10^{6}$ counts $/ \mathrm{kg} / \mathrm{keV} /$ year. Both predictions only consider single events, average over the $1-50 \mathrm{keV}$ energy range [135].
} 


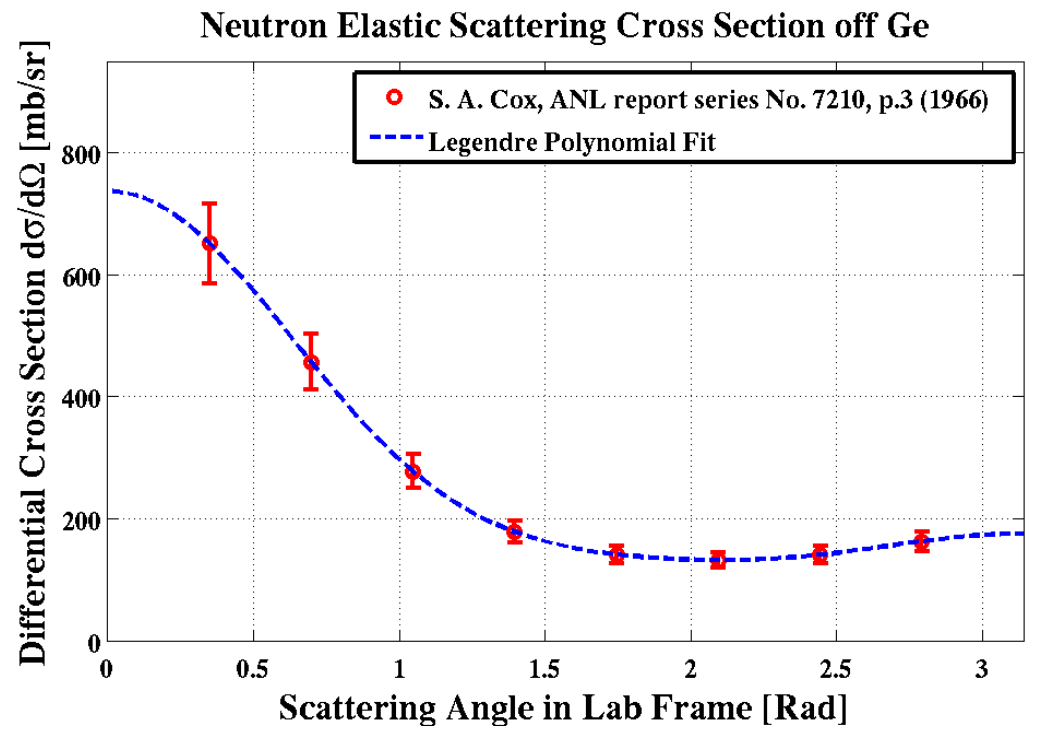

Figure 3.14: Neutron differential elastic scattering cross sections of Ge in the lab frame. Dotted blue curve is the best Legendre polynomial fit to the experimental points (red). Cross section data from 195 .

Table 3.2: Elastic Scatter Legendre Polynomial Coefficients [mb/sr]

\begin{tabular}{cccccccc}
\hline \hline & & & & & & & \\
& $\mathrm{B}_{1}$ & $\mathrm{~B}_{2}$ & $\mathrm{~B}_{3}$ & $\mathrm{~B}_{4}$ & $\mathrm{~B}_{5}$ & $\mathrm{~B}_{6}$ & $\mathrm{~B}_{7}$ \\
\hline & & & & & & & \\
Value & 237.07 & 208.61 & 188.04 & 72.208 & 31.015 & $5.7218 \mathrm{E}-2$ & $2.7658 \mathrm{E}-2$ \\
Error & 0.02 & 0.03 & 0.03 & 0.040 & 0.048 & $5.4514 \mathrm{E}-2$ & $6.0471 \mathrm{E}-2$ \\
\hline
\end{tabular}


is,

$$
\frac{d \eta_{n}}{d E_{N}}=j\left(E_{n}\right) \sigma_{G e} n_{G e} V
$$

for a Ge detector of volume $V$, and nuclear density $n_{G e}$. Since flux data is reported in discrete energy values, a Riemann sum approximation with energy slices of width $\Delta E_{n}$, is used over all incoming neutron energy values to get the total expected neutron rate, $\eta_{n}$ :

$$
\eta_{n}=\sum_{E_{n}} j\left(E_{n}\right) \Delta E_{n} \sigma_{G e} n_{G e} V .
$$

The neutron rate yields $\sim 3050$ neutron interactions per hour for the $100 \mathrm{~mm}$ diameter Ge iZIP detector.

Integrating Eq. 3.29 over the full range of scattering angles will give the total cross section. However, only scattering that results in $10-100 \mathrm{keV}$ recoil energy is relevant to this measurement. To determine the nuclear recoil energy, $E_{R}$, as a function of incoming neutron kinetic energy, $E_{n}$, and the center of mass frame scattering angle, $\theta$, Eq. 1.51 becomes,

$$
E_{R}=\frac{2 E_{n} m_{N} M_{T}}{\left(M_{T}+m_{N}\right)^{2}}(1-\cos \theta)
$$

where $M_{T}$ is the mass of the target material, in this case Ge. From Eq. 3.33, the minimum and maximum $\theta$ values for which $E_{R} \in[10 \mathrm{keV}, 100 \mathrm{keV}]$ can be determined for every value of $E_{n}$. Scattering angles can be converted from center of mass frame $(\theta)$ to lab frame $\left(\theta^{\prime}\right)$ via,

$$
\tan \left(\theta^{\prime}\right)=\frac{\sin (\theta)}{\cos (\theta)+M_{T} / m_{N}} .
$$

Since extrema scattering angles, $\theta_{\max }^{\prime}$ and $\theta_{\min }^{\prime}$ are dependent on incoming neutron energy, the total cross section described by Eq. 3.30 becomes energy dependent as well,

$$
\sigma_{G e}\left(E_{n}\right)=10^{-27} \int_{0}^{2 \pi} d \phi \int_{\cos \left(\theta_{\max }^{\prime}\left(E_{n}\right)\right)}^{\cos \left(\theta_{\min }^{\prime}\left(E_{n}\right)\right)} \sum_{L} B_{L} P_{L} \cos \left(\theta^{\prime}\right) d \cos \left(\theta^{\prime}\right) .
$$

The differential cross section given by Eq. 3.31 then becomes,

$$
\frac{d \eta_{n}}{d E_{N}}=j\left(E_{n}\right) \sigma_{G e}\left(E_{n}\right) n_{G e} V
$$

and total expected neutron rate described by Eq. 3.32 becomes,

$$
\eta_{n}=\sum_{E_{n}} j\left(E_{n}\right) \Delta E_{n} \sigma_{G e}\left(E_{n}\right) n_{G e} V
$$


This neutron rate yields $\sim 587$ neutron interactions per hour which result in a $10-100$ $\mathrm{keV}$ recoil energy in a $100 \mathrm{~mm}$ diameter Ge iZIP detector. This approximation does not consider any shielding provided by the lab building or experimental apparatus. The differential neutron interaction rate, $\frac{d \eta_{n}}{d E_{N}}$, for all resulting recoil energies and for recoil energies in the $10-100 \mathrm{keV}$ range, are compared in Fig. 3.15.

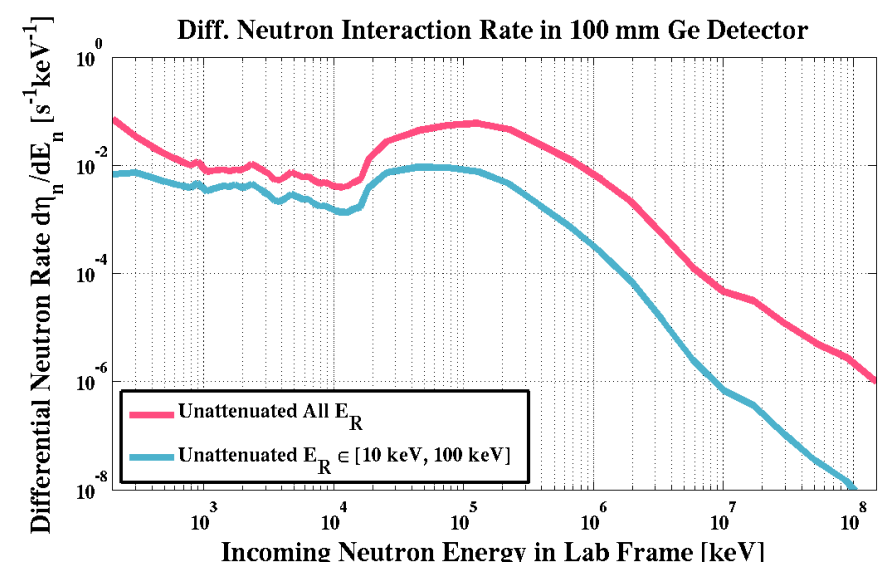

Figure 3.15: Differential neutron interaction rate $d \eta_{n} / d E_{n}$ for a $100 \mathrm{~mm}$ Ge iZIP when a full range of recoil energies is considered (salmon), compared to $d \eta_{n} / d E_{n}$ constrained to nuclear recoils in the $10-100 \mathrm{keV}$ energy range (teal).

\section{Shielding}

In 2011 R. Radpour, with the help of the test facility group and the UMN physics machine shop, designed, constructed, and tested a polyethylene neutron shield. Based on the neutron background estimates in Section 3.5, she determined that a $10 \mathrm{~cm}$ thick shield would theoretically reduce the neutron flux in the relevant energy region (1 $10 \mathrm{MeV}$ ) by a factor of $\sim 10$ [196]. Based on the materials available, and the need to completely surround the detector with minimal gaps, a $20 \mathrm{~cm}$ (8 in) thick shield was fabricated.

The bulk of the shield is comprised of 1" thick polyethylene sheets, supported by threaded rods attached to one of two rolling platforms which are secured together surrounding the fridge dewar. The sheets create four 8" thick walls which surround the dewar, and eight 22 " by 22 " by 1 " base sheets which rest on the conjoined platforms, 
beneath the dewar. From the outside, the shield forms a 42 " by 42 " by 55 " polyethylene box around the dewar. Since polyethylene sheets could not be placed across the top of the shield, nine circular layers of polyethylene were stacked on baffles within the upper portion of the cryostat; the inner polythene, secured with kapton tape, can be seen in the central image of Fig. 3.3 and the left image of Fig. 3.20. Initial studies with the polyethylene shielding compared $76 \mathrm{~mm}$ iZIP nuclear recoil events in a 10 $100 \mathrm{keV}$ phonon energy range with and without the shielding. Nuclear recoil events were selected using a linear cut with a variable y-intercept, called the neutron selection parameter (NSP). Results confirmed that the neutron flux was reduced by a factor of $\sim 10$ or better. For more information on the neutron shield analysis, see R. Radpour's thesis [196].

The polyethylene shield is expected to to reduce the neutron flux by,

$$
j\left(E_{n}\right)=j_{0}\left(E_{n}\right) e^{-\Sigma\left(E_{n}\right) d},
$$

where $j_{0}\left(E_{n}\right)$ is the initial neutron flux, $\Sigma$ is the energy-dependent attenuation factor, and $d$ is the distance traveled through the material by the neutron. The attenuation factor of the shield depends on the energy-dependent cross sections of hydrogen, $\sigma_{H}$, and carbon, $\sigma_{C}$ such that,

$$
\Sigma=\left(n_{H} \sigma_{H}+n_{C} \sigma_{C}\right) \times 10^{-24} \mathrm{~cm}^{-1},
$$

where the number densities of hydrogen and carbon, $n_{H}$ and $n_{C}$ both behave as,

$$
n_{A}=\frac{\rho_{\text {poly }} N_{0} b}{m_{c H_{2}}},
$$

where $\rho_{\text {poly }}=0.941 \mathrm{~g} / \mathrm{cm}^{3}$ is the density of polyethylene, $m_{\mathrm{CH}_{2}}$ is the molar mass of $\mathrm{CH}_{2}$, and $b$ is the number of the specific type of atoms in $\mathrm{CH}_{2}$, such that $b=1$ for carbon, and $b=2$ for hydrogen [196]. Energy-dependent cross sections for neutrons with incoming energy of $1-10 \mathrm{MeV}$ were fit with 6th order polynomials by Mesquita et al. [197,

$$
\sigma_{H}=\sum_{i=1}^{6} \mathcal{P}_{H, i}\left(E_{n}\right)^{i-1}, \quad \sigma_{C}=\sum_{i=1}^{6} \mathcal{P}_{C, i}\left(E_{n}\right)^{i-1},
$$

where $E_{n}$ is in $\mathrm{keV}$ and coefficients, $\mathcal{P}_{H, i}$ and $\mathcal{P}_{C, i}$ are listed in Table 3.3. Combining the Eqs. 3.39, 3.40, and 3.41 yields the attenuation factor for the polyethylene shield 
in,

$$
\Sigma=\sum_{i=1}^{6} \mathcal{P}_{\Sigma, i}\left(E_{n}\right)^{i-1},
$$

where $E_{n}$ is in $\mathrm{keV}$ and coefficients, $\mathcal{P}_{\Sigma, i}$ are listed in Table 3.3 .

Table 3.3: Polyethylene Cross Section and Attenuation Coefficients

\begin{tabular}{cccccccc}
\hline \hline & & & & & & & \\
Order $(i)$ & 1 & 2 & 3 & 4 & 5 & 5 & 7 \\
\hline & & & & & & & \\
$\mathcal{P}_{H, i}[\mathrm{~b}]$ & $6.94 \mathrm{E}-3$ & $-4.48 \mathrm{E}-3$ & $1.60 \mathrm{E}-3$ & $-3.13 \mathrm{E}-3$ & $3.32 \mathrm{E}-5$ & $-1.79 \mathrm{E}-6$ & $3.85 \mathrm{E}-8$ \\
$\mathcal{P}_{C, i}[\mathrm{~b}]$ & $9.70 \mathrm{E}-3$ & $-13.5 \mathrm{E}-3$ & $8.22 \mathrm{E}-3$ & $-2.33 \mathrm{E}-3$ & $3.30 \mathrm{E}-5$ & $-2.28 \mathrm{E}-6$ & $6.10 \mathrm{E}-6$ \\
$\mathcal{P}_{\Sigma, i}\left[\mathrm{~cm}^{-1}\right]$ & $9.38 \mathrm{E}-4$ & $-8.98 \mathrm{E}-4$ & $4.58 \mathrm{E}-4$ & $-1.19 \mathrm{E}-4$ & $1.60 \mathrm{E}-5$ & $-1.06 \mathrm{E}-6$ & $-2.50 \mathrm{E}-7$ \\
\hline
\end{tabular}

Including attenuation due to the shield, the differential cross section given by Eq. 3.31 then becomes,

$$
\frac{d \eta_{n}}{d E_{N}}=j\left(E_{n}\right) e^{-\Sigma\left(E_{n}\right) d} \sigma_{G e}\left(E_{n}\right) n_{G e} V,
$$

and total expected neutron rate described by Eq. 3.32 becomes,

$$
\eta_{n}=\sum_{E_{n}} j\left(E_{n}\right) e^{-\Sigma\left(E_{n}\right) d} \Delta E_{n} \sigma_{G e}\left(E_{n}\right) n_{G e} V .
$$

For a polyethylene shield of $20 \mathrm{~cm}$ thickness, this neutron rate yields $\sim 20$ neutron interactions per hour over the whole range of nuclear recoil energies, and $\sim 9$ neutron interactions per hour which result in a $10-100 \mathrm{keV}$ recoil energy in a $100 \mathrm{~mm}$ diameter Ge iZIP detector. This indicates a shield attenuation factor of $\sim 0.02$ for events with 10 $100 \mathrm{keV}$ recoil energies. The differential neutron interaction rate with shield attenuation is compared for all resulting recoil energies and for recoil energies in the $10-100 \mathrm{keV}$ range, in Fig. 3.16 .

Soon after polyethylene shield studies verified the neutron attenuation, plans began to fabricate a lead shielding component to reduce the gamma-ray background. Shield designs and testing were a team effort, including this author, H. Chagani, and A. Codoreanu of the CDMS group, and P. Ness of the UMN workshop. Initially Monte Carlo simulations, shown in Fig. 3.17, were conducted by H. Chagani, to determine the desired shield thickness. The shield was originally comprised of four layers of $1 / 8$ " thick 


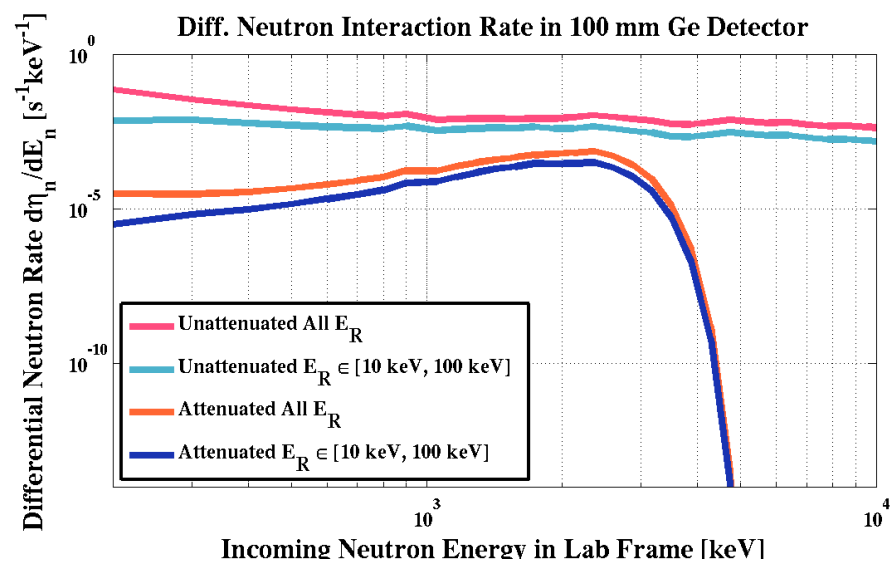

Figure 3.16: Comparison between unattenuated differential neutron interaction rates, and neutron rates when attenuated by a $20 \mathrm{~cm}$ thick polyethylene shield. Unattenuated $d \eta_{n} / d E_{n}$ for all $E_{R}$ (salmon) and for $E_{R} \in[10 \mathrm{keV}, 100 \mathrm{keV}]$ (teal) continue on for higher neutron energies, whereas attenuated $d \eta_{n} / d E_{n}$ for all $E_{R}$ (orange) and for $E_{R} \in$ [10 keV, $100 \mathrm{keV}]$ (navy) are effectively null for $E_{n}>5 \mathrm{MeV}$.

lead sheets, cut into manageable 9" - 11" by 48" strips (strip width varies to reduce clear line of sight). These strips are contained in four aluminum cages, which are secured to the $\mathrm{Al}$ frame and platforms of the neutron shield, creating four 44 " by 48 " by 0.5 " lead walls. To keep the malleable lead from buckling and creating gaps in the shield, thin $\mathrm{Al}$ sheets are used as an inner, middle, and outer layer, sandwiching the lead into place. Underneath the dewar, one of the 22 " by 22 " by 1 " polyethylene sheets was removed 8 to make room for a bottom 0.5 " thick layer of lead. As illustrated in Fig. 3.17, 0.5" of lead is expected to reduce the gamma background by roughly an order of magnitude in the 0 - $1 \mathrm{MeV}$ energy region, assuming full solid angle coverage [198]. Initial gamma shield studies performed with the HPGe detector found that the background was reduced by a factor of $\sim 3.5$ at most energies $(0-2.25 \mathrm{MeV})$, dropping to a factor of $\sim 2.0$ for the higher energy region $(>2.5 \mathrm{MeV})$. Fig. 3.18 demonstrates how these tests were conducted, with the HPGe detector set inside the shielding.

Due to the buckling of the malleable lead sheets, overlaps existed between sheets in each layer which made the shield bulkier than expected, as well as leaving some gaps

\footnotetext{
${ }^{8}$ Removing this polyethylene sheet was determined to have little effect on the incident neutron flux [196].
} 

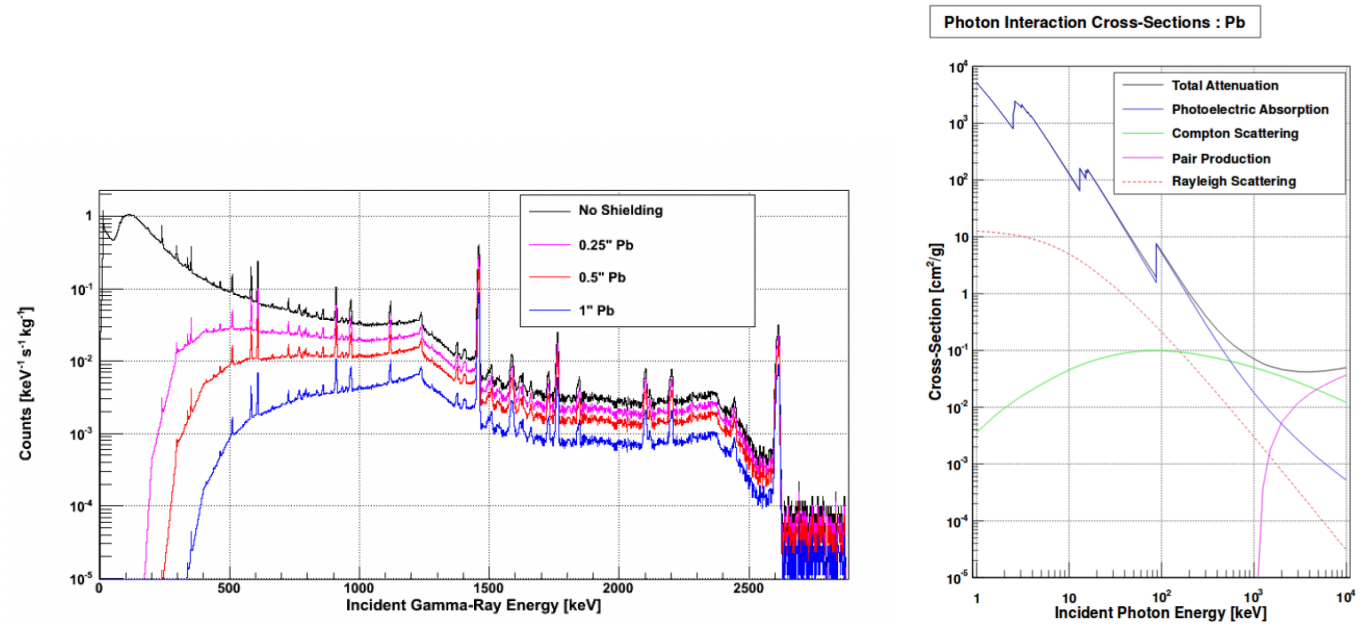

Figure 3.17: (LEFT) Incident photon energy spectra with various thicknesses of pure lead shielding. No shielding spectrum (black) recorded with HPGe detector. Lead shielding spectra (blue, red, and magenta) predicted with Monte Carlo simulation, assuming full solid angle coverage. (RIGHT) Cross-sections for gamma interactions in lead, used to predict the lead shield attenuation. For the $0-0.6 \mathrm{MeV}$ incident phonon energy region, photoelectric absorption is the dominant process, with sharp edges at the K-shell, L-shell, and M-shell binding energies. In the 0.6 - $5 \mathrm{MeV}$ region Compton scattering becomes the dominant process. Figures courtesy of H. Chagani. 
that could not be fixed by readjusting the sheets. To combat this, one lead sheet from each layer was trimmed such that each sheet fits without any overlap. In general this reduced the amount of cracks, but to increase the shielding (especially in places where the lead doesn't fit tightly against the adjacent pieces) an additional sheet of lead (also trimmed) was included. This extra layer was made from the remaining unused pieces of the shield. Since there was not a sufficient amount to make a complete fifth layer, one side simply has three 11"-wide pieces which cover the cracks in the previous layer. The sheets have been placed such that every layer gap is covered by at least 0.5 " of lead. Fig. 3.19 compares the spectrum of the standard four-layer shield to the trimmed five-layer shield, obtained with the HPGe detector. It can be seen by eye that there is a slight reduction in the event count with the new fifth layer shield. It appears as though the peaks have been significantly reduced in magnitude, though they have actually just been spread out. This is believed to be an unfortunate side effect of switching out the cord connecting the HPGe detector to the voltage bias. A spectra ratio is found to be roughly 1.1, suggesting a small but measurable change in event count.

Originally it was assumed that a significant portion of the background was from the cement floor, since it is likely to contain primordial radiogenic sources, and its relatively close to the detector. However, HPGe detector tests determined no significant difference between a spectrum with the full shield and a spectrum with the shield bottom removed; a ratio of the two spectra is roughly one, suggesting a negligible change in event counts between HPGe runs. This suggests that the bottom shield does not provide the dominant shielding for the detector 9 .

With the background spectrum unaffected by the bottom portion of the shield, efforts were focused on addressing the open top of the shield. As with the polyethylene neutron shield, this problem was difficult to solve, since any shield top must fit around the K100 fridge and provide sufficient coverage. The issue was addressed two-fold; some lead strips from the bottom of the shield (shown to have little impact) was removed and stacked on top of the polyethylene shielding, and 8" diameter, 0.5 " thick inner lead was added on top of polyethylene within the cryostat. HPGe tests with essentially all of the polyethylene sheets covered with a 0.5 " thick lead shield, showed a significant decrease

\footnotetext{
${ }^{9}$ Tests done by A. Emerick and K. Weir found that the shield bottom did have a significant affect on the event count, although a much greater shield thickness must be considered.
} 

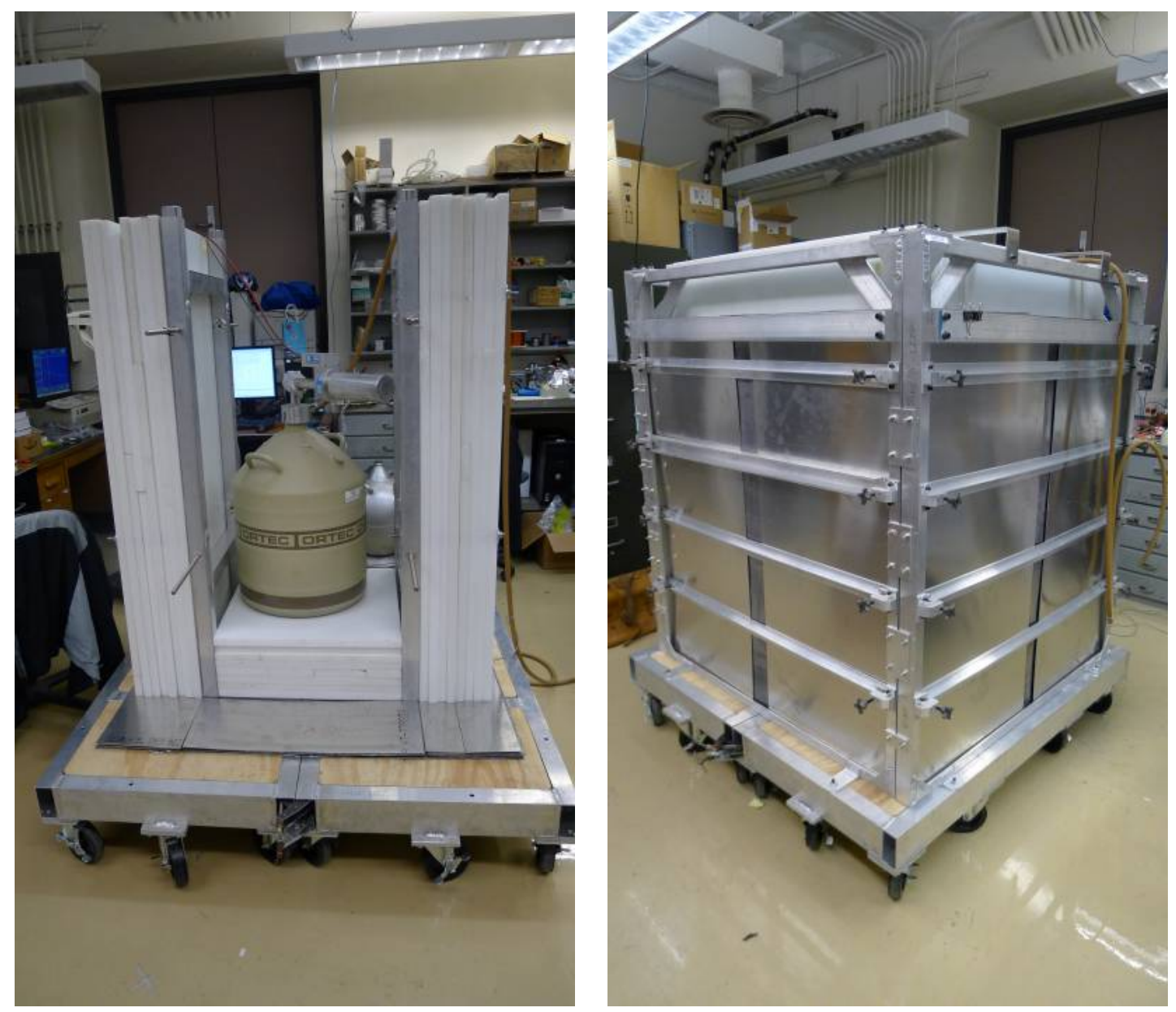

Figure 3.18: (LEFT) High-purity germanium (HPGe) detector in partially-constructed lead and polyethylene shielding mounted on aluminum frame on rolling platform. HPGe detector was used to study the attenuation factor of the lead gamma-ray shielding. (RIGHT) Fully constructed lead and polyethylene shielding, before gamma-ray shielding modifications. 


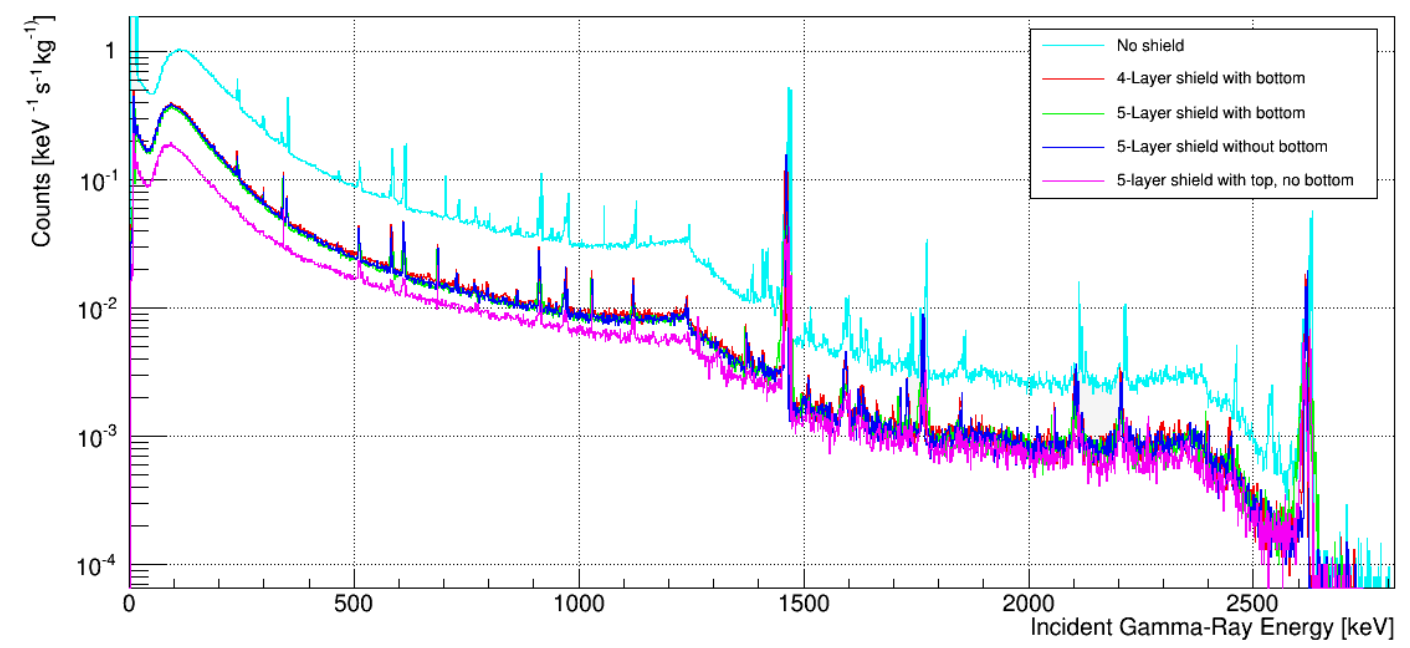

Figure 3.19: Gamma-ray background spectra taken with HPGe detector, demonstrating the effectiveness of the lead shield with various modifications.

in the background gamma spectrum: the low energy $(0-300 \mathrm{keV})$ region was reduced by a factor of $\sim 2$, and the spectrum below $2.5 \mathrm{MeV}$ was reduced by a factor of 1.25 or better.

Final modifications to both the neutron and gamma shields improved full angle coverage, by filling in the gap between the dewar and the rectangular shield with eight 1" thick polyethylene sheets covered with four 1/8" thick lead sheet, shown in Fig. 3.20 . This final version of the shield has been used by the test facility since late 2012. The final shield reduces the gamma-ray spectrum up to a factor of $\sim 5$ in the low energy region. Fig. 3.19 compares the gamma-ray spectra for the various shield modifications outlined here.

\section{Clean Room}

A majority of the background sources simply contribute to the detector event rate and leave the test facility experiment otherwise unaffected, but other isotopes can adhere to experimental hardware, resulting in a long-lasting radiogenic contamination. For example, radon is a noble gas with isotopes present in both the ${ }^{238} \mathrm{U}$ and ${ }^{232} \mathrm{Th}$ decay chains [199]. As a gas, radon can escape material and enter the atmosphere, where 


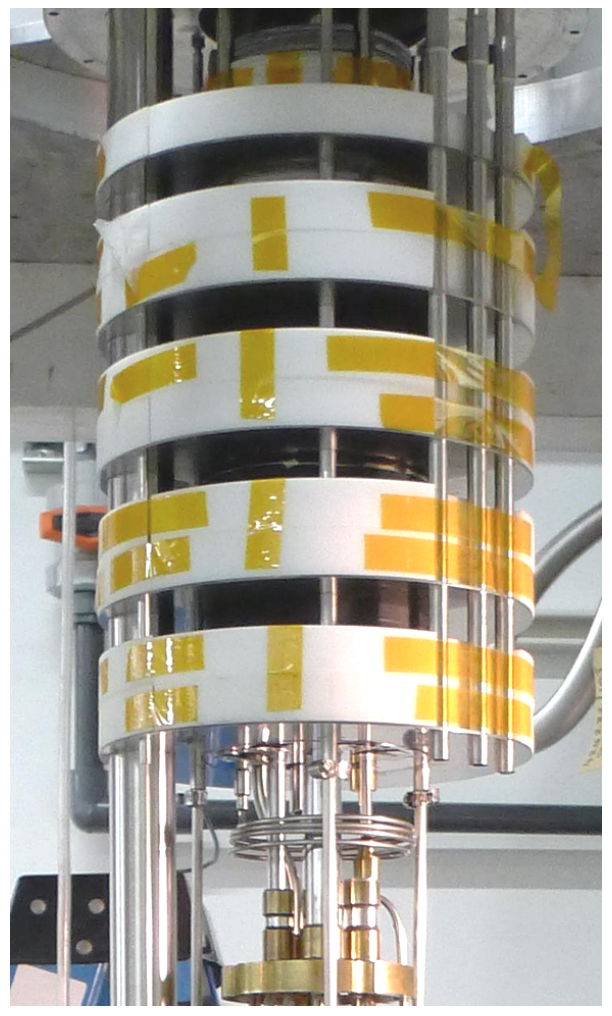

Figure 3.20: (LEFT) Nine inner layers of 12" diameter, 1" thick polyethylene shielding supported by baffles. At the top, additional polyethylene supports four layers of 4" diameter, 1/8" thick lead. (BOTTOM) Neutron and gamma shield erected around main bath, including 8" of polyethylene and 0.5 " lead top shielding between main bath and shield walls. Lead top shielding stacked atop polyethylene not shown.

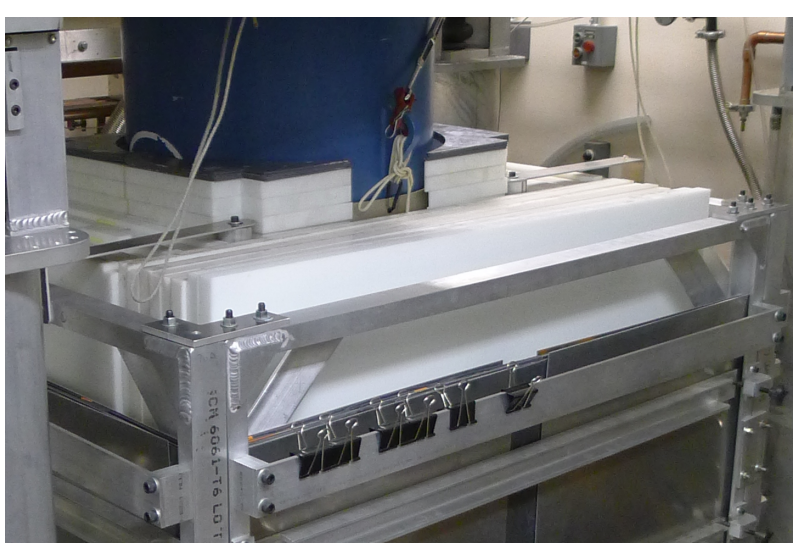


it can then attach itself to exposed surfaces, called "plate-out". From the ${ }^{238} \mathrm{U}$ decay chain, ${ }^{222} \mathrm{Rn}$ has a half-life of 3.82 days, making it possible for large quantities to escape and plate-out to nearby surfaces. This explains the typical ${ }^{222} \mathrm{Rn}$ air contamination level found in laboratory/dwelling, $40 \mathrm{Bqm}^{-3}$ [188.

Once adhered to a surface ${ }^{222} \mathrm{Rn}$ can decay into several isotopes which emit $\gamma$ and $\beta$ particles. Fortunately, most of these isotopes along the decay chain have short half-lives. However, the daughter isotope ${ }^{210} \mathrm{~Pb}$ has a half-life of $22 \mathrm{yrs}$, and its decay chain results in the production of a $1.62 \mathrm{MeV} \beta$, and a $5.3 \mathrm{MeV} \alpha$, among other products [199. If ${ }^{210} \mathrm{~Pb}$ decay occurred on a detector surface, the resulting products could appear as nuclear recoils and surface events.

To avoid contamination, detectors and cold hardware are stored in a class 10,000 clean room in the NE corner of the test facility lab. (Shown in Figure 3.2. Facility workers don clean suits, face masks, hair nets, and latex gloves in the clean room's anteroom before entering. Inside the clean room, detectors, towers, and other various cold hardware are stored in two N2 purge cabinets when not in use. Detector mounting and electrical checks occur on a class 1000 clean bench within the clean room. A second bench with an electrostatic discharge safe mat and laptop provides additional space for other clean room tasks, such as soldering cold electronics.

During detector installation procedure, the detector is double bagged in plastic before being removed from the clean room and placed in the "clean tent", a plastic enclosure that encapsulates the lower portion of the IVC can. Air passes through a HEPA filter before entering the clean tent, and a positive pressure keeps wandering dust and debris particles out. Measured to be of the order class 100, the clean tent provides a sufficient clean space for 1 - 2 people and installation equipment. As with the tower, all cold hardware and installation equipment is stored in the clean room to avoid passing along contaminant particles. Before every use, the inner walls of the clean tent are wiped down with isopropanol to remove any accumulated dust.

\section{$3.6100 \mathrm{~mm}$ iZIP Detectors}

The Minnesota facility has been testing prototype $100 \mathrm{~mm}$ detectors since 2011, testing a total of eleven $100 \mathrm{~mm}$ devices, often in multiple data runs. This section will summarize 
the tests conducted at Minnesota, providing a detailed description of detector G106a, a $100 \mathrm{~mm}$ Ge iZIP detector that will be the focus of Chapters 4 and 5 .

\subsubsection{Overview of Prototype $100 \mathrm{~mm}$ Detectors}

Initial prototype devices were two four-channel, ionization only (4Q) devices, called G101a and G102a. Both devices were fabricated at the Stanford Linear Accelerator Center (SLAC), using [100], n-type Ge crystals grown by Umicore. Crystals were patterned with four concentric equal-area charge channels on one side, and a single ground plane grid on the other. The purpose of these devices was to ensure that charge collection, resolution, and neutralization were not significantly worsened in the scaling up. G102a demonstrated distinct source peaks on each channel for three source configurations, and good collection efficiency at the SuperCDMS iZIP operating field strength $\left(1.6 \mathrm{Vcm}^{-1}[183]\right)$.

However G101a was found to be a defective detector, with significantly reduced event rates on two of the four channels, and a double peak from a single gamma source seen on one channel. It is possible that a scratch on the detector caused by the OGP scanner caused these issues; Regardless, after the first run at UMN, G101a was repolished and fabricated into a spiral detector, G101b.

The refabricated G101b possessed a spiral mask consisting of interleaved charge electrodes and grounded rails on both faces of the crystal. The detector featured two charge channels on each side (Qi and Qo), and no phonon channels. Starting in 2012, G101b was tested several times at UMN, with the goals of: investigating the effectiveness of a charge symmetry cut for identifying surface events induced by a ${ }^{210} \mathrm{~Pb}$ beta source and a ${ }^{241} \mathrm{Am}$ gamma source, continued studies on $100 \mathrm{~mm}$ neutralization, and determining the charge collection efficiency as a function of electric field strength using ${ }^{241} \mathrm{Am} 60$ $\mathrm{keV}$ gammas that interact close to the surface. G101b demonstrated sufficient neutralization (no decay in peak amplitude over 10 minutes of run time), good charge collection efficiency ( $>80 \%$ for $\geq \pm 2 \mathrm{~V}$ applied bias), and a significant portion of surface events could be identified with a symmetry cut on side 1 and side 2 charge signals.

Within a month of the initial G101b, the test facility studied its first $100 \mathrm{~mm}$ iZIP detector. Two Ge iZIPs were sent to UMN in early 2012, G103a and G106a. Discussed in detail in Section 3.6.2, both detectors had six phonon channels and two 
charge channels per face. Although the iZIP mask was the same for both detectors, the crystals themselves differed; G103a is a [111] orientation crystal purchased from Ortec, while G106a has a [100] orientation, and was purchased from Umicore. Focus will be given in this thesis to G106a, due to good performance and the sheer amount of data accumulated with this detector. Chapters 4 and 5 will focus on the $100 \mathrm{~mm}$ iZIP full characterization analysis, and therefore it will not be discussed here.

Following the success of the initial $100 \mathrm{~mm}$ iZIP devices, several other Ge iZIP detectors were fabricated and tested. G110 and G112 were fabricated by Texas A\&M University, using Ortec crystals, and G114 was fabricated at SLAC using another Umicore crystal. It should be noted that G103a, G110, and G112 all experienced low voltage charge breakdown, a phenomenon where distortions occur in the charge channel signals due to the crystal beginning to conduct current. Other $100 \mathrm{~mm}$ devices showed breakdown at $\pm>10 \mathrm{~V}$, if at all, but all three Ortec-boule detectors showed breakdown distortions at $\pm<6 \mathrm{~V}$; the cause of this is currently unknown.

Separate from SNOLAB iZIP development, USD1, a 4Q device fabricated by TAMU using a University of South Dakota grown Ge crystal, was tested in 2016. The device has the standard four concentric charge channels on one face, but a solid (non-gridded) ground channel on the other crystal face. The purpose of the test was to determine whether the USD-grown crystals would make suitable CDMS detectors. During the run, USD1's channel Q2 showed a high resistance during the LN2 stage electrical checkout, and proceeded to produce weak pulses throughout data taking. Of the remaining channels, only Q4, the outermost ring, produced a visible $60 \mathrm{keV}$ from the ${ }^{241} \mathrm{Am}$ sources. It is unknown why channels Q1 and Q3, which worked otherwise, did not display the peak; a triggering issue might be to blame, and another look at the detector is warranted. Results of the USD1 detector study revealed that the crystal did not see charge breakdown up to $\pm 13 \mathrm{~V}$, but that the detector's charge collection efficiency as a function of voltage bias was worse than other (Umicore and Ortec) detectors. The poor collection efficiency might have been related to neutralization. The crystal lost neutralization quickly, and attempts to re-neutralize the crystal did not seem effective. This could have been due to the flashing method utilized, or possibly the solid Al ground plane on the non-readout side of the detector.

Most recently, tests have been conducted on prototype HV Si and Ge detectors, 
S101 and G101c. Based on the success of CDMSlite, high voltage detectors, $100 \mathrm{~mm}$ $\mathrm{HV}$ detectors are comprised of six phonon channels per detector face: two outer rings, three middle channels (with $45^{\circ}$ rotation between faces), and an inner disk channel. The HV detectors do not utilize ionization channels, allowing more TES coverage and thus superior phonon collection than standard iZIPs.

High voltage studies with iZIP detectors began in 2015 with tests on G114 and G106a, using an iZIP v6 HV interface board fabricated by N. Mast based on designs implemented in CDMSlite. HV detector studies are ongoing at UMN, but initial results suggest a phonon collection efficiency of $15-22 \%$ [200] (larger than traditional iZIP collection efficiency of $\sim 13 \%$ [178, and meeting the expected value of $15 \%$ determined for the detector), and energy resolution of $0.6 \mathrm{keV}$ (at $0 \mathrm{~V}$ ).

\subsubsection{G106a}

G106a began as a $102.5 \mathrm{~mm}$ diameter, $35.0 \mathrm{~mm}$ thick, $1606 \mathrm{~g}$, orientation [100], high purity n-type germanium crystal, purchased from Umicore in 2012. The crystal has an etch pitch density of $3183-4900 \mathrm{~cm}^{-2}$, an impurity concentration of $8.10 \mathrm{E}+9$ $1.38 \mathrm{E}+10 \mathrm{~cm}^{-3}$, and Hall mobility of $22.7-26.5 \mathrm{~cm}^{2} / \mathrm{Vs}$. The crystal was fabricated at SLAC with a version 6 iZIP mask, specifically designed for testing at the UMN facility.

Each G106a QET consists of one $260 \mu \mathrm{m} \times 27 \mu \mathrm{m}$ W strip connected to eight $\sim 300$ $\mu \mathrm{m} \times 50 \mu \mathrm{m} \mathrm{Al}$ fins (4.5 $\mu \mathrm{m}$ of $\mathrm{Al} / \mathrm{W}$ overlap). Charge electrode lines are $50 \mu \mathrm{m}$ wide, with adjacent charge and phonon sensors separated by a $1.6 \mathrm{~mm}$ gap.

G106 has six phonon channels per face as shown in Fig. 3.21: an outer ring channel (A; from radius $44.8-50.0 \mathrm{~mm}$, total area $\sim 1550 \mathrm{~mm}^{2}$ ), four quadrant middle channels (B,C,D, and E; from radius $19.2-44.8 \mathrm{~mm}$, total area $\sim 1287 \mathrm{~mm}^{2}$ each) and inner disk channel ( $\mathrm{F}$; from radius $0.0-19.2 \mathrm{~mm}$, total area $\sim 1158 \mathrm{~mm}^{2}$ ). There is a $45^{\circ}$ channel offset between detector faces, for position reconstruction. The detector also has a $43.2 \mathrm{~mm}$ radius inner disk and $6.8 \mathrm{~mm}$ wide outer ring ionization electrodes on each detector face.

G106a has been the most tested detector at the UMN testing facility, with eleven data runs spanning over three years. G106a detector characterization will focus on data accumulated during 2015 - 2016, when filters where incorporated in the readout chain, resulting in cleaner phonon pulses than in previous G106a runs. 


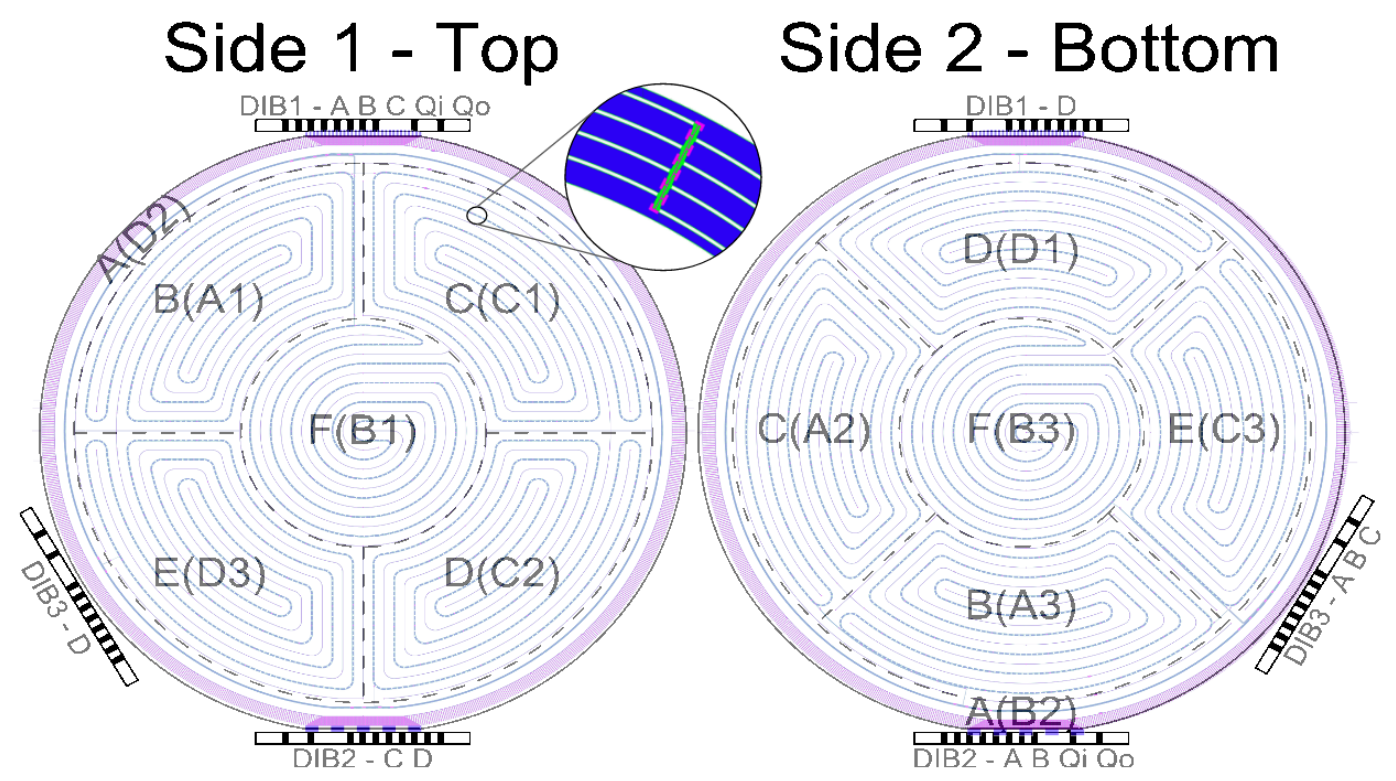

Figure 3.21: G106a side 1 and side 2 phonon channel layout overlaid on $100 \mathrm{~mm}$ iZIP mask. A zoom in on the phonon mask shows $100 \mathrm{~mm}$ iZIP QET design, with tungsten (green) TES strip overlapping aluminum fins (blue), all deposited on a layer of amorphous silicon (pink). 
Table 3.4: Table of $100 \mathrm{~mm}$ devices tested at UMN

\begin{tabular}{|c|c|c|c|}
\hline Device Name & Crystal & Mask Type & Fridge Runs \\
\hline G101a & Umicore, [100], n-type & SLAC 4Q & 11 \\
\hline G102a & Umicore, [100], n-type & SLAC 4Q & $20,22,23$ \\
\hline G101b & Umicore, [100], n-type & SLAC Spiral v2 & $28,36,37,41,44,45$ \\
\hline G103a & Ortec [111], p-type & SLAC iZIP v6 & 31,32 \\
\hline G106a & Umicore [100], p-type & SLAC iZIP v6 & $\begin{array}{c}33,34,35,39,46,52 \\
53,54,55,56,59(\mathrm{HV})\end{array}$ \\
\hline G110 & Ortec, [100], n-type & TAMU iZIP v6 & 52 \\
\hline G112 & Ortec, [100], p-type & TAMU iZIP v6 & 47 \\
\hline G114 & Umicore, [100] n-type & SLAC iZIP v6 & $43,57(\mathrm{HV})$ \\
\hline USD1 & USD & TAMU 4Q & 58 \\
\hline G101c & Umicore, [100], n-type & SLAC HV & 61 \\
\hline $\mathrm{S} 101$ & TOPSIL, [100], n-type & SLAC HV & 62 \\
\hline
\end{tabular}




\section{Chapter 4}

\section{0mm Detector Ionization Characterization}

The quality of a detector's ionization characteristics is described by several measurements: charge energy spectrum, neutralization, cross talk, and charge collection efficiency. This section aims to present evidence that G106a performs sufficiently in all of these regards, demonstrating the capabilities of $100 \mathrm{~mm}$ Ge iZIP detectors for use in the SuperCDMS SNOLAB experiment.

The data for this characterization, and the phonon characterization of the next chapter, come from UMN test facility Runs 54 and 56. Run 54 started in September of 2015 , with an ${ }^{241} \mathrm{Am}$ source, and occasionally an external ${ }^{133} \mathrm{Ba}$ source or ${ }^{252} \mathrm{Cf}$ neutron source. Initial data taking tested a wide range of applied biases (a process known as bias sweeping), with later data predominately using a \pm 4 and $\mp 4 \mathrm{~V}$ bias. The pre-MIDAS DAQ was used for Run 54 data taking. Run 56 started in January of 2016, with an internal ${ }^{210} \mathrm{~Pb}$ source, and occasionally an external ${ }^{133} \mathrm{Ba}$ source or ${ }^{252} \mathrm{Cf}$ sourc ${ }^{1}$ Run 56 data was taken with MIDAS DAQ, usually with a $\mp 4 \mathrm{~V}$ applied bias. For both runs, data series were limited to roughly 15 - 30 minutes, although there were some exceptions.

This section begins with a look at the quality cuts which must be applied to the

\footnotetext{
${ }^{1}$ Additional sources, such as ${ }^{88} Y$ and $\mathrm{PuBe}$, were used externally during some portions of this run, for projects which fall outside the scope of this thesis. However, none of the data accumulated when these sources were present is included in this analysis.
} 
charge RQ quantities as an initial analysis step. It then discusses the construction and desired characteristics of a charge spectrum. Next, it briefly covers the problem of cross talk, and the solution utilized by SuperCDMS. The chapter concludes with a look at G106a charge collection efficiency.

\subsection{Charge Quality Cuts}

Quality cuts are implemented to remove unwanted data events before analysis. Low quality or anomalous events are removed, because they are believed to not be representative of a given data sample. This could be due to the position of the event, amplitude of the event, degradation of detector performance, or noise in the system, to name a few examples. Cuts are typically implemented in the order presented here, unless otherwise stated.

\subsubsection{Charge $\chi^{2}$ Cut}

As discussed in section 2.2.3, charge pulse shape is dictated by the feedback components in the readout circuit, with little deviation expected from a roughly $1 \mu$ s rise time and $40 \mu \mathrm{s}$ fall time. This allows for charge templates to be constructed from the mean of a thousand well-behaved pulses, for use in the OF fitting algorithm. How well an individual pulse is reconstructed with the template is contained in its $\chi^{2} \mathrm{RQ}$ value, with $\chi^{2} \propto \sum_{n}\left|S_{n}-\hat{a} A_{n}\right|^{2}$, for an original signal $S_{n}$, template $A_{n}$ with maximized amplitude factor, $\hat{a}$. From this it can be seen that $\chi^{2}$ scales as $\hat{a}^{2}$, assuming a reasonable event reconstruction. Thus, well-behaved pulses tend to follow a parabolic relationship between $\chi^{2}$ and energy. Since the OF algorithm can only fit the pulse time shift to the nearest $\mathrm{ADC}$ bin $(0.4 \mu \mathrm{s}$ for charge, $0.8 \mu \mathrm{s}$ for phonons), the parabolic upward trend is broadened due to pulses fits with slight time shift inaccuracies.

However, alterations in signal could affect the pulse shape, increasing the $\chi^{2}$ value. Typically changes in signal are due to noise interference or pileup events. Pileup events, such as the event shown in Fig. 4.1, occur when two or more pulses occur within the time window of a single event. The OF algorithm fits to one pulse, and the second deviates heavily from the template, increasing $\chi^{2}$. To remove these poorly reconstructed events, a parabolic cut is introduced on both Qinner Side 1 and Side 2 values, (QiS1, and QiS2, 

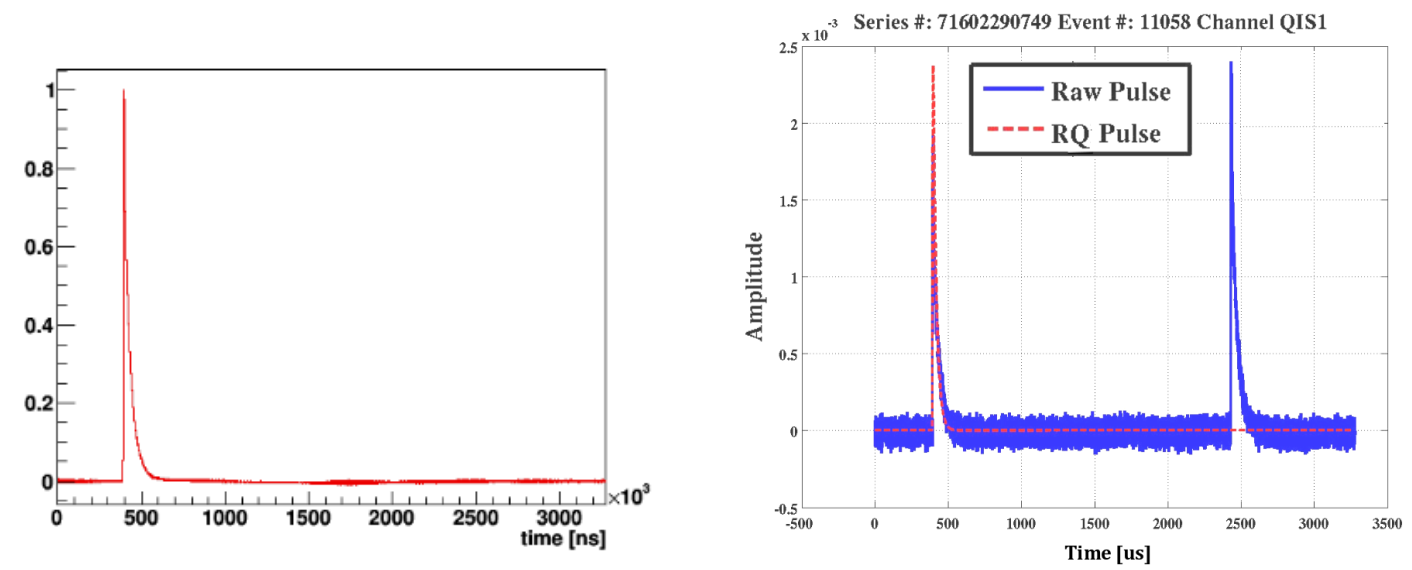

Figure 4.1: (LEFT) A charge template constructed by N. Mast from a thousand well behaved charge pulses. Template has typical $400 \mu \mathrm{s}$ (100 bins) pre-trigger region, a roughly $1 \mu$ s rise time and $40 \mu$ s fall time. Figure courtesy of N. Mast [201]. (RIGHT) A raw pileup QIS1 event (blue) is fit with a QIS1 template (red) using the OF fitting algorithm. Although the first pulse is reconstructed well by the fit, the second event is completely neglected. As a result, the $\chi^{2}$ of this fit will be high, and the event will be excluded. 
respectively), as shown in Fig. 4.2. Data must pass the $\chi^{2}$ cut on both sides to be considered well reconstructed.
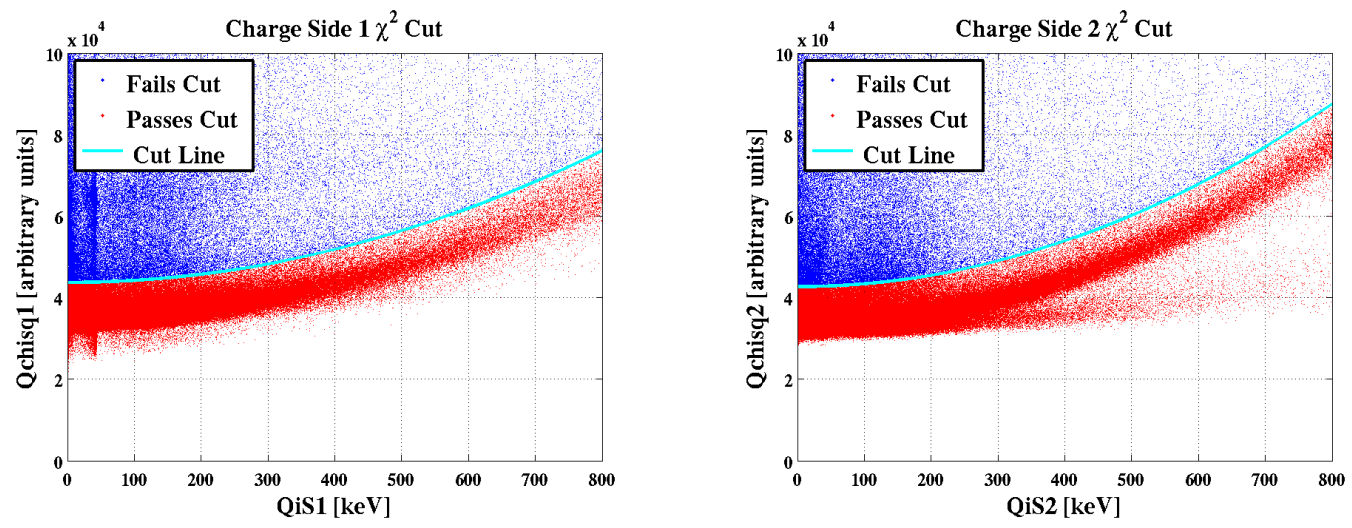

Figure 4.2: Charge $\chi^{2}$ versus Side 1 (LEFT) and Side 2 (RIGHT) calibrated ionization energy for G106a with $\mp 4 \mathrm{~V}$ applied bias. Events which pass the $\chi^{2}$ cut (red) fall below the parabolic cut line (cyan), and events which fail the cut (blue) fall above the line. An event must pass the cut on both detector sides to be considered a well constructed event.

\subsubsection{Charge Threshold Cut}

At low energies $(\lesssim 5 \mathrm{keV})$ it becomes difficult to distinguish actual events from noise events. Thus a charge threshold cut must be introduced, to remove events which are likely to be noise. To determine where the threshold should be set, a histogram is constructed of inner charge electrode amplitudes for events that are randomly triggered at the beginning of a data series. A Gaussian fit to the positive portion of the histogram is used to approximate the mean and the width of the noise event distribution. The threshold cut is made at $5 \sigma$ beyond the distribution mean. A threshold cut is made for both sides of the detector, since each side is read out by a different circuit, and thus noise environments can differ between sides. To pass the threshold cut the inner charge amplitudes of both detector sides must lie above their respective threshold values. Fig. 4.3 shows the randomly triggered event distributions, Gaussian fits, and thresholds for both sides of G106a. Threshold values are set at $2.8 \mathrm{keV}$ for Side 1, and $3.1 \mathrm{keV}$ for 
Side 2.
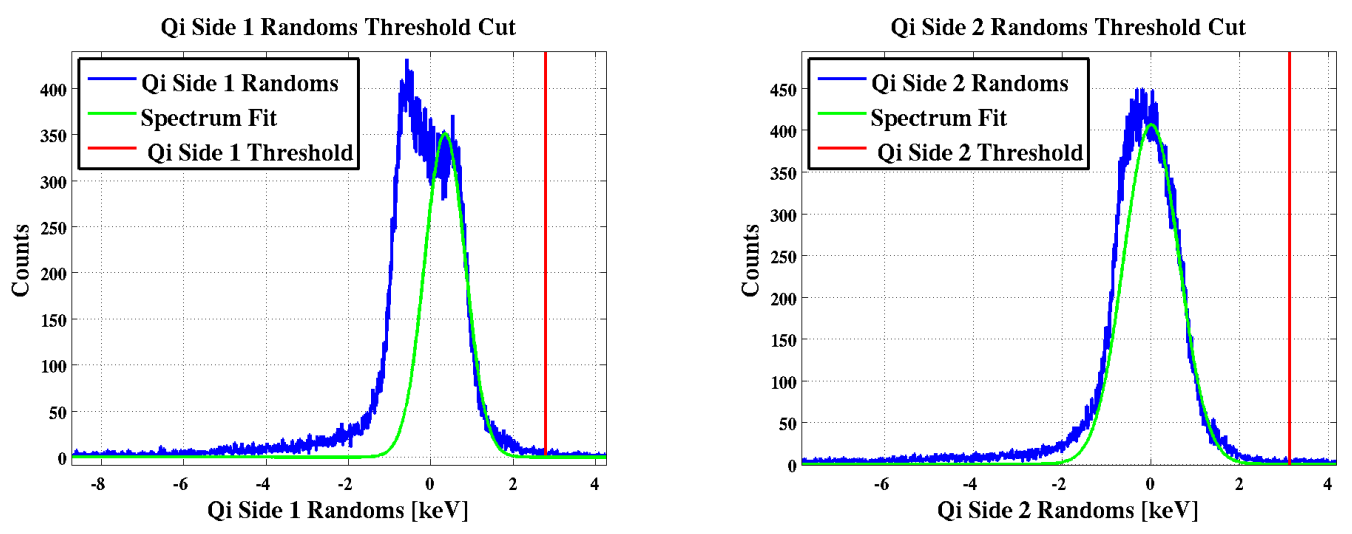

Figure 4.3: Distributions (blue) of Side 1 (LEFT) and Side 2 (RIGHT) inner charge amplitude distributions of beginning-of-run random events. A Gaussian (green) is fit to the positive portion of the distribution, to estimate the mean and width. A threshold cut (red) is made $5 \sigma$ beyond the mean.

\subsubsection{Charge Radial Partition Cut}

In an ideal experiment, the electric field applied across the detector would be uniform, but in the physical system the field deviates from uniformity at the outer edges of the crystal. A model of the electic field within a detector is shown in Fig. 4.4. The deviation reduces the ionization collection efficiency, since the electron/hole pairs are not fully collected.

The inner and outer charge channels allow one to easily circumvent this problem: events which occur predominantly in the inner channel reside within a fiducial volume with uniform electric field, whereas events with a significant outer channel energy cannot be assumed to be fully collected, and are thus removed. The cut which removes events with a significant outer channel energy component is called the charge radial partition cut, or the charge inner cut.

To create the cut, the zero time shift outer charge amplitude versus the inner charge amplitude is plotted for both sides. For slices of inner charge amplitude the mean and standard deviation of the outer amplitude is found. A polynomial function is fit to 


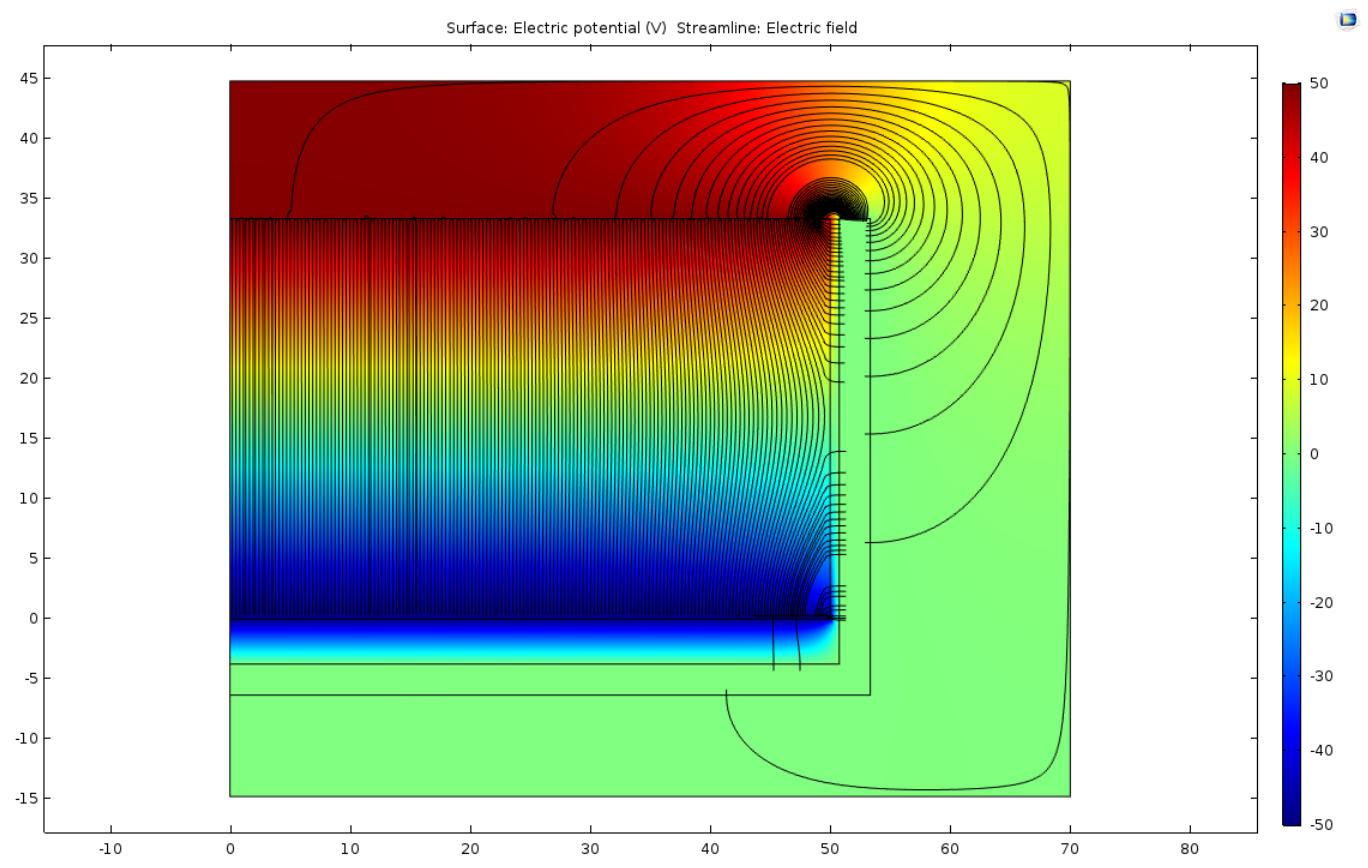

Figure 4.4: A COMSOL 202] simulation showing the electric field lines (black) and potential (color scale) of a Ge detector bias $\pm 50 \mathrm{~V}$ with a 30 mil gap between the crystal and the copper housing. Note the nonuniform electric field lines at the crystal's edge. Figure courtesy of N. Kurinsky [203]. 
the mean outer channel \pm twice the standard deviation, as shown in Fig. 4.5. Thus events with low amplitudes in the outer channels are contained within the polynomials. Events with significant outer channel component fall outside of the polynomial fit and are excluded. As with other cuts, an event must pass the radial charge partition cuts for both detector sides to not be cut.
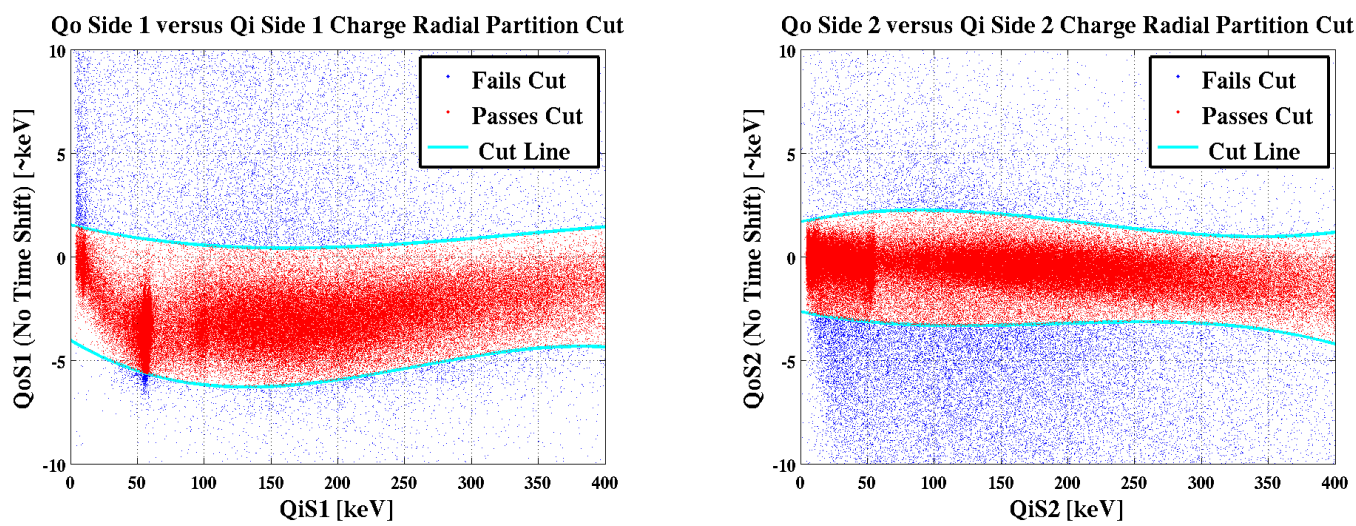

Figure 4.5: Charge outer amplitude (with zero time shift) versus inner amplitude for Side 1 (LEFT) and Side 2 (RIGHT). The mean and standard deviation of the outer amplitude are found for $10 \mathrm{keV}$ bands in inner charge amplitude, and a third-order polynomial function is fit for the mean outer channel \pm twice the standard deviation (cyan). Events within the polynomial fits (red) pass the cut, while events that fail (blue) are removed.

\subsubsection{Charge Baseline Cut}

As with $\chi^{2}$ values, the baseline value of a pulse can indicate data quality. The mean of the prepulse region of an event (the first $400 \mu$ s before the pulse occurs) is recorded as a baseline RQ value for each charge and phonon channel. Baseline is dependent on several variables such as channel noise, fridge temperature, and DAQ settings. It is expected for the baseline to drift during data runs, although significant deviations between adjacent series are often indicative of problems. If an individual event baseline deviates significantly from the typical value for the series, event pileup or excess noise is the likely culprit. These events can be removed with a charge baseline cut. 

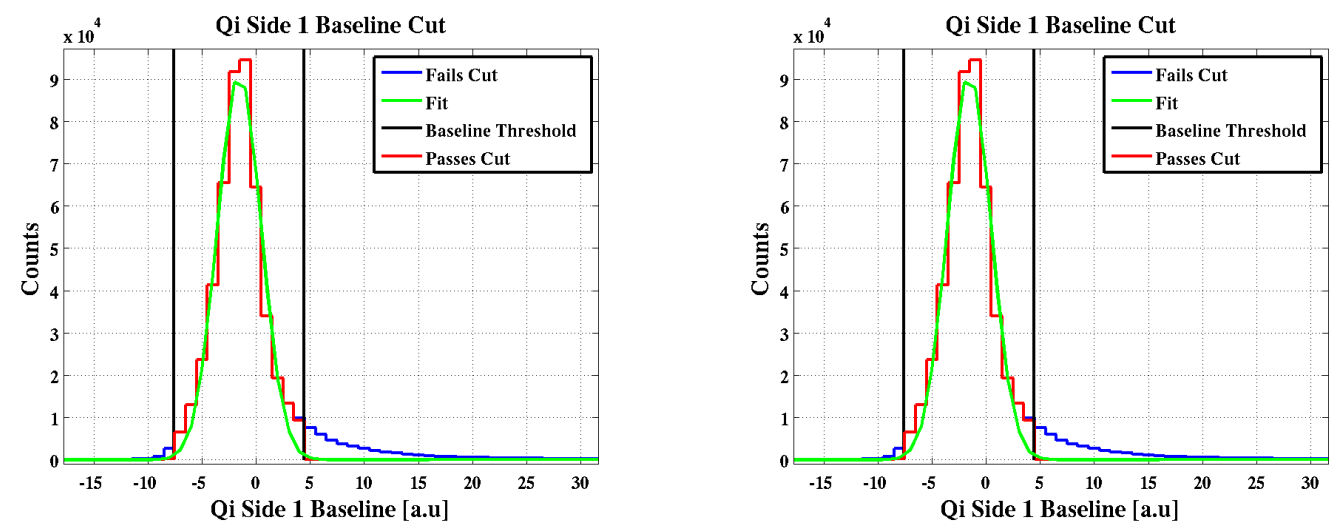

Figure 4.6: Distribution of mean-subtracted baseline values for (LEFT) Qi Side 1 and (RIGHT) Qi Side 2 after $\chi^{2}$ cut is applied. Distributions are fit with a Gaussian (green), and baseline thresholds are set to $\pm 3 \sigma$ (black). Baselines within the thresholds are kept (red), while events with baselines outside the thresholds are removed (blue).

Since baseline varies between series, a charge baseline cut must compare an individual event to the typical baseline of its series. To do so, the average baseline is calculated for QiS1 and QiS2 for each series. Shown in Fig. 4.6, a distribution of the difference between each event baseline and the series' average baseline is fit with a Gaussian. Baseline thresholds are set at $\pm 3 \sigma$ for the QiS1 and QiS2 distributions. Events which fall outside of either of these thresholds are cut.

\subsection{Calibration and Charge Energy Spectra}

In an ideal situation, the ionization amplitude RQ's could undergo a straightforward conversion from volts to a physical energy unit such as $\mathrm{keV}$, effectively providing the total ionization energy of the electrons and holes collected by the electrodes of the given channel. Unfortunately, this conversion requires an accurate measurement of the amplifier amplification, which involves temperature dependent capacitances and resistances, as well as parasitic effects. Without a precise measurement of FET gain, a straightforward conversion cannot be applied. As an alternative measure of calibration, radioactive sources are used to provide specific $\gamma$ lines of known energy. 
Depending on the purpose of the study, calibration values can be found for Qinner quantities or the sum of Qinner and Qouter, know as Qsum1 and Qsum2 for Side 1 and Side 2, respectively. Either calibration method is acceptable as long as the radial partition cut is applied to keep the outer channel contribution to the total signal minimal. For this thesis, calibration values are found for Qsum quantities when exploring the wide range of bias voltages (when observing the full charge signal is important), and Qinner quantities once the optimal voltage has been selected. To determine the calibration value, the charge Side 1 and Side 2 spectra are plotted with quality cuts applied to remove bad events. The energy collected from the electrons/holes depends on the applied voltage (due to charge trapping in the crystal), so only data series with the same applied voltage should be included in a spectrum for calibration. Typically, charge channel calibration values remain fairly consistent, even over long run periods (roughly $<10 \%$ change from series to series). However, individual series calibration values can be found and applied to improve the resolution of many-series energy spectra, assuming the source peak has a sufficient number of calibration source events above background to be identified in each individual series.

A high-quality ionization energy spectrum should clearly show peaks due to radioactive sources and be relatively consistent between charge channels. To calibrate the spectrum, a Gaussian (to fit the radioactive source peak) plus an exponential decay (to fit the low energy background) is fit to the source peak energy region, as shown in Fig. 4.7. The calibration value that can be applied to the entire spectrum will set the mean of the fitted peak to known energy of the source peak. If other peaks are present, they can be used to verify the calibration accuracy at other energies.

The most commonly used calibration peak is the ${ }^{241} \mathrm{Am} 60 \mathrm{keV}$ peak. However, photons from this source only have a penetration depth of $\sim 1 \mathrm{~mm}$ in Ge, and thus tend to produce surface events. The ionization energy of surface events will only be fully collected on the nearest side, due to the lateral surface electric field produced by the interleaved electrodes. Therefore, the detector side on which the ${ }^{241} \mathrm{Am}$ is held will see a reliable $60 \mathrm{keV}$ peak. The other side will see a peak smeared out, due to incomplete charge collection. This phenomenon can be most clearly seen in a Qsum1 versus Qsum2 plot, such as the right plot of Fig. 4.7. $60 \mathrm{keV}$ peak smearing in Qsum1 is due to gammas that escape after depositing a portion of their energy. Gammas are 

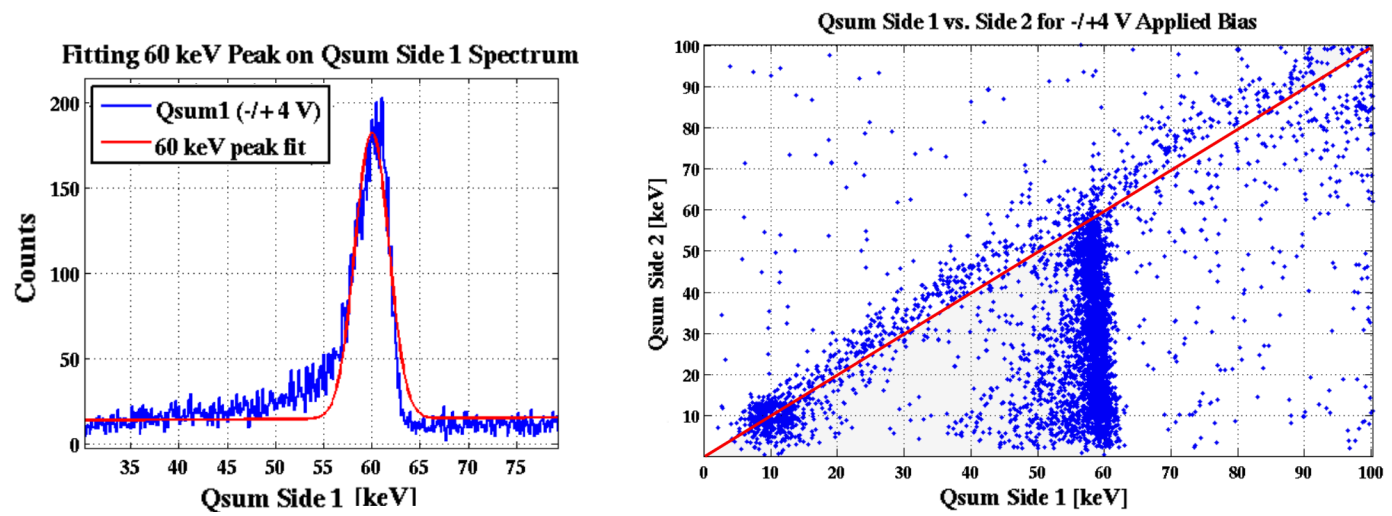

Figure 4.7: (LEFT) The $60 \mathrm{keV}$ peak of a Qsum1 spectrum (blue), fitted with a Gaussian (to fit peak) plus an exponential decay (to fit low energy background) (red). This fitting method is used for calibration and energy resolution studies. (RIGHT) Qsum2 versus Qsum1 demonstrates the incomplete charge collection of surface events. An ${ }^{241} \mathrm{Am}$ source held near Side 1 of the detector produces a $60 \mathrm{keV}$ peak in Qsum1. Due to incomplete charge collection, Qsum2 sees a source peak smudged out from $60 \mathrm{keV}$ down to $0 \mathrm{keV}$. $60 \mathrm{keV}$ peak smearing in Qsum1 is due to gammas that escape after depositing a portion of their energy. Gammas are more likely to scatter and escape near the surface, which explains why $60 \mathrm{keV}$ peak smearing in Qsum1 is more apparent for the smallest Qsum2 values. Events which occur in the bulk of the detector should deposit equal energy onto side 1 and side 2 ionization channels. These bulk events can be seen as the linear band of events with slope 1 (red line). 
more likely to scatter and escape near the surface, which explains why $60 \mathrm{keV}$ peak smearing in Qsum1 is more apparent for the smallest Qsum2 values. It is possible to calibrate the side farthest from the ${ }^{241} \mathrm{Am}$ using the $60 \mathrm{keV}$ peak, if a charge symmetry cut is first applied; discussed in detail in Section 5.3.4, a charge symmetry cut removes surface events by requiring near identical charge collection on both sides of the detector. Fig. 4.7 also demonstrates an alternative fitting method: if one side of the detector is properly calibrated, the other side can be calibrated by requiring bulk events to follow a linear trend with slope of one for Qsum1 versus Qsum2.

As an alternative to the $60 \mathrm{keV}{ }^{241} \mathrm{Am}$ peak, one might calibrate using the $356 \mathrm{keV}$ peak of the external ${ }^{133} \mathrm{Ba}$ source, or the $10.36 \mathrm{keV}$ peak that results from neutron activation with an external ${ }^{252} \mathrm{Cf}$ source. Both the $10.36 \mathrm{keV}$ and the $356 \mathrm{keV}$ peaks occur within the bulk of the crystal, making them accurate for calibrating both sides. Each source has its own advantages and disadvantages. ${ }^{241} \mathrm{Am}$ is an internal source, and thus there are no attenuation effects from passing through metal in the cryostat. Additionally, the $60 \mathrm{keV}$ peak resides in the energy region most relevant for CDMS detectors $(\sim 0-100 \mathrm{keV})$, while being easily distinguished from low energy background events. However, the internal ${ }^{241} \mathrm{Am}$ source is localized, not probing the entire detector volume, and it contributes to a higher event rate. The ${ }^{252} \mathrm{Cf} 10.36 \mathrm{keV}$ activation peak is useful for probing the low energy capabilities of detectors, with the added benefits of creating bulk events and having a minimal effect on event rate. However, it can sometimes be difficult to distinguish the ${ }^{252} \mathrm{Cf}$ activation peak from low energy background events. Also, the ${ }^{252} \mathrm{Cf}$ source works through neutron activation, where thermal neutron capture on ${ }^{70} \mathrm{Ge}$ results in a $10.36 \mathrm{keV}$ X-ray; this activation method cannot be used with Si detectors. As an external source, ${ }^{133} \mathrm{Ba}$ doesn't overwhelm the event rate, but the high energy source peak is outside of the relevant energy region. ${ }^{133} \mathrm{Ba}$ can be used with $\mathrm{Ge}$ and $\mathrm{Si}$, and it creates bulk events.

Because over eighty series over several months were used for this analysis, the following calibration method was used:

1. Calibrate QiS1 (or Qsum1) and Pt (total phonon) of each individual series by fitting the ${ }^{241} \mathrm{Am} 60 \mathrm{keV}$ peak with a Gaussian plus background. This serves as an approximate relative calibration between series. 
2. Calibrate QiS2 (or Qsum2) for each individual series such that the bulk events in QiS2 (Qsum2) versus QiS1 (Qsum1) plot have slope of one.

3. Determine quality cuts using the approximate relative calibration values found above. With quality cuts applied recalibrate QiS1 (Qsum1) and QiS2 (Qsum2) for each individual series by such that bulk events in QiS1 (Qsum1) versus Pt and QiS2 (Qsum2) versus Pt both have a slope of one in the high energy region dominated by electron recoils $(\sim 100-400 \mathrm{keV})$. This provides the final relative calibration between series.

4. Using all background data series (no ${ }^{252} \mathrm{Cf}$ source present to produce extra NR events) with quality cuts applied, calibrate QiS1 (Qsum1), QiS2 (Qsum2), and Pt by fitting $10.36 \mathrm{keV}$ peak with a Gaussian plus background.

5. Likely due to saturation of some phonon sensors, Pt demonstrates nonlinear calibration behavior in the $0-150 \mathrm{keV}$ region, when compared to charge signal amplitude. This can be corrected by applying a nonlinear calibration in that energy region. The nonlinearity and correction are shown in Fig. 4.8 .

6. The total phonon spectrum's $10.36 \mathrm{keV}$ peak should actually lie at $10.36(1+V / \epsilon)$ to account for Luke component of phonon energy. Multiply the total phonon calibration by the factor $(1+V / \epsilon)$ to get the true Pt calibration factor.

This method ensures that any changes in the system that might alter calibration factors over time have been accounted for, and that all energy RQ's were properly calibrated with respect to one another. For a given bias, calibration factors vary by as much as $30 \%$ over the whole Run 54 . However, calibration values typically stay within $<10 \%$ between consecutive series.

The careful reader might have noted that the charge quantity cuts outlined in Section 4.1 included figures of properly calibrated energy values. Often times analysis relies on a cyclical process of applying cuts, determining calibration values, modifying cuts to accurately reflect true energy values, and possibly re-calibrating. This process is particularly important in quality cuts which compare charge and phonon energies, or charge energies from different sides of the detector. 

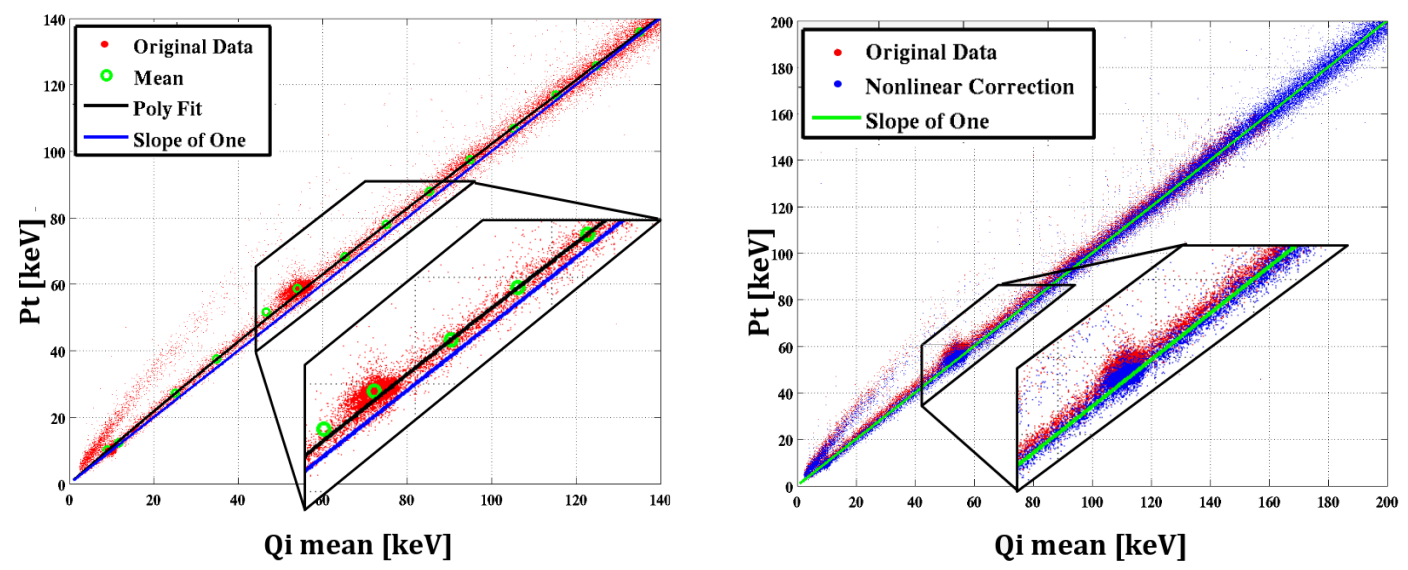

Figure 4.8: (LEFT) The nonlinearity of Pt with respect to the mean of QiS1 and QiS2, is made apparent by comparing Pt versus Qimean (red) to a line of slope one (blue). Fitting a fourth order polynomial (black) to the mean Pt values for slices of Qimean energy (green circles) yields a nonlinear correction. (RIGHT) Applying the nonlinear correction to the original data (red) yields data with a linear relationship between Pt and Qimean (blue), which follows a linear trend with slope of one (green). 



Figure 4.9: Qsum1 and Qsum2 spectra for (TOP) $\mp 2$ V, (TOP MIDDLE) $\mp 4$ V, (Bottom MIDDLE) $\pm 2 \mathrm{~V}$ (Bottom ) $\pm 4 \mathrm{~V}$ applied bias, calibrated using $60 \mathrm{keV}$ peak. Visible peaks include the $10.36 \mathrm{keV}$ peak from activation with ${ }^{252} \mathrm{Cf}$, the ${ }^{241} \mathrm{Am} 60 \mathrm{keV}$ peak, and the ${ }^{133} \mathrm{Ba} 356 \mathrm{keV}$ peak. Each spectrum is comprised of 2 - 5 data series, with $\chi^{2}$ cut applied to remove poorly fit events. 
Fig. 4.9 shows the Qsum1 and Qsum2 spectra for $\pm 4 \mathrm{~V}, \mp 4 \mathrm{~V}, \pm 2 \mathrm{~V}$, and $\mp 2 \mathrm{~V}$ applied bias, calibrated to the prominent $60 \mathrm{keV}$. As expected for well-behaved data, the spectra are consistent between detector sides. Both spectra show a prominent 10.36 $\mathrm{keV}$ peak, and a typical background hump centered around $200 \mathrm{keV}$ and falling off by $400 \mathrm{keV}$. Because the ${ }^{241} \mathrm{Am}$ source is near Side 1, Qsum1 has a prominent $60 \mathrm{keV}$ source peak. Qsum2 has a smeared out $60 \mathrm{keV}$ peak due incomplete charge collection of surface events. Since the external ${ }^{133} \mathrm{Ba}$ source was present during data taking, the $356 \mathrm{keV}$ source peak can be seen on the falling edge of the background hump for Qsum1 for $\mp 4 \mathrm{~V}$ data, and Qsum2 for $\pm 4 \mathrm{~V}$ data. Due to the oblique propagation of electrons (resulting in larger charge losses), hole-collecting sides of the detector typically have better resolution than electron-collecting sides. It should also be noted that the $356 \mathrm{keV}$ peak is not actually located at $356 \mathrm{keV}$ for the $\mp 4 \mathrm{~V}$ spectra. This possibly indicates some nonlinearity in the spectrum calibration. Calibrating these spectra to the $10.36 \mathrm{keV}$ peak (with only the $\chi^{2}$ cut applied) results in $3 \%-12 \%$ deviations in $60 \mathrm{keV}$ and $356 \mathrm{keV}$ peak locations from their expected values. Applying quality cuts improves peak resolution, and typically reduces the magnitude of these deviations, which is why calibration is done before and after cuts are applied.

\subsubsection{Charge Resolution}

Along with consistent and well-understood features, the energy resolution of the spectra's peaks is a significant indicator of the detector's performance. Detector resolution can limit the quality of many quantities, including the ionization yield used to distinguish recoil type. Resolution can also significantly influence the accuracy of physical models and simulations of the CDMS experiment. SuperCDMS SNOLAB is projected to have $100 \mathrm{eV}(110 \mathrm{eV})$ ionization energy resolution for $\mathrm{Ge}(\mathrm{Si})$ iZIPs at $0 \mathrm{keV}$ [135], based on ionization readout electronics studies [167]. Even though the UMN test facility is limited by prototype readout electronics and a high noise environment, demonstrating the lowest possible resolutions for $100 \mathrm{~mm}$ iZIPs is a longstanding goal.

To determine charge energy resolution the pulse-less noise traces $(0 \mathrm{keV})$, as well as $10.36 \mathrm{keV}, 60 \mathrm{keV}$, and $356 \mathrm{keV}$ peaks were fitted for $\mp 4 \mathrm{~V}$ data. Five 30-minute data series with the ${ }^{133} \mathrm{Ba}$ source present were used for the $356 \mathrm{keV}$ peak fit, and an additional six ${ }^{241}$ Am-only series were used for the other fits. Results, shown in Table 4.1 . 
Table 4.1: G106a Charge Energy Resolutions for 干4 V Applied Bias

\begin{tabular}{cccc}
\hline \hline & & & \\
Expected Energy & QiS1 & QiS2 & Qimean \\
Value $[\mathrm{keV}]$ & Peak Mean $\pm \sigma[\mathrm{keV}]$ & Peak Mean $\pm \sigma[\mathrm{keV}]$ & Peak Mean $\pm \sigma[\mathrm{keV}]$ \\
\hline & & & \\
0 & $-0.033 \pm 0.528$ & $-0.051 \pm 0.461$ & $-0.033 \pm 0.362$ \\
10.4 & $9.92 \pm 0.71$ & $10.3 \pm 0.9$ & $10.1 \pm 0.5$ \\
59.5 & $58.5 \pm 0.7$ & $53.3 \pm 1.6$ & $55.8 \pm 1.0$ \\
356 & $356 \pm 4$ & $358 \pm 16$ & $357 \pm 6$ \\
& & & \\
\hline
\end{tabular}

demonstrated that G106a charge resolution reaches as low as $\sim 300 \mathrm{eV}(1 \sigma$ width at 0 $\mathrm{keV}$ ) for the mean of QiS1 and QiS2, called Qimean, comparable to SuperCDMS Soudan Qsum resolutions 2 , which range from $291 \mathrm{eV}$ to $455 \mathrm{eV}$, depending on the iZIP (using the same type of readout electronics) [204]. Despite the test facility noise environment and readout system, $100 \mathrm{~mm}$ iZIPs show potential for reaching SuperCDMS SNOLAB resolution goals.

The study also demonstrated the effects of applied bias on resolution. Fig. 4.10 illustrates the change in resolution of the Qimean $10.36 \mathrm{keV}$ peak as a function of applied bias. A larger bias tends to increase the charge collection efficiency, resulting in improved resolution. Due to the oblique propagation of electrons, it is expected that the hole-collecting side (whichever side is negatively biased) will see better event resolution. This result is verified here.

\subsection{Cross Talk}

Cross talk occurs when the distinct charge channels influence one another in various ways. Typically crosstalk between inner and outer charge channels is due to mutual capacitance of the electrodes. Cross talk can be seen when comparing inner and outer

\footnotetext{
${ }^{2}$ Resolutions cited here are for the RQ value that is the maximum of either Qsum1 or Qsum2 for the event.
} 


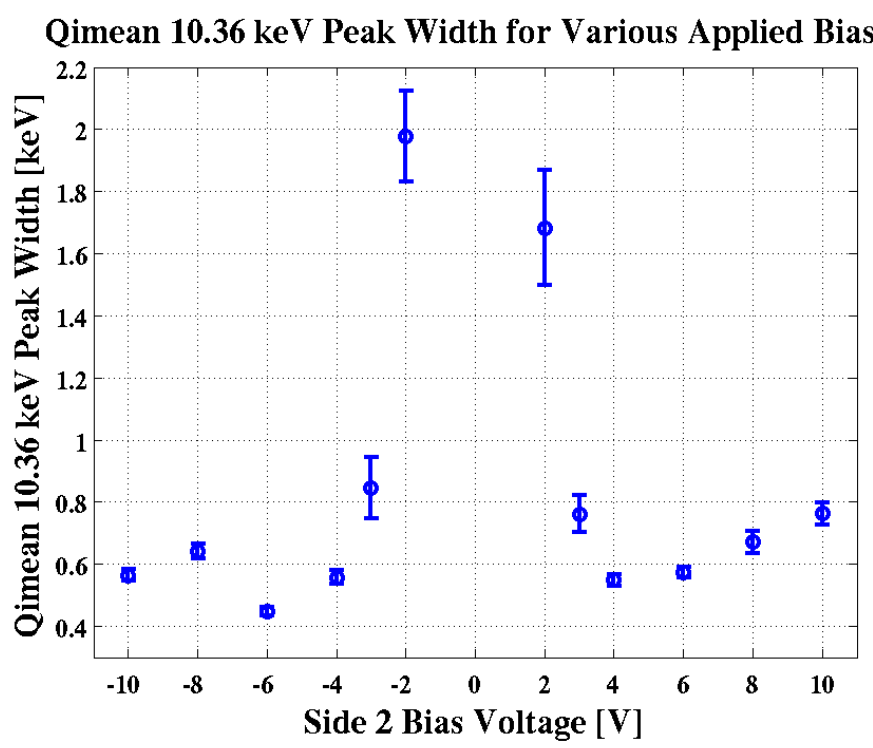

Figure 4.10: Qimean $10.36 \mathrm{keV}$ peak width $(1 \sigma)$ versus applied bias.

charge amplitudes. The left plots of Fig. 4.11 show the outer charge amplitude's distinct dependence on the inner channel, instead of being random with respect to the outer amplitude. Cross talk relationships are not strictly linear, but corrections assuming linearity are sufficient. Although cross talk is an expected phenomenon, a good iZIP detector should demonstrate a manageable level of cross talk that can be corrected.

Typically a cross talk correction for CDMS data is introduced in the OF fitting process. The ongoing effort to implement this method at the UMN facility is documented in Appendix B. Difficulties in template normalization and isolating the cross talk contribution of each channel's signal have resulted in unsuccessful utilization of the OF cross talk correction at UMN. Instead, cross talk corrections must be implemented by hand post-processing. A rotation in the Qi-Qo plane serves as the cross talk correction, with the angle of rotation, $\varphi$ determined by fitting a line to the linear relationship between outer and inner ionization channel signals. For a best-fit line with slope $\xi$, the angle of rotation is given by $\varphi=\arctan (\xi)$. The linear fit of Qo versus Qi and subsequent rotation are shown in Fig. 4.11. Both Side 1 and Side 2 cross talk corrections require a rotation of $<1^{\circ}$, and implementing the cross talk correction had no significant impact on data in the energy region of interest, $<100 \mathrm{keV}$. 

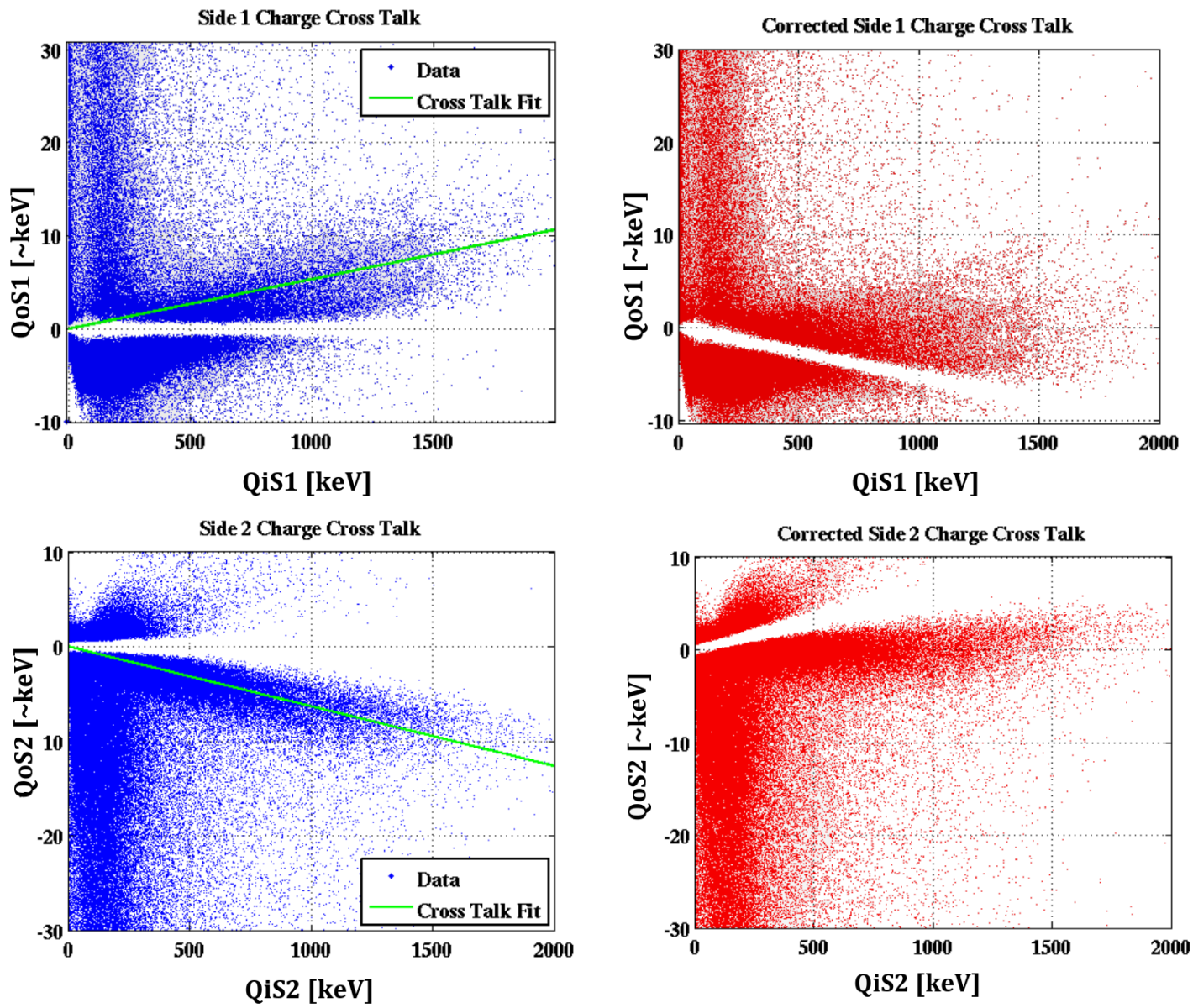

Figure 4.11: (LEFT) Cross talk is demonstrated here in the outer channel signal amplitude's near-linear dependence on the signal amplitude of the inner channel, for both (TOP) Side 1 and (BOTTOM) Side 2. A rotation is applied to correct for cross talk, with the angle of rotation determined by fitting a line (green) to the linear trend in Qo versus Qi (blue). (RIGHT) Qo versus Qi for (TOP) Side 1 and (BOTTOM) Side 2, with the cross talk correction rotation applied. 


\subsection{Neutralization and Timing Cut}

As a semiconductor detector is operated at milliKelvin temperatures, charge traps in the crystal gradually fill and become charged. Consequently, trapping centers start to interfere with the ionization signal readout, effectively reducing its charge collection. This is normal behavior for a detector, with the rate of collection efficiency degradation expected to be proportional to the total ionization energy deposited [186]. The effect of this loss of neutralization of the crystal is shown in the Side 1 charge signal amplitude versus time plots in Fig. 4.12. The $60 \mathrm{keV}$ peak of the Qsum1 spectrum is shown for several overlaid series, each 15 or 30 minutes long, and each with an applied bias of \pm 1 $\mathrm{V}, \mp 1 \mathrm{~V}, \pm 2 \mathrm{~V}, \mp 2 \mathrm{~V}, \pm 4 \mathrm{~V}$, or $\mp 4 \mathrm{~V}$. By visual inspection, peak decay can be seen in the $\pm 1 \mathrm{~V}, \mp 1 \mathrm{~V}$ within 10 minutes, but discerning a change in peak mean is more difficult for the higher biased data.

To quantify the change in peak location, a linear trend line is fit to Qsum1 versus time, providing an estimate for the rate of amplitude decay. Normalizing the initial peak mean to $60 \mathrm{keV}$ for each applied bias, rate of peak change and the estimated change in peak mean over a 30 minutes series are presented in Table 4.2 , Results indicate \pm 1 and $\mp 1 \mathrm{~V}$ data series follows a $\sim 3-5 \mathrm{keV}$ decay in peak mean over a 30 minute series. Applied biases of \pm 2 and $\mp 2 \mathrm{~V}$ see some improvement, but still observe a peak decay of $>1 \mathrm{keV}$ over a 30 minute series. Only biases of $\pm 4 \mathrm{~V} / \mp 4 \mathrm{~V}$ or greater see no significant peak decay within a 30 minutes series.

It is necessary that the detector can hold neutralization on time scales that allow for reasonable data collection ( $\gtrsim 10$ minutes for the UMN testing facility, due to increased event rate on the surface relative to underground facilities like Soudan or SNOLAB), and that a process referred to as flashing the detector restores neutralization.

Flashing occurs when the electrodes are grounded and LEDs with $940 \mathrm{~nm}$ wavelength irradiate the detector with a current of $0.6 \mathrm{~mA}$ in $100 \mu \mathrm{s}$ bursts with $5 \mathrm{~ms}$ pauses [205]. The wavelength corresponds to $1.32 \mathrm{eV}$, which is sufficient to excite electrons above the $0.8 \mathrm{eV}$ direct bandgap for $\mathrm{Ge}$, and the $1.1 \mathrm{eV}$ indirect bandgap for Si. The field produced by the trapping centers attracts the freed charges, re-neutralizing the detector. After $6 \mathrm{~s}$ of flashing the detector is allowed to cool back to base temperature before data collection is resumed. 

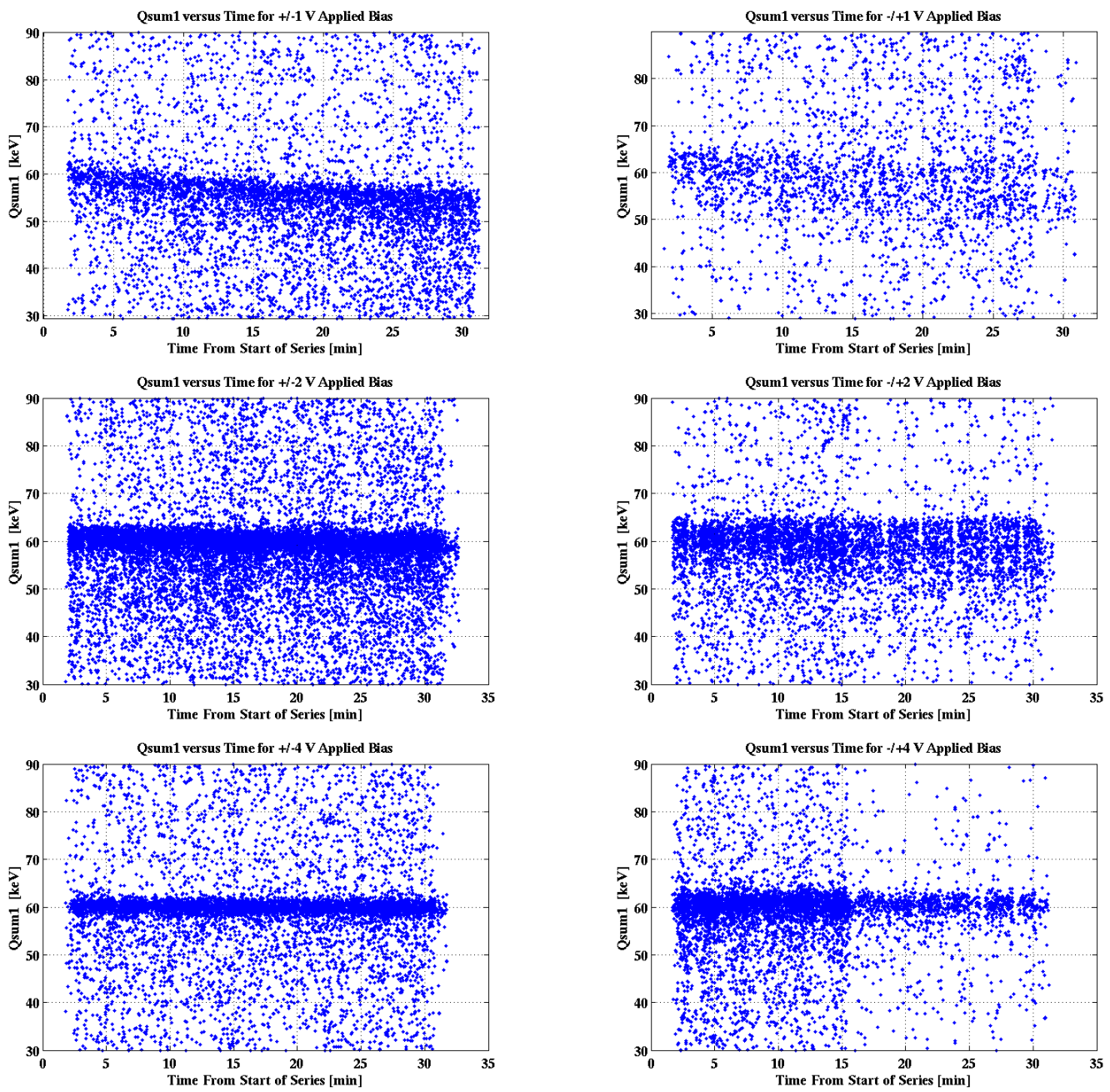

Figure 4.12: Side 1 Charge amplitude versus time for $\pm 1 \mathrm{~V}, \mp 1 \mathrm{~V}, \pm 2 \mathrm{~V}, \mp 2 \mathrm{~V}, \pm 4$ $\mathrm{V}$, and $\mp 4 \mathrm{~V}$ data. Plots are comprised of several series, typically of length 15 or 30 minutes, with standard ionization quality cuts applied. The $60 \mathrm{keV}$ peak can be seen as a distinct line, which begins to droop, indicating a loss of crystal neutralization. 


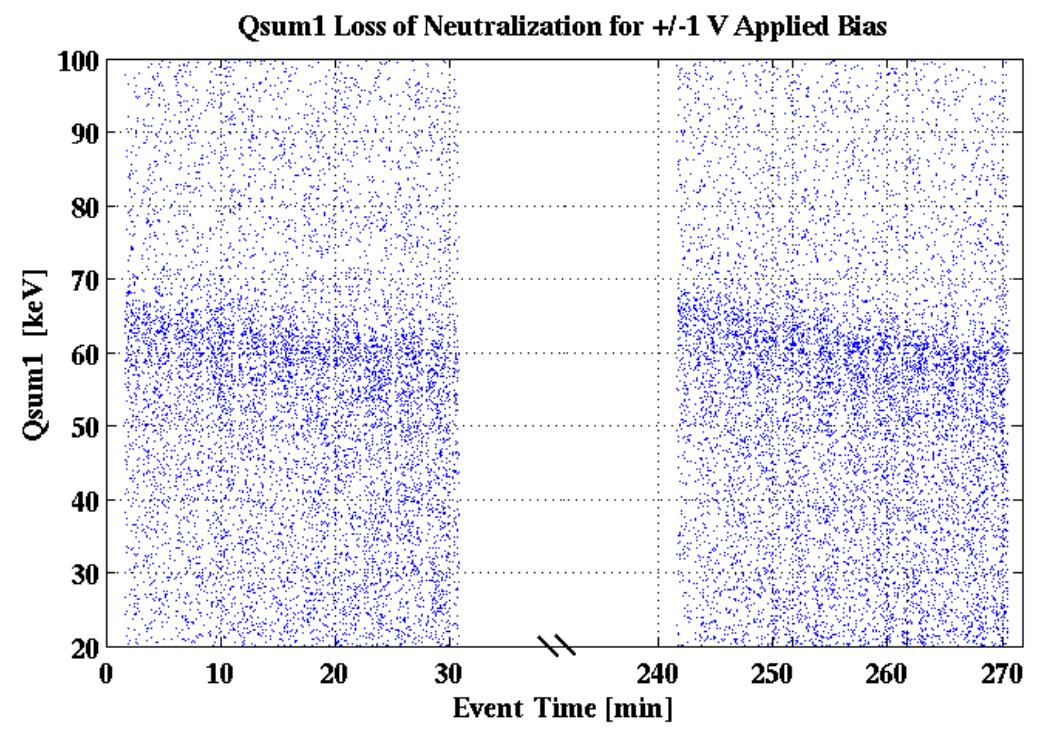

Figure 4.13: Two $\pm 1 \mathrm{~V}$ data series are shown in this qsum1 versus time plot. Loss of neutralization, indicated by the dropping $60 \mathrm{keV}$ event line, is apparent within $<10$ minutes of each series. Fortunately, grounding, flashing and cooling the detector, which occurs in the blank, event-less spaces between data series, neutralizes the crystal. As a result, the $60 \mathrm{keV}$ peak is returned to its original amplitude at the start of each series. 
Table 4.2: G106a 60 keV Peak Decay

\begin{tabular}{ccc}
\hline \hline Applied Bias & Rate of Peak Change $[\mathrm{eV} / \mathrm{min}]$ & $\begin{array}{c}\text { Estimated Peak Change } \\
\text { in } 30 \text { Minutes Series }[\mathrm{keV}]\end{array}$ \\
\hline & & \\
$\pm 4 \mathrm{~V}$ & $-1.62 \pm 4.51$ & $-0.0486 \pm 0.1353$ \\
$\pm 2 \mathrm{~V}$ & $-44.5 \pm 3.6$ & $-1.34 \pm 0.11$ \\
$\pm 1 \mathrm{~V}$ & $-145 \pm 6$ & $-4.35 \pm 0.19$ \\
$\mp 1 \mathrm{~V}$ & $-122 \pm 13$ & $-3.66 \pm 0.39$ \\
$\mp 2 \mathrm{~V}$ & $-57.4 \pm 6.4$ & $-1.72 \pm 0.19$ \\
$\mp 4 \mathrm{~V}$ & $7.42 \pm 6.04$ & $0.223 \pm 0.181$ \\
\hline
\end{tabular}

The charge signal versus event time plot shown in Fig. 4.13 demonstrates effective flashing of G106a over several iterations of data taking. De-neutralization can be clearly seen within $\sim 10$ minutes of the initial $\pm 1 \mathrm{~V}$ series. Several iterations of flashing, cooling, and data taking (at various applied biases) occur within the blank region of the figure. Each flashing neutralizes the crystal before the next data series is taken. Roughly 240 minutes after the initial series was started, and 30 minutes after the final flashing, another $\pm 1 \mathrm{~V}$ series begins and the $60 \mathrm{keV}$ peak has returned to the original position.

Given these results, G106a is determined to have sufficient neutralization hold time at $\mp 4 \mathrm{~V}$ when used as a SuperCDMS SNOLAB detector. Additionally, a timing quality cut can be added to the analysis, keeping neutralized data with an expected $60 \mathrm{keV}$ peak decay of $<1.0 \mathrm{keV}$. Understanding the neutralization hold time also increases the efficiency of test facility data taking, allowing the facility to take data series only as long as neutralization holds.

A majority of Run 54 data $\mp 4 \mathrm{~V}$ series are $<30$ minutes long and thus do not exhibit signs of deneutralization. However, it is important to remember that deneutralization of a crystal depends on the number of interactions in the crystal, and a single timing cut is only useful for data series with the same applied bias AND event rate. If no changes are made to the fridge shielding, and no strong sources are introduced/removed, it is safe to assume little change in event rate during Run $54 \mp 4 \mathrm{~V}$ series, and a single timing cut 
can be applied. By counting pulses which occur in start of series randoms, the Run 54 event rate is found to be $\sim 82 \mathrm{~Hz}$. However, a different G106a run with different sources present, Run 56, was found to have an event rate of $\sim 159 \mathrm{~Hz}$. As can be expected given the higher event rate, Run $56 \mp 4 \mathrm{~V}$ series show loss of neutralization after 20 minutes without flashing. Therefore a different timing cut would be applied to Run $56 \mp 4 \mathrm{~V}$ data than what is applied to Run $54 \mp 4 \mathrm{~V}$ data.

\subsection{Charge Collection Efficiency}

As discussed in Section 2.2.3, after a collision in the target material a cascade of electron/hole pairs are created and drift towards the detector faces, due to an applied electric field. Event-by-event discrimination between ER and NR recoils depends on near complete collection of these charge carries, since nuclear recoils have a quenched ionization signal relative to electron recoils. Therefore, assessing the charge collection efficiency of $100 \mathrm{~mm}$ iZIPs is essential for determining their potential for use in SuperCDMS SNOLAB.

As mentioned in previous sections, the charge collection efficiency characterizes the reduction in charge signal amplitude that occurs when electrons or holes enter charge carrier traps, or (if the bias is low enough) the electrons and holes are not separated quickly enough and recombine inside the crystal instead of reaching the surfaces electrodes. The rate at which carriers become trapped depends on the impurities of the crystal, the type of carrier, and the drift velocity of the carrier; the longer a carrier drifts about the crystal, the more likely it is to be caught in a trap. Studies of carrier propagation in indirect gap semiconductor detectors near $\sim 1 \mathrm{~K}$ with applied fields below $\sim 5 \mathrm{~V} / \mathrm{cm}$ determined that the magnitude of the drift velocity, $v_{d}$, is related to electric field, $E$, and characteristic range for carrier scattering, $l_{0}$, by,

$$
v_{d} \propto\left(e E l_{0}\right)^{1 / 5}
$$

with $l_{0}$ given by $l_{0}=\pi \hbar^{4} \rho_{\text {det }} / 2 m_{c}^{3} \Xi^{2}$, for a detector with density $\rho_{\text {det }}$, and deformation potential $\Xi$, and carrier with mass $m_{c}$ (hole effective mass is given as $m_{h}=0.5 m_{e}$ for $\mathrm{Si}$ and $m_{h}=0.35 m_{e}$ for Ge) [176]. Since the drift speed depends on the electric field, this naturally leads to charge collection efficiency also depending on the applied field. 
Assuming a uniform electric field, the charge collection efficiency, $\varepsilon_{c c}$ for a detector of active width, $W$, is given by,

$$
\varepsilon_{c c}=\left(\mu_{c} \tau_{c} E / W\right)\left(1-e^{W / \mu_{c} \tau_{c} E}\right),
$$

where $\mu_{c}$ and $\tau_{c}$ are the charge carrier mobility, and characteristic carrier lifetime, respectively [206].

Charge collection efficiency is characterized by the downward shift of source peaks (before energy calibration), as applied field is reduced. Typically the $60 \mathrm{keV}$ peak of the internal ${ }^{241} \mathrm{Am}$ source and the $356 \mathrm{keV}$ peak from the external ${ }^{133} \mathrm{Ba}$ source are used to complete these measurements. Because $60 \mathrm{keV}$ photons have a penetration depth of $\sim 1 \mathrm{~mm}$ in Ge, the ${ }^{241} \mathrm{Am}$ source is statistically more likely to produce surface events (occurring within the $\sim 10 \mu \mathrm{m}$ dead layer of the detector), than the $356 \mathrm{keV}$ photons from ${ }^{133} \mathrm{Ba}$, which have a penetration depth of $\sim 2 \mathrm{~cm}$ in Ge. Using both sources provides a useful comparison in collection efficiency, since $356 \mathrm{keV}$ events are more uniformly distributed throughout the crystal, whereas $60 \mathrm{keV}$ events are more focused near the ${ }^{241} \mathrm{Am}$ source location at one surface.

To determine the charge collection efficiency Gaussian fits are applied to the radioactive source peaks on both the Qsum1 and Qsum2 spectra for each desired voltage. Voltage bias is increased in steps of $0.5-2.0 \mathrm{~V}$, reaching a maximum of about $\pm 8-12 \mathrm{~V}$. The mean of the fit is then plotted as a function of the voltage bias applied across the detector, as shown in the lower right of Fig. 4.15. A detector has good charge efficiency if these mean values begin to plateau at an electric field strength of roughly $1 \mathrm{~V} / \mathrm{cm}(3$ - $4 \mathrm{~V}$ for a $33 \mathrm{~mm}$ thick crystal), indicating near-complete charge collection.

The maximum voltage used for the efficiency measurement is typically determined by voltage output limit of the DCRC, or by the evident plateauing of the efficiency measurement as voltage increases. However, some detectors experience a charge breakdown at high voltages, a phenomenon that introduces increased and unmanageable noise or distortion into the signal of affected channels, rendering it impossible to conduct trustworthy measurements. 


\subsubsection{Charge Breakdown}

Charge breakdown studies looked for breakdown symptoms due to voltages across the crystal as well as on individual sides between the inner (Qi) and outer (Qo) charge electrodes. A breakdown study is conducted by incrementally increasing the voltage bias by $0.5 \mathrm{~V}$ in the given polarity setup, and visually inspecting ionization and phonon output signal for consistent and repeatable signs of breakdown, typically pulse-less noise on phonon channels, spiky, distorted pulses on charge channels, and a slight (few mK) increase in tower temperature. An example of a phonon signal before and after breakdown distortion is shown in Fig. 4.14. These tests were performed with both positive and negative polarities, indicated with + , and - signs, respectively, in the results Table 4.3. The results reflect studies conducted using DCRCs 31 and 33 during Run 34, an ionization-focused G106a run from 2013.

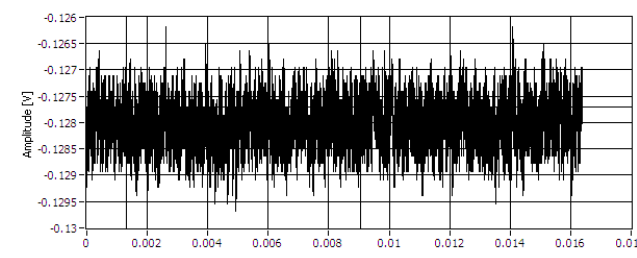

Figure 4.14: (TOP) A clean signal observed on Side 2 channel $\mathrm{D}$ with a $-11.5 \mathrm{~V}$ bias applied. (BOTTOM) Distortions typical of charge breakdown on Side 2 channel D, once

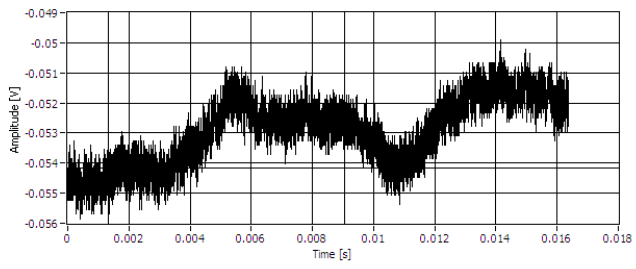
the bias has been increased to $-12.0 \mathrm{~V}$.

Overall, G106a shows little evidence of charge breakdown. A few instances of breakdown symptoms were exhibited around 9 and $11 \mathrm{~V}$ between charge electrodes on Side 2 , with full breakdown becoming apparent by $12 \mathrm{~V}$. Because no breakdown was seen between detector sides, it is unlikely that breakdown symptoms will hinder charge collection efficiency measurements, even at high voltages.

\subsubsection{Charge Collection Efficiency Measurements}

Charge collection efficiency was characterized using UMN Run 54 G106a data, with a cut to remove randoms and a $\chi^{2}$ cut applied. To accurately see the $60 \mathrm{keV}$ peak on 
side 2, a cut requiring approximate symmetry between Qsum1 and Qsum2 is applied as well. Side 1 studies were conducted with and without the symmetry cut, to see if any difference is apparent. Fig. 4.15 shows the Gaussian fit mean $\pm 1 \sigma$ versus bias for Side 1 and Side 2. Here $\sigma$ refers to the error on the fitted mean, and not to the width of the peak. The peak mean at $\pm 10 \mathrm{~V}$ data is normalized to one, indicating near-complete charge collection. Error bars are also normalized to reflect the standard deviation of the ratio $\operatorname{mean}(\mathrm{V}) / \operatorname{mean}(\mathrm{V}= \pm 10)$.

As expected given their typical drift trajectory in Ge, hole trapping (side with negative bias) is more effective than electron trapping (positive bias) for bulk events, although both polarities show similar trends. Because the ${ }^{241} \mathrm{Am}$ source is near Side 1, $60 \mathrm{keV}$ peak results are affected by a population of surface events (due to $\sim 1 \mathrm{~mm}$ penetration depth of $60 \mathrm{keV}$ photons) and events nearer to Side 1, whereas $356 \mathrm{keV}$ peak results are much less susceptible to this bias and provide a more robust estimate of the charge collection efficiency for bulk events.

Results indicate that $60 \mathrm{keV}$ events are better collected for both Side 1 and Side 2 when a \pm bias polarity is applied. Recall that $60 \mathrm{keV}$ events are produced by the shallow interactions of the ${ }^{241} \mathrm{Am}$ gammas, located on Side 1. As a result, holes produced in a $\mp$ bias polarity will drift towards Side 2, with a majority traversing a greater part of the detector thickness. Holes are less likely to hit the detector sides during this journey when compared to electrons with oblique propagation. Thus, it is unsurprising that collection efficiency of $60 \mathrm{keV}$ is greater when a \pm bias polarity. A charge symmetry cut has little affect on this result, because electrons/holes that make it into the bulk of the crystal $(>1 \mathrm{~mm})$ still must drift to the other detector side.

For both Side 1 and Side 2, bulk event collection efficiency reaches $\sim 90 \%$ for biases of magnitude $4 \mathrm{~V}$ or larger, corresponding to an electric field of $\sim 1.3 \mathrm{~V} / \mathrm{cm}$. This level of charge collection efficiency is sufficient for operation as a SuperCDMS SNOLAB detector. However, the bulk event collection efficiency is still greatly increasing at \pm 2 $\mathrm{V}$ and $\mp 2 \mathrm{~V}$ biases when compared to efficiency at $\pm 4 \mathrm{~V}$ or $\mp 4 \mathrm{~V}$. At these biases the efficiency has effectively plateaued, and increasing the bias further only results in a slight linear rise in efficiency. Thus \pm 4 and $\mp 4 \mathrm{~V}$ will be considered the optimal biases for near-complete charge collection efficiency with minimal Luke phonon contributions.

These can be compared to past bulk charge collection efficiency measurements, using 

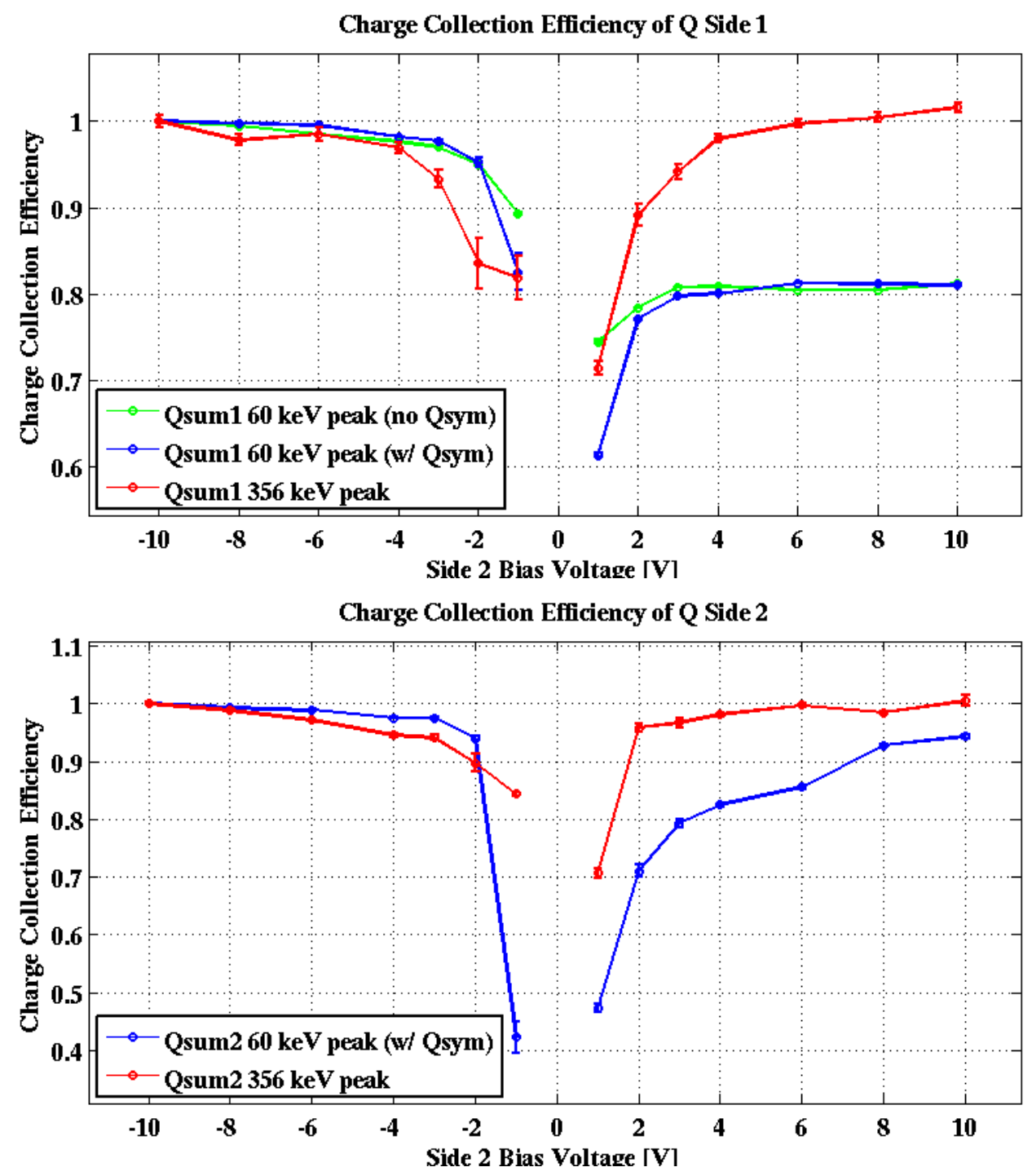

Figure 4.15: Charge collection efficiency for (TOP) Side 1 and (BOTTOM) Side 2 of G106a, using Run 54 bias sweep data. Efficiency measurements were taken with the 60 keV peak, with (blue) and without (Qsum1 only, green) a charge symmetry cut applied, and with the $356 \mathrm{keV}$ peak (red). Due to events mainly occurring in the bulk, the 356 $\mathrm{keV}$ measurements show a generally symmetric results between bias polarity. 
UMN Run 39 data for G106a Side 2, and data from G3D, a $76 \mathrm{~mm}$ diameter, $2.54 \mathrm{~cm}$ thick Ge ZIP detector studied in 2008 at Case Western Reserve University [208], that has phonon channels and charge channels on opposite detector faces. An ${ }^{241} \mathrm{Am}$ source was placed above the charge face on G3D, and electric field values are stated relative to the charge collection side. Fig. 4.16 compares G106a Side 2 charge collection efficiency using the $356 \mathrm{keV}$ (Run 54) and $60 \mathrm{keV}$ (Run 39 and 54) source peaks (charge symmetry cut applied to both), to G3D charge collection efficiency from the $60 \mathrm{keV}$ peak of Qi. As with previous results, the efficiency has been normalized to one at a field strength of $-2.96 \mathrm{~V} / \mathrm{cm}( \pm 10 \mathrm{~V}$ applied bias for G106a), although G3D and Run 39 results extend beyond this value, showing that the efficiency continues to increase. Run 39 and 54 studies using the $60 \mathrm{keV}$ peak match nicely for fields greater than $\pm 0.5 \mathrm{~V} / \mathrm{cm}$, indicating repeatable results for G106a.

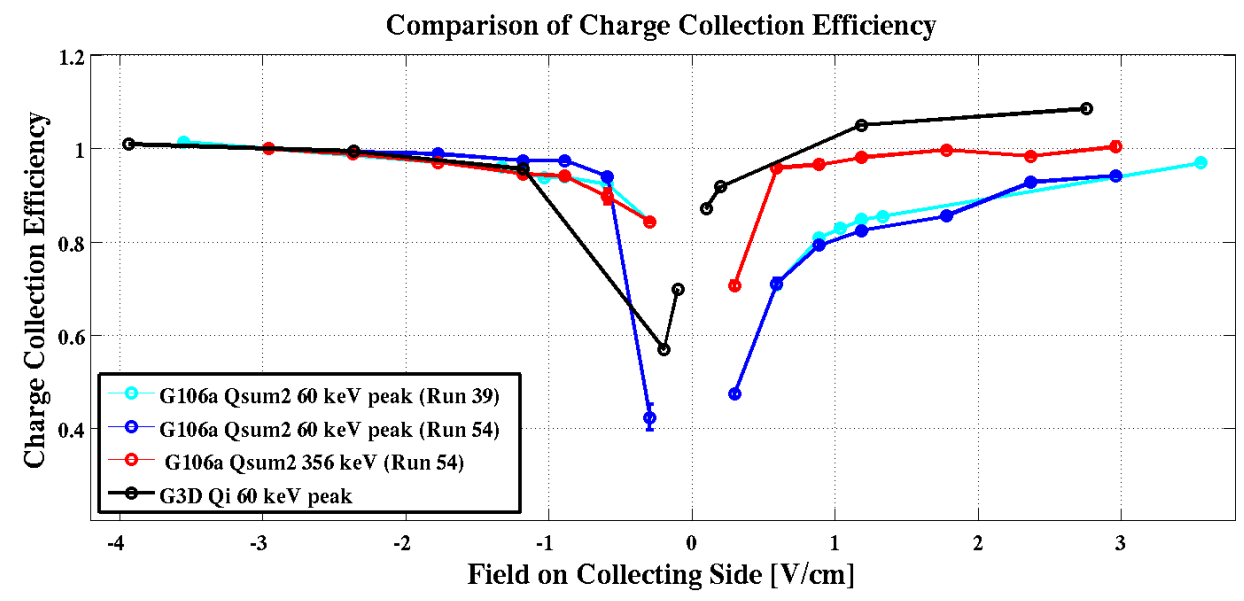

Figure 4.16: Previous charge collection efficiency studies, from G106a UMN Run 39 (cyan) and $76 \mathrm{~mm}$ diameter, $2.54 \mathrm{~cm}$ thick Ge ZIP G3D (black), are compared to Run 54 results (60 keV peak in blue, $356 \mathrm{keV}$ peak in red). Run 39 and 54 studies using the $60 \mathrm{keV}$ peak match nicely for fields greater than $\pm 0.5 \mathrm{~V} / \mathrm{cm}$, indicating repeatable results. When source location is taken into account, the larger detector volume of G106a does not demonstrate ill effects on charge collection efficiency when compared to G3D results, for fields greater than $\sim \pm 1 \mathrm{~V} / \mathrm{cm}$.

Although G3D results are sparse below $\pm 1 \mathrm{~V} / \mathrm{cm}$, results at larger net biases indicate similar trends in charge collection efficiency when a negative field is applied. G3D shows 
better collection efficiency for positive fields than any of the G106a results, but this is likely due to the ${ }^{241} \mathrm{Am}$ source being above the charge collection side of the detector. In this orientation, holes from shallow $60 \mathrm{keV}$ events will propagate the length of G3D for a positive field (+ ionization/- phonon bias), resulting in an increase collection efficiency. Ignoring this difference in overall magnitude of the collection efficiency in the positive field region, the trend of decreasing slope near $\sim 1.3 \mathrm{~V} / \mathrm{cm}$ is the same for G3D and G106a data. Thus, the larger detector volume does not demonstrate ill effects on charge collection efficiency in fields greater than $\sim \pm 1 \mathrm{~V} / \mathrm{cm}^{3}$

Thus the ionization characteristics of G106a, a preliminary $100 \mathrm{~mm}$ detector, suggests that they are suitable dark matter detectors. The next step in demonstrating 100 $\mathrm{mm}$ iZIP performance is to conduct full phonon characterization.

\footnotetext{
${ }^{3}$ Effects below this magnitude are hard to quantify, given the lack of G3D data, but this region is not of interest for SNOLAB operation anyway.
} 
Table 4.3: G106a Charge Breakdown Study [207]

\begin{tabular}{|c|c|c|}
\hline Side 1 Polarity & Side 2 Polarity & Results \\
\hline$+\mathrm{Qi},+\mathrm{Qo}$ & - Qi, - Qo & No charge breakdown detected across the crystal. \\
\hline - Qi, - Qo & $+\mathrm{Qi},+\mathrm{Qo}$ & Hints of breakdown (non-continuous signal) at $11-12 \mathrm{~V}$. \\
\hline + Qi, 0 Qo & 0 Qi, 0 Qo & No charge breakdown detected between electrodes. \\
\hline $0 \mathrm{Qi},+\mathrm{Qo}$ & 0 Qi, 0 Qo & No charge breakdown detected between electrodes. \\
\hline 0 Qi, 0 Qo & + Qi, 0 Qo & Breakdown began at $9 \mathrm{~V}$, full by $10 \mathrm{~V}$ on Side 2 . \\
\hline 0 Qi, 0 Qo & $0 \mathrm{Qi},+$ Qo & No charge breakdown detected between electrodes. \\
\hline 0 Qi, 0 Qo & - Qi, 0 Qo & No charge breakdown detected between electrodes. \\
\hline 0 Qi, 0 Qo & 0 Qi, - Qo & Breakdown began at $11 \mathrm{~V}$, full by $12 \mathrm{~V}$ on Side 2 . \\
\hline - Qi, 0 Qo & 0 Qi, 0 Qo & No charge breakdown detected between electrodes. \\
\hline 0 Qi, - Qo & 0 Qi, 0 Qo & No charge breakdown detected between electrodes. \\
\hline
\end{tabular}




\section{Chapter 5}

\section{0 mm Detector Phonon Characterization}

Since 2011, multiple prototype $100 \mathrm{~mm}$ and iZIP detectors have undergone charge characterization and analysis at the Minnesota test facility. Few prototype iZIPs have performed at sufficient levels for full phonon characterization, and no others have had the sheer amount of running time allowed as the prototype iZIP G106a. Thus, the results presented here represent the first instance of full phonon characterization of a $100 \mathrm{~mm}$ iZIP.

This chapter aims to demonstrate G106a's capabilities as an iZIP detector by presenting evidence of sufficient phonon performance. The chapter begins by discussing how the phonon readout electronics are set for optimized data taking. This includes a discussion on the transitional temperatures of G106a, and studies on the current and voltage characteristics of each phonon channel as it undergoes transition, known as $I_{b}-I_{s}$ studies. The chapter will then explore the construction of a phonon channel relative calibration and a Non-Stationary optimal filter (NSOF), using raw phonon pulses. Improvements in data quality due to the relative calibration and NSOF will be discussed next, including the implementation of phonon quality cuts and phonon spectrum analysis. The chapter will conclude with the construction of yield bands, demonstrating successful $100 \mathrm{~mm}$ iZIP surface event rejection and recoil discrimination. 


\subsection{Phonon Channel Setup}

Unlike charge channels, which function properly with little-to-no initial setup ${ }^{1}$ phonon channels must undergo a setup routine to put each channel into an optimal state. First, the SQUIDs must be "tuned", by adjusting closed loop operating parameters of the flux-based pre-amplifier circuit. Then, transition temperature regions are found for each phonon channel, as a means to determine an optimal QET bias to apply. Once SQUID and QET parameters are optimally set, phonon pulses can be read out.

\subsubsection{Tuning the SQUIDs}

SQUID tuning refers to the procedure of adjusting the closed loop operating parameters of the flux-based pre-amplifier circuit, including SQUID bias and lockpoint, to meet certain criteria, including: a noise performance of $\sim 1 \mathrm{pA} / \mathrm{rtHz}$ referenced relative to the input coil [209]; high SQUID responsivity with uniform response such that SQUID array resonance are not encountered and the electronics remain locked on one modulation cycle; and a bandwidth of $300-400 \mathrm{kHz}$ [210]. The closed loop refers to the feedback loop shown in Fig. 5.1

Here, responsitivity refers to the change in SQUID voltage due to a change in the input coil current. Since SQUID noise is assumed to be a fixed voltage independent of SQUID responsitivity, a higher responsitivity results in a higher signal-to-noise ratio, and thus better performance. Having a dynamic range with uniform response simply means having steady responsivity without large frequency-dependent increases or decreases over the voltage signal produced by a SQUID array while in a closed loop.

To have a dynamic range with uniform response, it must be free of resonances: points on the SQUID response curve where oscillations occur at $>\mathcal{O}(\mathrm{GHz})$ frequencies. A resonance is a characteristic of the SQUID array caused by feedback between different components within the device. When viewing the modulation curve (voltage variation across the SQUID as a function of magnetic flux through the SQUID), resonances appear as discontinuities, due to the relatively low bandwidth of the readout. SQUIDs fabricated for use in CDMS have been designed to minimize certain resonances on one

\footnotetext{
${ }^{1}$ Typically, only a long-term (1 - 12 hour) LED flash, referred to as a bake, is required before charge channels can be read out cleanly.
} 
slope of the modulation curve, at the expense of the other slope. Therefore the tuning process is designed to lock in the electronics on this slope of the modulation curve with optimal responsivity.

SQUID tuning works by using a triangle wave input signal to find the maximum modulation depth without discontinuities, by adjusting the SQUID bias. Then, a point on the modulation curve that the amplifier works to maintain, know as a lockpoint, is set. The lockpoint is typically selected to be the point of greatest increasing slope on the modulation curve, to maximize responsivity. Without closed loop feedback keeping deviations from the lockpoint minimal, current from the input coil would cause SQUID modulation to pass through at least one complete cycle, resulting in a distorted reproduction of changes in input coil current.

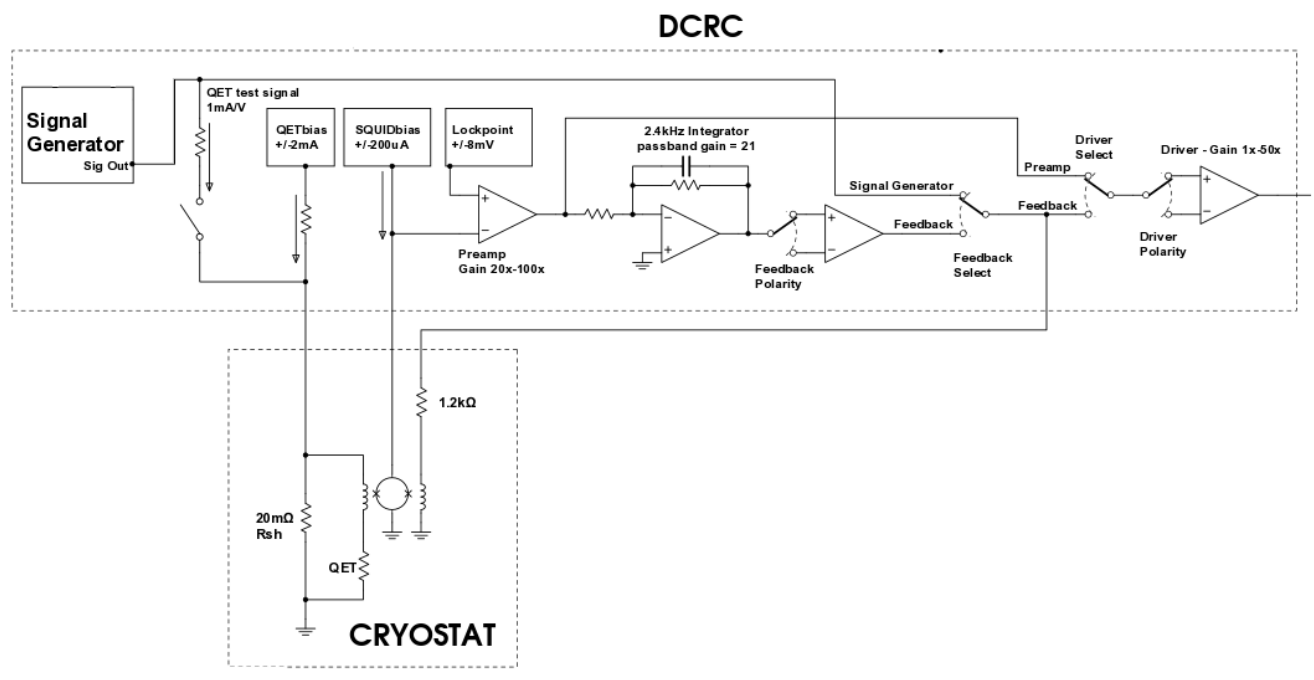

Figure 5.1: SQUID tuning setup schematic. Modified from D. Seitz.

The SQUID tuning procedure is summarized in the following steps, with a SQUID tuning setup schematic shown in Fig. 5.1. The procedure must be followed for each individual phonon channel, one at a time.

1. Using the MIDAS SQUID Tuning GUI, set each gain parameter to one and all other QET readout parameters to zero.

2. Select the 'Grounded/Open Loop' mode. 
3. Zap the SQUIDs (an automated process of driving the SQUIDs normal with a discharging capacitor) to remove any trapped flux.

4. Set signal source to 'Feedback'.

5. Send a $200 \mathrm{mVpp}, 100 \mathrm{~Hz}$ triangle wave test signal down the feedback line.

6. Once the test signal is visually confirmed in the responsivity window, switch the signal source to 'Pre-amp'.

7. Adjust the SQUID bias until the maximum modulation depth is reached. Modulation curve should look sinusoidal, with the top slightly flattened but no discontinuities. Fluctuations in the curve indicate trapped flux is still present.

8. Adjust the lockpoint setting until the point of greatest increasing slope of the modulation curve is at the $\mathrm{x}$-axis.

9. Switch from 'Pre-amp' back to the 'Feedback' signal source, and select either 'Inverting' or 'Non-inverting' polarity, whichever best reconstructs the original triangle wave. Selecting polarity will close the loop. The SQUIDs are now tuned and locked.

\subsection{2 $I_{b}-I_{s}$ and Transition Temperatures}

Once SQUIDs are tuned, measurements of phonon sensor critical temperatures, or $T_{c}$ 's, are possible. Each TES has it's own $T_{c}$, which can vary significantly from other TESs. When a single TES approaches superconducting, it shorts the remaining TESs, since current is distributed such that the greatest amount of current passes through TESs with the largest $T_{c}$. Thus the measured sensor $T_{c}$ of a channel is sensitive to the upper range of the TES critical temperature distribution.

Lower critical temperatures result in lower levels of phonon channel noise for the SuperCDMS SNOLAB electronics? Additionally, iZIP detectors depend on the dilution fridge first reaching temperatures below $T_{c}$ before an applied QET bias raises sensors to their transition regions. If this transition range falls below the fridge capabilities

\footnotetext{
${ }^{2}$ This does not hold true for the UMN facility, which is dominated by Johnson noise of the shunt resistor.
} 
then the detector will not function. Critical temperature measurements are performed for each phonon channel. They are performed by tuning and locking the SQUIDs, setting the QET bias to zero and sending a $10 \mathrm{mVpp}, 100 \mathrm{~Hz}$ triangle wave down the input line of a phonon channel, with the output gains set as large as possible while keeping the full triangle visible. Then the detector is heated above base temperature by setting the mixing chamber heater to $\sim 100 \mathrm{nW}$, and then incrementally increasing the heater by $2-5 \mathrm{nW}$, until a transition away from superconduction occurs, made apparent by the degradation of a triangle wave test signal. When the transition has fully occurred, the peak-to-peak amplitude of the triangle wave will have decreased by roughly an order of magnitude. Then the detector is allowed to cool until the transition to superconduction occurs. The rising and falling ranges of temperature for which the detector was in transition are recorded as the $T_{c}$ range. The system experiences a slight hysteresis effect, causing the decreasing temperature range to be slightly less than the increasing range. Current results set the range $69 \mathrm{mK}<T_{c}<85 \mathrm{mK}$ for the G106a, with little deviation in $T_{c}$ between detector faces. The complete measured temperature ranges are displayed in Table 5.1. All $T_{c}$ 's were measured using $10 \mathrm{mVpp}$ triangle wave at $100 \mathrm{~Hz}$ except for channel PD Side 1 (C2), which used $100 \mathrm{mVpp}$ triangle wave at 1 $\mathrm{kHz}$ to overcome high noise levels. The temperature of the mixing chamber was slowly raised using the mixing chamber heater. All temperatures are recorded with the tower thermometer.

$I_{b}-I_{s}$ measurement is effectively a measurement of the $T_{c}$ distribution of a phonon channel, by comparing the current through the sensor, $I_{s}$, to the QET biasing current, $I_{b}$. The relationship between $I_{s}$ and $I_{b}$ is given by,

$$
I_{s}=I_{b} \frac{R_{s h}}{R_{s}+R_{p}+R_{s h}}
$$

where $R_{s h} \approx 20 \mathrm{~m} \Omega$ is the shunt resistor, $R_{p}$ is the parasitic resistance, that is $\mathcal{O}(\mathrm{m} \Omega)$, and $R_{s}$ is the resistance of the sensor [168]. As $I_{b}$ is increased, the TESs in the phonon channel will transition from superconducting to normal state.

- Superconducting: For low enough values of $I_{b}$, the sensor is fully superconducting. Thus $R_{s}=0$ and,

$$
\left.\frac{d I_{s}}{d I_{b}}\right|_{s c}=\frac{R_{s h}}{R_{s h}+R_{p}}
$$


Table 5.1: G106a Transition Temperatures [207]

Channel (DIB) $\quad T_{c}$ Range (T increasing) $\pm 2 \mathrm{mK} \quad T_{c}$ Range ( $\mathrm{T}$ decreasing) $\pm 2 \mathrm{mK}$

PA Side 1 (D2)

$\begin{array}{ll}72-73 & 73-72 \\ 81-82 & 82-81 \\ 79-81 & 81-79 \\ 84-85 & 85-84 \\ 83-85 & 84-82 \\ 81-82 & 82-81 \\ 78-79 & 78-77 \\ 69-71 & 70-68 \\ 77-78 & 77-76 \\ 76-77 & 77-75 \\ 74-75 & 74-73 \\ 75-76 & 76-74\end{array}$

PB Side 1 (A1)

PC Side 1 (C1)

PD Side 1 (C2)

PE Side 1 (D3)

$83-85$

$4-82$

PF Side 1 (B1)

PA Side 2 (B2)

PB Side 2 (A3)

PC Side 2 (A2)

PD Side 2 (D1)

PE Side 2 (C3)

$75-76$

$6-74$

Fitting this portion of the $I_{b}-I_{s}$ curve provides an estimate for $R_{p}$, as shown in the red highlighted region of Fig. 5.2. In this region no power is dissipated in the sensor.

- Transitioning: In this region the sensor is in its superconducting transition, the width of which depends on the non-uniformity of the TES's critical temperature. The curved regions in the $I_{b}-I_{s}$ curves of Fig. 5.2 indicate a transitioning sensor. The power dissipation is roughly constant during transition, due to near-constant temperature during transition.

- Normal State: Once the sensor has reached the normal region, $R_{s} \approx 1 \Omega$, significantly larger than $R_{p}$ and $R_{s h}$. Using the parasitic resistance estimation found from the superconducting region, the resistance of the sensor in the normal 
region, $R_{n}$, is estimated to be,

$$
R_{n}=R_{s h}\left(\left(\left.\frac{d I_{s}}{d I_{b}}\right|_{n}\right)^{-1}-1\right)-R_{p}
$$

The blue and magenta highlighted regions of Fig. 5.2 demonstrate the linear dependence of the normal region for both positive and negative QET bias currents. In this region the power dissipated by the sensor follows $P=I_{s}^{2} R_{n}$, which is quadratic due to constant $R_{n}$.

\section{$I_{b}-I_{s}$ for G106a Channel D3}

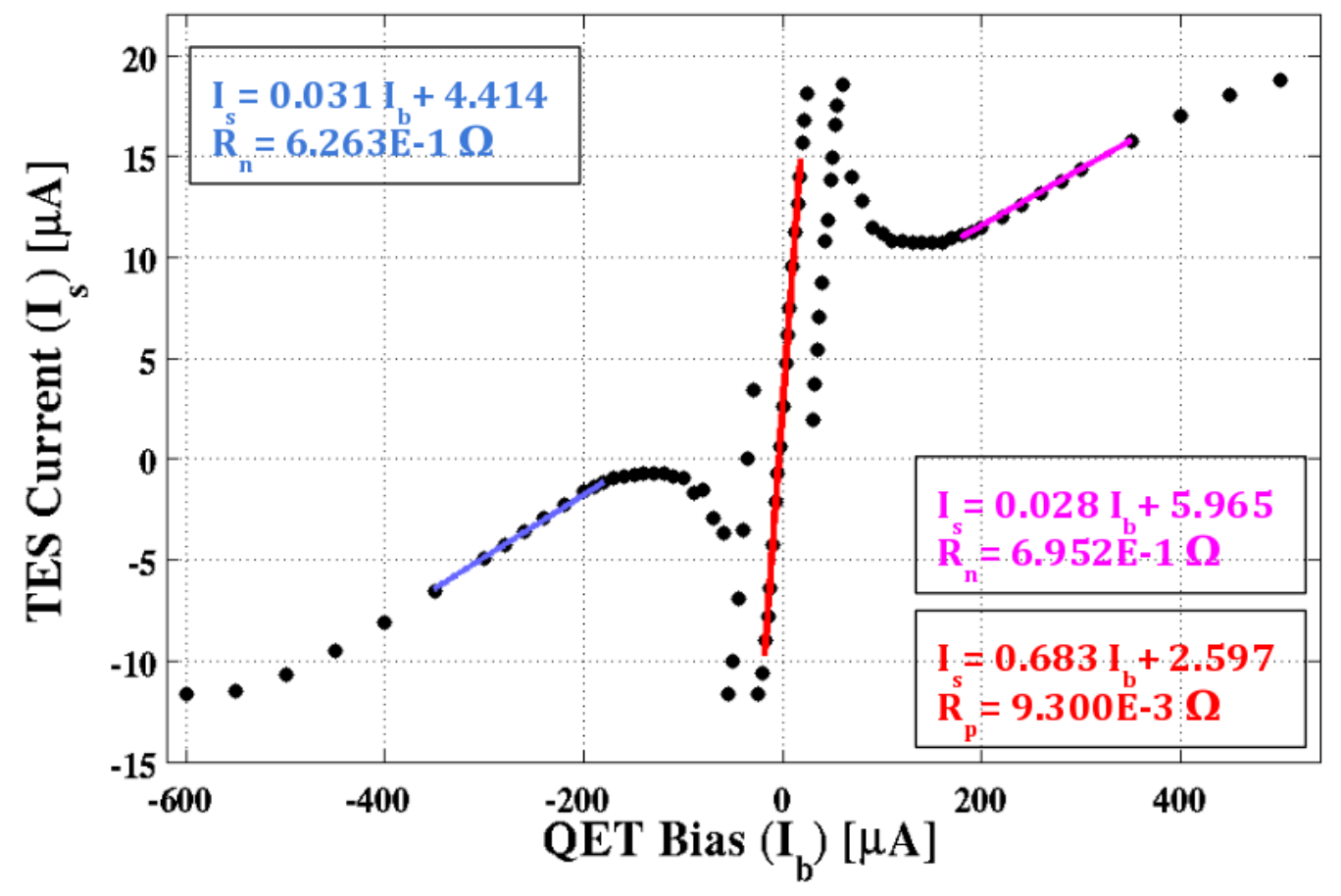

Figure 5.2: $I_{b}-I_{s}$ measurement for channel PE Side 1 (D3) of G106a, with superconducting region highlighted in red, and normal regions highlighted in blue (negative $I_{b}$ ) and magenta (positive $I_{b}$ ). Fitting the linear superconducting and normal regions allows for estimation of the parasitic and normal resistances of the sensor. The fits and estimated resistance values are shown in the upper left and lower right corners of the plot. 
The $I_{b}-I_{s}$ curve of G106a phonon channel PE Side 1 (D3) is shown in Fig. 5.2 . taken from UMN Run 34. The $I_{b}-I_{s}$ curves of all G106a phonon channels are shown in Fig. C.1 of Appendix C. To find the current passing through the sensor, the output voltage, $V_{o}$ is recorded, and then converted to sensor current using,

$$
I_{s}=\frac{V_{o}}{R_{f b} \times G_{d} \times G_{l o o p} \times G_{D C R C} \times G_{o}},
$$

where $R_{f b}=1233 \Omega$ is the feedback resistor, $G_{d}=1$ is the driver gain, $G_{\text {loop }}=10.5$ is the SQUID loop gain due to the inductor coil ratio, $G_{D C R C}$ is the DCRC gain (typically set to 2 or 5 , depending on the DCRC version), and $G_{o}$ is the output gain, typically set to 1 or 2 .

Most sensors demonstrate a parasitic resistance of $R_{p}<10 \mathrm{~m} \Omega$, and normal sensor resistances within $0.5-1 \Omega$, within the realm of acceptable values. Channel PD Side 1 (C2), was outside of expected range, with a parasitic resistance roughly an order of magnitude greater than expected, and normal resistance roughly 2 - 3 times larger than expected. Initial $I_{b}-I_{s}$ curves were taken during Run 34 for channel PD Side 1, and they were repeated in Run 35 using a different SQUET card to ensure cold hardware was not the cause.

Fig. 5.3 shows $R_{s}$ plotted versus $I_{b}$ for phonon channel PE Side 1 (D3) of G106a, showing the sensor resistance increasing from zero in the superconducting region and plateauing towards the positive and negative QET bias $R_{n}$ values estimated in the previous $I_{b}-I_{s}$ curve. The $R_{s}$ versus $I_{b}$ plots for all G106a phonon channels, except channel PD Side 1 (C2), are shown in Fig. C.2 of Appendix C. Positive and negative QET bias curves are fit to the data, shown in blue and pink. From the fit curve, $R_{p}$ and $R_{n}$ are found, to ensure that the values are consistent with those found prior. Channel PD Side 1 (C2) was excluded due to difficulty procuring consistent values.

For each channel, the optimized QET bias that will be used for data-taking is set at the QET bias corresponding to the start of the transition region, where $R_{s} \sim R_{n} / 4$. Accounting for parasitic resistance, this value is more specifically,

$$
R_{s}=\left(\frac{R_{n}-R_{p}}{4}\right)+R_{p}
$$

although $R_{p}$ is assumed to be negligible in most cases [211]. The optimized positive and negative QET bias for each channel is included next to the $R_{s}$ versus $I_{b}$ curves in Figs. 


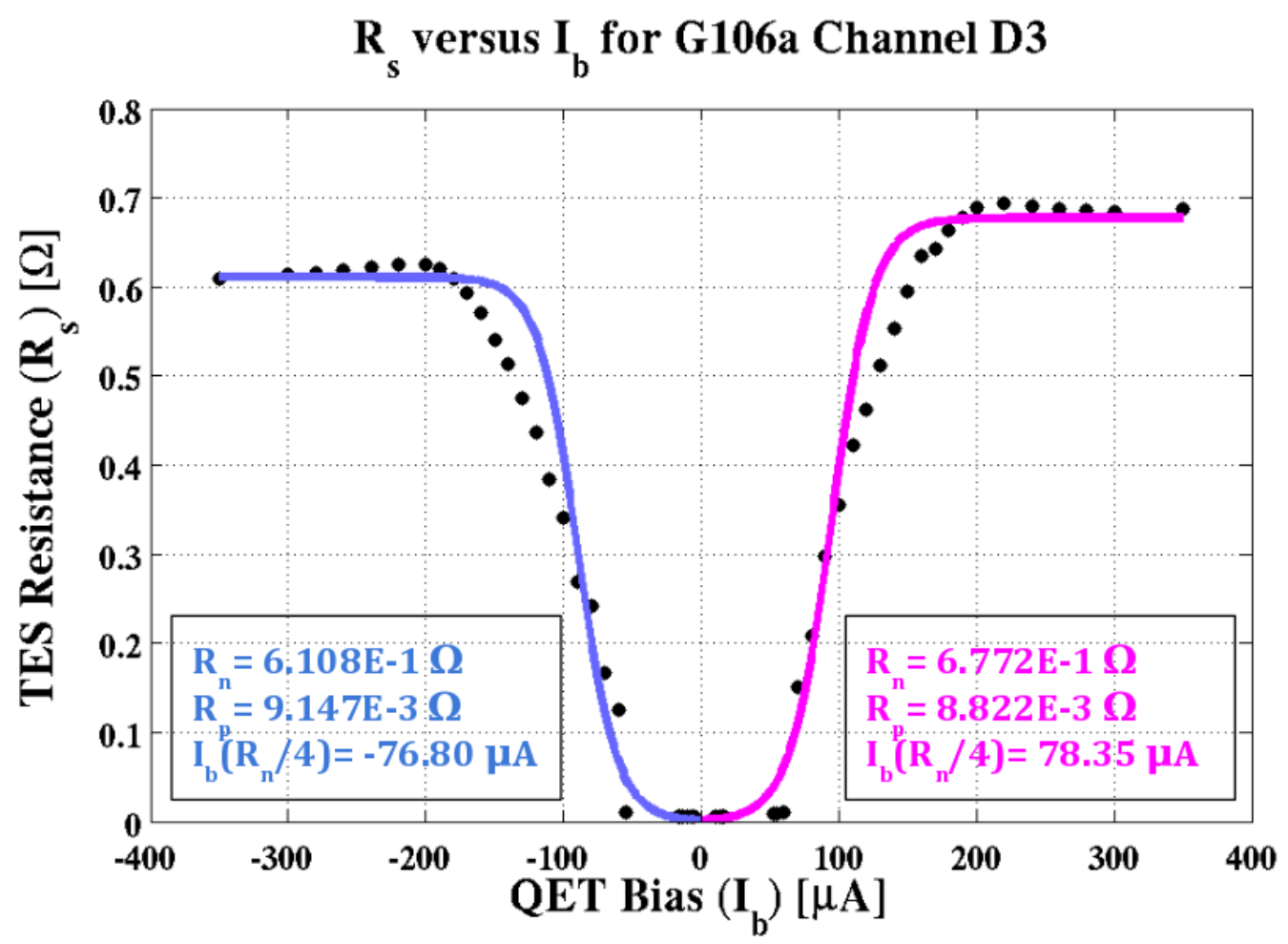

Figure 5.3: $R_{s}$ plotted versus $I_{b}$ for G106a phonon channel PE Side 1 (D3). Plot shows sensor resistance increasing from zero in the superconducting region and plateauing towards the positive and negative QET bias $R_{n}$ values. Positive and negative QET bias curves are fit to the data, shown in blue and pink. From the fit curve, $R_{p}$ and $R_{n}$ are found, along with the optimized QET bias corresponding to the start of the transition region, where $I_{b}=R_{n} / 4$. Estimated resistance values and optimized QET bias are shown in the bottom corners of the plot. 
5.3 and C.2. With these values, and the proper SQUID settings outlined in Section 5.1.1, proper phonon data can be taken.

\subsection{Raw Phonon Pulse Studies}

Since initial 100mm iZIP studies began in 2011, a substantial effort has been placed on optimizing $100 \mathrm{~mm}$ phonon signals. Some efforts, such as the introduction of RF filters in the signal readout pipeline, improved the noise performance of the phonon readout. Others, such as the efforts put forth in this section, aimed to improve signals through careful improvements on phonon signal processing.

This section covers phonon channel analysis which specifically looks at raw phonon signals in an attempt to improve phonon data processing at the UMN test facility. Since Run 56 marked the first test facility run using the full capabilities of the MIDAS DAQ, initial raw phonon studies set forth to ensure MIDAS was working properly, and passing raw events to processing as expected. These studies set forth to verify the following:

- That the processing RQ outputs corresponded to the correct raw event.

- That the OF processing algorithm was correctly determining the proper fit for each raw event.

- That OF templates accurately reflected UMN facility raw phonon pulse shapes.

The first two items were both verified by creating a replica of the Optimal Filter processing in MATLAB. The replication script inputs a single raw event (phonon or charge) and the noise and template information contained in the series filter file (discussed in Section 3.4, and outputs the amplitude, time shift, and $\chi^{2}$ reduced quantity values. An example of the MATLAB replication script output for a single event is shown in Fig. 5.4. Fit values derived by this replication script could then be compared to RQ values processed with the standard ROOT-based CDMS processing package. For the few hundred total phonon events studied, amplitude and time shift fit values found by the replication script matched within $1 \%$ of the processing RQ values, indicating that CDMS processing was outputting RQ's that corresponded to the correct raw event. 
Slight differences between $\chi^{2}$ values, typically $<5 \%$, are attributed to rounding differences between MATLAB and ROOT. Overall, the study indicated that CDMS raw event processing was well understood and repeatable.
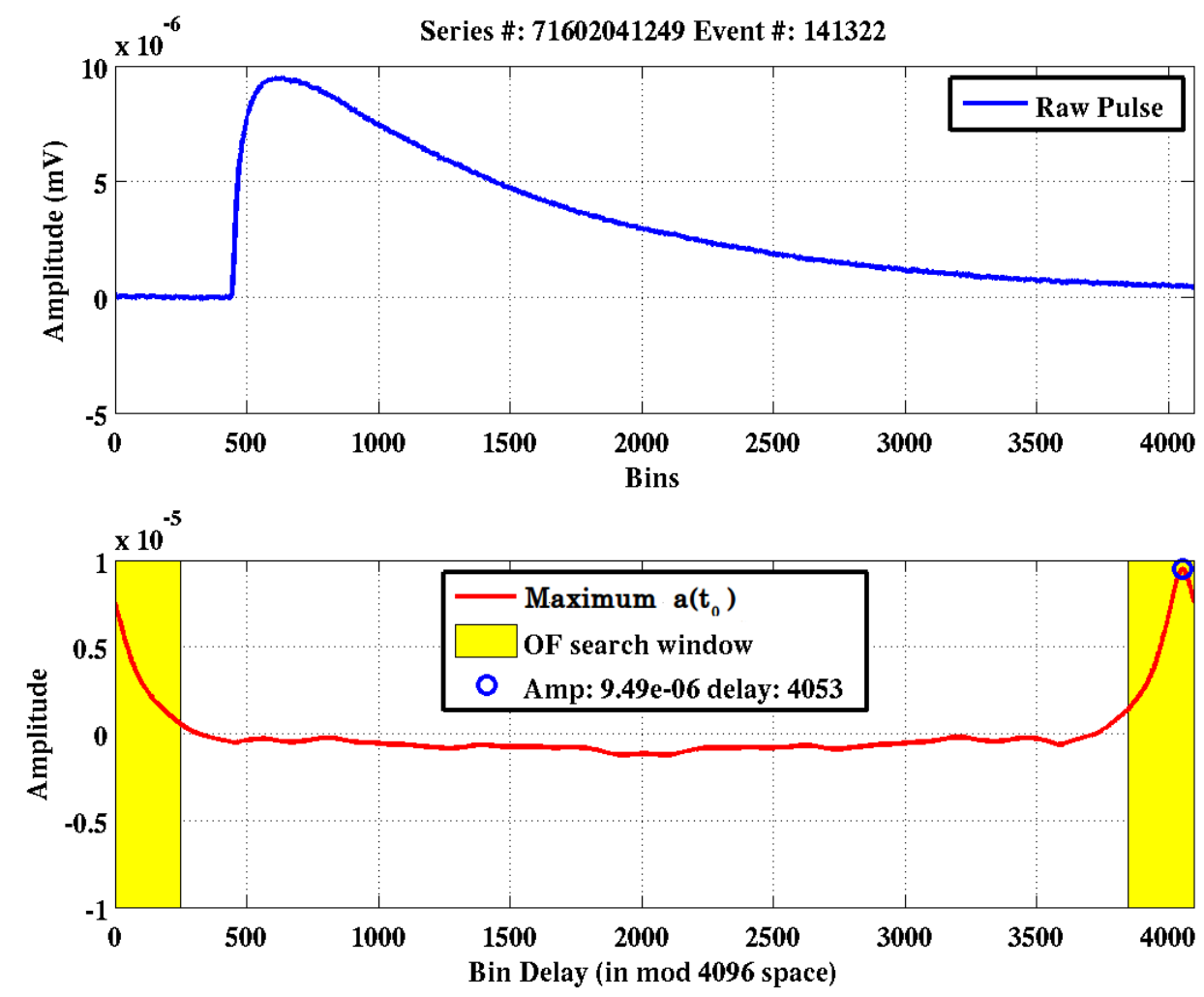

Figure 5.4: A total phonon raw pulse (blue, TOP) from UMN test facility data is input to the OF replication script in MATLAB. The replication script clearly illustrates the OF algorithm, by depicting the resulting $\hat{a}\left(t_{0}\right)$ amplitude function (red, BOTTOM) and search window (highlighted in yellow). This allows the user to verify that the selected maximum amplitude (blue circle) is the correct choice.

As shown in Fig. 5.6, the replication script also allowed for a direct comparison between a raw event, and the reconstructed event, providing insight to the goodnessof-fit that went beyond the somewhat esoteric $\chi^{2}$ value. Studies indicated that the OF processing was correctly determining amplitude and time shift RQ values which minimized $\chi^{2}$, but it was using a template that didn't match the shape of raw pulses. 
Thus, reconstructed pulses were not an accurate representation of their raw counterpart. In particular, the old template had a slower rising edge and a bloated falling edge, which hindered accurate fitting. It is uncertain how this template was developed, and how long ago. It was likely developed using phonon pulses from a detector smaller than G106a, which have a slightly different shape than pulses seen in G106a (or other 100 mm iZIPs). This would explain why the template is not optimal for G106a pulses.

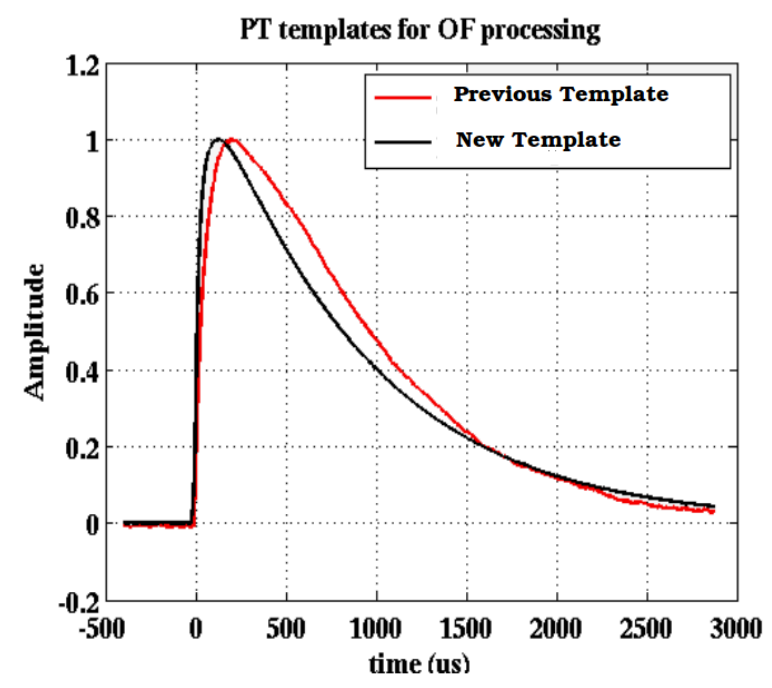

Figure 5.5: Total phonon templates used for UMN test facility OF processing. The previous template (red) did not accurately represent raw pulses, and was thus replaced by a new template (black), created from averaging a thousand well-behaved raw events.

N. Mast of the UMN group created new templates for each phonon channel, as well as a total phonon template. As with his cross talk templates, discussed in Appendix B phonon template construction begins with raw events whose RQ values pass basic quality cuts ( $\chi^{2}$ cuts, side 1 and side 2 charge symmetry cut), normalized to peak height of one. Additional quality cuts are applied to the raw data to ensure that remaining pulses are smooth, free of pileup, and have well-behaved pre-trigger and post-trigger baselines [201]. The mean of a thousand normalized events which pass this criteria forms the new template.

Fig. 5.5 compares the original total phonon template to the new template created by N. Mast. Fig. 5.6 compares raw event reconstruction with both templates to the original raw event. For the majority of the $\sim 200$ raw total phonon events studied, the newly constructed template fit with a lower $\chi^{2} \mathrm{RQ}$, indicating a better fit than the original template. For the remainder of this phonon analysis, the new templates will be used for each phonon channel and the total phonon events. 


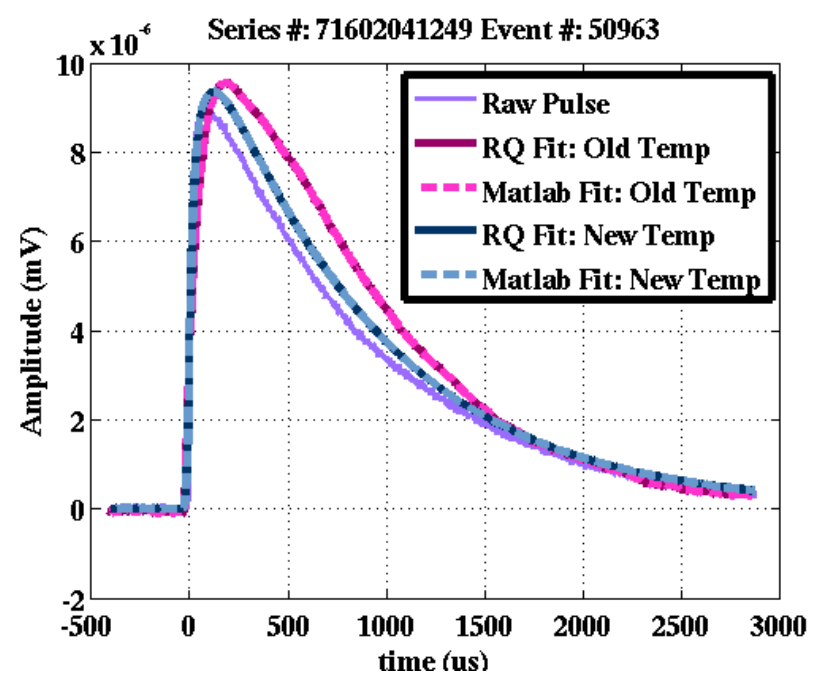

Figure 5.6: A reconstructed event, using the old and newly constructed total phonon template, to the raw pulse (purple). The fit constructed using the RQ value calculated in CDMSBats is shown in solid maroon and navy lines for the old and new template, respectively. The fit constructed using the MATLAB algorithm is shown in dotted pink and light blue lines for the old and new template, respectively.

\subsubsection{Constructing Relative Calibration}

In tandem with the raw pulse studies previously described, efforts to determine the relative calibration between phonon channels was underway. Just as Qsum1 and Qsum2 require separate calibration factors to align their spectrum peaks with the proper calibration source energy, each phonon channel needs its own calibration value. Fig. 5.7 depicts the phonon signals of all twelve channels before a relative calibration is applied. Sensitivities of $76 \mathrm{~mm}$ phonon sensors can vary by $\sim 10-15 \%$ [186]. However, the total phonon energy of each event is distributed to the phonon channels in a position-dependent way, and thus calibration methods cannot rely on the complete energy collection of calibration peaks, as charge calibration does.

However, relative phonon calibration can still be achieved by comparing the collections of phonons that have lost directional information. Recall from Section 2.2.4. that $\sim 800 \mu$ s after the initial recoil in the crystal, the remaining uncollected phonons have undergone multiple ballistic scatters and have lost directional information about the original event. These phonons are evenly dispersed throughout the detector (i.e. they have lost any position dependence), and they are equally likely to hit any channel surface. Since each channel has the same TES surface coverage, each channel should see equal signals from these late phonons. Therefore, relative phonon calibrations can 
be found by matching the phonon pulse tails, which correspond to these late phonon signals.

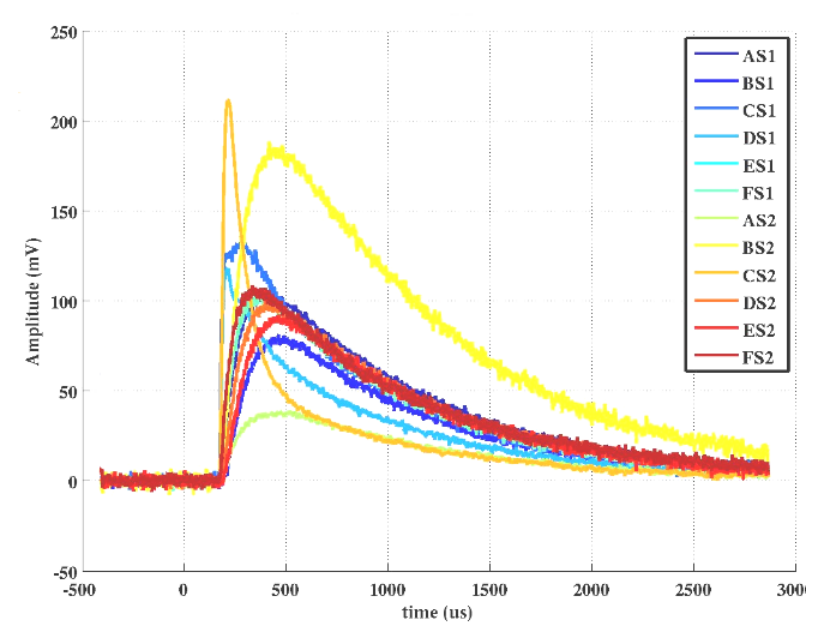

Figure 5.7: The twelve phonon channel pulses from an example event without relative calibrations applied. Peak tails $(\sim 800 \mu$ s after rising edge) containing signal from collected athermal phonons are not aligned.

Events used for relative calibration calculations must first pass typical RQ quality cuts, including $\chi^{2}$, radial charge partition, and charge symmetry cuts. To avoid events with low signal-to-noise ratio, surface events from the ${ }^{241} \mathrm{Am}$ source, or high energy events with the potential to saturate phonon channels, an energy cut is applied, requiring Qsum1 to fall within $80-180 \mathrm{keV}$. For a given data series, $\sim 300$ raw events which pass these quality cuts (and are not randoms) are considered for relative calibration, to cut down on processing time. Series with less than 300 quality events undergo relative calibration with whatever events are available, though this is atypical.

Next, a series of raw pulse quality cuts are applied, to remove pulses with a nonstandard or unwanted shape. The most easily identified unwanted event is a railed event. For various reasons, such as improper settings, or changes in the fridge environment, a channel can rail, or flat line, during data taking. Once a channel is railed, it typically remains railed until the series ends. Sometimes, flashing between series is enough to rectify a railed channel. Other times the DAQ must be reset to reanimate the lost channel.

The prepulse or pretrigger region is defined as the time in the trace before the pulse occurs. Typically for phonons the first 500 ADC bins $(400 \mu \mathrm{s})$ of a trace is the prepulse region. However, recoils that occur nearer to channels not designated as a trigger 
channel can result in well-behaved events that occur slightly before the 500th bin, since the pulse start time is dependent only on the pulses seen in channels designated as trigger channels. Additionally, a well known glitch in DCRC RevC data writing causes traces to contain (seemingly random) single-bin spikes in amplitude within the first 10 bins. To account for both these issues, the prepulse region is considered to be the 10th to the 400 th $\mathrm{ADC}$ bin $(8-320 \mu \mathrm{s})$, for the purpose of these raw pulse quality cuts.

Raw pulse cuts depend on the quantity, $\sigma_{C}$,

$$
\sigma_{C}=\sqrt{\frac{1}{b_{2}-b_{1}-1} \sum_{i=b_{1}}^{b_{2}}\left|C_{i}-\mu_{C}\right|^{2}},
$$

the standard deviation of the $\mathrm{ADC}$ bin region $\left[b_{1}, b_{2}\right]$ of a given channel's signal, $C$. Here $\mu_{C}$ is the signal mean in the region, given by,

$$
\mu_{C}=\frac{1}{b_{2}-b_{1}} \sum_{i=b_{1}}^{b_{2}} C_{i} .
$$

Let $\tilde{\sigma}_{C}$ represent the particular prepulse case where the ADC bin region is $[10,400]$. Typically the prepulse region of a well-behaved event will be mostly flat, with small amounts of noise contributing to a standard deviation of $\mathcal{O}(10 \mathrm{mV})$. Railed events are easily removed by cutting out all events with that have a null standard deviation in their prepulse region, indicating no noise present.

The standard deviation of the prepulse can also be used cut on post-muon events. When a muon recoils within the detector, the energy produced saturates phonon and charge channels quickly, creating events that cannot be fit well by the template and are typically removed by the charge $\chi^{2}$ cuts. Due to the differences in charge carrier and phonon drift speeds, ionization signals quickly recover from muon events, whereas phonon channels require more time.

Depicted in Fig. 5.8, the phonon channels are still collecting phonons from the muon event when the post-muon event occurs, resulting in a downward sloping prepulse region typical of post-muon phonon traces. As can also be seen by the figure, the resulting charge event shows no indication of abnormality. Because charge has already recovered from the muon, charge quality cuts will not remove post-muon events. Fortunately, postmuon events have a prepulse standard deviation of $\mathcal{O}(100 \mathrm{mV})$ or larger, depending on 


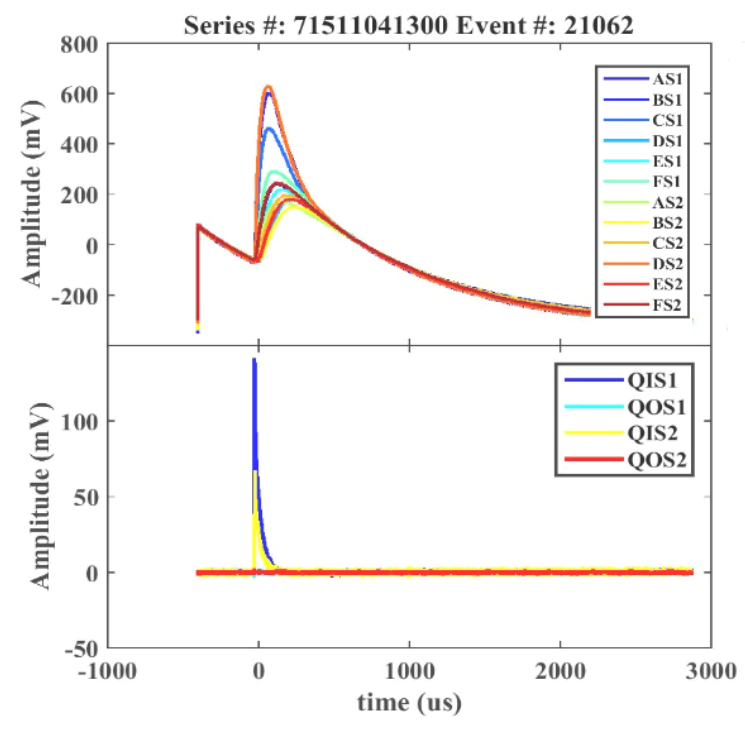

Figure 5.8: The phonon (TOP) and charge (BOTTOM) channel traces for a post-muon event. Charge channels have already recovered from the muon recoil event, and have no noticeable disfigurations. The phonon channels are still collecting thermalized phonons from the muon event when the postmuon event occurs, resulting in a downward sloping prepulse region typical of post-muon phonon traces.

their time delay relative to the original muon event. Cutting out events with a prepulse standard deviation of $\tilde{\sigma}_{C}>100 \mathrm{mV}$ removes all substantial post-muon events.

As a final cut, $\sigma_{C}$ of the tail region (ADC bins 1512-3637) is calculated for all raw events that passed the RQ quantity cuts. The events with a standard deviation larger than the 70th percentile are removed as well. This cut works to remove the noisiest events, or those with odd tail behaviors. These odd behaviors can include noise pickup, or pileup events where the secondary event has low enough energy to pass the $\chi^{2}$ cut, to name a few examples.

A straightforward method to perform the relative calibration is to select a base channel, and align the tails of the other channels with the tail of that channel, by applying a calibration factor to each channel. For the $n$th event, a goodness of fit, $\chi_{C_{n}, B_{n}}^{2}$, between base channel signal, $B_{n}$, and channel signal, $C_{n}$, with calibration factor, $\mathcal{F}$, is given by,

$$
\chi_{C_{n}, B_{n}}^{2}(\mathcal{F})=\sum_{i=b_{1}}^{b_{2}} \frac{\left(\mathcal{F} C_{n, i}-B_{n, i}\right)^{2}}{\left(b_{2}-b_{1}\right)\left(\left(\mathcal{F} \tilde{\sigma}_{C_{n}}\right)^{2}+\left(\tilde{\sigma}_{B_{n}}\right)^{2}\right)},
$$

where the sum is occurring over the tail region $[b 1, b 2]=[1512,3637]$. The total goodness of fit between channel $C$ and base channel $B$, over all $N$ raw events which 
pass the quality cuts, is then given by,

$$
\chi_{C, B}^{2}(\mathcal{F})=\sum_{n=1}^{N} \chi_{C_{n}, B_{n}}^{2}(\mathcal{F}) .
$$

A relative calibration MATLAB script scans through potential calibration factors $\mathcal{F} \in$ $[0.01,10.0]$, finding the calibration value which minimizes $\chi_{C, B}^{2}$ for each channel, relative to each possible base channel; this calibration value will be called $\hat{\mathcal{F}}$.

It is possible that some events deviate from typical tail behavior, despite the quality cuts already applied. As a final measure to remove such events, any events with a

$\chi_{C_{n}, B_{n}}^{2}(\hat{\mathcal{F}})$ greater than four times the mean of a $\chi_{C_{n}, B_{n}}^{2}(\hat{\mathcal{F}})$ will be removed. After this final cut, $\chi_{C, B}^{2}(\mathcal{F})$ will be minimized again, determining a final $\hat{\mathcal{F}}$ calibration factor for each channel relative to each potential base channel.

\subsubsection{Stability of the Relative Calibration}

The calculated $\hat{\mathcal{F}}$ factors can be compared across different series in a detector run to look at calibration stability. It is expected for calibration factors to change if settings in the readout electronics are altered, or in the case of significant changes in the thermal profile of the fridge. Based on studies of SuperCDMS Soudan $76 \mathrm{~mm}$ iZIP data, calibration values are expected to deviate within $\sim 5 \%$ over time, with larger deviations possible on series with low statistics [212].

When considering which base channel to select for an official relative calibration (that is, the relative calibration that will be used in data reprocessing), significant deviations between series is indicative of a poor base channel. This could be due to noise periodically affecting the channel, or other issues with electronics. A good base channel should be stable (within $\sim 10 \%$ ) over the relevant data series.

Fig. 5.9 monitors the change in calibration values and deviation in value from the mean, of each series with respect to base channel PBS1, for a 15 hour data taking period in Run 54. Fig. C.3 in Appendix C monitors the change in calibration values of each series with respect to each base channel, for the same 15 hour data taking period. The clear trends in most channels relative to base channels PDS1, PES1, PAS2, PES2 and PFS2 (shown most clearly in the right side plots of Fig. C.3) indicate that these channels undergo some instability, and should not be used as a base channel. Otherwise, 



Figure 5.9: (TOP) Calibration values over time for all channels with respect to base channel PBS1. (BOTTOM) Calibration value deviation from mean over time for all channels with respect to base channel PBS1. Series used include those of a 15 hour data taking period in Run 54 . 
most channels show stability sufficient for use as the base channel, with calibration values remaining within $10 \%$ on all channels within the 15 hour period during Run 54. Channel PBS1 was selected to be the base channel, due to its stability over the 1-month, Run 54 data taking period considered for this analysis. Shown in Fig. 5.10, most calibration values calculated with reference to base channel PBS1 only deviate within $5 \%$ of the mean value, over the course of a month. This level of stability (with $\sim 5 \%$ deviations from the mean) is observed in other UMN facility runs, including Run 56. Even the most extreme deviations remain within $15 \%$ of the mean. As mentioned previously, it is likely that these extreme deviations were due to periodic noise in a channel, or low statistics in a particular series, which cannot be avoided in this series-by-series calibration approach.

Implementing the relative calibration into processing requires entering an array of calibration values into a processing parameter file. The factors will then be applied to the raw traces of each corresponding channel before each channel is summed into the total phonon trace, which then undergoes normal processing algorithms. The relative calibration values will not affect the processing of individual channels.

Because calibration values must be manually entered into processing files, it is not prudent to use the optimal $\hat{\mathcal{F}}$ values for each individual series, given that hundreds of series are produced in a given data run. Instead, the mean of these values across all series can be used as an easy alternative, with minimal effects on the total phonon amplitude. The total phonon signal, $P T$, is given by,

$$
P T=\sum_{i=1}^{12} \hat{\mathcal{F}}_{i} C_{i},
$$

for a $100 \mathrm{~mm}$ iZIP with 12 channels. If the calibration of a single channel, $C_{a}, a \in[1,12]$ is altered by some $\delta \hat{\mathcal{F}}_{a}$, then the total signal becomes,

$$
P T=P T_{0}+\delta P T=\sum_{i=1}^{12} \hat{\mathcal{F}}_{i} C_{i}+\delta \hat{\mathcal{F}}_{a} C_{a}
$$

From this, the percent change in $P T$ from its original value is,

$$
\delta P T / P T_{0}=\frac{\delta \hat{\mathcal{F}}_{a} C_{a}}{\left(\sum_{i=1}^{12} \hat{\mathcal{F}}_{i} C_{i}\right)}
$$

From Run 54 phonon data, an individual channel contributes $0.04 \%$ to $49.4 \%(0.03 \%$ to $47.1 \%$ ) of a well-behaved total phonon event before (after) calibration is applied, 

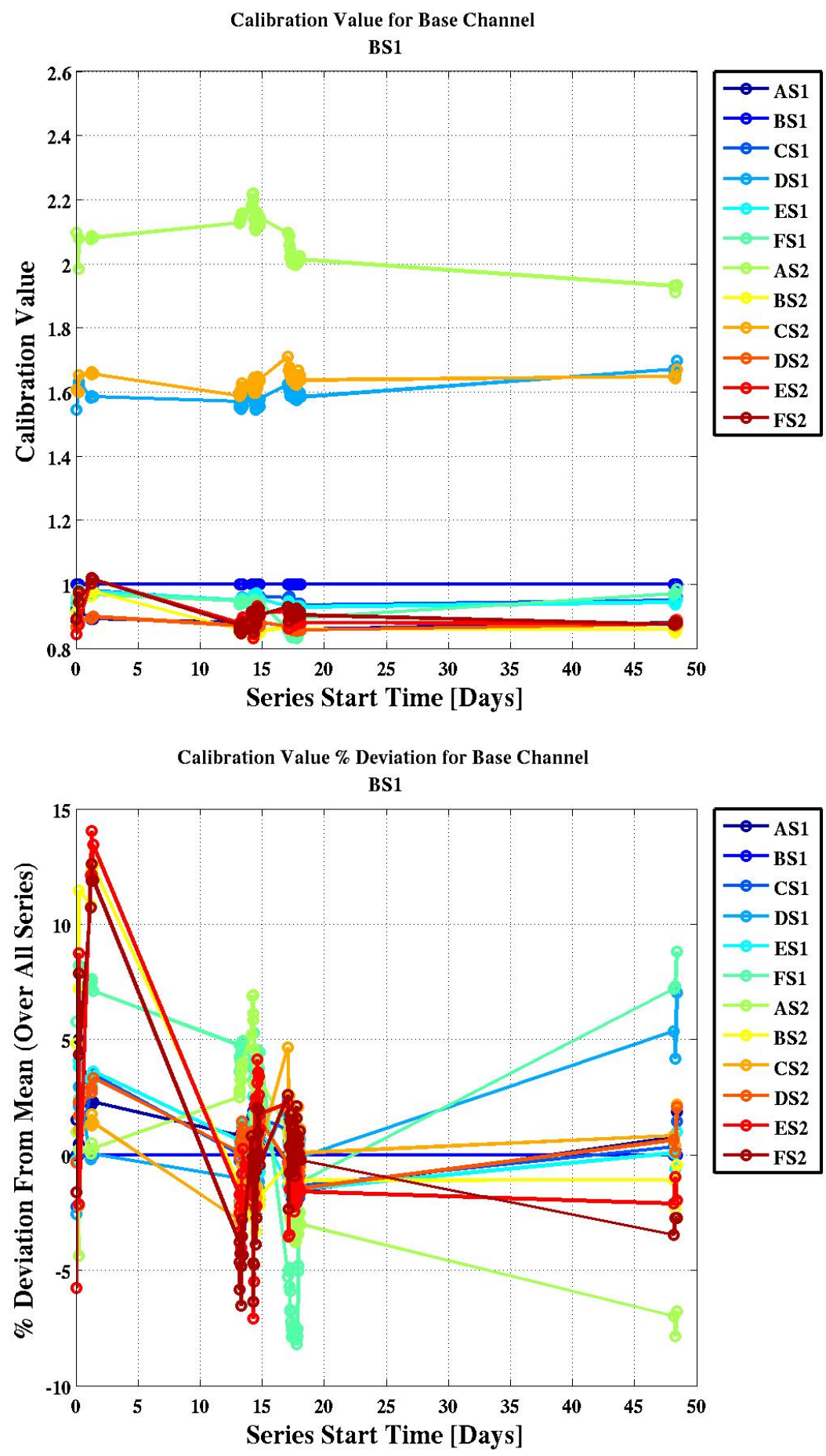

Figure 5.10: (TOP) Calibration values and (BOTTOM) values deviation from mean over time for all channels with respect to base channel PBS1. Includes data taken over the whole, 1-month, Run 54 data taking period. 
and PBS1 base channel calibration values range from 0.872 to 2.07 , with up to $14.0 \%$ deviation from mean values. Therefore, the percent change in $P T$ from its original (calibrated) value is restricted to fall within $-4 \%$ to $5 \%$. Because so many channels contribute to the total signal, small deviations in the calibration of a single channel do not significantly affect the total outcome.

\subsubsection{Effects of the Relative Calibration}

The effects of the relative calibration can be understood without reprocessing pulse data. Like Qsum1 and Qsum2, define Psum as the sum of all phonon channel RQ amplitudes, and define PsumC as the sum of all phonon channel RQ amplitudes, with the PBS1 base channel calibration factors applied. Fig. 5.11 shows the Psum and PsumC spectrum for seventy series from data Run 54. Both spectra are comprised of series individually calibrated to the $10.36 \mathrm{keV}$ activation peak, and both spectra have the charge quality cuts applied. The PsumC spectrum shows improved resolution for both calibration peaks present.

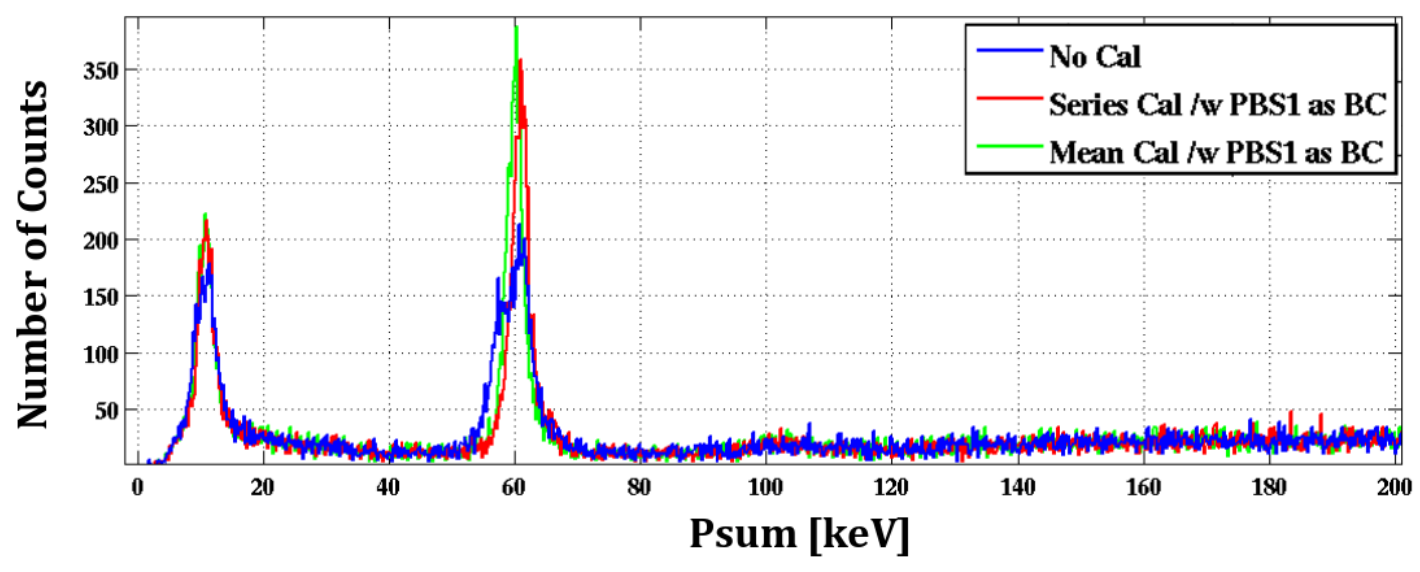

Figure 5.11: Spectra of Psum (blue), PsumC calibrated using the individual series factors (red), and PsumC calibrated the mean of the series factors (green) for seventy Run 54 data series. Both $10.36 \mathrm{keV}$ activation peak and $60 \mathrm{keV}$ source peak are visible. Absolute calibration applied to align $10.36 \mathrm{keV}$ peak.

A closer inspection of the $10.36 \mathrm{keV}$ activation peak and $60 \mathrm{keV}{ }^{241} \mathrm{Am}$ peak are shown in Fig. 5.12. Figure shows Psum spectrum, PsumC using optimal calibration 
values for each individual series and PsumC using mean calibration values. All peaks are fit with a Gaussian plus flat background. Gaussian fits indicate that using the mean (individual) calibration values improve the $60 \mathrm{keV}$ peak resolution $54 \%$, from $2.849 \mathrm{keV}$ to $1.323 \mathrm{keV}(1.378 \mathrm{keV})$, and improves $10.36 \mathrm{keV}$ peak resolution 26\%, from $1.659 \mathrm{keV}$ to $1.223 \mathrm{keV}(1.236 \mathrm{keV})$. A $4.0 \%$ (1.1\%) difference in $60 \mathrm{keV}(10.36 \mathrm{keV})$ resolution between the mean and individually calibrated spectra further indicate that small deviations in calibration values are not significant. PsumC calibrated with the mean factors shows better resolution than individual series factor calibrations, likely due to poor calibration factors found for low statistic series.
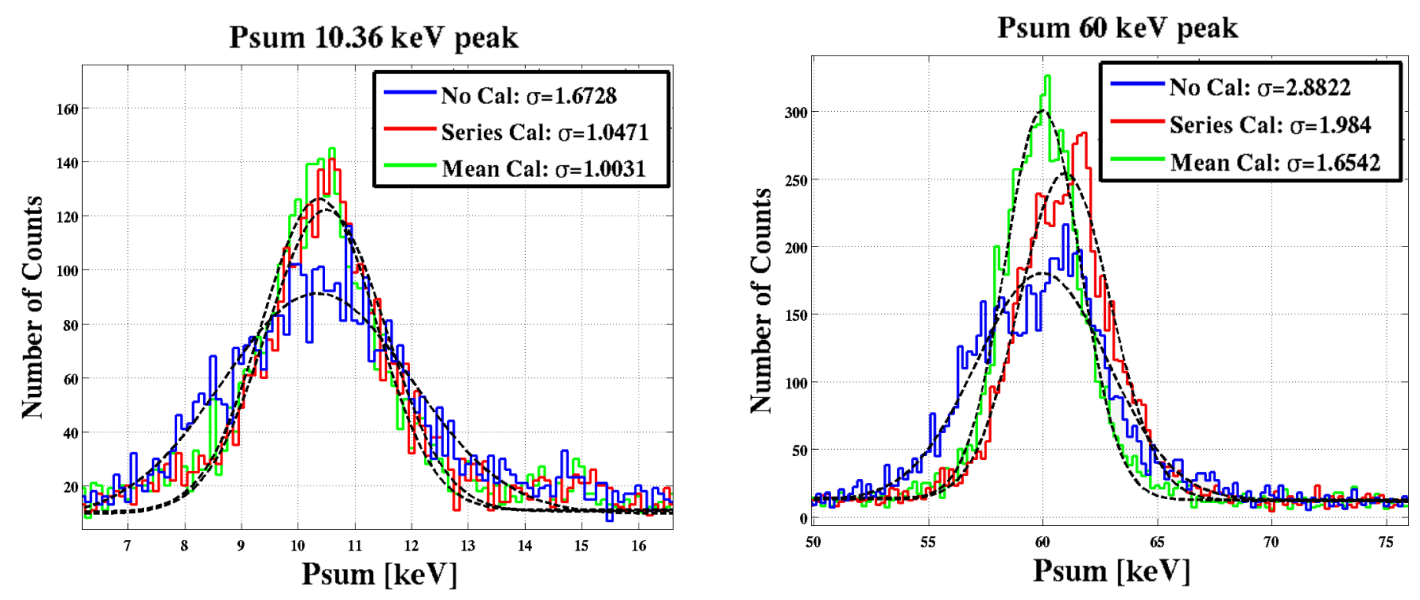

Figure 5.12: Fitting the (LEFT) $10.36 \mathrm{keV}$ activation peak and (RIGHT) $60 \mathrm{keV}$ source peak for uncalibrated Psum (blue), PsumC calibrated using the individual series factors (red), and PsumC calibrated the mean of the series factors (green).

\subsubsection{Constructing Non-Stationary Optimal Filter}

The theory of the Non-Stationary Optimal Filter has already been discussed in Section 3.4.2, but will be summarized again here. Position-dependence of the primary phonons produces fluctuations in the first $100 \mu$ s of the signal, causing the peak region to deviate from the general phonon signal shape. The NSOF aims to deweight this portion in the $\chi^{2}$, by treating the deviations as noise. Since the "noise" occurs within the first $100 \mu \mathrm{s}$, it will be correlated in frequency space. Therefore, the entire covariance matrix must 
be calculated for the NSOF fitting algorithm, to account for the correlated frequency components. This understandably requires heavier computation than the standard OF algorithm, which assumes uncorrelated, white spectrum noise and a well-understood pulse shape, and thus requires only the diagonal covariance matrix elements.

A script was developed in MATLAB by M. Pyle and Y. Ricci to construct the covariance matrix, requiring several hundred, well behaved total phonon pulses, and a baseline noise PSD. Total phonon events must have their relative calibrations applied, and must satisfy the following requirements:

- Must pass charge quality cuts, including $\chi^{2}$, charge radial partition, and charge symmetry cuts

- Must have Qsum1 energy within 80 - $180 \mathrm{keV}$

- Must be free of phonon pileup events

- Must not be a post-muon event $\left(\sigma_{T}<50 \mathrm{mV}\right)$
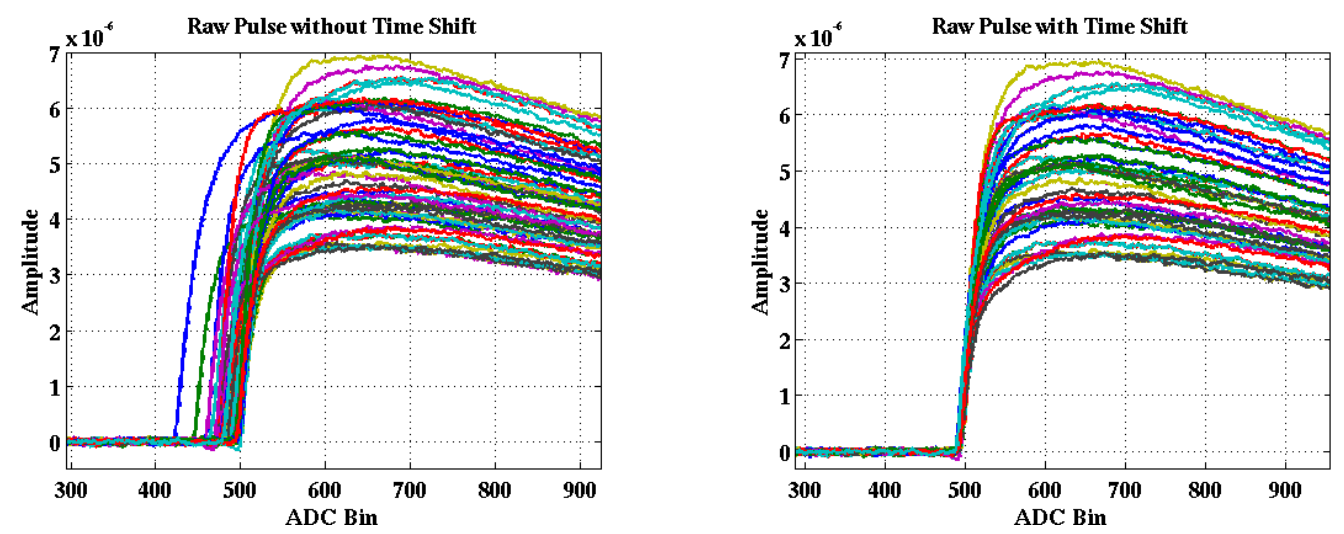

Figure 5.13: A sample of raw pulses (LEFT) before and (RIGHT) after they are shifted over by their time shift RQ value (converted to ADC bins). With the pulses all correctly aligned to start at the 500th bin, the pulses can be used to construct the covariance matrix.

Since the NSOF looks for small variances in the rising edge of the raw pulses, it is essential that pulses input into the covariance matrix script have been properly aligned, 
such that they begin at the same time (or in this case, the same bin). Fortunately, the time shift RQ value, PTOFdelay, can be converted into ADC bins and used to shift pulses the appropriate amount. Fig. 5.13 shows the rising edge of raw pulses before and after they are shifted into alignment.
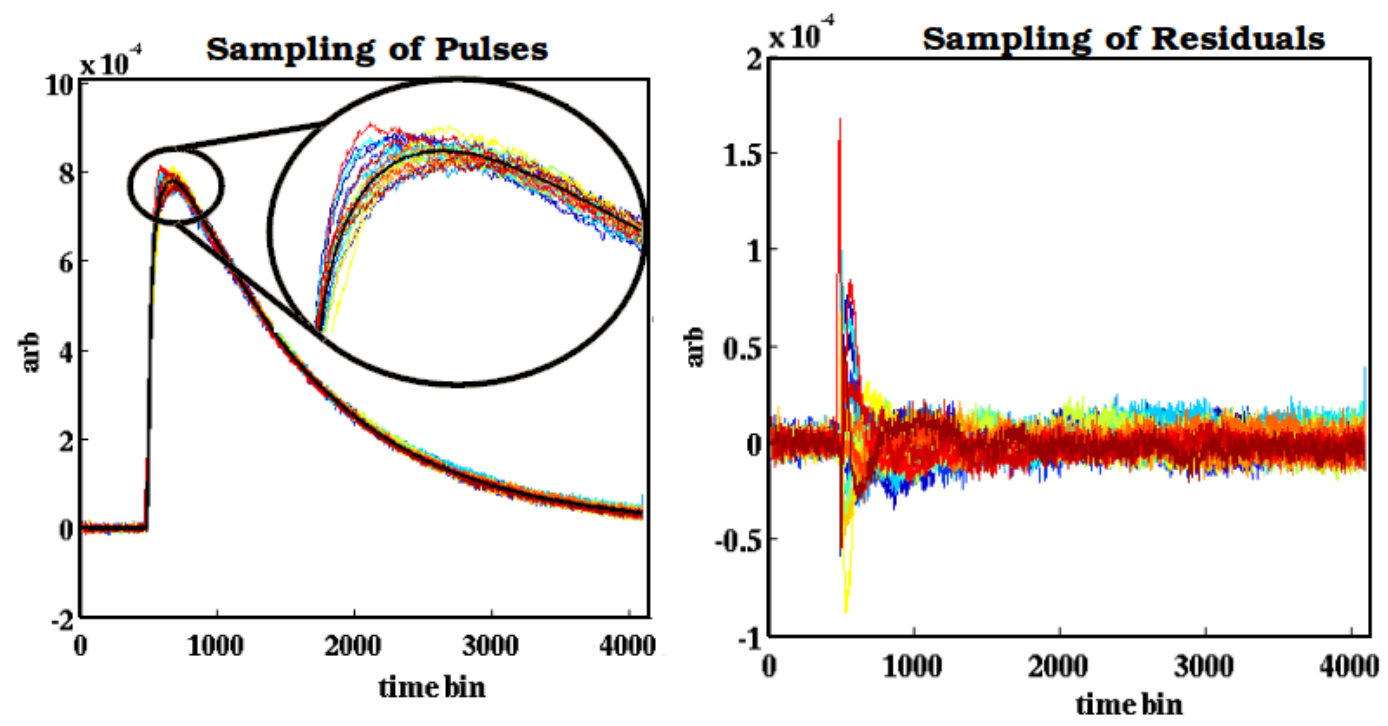

Figure 5.14: (LEFT) A sampling of a hundred raw, total phonon events, normalized to a maximum amplitude of one. Although they appear similar in shape, a zoom-in near the peak shows the subtle differences in the rising edge of each event. The mean of each event is shown in black. (RIGHT) The discrepancies in each peak's rising edge are made more apparent in the trace residuals, the remainder once the mean has been subtracted from the pulse. These residuals are a result of position dependence in the event. They are treated as noise and de-weighted in the Non-Stationary Optimal Filter processing algorithm.

$M$ properly aligned phonon pulses of $N$ ADC bins (4096 for UMN facility), which fulfill these requirements and are not random triggers will be passed into the script in a $N \times M$ matrix, along with a PSD constructed from their initial random trigger events. Each pulse will be scaled to one, and a pulse template will be constructed from the mean of all events. The residuals of each pulse (i.e. the position-dependent deviations treated as noise), can be seen by subtracting the template from each raw 
pulse, as shown in Fig. 5.14. Then the covariance matrix is calculated for the Fourier transform of the scaled pulses, and the baseline noise is subtracted, as illustrated in Fig. 5.15. The covariance matrix is sparsified and a frequency cutoff is applied, to only keep the relevant low frequency cross-correlations. The covariance matrix output can then be put into the CDMS processing templates files, where it can be accessed by NSOF processing algorithms.

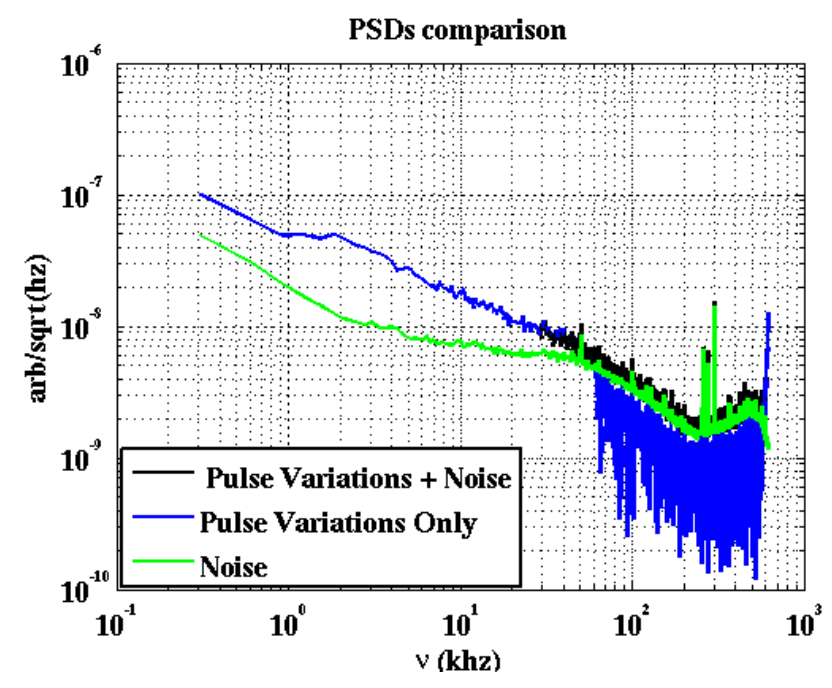

Figure 5.15: From the initial randoms taken at the start of each series, a PSD is found on pulseless (only noise) traces. As input into covariance constructing script, the stationary noise PSD (green) is subtracted from the total pulse variations including noise (black). What remains is the pulse variations only (blue), without the presence of stationary noise.

Determining quality cuts, applying cuts to data, aligning pulses and calculating the covariance matrix are all time consuming processes, and thus the covariance matrix is not found for each series 3 . Like the relative calibration, it is assumed that the phonon signal position-dependence is relatively stable over time. For the Soudan Run 133, which spanned months, a single covariance matrix was calculated for each detector. At the UMN test facility, the covariance matrix calculated from the Run 54 data series will be applied to all data included in this analysis.

\subsection{Reprocessing with Relative Calibration and NSOF}

With the Run 54 covariance matrix included in the processing files, select series were reprocessed with the relative calibration and NSOF. Although processing can be done

\footnotetext{
${ }^{3}$ Additionally, each matrix, once sparsified, is still $>1$ Gigabyte in size.
} 
concurrently in the HTCondor system, each minute-long dump file can take over an hour to processes completely. Therefore, only the 70 series included in the relative calibration stability (shown in Fig. 5.10) were reprocessed. These series include data

taken with only the internal ${ }^{241} \mathrm{Am}$ source, as well as data taken with the external ${ }^{252} \mathrm{Cf}$ source, to allow comparisons between neutron-rich data to typical background neutron data. Several data series taken with an external ${ }^{133} \mathrm{Ba}$ source were also reprocessed, such that the effects of the NSOF on phonon energy resolution and various energies can be studied. All reprocessed data has an applied bias of $\mp 4 \mathrm{~V}$, as this bias was deemed to have near-complete charge collection efficiency.

Data reprocessed with the NSOF algorithm will still undergo OF fitting, and will maintain the standard optimal filter phonon RQ values (PAS1OFamps, PTOFdelay, PDS2OFchisq are a few examples). The NSOF algorithm will output PTNFamps, PTNFdelay, and PTNFchisq values. Let PtNF and PtOF represent the calibrated total phonon signal amplitudes for the NSOF and OF fit, respectively.

\subsubsection{Improvement to Raw Pulse Fitting}

Directly comparing the NSOF and standard OF template fit to well-behaved raw pulses provides evidence that the NSOF fit performs better. Fig. 5.16 illustrates this point by comparing the fits for two well-behaved total phonon pulses of different energies. These pulses pass the charge quality cuts outlined in Section 4.1, as well as the raw pulse cuts applied to events used for covariance matrix construction, discussed in Section 5.2 .4 .

Although fit comparison of individual pulses provides anecdotal evidence in support of the NSOF, it cannot provide an overview of how entire series are affected by the NSOF fit. A comparison of the OF and NSOF $\chi^{2}$ RQ value distributions could be performed, but subtle differences in how the two $\chi^{2}$ values are calculated means they cannot be directly compared. Instead, a similar comparison can be made, by comparing integrated pulses. A basic RQ value calculated for each raw pulse is the integrated pulse value; this value is the sum of the amplitude of each ADC bin, over all bins in the pulse. The same technique can be applied to the fitted pulses, but summing over every bin in the template after it was scaled by the OF or NSOF amplitude RQ. The better the fit, the closer the integrated template value should match to the integrated raw pulse value. If a ratio is taken of the fitted template integration value over the raw pulse integration 

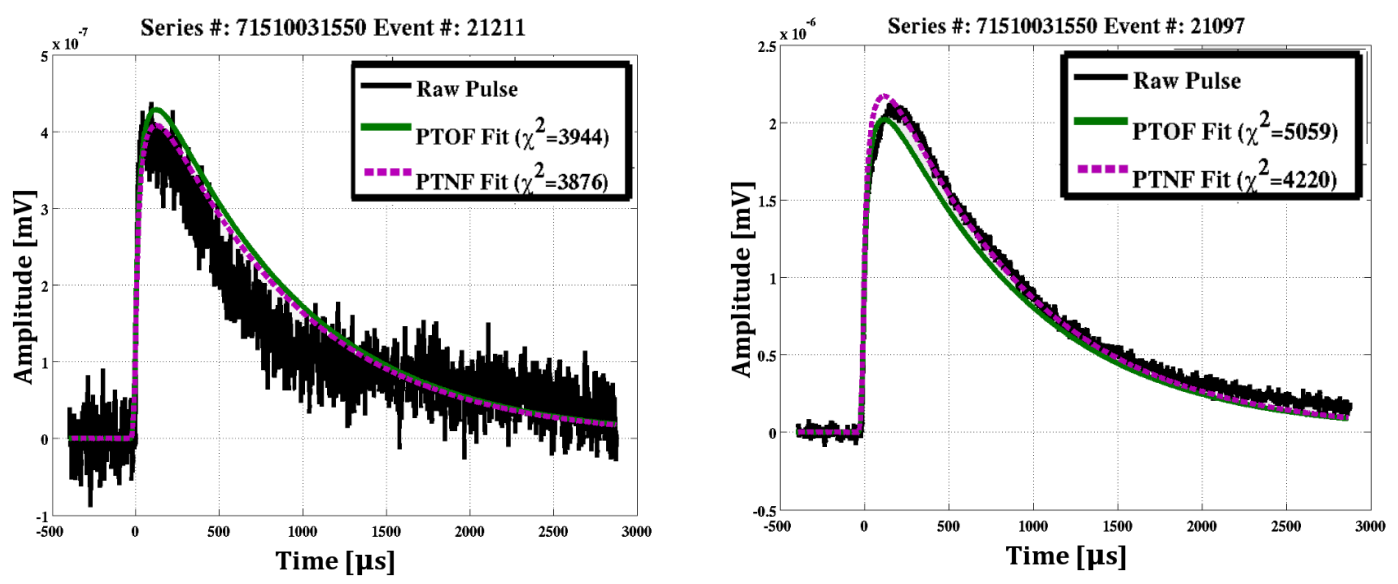

Figure 5.16: Comparing the NSOF (dotted pink) and standard OF (solid green) template fits for two typical raw total phonon pulses of energy $14 \mathrm{keV}$, and $73 \mathrm{keV}$, respectively. Comparing fits by eye indicates a superior fit by the NSOF algorithm.

value, a good template fit would have a ratio near one.

Fig. 5.17 shows the ratio of the template fit integration over the raw pulse integration value, for both the NSOF and the OF fits, with standard RQ quality cuts and raw pulse quality cuts applied 4 . Both fits form a Gaussian distribution around one, the perfect fit ratio value. Fitting these distributions with a Gaussian reveals a $\sim 33 \%$ improvement in reconstruction accuracy when the NSOF is used, with a PTOFamp peak mean of $1.071( \pm 0.001)$ and a PTNFamp peak mean of $1.0360( \pm 0.001)$. The fits also show a $\sim 31 \%$ improvement in precision, from a resolution of $\sigma_{O F}=6.670 \mathrm{e}-2( \pm 0.142 \mathrm{e}-2)$ for the OF fit to $\sigma_{N S O F}=4.576 \mathrm{e}-2( \pm 0.076 \mathrm{e}-2)$ for the NSOF.

Although NSOF demonstrates a clear improvement in total phonon fitting, not all raw pulses are accurately reconstructed with the NSOF. In particular, the post-muon phonon pulses tend to be fit poorly with OF and NSOF, as shown in Fig. 5.18. Fitting

\footnotetext{
${ }^{4}$ Including this comparison begs the question: 'Why not use the pulse integral quantity for all phonon characterization?' In most cases the pulse integral quantity is actually a worse energy estimate than both the OF and NSOF fit amplitudes. Unlike OF and NSOF quantities, the integral quantity doesn't account for noise in the signal. However, the comparison of Fig. 5.17 is simply one piece of evidence suggesting that the NSOF fit more consistently reconstructs the raw pulse integral than the OF fit.
} 


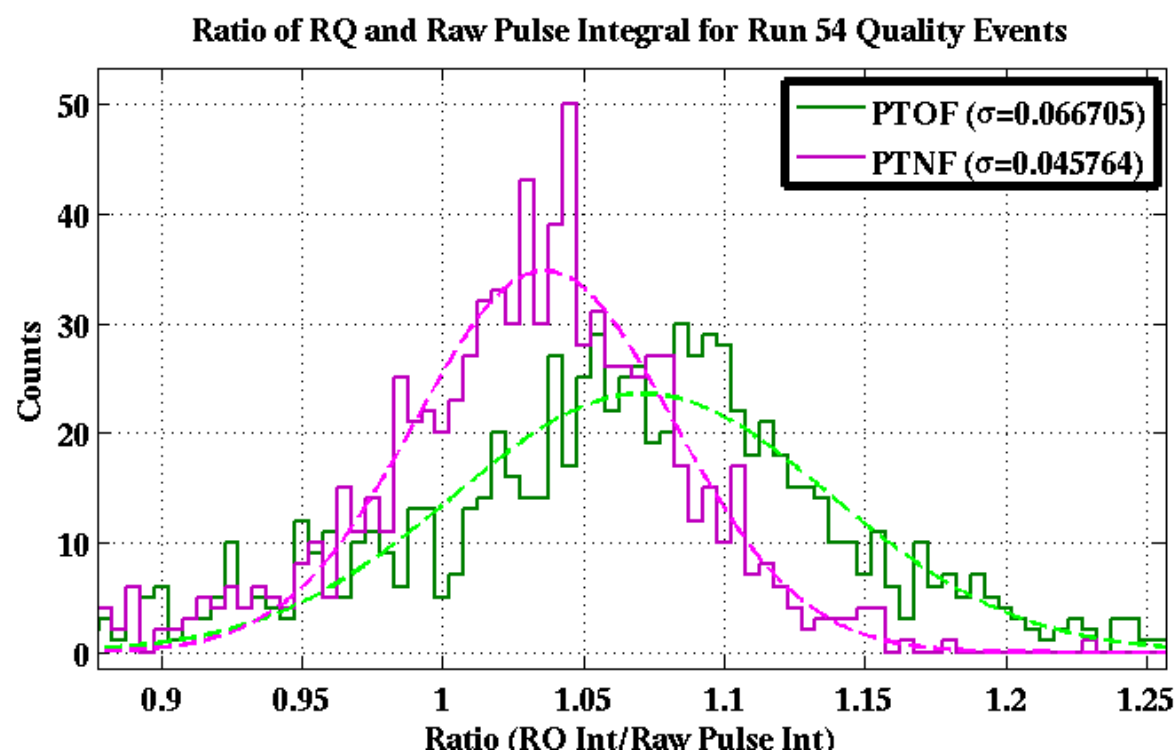

Figure 5.17: The ratio of the template fit integration over the raw pulse integration value, for both the NSOF (pink solid line) and the OF (green solid line) total phonon RQ's. Gaussian fits to PTNFamp distribution (pink dotted) has mean of $1.0360( \pm 0.001)$ and resolution of $4.576 \mathrm{e}-2( \pm 0.076 \mathrm{e}-2)$. This is a significant improvement in pulse reconstruction accuracy and precision when compared to the PTOFamp distribution Gaussian fit (green dotted), with a mean of $1.071( \pm 0.001)$ and resolution of $6.670 \mathrm{e}-2( \pm 0.142 \mathrm{e}-2)$.

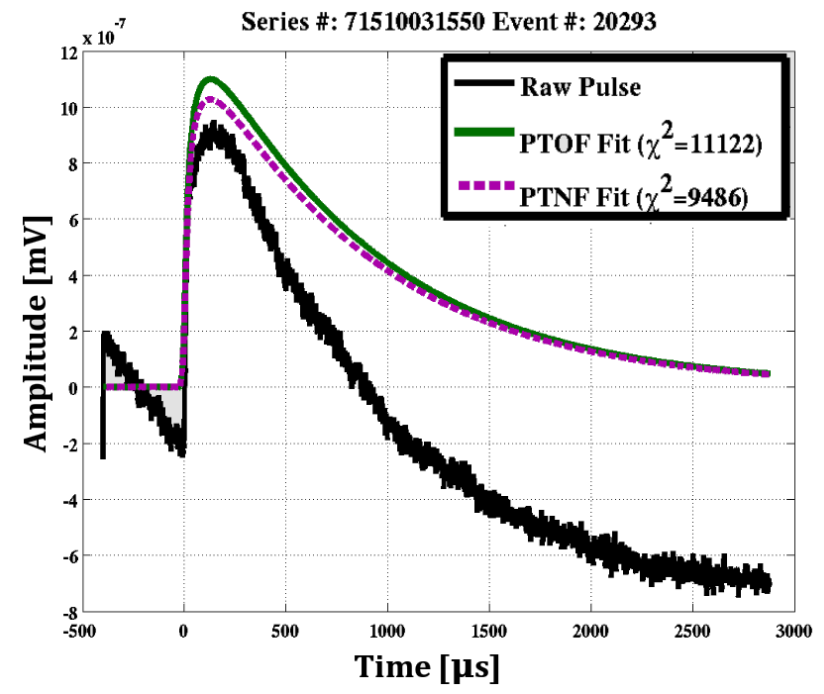

Figure 5.18: A post-muon, total phonon pulse (black), if fit with the standard OF (green solid), and the NSOF (pink dotted) algorithms, using the same template. Neither algorithm provides a close fit to the raw pulse, due to the pulse deformations caused by the thermal phonons produced in an earlier muon interaction. 
poorly behaved events is not a priority, but this does highlight the issue of fitting postmuon events.

\subsubsection{Phonon Quality Cuts}

\section{Phonon $\chi^{2}$ Cut}

As previously mentioned in Sections 5.2.1 and 5.3.1. post-muon events typically have well-behaved charge traces, but poorly-fit phonon traces with a downward sloping trend, caused by the slow collection of muon-produced thermal phonons. These events typically pass charge quality cuts, requiring additional raw pulse cuts to be removed. In past UMN test facility analysis, these events were typically allowed to pass through the cuts. The reasons for this were two-fold. Early $100 \mathrm{~mm}$ iZIP studies focused on charge characterization and the base-level functioning of phonon channels, with little emphasis placed on full characterization. In addition, the noisy phonon pulses experienced before filters were added to the electronics readout made identifying poor phonon pulses difficult, and made phonon characterization near impossible. Cleaner readout means full phonon characterization is possible for the first time at the UMN test facility. This also means that phonon quality cuts can now be developed and implemented.

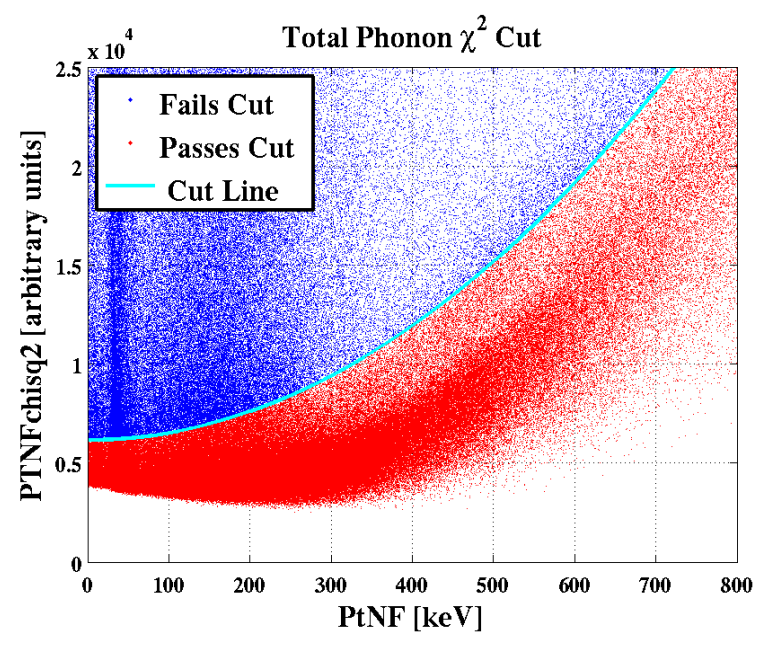

Figure 5.19: Total Phonon $\chi^{2}$ calibrated ionization energy for G106a with $\pm 4 \mathrm{~V}$ applied bias. Events which pass the $\chi^{2}$ cut (red) fall below the parabolic cut line (cyan), and events which fail the cut (blue) fall above the line. An event passing this cut must also pass the Side 1 and Side 2 charge $\chi^{2}$ cuts to be considered a well constructed event.

Like charge fits, the quality of phonon template fits is quantified by the $\chi^{2} \mathrm{RQ}$ value. Similar to the charge case, the phonon $\chi^{2} \mathrm{RQ}$ is expected to have a parabolic relationship 
with total phonon energy for well-fit events. Since the NSOF and OF algorithms can only fit the pulse time shift to the nearest ADC bin ( $0.8 \mu$ s for phonons), the parabolic upward trend is also broadened due to pulses fits with slight time shift inaccuracies.

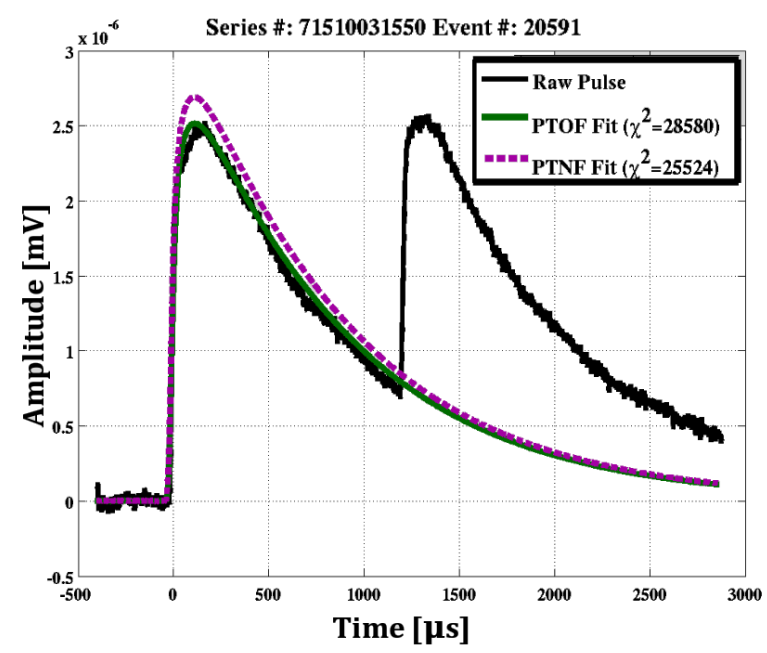

Figure 5.20: A raw total phonon pileup event (black) is fit with a total phonon template, using the NSOF (dotted pink) and OF (solid green) fitting algorithms. Although the first pulse is reconstructed well by the fits (particularly OF, which does not deweight the rising edge "noise"), the second pulse is completely neglected. As a result, the $\chi^{2}$ of this fit is high, and the event will be removed.

Anomalous events, such as post-muon events (See Fig. 5.18), or pileup events (see Fig. 5.20, have $\chi^{2}$ values much larger than expected for their corresponding amplitude. To remove these poorly reconstructed events, a parabolic cut line is introduced to PTNFchisq versus PtNF, as shown in Fig. 5.19.

\section{Phonon Baseline Cut}

As with charge events, variation in phonon event baseline can indicate poor quality data, with pileup events and excess channel noise as typical culprits of changing baseline. For ionization events, a cut was constructed to remove events which deviated from the typical series baseline for both QiS1 and QiS2. A similar method can be applied to phonon channels, with baseline thresholds set for PFS1 and PFS2 baseline distributions. The $\mathrm{PF}$ channel is the central phonon channel of each side, making it the best representation of baseline deviations for each side.

The phonon baseline cut compares an individual event to the typical baseline of its series. To do so, the average baseline is calculated for PFS1 and PFS2 for each series. 

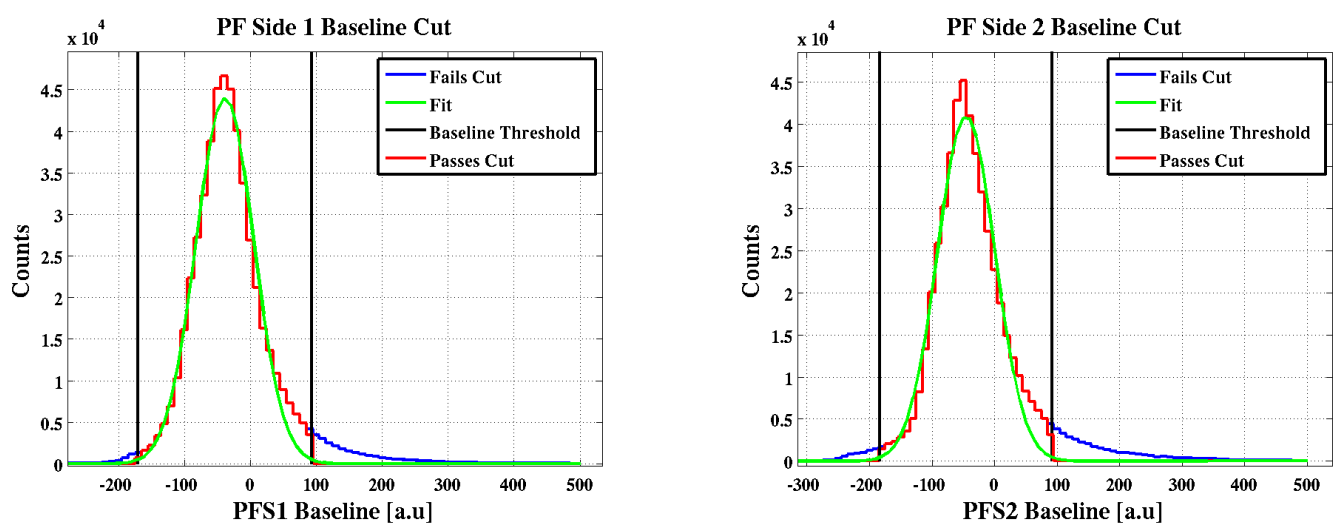

Figure 5.21: Distribution of mean-subtracted baseline values for (LEFT) PF Side 1 and (RIGHT) PF Side 2 with the $\chi^{2}$ cut applied. Distributions are fit with a Gaussian (green), and baseline threshold are set to $\pm 3 \sigma$ (black). Baselines within the thresholds are kept (red), while events with baselines outside the thresholds are removed (blue).

Shown in Fig. 5.21, the distribution of the difference between each event baseline and the series' average baseline is fit with a Gaussian ${ }^{5}$. Baseline thresholds are set at $\pm 3 \sigma$ for the PFS1 and PFS2 distributions. Events which fall outside of these thresholds are cut.

\subsubsection{Phonon Spectra}

As with charge spectra, total phonon spectra require absolute calibration values, that can change slightly over time. Fortunately, the same fitting methods can be utilized, with Gaussian plus background fit to $10.36 \mathrm{keV}$ and $60 \mathrm{keV}$ calibration peaks present in the PtNF amplitude spectra. However, calibrating the means of these peaks to exactly $10.36 \mathrm{keV}$ or $60 \mathrm{keV}$ would be incorrect, as this does not account for the Luke phonons observed in the total phonon energy measurement. Recall from Section 2.2.5, that the total phonon energy measured in the detector, $E_{T}$ is given by,

$$
E_{T}=E_{r}+e N_{e / h} V
$$

\footnotetext{
${ }^{5}$ The distributions are not centered around zero due to post-muon events with elevated baselines being included in the average baseline calculation.
} 
where recoil energy, $E_{r}$, represents the total energy deposited in the initial interaction. Also recall that for an ER, the charge energy, $E_{Q}=\epsilon_{\gamma} N_{e / h}$ would also represent the total energy deposited by the initial interaction (assuming 100\% charge collection efficiency). Therefore, for ER events, such as the $10.36 \mathrm{keV}$ and $60 \mathrm{keV}$ gamma events from calibration sources, it can be assumed that $E_{Q}=E_{r}$. For an ER, $E_{T}$ can be rewritten as,

$$
E_{T, e r}=E_{Q}+\frac{e}{\epsilon_{\gamma}} E_{Q} V=E_{Q}\left(1+\frac{e}{\epsilon_{\gamma}} V\right) .
$$

The factor $\left(1+\frac{e}{\epsilon_{\gamma}} V\right)$ is the Luke gain. For the $\mp 4 \mathrm{~V}$ applied data used in this analysis, the Luke gain is $11 / 3$, since $\epsilon_{\gamma}=3$ for Ge crystals. Therefore, the calibration sources that deposit $10.36 \mathrm{keV}, 60 \mathrm{keV}$, and $356 \mathrm{keV}$, will result in total phonon energy peaks of $38 \mathrm{keV}, 218 \mathrm{keV}$, and $1305 \mathrm{keV}$, respectively.

For clarity as the analysis moves forward three different energy scales will be used when describing phonon energy, each with its corresponding unit:

1. Total Phonon Energy: No assumption is made on recoil type. Measured $E_{T}$ is reported in units $\mathrm{keV}_{t}$

2. Electron Equivalent Energy: An electron recoil is assumed, and the yield is assumed to be unity. The electron-equivalent recoil energy, $E_{R, e e}=E_{T} /\left(1+\frac{e}{\epsilon_{\gamma}} V\right)$ is reported in units of $\mathrm{keV}_{e e}$

3. Nuclear-Recoil Equivalent Energy:A nuclear recoil is assumed and the yield depends on $E_{R, n r}$. The nuclear-recoil-equivalent recoil energy, $E_{R, n r}=E_{T} /(1+$ $\left.Y\left(E_{R, n r}\right) \frac{e}{\epsilon_{\gamma}} V\right)$ is reported in units of $\mathrm{keV}_{n r}$

Using these units, it becomes correct to say that the $10.36 \mathrm{keV}, 60 \mathrm{keV}$, and 356 $\mathrm{keV}$ deposited source energy will result in total phonon energy peaks of $38 \mathrm{keV}_{t}, 218$ $\mathrm{keV}_{t}$, and $1305 \mathrm{keV}_{t}$, respectively.

A comparison of spectra produced by the NSOF (PtNF) and OF (PtOF) fits is shown in Fig. 5.22. The top figure compares the total phonon spectra, with individual series calibrated against the $38 \mathrm{keV}$ peak expected from ${ }^{252} \mathrm{Cf}$ activation when a $\mp 4 \mathrm{~V}$ bias is applied. Ionization and phonon RQ quality cuts have been applied. The higher, tighter calibration peaks indicate that the NSOF improves resolution over the standard OF. 

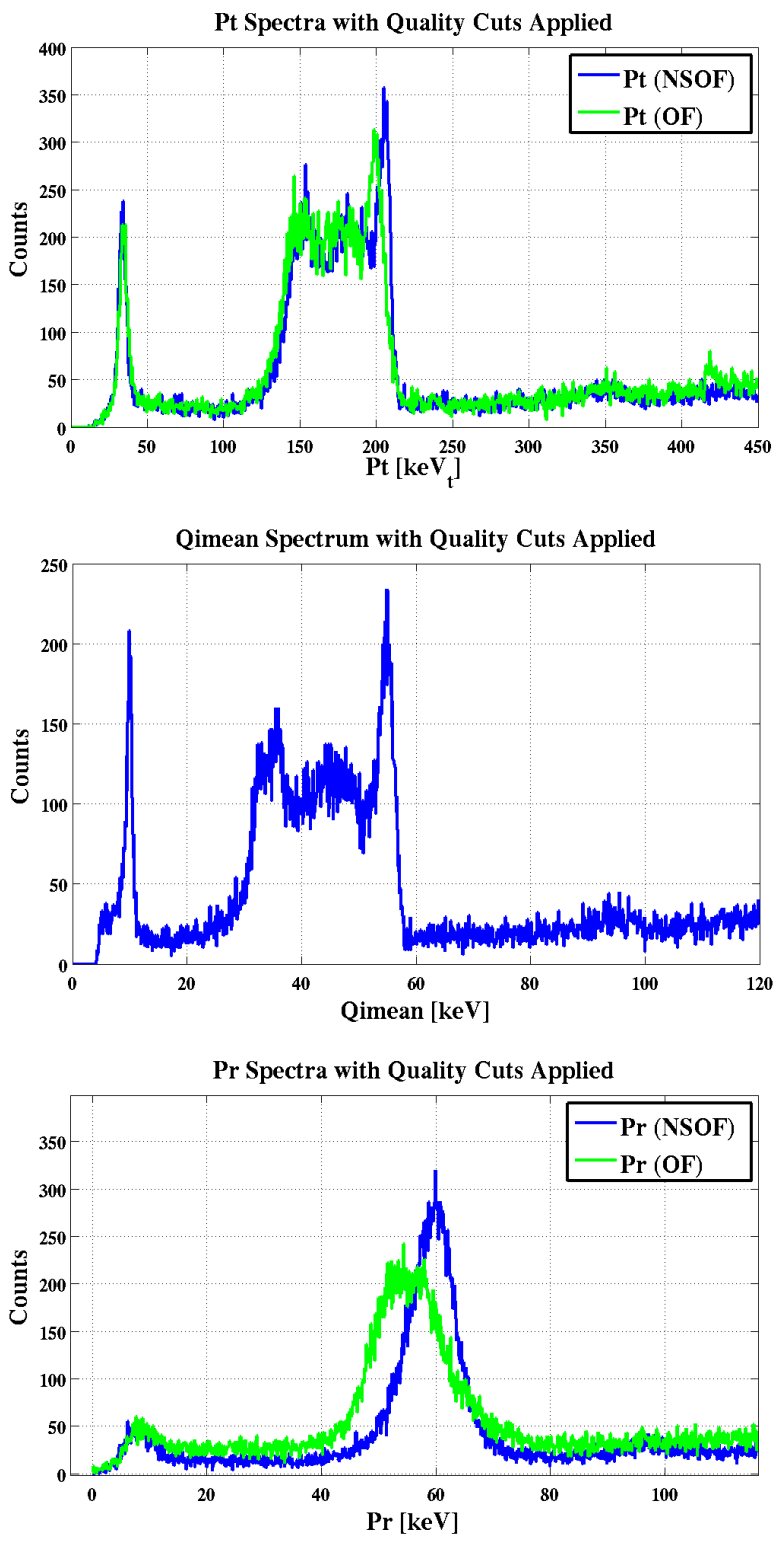

Figure 5.22: (TOP) Total phonon energy spectra, comparing the NSOF (blue) fitting to the OF (green) fitting. (MIDDLE) The Qimean energy spectrum. (BOTTOM) Recoil energy spectra, derived from the total phonon energy and Qimean. As with total energy, the NSOF (blue) fitting and standard OF (green) fitting are compared. All charge and phonon RQ quality cuts applied. 
Since yield has not yet been calculated, one can use an equivalent relationship to determine $E_{R}$ that is independent of recoil type,

$$
E_{R}=E_{T}-\frac{e V}{\epsilon_{\gamma}} E_{Q}
$$

where Qimean has been selected as the measured value to most accurately represent $E_{Q}$. Past CDMS analyses typically use Qimean in this manner, though other, similar quantities have also been chosen. In terms of measured RQ's, the recoil energy for NSOF and OF is given by,

$$
\begin{gathered}
\operatorname{PrNF}=\operatorname{PtNF}-\left(8 / \epsilon_{\gamma}\right) \text { Qimean, } \\
\operatorname{PrOF}=\operatorname{PtOF}-\left(8 / \epsilon_{\gamma}\right) \text { Qimean },
\end{gathered}
$$

for $\mp 4 \mathrm{~V}$ data. Comparison of recoil energy quantities derived from PtNF and PtOF values, is shown in the bottom of Fig. 5.22, The broad feature that appears at $\sim 200$ $\mathrm{keV}_{t}$ in the total phonon spectra, and $\sim 50 \mathrm{keV}$ in the Qimean spectrum is comprised of events from $60 \mathrm{keV}$ gammas. Since a cut on charge symmetry has not been applied, both bulk and surface events (with reduced charge collection) are contributing to the spectra.

\section{Phonon Resolution}

To determine total phonon energy resolution the noise blob $\left(0 \mathrm{keV}_{t}\right)$, as well as $38 \mathrm{keV}_{t}$, $218 \mathrm{keV}_{t}$, and $1305 \mathrm{keV}_{t}$ will be fit with a Gaussian plus background. Five 30-minute data series with the ${ }^{133} \mathrm{Ba}$ source present were used for the $356 \mathrm{keV}$ peak fit, and an additional six ${ }^{241} \mathrm{Am}$ only series were used for the other peak fits. Results, shown in Table 5.2, demonstrated that G106a PtNF resolution sees significant improvement in resolution over PtOF.

Phonon resolution reaches as low as $\sim 1 \mathrm{keV}$, significantly higher than the SuperCDMS Soudan total phonon (NSOF) resolutions, which range from $181 \mathrm{eV}$ to $702 \mathrm{eV}$, depending on the iZIP. Phonon resolution becomes comparable to Soudan detectors for energies about $\sim 100 \mathrm{keV}_{t}$ or greater. Despite the higher noise in the UMN facility setup, the $100 \mathrm{~mm}$ iZIPs can match the performance observed with smaller detectors at SuperCDMS Soudan. To further improve on the phonon energy resolution, improvements in the phonon readout system will be implemented for SuperCDMS SNOLAB. 
Table 5.2: G106a Total Phonon Energy Resolutions for $\mp 4$ V Applied Bias

\begin{tabular}{ccc}
\hline \hline & & \\
Expected Energy & PtNF & PtOF \\
Value $\left[\mathrm{keV}_{t}\right]$ & Peak Mean $\pm \sigma\left[\mathrm{keV}_{t}\right]$ & Peak Mean $\pm \sigma\left[\mathrm{keV}_{t}\right]$ \\
\hline & & \\
0 & $-0.216 \pm 1.167$ & $-0.354 \pm 1.232$ \\
37.99 & $38.19 \pm 2.61$ & $40.15 \pm 2.95$ \\
218.2 & $221.2 \pm 2.9$ & $241.3 \pm 6.5$ \\
1305 & $1305 \pm 27$ & $1305 \pm 42$ \\
\hline
\end{tabular}

Phonon resolution studies also demonstrated the effects of applied bias on resolution. Fig. 5.23 illustrates the change in resolution of the PtNF $10.36 \mathrm{keV}_{\text {ee }}$ peak as a function of applied bias; included error bars reflect the uncertainty of the Gaussian fit to the peak. This study was conducted with a different NSOF covariance matrix, calculated from Run 56 data. Although the resulting PtNF values might differ slightly from those calculated with a Run 54 covariance matrix, general trends in resolution should be unaffected. As with charge resolution, PtNF resolution tends to decrease as Side 2 bias magnitude increases from $1 \mathrm{~V}$ to roughly $3 \mathrm{~V}$. This is expected, since charge collection efficiency improves with increasing bias until it reaches a maximum around $3-4 \mathrm{~V}$. The outlier peak width at $6 \mathrm{~V}$ is due to low-statistics, the uncertainty of which is not accounted for in the included error bars. Once the Luke gain contribution is applied, biases larger than $3 \mathrm{~V}$ will see a large degradation in $\mathrm{keV}_{t}$ energy resolution.

\subsubsection{Ionization Yield with Surface Event Rejection}

Up to this point, results presented in the ionization and phonon $100 \mathrm{~mm}$ characterization chapters have mostly used data taken during UMN Run 54. This run featured the easily identifiable $60 \mathrm{keV}{ }^{241} \mathrm{Am}$ source peak, a strong $10.36 \mathrm{keV}$ peak from frequent activation with ${ }^{252} \mathrm{Cf}$, and consistent DAQ settings leading to little variation in relative and absolute calibration values over multiple data series.

However, Run 54 only provides a single source of surface events; the ${ }^{241}$ Am source provides surface $\gamma$ particles, but none of the sources used create surface $\beta$ particles. 


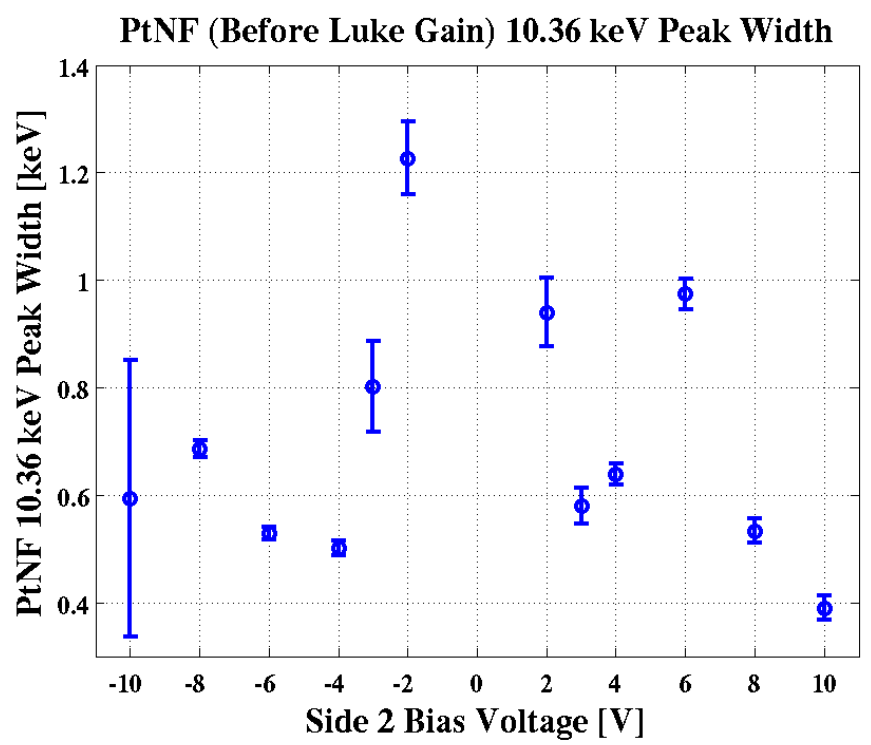

Figure 5.23: PtNF $10.36 \mathrm{keV}_{\text {ee }}$ peak resolution versus applied bias, before the Luke gain contribution is included.

Therefore, only using Run 54 data would provide an incomplete picture of the surface event rejection capabilities of G106a. To remedy this, a portion of Run 56 data is used, with an internal ${ }^{210} \mathrm{~Pb}$ present to provide surface $\beta$ 's. Due to some issues with prototype DAQ software implementation during Run 56, data from this run suffers from frequently changing relative calibration values. Additionally, Run 56 did not use the ${ }^{241} \mathrm{Am}$ internal source, and had infrequent ${ }^{252} \mathrm{Cf}$ activation, leading to sporadic presence of the $10.36 \mathrm{keV}$ peak. Fortunately, several series were taken with the external ${ }^{133} \mathrm{Ba}$ source, providing the $356 \mathrm{keV}$ peak for calibration. Additionally, several of these series occur within a two week period of stability with little change in DAQ settings.

Thus, eight Run 56 data series from early March 2016 will be added to the yield and surface event rejection analysis. Following the same procedures outlined in this chapter, relative calibration values were calculated for these series, using PBS1 as the base channel. With this relative calibration applied, a phonon covariance matrix was calculated for Run 56 data as well. All eight series were then reprocessed with the proper relative calibration and the NSOF algorithm. Absolute calibration for charge and phonon spectra were found by fitting the $356 \mathrm{keV}$ peak. As discussed in Section 
4.4. Run 56 data will have a different timing cut than Run 54 data, due to its increased event rate $(\sim 159 \mathrm{~Hz}$, compared to $\sim 82 \mathrm{~Hz}$ for Run 54$)$.

Yield values for the OF and NSOF can extracted from Qimean and recoil energy,

$$
\begin{array}{r}
Y N F=\text { Qimean } / \operatorname{Pr} N F \\
Y O F=\text { Qimean } / \operatorname{PrOF} .
\end{array}
$$

The discrimination power of ionization yield is most easily conveyed in a plot of yield versus recoil energy. The ER and NR events form distinct bands in yield, to which functional forms can be fit. However, finding distinct bands in yield versus recoil energy plots in Fig. 5.24 proves difficult. This is because surface events, which fall between the bands, have not yet been cut. Recall that surface events are the result of an incident particle (typically a $\beta$ or $\gamma$ particle) scattering within $\sim 10 \mu \mathrm{m}$ of the crystal surface. When this occurs, the initial charge carrier cloud can self-shield against the applied field, and carriers will be collected immediately by the nearest electrode. These events have a suppressed ionization signal and thus a reduced yield for their given recoil energy, making them easy to mistake for nuclear recoil events.

In Fig. 5.24 , surface events are caused by $\beta$ particles from the ${ }^{210} \mathrm{~Pb}$ lead source present for Run 56, and poorly-penetrating $60 \mathrm{keV} \gamma$ 's from the ${ }^{241} \mathrm{Am}$ source present for Run 54. To demonstrate G106a's surface event rejection capabilities, both types of surface events must be removed effectively. Most importantly, the surface cut applied must thoroughly remove surface events which fall within the NR band region and risk being mistaken for a neutron or WIMP.

\section{The Surface Cut}

Surface events are removed by taking advantage of the single-side collection of surface event charge carriers. Ionization signals of events which occur in the detector bulk will be near-identical on both sides, whereas surface events will be collected only on one side. In a plot comparing the ionization measurements of both detector sides, such as Fig. 5.25, charge-symmetric bulk events will manifest as a line of unity slope. Surface

events will fall outside of this symmetric region, as illustrated by the vertical blob of Side $160 \mathrm{keV}$ events which span from the symmetric region down to zero Side 2 charge signal. 

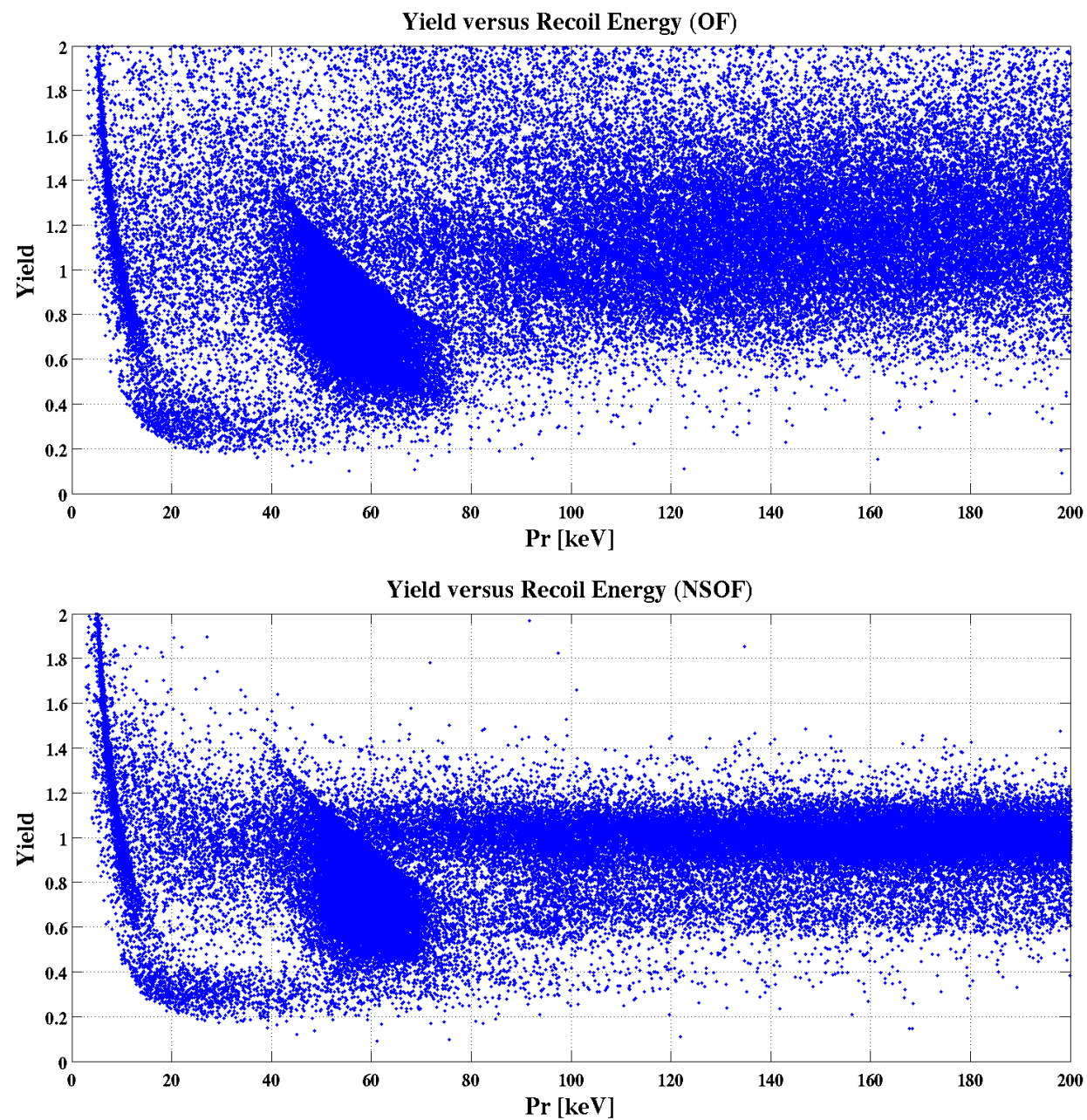

Figure 5.24: Yield versus recoil energy for (TOP) OF and (BOTTOM) NSOF total phonon processing. Previously discussed quality cuts have been applied, including charge and phonon $\chi^{2}$, charge threshold, charge radial partition, and charge and phonon baseline cuts. Run 54 and Run 56 data included. 


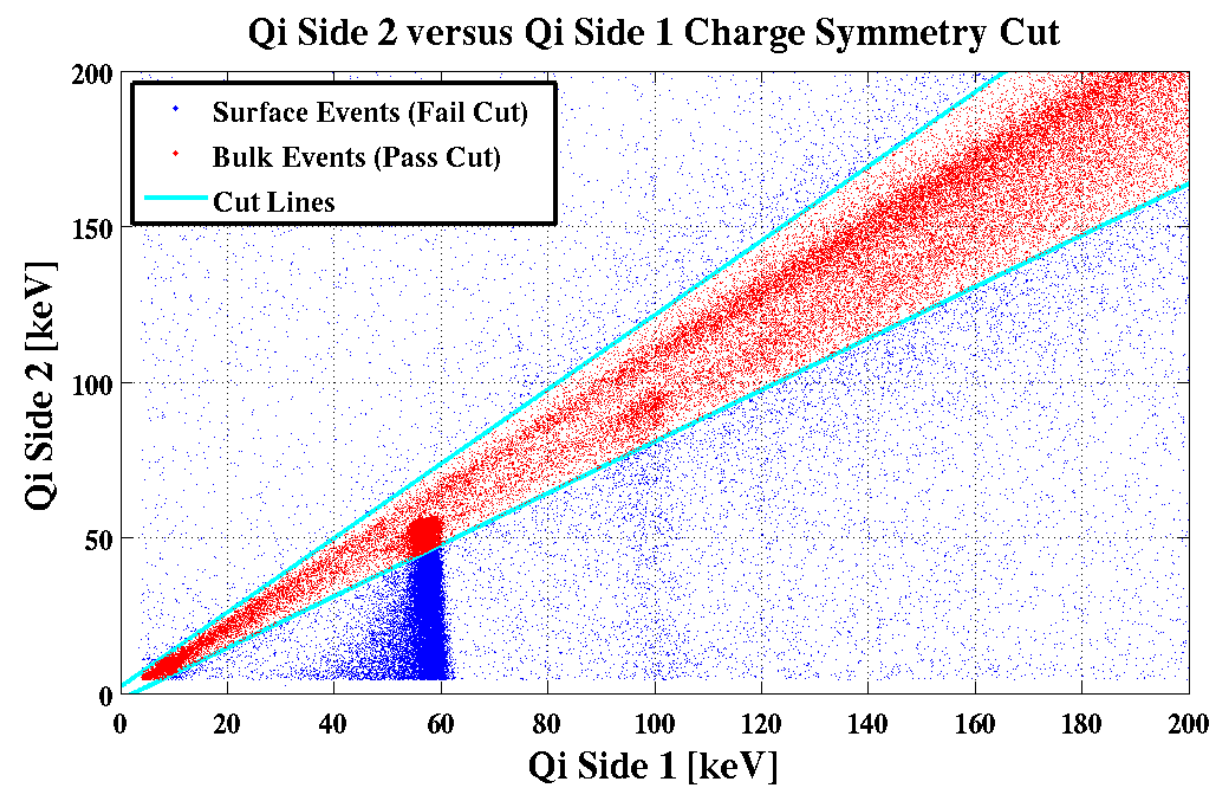

Figure 5.25: Side 2 charge signal (QiS2) versus Side 1 charge signal (QiS1), showing a charge-symmetric data region with slope of one, and non-symmetric data, such as surface events. Events from the ${ }^{241} \mathrm{Am}$ source $60 \mathrm{keV} \gamma$ 's created a vertical blob of events extending from the symmetric region downward to a null QiS2 signal. A charge symmetry cut is applied (cyan lines), removing surface events (blue) while keeping symmetric bulk events (red). 
The surface cut, also called the charge symmetry cut, is comprised of two lines which surround the symmetric event region; all events outside of the lines fail the cut and are removed. The cut-line parameters are determined by fitting Gaussian forms to QiS2 energy distributions, for consecutive slices of QiS1 values spanning $100 \mathrm{keV}-400 \mathrm{keV}$ in $10 \mathrm{keV}$ increments. A high energy range is selected to avoid the surface events present in the $60 \mathrm{keV}$ peak energy region. From the Gaussian mean and sigma values, $\mu$ and $\sigma$ receptively, cut lines are fit to QiS2 $=(\mu-0.2 \sigma)$ QiS1-2 and QiS2 $=(\mu+0.2 \sigma)$ QiS1 +2 , enclosing the symmetric region which passes the cut. If charge calibration has been done properly, a line fit to just the $\mu$ terms should have a slope of one. The $2 \mathrm{keV}$ intercept and $0.2 \sigma$ coefficient were selected to ensure that low energy events were not needlessly cut, and that the cut fully enclosed the symmetric event region without extending too far beyond. The effects of the surface cut on ionization and phonon spectra can be seen in Fig. 5.26. With the surface cut applied, the broad feature that had been present in Pt and Qimean spectra (see Fig. 5.22), and which contained bulk and surface events from the $60 \mathrm{keV}$ gammas, is now a narrow peak comprised of bulk events.

The effect of the surface cut can be seen on the yield bands in Fig. 5.27. Distinct ER and NR bands are now discernible, particularly in the NSOF case. The space between the bands still contains a sparse few events, indicating that not all surface events have been removed. The efficiency of the surface cut will be explored in Section 5.3.6, once ER and NR band regions have been determined by fitting the bands with functional forms.

\section{Modeling Yield Bands}

Before looking at yield results obtained for G106a, it can be helpful to understand what can be expected. Yield depends on measured charge and phonon quantities, and thus is limited by the resolution of these quantities. Since both charge and phonon quantities have been shown to have energy-dependent resolutions, it can be expected that yield resolution will be energy dependent as well.

A study by D. Jardin on Soudan R133 $76 \mathrm{~mm}$ iZIP data determined that both phonon and ionization energy resolution, $\sigma_{E}$, related to energy, $E$, through the functional form,

$$
\sigma_{E}=\sqrt{\alpha+\beta E+\gamma E^{2}},
$$



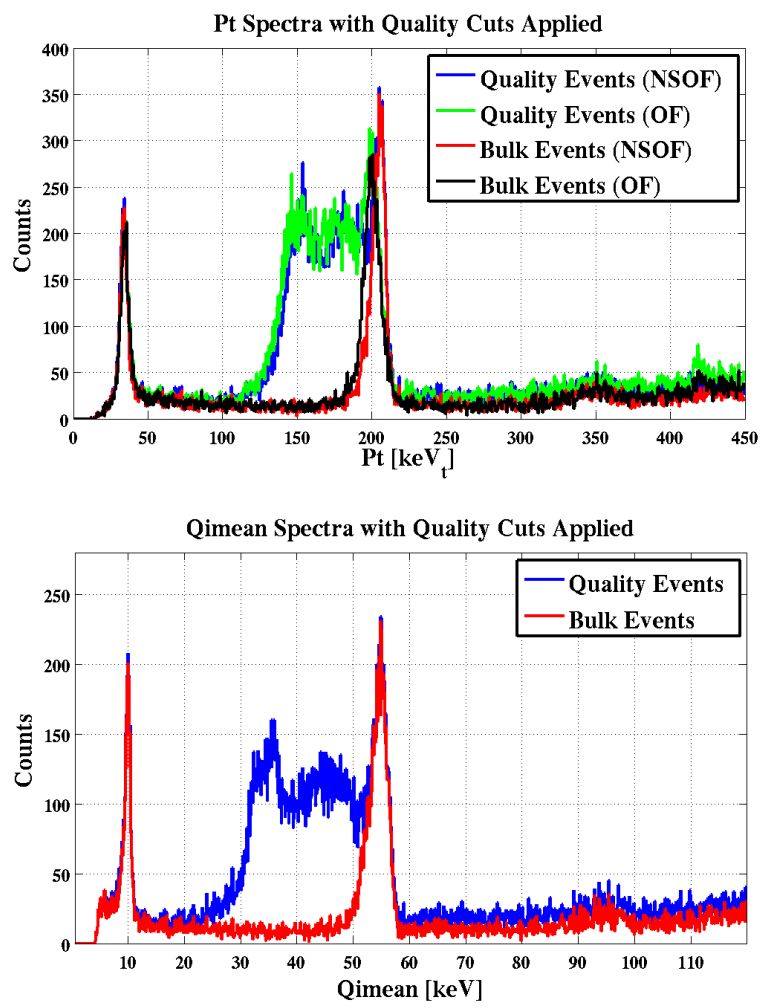

Pr Spectra with Quality Cuts Applied

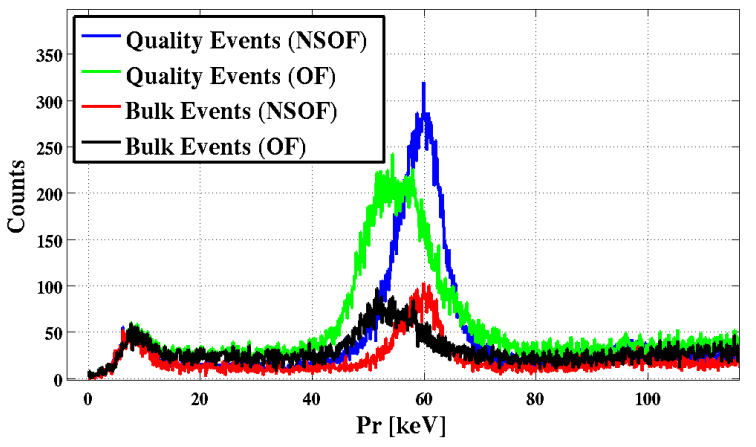

Figure 5.26: (TOP) Total phonon energy spectra, comparing PtNF with (red) and without (blue) surface cut applied to the PtOF with (black) and without (green) surface cut. (MIDDLE) The Qimean energy spectrum, with (blue) and without (red) surface cut applied. (BOTTOM) Recoil Energy energy spectra, comparing PrNF with (red) and without (blue) surface cut applied to the PrOF with (black) and without (green) surface cut. All charge and phonon RQ quality cuts applied. 

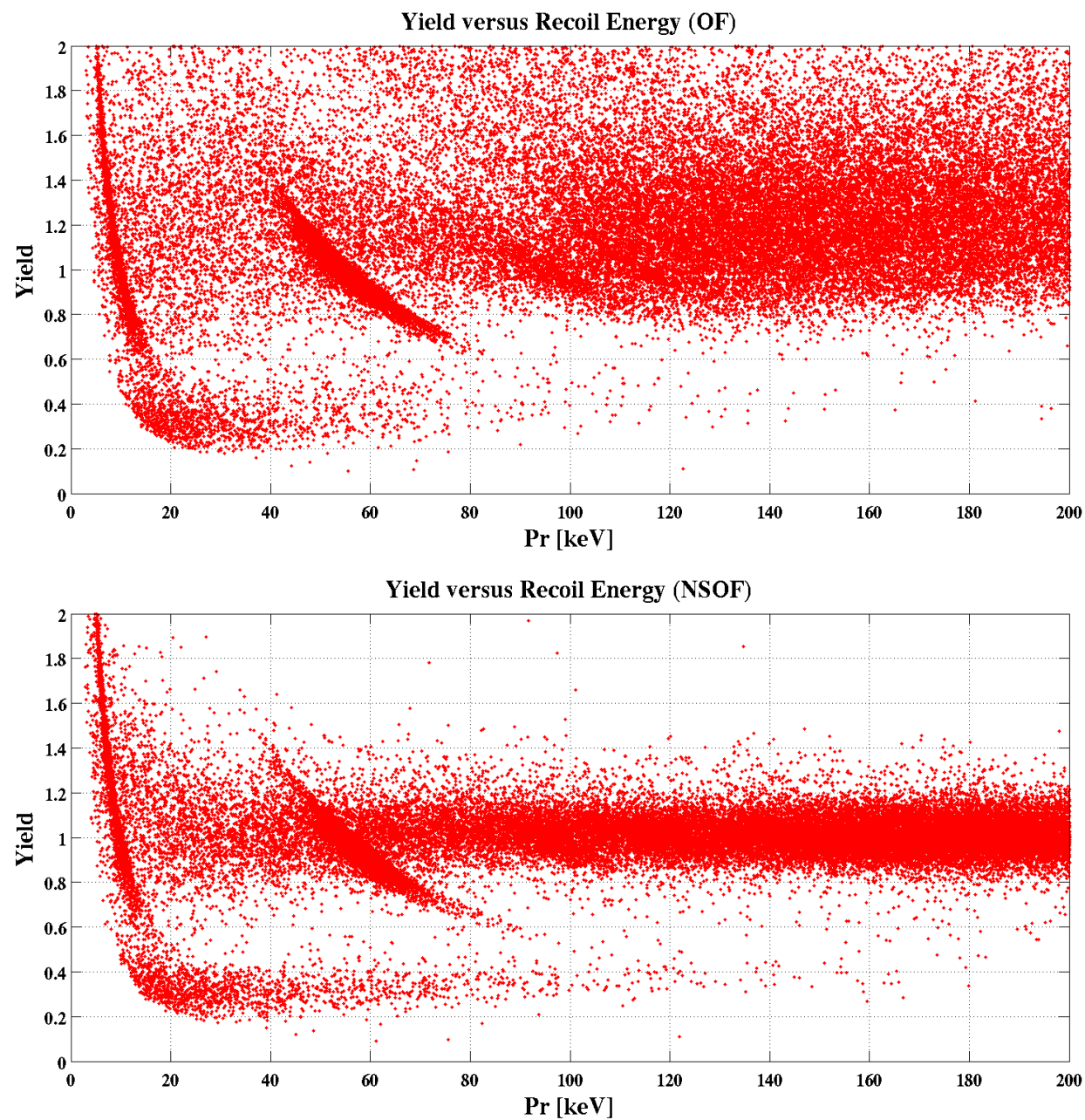

Figure 5.27: Yield versus recoil energy for (TOP) OF and (BOTTOM) NSOF total phonon processing. Previously discussed quality cuts have been applied, including charge and phonon $\chi^{2}$, charge threshold, charge radial partition, and charge and phonon baseline cuts. The surface cut has also been applied. 
where $\alpha, \beta$, and $\gamma$, are parameters computationally found for each of the fifteen SuperCDMS detectors [204]. Results were determined by fitting to the $0 \mathrm{keV}$ noise event distribution and four bulk event source peaks, up to $511 \mathrm{keV}$ with a Gaussian, and comparing the resulting $\sigma_{E}$ to the peaks mean energy. The maximum Qsum value (Qsum1 or Qsum2) was chosen to represent the ionization energy. Total phonon energy, using the NSOF RQ, represents the phonon energy.

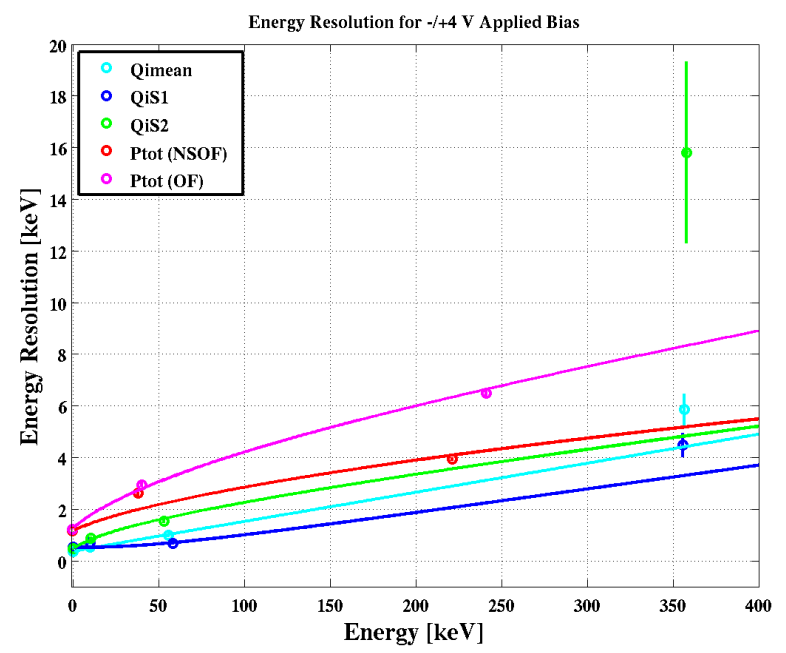

Figure 5.28: Zero-energy events, $10.36 \mathrm{keV}, 60 \mathrm{keV}$, and $356 \mathrm{keV}$ source peaks are fit with a Gaussian plus exponential decay background on QiS1, QiS2, Qimean, PTNF, and PTOF. The resulting peak mean and resolution values are used in a weighted fit to determine the functional form parameters in Eq. 5.20. Phonon energy values are in units $\mathrm{keV}_{t}$.

The study was repeated with G106a test facility data with $\mp 4 \mathrm{~V}$ applied bias data. Noise event distribution (0 keV), $10.36 \mathrm{keV}, 60 \mathrm{keV}$, and $356 \mathrm{keV}$ peaks were fitted with a Gaussian (to fit peak events) plus an exponential decay function (to fit background events). The peak means are shown in Fig. 5.28, with the functional forms overlaid. Resulting forms, shown in Fig. 5.29, demonstrate that G106a has a charge and phonon resolution comparable to SuperCDMS Soudan resolutions, despite the test facility noise environment and readout system.

Given ionization and total phonon resolutions, $\sigma_{Q}$ and $\sigma_{T}$, respectively, ionization and total phonon data can be modeled. For a given recoil energy, $E_{R}$, and a given yield, $Y$, charge and total phonon energies are given by,

$$
\begin{array}{r}
E_{Q}=Y E_{R} \\
E_{T}=\left[1+Y\left(\frac{e V}{\epsilon_{\gamma}}\right)\right] E_{R} .
\end{array}
$$



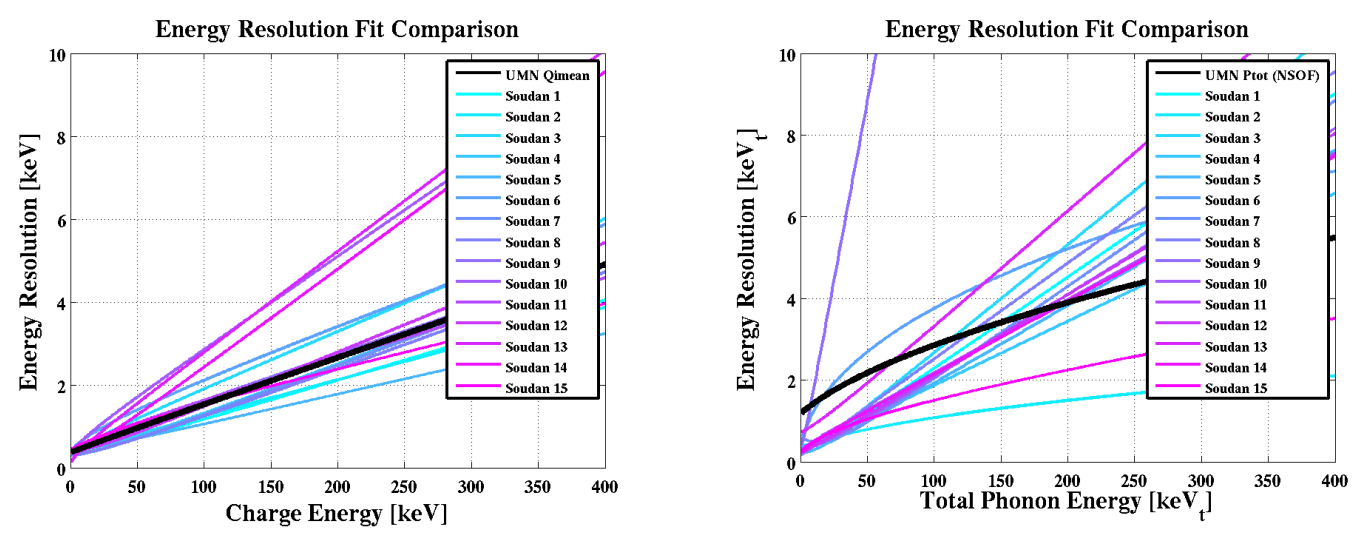

Figure 5.29: Comparing the (LEFT) ionization and (RIGHT) total phonon energy resolution functional forms of G106a (black) to the SuperCDMS Soudan $76 \mathrm{~mm}$ iZIPs (colored lines). UMN functional forms are fit to $\mp 4 \mathrm{~V}$, Qimean and PtNF peaks. Soudan functional forms are fit to Run $133 \pm 2 \mathrm{~V}$ data, using maximum Qsum and PtNF.

Assuming a Gaussian distribution about these ideal values, the probability density of the charge and phonon energy distributions are given by

$$
\begin{array}{r}
f\left(x \mid \sigma_{Q}, E_{R}, Y\right)=\frac{1}{\sqrt{2 \pi \sigma_{Q}^{2}}} e^{-\frac{\left(x-Y E_{R}\right)^{2}}{2 \sigma_{Q}^{2}},} \\
f\left(x \mid \sigma_{T}, E_{R}, Y\right)=\frac{1}{\sqrt{2 \pi \sigma_{T}^{2}}} e^{-} \frac{\left(x-\left[1+Y\left(\frac{e V}{\epsilon_{\gamma}}\right)\right] E_{R}\right)^{2}}{2 \sigma_{T}^{2}} .
\end{array}
$$

To model ER and NR yield bands using G106a energy resolutions, one must first have a general idea of event distribution for both bands. Based on the results of Section 5.3.5 event count is expected to exponentially decay in both bands with increasing recoil energy, with the ER band having a distribution estimated by $82 \times e^{-E_{R} / 304}$ events/bin, and the NR band having a distribution estimated by $93 \times e^{-E_{R} / 29}$ events/bin, for recoil energy bins of width $1 \mathrm{keV}$. From these distributions, one can determine the number of events in each $1 \mathrm{keV}$-wide bin for each band, over the whole $0-200 \mathrm{keV}$ region of interest. Events are draw from these exponential distributions, and each event is assigned a random recoil energy within the $1 \mathrm{keV}$ width. If the event is from the ER distribution it is given an ideal yield of exactly one. For a NR event, the expected yield value is determined from a simplified recoil energy-dependent Lindhard model [213] 
[214],

$$
Y_{L}=\frac{k g(\epsilon)}{1+k g(\epsilon)}
$$

where, for materials with ordering number $Z$ and atomic mass $A$ (in this case Ge), $g(\epsilon$ ) is well fitted by [84,

$$
\begin{array}{r}
g(\epsilon)=3 \epsilon^{0.15}+0.7 \epsilon^{0.6}+\epsilon, \\
\epsilon=11.5 E_{R} Z^{-7 / 3}, \quad k=0.133 Z^{2 / 3} A^{-1 / 2} .
\end{array}
$$

Let this ideal yield value be labeled $Y^{*}$.

For each $E_{R}$ value, ideal charge and phonon values, called $E_{Q}^{*}$ and $E_{T}^{*}$ for clarity, are found using Eqs. 5.21 and 5.22, they assume perfect resolution and signal collection. From these ideal energy values, the random $E_{R}$ value, and the already assigned ideal yield, a random $E_{Q}$ and $E_{T}$ value are found, as determined by thedrawn from the charge and phonon energy distributions in Eqs. 5.23 and 5.24. The $E_{Q}$ and $E_{T}$ values reflect what the system is likely to measure, given a recoil of energy $E_{R}$.

The reconstructed yield of $E_{Q}$ and $E_{T}$ is then,

$$
Y=\frac{E_{Q}}{E_{T}-\frac{e V}{\epsilon_{\gamma}} E_{Q}}
$$

The yield values of the model highlight the limitations due to energy resolution, but they still represent a perfect system, without leakage events, fluctuations in calibration, or changes in fridge or readout system. To illustrate this point, compare the $\pm 1.5 \mathrm{~V}$ applied bias Soudan data to modeled data that uses a typical $76 \mathrm{~mm}$ iZIP's energy resolutions. The modeled data forms tighter bands, particularly for the NR band.

Fig. 5.31 compares yield versus recoil energy of G106a data, to data modeled from G106a's energy resolution. As with the Soudan data comparison, the modeled data forms tighter bands. It is important to note that G106a modeled data forms wider bands at lower energy than Soudan modeled data, with the bands intersecting at a higher recoil energy. The cause is two-fold: increasing the applied bias will widen the bands due to an increase in Luke gain, and poor phonon resolution at low energies widens the low-energy region of both G106a yield bands. Thus, G106a data cannot be expected to demonstrate event discrimination capabilities identical to Soudan data, particularly at low recoil energies. As with the Soudan data, the model's bands are 

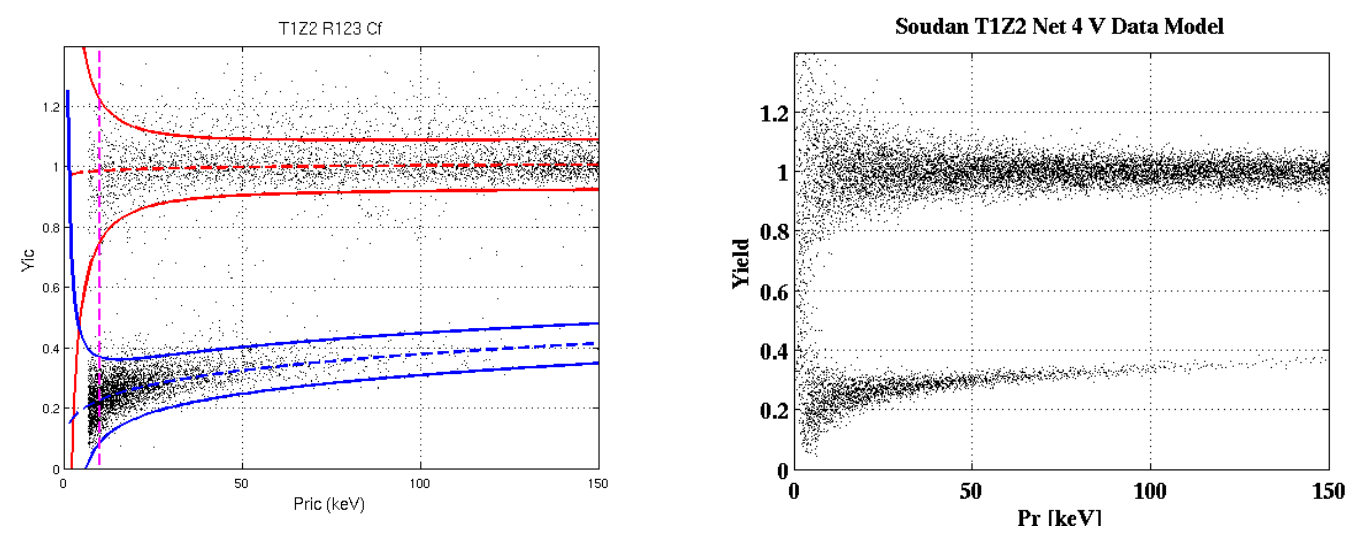

Figure 5.30: (LEFT) Ionization yield versus recoil energy for typical $76 \mathrm{~mm}$ iZIP detector (T1Z2) ${ }^{252} \mathrm{Cf}$ calibration data from Soudan R123 (black), with a $\pm 2 \mathrm{~V}$ applied bias. Data is predominately divided into ER band (red) and NR band (blue). Figure courtesy of J. Filippini [215]. (RIGHT) Modeled Soudan data with an applied bias difference of $4 \mathrm{~V}$, and energy resolutions of a selected Soudan detector T1Z2.

generally thinner than those of the actual data, particularly the NR band. The $60 \mathrm{keV}$ and $10.36 \mathrm{keV}$ events also form wide vertical bands in the actual data, not included in the model. A combination of the wide $10.36 \mathrm{keV}$ source band and wider ER and NR bands in general results in the ER and NR bands merging around $E_{R}=20 \mathrm{keV}$, whereas model bands merge at $<10 \mathrm{keV}$. The bands merging at such a high energy will limit the NR discrimination power of this data, as past CDMS yield analysis worked down to a recoil energy threshold of $\sim 10 \mathrm{keV}$.

Modeling can also demonstrate the effects of applied bias on yield resolution. In Sections 4.2.1 and 5.3.3, the $10.36 \mathrm{keV}^{6}$ peak resolution was determined for Qimean, $\sigma_{Q}$, and PtNF, $\sigma_{T}$, for $\pm 10 \mathrm{~V}, \pm 8 \mathrm{~V}, \pm 6 \mathrm{~V}, \pm 4 \mathrm{~V}, \pm 2 \mathrm{~V}, \mp 2 \mathrm{~V}, \mp 4 \mathrm{~V}, \mp 6 \mathrm{~V}, \mp 8 \mathrm{~V}$, $\mp 10 \mathrm{~V}$ data. Yield resolution, $\sigma_{Y}$, at $E_{R}=10.36 \mathrm{keV}$ can be found from these values, the resolution of $E_{R}, \sigma_{R}$, and yield,

$$
\sigma_{R}^{2}=\sigma_{T}^{2}+\left(\frac{e V}{\epsilon_{\gamma}}\right)^{2} \sigma_{Q}^{2},
$$

\footnotetext{
${ }^{6}$ Here $10.36 \mathrm{keV}$ refers to the collected charge energy. It should be acknowledged that the total phonon energy differ from this value, due to bias-dependent Luke gain.
} 

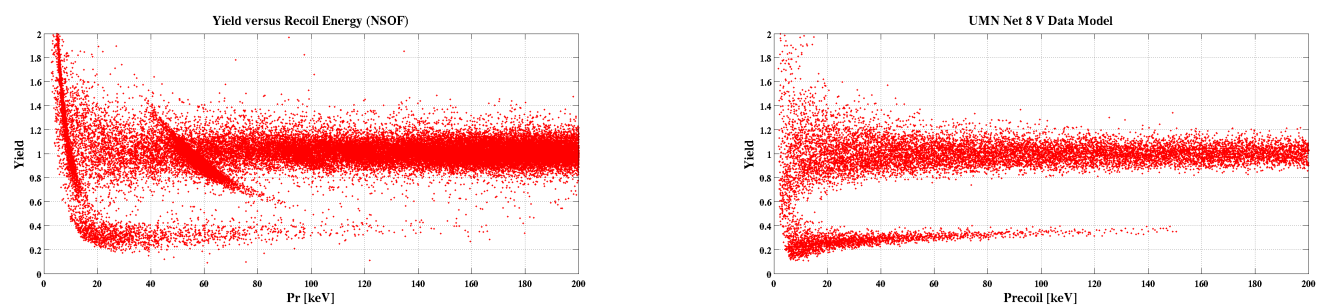

Figure 5.31: (LEFT) Ionization yield versus recoil energy for G106a ${ }^{252} \mathrm{Cf}$ calibration data, with a $\mp 4 \mathrm{~V}$ applied bias. Only events which pass quality cuts and surface cut are shown. (RIGHT) Modeled UMN data with an applied bias difference of $8 \mathrm{~V}$, and energy resolutions of G106a at $\mp 4 \mathrm{~V}$.

$$
\sigma_{Y}^{2}=Y^{2}\left[\left(\frac{\sigma_{Q}}{E_{Q}}\right)^{2}+\left(\frac{\sigma_{R}}{E_{R}}\right)^{2}\right] .
$$

Using Eq. 5.29 with measured Qimean and PtNF $10.36 \mathrm{keV}$ peak width values, the PrNF $10.36 \mathrm{keV}$ peak width can be predicted. Fig. 5.32 shows the predicted PrNF $10.36 \mathrm{keV}$ peak width as a function of applied bias.

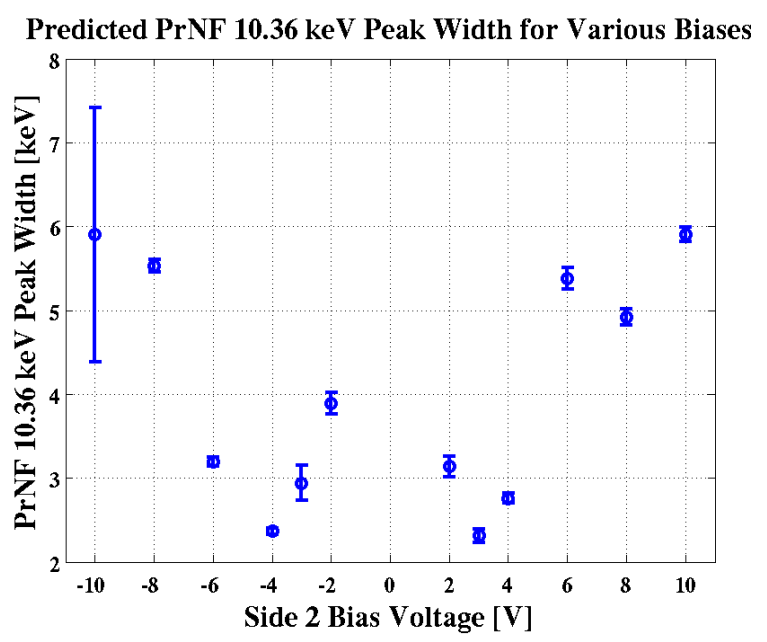

Figure 5.32: Predicted PrNF 10.36 $\mathrm{keV}$ peak width versus applied bias. Predicted peak width is derived using Eq. 5.29 , from measured values of the $10.36 \mathrm{keV}$ peak width in Qimean and PtNF spectra, shown in Figs. 4.10 and 5.23 . respectively.

Fig. 5.33 shows how the ER and NR band thicknesses at $E_{R}=10.36 \mathrm{keV}_{e e}$ are expected to vary with applied bias. Band thickness is set at three (two) times the standard deviation of the yield for the ER (NR) band. Yield band thickness is derived using Eq. 5.30, from measured values of the $10.36 \mathrm{keV}$ peak width in Qimean and PtNF spectra, shown in Figs. 4.10 and 5.23 , respectively. The plots of Fig. 5.33 indicate that 
yield bands are thinest, and thus easiest to distinguish, for net applied voltages around 4 - $12 \mathrm{~V}$. Although the applied bias of $\mp 4 \mathrm{~V}$ was selected due charge collection efficiency, the chosen bias fortuitously lands within the optimal yield resolution region.
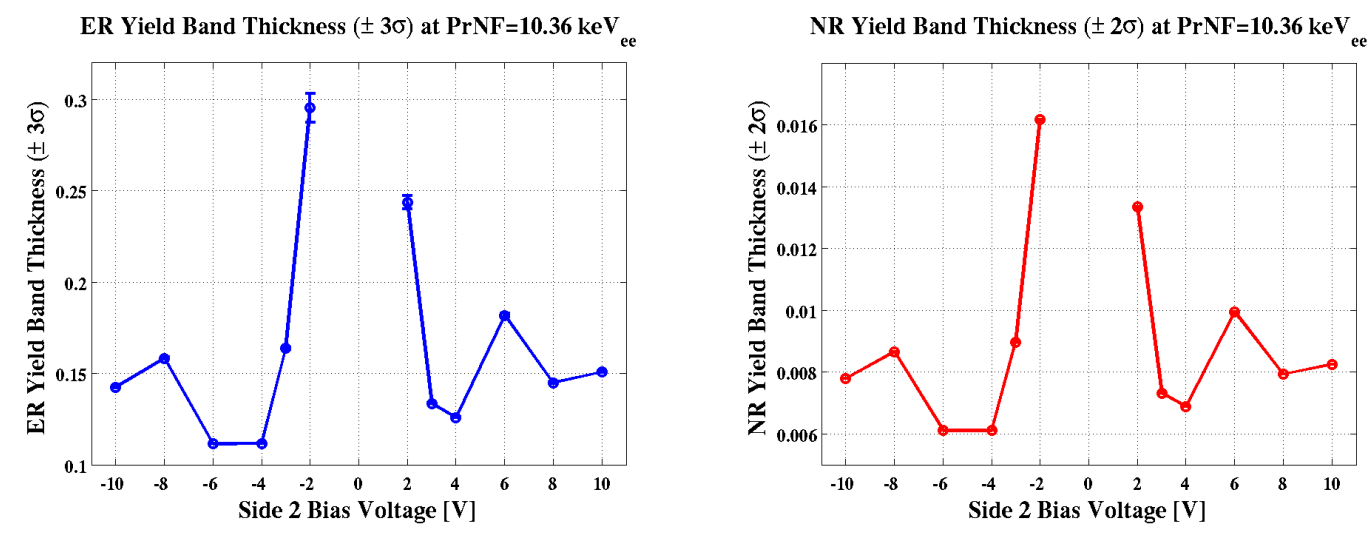

Figure 5.33: Predicted thickness of yield (LEFT) ER and (RIGHT) NR bands at a recoil energy of $10.36 \mathrm{keV}_{e e}$ versus applied bias. Yield band thickness is derived using Eq. 5.30, from measured values of the $10.36 \mathrm{keV}$ peak width in Qimean and PtNF spectra, shown in Figs. 4.10 and 5.23 , respectively.

\subsubsection{Fitting Yield Bands}

Once the surface event cut has been applied, remaining data forms distinct ER and $\mathrm{NR}$ regions in the yield versus recoil energy plot. It is useful in surface event rejection studies to quantify these regions, by fitting form functions to the data. The nuclear (electron) recoil bands are derived by first fitting the mean and standard deviation of the yield distribution for a set of twelve logarithmically spaced recoil energy bins, ranging from $25-120 \mathrm{keV}(4-400 \mathrm{keV})$. These energy ranges were chosen based on previous SuperCDMS Soudan analysis, which used 5 - $100 \mathrm{keV}$ for NR band [216] and 5 - $400 \mathrm{keV}$ for ER band [217]. This range was modified to account for the charge threshold limits, and the $10.36 \mathrm{keV}_{e e}$ events interfering with the $\mathrm{NR}$ band fit at low energies. To avoid NR events entering the ER band fit and vice-versa, the yield events in the range $0-0.5(0.5-2)$ were considered for the NR (ER) fit. To further avoid cross-contamination of event type, only ${ }^{252} \mathrm{Cf}$ data is used to fit the $\mathrm{NR}$ band, and only 
data without ${ }^{252} \mathrm{Cf}$ present is used to fit the ER band.

The results of the Gaussian fits, means and widths, are then fit with a recoil-energy dependent function,

$$
\begin{array}{r}
\mu_{Y}=\alpha E_{R}^{\beta}, \\
\sigma_{Y}=\frac{\alpha^{2} E_{R}^{\beta}+\gamma}{E_{R}},
\end{array}
$$

for both $\mathrm{ER}$ and NR bands [218]. Once parameters $\alpha, \beta$, and $\gamma$ are determined from the band fits, the NR band is defined as $\mu_{Y, N R} \pm 2 \sigma_{Y, N R}$ [219], and the ER band is defined as $\mu_{Y, E R} \pm 3 \sigma_{Y, E R}$ [161].

Yield bands are fit to OF and NSOF data in Fig. 5.34. It is clear that the OF data produced bands too wide to be accurately distinguished, indicating poor discrimination capabilities. Only the NSOF results will be used in the surface event rejection study. Accurately reflecting the data, the NSOF fitted bands merge just below PrNF $=20 \mathrm{keV}$. Despite the $60 \mathrm{keV}$ source events appearing significantly wider than the surrounding ER band, the fitted bands are not widened due to the presence of such events, but instead follow the general widening trend at low energies.

\subsubsection{Surface Event Rejection}

Fig. 5.35 demonstrates the surface event rejection capabilities of the charge symmetry cut. Surface events, colored blue, make up the bulk of data in the 0.4 - 0.7 yield region. Of the 43,412 events which pass the data quality cuts (excluding the surface event cut) in a $10-100 \mathrm{keV}$ recoil energy range, 17,539 are removed via the surface event cut. Data in the $10-20 \mathrm{keV}$ range which fall into both bands are excluded from the analysis, as it is difficult to determine whether they are ER or NR events. However, of the 107 events that fall in both bands and pass quality cuts, 103 events come from ${ }^{252} \mathrm{Cf}$ data, and 106 pass the surface event cut. Thus, this region contributes at most two non- ${ }^{252} \mathrm{Cf}$ NR events which pass the surface cut. The breakdown of total surface event rejection efficiency is provided in Table 5.3 . overall results indicate high rejection efficiency $(>96 \%)$ for non- ${ }^{252} \mathrm{Cf}$ NR events, and events between bands, called surface leakage events. The rejection efficiency of non- ${ }^{252} \mathrm{Cf}$ surface leakage events for the combined run data set is $99.2 \%$, for the $10-100 \mathrm{keV}$ recoil energy region, and $98.7 \%$ rejection for all $\left({ }^{252} \mathrm{Cf}\right.$ and 

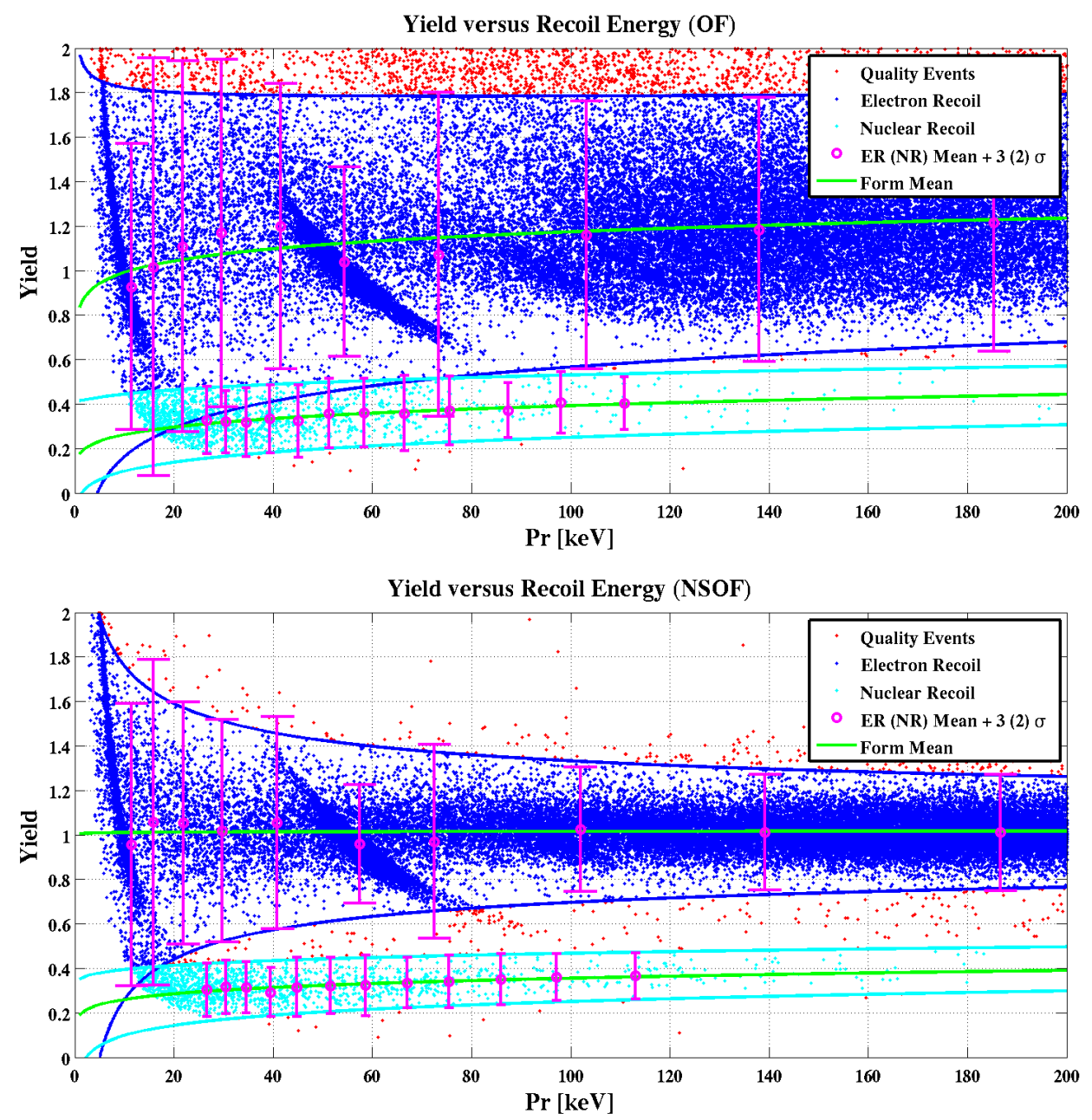

Figure 5.34: Yield versus recoil energy for (TOP) OF and (BOTTOM) NSOF total phonon processing, with quality cuts and surface event cut applied. ER (blue) and NR (cyan) bands are fit to the mean plus standard deviation (pink) of the yield distributions for a set of twelve logarithmically spaced energy bins. 
non- ${ }^{252} \mathrm{Cf}$ ) events from $10-100 \mathrm{keV}$. However, the non- ${ }^{252} \mathrm{Cf}$ NR events and surface leakage events can be accounted for. The remaining surface leakage events are expected to simply be bulk ER and NR events which fall outside the $2 \sigma$ and $3 \sigma$ distribution cutoffs. To confirm this, a combination Gaussian-Cauchy distribution 7 is fit to the yield distributions within the $\mathrm{ER}$ and $\mathrm{NR}$ bands, for $10 \mathrm{keV}$-wide recoil energy slices. For each slice, the percentage of the ER and NR distribution fits which fall between the bands is calculated, as well as the number of events within each distribution, in order to calculate the expected number of leakage events for each slice. Of the 123 surface leakage events which pass quality and surface cuts in this energy region, 34 of these events are expected to be bulk neutrons from the high-yield end of the NR distribution, given the 1,530 NR events observed, and the $2 \sigma$ NR band fit. An additional 76 events are expected to be bulk electron recoils from the low-yield end of the ER distribution, given the 15,796 ER events observed, and the $3 \sigma$ ER band fit. Therefore 110 events in the surface leakage region between ER and NR bands are expected, which is consistent with the observed 123 events within $1 \sigma$ Poisson uncertainty.

Assuming the same ${ }^{210} \mathrm{~Pb}$ background as Soudan is achieved at SuperCDMS SNO$\mathrm{LAB}$, a statistics-limited leakage fraction upper limit for surface leakage events occurring in the $8-115 \mathrm{keV}_{r}$ region of the NR band is $1.7 \mathrm{E}-5$ (90\% C.L.) for $100 \mathrm{~mm}$ iZIPs [162]. Although the UMN test facility is limited by ambient neutrons, prohibiting a direct comparison of leakage events in the NR band, the observed leakage fraction of 8.0E3 between ER and NR bands shows promise for reaching the $100 \mathrm{~mm}$ iZIP rejection capabilities projected for SuperCDMS SNOLAB.

The 23 non- ${ }^{252} \mathrm{Cf}$ NR events which are uncut fall within the expected background of $\sim 28 \pm 12$ neutrons for the non- ${ }^{252} \mathrm{Cf}$ live time of $3.16 \pm 1.40$ hours, approximated by the expected neutron rate given by Eq. 3.44. Live time is not explicitly known, but instead estimated given event rate for both data runs, the run time of each series, and the total number of events in each series. Total live time of data included in this study (considering quality cuts on time) is $8.63 \pm 4.70$ hours, with the large uncertainty due to fluctuations in dead time during Run 54 data taking.

It is clear from Fig. 5.35 that the rejection efficiency is energy dependent. Fig. 5.36

\footnotetext{
7 A combination distribution was selected since it most accurately fit the tail-ends of both yield distributions, which were overestimated by a Cauchy distribution, and underestimated by a Gaussian distribution.
} 


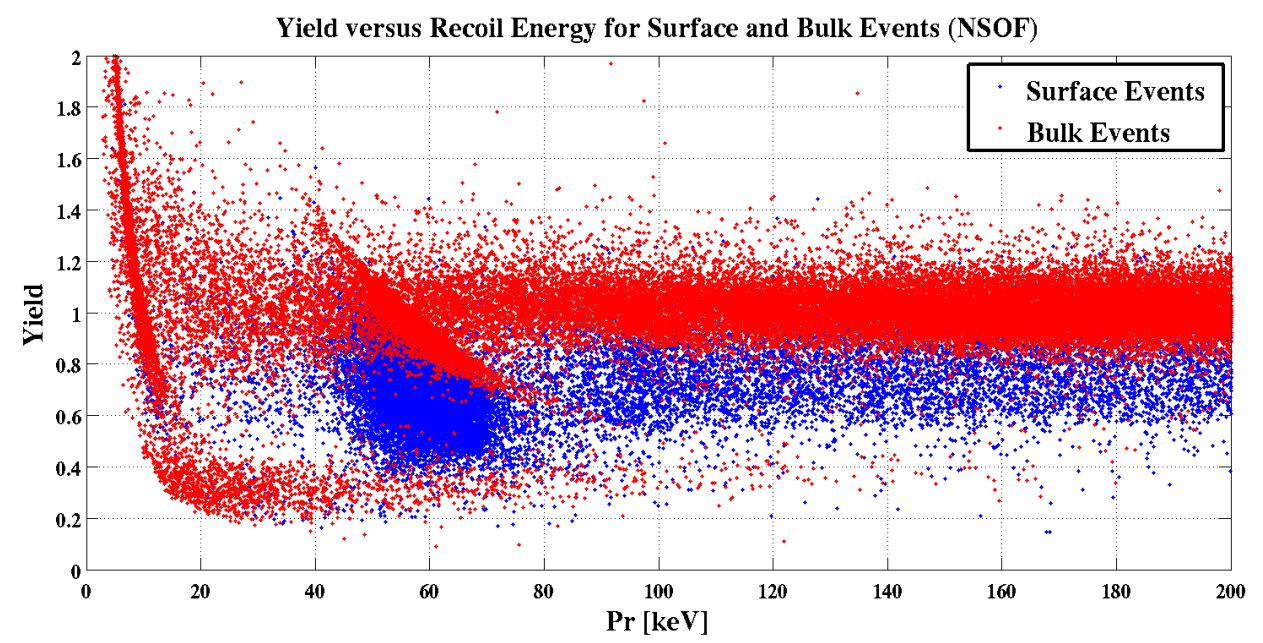

Figure 5.35: Bulk events (red) comprise the ER and NR yield bands. Events which fail the charge symmetry cut and are thus surface events (blue), tend to bleed out of the ER band, into the NR band region.

shows rejection efficiency versus recoil energy for various event types. ER and NR with ${ }^{252} \mathrm{Cf}$ events follow a general trend of low surface event rejection at low energies, peaking at $60 \mathrm{keV}$ and then dropping for higher PrNF values. This is expected, since ER and true NR events should be bulk events, though it is likely that some $60 \mathrm{keV}$ surface events are originally present in both bands before the surface cut is applied. The rise of ${ }^{252} \mathrm{Cf} \mathrm{NR}$ surface event rejection at higher energies is also expected, as the number of expected true NR events diminishes at higher recoil energies. Non- ${ }^{252} \mathrm{Cf}$ and leakage events (events between the bands) all demonstrate near 100\% surface event rejection above $40 \mathrm{keV}$, expected since these events are believed to predominately be surface ER events with diminished yield.

Yield distributions provide another way to observe the effect of the surface event cut. The left-side plots Fig. 5.37 show the yield distributions of Run $54\left({ }^{252} \mathrm{Cf}\right.$ and non- ${ }^{252} \mathrm{Cf}$ ), Run 56, and combined runs, with and without the surface cut applied. Distributions focus on the $10-100 \mathrm{keV}$ recoil energy range. It is clear from these distributions that events within the leakage region between bands, and non- ${ }^{252} \mathrm{Cf}$ events in the NR band are the events predominately removed by the cut. The surface event rejection efficiency as a function of yield for each data type is shown the right column 


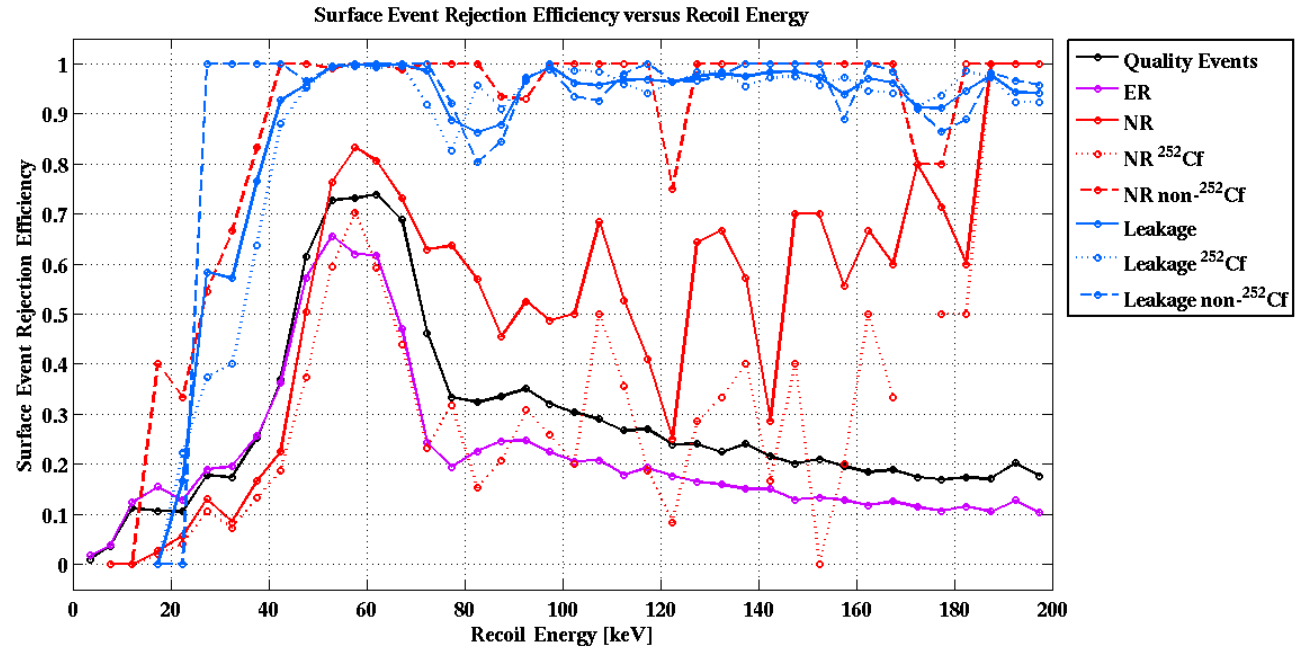

Figure 5.36: The surface event rejection efficiency versus recoil energy for all events (solid black), ER events (solid blue), NR events (solid red, ${ }^{252} \mathrm{Cf}$ dotted red, Non- ${ }^{252} \mathrm{Cf}$ dashed red), Leakage Events (solid green, ${ }^{252} \mathrm{Cf}$ dotted green, Non- ${ }^{252} \mathrm{Cf}$ dashed green)

of Fig. 5.37. Surface event rejection remains about $80 \%$ between ER and NR bands, and is typically above $90 \%$. Surface event rejection of ${ }^{252} \mathrm{Cf}$ events within the NR band region is below $20 \%$, and surface event rejection of ER events is typically below $10 \%$. 

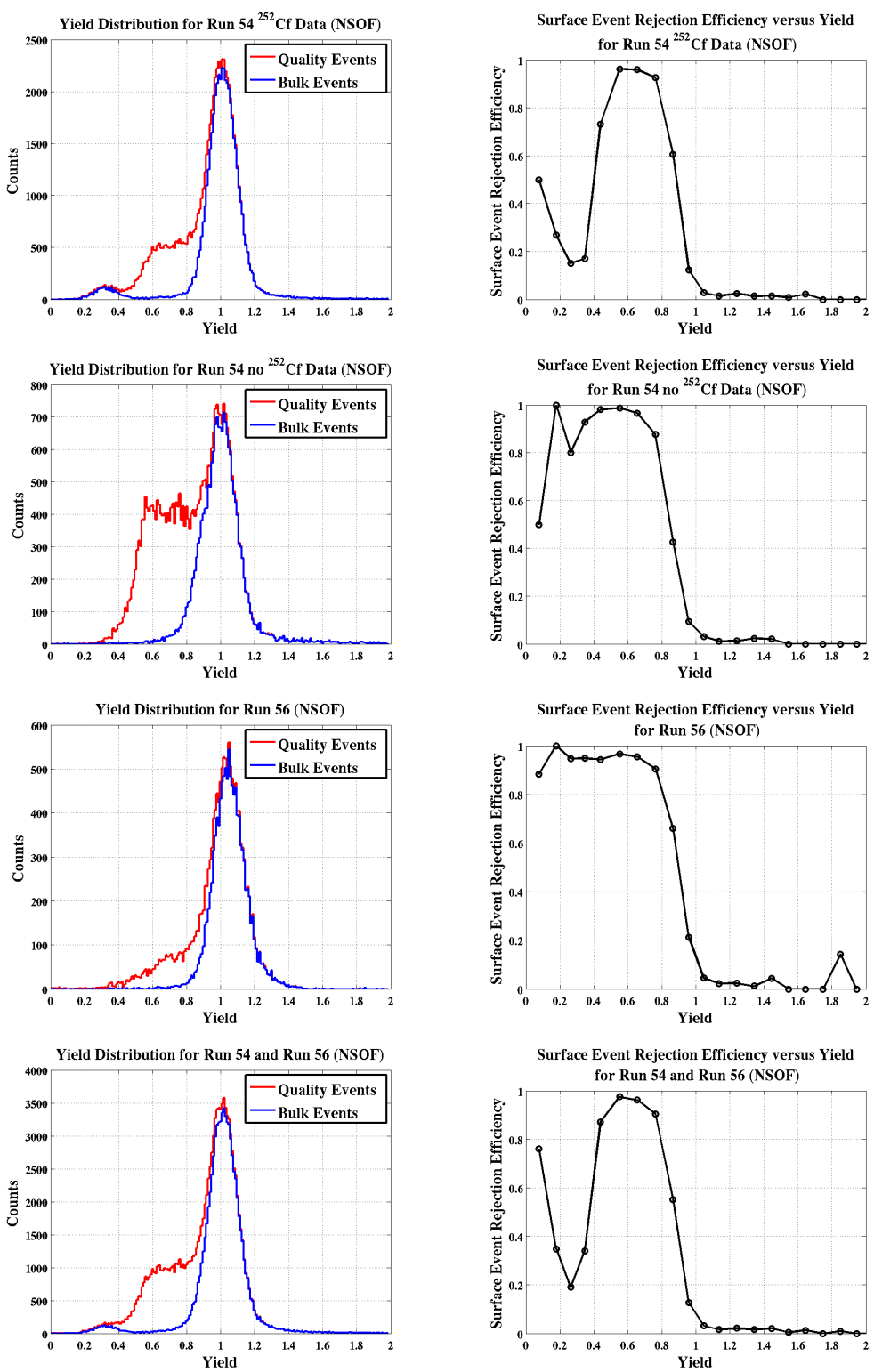

Figure 5.37: (LEFT) Yield distributions demonstrating surface event rejection efficiency of the charge symmetry cut in the $10-100 \mathrm{keV}$ recoil energy range. Distribution of all quality data (blue) is compared to distribution with surface event cut applied (red). (RIGHT) Surface event rejection efficiency versus yield. Both plots are presented for (TOP) Run $54{ }^{252} \mathrm{Cf}$ data (TOP MIDDLE) Run 54 non- ${ }^{252} \mathrm{Cf}$ data (BOTTOM MIDDLE) Run 56 data (BOTTOM) Run 56 and Run 54 data. 
Table 5.3: Surface Event Rejection Efficiency for 10 - $100 \mathrm{keV}$ Events

\begin{tabular}{|c|c|c|c|}
\hline Run 54 & Total Count & Pass Surface Cut & Rejection Efficiency \\
\hline All Events & 41982 & 16510 & $60.67 \%$ \\
\hline ER Events & 29725 & 14801 & $50.21 \%$ \\
\hline NR Events & 2617 & 1526 & $41.69 \%$ \\
\hline${ }^{252} \mathrm{Cf}$ NR Events & 1976 & 1507 & $23.73 \%$ \\
\hline Non- ${ }^{252}$ Cf NR Events & 641 & 19 & $97.04 \%$ \\
\hline Leakage Events & 9574 & 118 & $98.77 \%$ \\
\hline${ }^{252}$ Cf Leakage Events & 4175 & 79 & $98.11 \%$ \\
\hline Non- ${ }^{252} \mathrm{Cf}$ Leakage Events & 5399 & 39 & $99.28 \%$ \\
\hline Run 56 & Total Count & Pass Surface Cut & Rejection Efficiency \\
\hline All Events & 1430 & 1029 & $28.04 \%$ \\
\hline ER Events & 1292 & 995 & $22.99 \%$ \\
\hline NR Events & 35 & 4 & $88.57 \%$ \\
\hline Leakage Events & 76 & 5 & $93.42 \%$ \\
\hline Run 54 and 56 & Total Count & Pass Surface Cut & Rejection Efficiency \\
\hline All Events & 43412 & 17539 & $59.60 \%$ \\
\hline ER Events & 31017 & 15796 & $49.07 \%$ \\
\hline NR Events & 2652 & 1530 & $42.31 \%$ \\
\hline${ }^{252} \mathrm{Cf}$ NR Events & 1976 & 1507 & $23.73 \%$ \\
\hline Non- ${ }^{252}$ Cf NR Events & 676 & 23 & $96.60 \%$ \\
\hline Leakage Events & 9650 & 123 & $98.73 \%$ \\
\hline${ }^{252}$ Cf Leakage Events & 4175 & 79 & $98.11 \%$ \\
\hline Non- ${ }^{252} \mathrm{Cf}$ Leakage Events & 5475 & 44 & $99.20 \%$ \\
\hline
\end{tabular}




\section{Chapter 6}

\section{$150 \mathrm{~mm}$ Si Contact-Free Detector}

\subsection{Project Motivation}

Based on the success of CDMSlite, SuperCDMS SNOLAB is implementing higher voltage detectors [135] that take advantage of Luke phonon production to reach higher signal to noise ratios. However, current leakage reduces the sensitivity of these detectors, particularly at the $70 \mathrm{~V}$ operating bias of the CDMSlite detector [136]. Prototype contact-free bias and readout electrodes were designed, fabricated, and tested at the UMN test facility, to pursue the contact-free electrodes operational capabilities in the CDMSlite operating bias range of $60-100 \mathrm{~V}$ applied bias. Designs were based off of an initial study conducted by SuperCDMS collaborators at Texas A\&M University, which paired a contact-free bias electrode with a mask of athermal phonon sensors deposited on the opposite side of a $0.25 \mathrm{~kg}$ Ge substrate [220]. The UMN design serves as an initial study on the feasibility of a fully contact-free setup, and does not feature phonon sensors.

Thus far, Ge has been the dominate target material for prototype SuperCDMS SNOLAB detectors tested at UMN (listed in Section 3.6). However, SuperCDMS SNOLAB will implement several iZIP and high voltage Si detectors during its initial stages of operation. Additionally, Si crystals offer some benefits over Ge. Since Si does not face the same restrictions on crystal size as Ge it is possible for future Si detectors to be $150 \mathrm{~mm}$ $(6 ")$ in diameter and $4 / 3$ " in thickness (provided sufficient charge collection efficiency at such thicknesses), giving these detectors the same mass as 4" Ge detectors $(1.37 \mathrm{~kg})$ and 
thus a similar fiducial exposure. For spin-independent scattering, the lower atomic mass of Si results in a less sensitive detector than Ge since cross section scales as $A^{2}$, where $\mathrm{A}$ is the mass number. However, for low mass WIMPs $\left(\lesssim 40 \mathrm{GeV} / \mathrm{c}^{2}\right)$, the reduced mass of the WIMP-silicon system is larger than that of Ge, and thus the average recoil energy at this mass will be larger for Si.

This mass range has received particular interest recently due to a hint of signal of low mass WIMPs presented by collaborations such as CDMS and DAMA/Libra 143. Recent CDMS-II results explore these energy regions with Si detectors, with three candidate WIMP-scattering events found and with an expected background of 0.7 events [133. $150 \mathrm{~mm}$ Si detectors would allow SuperCDMS SNOLAB to continue probing this region of WIMP mass. SuperCDMS is particularly advantaged to look at such low energies due to its ability to increase phonon production with increased ionization bias voltage, resulting in observable sub-keV events.

Therefore, UMN contact-free designs were outfitted for use with a $150 \mathrm{~mm}$ diameter Si crystal, to explore the feasibility of both larger Si crystals, and contact-free designs. The preliminary study focuses on ionization characterization, particularly neutralization and charge collection efficiency, and performance as a high voltage detector. Based on these results, subsequent studies can be conducted with designs expanded to incorporate phonon sensors.

\subsection{Designs}

Detector housing and additional new cold hardware designs were created using Autodesk Inventor CAD software [221], and fabricated in the UMN College of Science and Engineering Shop. Electrode designs were created with the guidance of V. Mandic and N. Mirabolfathi, and detector housings and other cold hardware designs were based on previous test facility designs created by H. Chagani and J. Zhang. Much of the modified room-temperature electronics used in the contact-free readout were designed and fabricated by N. Mast. 


\subsubsection{Detector Designs}

Prototype contact-free housing designs were fit to $150 \mathrm{~mm}$ diameter, $33 \mathrm{~mm}$ thick, high purity Si crystal, significantly larger than the $100 \mathrm{~mm}$ diameter, $33 \mathrm{~mm}$ thick Ge crystals commissioned for use in SuperCDMS SNOLAB, though both crystals have similar masses and thus a similar fiducial exposure. A single-channel, charge-only sensor detector was fabricated and tested using an n-type, $[100] \pm 2^{\circ}$ orientation, high purity, high resistivity $(>15,000 \Omega \cdot \mathrm{cm})$ silicon crystal purchased from TopSil and polished by Shaw.

\subsubsection{Contact-Free Electrodes}

Two electrodes were fabricated from aluminum for the contact-free design; one to apply a voltage bias to the crystal, and one to readout the charge signal. The contact-free electrode designs, and the fabricated electrodes, are shown in Fig. 6.1. The cylindrical electrodes are comprised of a 5.906" outer diameter, 0.132" thick top lip which secures the electrode to its housing, and a solid 5.606" diameter cylinder which extends 0.33 " below the bottom edge of the lip. For both readout and bias electrodes, the surface of the electrode, referred to as the electrode face, is parallel to the crystal surface, with a gap of $<0.5 \mathrm{~mm}$ between them. In order to minimize the effects on the bulk bias field, two shallow recesses along the radial periphery of the electrode face provide space for small circuit boards. An IR (940 $\mathrm{nm})$ and a UV (310 nm) LED are mounted on each board, for purposes of detector neutralization. Gaps in the electrode top lip provided space for wires to connect LEDs to power supply through the DIB, secured on the electrode housings.

Dodecagonal housings were fabricated for the crystal and each electrodes from OFHC copper. Distinct top and bottom-side lips allowed the electrode and crystal housings to stack securely together. Copper spacers of $\sim 100 \mu \mathrm{m}$ thickness are used to ensure that the electrodes and crystal are secured within the desired gap distance of $<0.5$ $\mathrm{mm}$. The housings were stacked with an average gap of $\approx 0.3 \mathrm{~mm}$ for readout side and $\approx 0.4 \mathrm{~mm}$ for bias side. Given a 11.9 dielectric coefficient for $\mathrm{Si}$, these gaps imply that the bias across the crystal is $\sim 79 \%$ of the total applied bias, and yield a calculated detector capacitance of $\approx 140 \mathrm{pF}$. Direct measurements of detector stack capacitance 
give $139 \mathrm{pF}$ at $10 \mathrm{kHz}$. This is comparable to typical SuperCDMS detector electrode capacitance $(\sim 100 \mathrm{pF})$ [167]. Copper bottom and top lids were also fabricated from OFHC $\mathrm{Cu}$ to provide shielding. Additionally the top lid is designed to mate with a CDMS II tower. The detector stack is secured together with brass threaded rods. Fig. 6.1 illustrates the detector stack design, and assembled bias and readout electrodes in their housings. The first three images included in Fig. 6.2 illustrate the process of mounting the crystal, and the final three images illustrate the process of mounting the electrodes and accompanying electronics in their housings.

\subsubsection{LEDs}

Pairs of LEDs are mounted on small $(0.5 " \times 0.3 ")$ circuit boards and located in shallow recesses on the surface of each bias electrode, shown in Fig. 6.3, to neutralize any regions of accumulated charge in the crystal by flashing. The chips were placed near the radial periphery of the electrode in order to minimize effects on the bulk bias field.

Each board was outfitted with an IR $(940 \mathrm{~nm}, 1.3 \mathrm{eV})$ and a UV $(310 \mathrm{~nm}, 4.0 \mathrm{eV})$ LED in order to target the Ge direct and indirect band gaps. Standard SuperCDMS IR LEDs have sufficient energy to excite electrons through the direct band gap of Ge, $E_{\Gamma 1}=0.8 \mathrm{eV}$, and the indirect band gap of $\mathrm{Si}, E_{g}=1.12 \mathrm{eV}$, as illustrated in the band structures of Fig. 6.4. SuperCDMS detectors are traditionally illuminated with IR LEDs mounted in the detector housing, a setup found to successfully neutralize Ge detectors during routine operation. However, past attempts at neutralizing Si detectors have been less successful, possibly due to relying on electron excitation through the indirect band gap [223. Newly-added UV LEDs have sufficient energy to excite electrons through the direct band gap of Si, $E_{\Gamma 1}=3.4 \mathrm{eV}$. Studies comparing the effectiveness of UV and IR LEDs on crystal neutralization are described in Section 6.3.7.

\subsubsection{Electronics and Warm Hardware Designs}

Electronic signals are passed from each electrode DIB to the the tower through a flexible side coax comprised of adapted SCAB board and SCUHBA with grounding plane, connected via eleven low-noise copper coaxial wires. Two $25 \mathrm{~cm}$ long flexible side coaxes were used. The readout side coax was modified to fit an upside-down DIB without 

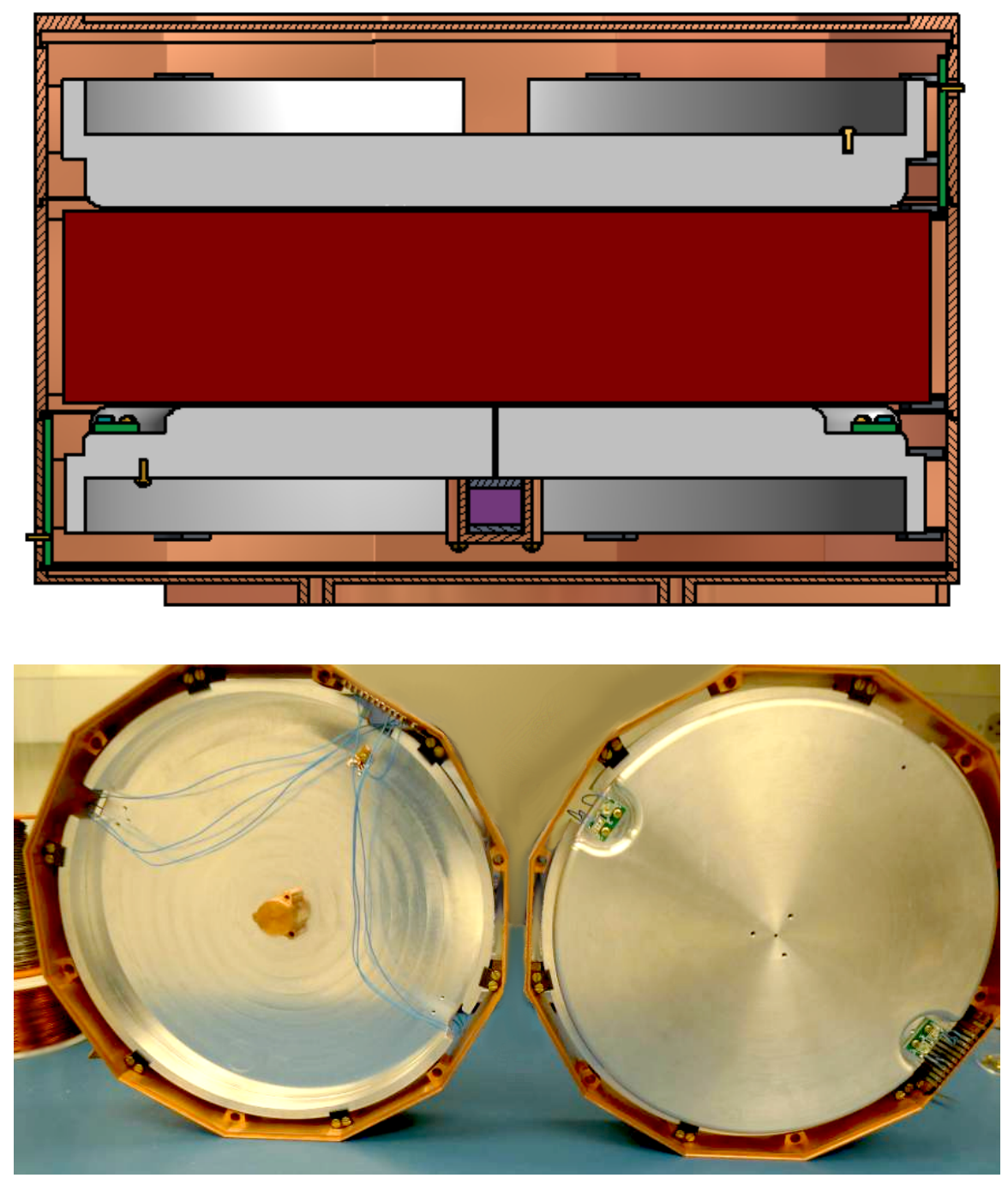

Figure 6.1: (TOP) Contact-free detector design, with two aluminum electrodes (grey) surrounding a $150 \mathrm{~mm}$ Si crystal (red) with a $<0.5 \mathrm{~mm}$ gap between faces, all secured within copper housing. Both electrodes feature two circuit boards with LEDs on their face side (shown in green on readout side), and are connected to DIBs (green) mounted on their housing. Additionally, the readout electrode features a collimated ${ }^{241} \mathrm{Am}$ source (purple) in a copper source holder at the center of the electrode. Wire (not shown) extending from the DIB to the small screw (brass) secured in each electrode will provide applied bias and electrode readout. (BOTTOM) Fabricated electrodes in housings. Top side of readout electrode (on left) shows ${ }^{241} \mathrm{Am}$ copper source holder, charge signal readout and LED wiring leading to housing-mounted DIB. Face side of bias electrode (on right) shows UV and IR LEDs each mounted on two small circuit boards. 

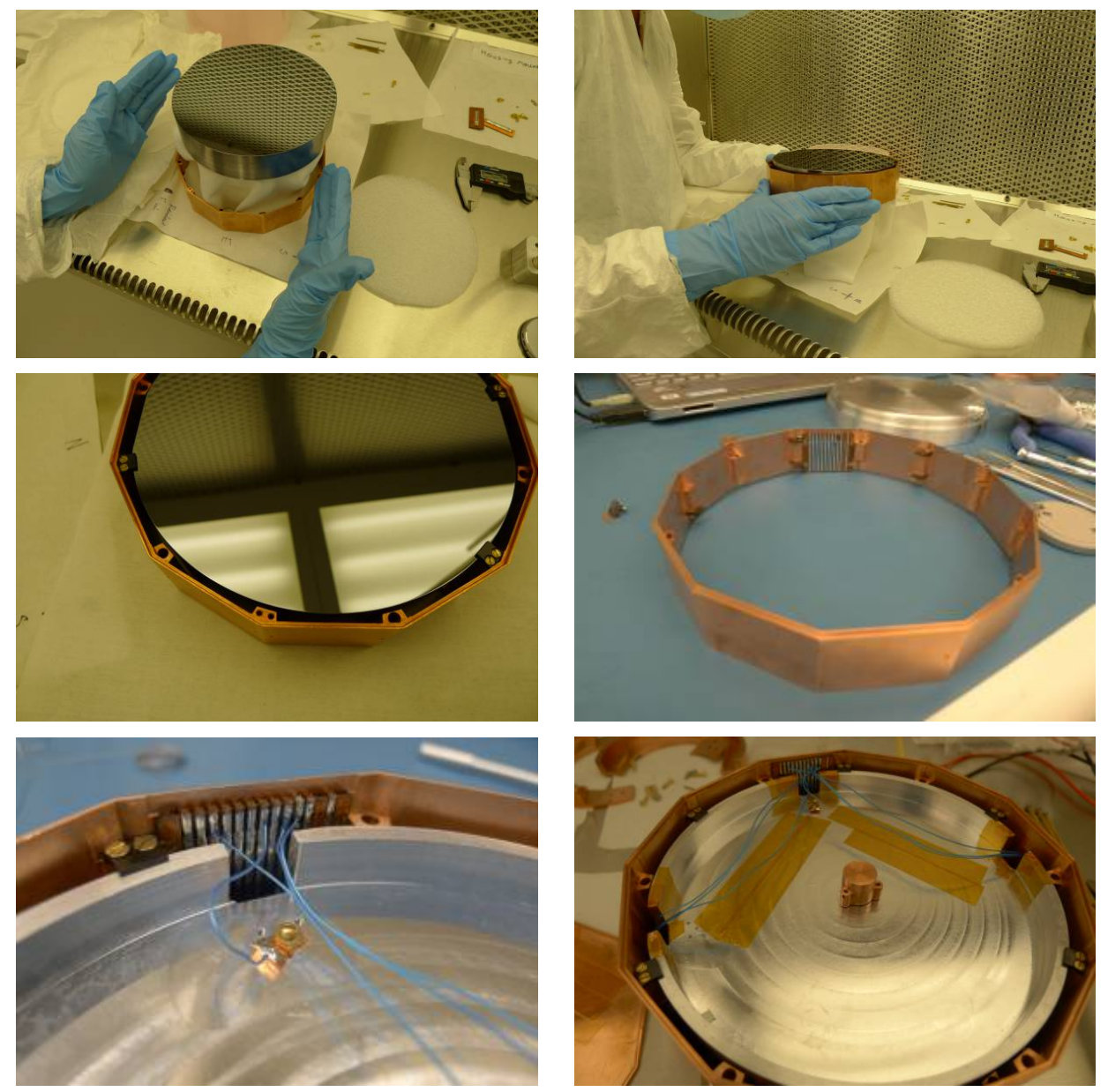

Figure 6.2: Stages of mounting crystal and electrodes into housings. (TOP LEFT) In preparation for crystal mounting, cirlex clamps are installed on the bottom of the crystal housing, which is then set on the clean bench. Polished crystal is set on stand (with protective kim wipe) in the center of the housing. (TOP RIGHT) Housing is slowly raised until bottom clamps catch on the crystal, raising it above the stand. (MIDDLE LEFT) Housing and crystal are set on the clean bench, and top clamps are carefully installed to secure the crystal in its housing. (MIDDLE RIGHT) DIBs are installed onto both electrode housings before electrodes are added. (BOTTOM LEFT) After electrodes have been secured in place with clamps, wires are soldered, connecting the DIB channels to LED boards and the bias/readout screw secured to the electrode face with additional copper tape for good contact. (BOTTOM RIGHT) The ${ }^{241} \mathrm{Am}$ source is added to the readout electrode, and kapton tape is places around the wires for additional protection and insulation. 


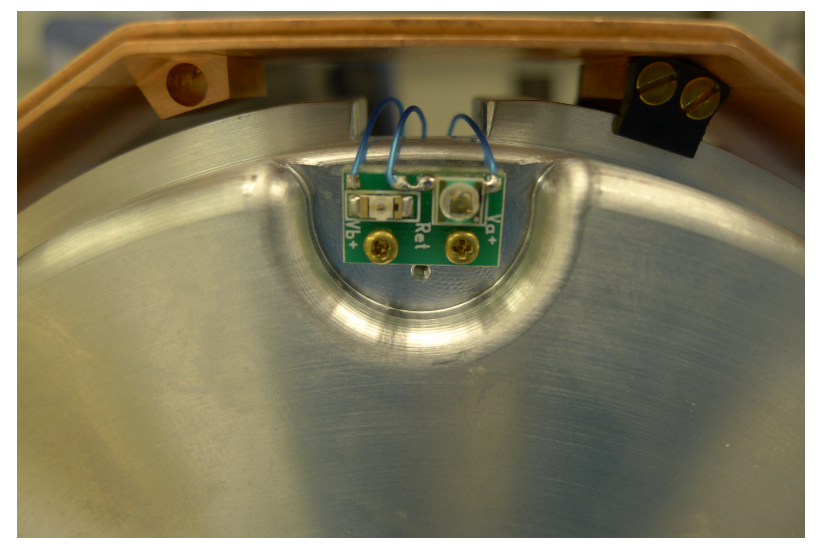

Figure 6.3: UV and IR LEDs mounted to a custom-made PCB board are attached to the contractfree electrode. Boards are wired to LED and phonon return lines on the DIB.
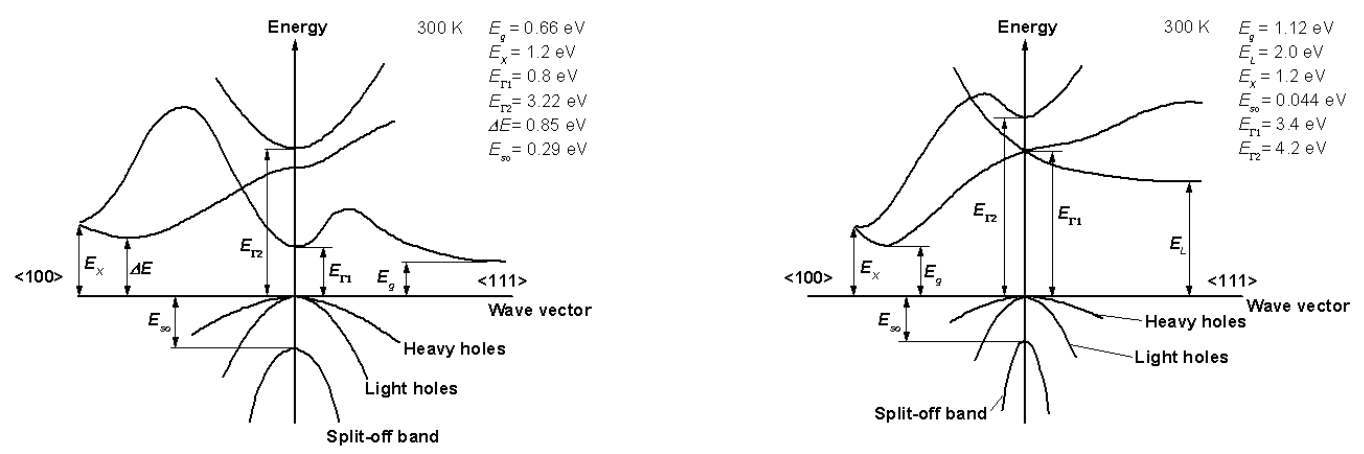

Figure 6.4: (LEFT) Band structure of Ge and (RIGHT) Si at $300 \mathrm{mK}$. Typical CDMS $940 \mathrm{~nm}(1.3 \mathrm{eV})$ LED's have sufficient energy to excite electrons through the direct band gap of Ge, $E_{\Gamma 1}=0.8 \mathrm{eV}$, and the indirect band gap of Si, $E_{g}=1.12 \mathrm{eV}$. Newly-added $310 \mathrm{~nm}$ UV LEDs $(4.0 \mathrm{eV})$ have sufficient energy to excite electrons through the direct band gap of Si, $E_{\Gamma 1}=3.4 \mathrm{eV}$. Figures courtesy of Ioffe Physico-Technical Institute [222]. 
strenuous cable twisting. Previous tests indicate the flexible side coax noise performance is similar to that of typical CDMS vacuum side coax during normal operation, and the that cables have do not significantly reduce the energy resolution of the ionization measurement 224]. Fig. 6.6 depicts the process of mounting the completed detector stack on the tower with the flexible side coaxes.

The readout electrode is controlled and read out via the standard setup, with a DCRCs [225] connected to a computer running MIDAS-based data acquisition software. A second DCRC is used to control the LEDs on the bias electrode; all unused bias-side channels were disconnected via an adapter board designed by N. Mast, which is inserted between the DCRC and the D-sub cables leading to the Ebox. This adapter board also connects the bias electrode to an external power supply. A schematic of the contact-free bias and readout electronics is shown in Fig. 6.5.

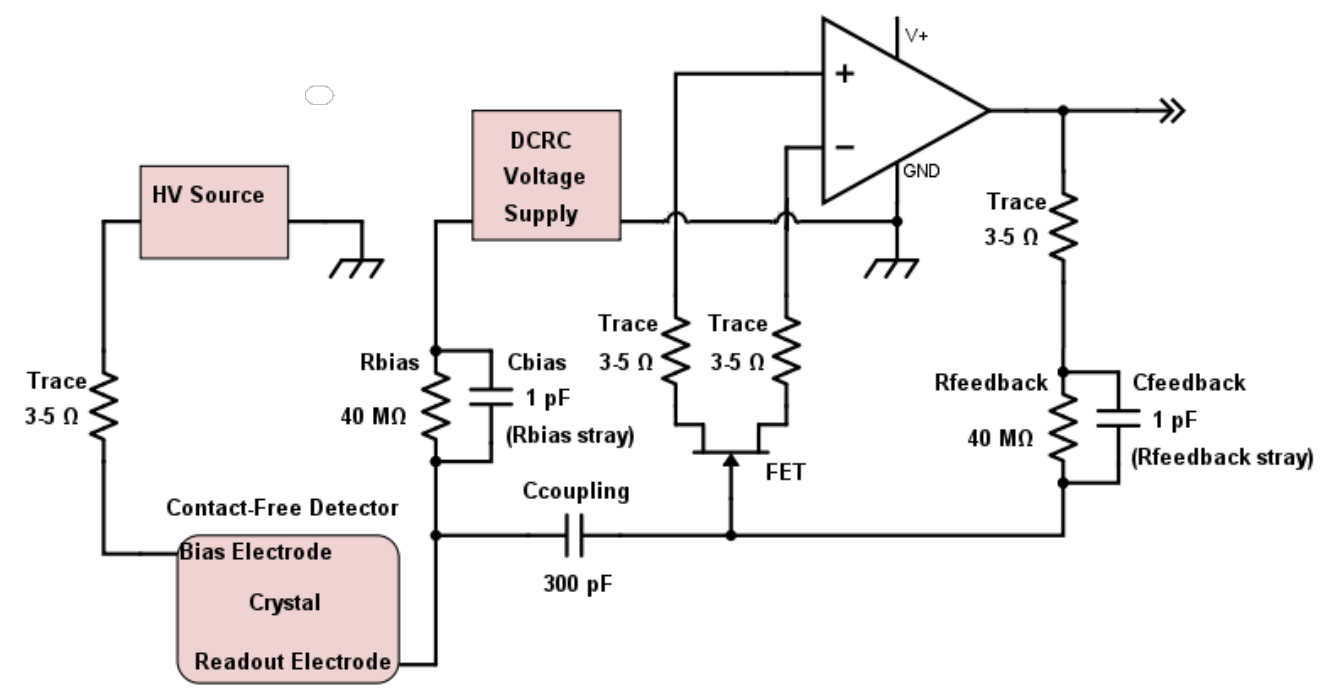

Figure 6.5: Schematic of the Run 60 contact-free electronics setup. Readout electrode DIB passes through typical CDMS ionization readout circuit, comprised of coaxial cables, SQUET card, stripline, and MIDAS-controlled DCRC. 


\subsubsection{Modifications to Inner Vacuum Chamber}

To accommodate the increased bulk of the $150 \mathrm{~mm}$ designs, modifications were made to the UMN test facility IVC. A new $30 \mathrm{mK}$ (innermost) can, comprised of a can tube (sides), top lid, bottom flange, and connecting adapter plate to secure the detector tower into the fridge, were fabricated with $\mathrm{OFHC} \mathrm{Cu}$. The new can has an increased diameter, from 4.50" to 7.44". Although this size can accommodate the $150 \mathrm{~mm}$ detectors and fit within the surrounding $100 \mathrm{mK}$ can, the $600 \mathrm{mK}$ rod, connecting the still to the 600 $\mathrm{mK}$ tower stage, had to be removed. Increased base temperature is an expected effect of this change, however the higher temperature should still be sufficient for charge-only operation.

Increase detector size and modified $30 \mathrm{mK}$ can diminished the clearance space inside the IVC, with only $14 \mathrm{~mm}$ between the tower and inner $30 \mathrm{mK}$ wall $(9 \mathrm{~mm}$ at $30 \mathrm{mK}$ can entrance), and $10 \mathrm{~mm}$ between $30 \mathrm{mK}$ and $100 \mathrm{mK}$ cans, increasing the risk of a thermal touch between temperature stages. Limited space also made tower installation particularly difficult. To accommodate the increased bulk of the tower and $30 \mathrm{mK}$ can:

- Stripeline fasteners had to be removed from the IVC during installation.

- Wires connecting to the tower thermometer were rerouted from their standard position between the $30 \mathrm{mK}$ and $100 \mathrm{mK}$ cans to inside the $30 \mathrm{mK}$ can, passing through a hole in the top lid.

- Kapton tape was applied to the outward faces of the flexible side coaxes, to provide additional protection in the event of contact with the $30 \mathrm{mK}$ can inner wall.

With these accommodations and careful installation, the contact-free data run saw no thermal or electrical short. Fig. 6.7 depicts the close proximity of the tower and $30 \mathrm{mK}$ can.

\subsubsection{Cryogenics and Data Run}

The 150 mm contact-free run, UMN Run 60, lasted from September 12th to October 2nd 2016, with data taken from September 19th onwards. In that time over 200 data series were taken, with applied bias voltages ranging from -110 to $+110 \mathrm{~V}$. 

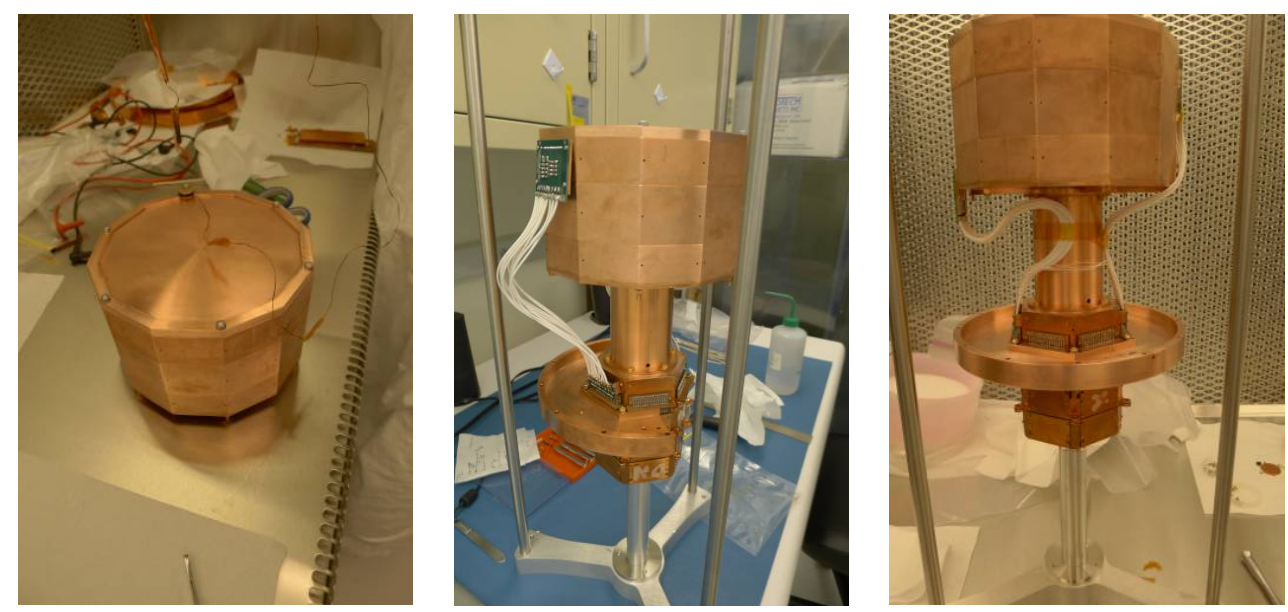

Figure 6.6: (LEFT) Assembled electrodes and crystal housings are stacked with top and bottom lids. Stack is secured with threaded rods which pass through the top lid and stack to secure into the bottom lid. Tower thermometer is secured to the top lid using one of the threaded rods. (MIDDLE) Brass screw secure bottom lid of stack to tower. Flexible side coax is attached to electrode DIB and tower SCUHBA. One half of the new adapter plate is installed on the tower. (RIGHT) Installation of flexible side coaxes and adapter plate complete. Coax wires are secured around tower spool with floss and kapton tape. Finished tower is ready for installation in the fridge.

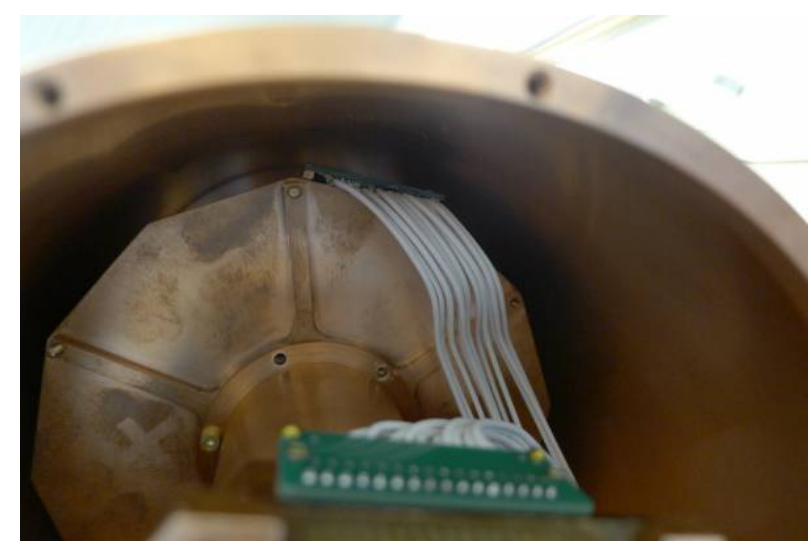

Figure 6.7: Tower successfully installed in new $30 \mathrm{mK}$ can, with sufficient space to avoid contact between can walls and outward facing flexible side coax SCAB. Additional kapton tape was used to cover the flexible side coax components to provide additional protection. 
During the run the base tower temperature ranged from 77 - $94 \mathrm{mK}$, and base mixing chamber temperature ranged from $54-61 \mathrm{mK}$. This is slightly elevated from the typical base temperature of around $30 \mathrm{mK}$, and was expected due to modification made to the inner dilution cans to accommodate a larger detector and lack of thermal sinking of the $600 \mathrm{mk}$ stage of the tower..

\subsection{Results}

This section discusses the data processing and analysis of the $150 \mathrm{~mm}$ contact-free run, including solutions developed in-situ to accommodate for unexpected processing issues and the ringdown-like shape of event pulses. The section also discusses post-run attempts to determine the cause of this pulse shape.

\subsubsection{Time Domain Fit}

At the start of the contact-free run, additions had been made to standard CDMSBats processing, to allow for a single-charge channel, two-phonon channel (one per side) detector to be processed. A DAQ setting allows for phonon A channels to read out IR LED channels, resulting in pulse-less noise during data taking. Raw charge data underwent the standard OF processing, without cross talk. RQ output included QOFvolts, QOFdelay, and QOFchisq, representing charge amplitude, time delay, and $\chi^{2}$ fit value, respectively.

During the initial week of Run 60 data taking, it became apparent that processed data fell into two regimes, each with a different QOFvolts RQ calibration value. Further investigation revealed that this regime splitting was a product of the standard CDMSBats Optimal Filter responding to a 'bin swapping' issue believed to be caused by the DCRCs. Although this issues is known from past detector runs, this was the first time it manifest in a way that altered the amplitude calibration of the OF fit. Regardless of the cause, a reasonable fix for the bin swapping issue could not be found. Working around the issue also became impossible, since the OF processing could switch regimes suddenly, such as the mid-series regime switch shown in Fig. 6.8.

Instead of the OF algorithm, data is processed with a slightly modified version, in which the fitting is performed in the time-domain instead of the frequency domain. 
As with the OF fit, output charge signal traces are fit to a template constructed from characteristic events, optimizing trace amplitude and time delay from trigger quantities to minimize fit $\chi^{2}$, as demonstrated in Fig. 6.9. For all time delay values within the standard search window an amplitude is found to minimize a simple $\chi^{2}$ value. The delay value and corresponding amplitude with the overall minimal $\chi^{2}$ is selected, using Minuit to perform the fit. Energy spectra are constructed from trace amplitude values, calibrated to source peaks. Poor quality events can be removed from analysis through cuts on $\chi^{2}$ and time delay quantities. The time domain fit processing passes along RQ values QTDFvolts (amplitude in volts), QTDFdelay (time delay in seconds), and QTDFchisq $\left(\chi^{2}\right.$ value, in volts $\left.{ }^{2}\right)$.

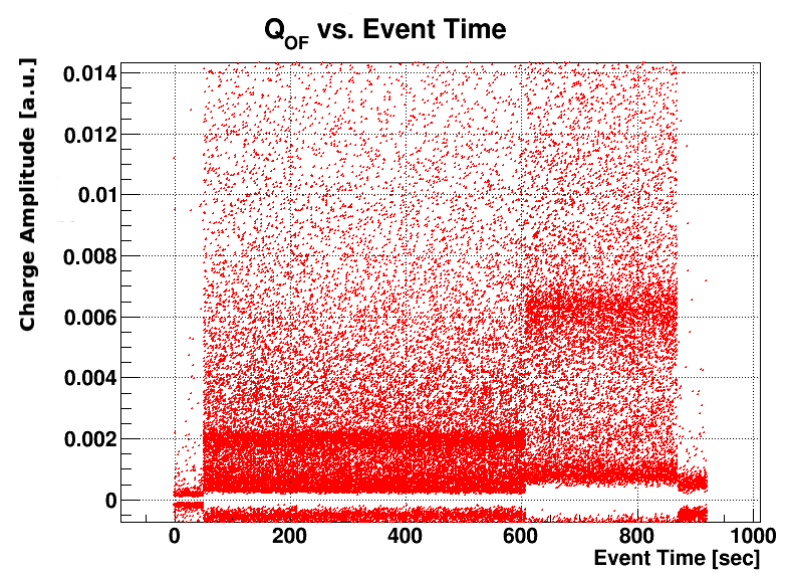

Figure 6.8: An example of the split regimes that occur in charge amplitude. Figure shows uncalibrated charge amplitude versus time. After $\sim 600 \mathrm{~s}$ of data taking, the amplitude enters a new regime, with the uncalibrated $60 \mathrm{keV}$ peak increasing from $\sim 0.002$ a.u. to $\sim 0.006$ a.u. for the remainder of the data series.

\subsection{2 $17 \mathrm{kHz}$ Ringdown}

The falling edge of the ionization pulse is generally an exponential with time constant set to $40 \mu \mathrm{s}$ by the feedback branch of the readout circuit. The modified electronics used with this detector introduced a $17 \mathrm{kHz}$ pole in this branch which caused the ringing observed in the tail, shown in Fig. 6.9. Multiple attempts to determine the source of this behavior during the run were unsuccessful. Fortunately, comparison between raw pulses determined that the energy information is contained in the initial amplitude of the trace, which is expected to be linearly proportional to induced charge. As expected, the pulse shape was observed to scale linearly with pulse amplitude. The template 
constructed for processing includes the $17 \mathrm{kHz}$ ringdown, which allowed data taking to proceed.
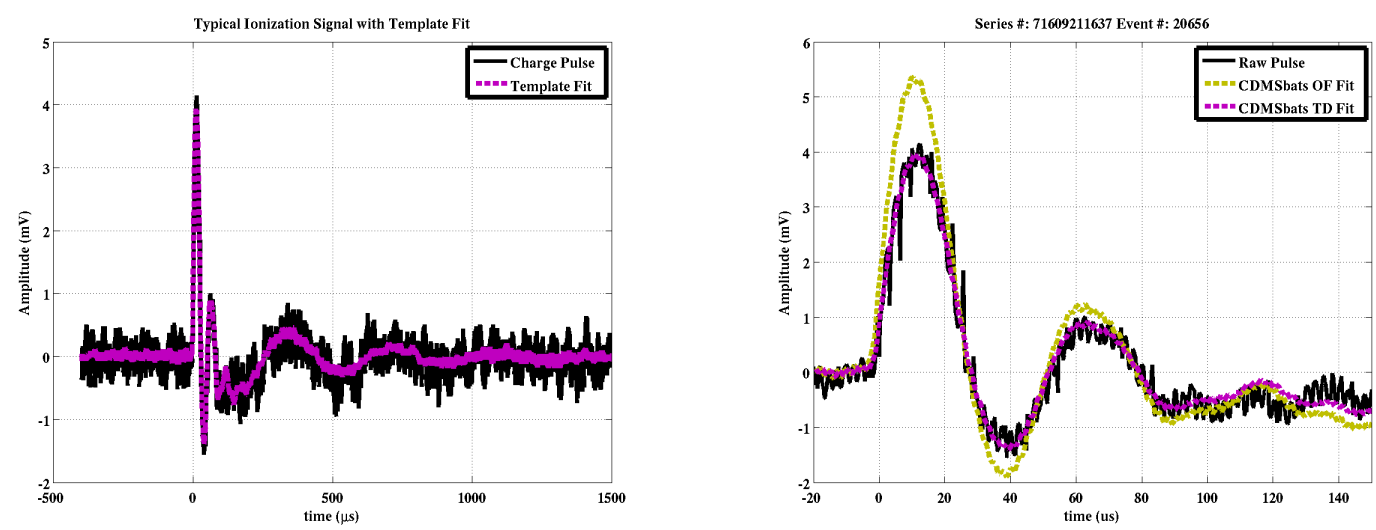

Figure 6.9: (LEFT) Typical ionization pulse signal with $17 \mathrm{kHz}$ ringdown in pulse tail shown in black, overlaid with fitted pulse template in magenta. Fitted template amplitude, time delay, and $\chi^{2}$ quantities are used for data analysis.(RIGHT) Zoom in of raw pulse comparing time-domain fit (magenta) to standard CDMS OF fit (yellow).

\section{$17 \mathrm{kHz}$ Studies}

Starting in mid February 2017, post-run bench tests were conducted on the charge readout circuit. A spare SQUET and stripline were connected to the room-temperature $150 \mathrm{~mm}$ stack (mounted on the tower), as well as to a DCRC. A rerouting board between the stripline and DCRC provided the pin-reversal that would normally occur due to passing through the Ebox.

To simulate an event, an Agilent 81101A $50 \mathrm{MHz}$ pulse generator passes a $1 \mathrm{~V}, 200 \mathrm{~Hz}$ square through a $33 \mathrm{pF}$ capacitor ${ }^{1}$ at the readout electrode, which then passes through the coax, SQUET board, stripline and DCRC. In an attempt to pinpoint the source of the ring down phenomenon, simulated pulses were passed through either a vacuum side coax or a flexible side coax, both with and without the coax being connected to the detector stack. The output waveform was monitored directly from the DCRC analog

$133 \mathrm{pF}$ and $50 \mathrm{pF}$ values were tried as the signal-injecting capacitor, but the effects on peak shape and amplitude were deemed nonsignificant. Various signal generators were also tested, though the Agilent had the most consistent, understandable results. 
section using an oscilloscope. In particular, the scope measured the voltage from analog ground to a test point on a Rev C.0 DCRC board; during data taking, this voltage would be recorded and digitized by the DCRC ADC.

Fig. 6.10 shows example pulse shapes observed using both the standard vacuum side coax and the flexible side coax. On the rising and falling edges of the square wave a significant $\sim 4 \mathrm{kHz}$ oscillation is present when using the flexible coax, that is not present for the vacuum side coax. This observation was independent of whether the flexible coax was connected to the detector stack or not. This observation was independent of whether the coax was connected to the detector stack or not. Overall, the flexible side coax has a ringdown of $\sim 4 \mathrm{kHz}$ with a decay of $1.5 \mathrm{~ms}$, whether or not the coax is connected to the detector. The vacuum side coax was characterized with the expected decay of $250 \mu \mathrm{s}$. This indicates that the flexible side coax was responsible for the 17 $\mathrm{kHz}$ ringdown phenomenon, although the exact cause is not known.
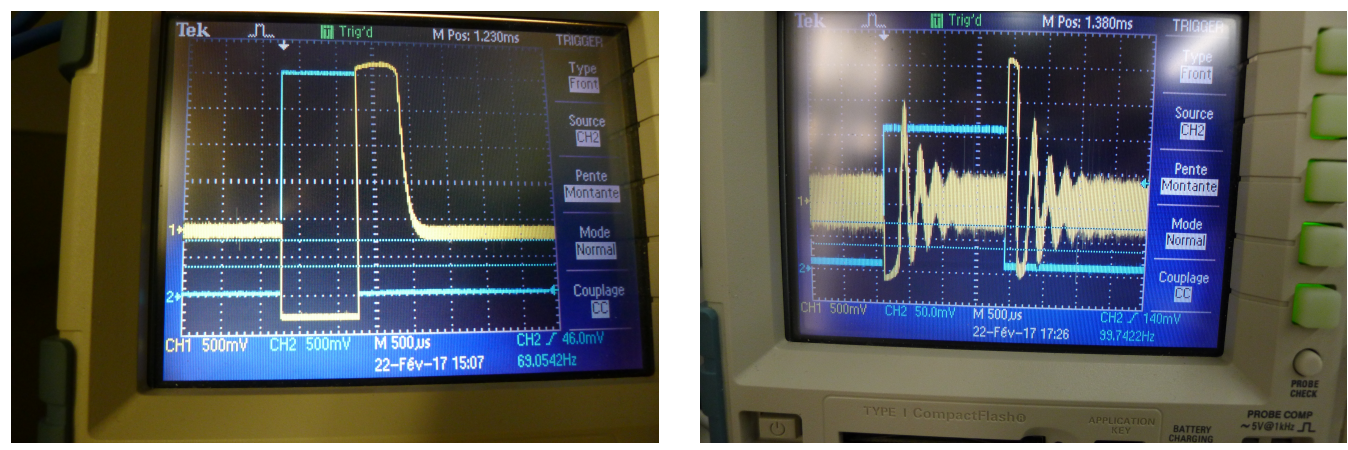

Figure 6.10: Example pulse shapes observed during $17 \mathrm{kHz}$ ringdown studies, using (LEFT) the standard vacuum side coax and (RIGHT) the flexible side coax. The simulated input pulse is shown in light blue, and the response measured from the DCRC is shown in yellow. The vacuum side coax shows no oscillations and decays with $250 \mu \mathrm{s}$ decay time. On the rising and falling edges of the square wave, the flexible side coax has a ringdown of $\sim 4 \mathrm{kHz}$ with a decay of $1.5 \mathrm{~ms}$, whether or not the coax is connected to the detector.

Measurements of the side coax electrical properties were conducted on several occasions, but no deviations from the standard coax component values were found. The 
flexible coax was found to have higher capacitances to ground than the standard vacuum coax, 25 - $35 \mathrm{pF}$, compared to $3.0-6.5 \mathrm{pF}$ at $20 \mathrm{kHz}$, respectively. A plausible explanation for the observed ringdown is a change in the $\mathrm{RC}$ time constant of the amplifier circuit, due to the increased capacitance. However, N. Mast's SPICE simulations modeling the readout circuit have not shown oscillation with these values. Capacitances between coax channels were also measured, with measured values of $2-5 \mathrm{pF}$ for the vacuum coax and $14-16 \mathrm{pF}$ for the flexible coax. In an effort to reproduce the oscillation using the vacuum coax, a $30 \mathrm{pF}$ capacitor was used to introduce extra capacitance to ground on the the various lines, but this had little effect on the pulse shape.

\subsubsection{Quality Cuts}

Minimal quality cuts were applied to the data, including a $\chi^{2}$ cut and a cut restricting the time delay quantity to within $\pm 5 \mu$ s of the initial trigger time. The time delay cut was imposed to remove events in which the template was fit to a secondary ringdown peak, which result in lower reported peak amplitude.

As with $100 \mathrm{~mm}$ iZIP data, $\chi^{2}$ cuts fit the parabolic trend of $\chi^{2}$ versus charge amplitude, removing events with high $\chi^{2}$ values. $\chi^{2}$ cuts are applied on a series-byseries basis, due to frequent deviations in noise between series during Run 60. Fig. 6.11 shows a typical $\chi^{2}$ cut applied to a series with $+20 /-10 \mathrm{~V}$ applied bias. The red band contains pulses which are fit well by the template, while the blue band near low charge energies contains poorly-fit noise events above the trigger threshold.

\subsubsection{Leakage Current}

To understand the noise environment during detector testing, Power Spectral Densities (PSDs) are constructed from 1000 random trigger events taken at the beginning of each data series. Fig. 6.12 shows resulting PSDs from sample data series representing a wide range of crystal biases used. Note that the total crystal bias is taken to be the DCRC voltage bias subtracted from the power supply bias. Noise was higher than in typical detector tests at the UMN facility, but the signal amplitude triggering threshold is raised above noise by the data acquisition software, such that noise is mostly excluded from data. 


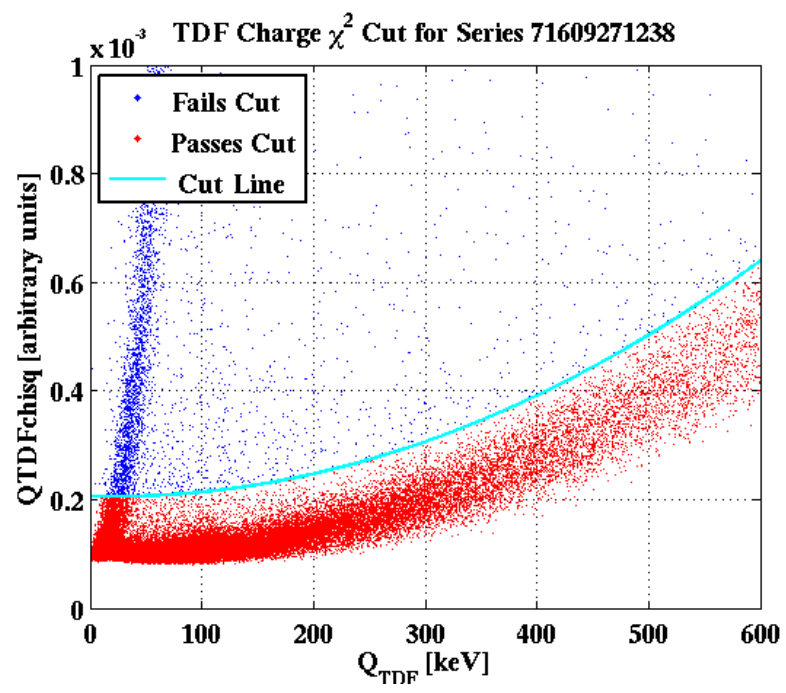

Figure 6.11: Charge $\chi^{2}$ versus calibrated ionization energy for $\mathrm{Si}$ $150 \mathrm{~mm}$ contact-free detector series with $+20 /-10 \mathrm{~V}$ applied bias. Events which pass the $\chi^{2}$ cut (red) fall below the parabolic cut line (cyan), and events which fail the cut (blue) fall above the line. Due to changing noise levels, $\chi^{2}$ cuts are applied on a series-by-series basis.

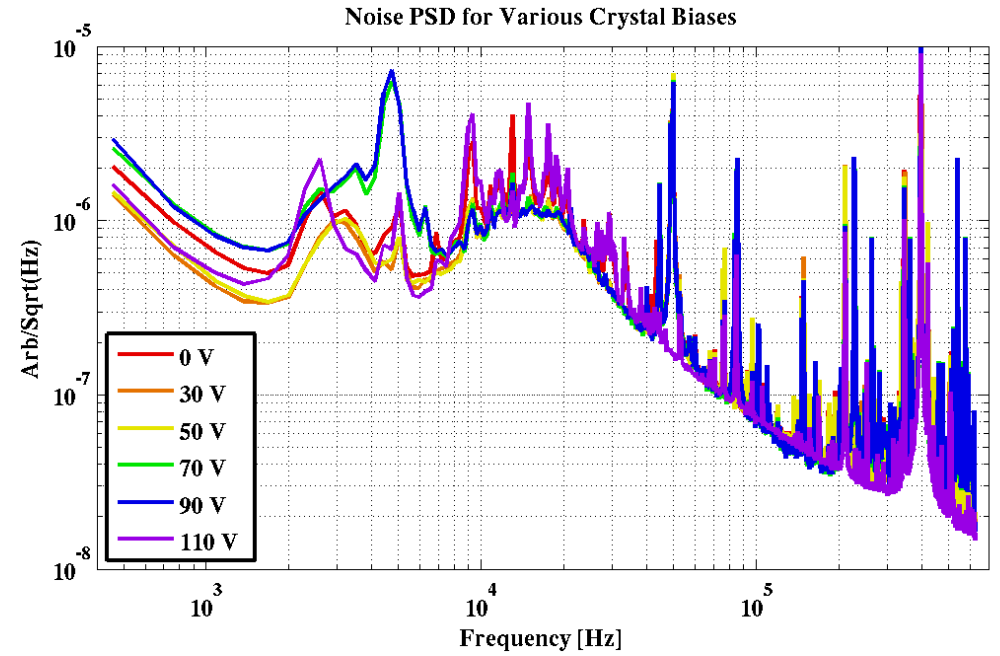

Figure 6.12: PSDs of 1000 initial random triggers taken at the start of $0 \mathrm{~V}, 30 \mathrm{~V}, 50$ $\mathrm{V}, 70 \mathrm{~V}, 90 \mathrm{~V}$, and $110 \mathrm{~V}$ biased data series. Results indicate similar noise across all voltage biases, with some deviation between biases in the low frequency $\left(<10^{4} \mathrm{~Hz}\right)$ range. Changes in noise do not follow the expected patterns due to leakage current through the crystal, which would increase with bias, possibly indicating that variations in noise are due to the state of readout electronics during data taking, more so than applied bias. 
CDMSlite voltage bias was limited by observed leakage current through the crystal for fields as low as 24 Volts/cm 220, which increased phonon shot noise and reduced the applied bias at the crystal. The contact-free design of this device was used to investigate the leakage phenomenon. By removing contact between the electrode and crystal bulk, carrier injection from the contacts is removed as a source of leakage. Leakage current, drawn from the biasing power supply, was measured with a test resistor on a custom designed electronics biasing board. The primary voltage drop was across a $400 \mathrm{M} \Omega$ bias resistor.

The leakage currents observed were time dependent, with the measured value falling off exponentially in the first few minutes after applying the bias. Table 6.3.4 includes leakage measurements taken at base temperature $(\sim 80 \mathrm{mK})$ and after warm up $(\sim 300$ $\mathrm{K})$. Leakage measurements were generally taken within those first few minutes, and thus represent upper limits to the leakage. The values measured were all relatively low, resulting in reductions in the applied bias of $2-10 \%$. This shows the feasibility of using such contact-free electrodes to reduce the leakage currents observed at high bias potentials.

\begin{tabular}{ccccc}
\hline External Bias (V) & \multicolumn{2}{c}{ Leakage current $(\mathrm{nA})$} & \multicolumn{2}{c}{ Bias Reduction $(\mathrm{V})$} \\
& at $\sim 80 \mathrm{mK}$ & at $\sim 300 \mathrm{~K}$ & at $\sim 80 \mathrm{mK}$ & at $\sim 300 \mathrm{~K}$ \\
\hline 30 & 2.3 & - & 1.0 & - \\
50 & 2.0 & 1.4 & 0.88 & 0.62 \\
70 & $<6.9$ & 2.4 & $<3.0$ & 1.1 \\
90 & $<17$ & 2.7 & $<7.5$ & 1.2 \\
110 & - & 3.7 & - & 1.6 \\
-50 & -5 & -2.2 & -2.2 & -0.97 \\
-70 & - & -2.9 & - & -1.3 \\
-90 & - & -3.5 & - & -1.5 \\
-110 & - & -4.3 & - & -1.9 \\
\hline
\end{tabular}

\subsubsection{Ionization Spectra}

The $60 \mathrm{keV}$ gamma line from an ${ }^{241} \mathrm{Am}$ source was used for energy calibration. The source was mounted on the center of the readout electrode with a 0.008 " diameter hole 
in a lead disc collimating the source down the crystal axis. At low bias, some liberated charges recombine before traversing the crystal, reducing the induced charge signal. At higher bias, nearly all liberated charges are fully collected. Distinguishing the $60 \mathrm{keV}$ peak from noise was found to be particularly difficult when low negative biases $(-20$ $0 \mathrm{~V}$ ) were applied to the detector. This is likely due to either a triggering threshold asymmetry, or an asymmetry in the analysis algorithm; this asymmetry would cause an absence of small negative amplitude events post-processing which extends close to the $60 \mathrm{keV}$ energy region. Although low negative bias data was included in analysis for completeness, the accuracy of the results at these biases should be considered not reliable. The suspect regions will be highlighted red in analysis plots, to keep this apparent.

The effects of the $\chi^{2}$ cut are demonstrated in Fig. 6.13, which shows the $Q_{T D F}$ spectrum of a $10 \mathrm{~V}$ net bias series with and without the cut applied. The effects of the cut are unclear when looking at the whole spectrum (shown on the left), but become apparent when the spectrum contains only the first five minutes of data (shown on the right). The $\chi^{2}$ cut removes poorly-fit noise from the low energy region $(\sim 0-40 \mathrm{keV})$, while the $60 \mathrm{keV}$ peak is unaffected. Loss of neutralization results in the $60 \mathrm{keV}$ peak falling into the low energy region after the first five minutes of data taking, making it difficult to distinguish from noise. A more detailed analysis on the $150 \mathrm{~mm}$ Si detector's neutralization is included in Section 6.3.7

Several data series were taken with an external, $0.433 \mathrm{mCi}{ }^{57} \mathrm{Co}$ source present, causing a smeared peak around $100 \mathrm{keV}$, due to the $122 \mathrm{keV}{ }^{57} \mathrm{Co} \gamma$ 's recoiling off the crystal after interacting with the intervening cryostat material. Fig. 6.14 shows the charge energy spectra when $40 \mathrm{~V}$ is applied, for data with and without the ${ }^{57}$ Co source present. Calibration was performed by fitting the $60 \mathrm{keV}$ peaks of each series. The 60 $\mathrm{keV}$ peak, smeared $122 \mathrm{keV}$ peak, and Compton edges of both sources can be seen.

Spectra are fairly consistent across data series, with some deviations based on applied bias, location of ${ }^{57} \mathrm{Co}$ source (if present), charge trigger threshold, and loss of neutralization. Fig. 6.15 shows the spectra of three ${ }^{241}$ Am-only series, with net biases of $10 \mathrm{~V}, 50 \mathrm{~V}$, and $90 \mathrm{~V}$, respectively, taken with the same trigger threshold. Each series has been individually calibrated using the first 5 minutes of the data series (shown in the 

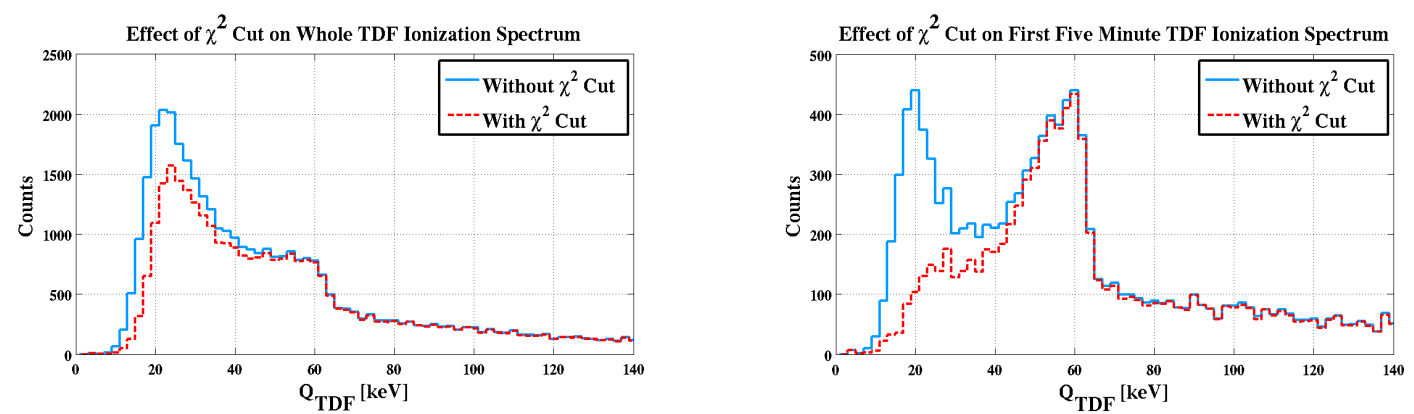

Figure 6.13: $Q_{T D F}$ spectrum with (red dashed) and without (blue solid) the $\chi^{2}$ cut applied, for (LEFT) the whole $10 \mathrm{~V}$ net bias data series and (RIGHT) the first five minutes of the same data series. The $\chi^{2}$ cut removes poorly-fit noise from the low energy region $(\sim 0-40 \mathrm{keV})$, while the $60 \mathrm{keV}{ }^{241} \mathrm{Am}$ peak is unaffected. Loss of neutralization results in the ${ }^{241} \mathrm{Am}$ peak falling into the low energy region after the first five minutes of data taking, making it difficult to distinguish from noise.

left plot), with quality cuts applied. The three spectra of the left plot have similar features, with slight differences in the $60 \mathrm{keV}$ peak resolution. The effects of bias on charge collection efficiency (i.e. absolute calibration values) and charge energy resolution are discussed in Section 6.3.6.

The right-side plot of Fig. 6.15 shows spectra constructed from the 5 - 10 minute region of the same three data series. The spectrum features of the $50 \mathrm{~V}$ and $90 \mathrm{~V}$ series match those seen in the first five minutes, with the ${ }^{241} \mathrm{Am}$ peaks still located around 60 $\mathrm{keV}$. The crystal shows no signs of neutralization loss in the $5-10$ minute region of the $50 \mathrm{~V}$ and $90 \mathrm{~V}$ data series. The $10 \mathrm{~V}$ spectrum demonstrates loss of neutralization in the 5 - 10 minute region, with the ${ }^{241} \mathrm{Am}$ peak falling to $\sim 30 \mathrm{keV}$. The effects of bias on neutralization are discussed in Section 6.3.7.

\subsubsection{Charge Collection Efficiency and Energy Resolution}

Performing Gaussian plus background fits to the $60 \mathrm{keV}$ peak yields the dependence of the charge signal amplitude distributions as a function of applied bias voltage. The relative charge collection efficiency is found by normalizing the maximum amplitude to one. 


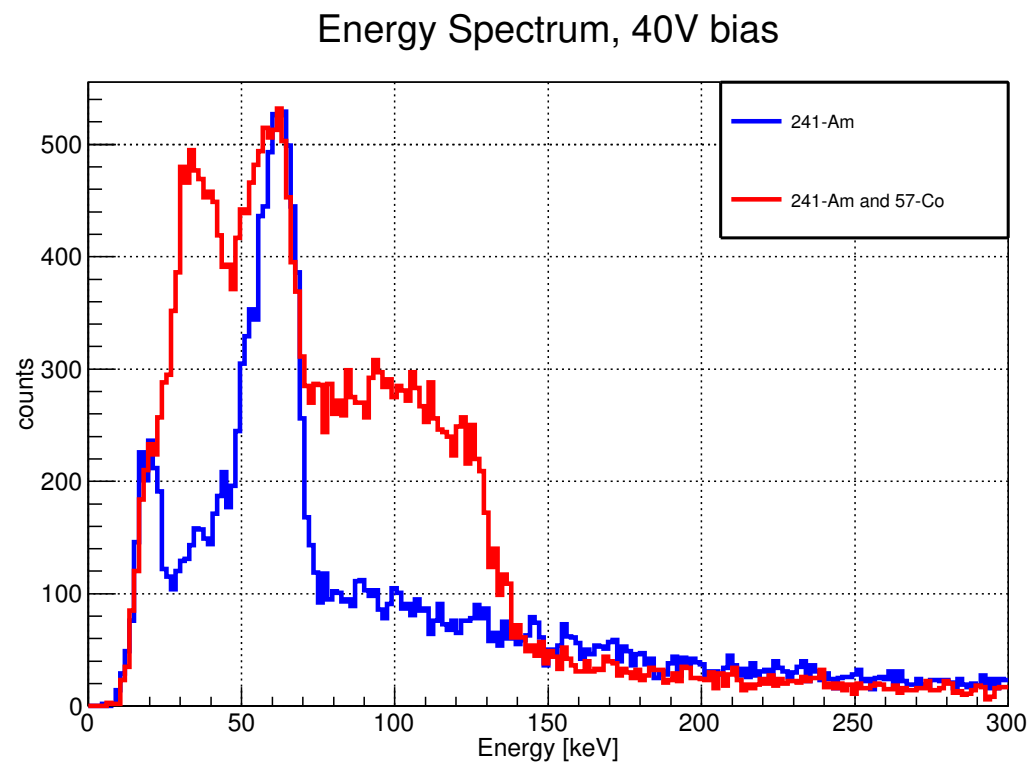

Figure 6.14: Energy spectra taken with an ${ }^{241} \mathrm{Am}$ source mounted near the detector and with (red) and without (blue) a ${ }^{57}$ Co source exterior to the cryostat. The $60 \mathrm{keV}$ peak of ${ }^{241} \mathrm{Am}$ and the $122 \mathrm{keV}$ edge of ${ }^{57} \mathrm{Co}$ as well as their Compton edges can be seen. Note that the ${ }^{57} \mathrm{Co}$ spectrum is smeared to low energies due to the interaction with intervening cryostat material. Figure courtesy of N. Mast. 

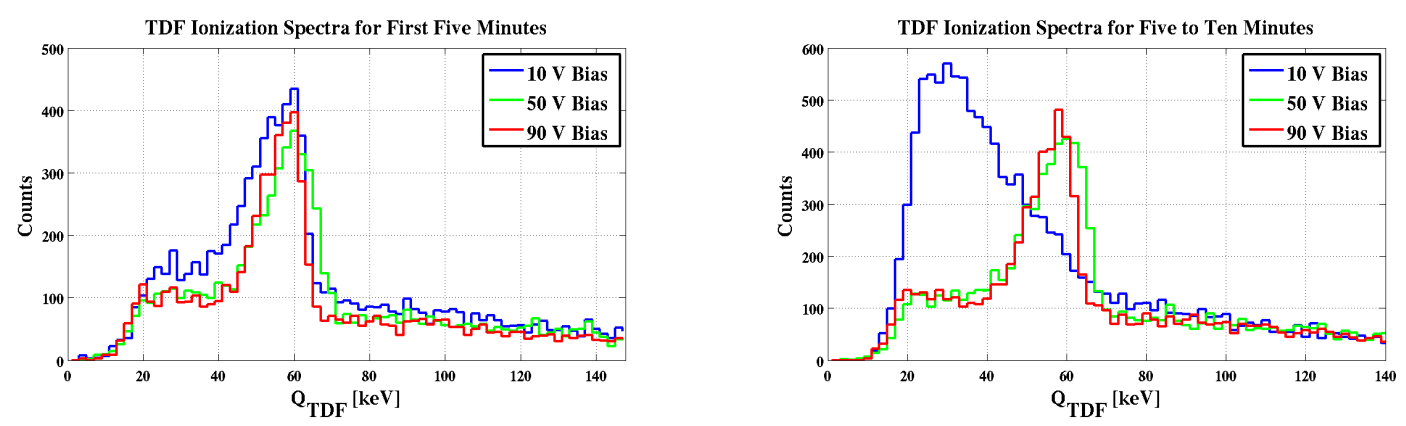

Figure 6.15: Individually calibrated $Q_{T D F}$ spectra constructed from the (LEFT) 0 - 5 minute region and (RIGHT) 5 - 10 minute region of a $10 \mathrm{~V}$ biased (blue), $50 \mathrm{~V}$ biased (green) and $90 \mathrm{~V}$ biased (red) data series. The 0 - 5 minute region spectra share similar features, with slight differences in the $60 \mathrm{keV}$ peak resolution. The $50 \mathrm{~V}$ and $90 \mathrm{~V}$ spectra constructed from the 5 - 10 minute region also share features from the first 5 minute spectra, showing no signs of loss of neutralization for these series. The 10 $\mathrm{V}$ spectrum demonstrates loss of neutralization in the 5 - 10 minute region, with the ${ }^{241}$ Am peak falling to $\sim 30 \mathrm{keV}$.

Results shown in Fig. 6.16, normalized relative to the efficiency at $-60 \mathrm{~V}(-18 \mathrm{~V} / \mathrm{cm})$, follow the seagull-shape present in $100 \mathrm{~mm}$ charge collection efficiency measurements. For a positive (negative) field, 90\% collection efficiency occurring at biases of $\sim 7 \mathrm{~V}$ $(\sim 7-24 \mathrm{~V})$, or electric fields of $\sim 2 \mathrm{~V} / \mathrm{cm}(\sim 2-7 \mathrm{~V} / \mathrm{cm})$. The lower population of measurements in the $[-6,0] \mathrm{V} / \mathrm{cm}$ region are suspected to have poor neutralization, but this has not yet been verified. Charge collection efficiency measurements of the contactfree detector is compared to a previous measurement of S10C, a $76 \mathrm{~mm}$ diameter, 2.54 cm thick Si ZIP detector studied in 2007 at Case Western Reserve University [226], which also fit the $60 \mathrm{keV}$ peak due to an ${ }^{241} \mathrm{Am}$ source on the charge collection side of the detector. S10C results have been normalized relative to the efficiency at -10 $\mathrm{V}(-4 \mathrm{~V} / \mathrm{cm})$. The contact-free detector results align nicely with the S10C measured charge collection efficiency, with both demonstrating $90 \%$ collection efficiency around $2 \mathrm{~V} / \mathrm{cm}$, indicating that larger Si crystals may be operated with similar ionization signal performance. Since the mean free path of $60 \mathrm{keV}$ photons in silicon is $\sim 1.3$ $\mathrm{cm}$, the events occur at a variety of depths in the crystal, not just near the surface. 


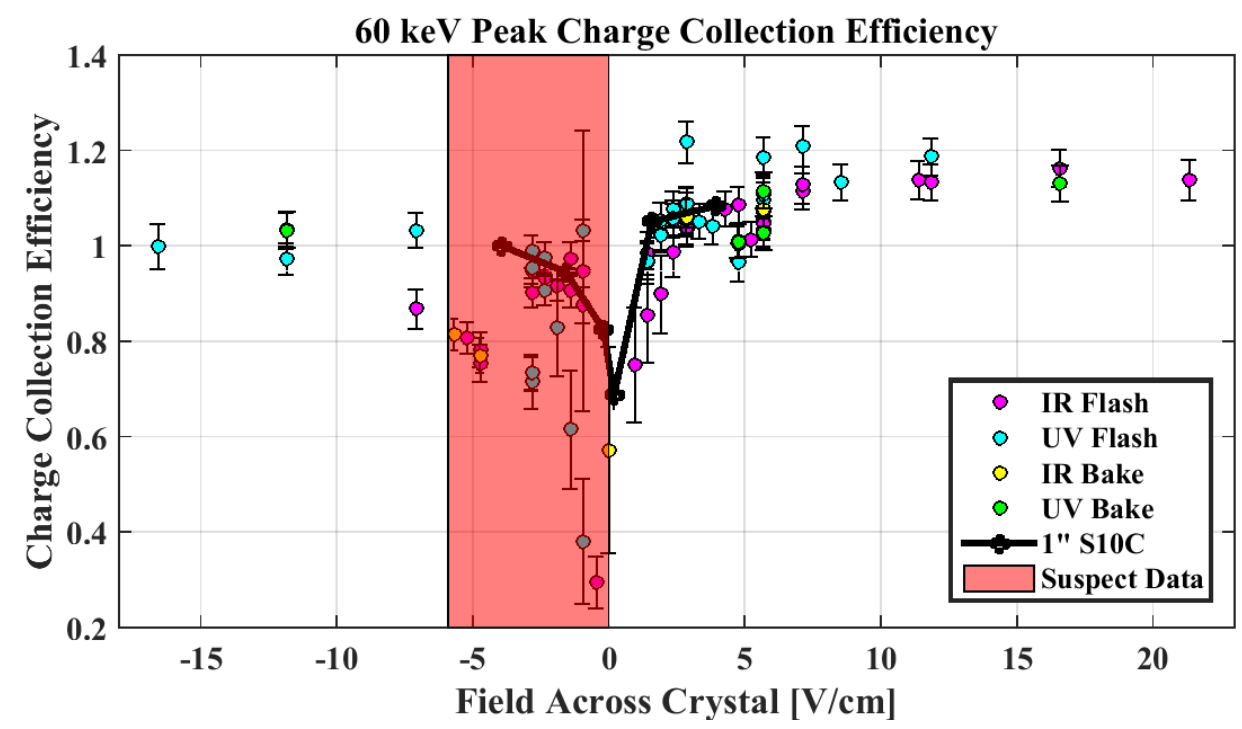

Figure 6.16: Charge collection efficiency as a function of applied bias. Results of broken down into categories of LED type used for most recent flashing or baking (IR flash in magenta, UV flash in cyan, IR bake in yellow, UV bake in green). Suspect data is highlighted in red. Contact-Free detector results are compared to a 2007 measurement of the $2.54 \mathrm{~cm}$ thick Si detector, S10C (black). 
This explains why the $150 \mathrm{~mm}$ Si charge collection efficiency more closely resembles the G106a collection efficiency of $356 \mathrm{keV}$ bulk events than the $60 \mathrm{keV}$ surface events, compared in Fig. 4.15 .

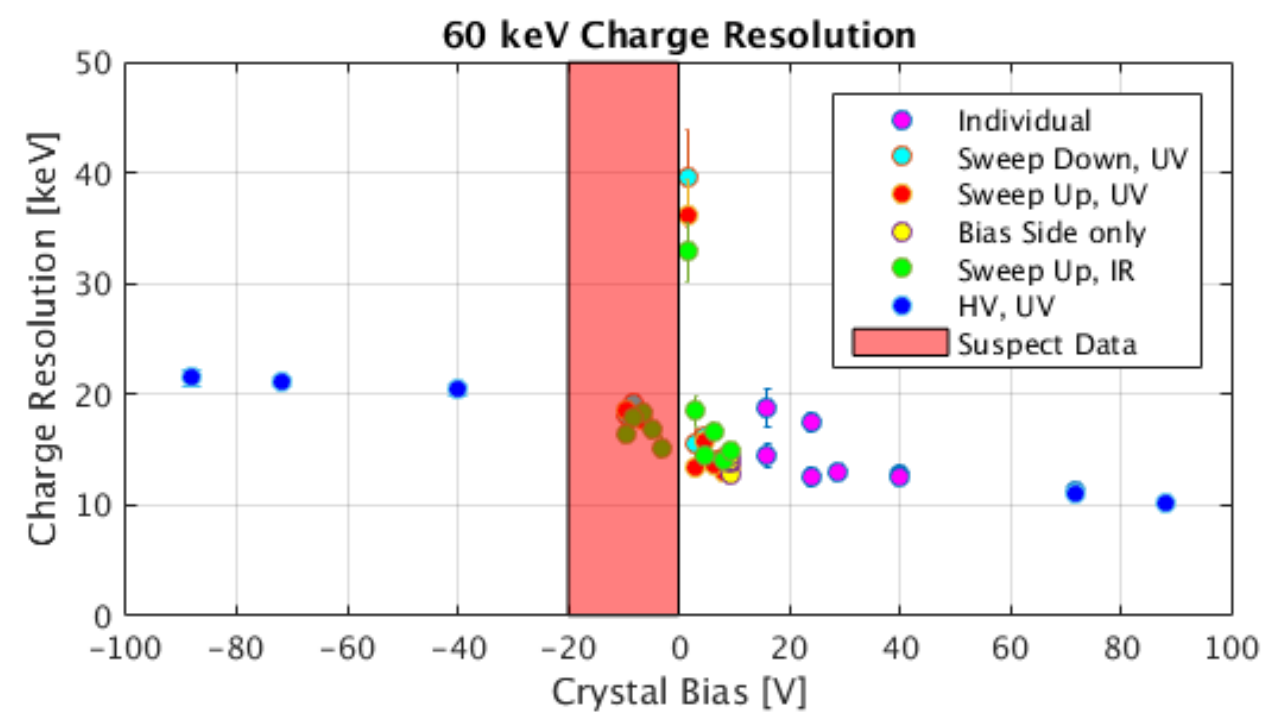

Figure 6.17: Charge energy resolution as a function of applied bias. Suspect data is highlighted in red. Figure courtesy of N. Mast.

The standard deviation of the fitted $60 \mathrm{keV}$ peaks are reported as the associated charge resolution for each corresponding bias. Above $\sim 3 \mathrm{~V}$, the charge resolution of this detector is $10-15 \mathrm{keV}$. The resolution suffers for negative biases due to the spectral asymmetry previously mentioned. It is unknown how significantly the $17 \mathrm{kHz}$ ringdown, or the time domain fit affect the resolution, if at all. Future studies must be conducted to determine the energy resolution of the $150 \mathrm{~mm}$ contact-free setup under more optimal circumstances.

Charge collection efficiency and energy resolution do not depend on the source of applied bias (DCRC, HV external supply, or both), nor do they depend on the type of flashing or baking (IR or UV LED) proceeding data taking. Although this behavior is expected, it provides further evidence that the detector is behaving as expected. 


\subsubsection{Neutralization}

Loss of neutralization can be measured quantitatively through the ${ }^{241} \mathrm{Am} 60 \mathrm{keV}$ peak hold time, that is the number of seconds a well-understood calibration peak holds stable before loss of neutralization causes reduced peak amplitude. The effect of this loss of neutralization of the crystal is shown in the charge signal amplitude versus time plot in Fig. 6.18, As discussed in G106a analysis, this is normal behavior for a detector, though it is expected that the detector can hold neutralization on time scales that allow for reasonable data collection ( $\sim 10$ minutes for the testing facility), and that flashing the detector restores neutralization. Recall that for typical CDMS detectors, the ionization electrodes are grounded and $940 \mathrm{~nm}$ IR LEDs illuminate the detector with a current of $0.6 \mathrm{~mA}$ in $100 \mu \mathrm{s}$ burst with $5 \mathrm{~ms}$ pauses [205]. After $6 \mathrm{~s}$ of flashing the detector is allowed to cool back to base temperature before data collection is resumed.

The charge signal versus time plot in Fig. 6.18 compares four consecutive 20 minute data series of the same bias $(40 \mathrm{~V})$, interspersed with 30 seconds of $0.6 \mathrm{~mA}$ IR (top) and $0.8 \mathrm{~mA} \mathrm{UV}$ (bottom) flashing. Both LED types show successful neutralization of the crystal with flashing, indicated by the ${ }^{241} \mathrm{Am} 60 \mathrm{keV}$ peak returning to its initial amplitude after the flash and cooldown period. The UV LED flashing resulted in a mean $60 \mathrm{keV}$ peak hold time of 435 seconds, with a 240 second reduction in hold time between the initial and subsequent data series, whereas the IR flashing resulted in a 60 $\mathrm{keV}$ peak hold time that remained consistently around 810 seconds. This suggest that the crystal is more neutralized after flashing with the IR LED (at $0.6 \mathrm{~mA}$ ) than with the UV LED (at $0.8 \mathrm{~mA}$ ). Perhaps higher current through the UV LED would improve its performance, but further UV LED testing was not possible within the time allotted for the initial contact-free detector run.

\section{Effects of Voltage Bias on Crystal Neutralization}

To study the effects of LEDs on crystal neutralization the ${ }^{241} \mathrm{Am} 60 \mathrm{keV}$ peak, and the ${ }^{57} \mathrm{Co} 122 \mathrm{keV}$ peak when possible, were fit incrementally over time with a Gaussian over an exponential background to look for peak decay. Due to incremental fitting, peak hold time quantities are accurate within \pm 60 seconds. Fig. 6.19 compares the hold time of the ${ }^{241} \mathrm{Am} 60 \mathrm{keV}$ peak to crystal bias. Since the external bias must be 

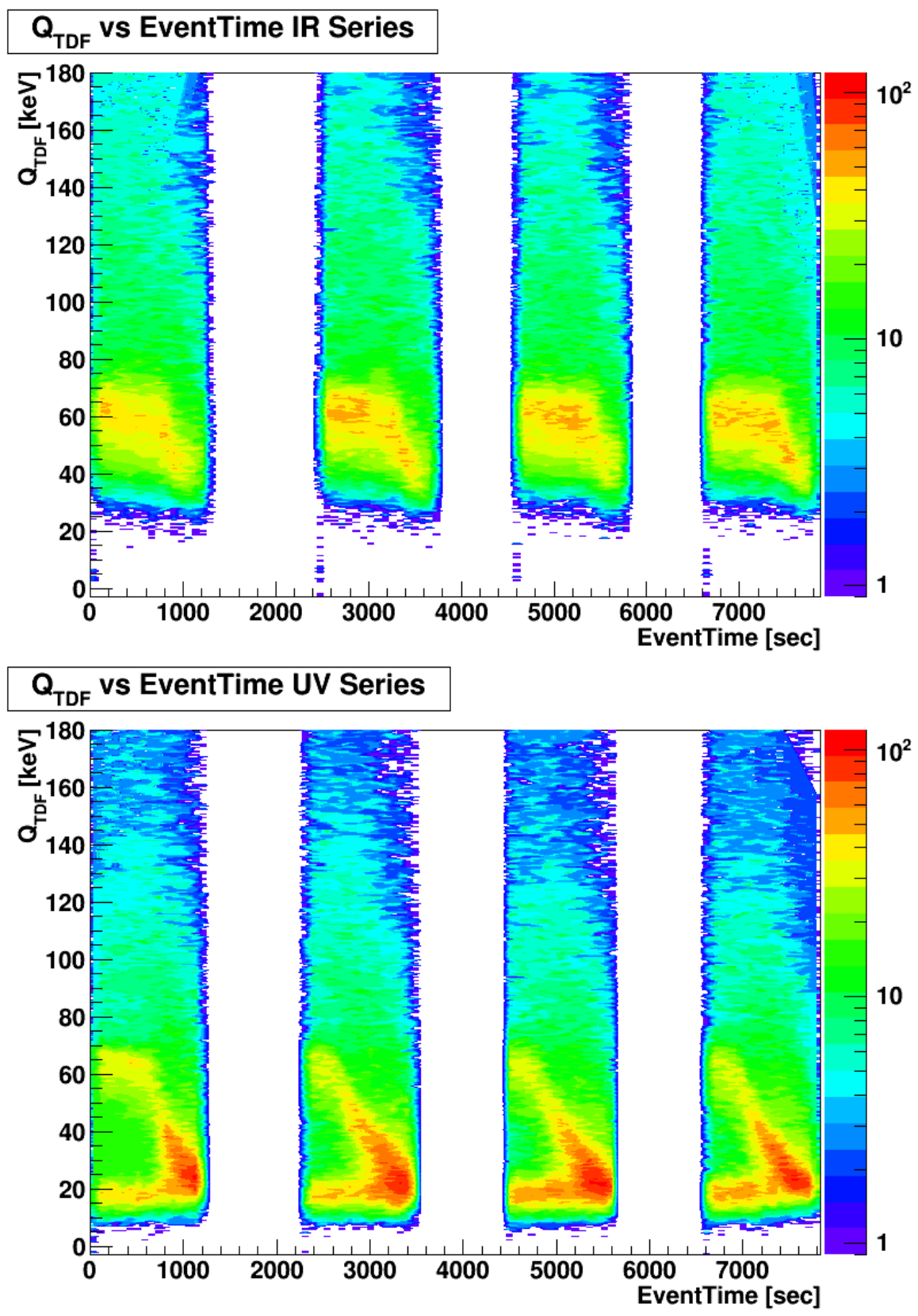

Figure 6.18: Charge neutralization after IR (TOP) and UV (BOTTOM) flashing. Both show successful neutralization of the crystal after flashing. Data from IR flashing shows ${ }^{241} \mathrm{Am} 60 \mathrm{keV}$ peak hold time consistent around 810 seconds, whereas UV flashing data has a mean peak hold time of 435 seconds. 
set by turning a dial, the exact time the detector was biased with the external supply before data taking, and the HV power supply 'charging' time (while the dial is being turned to the final value) are two significant sources of uncertainty that have not been accounted for. The external voltage supply was turned up slowly to avoid overwhelming the system; In most instances the external voltage was increased at a steady rate of 5 $10 \mathrm{~V} / \mathrm{s}$ until it reached its final value, and the detector was fully biased for $\sim 1$ minute before data taking began. Results suggest a roughly linear relationship between bias and peak hold time. Fig. 6.19 also suggests that flashing with the IR LED results in a longer hold time than with the UV LED for bias of $30 \mathrm{~V}$ or less.

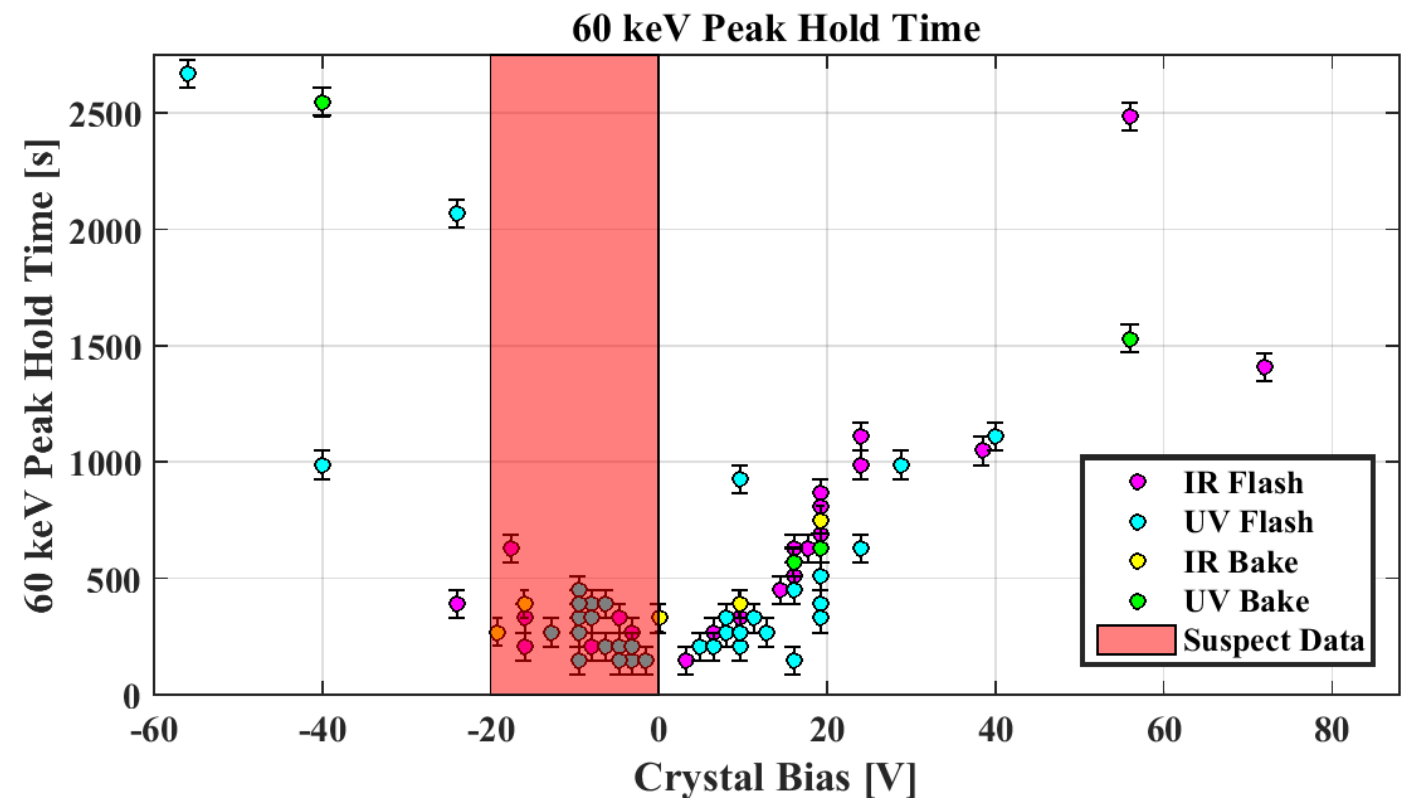

Figure 6.19: $60 \mathrm{keV}$ peak hold time measurements for bias voltages up to $\approx \pm 70$ Volts. Peak hold time increases linearly with bias, with some variation favoring IR LED flashing over UV LED flashing for increased hold time.

Overall, of the 118 ( 70 for $\pm \mathrm{V}, 48$ for $\mp \mathrm{V}$ ) data series with biases ranging from -110 $\mathrm{V}$ to $+110 \mathrm{~V}$ used for neutralization studies, $37(26 \pm \mathrm{V}, 11 \mp \mathrm{V})$ series had a $60 \mathrm{keV}$ peak hold time $\geq 10$ minutes limit set by the testing facility for sufficient neutralization. Although only $13(11 \pm \mathrm{V}, 2 \mp \mathrm{V})$ of the $92(55 \pm \mathrm{V}, 37 \mp \mathrm{V})$ series with a bias magnitude of $<30 \mathrm{~V}$ had sufficient peak hold time, $24(15 \pm \mathrm{V}, 9 \mp \mathrm{V})$ of the $26(15 \pm \mathrm{V}, 11 \mp \mathrm{V})$ 
series with a bias of $\geq 30 \mathrm{~V}$ had a peak hold time of 10 minutes or longer, with a maximum achieved hold time of over 40 minutes.

\section{$6.4150 \mathrm{~mm}$ Contact-Free Initial Run Conclusions and Next Steps}

The prototype contact-free detector designs with $150 \mathrm{~mm}$ diameter Si crystal show sufficient charge collection efficiency and crystal neutralization for operation as future generation dark matter detectors. Furthermore, low current leakage values indicate the potential for contact-free technology to increase detector sensitivity with high voltage use. Future steps call for the design, fabrication, and implementation of phonon sensors for use with electrodes, as well as developing multiple charge channels for positiondependent readout. As a next step, a $150 \mathrm{~mm}$ Si 5Q device has been fabricated for testing at the UMN test facility.

\subsection{1 $150 \mathrm{~mm}$ Si 5Q Device}

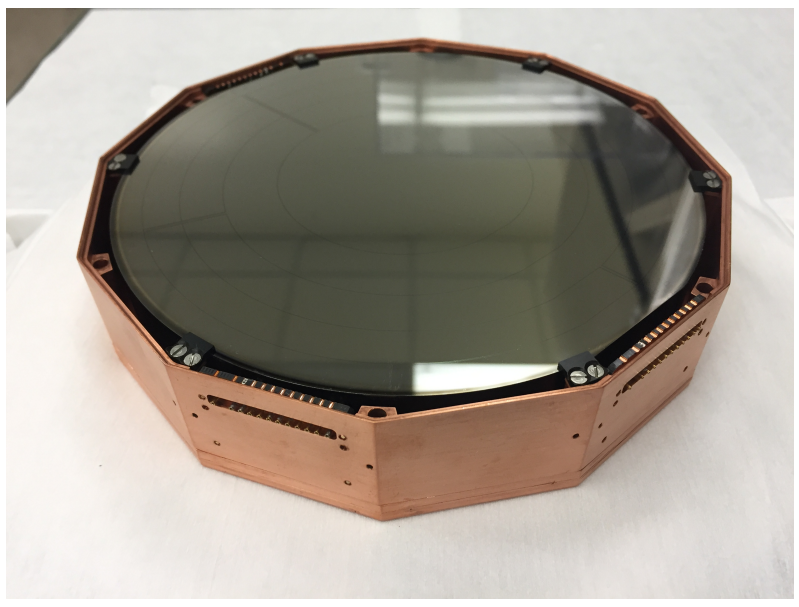

Figure 6.20: Prototype Si $150 \mathrm{~mm}$ $5 \mathrm{Q}$ device, to be tested at the UMN testing facility during Fall 2017. Although faint in this image, the $5 \mathrm{Q}$ mask can be seen on the crystal surface. The charge channels are read out through 3 DIBs on the $\mathrm{HPCu}$ housing.

A $150 \mathrm{~mm}$ (6") diameter, $25 \mathrm{~mm}$ (1") thick Si ionization detector with 5Q mask is planned for Fall 2017 testing at the UMN testing facility. The crystal will have five concentric electrodes on each face, with a contact-free electrode on the opposite side for grounding and applying voltage. The 5Q device, in its 3-DIB, OFHC Cu housing, is shown in Fig. 6.20. The contact-free bias electrode will be modified to hold four 
${ }^{241}$ Am sources, one over each channel, excluding the outermost channel. To avoid the $17 \mathrm{kHz}$ pulse-tail ringing caused by the flexible side coaxes, standard CDMS vacuum side coaxes will be used with extender plugs that are currently in development. Adaptor plate and $30 \mathrm{mK}$ can designed for initial $150 \mathrm{~mm}$ Si detector will be reused, as will the bias electrode, and electrode housing.

\subsection{Projected Sensitivity of $150 \mathrm{~mm}$ Si Detectors use at SuperCDMS SNOLAB}

Although years of research, development, and testing would be required before contactfree detectors comprised of $150 \mathrm{~mm}$ Si crystals could be utilized in the SuperCDMS SNOLAB main experiment, the sensitivity of such an experiment can be projected with a few reasonable assumptions.

The following section begins with an overview of calculating the maximum likelihood over a WIMP mass and coupling constant parameter space. The section then discusses the underlying assumptions required for the computational space in the Effective Field Theory (EFT) formalism [104 105] 227]. The section concludes by estimating sensitivities to different types of couplings of WIMPs to nucleons of a Si-based experiment at SNOLAB.

\subsubsection{Calculating Maximum Likelihood}

Maximum Likelihood calculations will begin with a Poisson likelihood function split into $n$ energy bins, in order to incorporate spectral information of the experiment exposure and expected background. A dark-matter only likelihood, $\mathcal{L}^{D M}$, is given by,

$$
\mathcal{L}^{D M}=\prod_{k=1}^{n} \frac{1}{N_{k} !} \eta_{k}^{N_{k}} e^{-\eta_{k}},
$$

where $N_{k}$ is number of detected events, and $\eta_{k}$ is the expected number of events, for the $k$ th energy bin. The expected number of events for a given energy bin depends on the chosen WIMP mass, $M_{\chi}$, and nonzero coupling coefficients, $c_{i}^{0}$ and $c_{i}^{1}$,

$$
\eta_{k}\left(\left\{M_{\chi}, c_{i}^{0}, c_{i}^{1}\right\}\right)=\int_{E_{k}} \frac{d R}{d E_{R}} d E_{R}
$$


The isoscalar and isovector coupling constants, $c_{i}^{0}$ and $c_{i}^{1}$, respectively, are the coupling coefficients for the $i$ th of eleven possible EFT interaction operators, as discussed in Section 1.4.3. The differential event rate, $\frac{d R}{d E_{R}}$, is given by Eq. 1.61 .

The likelihood function can be expanded to include any known energy-dependent backgrounds, with $b_{k}$ background events in the $k$ th bin. In this case, the likelihood function becomes,

$$
\mathcal{L}^{D M+b k g}=\prod_{k=1}^{n} \frac{1}{N_{k} !}\left(\eta_{k}+b_{k}\right)^{N_{k}} e^{-\left(\eta_{k}+b_{k}\right)},
$$

When considering all possible operators, the parameter space, $\left\{M_{\chi}, c_{i}^{0}, c_{i}^{1}\right\}$ contains 23 variables. To simplify matters, likelihoods can be explored over the three-dimensional subspaces which correspond to an individual operator. Bayes' Theorem can then be applied to the calculation, to determine which operators fit most consistently with the data. Given a hypothesis (operator), $H$, with a prior $\operatorname{Pr}(H)$ (the initial degree of confidence in $H$ ), the Bayesian evidence, $\mathcal{Z}$, is given by,

$$
\mathcal{Z}=\int d H \mathcal{L}\left(N_{k} \mid H\right) \operatorname{Pr}(H)
$$

where $\mathcal{L}\left(N_{k} \mid H\right)$ is the likelihood of obtaining data $N_{k}$, given $H$, and $\int d H$ is the integration over the parameter space of all parameters that make up $H$. Then the posterior probability distribution $\mathcal{P}\left(H \mid N_{k}\right)$, which describes the probability of $H$ accounting for acquired data $N_{k}$, is given by,

$$
\mathcal{P}\left(H \mid N_{k}\right)=\frac{\mathcal{L}\left(N_{k} \mid H\right) \operatorname{Pr}(H)}{\mathcal{Z}} .
$$

In the case of EFT operator probabilities, the Bayesian evidence of a single operator, $\mathcal{O}_{i}$, is calculated by,

$$
\mathcal{Z}_{i}=\int d M_{\chi} d c_{i}^{0} d c_{i}^{1} \mathcal{L}\left(N_{k} \mid\left\{M_{\chi}, c_{i}^{0}, c_{i}^{1}\right\}\right) \operatorname{Pr}\left(\left\{M_{\chi}, c_{i}^{0}, c_{i}^{1}\right\}\right),
$$

where a flat prior is assumed for each parameter. Operators with the highest Bayesian evidence are considered most consistent with the data, and most likely to have nonzero coupling coefficients.

Given the evidence for the EFT operators, the posterior probability distribution is,

$$
\mathcal{P}\left(\left\{M_{\chi}, c_{i}^{0}, c_{i}^{1}\right\} \mid N_{k}\right)=\frac{\mathcal{L}\left(N_{k} \mid\left\{M_{\chi}, c_{i}^{0}, c_{i}^{1}\right\}\right) \operatorname{Pr}\left(\left\{M_{\chi}, c_{i}^{0}, c_{i}^{1}\right\}\right)}{\mathcal{Z}} .
$$


More information on analyzing direct dark matter detection data within the whole EFT parameter space can be found in the work of H. E. Rogers et al. [227], and K. Shneck's thesis [228].

\section{Computational Likelihood Calculations}

Likelihood calculations are conducted using a collection of MATLAB functions called by a nested sampling Monte Carlo software package MULTINEST [229] 230] 231]. MULTINEST is a Bayesian inference tool, used to compare or select models, and estimate model parameters. MATLAB and MULTINEST scripts were modified from original code developed by H. E. Rogers [227], drawing from the work of R. Catena and P. Gondolo [232, and a similar Mathematica [233] package developed by N. Annand et al. [105]. Static parameters are set at the beginning of the calculation, including detector target material(s), total fiducial exposure (in $\mathrm{kg} \cdot$ days), the relevant energy region (in $\mathrm{keV}$ ) which may include energy-dependent cut efficiencies, an array of observed event count, and the number of background events. Known background spectra can also be included. MULTINEST scans the desired regions of WIMP mass, $M_{\chi}$, and nonzero coupling constants, $c_{1}^{0}$, and $c_{1}^{1}$, calculating the expected number of events using Eq. 6.2, for each energy bin in the selected region, and converging on regions of parameter space with non-zero likelihood.

In the case that a total (single energy bin) background estimate is given, all possible background configurations within the energy range are considered, provided that the total background event count remains within $2 \sigma$ of the original background estimate. The background configuration which results in the maximum likelihood is selected. However, this method is computational intensive; for background estimate of $B$ and number of energy bins, $n$, the number of possible background combinations is $(n+B-$ $1) ! /((n-1) ! B !)$. For $n=100$ bins, completing the computation in a practical amount of time requires the background $(\mathrm{B}+2 \sigma)$ must be 4.49 counts or less. Likelihoods can still be computed for expected backgrounds larger than 4.49, but a background spectrum must also be included to remain computationally feasible.

Whether the background, $b_{k}$ is determined via included spectrum or maximized likelihood, the signal plus background likelihood is calculated using Eq. $6.3 \mathrm{~b}^{2}$ for the

\footnotetext{
${ }^{2}$ Likelihood with a total background $\mathrm{B}$ and error $\sigma$ will have an additional factor of
} 
3D parameter space of each individual operator. From each operator's 3D likelihood, the Bayesian evidence is calculated using Eq. 6.6, to determine which operators or operator combinations best fit the provided data, and therefore which best represent the dark matter interaction. 3D likelihoods can be marginalized down to multiple 2D or 1D likelihoods, making them easier to display graphically. The range for each flat prior used in Bayesian evidence calculations are determined from calculations of relative operator strength by H. E. Rogers [234]. From the likelihoods $2 \sigma$ sensitivities can be found for the parameters of a given operator, demonstrating the sensitivity capabilities of the $150 \mathrm{~mm}$ diameter Si detectors.

\subsubsection{Assumptions}

Several educated assumptions must be made to calculate a reasonable maximum likelihood for SuperCDMS-SNOLAB filled with $150 \mathrm{~mm}$ Si detectors. When assumptions must be made, best-case scenarios are selected within reason.

Assumptions can be broken down into two categories: exposure and background. Assumptions relating to exposure include the total number of detectors used in the experiment, the energy-dependent efficiency of the detectors, and the live time to be considered. The background is simply the expected number of background events, given the detector characteristics and live time.

\section{Fiducial Volume, Efficiency, and Live Time}

Current SuperCDMS-SNOLAB designs include an innermost (base-temperature) can with inner diameter of 36.25 " and inner height of 16.31 ", with a capacity to hold up to 31 towers of six $100 \mathrm{~mm}$ iZIPs each. With detector housing heights of $\sim 1.3$ ", detectors are not filling all of the vertical space within the can, a portion of which contains detector connections to the towers These are reasonable designs for the present, but near-future designs in which such connections to the towers require only a small portion of the inner can space are not inconceivable. In this case, it is possible to fill the inner can volume with a 21 across by 12 deep arrangement of $150 \mathrm{~mm}$ Si detectors in their OFHC $\mathrm{Cu}$ housing, as shown in Fig. 6.21. Since the $150 \mathrm{~mm}$ housing is comprised of twelve $1 / \sqrt{2 \pi \sigma} e^{-\left(\sum_{k}^{n} b_{k}-B\right)^{2} / 2 \sigma^{2}}$ applied. 
sides, it is also conceivable that each detector readout cable could pass information down from one side of the housing, to avoid overlapping readout electronics. This filled can arrangement would allow for $252 \mathrm{Si}$ detectors, with a combined mass of $342 \mathrm{~kg}$. Comparatively, current SNOLAB capacity would allow for a maximum of 186 detectors, or $255 \mathrm{~kg}$ maximum combined mass, in the case that only $100 \mathrm{~mm}$ Ge iZIPs are used.
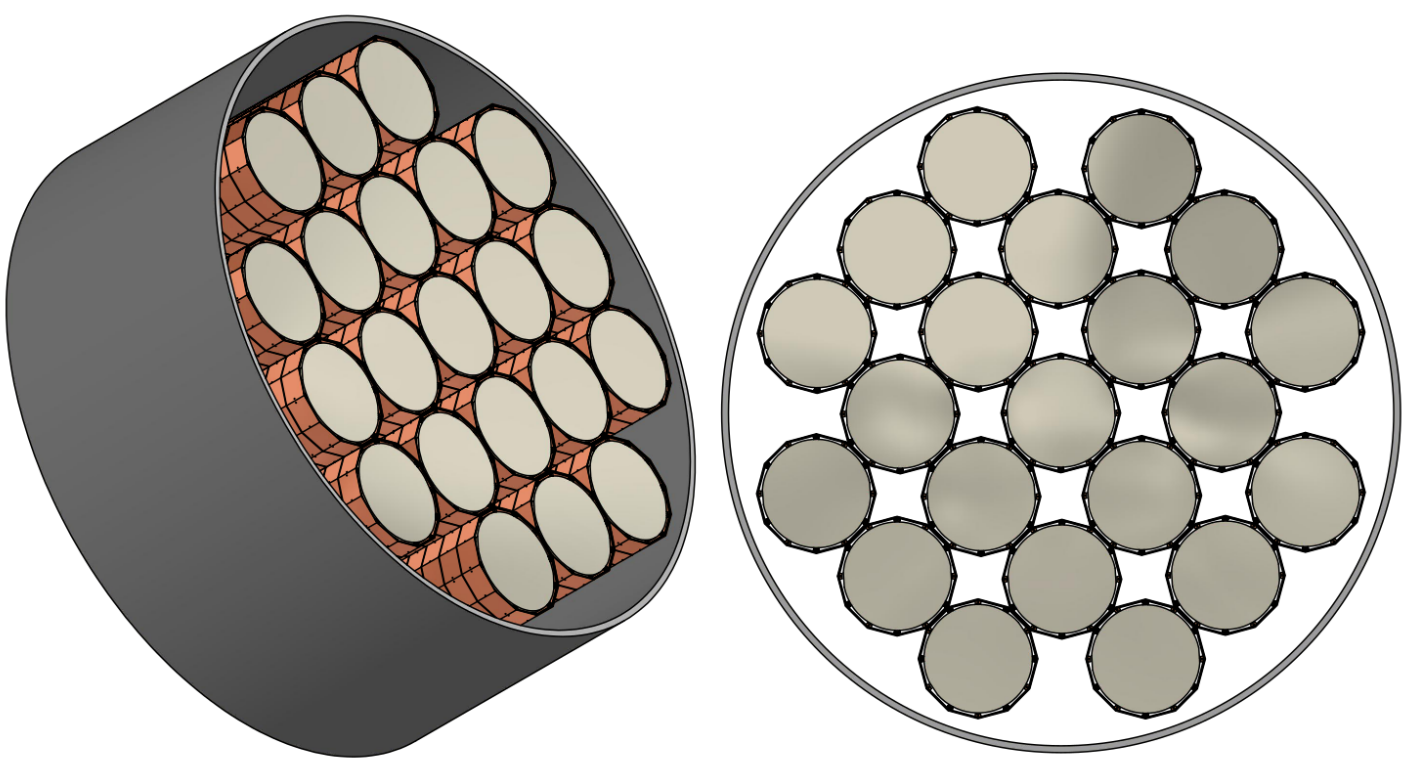

Figure 6.21: Drawing of a side and top view of the 36.25 " inner diameter, 16.3125 " tall SNOLAB mixing chamber can (grey), filled with a 21 across by 12 deep arrangement of $150 \mathrm{~mm}$ diameter Si detector (light grey), in OFHC Cu housings (copper).

The fiducial volume of each detector depends on the detector type. As outlined in the G106a analysis, a radial partition cut and surface event cut are applied to iZIPs to ensure that only bulk events are considered in final analysis. Surface events cuts remove events that occur within $\sim 1 \mathrm{~mm}$ of the detector face, and the outer ring of iZIP electrodes covers the outer $\sim 7 \mathrm{~mm}$ radius of the crystal. Of the $583.2 \mathrm{~cm}^{3}$ detector volume, roughly $77 \%$ or $450.3 \mathrm{~cm}^{3}$ remains after these cuts are applied.

Detector efficiencies will also vary based on detector type. Cut efficiencies on SuperCDMS data resemble step functions, with a near constant value for all energies within an energy region above the energy threshold. Energy thresholds will be limited by the 
projected energy thresholds of the initial SuperCDMS-SNOLAB detectors, based on the current understanding of prototype $100 \mathrm{~mm}$ detectors. For iZIP Si detectors, the threshold is set to $166 \mathrm{eV}$ [135]. Additionally, the iZIP expected background, shown in Fig. 6.22, is large for recoil energies below a few $\mathrm{keV}$, due to the diminished NR discrimination of yield at those energies. To account for this, the lower iZIP energy limit will be set to $5 \mathrm{keV}$. The energy region of interest is also detector type dependent. For this analysis, the energy region of iZIPs will extend up to $100 \mathrm{keV}$.

In addition to the fiducial volume cuts, an ionization yield cut will be applied to the iZIP data. To model this, the efficiency will be set to 0.50 , a conservative efficiency approximation which combines yield, surface, and radial fiducial volume cuts [235]. Projected SuperCDMS SNOLAB sensitivity studies place the expected iZIP overall cut efficiency at 0.75 [135], but the previously stated, more cautious efficiency estimate will be used for this study. Therefore, the efficiency for this study will be modeled as step functions, with a value of 0.50 within the energy range $5 \mathrm{keV}-100 \mathrm{keV}$.

The final assumption that goes into the total exposure value is live time. For this study, a live time of 365 days is selected to represent a reasonable run time with the detectors installed and operating at SNOLAB. Therefore the total exposure is given by, Exposure $=$ Total mass $\times$ Efficiency $\times$ Live time, or,

$$
\text { Exposure }=62,589 \mathrm{~kg} \cdot \text { days, } 5 \mathrm{keV}-100 \mathrm{keV} \text {. }
$$

\section{Expected Background}

Background event rates observed by the $150 \mathrm{~mm}$ Si detectors are assumed to resemble the expected event rate of $100 \mathrm{~mm}$ Si SNOLAB detectors. SNOLAB event rates are determined from Monte Carlo simulations of background spectra, including contributions from:

- ${ }^{3} \mathrm{H}$, a spallation product from high-energy cosmic-ray secondaries interacting with crystal nuclei. ${ }^{3} \mathrm{He}$ emits $\beta$-particles with an end-point energy of $18.6 \mathrm{keV}$.

- ${ }^{32} \mathrm{Si}$, a spallation product from high-energy cosmic-ray secondaries interacting with argon in the atmosphere, which then travels to the surface via aqueous transport. Concentration of ${ }^{32} \mathrm{Si}$ in detectors depends on source and location or crystal material. ${ }^{32} \mathrm{Si}$ emits $\beta$-particles with an end-point energy of $224 \mathrm{keV}$. 
- Compton scatters from gamma rays, produced from cavern environment materials via ${ }^{238} \mathrm{U}$ and ${ }^{232} \mathrm{Th}$ decay chains, and ${ }^{40} \mathrm{~K}$ decay, and leaking through experiment shielding.

- Surface betas, the result of daughter isotopes of decaying ${ }^{210} \mathrm{~Pb}$ surface contamination, caused by exposure of the detectors and housing materials to ${ }^{222} \mathrm{Rn}$ and its progeny during fabrication, testing and installation.

- Surface ${ }^{206} \mathrm{~Pb}$ (part of the ${ }^{210} \mathrm{~Pb}$ decay chain) recoils incident on a detector face with sufficient energy to potentially create a NR in the dark matter signal region.

- Neutrons, produced via spontaneous fission of ${ }^{238} \mathrm{U}$ and $(\alpha, n)$ reactions in the rock due to $\alpha$-emitting isotopes within the ${ }^{238} \mathrm{U}$ and ${ }^{232} \mathrm{Th}$ decay chains.

- Coherent elastic neutrino-nucleus scattering ( $\mathrm{CE} \nu \mathrm{NS})$ off detector nuclei, with the primary solar neutrino background produced by the decay of ${ }^{8} \mathrm{~B}$ at the end of the pp-III solar fusion reaction chain.

Assuming $150 \mathrm{~mm}$ Si detectors and housing will undergo similar fabrication, testing, and installation procedures as SNOLAB detectors, all discussed backgrounds will be present in the hypothetical $150 \mathrm{~mm}$ Si experiment. Projected backgrounds are stated in rates of [events $\left./ \mathrm{kg} \cdot \mathrm{yr} \cdot \mathrm{keV}_{n}\right]$, and thus can be scaled up with increased detector mass. Projected backgrounds caused by cosmogenic or cavern sources will not be significantly affected by switching to the $150 \mathrm{~mm}$ detectors. Material contamination sources, particularly background sources which depend on line-of-sight, such as the ${ }^{210} \mathrm{~Pb}$ decay products, may be affected by the change of detector and housing geometry. However, it is assumed that these effects will not be significant, and this study will use the same background model used for the SuperCDMS SNOLAB experiment.

To model the effect of applying the analysis cuts to the data, each background spectrum is multiplied with the appropriate energy-dependent cut-passing efficiency as determined in [235]. Analysis cuts not only include the fiducial volume cuts already outlined, but an additional iZIP-only yield cut that rejects bulk ER events, reducing bulk ER backgrounds by a factor of up to $\sim 10^{6}$ in iZIPs. Fig. 6.22 shows these simulated spectra before and after fiducial cuts are applied. 

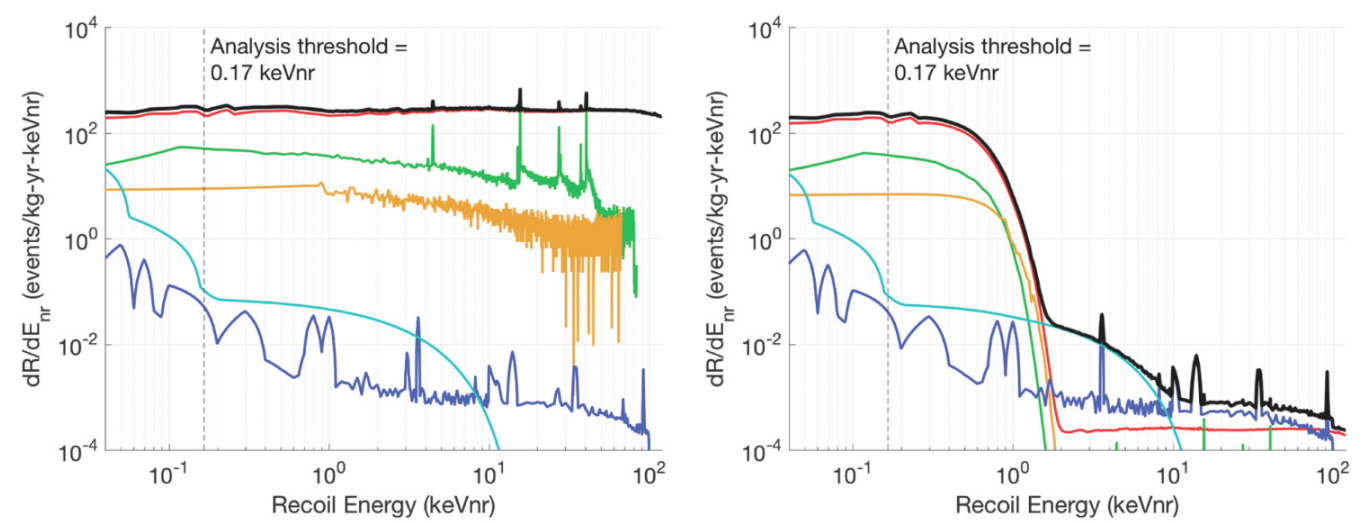

Figure 6.22: Background spectra, before (LEFT) and after (RIGHT) analysis cuts for Si iZIP, shown as a function of nuclear recoil energy $\left(\mathrm{keV}_{n r}\right)$. Thick black lines represent the total background rates. Electron recoils from Compton gamma rays, ${ }^{3} \mathrm{H}$, and ${ }^{32} \mathrm{Si}$ are grouped together (red), and other components include surface betas (green), surface ${ }^{206} \mathrm{~Pb}$ recoils (orange), neutrons (blue), and $\mathrm{CE} \nu \mathrm{NS}$ (cyan). Figure courtesy of [135].

Total background event rates are determined by integrating the spectra over the energy ranges of interest. Background estimates are then scaled by the relative difference between projected SNOLAB efficiencies, and $150 \mathrm{~mm}$ detector efficiencies used in this study. Given the fiducial mass $(252.3 \mathrm{~kg})$ and energy range of the Si iZIP detector, the expected background is 19 events, or $\sim 0.08$ events per detector, in one year of observation.

\section{Simulated Data}

The observed data used for likelihood calculations, $N_{k}$, must be simulated. Since a projected exclusion sensitivity is desired, simulated data will contain only background events, without any signal events. Since background spectra are available, and the expected background event rate is known, observed data can be drawn randomly from a probability distribution of the expected background spectra within the energy region of interest $(5-100 \mathrm{keV})$, until a randomly assigned energy is acquired for each of the expected background events. The expected background and simulated "observed" data are shown in Fig. 6.23. 


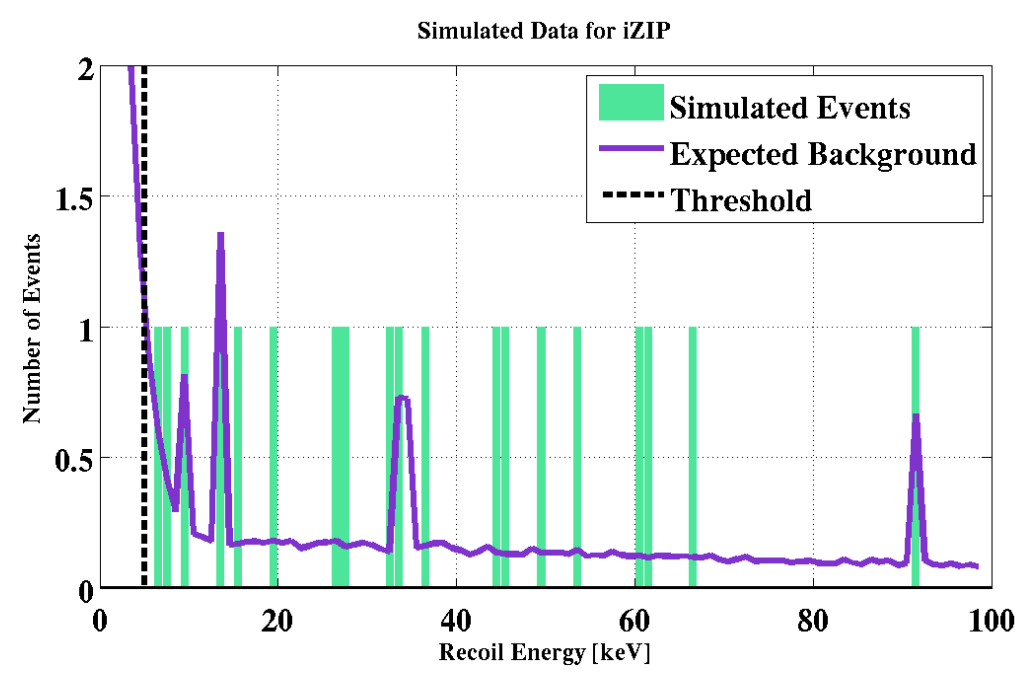

Figure 6.23: Expected background spectrum (purple solid), energy threshold (dotted black), and simulated events (green) for $150 \mathrm{~mm}$ Si iZIP likelihood calculation results.

\subsubsection{Results}

The 3D likelihoods can be marginalized into 2D parameter space likelihoods by integrating over one of the parameters. The resulting $2 \mathrm{D}$ marginalized likelihoods are presented in Fig. 6.24. For all operators except Operator 1, priors are set such that the maximum coupling coefficient results in 50 expected signal events for a WIMP mass of $5 \mathrm{GeV} / \mathrm{c}^{2}$, assuming all other coefficients are zero. With this prior range, calculations for Operator 1 were unable to successfully complete for unknown reasons, and thus the prior range was increased to such that the maximum coefficient results in 500 signal events for $M_{\chi}=5 \mathrm{GeV} / \mathrm{c}^{2}$, in order to sidestep this issue.

For all operators, a band of high-likelihood is present in the low WIMP mass region $\left(<5 \mathrm{GeV} / \mathrm{c}^{2}\right)$, representing a parameter space region to which the experiment is not sensitive. Aside from this shared feature, operators fall into two categories of behavior. Operators 4, 6, 7, 9, and 10 show evidence of destructive interference between isoscalar and isovector terms for coupling coefficient ratios of $c_{i}^{0} / c_{i}^{1} \sim 1$, as indicated by the bands of high likelihood in the $c_{i}^{1}$ versus $c_{i}^{0}$ plots in Fig. 6.24. Due to this interference, which causes low signal event rates regardless of WIMP mass, the coupling versus mass 2D likelihoods of these operators show no significant constraint on coupling coefficient, 
aside from requiring a positive $c_{i}^{1}$ value.

The remaining operators $(1,3,5,8$, and 11$)$, do not show significant interference between isoscalar and isovector coupling, but instead show increased likelihood for weak couplings in the $M \chi>5 \mathrm{GeV} / \mathrm{c}^{2}$ parameter space region. This is expected, since the simulated data is well explained by the expected background without any significant dark matter signal. In general, these results are in agreement with those presented by H. E. Rogers et al. [227, though a different range of parameter space is explored here.

In addition to $3 \mathrm{D}$ Likelihood calculations, a 2D likelihood calculation was done for the spin-independent WIMP-neutron interaction coefficient, $c_{1}^{n}$, related to the spinindependent isoscalar and isovector coefficients by,

$$
c_{1}^{n}=\frac{1}{2}\left(c_{1}^{0}-c_{1}^{1}\right) .
$$

Oftentimes this parameter space is instead expressed in terms of the WIMP-neutron spin-independent cross section. Given an Operator 1 coupling coefficient, $c_{1}^{\tau}$, the corresponding cross section is given by,

$$
\sigma_{1}^{\tau}=\frac{\left(A m_{N}\right)^{2}}{4 \pi\langle V\rangle^{4}(1+A)^{2}}\left(c_{1}^{\tau}\right)^{2},
$$

where $\mathrm{A}$ is the number of nucleons in the target material, and $\langle V\rangle=246.2 \mathrm{GeV}$ is the Higgs vacuum expectation value, which is used in this capacity to represent the electroweak scale and define dimensionless coefficients. The EFT-calculated projected sensitivity, is shown in Fig. 6.25, in terms of the WIMP mass and spin-independent cross section, $\sigma_{1}^{n}$. The $150 \mathrm{~mm}$ Si iZIP projected sensitivity probes spin-independent cross sections roughly an order of magnitude lower than that of SuperCDMS SNOLAB $100 \mathrm{~mm}$ Si iZIPs in the $\sim 1-5 \mathrm{GeV} / \mathrm{c}^{2}$ mass region. The sharp cutoff below $1 \mathrm{Gev} / \mathrm{c}^{2}$ is likely due to the step function behavior of the efficiency used for this prediction. 

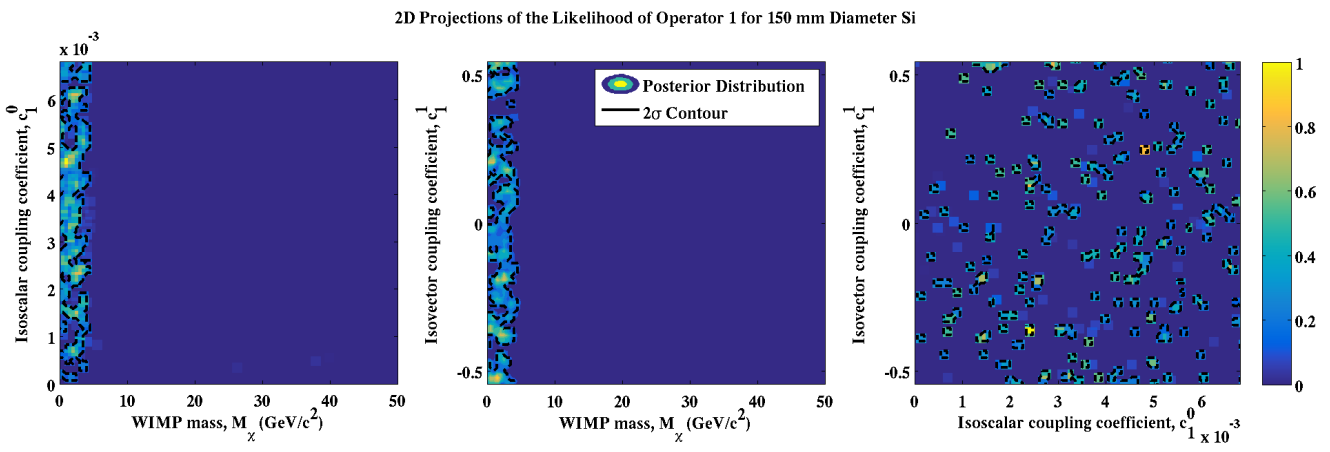

2D Projections of the Likelihood of Operator 3 for $150 \mathrm{~mm}$ Diameter Si
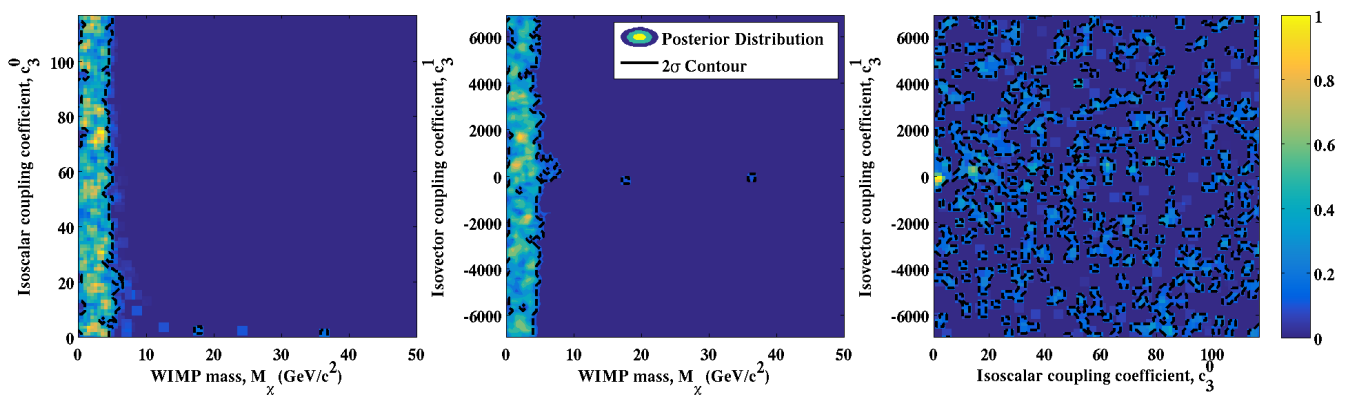

2D Projections of the Likelihood of Operator 4 for $150 \mathrm{~mm}$ Diameter Si
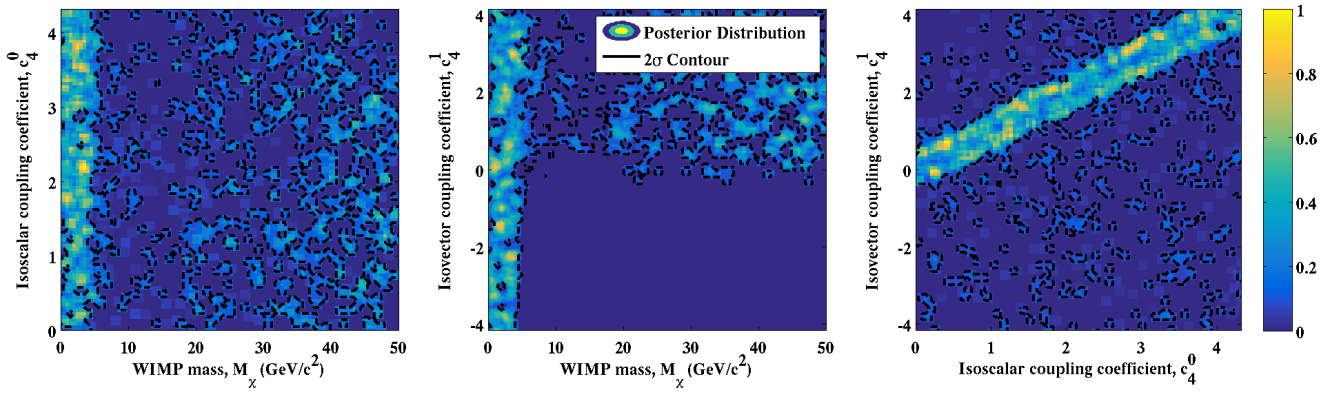

2D Projections of the Likelihood of Operator 5 for $150 \mathrm{~mm}$ Diameter $\mathrm{Si}$
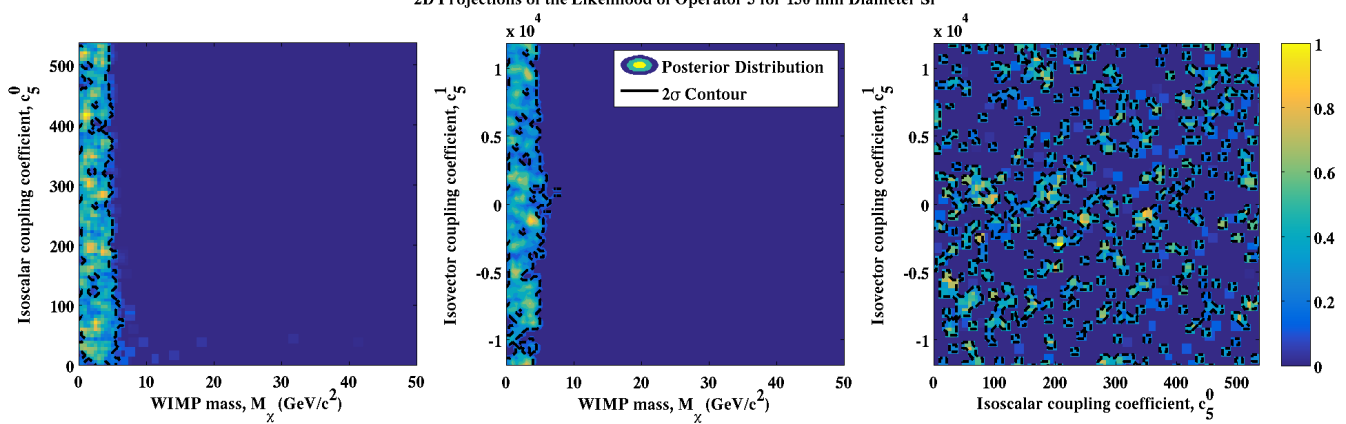

Figure 6.24: 2D marginalized likelihoods for $150 \mathrm{~mm}$ Si iZIP detectors with $2 \sigma$ contours, for Operators 1, 3, 4, and 5. Continued on next page. 

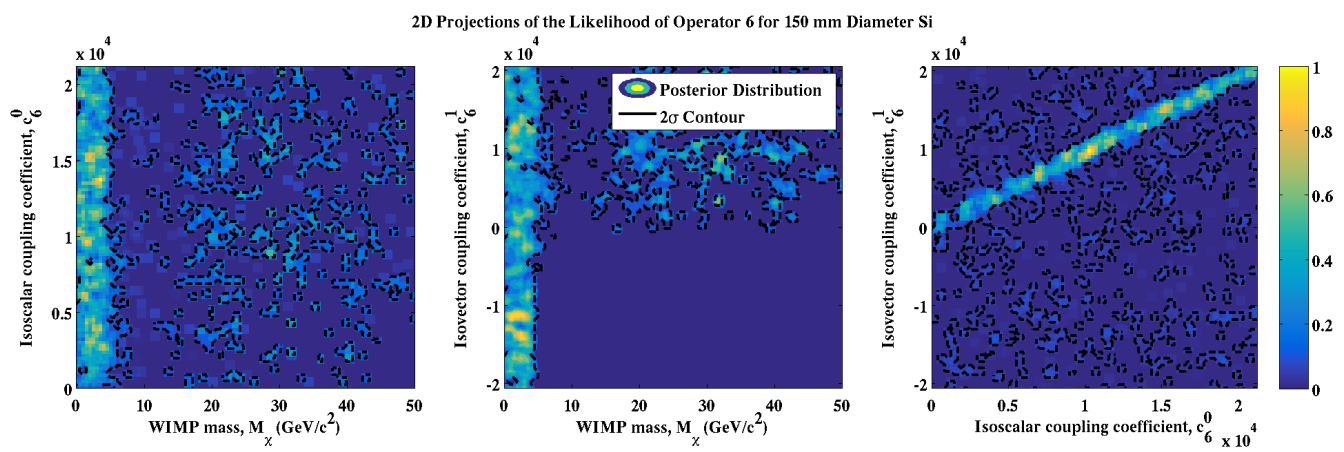

2D Projections of the Likelihood of Operator 7 for $150 \mathrm{~mm}$ Diameter Si
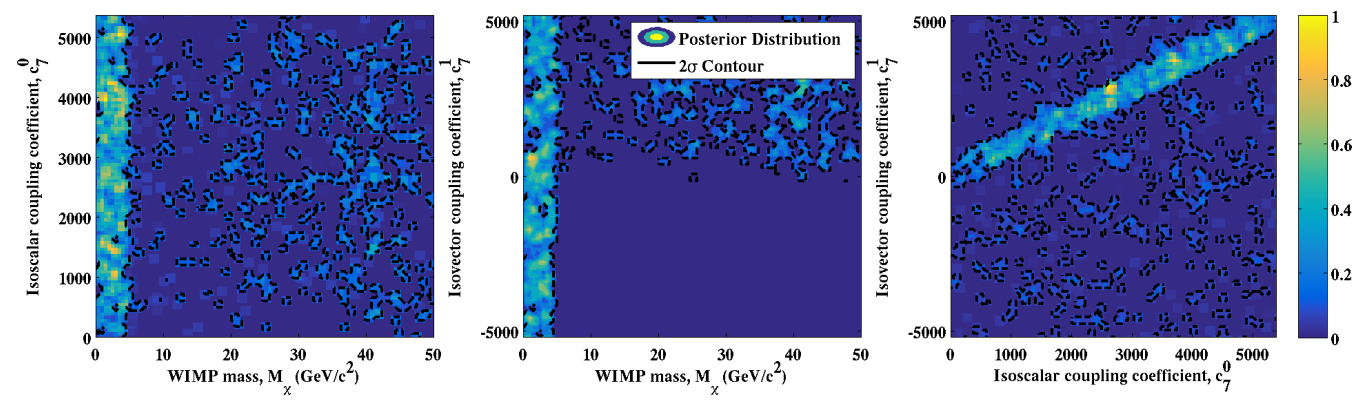

2D Projections of the Likelihood of Operator 8 for $150 \mathrm{~mm}$ Diameter Si
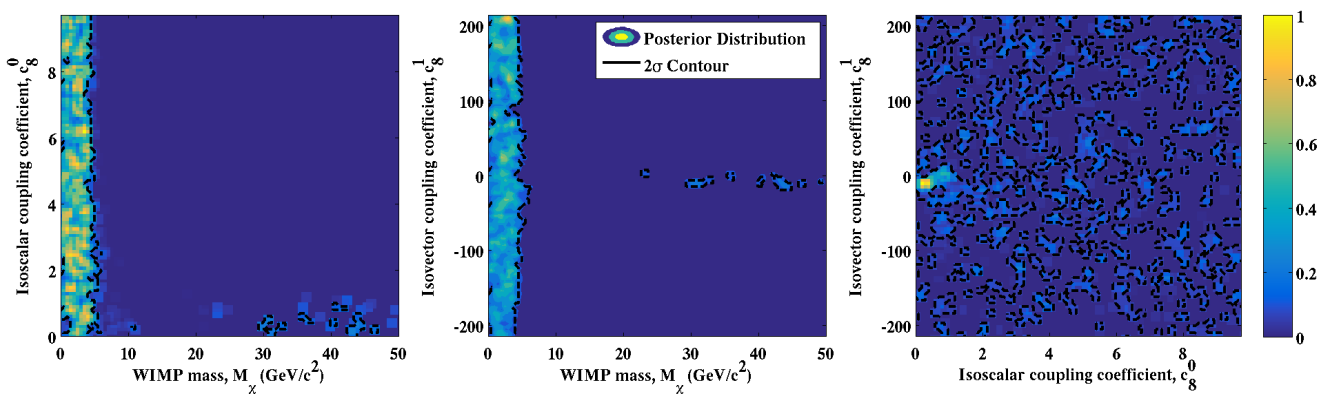

2D Projections of the Likelihood of Operator 9 for $150 \mathrm{~mm}$ Diameter Si
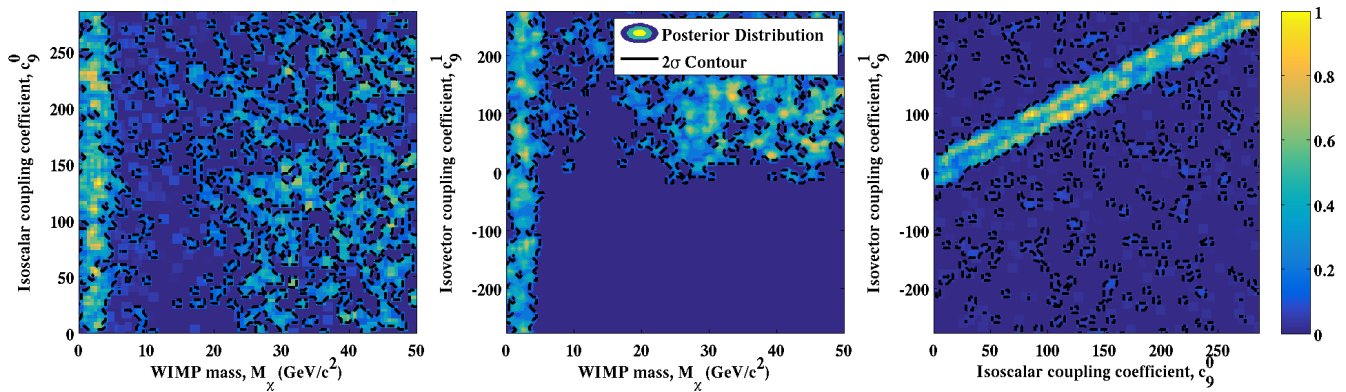

Figure 6.24: Continued from previous page. 2D marginalized likelihoods for $150 \mathrm{~mm} \mathrm{Si}$ iZIP detectors with $2 \sigma$ contours, for Operators 6-9. Continued on next page. 
2D Projections of the Likelihood of Operator 10 for $150 \mathrm{~mm}$ Diameter $\mathrm{Si}$
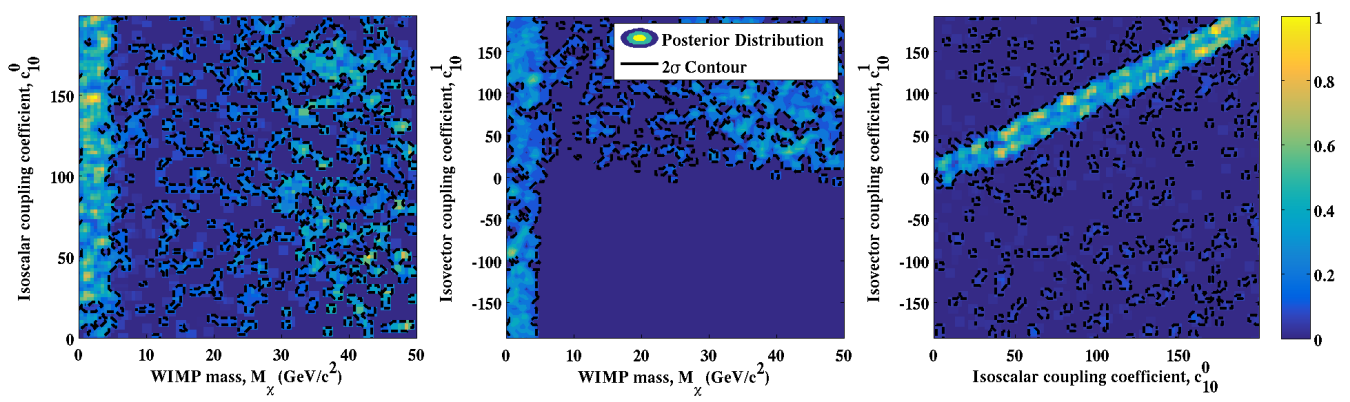

2D Projections of the Likelihood of Operator 11 for $150 \mathrm{~mm}$ Diameter Si
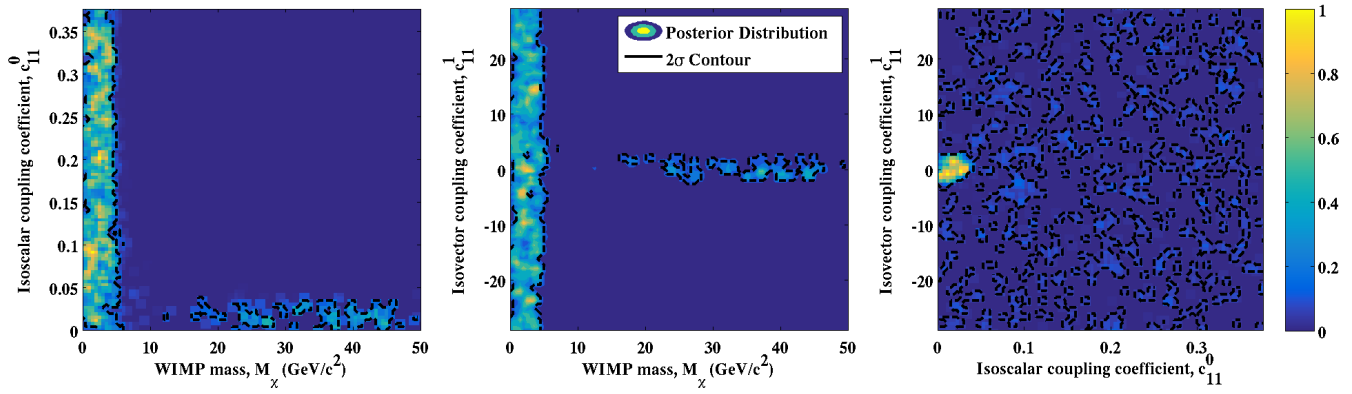

Figure 6.24: Continued from previous page. 2D marginalized likelihoods for $150 \mathrm{~mm} \mathrm{Si}$ iZIP detectors with $2 \sigma$ contours, for Operators 10 and 11 . 


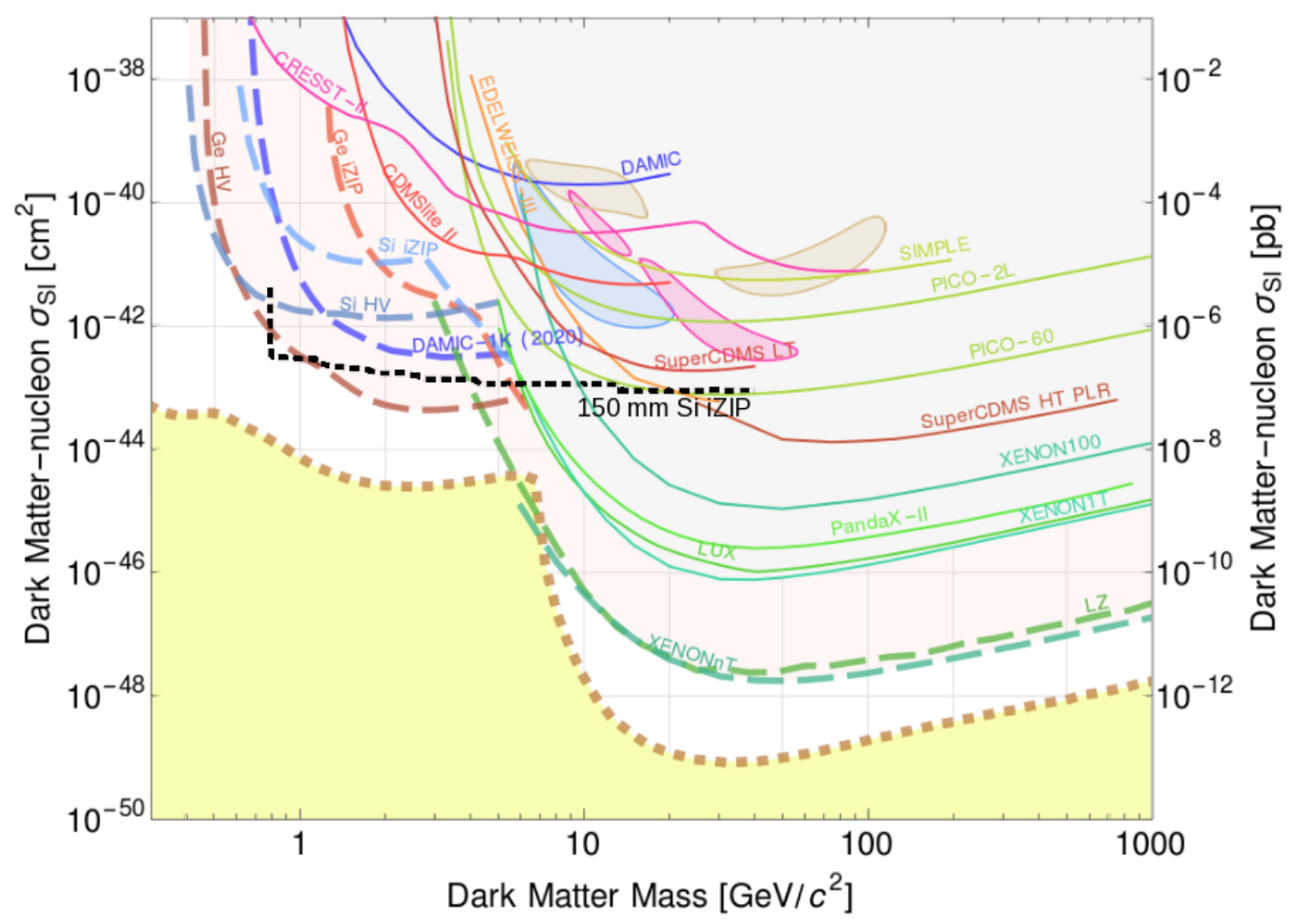

Figure 6.25: EFT-calculated projected sensitivities for SuperCDMS SNOLAB cryostat filled with $150 \mathrm{~mm}$ diameter Si detectors (black dotted), compared to current experimental limits (solid lines), projected sensitivities (dashed lines), and candidate regions (shaded) on spin-independent cross sections as a function of WIMP mass for various experiments. For full list of limits and projected sensitivity sources, see Fig. 1.13 


\section{Chapter 7}

\section{Conclusion and Future Outlook}

The future of dark matter detection depends on a global effort, with various experimental groups worldwide probing their own regions of the vast parameter space: crystalbased experiments such as SuperCDMS, CRESST, and DAMA/LIBRA, and liquidnoble detectors, such as XENON1T and DEAP-3600, continue pushing sensitivities to lower cross sections at the extremes WIMP mass parameter space. Continuing down

this path of progress requires constant evolution, from the technology and scope of the experiments, to the underlying theory that drives them. From a newly discovered phenomenon with no known source in the 1930's, the theory behind dark matter has exploded into a broad horizon of possibilities. As experimental data catches up to proposed theory, either in support or dismissal, alternatives and new options must be at the ready. Historically pursued potential solutions, such as Modified Newtonian Dynamics, MACHOs, and light neutrinos, have added to academic discourse and understanding, even if they could not stand up to scientific scrutiny as stand-alone explanations. Current pursuits of Axions, Asymmetric Dark Matter, and Supersymmetric WIMP candidates continue to push the envelope in the realm beyond the standard model. Whether they reveal the nature of dark matter or not, these avenues of approach will certainly prove fruitful to the Astrophysics community.

To proceed down their own path of progress, the SuperCDMS collaboration must continue combining novel ideas with the persistence to see them to fruition. This modus operandi has been apparent since the group's inception, with the implementation of the transition edge sensor, and it continues at the forefront of CDMS research. Interleaved 
sensors for recoil discrimination, position dependence mitigation through the NSOF, increased low-mass WIMP sensitivity from applied high voltage, and multidimensional EFT analysis of experimental results are only a few examples of the progress that occurs when creative thinking meets indomitable spirit. This approach to problem solving will only become more crucial as SuperCDMS SNOLAB shifts from fabrication and assembly to operation and eventual data taking. As presented in this thesis, prototype versions of the $100 \mathrm{~mm}$ diameter iZIPs intended for use at SNOLAB have demonstrated sufficient charge collection efficiency, neutralization and surface event rejection for operation in the experiment, but there is still much to be done. Identifying, understanding, and reducing backgrounds and contaminants; maintaining consistent, low-noise electronics and dilution unit operation; and ensuring quality across new detectors are only a few of the tasks at hand. Some support in this undertaking comes from the Cryogenic Underground TEst facility (CUTE), which will provide underground (and thus low background and low contamination) $100 \mathrm{~mm}$ detector characterization and background studies at SNOLAB.

As SuperCDMS SNOLAB moves forward with CUTE, the UMN testing facility must evolve beyond $100 \mathrm{~mm}$ detector characterization, to more $\mathrm{R} \& \mathrm{D}$ projects. This evolution has already begun with the $150 \mathrm{~mm}$ Si contact-free device testing, and will continue with the $150 \mathrm{~mm}$ Si 5Q device. Tentative plans are underway to test other novel prototype detectors, further down the time line; such detectors include $>100 \mathrm{~mm}$ diameter $\mathrm{Ge}$ crystals in development at University of South Dakota, as well as 3 - 4" diameter bonded Si wafer detectors fabricated at TAMU. The UMN test facility can also utilize current $100 \mathrm{~mm}$ SuperCDMS detectors for conducting new science measurements. Initial data has already been taken and analysis is ongoing for a study of NR's following thermal neutron capture, with external coincidence triggering on the escaping capture gammas by NaI detectors. The goal of the study is to precisely measure $100-700 \mathrm{eV}$ nuclear recoils in Ge, aiding future sub-GeV WIMP dark matter detectors by providing insight into the a potential thermal neutron background and offering detector calibrations near a $1 \mathrm{GeV}$ dark matter mass. UMN data with a ${ }^{88} \mathrm{Y}$ source has also been taken for future Multiple Interaction Surface Scatter (MISS) studies. MISS events are ER events which scatter on both surfaces, leading to suppressed yield in the NR range, and symmetric charge signals. The studies seek to understand the expected MISS background and 
possibly develop effective MISS event discrimination techniques that can be applied to SuperCDMS SNOLAB data. By continuing studies which probe the sensitivities and backgrounds of current detectors, and by aiding in the development of future generation dark matter detectors, the UMN testing facility, as part of the larger SuperCDMS collaboration, can continue to shed light on the dark matter problem. 


\section{References}

[1] F. Zwicky, Helv. Phys. Acta 6, 110 (1933).

[2] V. C. Rubin, N. Thonnard and W. K. Ford, Jr., Astrophys. J. 238, 471 (1980).

[3] Y. Sofue and V. Rubin, Ann. Rev. Astron. Astrophys. 39, 137 (2001) [astro$\mathrm{ph} / 0010594]$.

[4] Weinberg, Steven. Cosmology. Oxford: Oxford UP, 2008. Print.

[5] B. Fields and S. Sarkar, astro-ph/0601514.

[6] R. H. Cyburt, B. D. Fields, K. A. Olive and T. H. Yeh, Rev. Mod. Phys. 88, 015004 (2016) doi:10.1103/RevModPhys.88.015004 [arXiv:1505.01076 [astro-ph.CO]].

[7] K. A. Olive et al. [Particle Data Group], Chin. Phys. C 38, 090001 (2014). doi:10.1088/1674-1137/38/9/090001

[8] M. Fukugita and P. J. E. Peebles, Astrophys. J. 616, 643 (2004) doi:10.1086/425155 [astro-ph/0406095].

[9] P. A. R. Ade et al. [Planck Collaboration], Astron. Astrophys. 594, A13 (2016) doi:10.1051/0004-6361/201525830 [arXiv:1502.01589 [astro-ph.CO]].

[10] C. L. Bennett et al. [WMAP Collaboration], arXiv:1212.5225 [astro-ph.CO].

[11] G. Hinshaw et al. [WMAP Collaboration], arXiv:1212.5226 [astro-ph.CO].

[12] D. Scott and G. F. Smoot, arXiv:1005.0555 [astro-ph.CO]. 
[13] G. Bertone, D. Hooper and J. Silk, Phys. Rept. 405, 279 (2005) [arXiv:hep$\mathrm{ph} / 0404175]$.

[14] R. Gavazzi, T. Treu, J. D. Rhodes, L. V. Koopmans, A. S. Bolton, S. Burles, R. Massey and L. A. Moustakas, Astrophys. J. 667, 176 (2007) [astro-ph/0701589].

[15] R. Massey, T. Kitching and J. Richard, Rept. Prog. Phys. 73, 086901 (2010) [arXiv:1001.1739 [astro-ph.CO]].

[16] M. Markevitch, A. H. Gonzalez, D. Clowe, A. Vikhlinin, L. David, W. Forman, C. Jones and S. Murray et al., Astrophys. J. 606, 819 (2004) [astro-ph/0309303].

[17] M. Milgrom, Astrophys. J. 270, 365 (1983). doi:10.1086/161130

[18] R. H. Sanders and E. Noordermeer, Mon. Not. Roy. Astron. Soc. 379, 702 (2007) doi:10.1111/j.1365-2966.2007.11981.x [astro-ph/0703352 [ASTRO-PH]].

[19] G. W. Angus, B. Famaey and D. A. Buote, Mon. Not. Roy. Astron. Soc. 387, 1470 (2008) doi:10.1111/j.1365-2966.2008.13353.x [arXiv:0709.0108 [astro-ph]].

[20] P. Tisserand et al. [EROS-2 Collaboration], Astron. Astrophys. 469, 387 (2007) [astro-ph/0607207].

[21] T. Harada, C. M. Yoo and K. Kohri, Phys. Rev. D 88, no. 8, 084051 (2013) Erratum: [Phys. Rev. D 89, no. 2, 029903 (2014)] doi:10.1103/PhysRevD.88.084051, 10.1103/PhysRevD.89.029903 [arXiv:1309.4201 [astro-ph.CO]].

[22] B. P. Abbott et al. [LIGO Scientific and Virgo Collaborations], Phys. Rev. Lett. 116, no. 6, 061102 (2016) doi:10.1103/PhysRevLett.116.061102 [arXiv:1602.03837 $[\mathrm{gr}-\mathrm{qc}]]$.

[23] B. Carr, F. Kuhnel and M. Sandstad, Phys. Rev. D 94, no. 8, 083504 (2016) doi:10.1103/PhysRevD.94.083504 [arXiv:1607.06077 [astro-ph.CO]].

[24] P. Sikivie, arXiv:1210.0040 [astro-ph.CO].

[25] G. Raffelt, Nucl. Phys. Proc. Suppl. 72, 43 (1999) doi:10.1016/S09205632(98)00501-5 [hep-ph/9805400]. 
[26] K. van Bibber and G. Carosi, arXiv:1304.7803 [physics.ins-det].

[27] S. J. Asztalos et al. [ADMX Collaboration], Phys. Rev. Lett. 104, 041301 (2010) doi:10.1103/PhysRevLett.104.041301 [arXiv:0910.5914 [astro-ph.CO]].

[28] B. M. Brubaker et al., Phys. Rev. Lett. 118, no. 6, 061302 (2017) doi:10.1103/PhysRevLett.118.061302 [arXiv:1610.02580 [astro-ph.CO]].

[29] L. J. Rosenberg, Proc. Nat. Acad. Sci. (2015). doi:10.1073/pnas.1308788112

[30] K. C. Y. Ng, S. Horiuchi, J. M. Gaskins, M. Smith and R. Preece, Phys. Rev. D 92, no. 4, 043503 (2015) doi:10.1103/PhysRevD.92.043503 [arXiv:1504.04027 [astroph.CO]].

[31] A. Boyarsky, O. Ruchayskiy and M. Shaposhnikov, Ann. Rev. Nucl. Part. Sci. 59, 191 (2009) doi:10.1146/annurev.nucl.010909.083654 [arXiv:0901.0011 [hep-ph]].

[32] M. Drees and G. Gerbier, arXiv:1204.2373 [hep-ph].

[33] C. Munoz, EPJ Web Conf. 13, 60100 (2017) doi:10.1051/epjconf/201713601002 [arXiv:1701.05259 [hep-ph]].

[34] H. Baer, V. Barger and A. Mustafayev, JHEP 1205, 091 (2012) doi:10.1007/JHEP05(2012)091 [arXiv:1202.4038 [hep-ph]].

[35] J. R. Ellis, T. Falk, K. A. Olive and M. Srednicki, Astropart. Phys. 13, 181 (2000) Erratum: [Astropart. Phys. 15, 413 (2001)] doi:10.1016/S0927-6505(99)00104-8 [hep-ph/9905481].

[36] V. Khotilovich, R. L. Arnowitt, B. Dutta and T. Kamon, Phys. Lett. B 618, 182 (2005) doi:10.1016/j.physletb.2005.04.078 [hep-ph/0503165].

[37] J. R. Ellis, T. Falk, G. Ganis, K. A. Olive and M. Srednicki, Phys. Lett. B 510, 236 (2001) doi:10.1016/S0370-2693(01)00541-X [hep-ph/0102098].

[38] A. Djouadi, M. Drees and J. L. Kneur, Phys. Lett. B 624, 60 (2005) doi:10.1016/j.physletb.2005.08.010 [hep-ph/0504090]. 
[39] J. L. Feng, K. T. Matchev and T. Moroi, Phys. Rev. Lett. 84, 2322 (2000) doi:10.1103/PhysRevLett.84.2322 [hep-ph/9908309].

[40] J. R. Ellis, K. A. Olive and Y. Santoso, Astropart. Phys. 18, 395 (2003) doi:10.1016/S0927-6505(02)00151-2 [hep-ph/0112113].

[41] L. Ackerman, M. R. Buckley, S. M. Carroll and M. Kamionkowski, Phys. Rev. D 79, 023519 (2009) [arXiv:0810.5126 [hep-ph]].

[42] D. Hooper, N. Weiner and W. Xue, Phys. Rev. D 86, 056009 (2012) doi:10.1103/PhysRevD.86.056009 [arXiv:1206.2929 [hep-ph]].

[43] J. Kumar, PoS ICHEP 2010, 438 (2010) [arXiv:1012.0078 [hep-ph]].

[44] J. L. Feng and J. Kumar, Phys. Rev. Lett. 101, 231301 (2008) doi:10.1103/PhysRevLett.101.231301 [arXiv:0803.4196 [hep-ph]].

[45] K. Petraki and R. R. Volkas, Int. J. Mod. Phys. A 28, 1330028 (2013) doi:10.1142/S0217751X13300287 [arXiv:1305.4939 [hep-ph]].

[46] D. E. Kaplan, M. A. Luty and K. M. Zurek, Phys. Rev. D 79, 115016 (2009) doi:10.1103/PhysRevD.79.115016 [arXiv:0901.4117 [hep-ph]].

[47] N. Nagata, K. A. Olive and J. Zheng, JCAP 1702, no. 02, 016 (2017) doi:10.1088/1475-7516/2017/02/016 [arXiv:1611.04693 [hep-ph]].

[48] M. Ibe, S. Matsumoto and T. T. Yanagida, Phys. Lett. B 708, 112 (2012) doi:10.1016/j.physletb.2012.01.032 [arXiv:1110.5452 [hep-ph]].

[49] K. M. Zurek, Phys. Rept. 537, 91 (2014) doi:10.1016/j.physrep.2013.12.001 [arXiv:1308.0338 [hep-ph]]. https://arxiv.org/abs/1308.0338

[50] M. Nojiri et al., IPMU News J.5, 4 (2009)

[51] G. Aad et al. [ATLAS Collaboration], JHEP 1409, 176 (2014) doi:10.1007/JHEP09(2014)176 [arXiv:1405.7875 [hep-ex]].

[52] S. Chatrchyan et al. [CMS Collaboration], JHEP 1406, 055 (2014) doi:10.1007/JHEP06(2014)055 [arXiv:1402.4770 [hep-ex]]. 
[53] K. A. Olive, PoS PLANCK 2015, 093 (2015) [arXiv:1510.06412 [hep-ph]].

[54] G. Aad et al. [ATLAS Collaboration], Phys. Lett. B 710, 67 (2012) doi:10.1016/j.physletb.2012.02.051 [arXiv:1109.6572 [hep-ex]].

[55] S. Chatrchyan et al. [CMS Collaboration], Phys. Rev. Lett. 107, 221804 (2011) doi:10.1103/PhysRevLett.107.221804 [arXiv:1109.2352 [hep-ex]].

[56] K. A. Olive, PoS DSU 2015, 035 (2016) [arXiv:1604.07336 [hep-ph]].

[57] E. Aprile et al. [XENON100 Collaboration], Phys. Rev. Lett. 109, 181301 (2012) doi:10.1103/PhysRevLett.109.181301 [arXiv:1207.5988 [astro-ph.CO]].

[58] D. S. Akerib et al. [LUX Collaboration], Phys. Rev. Lett. 112, 091303 (2014) doi:10.1103/PhysRevLett.112.091303 [arXiv:1310.8214 [astro-ph.CO]].

[59] D. C. Malling et al., arXiv:1110.0103 [astro-ph.IM].

[60] N. Fornengo, Adv. Space Res. 41, 2010 (2008) [arXiv:astro-ph/0612786].

[61] M. Ackermann et al. [Fermi-LAT Collaboration], JCAP 1509, no. 09, 008 (2015) doi:10.1088/1475-7516/2015/09/008 [arXiv:1501.05464 [astro-ph.CO]].

[62] A. Albert et al. [Fermi-LAT and DES Collaborations], Astrophys. J. 834, no. 2, 110 (2017) doi:10.3847/1538-4357/834/2/110 [arXiv:1611.03184 [astro-ph.HE]].

[63] H. Abdallah et al. [H.E.S.S. Collaboration], Phys. Rev. Lett. 117, no. 11, 111301 (2016) doi:10.1103/PhysRevLett.117.111301 [arXiv:1607.08142 [astro-ph.HE]].

[64] A. Abramowski et al. [H.E.S.S. Collaboration], Phys. Rev. D 90, 112012 (2014) doi:10.1103/PhysRevD.90.112012 [arXiv:1410.2589 [astro-ph.HE]].

[65] H. Abdalla et al. [H.E.S.S. Collaboration], Phys. Rev. Lett. 117, no. 15, 151302 (2016) doi:10.1103/PhysRevLett.117.151302 [arXiv:1609.08091 [astro-ph.HE]].

[66] C. Weniger, JCAP 1208, 007 (2012) doi:10.1088/1475-7516/2012/08/007 [arXiv:1204.2797 [hep-ph]].

[67] A. Abramowski et al. [H.E.S.S. Collaboration], Phys. Rev. Lett. 110, 041301 (2013) doi:10.1103/PhysRevLett.110.041301 [arXiv:1301.1173 [astro-ph.HE]]. 
[68] M. Ackermann et al. [Fermi-LAT Collaboration], Phys. Rev. D 91, no. 12, 122002 (2015) doi:10.1103/PhysRevD.91.122002 [arXiv:1506.00013 [astro-ph.HE]].

[69] M. G. Aartsen et al. [IceCube Collaboration], arXiv:1705.08103 [hep-ex].

[70] M. G. Aartsen et al. [IceCube Collaboration], Eur. Phys. J. C 76, no. 10, 531 (2016) doi:10.1140/epjc/s10052-016-4375-3 [arXiv:1606.00209 [astro-ph.HE]].

[71] A. Albert et al., Phys. Lett. B 769, 249 (2017) doi:10.1016/j.physletb.2017.03.063 [arXiv:1612.04595 [astro-ph.HE]].

[72] K. Frankiewicz [Super-Kamiokande Collaboration], arXiv:1510.07999 [hep-ex].

[73] M. G. Aartsen et al. [IceCube Collaboration], Eur. Phys. J. C 77, no. 3, 146 (2017) doi:10.1140/epjc/s10052-017-4689-9 [arXiv:1612.05949 [astro-ph.HE]].

[74] S. Adrian-Martinez et al. [ANTARES Collaboration], Phys. Lett. B 759, 69 (2016) doi:10.1016/j.physletb.2016.05.019 [arXiv:1603.02228 [astro-ph.HE]].

[75] K. Choi et al. [Super-Kamiokande Collaboration], Phys. Rev. Lett. 114, no. 14, 141301 (2015) doi:10.1103/PhysRevLett.114.141301 [arXiv:1503.04858 [hep-ex]].

[76] M. G. Aartsen et al. [IceCube Collaboration], Eur. Phys. J. C 77, no. 2, 82 (2017) doi:10.1140/epjc/s10052-016-4582-y [arXiv:1609.01492 [astro-ph.HE]].

[77] A. Albert et al. [ANTARES Collaboration], Phys. Dark Univ. 16, 41 (2017) doi:10.1016/j.dark.2017.04.005 [arXiv:1612.06792 [hep-ex]].

[78] A. Tan et al. [PandaX-II Collaboration], Phys. Rev. Lett. 117, no. 12, 121303 (2016) doi:10.1103/PhysRevLett.117.121303 [arXiv:1607.07400 [hep-ex]].

[79] S. W. Barwick et al. [HEAT Collaboration], Astrophys. J. 482, L191 (1997) [astro$\mathrm{ph} / 9703192]$.

[80] J. J. Beatty et al., Phys. Rev. Lett. 93, 241102 (2004) doi:10.1103/PhysRevLett.93.241102 [astro-ph/0412230].

[81] O. Adriani et al. [ PAMELA Collaboration], arXiv:1308.0133 [astro-ph.HE]. 
[82] L. Accardo et al. [AMS Collaboration], Phys. Rev. Lett. 113, 121101 (2014). doi:10.1103/PhysRevLett.113.121101

[83] O. Adriani et al. [PAMELA Collaboration], Phys. Rev. Lett. 106, 201101 (2011) doi:10.1103/PhysRevLett.106.201101 [arXiv:1103.2880 [astro-ph.HE]].

[84] J. D. Lewin and P. F. Smith, Astropart. Phys. 6, 87 (1996).

[85] A. K. Drukier, K. Freese and D. N. Spergel, Phys. Rev. D 33, 3495 (1986). doi:10.1103/PhysRevD.33.3495

[86] F. Brimioulle, S. Seitz, M. Lerchster, R. Bender and J. Snigula, Mon. Not. Roy. Astron. Soc. 432, 1046 (2013) doi:10.1093/mnras/stt525 [arXiv:1303.6287 [astroph.CO]].

[87] T. G. Brainerd, R. D. Blandford and I. Smail, Astrophys. J. 466, 623 (1996) doi:10.1086/177537 [astro-ph/9503073].

[88] J. F. Navarro, C. S. Frenk and S. D. M. White, Astrophys. J. 462, 563 (1996) doi:10.1086/177173 [astro-ph/9508025].

[89] J. Binney and S. Tremaine, Galactic Dynamics, 2nd ed. (Princeton University Press, Princeton, 2008).

[90] P. Salucci, F. Nesti, G. Gentile and C. F. Martins, Astron. Astrophys. 523, A83 (2010) doi:10.1051/0004-6361/201014385 [arXiv:1003.3101 [astro-ph.GA]].

[91] S. Garbari, C. Liu, J. I. Read and G. Lake, Mon. Not. Roy. Astron. Soc. 425, 1445 (2012) doi:10.1111/j.1365-2966.2012.21608.x [arXiv:1206.0015 [astro-ph.GA]].

[92] F. Nesti and P. Salucci, JCAP 1307, 016 (2013) doi:10.1088/14757516/2013/07/016 [arXiv:1304.5127 [astro-ph.GA]].

[93] O. Bienaym et al., Astron. Astrophys. 571, A92 (2014) doi:10.1051/00046361/201424478 [arXiv:1406.6896 [astro-ph.GA]].

[94] J. I. Read, J. Phys. G 41, 063101 (2014) doi:10.1088/0954-3899/41/6/063101 [arXiv:1404.1938 [astro-ph.GA]]. 
[95] F. J. Kerr and D. Lynden-Bell, Mon. Not. Roy. Astron. Soc. 221, 1023 (1986).

[96] P. J. McMillan and J. J. Binney, Mon. Not. Roy. Astron. Soc. 402, 934 (2010) doi:10.1111/j.1365-2966.2009.15932.x [arXiv:0907.4685 [astro-ph.GA]].

[97] M. J. Reid et al., Astrophys. J. 700, 137 (2009) doi:10.1088/0004-637X/700/1/137 [arXiv:0902.3913 [astro-ph.GA]].

[98] M. J. Reid et al., Astrophys. J. 783, 130 (2014) doi:10.1088/0004-637X/783/2/130 [arXiv:1401.5377 [astro-ph.GA]].

[99] R. Schoenrich, J. Binney and W. Dehnen, Mon. Not. Roy. Astron. Soc. 403, 1829 (2010) doi:10.1111/j.1365-2966.2010.16253.x [arXiv:0912.3693 [astro-ph.GA]].

[100] K. Freese, M. Lisanti and C. Savage, Rev. Mod. Phys. 85, 1561 (2013) doi:10.1103/RevModPhys.85.1561 [arXiv:1209.3339 [astro-ph.CO]].

[101] Y. Y. Mao, L. E. Strigari, R. H. Wechsler, H. Y. Wu and O. Hahn, Astrophys. J. 764, 35 (2013) doi:10.1088/0004-637X/764/1/35 [arXiv:1210.2721 [astro-ph.CO]].

[102] M. C. Smith et al., Mon. Not. Roy. Astron. Soc. 379, 755 (2007) doi:10.1111/j.1365-2966.2007.11964.x [astro-ph/0611671].

[103] T. Piffl et al., Astron. Astrophys. 562, A91 (2014) doi:10.1051/00046361/201322531 [arXiv:1309.4293 [astro-ph.GA]].

[104] A. L. Fitzpatrick, W. Haxton, E. Katz, N. Lubbers and Y. Xu, JCAP 1302, 004 (2013) doi:10.1088/1475-7516/2013/02/004 [arXiv:1203.3542 [hep-ph]].

[105] N. Anand, A. L. Fitzpatrick and W. C. Haxton, Phys. Rev. C 89, no. 6, 065501 (2014) doi:10.1103/PhysRevC.89.065501 [arXiv:1308.6288 [hep-ph]].

[106] M. Kuhlen, A. Pillepich, J. Guedes and P. Madau, Astrophys. J. 784, 161 (2014) doi:10.1088/0004-637X/784/2/161 [arXiv:1308.1703 [astro-ph.GA]].

[107] R. J. Gaitskell, Ann. Rev. Nucl. Part. Sci. 54, 315 (2004).

[108] P. F. Smith and J. D. Lewin, Phys. Rept. 187, 203 (1990). 
[109] T. A. Girard et al., doi:10.1142/9789812701848_0034 hep-ex/0504022.

[110] T. Girard et al. [SIMPLE Collaboration], PoS IDM 2010, 055 (2011) [arXiv:1101.1885 [astro-ph.CO]].

[111] C. Amole et al. [PICO Collaboration], Phys. Rev. Lett. 114 (2015) no.23, 231302 doi:10.1103/PhysRevLett.114.231302 [arXiv:1503.00008 [astro-ph.CO]].

[112] C. Amole et al. [PICO Collaboration], Phys. Rev. D 93, no. 6, 061101 (2016) doi:10.1103/PhysRevD.93.061101 [arXiv:1601.03729 [astro-ph.CO]].

[113] C. Amole et al. [PICO Collaboration], Phys. Rev. D 93, no. 5, 052014 (2016) doi:10.1103/PhysRevD.93.052014 [arXiv:1510.07754 [hep-ex]].

[114] C. Amole et al. [PICO Collaboration], Phys. Rev. Lett. 118, no. 25, 251301 (2017) doi:10.1103/PhysRevLett.118.251301 [arXiv:1702.07666 [astro-ph.CO]].

[115] K. S. Olsen [DEAP/CLEAN Collaboration], arXiv:0906.0348 [hep-ex].

[116] P.-A. Amaudruz et al. [DEAP-3600 Collaboration], arXiv:1707.08042 [astroph.CO].

[117] P. Beltrame [on behalf of the XENON Collaboration], arXiv:1305.2719 [astroph.CO].

[118] E. Aprile et al. [XENON Collaboration], arXiv:1708.07051 [astro-ph.IM].

[119] E. Aprile et al. [XENON Collaboration], JCAP 1604, no. 04, 027 (2016) doi:10.1088/1475-7516/2016/04/027 [arXiv:1512.07501 [physics.ins-det]].

[120] E. Aprile et al. [XENON Collaboration], arXiv:1705.06655 [astro-ph.CO].

[121] J. Monroe [DMTPC Collaboration], AIP Conf. Proc. 1441, 515 (2012) [arXiv:1111.0220 [physics.ins-det]].

[122] J. B. R. Battat et al. [DRIFT Collaboration], Astropart. Phys. 91, 65 (2017) doi:10.1016/j.astropartphys.2017.03.007 [arXiv:1701.00171 [astro-ph.IM]].

[123] S. Ahlen et al., Phys. Lett. B 695, 124 (2011) doi:10.1016/j.physletb.2010.11.041 [arXiv:1006.2928 [hep-ex]]. 
[124] R. Bernabei et al. [DAMA and LIBRA Collaborations], Eur. Phys. J. C 67, 39 (2010) [arXiv:1002.1028 [astro-ph.GA]].

[125] R. Agnese et al. [SuperCDMS Collaboration], Phys. Rev. Lett. 112, no. 24, 241302 (2014) doi:10.1103/PhysRevLett.112.241302 [arXiv:1402.7137 [hep-ex]].

[126] J. R. T. de Mello Neto et al. [DAMIC Collaboration], PoS ICRC 2015, 1221 (2016) [arXiv:1510.02126 [physics.ins-det]].

[127] A. Aguilar-Arevalo et al. [DAMIC Collaboration], Phys. Rev. D 94, no. 8, 082006 (2016) doi:10.1103/PhysRevD.94.082006 [arXiv:1607.07410 [astro-ph.CO]].

[128] E. Armengaud et al. [EDELWEISS Collaboration], Phys. Lett. B 702, 329 (2011) [arXiv:1103.4070 [astro-ph.CO]].

[129] E. Armengaud et al. [EDELWEISS Collaboration], Phys. Rev. D 86, 051701 (2012) doi:10.1103/PhysRevD.86.051701 [arXiv:1207.1815 [astro-ph.CO]].

[130] Q. Arnaud et al. [EDELWEISS Collaboration], arXiv:1707.04308 [physics.ins-det].

[131] E. Armengaud et al. [EDELWEISS Collaboration], JCAP 1605, no. 05, 019 (2016) doi:10.1088/1475-7516/2016/05/019 [arXiv:1603.05120 [astro-ph.CO]].

[132] Z. Ahmed et al. [CDMS-II Collaboration], Science 327, 1619 (2010) [arXiv:0912.3592 [astro-ph.CO]].

[133] R. Agnese et al. [CDMS Collaboration], Phys. Rev. D 88, 031104 (2013) Erratum: [Phys. Rev. D 88, no. 5, 059901 (2013)] doi:10.1103/PhysRevD.88.031104, 10.1103/PhysRevD.88.059901 [arXiv:1304.3706 [astro-ph.CO]].

[134] R. Agnese et al. [SuperCDMS Collaboration], Phys. Rev. Lett. 112, no. 4, 041302 (2014) doi:10.1103/PhysRevLett.112.041302 [arXiv:1309.3259 [physics.ins-det]].

[135] R. Agnese et al. [SuperCDMS Collaboration], [arXiv:1610.00006 [physics.ins-det]].

[136] R. Agnese et al. [SuperCDMS Collaboration], Phys. Rev. Lett. 116, no. 7, 071301 (2016) doi:10.1103/PhysRevLett.116.071301 [arXiv:1509.02448 [astro-ph.CO]]. 
[137] H. Qui, Exclusion Limit from a Profile Likelihood Ratio Analysis on the HT unblinded data, Ph.D. Thesis, Southern Methodist University, 2017.

[138] D. S. Akerib et al. [LUX Collaboration], Phys. Rev. Lett. 116, no. 16, 161301 (2016) doi:10.1103/PhysRevLett.116.161301 [arXiv:1512.03506 [astro-ph.CO]].

[139] G. Angloher et al. [CRESST Collaboration], Eur. Phys. J. C 76, no. 1, 25 (2016) doi:10.1140/epjc/s10052-016-3877-3 [arXiv:1509.01515 [astro-ph.CO]].

[140] J. Barreto et al. [DAMIC Collaboration], Phys. Lett. B 711, 264 (2012) doi:10.1016/j.physletb.2012.04.006 [arXiv:1105.5191 [astro-ph.IM]].

[141] D. C. Malling et al., arXiv:1110.0103 [astro-ph.IM].

[142] G. Angloher et al., Eur. Phys. J. C 72, 1971 (2012) doi:10.1140/epjc/s10052-0121971-8 [arXiv:1109.0702 [astro-ph.CO]].

[143] C. Savage, K. Freese, P. Gondolo and D. Spolyar, JCAP 0909, 036 (2009) doi:10.1088/1475-7516/2009/09/036 [arXiv:0901.2713 [astro-ph.CO]].

[144] R. Agnese et al. [CDMS Collaboration], Phys. Rev. Lett. 111, no. 25, 251301 (2013) doi:10.1103/PhysRevLett.111.251301 [arXiv:1304.4279 [hep-ex]].

[145] F. Ruppin, J. Billard, E. Figueroa-Feliciano and L. Strigari, Phys. Rev. D 90, no. 8, 083510 (2014) doi:10.1103/PhysRevD.90.083510 [arXiv:1408.3581 [hep-ph]].

[146] B. Cabrera, L. M. Krauss and F. Wilczek, Phys. Rev. Lett. 55, 25 (1985). doi:10.1103/PhysRevLett.55.25

[147] B. Sadoulet, Conf. Proc. C 861214, 260 (1986).

[148] N. Wang [CDMS Collaboration], Physica B 3, 165 (1990).

[149] D. Abrams et al. [CDMS Collaboration], Phys. Rev. D 66, 122003 (2002) [astro$\mathrm{ph} / 0203500]$.

[150] R. Clarke, An Athermal-Phonon-Mediated Dark Matter Detector with Surface Discrimination, Ph.D. Thesis, Stanford University, 1999. 
[151] R. Abusaidi et al. [CDMS Collaboration], Phys. Rev. Lett. 84, 5699 (2000) doi:10.1103/PhysRevLett.84.5699 [astro-ph/0002471].

[152] S.R. Golwala, Exclusion Limits on the WIMP-Nucleon Elastic-Scattering Cross Section from the Cryogenic Dark Matter Search, Ph.D. Thesis, U.C. Berkeley, 2000.

[153] R. Bernabei et al. [DAMA Collaboration], Phys. Lett. B 480, 23 (2000). doi:10.1016/S0370-2693(00)00405-6

[154] T. Shutt et al. [CDMS Collaboration], IEEE Trans. Nucl. Sci. 37, 547 (1990). doi:10.1109/23.106675

[155] D. S. Akerib et al. [CDMS Collaboration], Phys. Rev. D 68, 082002 (2003) doi:10.1103/PhysRevD.68.082002 [hep-ex/0306001].

[156] D. S. Akerib et al. [CDMS Collaboration], Phys. Rev. Lett. 93, 211301 (2004) doi:10.1103/PhysRevLett.93.211301 [astro-ph/0405033].

[157] A. Benoit et al., Phys. Lett. B 545, 43 (2002) doi:10.1016/S0370-2693(02)02238-4 [astro-ph/0206271].

[158] D. S. Akerib et al. [CDMS Collaboration], Phys. Rev. D 72, 052009 (2005) doi:10.1103/PhysRevD.72.052009 [astro-ph/0507190].

[159] D. S. Akerib et al. [CDMS Collaboration], Phys. Rev. Lett. 96, 011302 (2006) doi:10.1103/PhysRevLett.96.011302 [astro-ph/0509259].

[160] Z. Ahmed et al. [CDMS Collaboration], Phys. Rev. Lett. 102, 011301 (2009) doi:10.1103/PhysRevLett.102.011301 [arXiv:0802.3530 [astro-ph]].

[161] R. Agnese et al. [SuperCDMS Collaboration], Phys. Rev. D 92, no. 7, 072003 (2015) doi:10.1103/PhysRevD.92.072003 [arXiv:1504.05871 [hep-ex]].

[162] R. Agnese et al. [SuperCDMSSoudan Collaboration], Appl. Phys. Lett. 103, 164105 (2013) doi:10.1063/1.4819835, 10.1063/1.4826093 [arXiv:1305.2405 [physics.ins-det]].

[163] C. E. Aalseth et al. [CoGeNT Collaboration], Phys. Rev. D 88, 012002 (2013) doi:10.1103/PhysRevD.88.012002 [arXiv:1208.5737 [astro-ph.CO]]. 
[164] Z. Ahmed et al. [CDMS-II Collaboration], Phys. Rev. Lett. 106, 131302 (2011) doi:10.1103/PhysRevLett.106.131302 [arXiv:1011.2482 [astro-ph.CO]].

[165] J. Angle et al. [XENON10 Collaboration], Phys. Rev. Lett. 107, 051301 (2011) Erratum: [Phys. Rev. Lett. 110, 249901 (2013)] doi:10.1103/PhysRevLett.110.249901, 10.1103/PhysRevLett.107.051301 [arXiv:1104.3088 [astro-ph.CO]].

[166] M. Pepin, Low-Mass Dark Matter Search Results and Radiogenic Backgrounds for the Cryogenic Dark Matter Search, Ph.D. Thesis, University of Minnesota, 2016.

[167] A. Phipps, Ionization Collection in Detectors of the Cryogenic Dark Matter Search, Ph.D. Thesis, U.C. Berkeley, 2016.

[168] V. Mandic, First Results from the Cryogenic Dark Matter Search Experiment at the Deep Site, Ph.D. Thesis, University of California, Berkeley, 2004.

[169] S. Hertel, Advancing the Search for Dark Matter: From CDMS II to SuperCDMS, Ph.D. Thesis, Massachusetts Institute of Technology, 2012.

[170] Z. He, Nucl. Instrum. Methods Phys. Res. A 463 (2001) 250.

[171] S. Ramo, Proc. Ire. 27, 584 (1939). doi:10.1109/JRPROC.1939.228757

[172] D. Yvon et al., "Low Noise Voltage and Charge Preamplifiers for Phonon and Ionization Detectors at Very Low Temperature," Nucl. Instrum. and Meth., A368, 778 (1996)

[173] T. Saab, Search for Weakly Interacting Massive Particles with the Cryogenic Dark Matter Search Experiment, Ph.D. Thesis, Stanford University, 2002.

[174] B. S. Neganov and V. N. Trofimov, Otkrytia i izobreteniya, 215 (1985), USSR Patent No 1037771.

[175] P.N. Luke, J. Appl. Phys. 64, 6858 (1988).

[176] B. Cabrera, M. Pyle, R. Moffatt, K. Sundqvist and B. Sadoulet, arXiv:1004.1233 [astro-ph.IM]. 
[177] N. E. Booth, "Quasiparticle Trapping And The Quasiparticle Multiplier," Submitted to: Appl.Phys.Lett..

[178] M. Fritts, "Phonon Collection Efficiency for G106a in K100 Run 055", (2016). Internal note.

[179] Pobell, Frank. Matter and Methods at Low Temperatures. 3rd ed. Berlin: SpringerVerlag, 2007. Print.

[180] B. A. Hines, K. M. Sundqvist, D. N. Seitz and M. E. Huber, IEEE Trans. Appl. Supercond. 21, no. 3, 5672549 (2010). doi:10.1109/TASC.2010.2091617

[181] S. Yellin, Phys. Rev. D 66, 032005 (2002) doi:10.1103/PhysRevD.66.032005 [physics/0203002].

[182] S. Yellin, arXiv:0709.2701 [physics.data-an].

[183] J. Zhang, A Dark Matter Search Using the Final CDMS-II Data and $100 \mathrm{~mm}$ SuperCDMS Germanium Detector Ionization Test, Ph.D. Thesis, University of Minnesota, 2014.

[184] Maximum Integrated Data Acquisition System [Computer Software]. https://midas.triumf.ca/MidasWiki/index.php

[185] R. Basu Thakur, The Cryogenic Dark Matter Search Low Ionization-Threshold Experiment, Ph.D. Thesis, Department of Physics, University of Illinois at UrbanaChampaign, 2015.

[186] J.P. Filippini, A Search for WIMP Dark Matter Using the First Five-Tower Run of the Cryogenic Dark Matter Search, Ph.D. Thesis, University of California, Berkeley, 2008.

[187] "Detector User Manual: Gamma and X-ray Detectors", Princeton Gamma-Tech Instruments, INC: Rocky Hill, N.J. 2005

[188] G. Heusser, Ann. Rev. Nucl. Part. Sci. 45, 543 (1995). doi:10.1146/annurev.ns.45.120195.002551 
[189] A. Emerick, Characterization of the Natural Gamma Ray Background Emission at the CDMS Detector Testing Facility. University of Minnesota, 2012.

[190] L.P. Ekstrom and R.B. Firestrone, WWW Table of Radioactive Isotopes, database version 2/28/99 from URL http://ie.lbl.gov/toi/index.htm

[191] A. Reisetter. Monte-Carlo simulation of Soudan Mine neutron energy spectrum. Private communication.

[192] Gordon, M.s., et al. IEEE Trans. on Nucl. Sci., vol. 51, no. 6, 2004, pp. 34273434., doi:10.1109/tns.2004.839134.

[193] M. Kole, M. Pearce and M. Muoz Salinas, Astropart. Phys. 62, 230 (2015) doi:10.1016/j.astropartphys.2014.10.002 [arXiv:1410.1364 [astro-ph.IM]].

[194] J. A. Formaggio and C. J. Martoff, Ann. Rev. Nucl. Part. Sci. 54, 361 (2004). doi:10.1146/annurev.nucl.54.070103.181248

[195] S. A. Cox Prog: Argonne National Laboratory report series, No.7210, p.3 (1966). Data retrieved via EXFOR, http://www-nds.iaea.org/EXFOR/11519.008

[196] R. Radpour, "Design, Construction, and Assessment of a Neutron Shield for CDMS Test Facilities." Thesis. University of Minnesota, 2011.

[197] C.H. Mesquita, T.M. Filho, and M.M.Hamada. IEEE Trans. on Nucl. Sci., vol. 50, no 4,2003, pp. 11701175.

[198] H. Chagani and A. Kennedy, "Brief Feasibility Study of Gamma Shield for K100", (2011). Internal note.

[199] A. Sonzogni, Nudat 2, (2016).

[200] M. Fritts, "Phonon Collection Efficiency for S101(HV\#4) in K100 Run 062", (2017). Internal note.

[201] N. Mast, "Optimal Filter Template Development for K100 Data", (2016). Internal note.

[202] COMSOL [Computer Software]. https://www.comsol.com/ 
[203] N. Kurinsky, "COMSOL Grid Models", (2016). Internal note.

[204] D. Jardin, "Charge and Phonon Resolution", (2015). Internal note.

[205] H. Chagani, D. A. Bauer, D. Brandt, P. L. Brink, B. Cabrera, M. Cherry, E. D. C. eSilva and G. G. Godfrey et al., "Ionization Measurements of SuperCDMS SNOLAB 100 mm Diameter Germanium Crystals," J. Low. Temp. Phys. 167, 1125 (2012).

[206] Emery, F. E., and T. A. Rabson. Physical Review, vol. 140, no. 6A, 1965, doi:10.1103/physrev.140.a2089.

[207] R. Radpour et al, "Run 34 Summary", (2013). Internal note

[208] C. N. Bailey, The Cryogenic Dark Matter Search: First 5-Tower Data And Improved Understanding Of Ionization Collection, Ph.D. Thesis, Case Western Reserve University, 2010.

[209] D. S. Akerib et al., Nucl. Instrum. Meth. A 591, 476 (2008). doi:10.1016/j.nima.2008.03.103

[210] D. Seitz, "Soudan SQUID Tuning Procedure", (2010). Internal note.

[211] H. Chagani, "IbIs Curves \& Resultant QET Biases", (2014). Internal note.

[212] A.J. Anderson, "Time-dependence of Relative Phonon Calibration", (2013). Internal note.

[213] J. Lindhard et al., K. Dan. Vidensk. Selk., Mat.-Frys. Medd. 33 (1963) No. 10; 36 (1968) No. 10.

[214] T. Bruch, "Comparison of the NR band mean for R125-R128 with the Lindhard prediction", (2009). Internal note.

[215] J. Filippini, "First Look at R123 Bands", (2007). Internal note.

[216] S. Scorza, "Nuclear Recoil Bands", (2012). Internal note.

[217] S. Scorza, "Electronic Recoil Bands", (2012). Internal note. 
[218] T. Bruch, "Definition of the ER bands and yield distributions for mean and gradient corrected data", (2009). Internal note.

[219] A. N. Villano, "Nuclear Recoil Bands in c58 Re-Analysis", (2011). Internal note.

[220] N. Mirabolfathi, H. R. Harris, R. Mahapatra, K. Sundqvist, A. Jastram, B. Serfass, D. Faiez and B. Sadoulet, Nucl. Instrum. Meth. A 855, 88 (2017) doi:10.1016/j.nima.2017.02.032 [arXiv:1510.00999 [physics.ins-det]].

[221] Autodesk Inventor [Computer Software]. https://www.autodesk.com/

[222] New Semiconductor Materials. Characteristics and Properties. [Electronic Archive]. http://www.ioffe.ru/SVA/NSM/

[223] V. Mandic. Success of Si Crystal Neutralization with IR LEDs. Private communication.

[224] Codoluto, Daniel. (2015). SuperCDMS 150-mm Silicon Crystal Detector Housing Flexible Side Coax Assembly Board Component and Fixture Adaptation. University of Minnesota Digital Conservancy, http://hdl.handle.net/11299/172022.

[225] Hansen et al., The Cryogenic Dark Matter Search test stand warm electronics card, in Nuclear Science Symposium Conference Record (NSS/MIC), 2010 IEEE (2010) 1329.

[226] C. N. Bailey, "S06C and S10C Seagull Plots", (2007). Internal note.

[227] H. Rogers, D. G. Cerdeno, P. Cushman, F. Livet and V. Mandic, Phys. Rev. D 95, no. 8, 082003 (2017) doi:10.1103/PhysRevD.95.082003 [arXiv:1612.09038 [astroph.CO]].

[228] K. Schneck, Search For Low-Mass Dark Matter With Supercdms Soudan And Study Of Shorted Electric Field Configurations In CDMS Detectors, Ph.D. Thesis, Department of Physics, Stanford University, 2014.

[229] F. Feroz, M. P. Hobson and M. Bridges, Mon. Not. Roy. Astron. Soc. 398, 1601 (2009) doi:10.1111/j.1365-2966.2009.14548.x [arXiv:0809.3437 [astro-ph]]. 
[230] F. Feroz and M. P. Hobson, Mon. Not. Roy. Astron. Soc. 384, 449 (2008) doi:10.1111/j.1365-2966.2007.12353.x [arXiv:0704.3704 [astro-ph]].

[231] F. Feroz, M. P. Hobson, E. Cameron and A. N. Pettitt, arXiv:1306.2144 [astroph.IM].

[232] R. Catena and P. Gondolo, JCAP 1508, no. 08, 022 (2015) doi:10.1088/14757516/2015/08/022 [arXiv:1504.06554 [hep-ph]].

[233] Wolfram Mathematica [Computer Software]. https://midas.triumf.ca/MidasWiki/index.php

[234] H. E. Rogers, "Effective Field Theory Operator Comparison for CDMS II Ge and CDMSlite", (2017). Internal note.

[235] SuperCDMS Collaboration, "Summary of First iZIP High Threshold Analysis", (2017). Internal note. 


\section{Appendix A}

\section{Acronyms, Variables, and Data Quantities}

Care has been taken in this thesis to minimize the use of jargon and acronyms, but this cannot always be achieved. This appendix contains a table of acronyms, variables, and data quantities, with their meaning.

\section{A.1 Acronyms}

Table A.1: Acronyms

\begin{tabular}{|l|l|}
\hline Acronym & Meaning \\
\hline \hline ADC & Analogue-to-Digital Converter \\
ADMX (-HF) & Axion Dark Matter eXperiment (High Frequency) \\
BAO & Baryon Acoustic Oscillations \\
BLIP & Berkeley Large Ionization and Phonon mediated (detector) \\
CCD & Charge-coupled device \\
CDMS & Cryogenic Dark Matter Search \\
CDMSlite & CDMS Low Ionization Threshold Experiment \\
CE $\nu$ NS & Coherent Elastic Neutrino-Nucleus Scattering \\
\hline \hline \multicolumn{2}{|c}{ Continued on next page } \\
\hline
\end{tabular}


Table A.1 - continued from previous page

\begin{tabular}{|c|c|}
\hline Acronym & Meaning \\
\hline DAQ & Data Acquisition \\
\hline DCRC & Detector Control and Readout Cards \\
\hline DIB & Detector Interface Boards \\
\hline EFT & Effective Field Theory \\
\hline ER & Electron Recoil \\
\hline ETF & ElectroThermal Feedback \\
\hline FEB & Front-end Electronics Boards \\
\hline FFT & Fast Fourier Transform \\
\hline GUI & Graphic User Interface \\
\hline GUT & Grand Unified Theory \\
\hline HPGe & High Purity Ge \\
\hline HV & High voltage \\
\hline IGH & Intelligent Gas Handling \\
\hline IR & InfaRed \\
\hline IVC & Inner Vacuum Can \\
\hline iZIP & interleaved Z-dependent Ionization and Phonon (detector) \\
\hline$(\mathrm{J}) \mathrm{FET}$ & (Junction gate) Field-Effect Transistor \\
\hline K100 & Kelvinox 100 Dilution Refrigerator \\
\hline LED & Light Emitting Diode \\
\hline LHe & Liquid ${ }^{4} \mathrm{He}$ \\
\hline LHC & Large Hadron Collider \\
\hline LN2 & Liquid $\mathrm{N}_{2}$ \\
\hline LSP & Lightest Supersymmetric Particle \\
\hline LSR & Local Standard of Rest. \\
\hline MACHO & MAssive Compact Halo Object \\
\hline MIDAS & Maximum Integrated Data Acquisition Systems \\
\hline MSSM & Minimal SuperSymmetric Model \\
\hline mSUGRA & minimal SUper GRAvity \\
\hline mwe & meters water equivalent \\
\hline
\end{tabular}


Table A.1 - continued from previous page

\begin{tabular}{|c|c|}
\hline Acronym & Meaning \\
\hline NR & Nuclear Recoil \\
\hline NSOF & Non-Stationary Optimal Filter \\
\hline NSP & Neutron Selection Parameter \\
\hline NTD & Neutron Transmutation Doped \\
\hline NTL & Neganov-Trofimov-Luke \\
\hline $\mathrm{OF}$ & Optimal Filter \\
\hline OFHC & Oxygen-Free High thermal Conductivity \\
\hline (o)ZIP & (original) Z-dependent Ionization and Phonon (detector) \\
\hline PCB & Printed Circuit Board \\
\hline POE & Power Over Ethernet \\
\hline RQ & Reduced Quantity \\
\hline RTF & Receiver-Trigger-Filter (board) \\
\hline $\mathrm{SCAB}$ & Side Coax Assembly Board \\
\hline SHM & Standard Halo Model \\
\hline SIS & Singular Isothermal Sphere (profile) \\
\hline SCUHBA & Side Coax Upper Heatsink Board Assembly \\
\hline SLAC & Stanford Linear Accelerator Center \\
\hline SM & Standard Model \\
\hline $\mathrm{SNO}$ & Sudbury Neutrino Observatory (LAB or experiment) \\
\hline SQUET & SQUID and FET electronics \\
\hline SQUID & Superconducting Quantum Interference Device \\
\hline SUF & Stanford Underground Facility \\
\hline SUSY & Supersymmetry \\
\hline TAMU & Texas A\&M University \\
\hline $\mathrm{TDF}$ & Time Domain Fit \\
\hline TES & Transition Edge Sensor \\
\hline UMN & University of Minnesota \\
\hline UV & UltraViolet \\
\hline QET & Quasiparticle assisted Electrothermal feedback TES \\
\hline
\end{tabular}


Table A.1 - continued from previous page

\begin{tabular}{|l|l|}
\hline Acronym & Meaning \\
\hline \hline WIMP & Weakly Interactive Massive Particle \\
WMAP & Wilkinson Microwave Anisotropy Probe \\
\hline \hline
\end{tabular}

\section{A.2 Variables}

Table A.2: Variables

\begin{tabular}{|l|l|}
\hline Variables & Meaning \\
\hline \hline$a$ & Pulse amplification factor. \\
$\hat{a}$ & Pulse amplification factor which minimizes $\chi^{2}$. \\
& Time dependent scale factor that describes the expansion of space- \\
$A$ & time. \\
$A_{i}$ & Atomic mass. \\
$B_{n}$ & Digitized pulse template. \\
$c_{i}^{0}$ & Base channel signal. \\
$c_{i}^{1}$ & Isoscalar coupling constant. \\
$c_{v}$ & Isovector coupling constant. \\
$C_{C}$ & Heat capacity. \\
$C_{D}$ & Coupling capacitance. \\
$C_{F}$ & Detector capacitance. \\
$C_{p}$ & Feedback capacitance. \\
$C_{n}$ & Parasitic capacitance. \\
$\mathcal{C}$ & Channel signal. \\
$\mathcal{C}_{\ell}$ & Normalization constant. \\
$\chi, \bar{\chi}$ & Angular power spectrum. \\
$\chi^{2}$ & WimP nonrelativistic field. \\
$\mathcal{D}_{\ell}^{T T}$ & Goodness-of-fit. \\
$\Delta$ & Temperature-temperature band power map. \\
\hline \hline & Energy gap. \\
\hline
\end{tabular}


Table A.2 - continued from previous page

\begin{tabular}{|c|c|}
\hline Symbol & Meaning \\
\hline$\Delta \pi$ & Osmotic pressure difference. \\
\hline$e$ & Elementary charge. \\
\hline$e^{+}, e^{-}$ & Electron or positron. \\
\hline E & Electric field. \\
\hline$E_{0}$ & Zero point energy. \\
\hline$E_{F}$ & Fermi energy. \\
\hline$E_{g}$ & Indirect band gap energy. \\
\hline$E_{\text {gap }}$ & Bandgap energy. \\
\hline$E_{\Gamma 1}$ & Direct band gap energy. \\
\hline$E_{K E}$ & Kinetic energy of charge carriers. \\
\hline$E_{N T L}$ & Neganov-Trofimov-Luke phonon energy component. \\
\hline$E_{P}$ & Primary phonon energy. \\
\hline$E_{Q}$ & Ionization energy. \\
\hline$E_{R}, E_{R, e e}, E_{R, n r}$ & $\begin{array}{l}\text { Recoil energy, electron equivalent recoil energy, and nuclear-recoil } \\
\text { equivalent energy, respectively. }\end{array}$ \\
\hline$E_{r e l}$ & Relaxation phonon energy. \\
\hline$E_{T}$ & Total phonon energy. \\
\hline$E_{v}$ & Component of electric field in direction of $\mathrm{v}$. \\
\hline$\varepsilon$ & Efficiency. \\
\hline$\varepsilon_{c c}$ & Charge collection efficiency. \\
\hline$\epsilon_{3}$ & Binding energy of ${ }^{3} \mathrm{He}$ \\
\hline$\epsilon_{\gamma}$ & Material-dependent electron/hole pair production energy. \\
\hline$\eta_{k}$ & Expected number of events in the $k$ th energy bin. \\
\hline$f_{A}$ & Axion decay constant. \\
\hline$f_{n}$ & Bin index over full pulse time. \\
\hline$F, F_{i, j}^{\tau, \tau^{\prime}}$ & Form factor. \\
\hline $\mathcal{F}$ & Phonon channel relative calibration factor. \\
\hline$\hat{\mathcal{F}}$ & Phonon channel relative calibration factor which minimizes $\chi^{2}$. \\
\hline$g_{A \gamma}$ & Axion-phonon coupling constant. \\
\hline
\end{tabular}


Table A.2 - continued from previous page

\begin{tabular}{|c|c|}
\hline Symbol & Meaning \\
\hline$g^{i j}$ & $\begin{array}{l}\text { Spatial portion of Robertson-Walker metric in Cartesian coordi- } \\
\text { nates. }\end{array}$ \\
\hline$G$ & Gravitational constant. \\
\hline$G_{d}$ & Driver gain. \\
\hline$G_{D C R C}$ & DCRC gain. \\
\hline$G_{\text {loop }}$ & Phonon readout loop gain. \\
\hline$G_{o}$ & Output gain. \\
\hline$\gamma$ & Angle of reflection of a light ray. \\
\hline$h$ & Hubble parameter for present time. \\
\hline$h, \hbar$ & Planck constant. \\
\hline$H$ & Enthalpy. \\
\hline$H(t)$ & Hubble parameter. \\
\hline$i$ & Current or image current. \\
\hline$I_{b}$ & Bias current. \\
\hline$I_{s}$ & Current passing through the sensor. \\
\hline$J$ & PSD. \\
\hline$J^{i}$ & Angular momentum. \\
\hline$k$ & $+1,-1,0$ for closed, open, or flat universe, respectively. \\
\hline$\vec{k},\left(\vec{k}^{\prime}\right)$ & Incoming (outgoing) nucleon momentum \\
\hline$k_{B}$ & Boltzman constant. \\
\hline$\kappa$ & Thermal conductivity coefficient. \\
\hline$l_{0}$ & Characteristic range for carrier scattering. \\
\hline $\mathcal{L}$ & $\begin{array}{l}\text { Likelihood, } \mathcal{L}^{D M} \text { for dark matter signal only and } \mathcal{L}^{D M+b k g} \text { for } \\
\text { signal and background. }\end{array}$ \\
\hline $\mathcal{L}_{\text {int }}$ & Interaction Lagrangian. \\
\hline$L_{3}$ & Latent heat of evaporation. \\
\hline$L_{i}$ & Input loop inductance \\
\hline$L_{f b}$ & Feedback loop inductance. \\
\hline$\lambda(x \leftrightarrow y)$ & Conversion rate between particles $x$ and $y$. \\
\hline
\end{tabular}


Table A.2 - continued from previous page

\begin{tabular}{|c|c|}
\hline Symbol & Meaning \\
\hline$m_{A}$ & Axion mass. \\
\hline$m_{c}$ & $\begin{array}{l}\text { Carrier mass. Electron mass given by } m_{e} \text {, hole effective mass given } \\
\text { by } m_{h} \text {. }\end{array}$ \\
\hline$m_{N}$ & Nucleon mass. \\
\hline$M$ & $\begin{array}{l}\text { Mass. Can be a stand alone value, or part of a distribution, such } \\
\text { as } M(r) \text {. }\end{array}$ \\
\hline$M_{\chi}$ & WIMP mass. \\
\hline$M_{\odot}$ & Mass of the Sun. \\
\hline$M_{T}$ & Target mass. \\
\hline $\mathcal{M}$ & Total exposed mass. \\
\hline$\mu$ & Reduced mass. \\
\hline $\begin{array}{l}\mu_{c} \text { Charge carrier mo- } \\
\text { bility. }\end{array}$ & \\
\hline$\mu_{C}$ & Mean of channel $C$ signal region. \\
\hline$\mu_{Y}$ & $\begin{array}{l}\text { Yield mean. Can refer to ER band mean }\left(\mu_{Y, E R}\right) \text { or } \mathrm{NR} \text { band } \\
\text { mean }\left(\mu_{Y, N R}\right) \text {. }\end{array}$ \\
\hline$\langle n\rangle$ & Average number. \\
\hline$n_{i}$ & Random pulse noise. \\
\hline$n_{n}$ & Neutron number density. \\
\hline$n_{N}$ & Nucleon number density. \\
\hline$n_{0}$ & mean WIMP number density. \\
\hline$n_{p}$ & Proton number density. \\
\hline$n_{X}$ & $\begin{array}{l}\text { Number density of some element } \mathrm{X} \text {. For example, } n_{H e} \text { is the num- } \\
\text { ber density of He nuclei. }\end{array}$ \\
\hline$N$ & Number of ADC bins in a signal event. \\
\hline$N, \bar{N}$ & Nucleon nonrelativistic field. \\
\hline$N_{0}$ & Avagadro's Number \\
\hline$N_{\text {det }}$ & Total number of detected events. \\
\hline$N_{e / h}$ & Number of electron/hole pairs. \\
\hline
\end{tabular}


Table A.2 - continued from previous page

\begin{tabular}{|c|c|}
\hline Symbol & Meaning \\
\hline$N_{k}$ & Number of detected events in the $k$ th energy bin. \\
\hline$\nu$ & Frequency. \\
\hline$\nu(x)$ & Position-dependent probability density. \\
\hline $\mathcal{O}$ & EFT operator. \\
\hline$\omega$ & Angular frequency. \\
\hline$\Omega$ & $\begin{array}{l}\text { Density parameters. } \Omega_{m}, \Omega_{b}, \Omega_{l u m}, \Omega_{r}, \Omega_{\Lambda} \text {, and } \Omega_{k} \text { represent non- } \\
\text { relativistic matter, baryonic matter, luminous matter, relativistic } \\
\text { matter, vacuum energy, and universe shape, respectively. }\end{array}$ \\
\hline$\vec{p},\left(\vec{p}^{\prime}\right)$ & Incoming (outgoing) WIMP momentum \\
\hline$p(t)$ & Pressure. \\
\hline$P$ & Power. \\
\hline$P T$ & Total phonon signal. \\
\hline$\Phi$ & Newtonian potential. \\
\hline$q$ & Charge. \\
\hline$\vec{q}$ & Momentum transfer invariance. \\
\hline$\dot{Q}$ & Cooling Power. \\
\hline$r$ & Radius or distance. \\
\hline$r_{0}$ & King's radius. \\
\hline$r_{L}$ & Radius of luminous matter within a galaxy. \\
\hline$r_{s}$ & Scale radius. \\
\hline$R$ & Resistance. \\
\hline$R_{F}$ & Feedback resistance (ionization readout). \\
\hline$R_{f b}$ & Feedback resistance (phonon readout). \\
\hline$R_{B}$ & Bias resistance. \\
\hline$R_{n}$ & Sensor resistance when in normal temperature region. \\
\hline$R_{p}$ & Parasitic resistance. \\
\hline$R_{s}$ & Resistance of the sensor \\
\hline$R_{s h}$ & Shunt resistance. \\
\hline$R_{T E S}$ & TES resistance. \\
\hline
\end{tabular}


Table A.2 - continued from previous page

\begin{tabular}{|c|c|}
\hline Symbol & Meaning \\
\hline$R_{\mu} \nu$ & Ricci tensor. \\
\hline$\rho$ & $\begin{array}{l}\text { WIMP density. Local WIMP density given by } \rho_{0} \text {, and galactic } \\
\text { central density given by } \rho_{c} \text {. }\end{array}$ \\
\hline$\rho_{\text {det }}$ & Detector's crystal density. \\
\hline$\tilde{\rho}_{\alpha \beta}$ & Noise correlation. \\
\hline$\varrho(t)$ & $\begin{array}{l}\text { Proper energy density. } \varrho_{m}, \varrho_{r} \text {, and } \varrho_{\Lambda} \text { represent energy density } \\
\text { of non-relativistic matter, relativistic matter, and vacuum energy, } \\
\text { respectively. }\end{array}$ \\
\hline$s$ & Truncated radius. \\
\hline$S_{i}$ & Digitized pulse. \\
\hline$\vec{S}$ & Spin. \\
\hline$\sigma$ & Cross section. \\
\hline$\vec{\sigma}$ & Pauli sigma matrices. \\
\hline$\sigma_{C}$ & Standard deviations of channel $C$ signal region. \\
\hline$\sigma_{E} \sigma_{Q}, \sigma_{T}, \sigma_{R}$ & $\begin{array}{l}\text { Generic, ionization, total phonon, and recoil energy resolutions, } \\
\text { respectively. }\end{array}$ \\
\hline$\sigma_{v}$ & 1D radial velocity dispersion. \\
\hline$\sigma_{Y}$ & $\begin{array}{l}\text { Yield resolution. Can refer to ER band resolution }\left(\sigma_{Y, E R}\right) \text { or NR } \\
\text { band resolution }\left(\sigma_{Y, N R}\right) \text {. }\end{array}$ \\
\hline$\tilde{\sigma}_{\alpha}$ & Noise variance. \\
\hline$t$ & Time. \\
\hline$t_{0}$ & Template time shift with respect to signal. \\
\hline$t_{L}$ & Time of last scattering. \\
\hline$T$ & Temperature. \\
\hline $\mathcal{T}$ & Total time of exposure. \\
\hline$T_{c}$ & Transition (critical) temperature. \\
\hline$T_{F}$ & Fermi Temperature. \\
\hline$T_{s}$ & Substrate (crystal) temperature. \\
\hline$T_{s t}$ & Still Temperature. \\
\hline
\end{tabular}


Table A.2 - continued from previous page

\begin{tabular}{|c|c|}
\hline Symbol & Meaning \\
\hline$T(\theta, \phi)$ & Temperature anisotropies of the CMB. \\
\hline$T^{i j}$ & Energy-Momentum tensor. $i, j \in\{0,1,2,3\}$. \\
\hline$\theta$ & Scattering angle. \\
\hline$\Theta_{0}$ & Local standard of rest. \\
\hline$\tau_{0}$ & Return-to-equilibrium time constant. \\
\hline $\begin{array}{ll}\tau_{c} & \text { Characteristic } \\
\text { charge } & \text { carrier life- } \\
\text { time. } & \end{array}$ & \\
\hline$\tau_{d}$ & Rate of anharmonic decay. \\
\hline$\tau_{s}$ & Time between isotropic scatters. \\
\hline$\tau_{E T F}$ & TES sensor cool down time. \\
\hline$v$ & Velocity. \\
\hline$\vec{v}$ & Incoming velocity invariance. \\
\hline$v_{0}$ & Characteristic WIMP velocity. \\
\hline$v_{\odot}$ & Solar peculiar velocity. \\
\hline$v_{\oplus}$ & Earth's orbital velocity. \\
\hline$v_{c}$ & Galactic circular velocity. \\
\hline$v_{d}$ & Magnitude of charge carrier drift velocity. \\
\hline$v_{E}$ & Earth's velocity relative to halo frame. \\
\hline$v_{e s c}$ & Local galactic escape velocity. \\
\hline$v_{\max }$ & Maximum velocity for which a WIMP will escape, in the lab frame. \\
\hline$v_{\min }$ & Minimum velocity for which a nuclear recoil can occur. \\
\hline$\tilde{V}_{\alpha \beta}$ & Covariance matrix. \\
\hline$V$ & Volume. \\
\hline$\langle V\rangle$ & Higgs vacuum expectation value. \\
\hline$V_{b}$ & Voltage Bias. \\
\hline$V_{m}$ & Molar volume. \\
\hline$V_{o}$ & Output voltage. \\
\hline$w$ & Time independent value relating $\rho(t)$ and $p(t)$. \\
\hline
\end{tabular}


Table A.2 - continued from previous page

\begin{tabular}{|l|l|}
\hline Symbol & Meaning \\
\hline \hline$w_{\max }$ & OF sampling window maximum. \\
$w_{\min }$ & OF sampling window minimum. \\
$W$ & Active width of detector. \\
$x$ & Concentration of ${ }^{3}$ He in dilute phase. \\
$\xi$ & Slope of best-fit to G106a cross talk. \\
$\Xi$ & Detector deformation potential. \\
$Y$ & Ionization yield. \\
$Y_{L}$ & Expected yield from Lindhard model. \\
$Y_{l, m}$ & Laplace's spherical harmonics. \\
$Y_{p}$ & Fraction weight abundance. \\
$Z$ & Atomic ordering number. \\
\hline \hline
\end{tabular}

\section{A.3 Data Quantities}

* = AS1, BS1, CS1, DS1, ES1, FS1, AS2, BS2, CS2, DS2, ES2, FS2, T

\# = IS1, OS1, IS2, OS2, or blank for contact-free

Table A.3: Data Quantities

\begin{tabular}{|l|l|}
\hline Data Quantity & Meaning \\
\hline \hline Q\#bs & Charge channel prepulse region baseline. \\
Q\#OFvolts & Optimal Filter charge channel amplitude, in volts. \\
Q\#OFvolts0 & Optimal Filter charge channel amplitude with time delay fixed at \\
& zero, in volts. \\
Q\#\#OFdelay & $\begin{array}{l}\text { Optimal Filter charge channel time shift between actual pulse and } \\
\text { template start time, in seconds. }\end{array}$ \\
Q\#OFchisq & Optimal Filter charge channel goodness-of-fit. \\
QTDFvolts & Time Domain Fit charge channel amplitude, in volts. \\
QTDFdelay & Time Domain Fit charge channel time shift between actual pulse \\
& and template start time, in seconds. \\
\hline \hline
\end{tabular}

Continued on next page 
Table A.3 - continued from previous page

\begin{tabular}{|c|c|}
\hline Data Quantity & Meaning \\
\hline QTDFchisq & Time Domain Fit charge channel goodness-of-fit. \\
\hline $\mathrm{P}^{*} \mathrm{bs}$ & Phonon channel prepulse region baseline. \\
\hline $\mathrm{P}^{*}$ OFamps & Optimal Filter phonon channel amplitude, in Amps. \\
\hline $\mathrm{P}^{*} \mathrm{OFamps} 0$ & $\begin{array}{l}\text { Optimal Filter phonon channel amplitude with time delay fixed } \\
\text { at zero, in Amps. }\end{array}$ \\
\hline $\mathrm{P}^{*}$ OFdelay & $\begin{array}{l}\text { Optimal Filter phonon channel time shift between actual pulse } \\
\text { and template start time, in seconds. }\end{array}$ \\
\hline $\mathrm{P}^{*} \mathrm{OF}$ chisq & Optimal Filter phonon channel goodness-of-fit. \\
\hline $\mathrm{P}^{*}$ NFamps & $\begin{array}{l}\text { Non-Stationary Optimal Filter phonon channel amplitude, in } \\
\text { Amps. }\end{array}$ \\
\hline $\mathrm{P}^{*}$ NFamps0 & $\begin{array}{l}\text { Non-Stationary Optimal Filter phonon channel amplitude with } \\
\text { time delay fixed at zero, in Amps. }\end{array}$ \\
\hline $\mathrm{P}^{*}$ NFdelay & $\begin{array}{l}\text { Non-Stationary Optimal Filter phonon channel time shift between } \\
\text { actual pulse and template start time, in seconds. }\end{array}$ \\
\hline $\mathrm{P}^{*} \mathrm{NF}$ chisq & Non-Stationary Optimal Filter phonon channel goodness-of-fit. \\
\hline QiS1, QiS2, Qimean & $\begin{array}{l}\text { Side } 1 \text {, Side } 2 \text {, and mean calibrated inner charge channel signal } \\
\text { amplitude, in } \mathrm{keV}\end{array}$ \\
\hline QoS1, QoS2 & $\begin{array}{l}\text { Side } 1 \text {, and Side } 2 \text { calibrated outer charge channel signal ampli- } \\
\text { tude, in keV }\end{array}$ \\
\hline Qsum1, Qsum2 & $\begin{array}{l}\text { Side } 1 \text {, and Side } 2 \text { calibrated charge channel signal amplitude, in } \\
\mathrm{keV}\end{array}$ \\
\hline $\mathrm{PtOF}$ & Total calibrated phonon signal using OF, in keV \\
\hline PtNF & Total calibrated phonon signal using NSOF, in $\mathrm{keV}$ \\
\hline $\mathrm{PrOF}$ & Recoil energy determined using $\mathrm{OF}$, in $\mathrm{keV}$ \\
\hline PrNF & Recoil energy determined using NSOF, in $\mathrm{keV}$ \\
\hline Psum & $\begin{array}{l}\text { Total phonon channel signal amplitude with absolute calibration, } \\
\text { in } \mathrm{keV}\end{array}$ \\
\hline PsumC & $\begin{array}{l}\text { Total phonon channel signal amplitude with absolute and relative } \\
\text { calibrations, in } \mathrm{keV}\end{array}$ \\
\hline
\end{tabular}


Table A.3 - continued from previous page

\begin{tabular}{|l|l|}
\hline Data Quantity & Meaning \\
\hline \hline YOF & Yield determined from OF \\
YNF & Yield determined from NSOF \\
QOF & Calibrated contact-free charge amplitude using OF, in keV \\
QTDF $_{T}$ & Calibrated contact-free charge amplitude using TDF, in keV \\
\hline \hline
\end{tabular}




\section{Appendix B}

\section{CDMS Cross Talk Correction with OF Fitting Algorithm}

Standard CDMS cross talk correction is implemented in the OF fitting process. Recall from Section 3.4.1, a system of inner and outer signals, $S_{I}$ and $S_{O}$, being fit with two templates can be represented as such,

$$
\left[\begin{array}{c}
S_{I} \\
S_{O}
\end{array}\right]=\left[\begin{array}{cc}
A_{I} & A_{O x} \\
A_{I x} & A_{O}
\end{array}\right]\left[\begin{array}{c}
a_{I} \\
a_{O}
\end{array}\right]+\left[\begin{array}{c}
n_{I} \\
n_{O}
\end{array}\right],
$$

where $A_{I x}$ and $A_{O x}$ represent the cross talk templates: that is, $A_{I x}$ is the template for the outer charge signal from an inner charge event (and vice versa). The amplitudes for which $\chi^{2}$ is minimized for a given time shift $t_{0}$ are given by,

$$
\left[\begin{array}{c}
\hat{a}_{I} \\
\hat{a}_{O}
\end{array}\right]=\mathbf{P}^{-1} \cdot\left[\begin{array}{c}
q_{I}\left(t_{0}\right) \\
q_{O}\left(t_{0}\right)
\end{array}\right],
$$

where $\mathbf{P}$ and $\mathbf{q}\left(t_{0}\right)$ are given in Eq. 3.25. CDMS processing algorithms will calculate $\hat{a}_{I}$ and $\hat{a}_{O}$ for all possible $t_{0}$ within the given window. With two distinct amplitudes it is unclear what value of $t_{0}$ to select if using the tradition OF method. Instead, the $t_{0}$ value with minimizes $\chi^{2}$ via $\mathrm{Eq} 3.26$ is used to determine the final amplitude values.

Therefore, cross talk between inner and outer charge channels can be properly accounted for in the fitting algorithm if cross talk templates are available. Fig. B.1 shows cross talk templates created by N. Mast for the UMN test facility using G106a background data. 

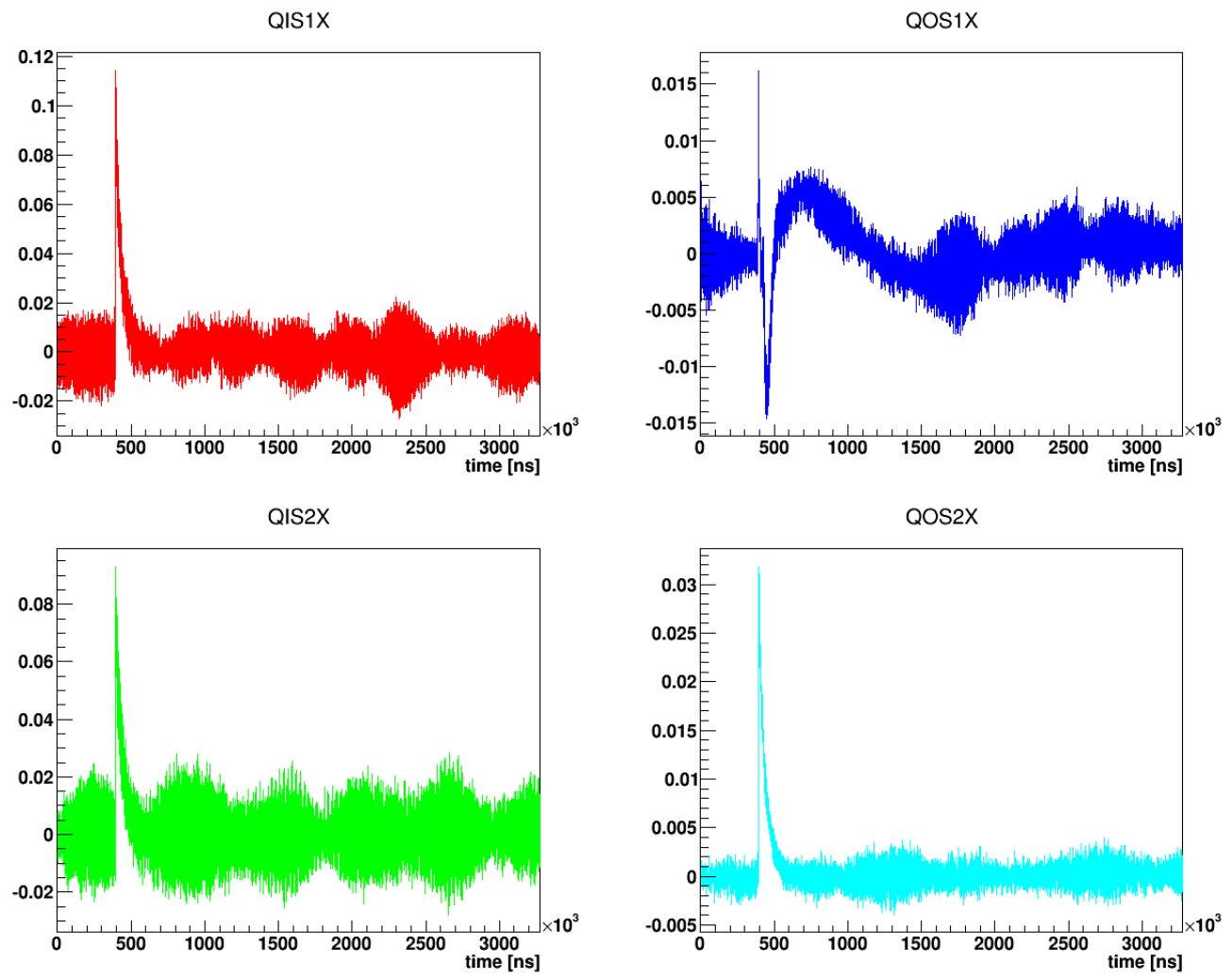

Figure B.1: Cross talk templates constructed from a thousand well behaved pulses. Templates represent the typical signal seen in an inner (outer) charge channel due to an outer (inner) event. For example, template named QIS1X represents typical signal in QIS1 from QOS1 event. Only the QOS1X template has the form typically seen in a cross talk template, raising suspicions that the other templates do not properly isolate cross talk contribution from true event signals. Figure courtesy of N. Mast. 
Cross talk template construction begins with the same steps as general template construction. Raw events whose RQ values pass basic quality cuts ( $\chi^{2}$ cuts, Side 1 and Side 2 charge symmetry cut, and a radial partition cut) are pulled from the raw pulse data files, and normalized to peak height of 1.0. Additional quality cuts are applied to the raw data to ensure that remaining pulses have the following qualities:

- Consistent baseline at beginning and end of pulse

- Smooth and flat prepulse region

- Smooth tail region

- Consistent pulse start time location

- Single pulse in trace (no pileup)

In the particular case of cross talk templates, raw events of inner (outer) charge channels are pulled when a pulse is seen in the corresponding outer (inner) channel. To accomplish this, a cut is implemented requiring the ratio of inner-to-outer amplitudes to fall below 0.1 on a given side 201]. Unfortunately, of the four constructed templates, shown in Fig. B.1, only the QOS1X template has the form typically seen in a CDMS cross talk template, with a sharp upward and downward peak followed by an upward hump. The peak-like shape of the other three templates raises suspicions that the inner-to-outer amplitude ratio requirement of $<0.1$ was insufficient to isolate cross talk contribution from true event signals. However, it could also simply be that this is form of cross talk signals for the UMN facility.

Attempts to implement the OF cross talk correction using the above templates have thus far been unsuccessful; in recent attempts the magnitude of the cross talk has increased when the OF correction is implemented. The cause of this could be due to the suspicious shape of three of the templates, or possibly incorrect cross talk template normalization with respect to the non-cross talk event templates. Cross talk correction studies are still ongoing. 


\section{Appendix $\mathrm{C}$}

\section{Additional Figures}

This appendix contains additional plots that did not fit within the main text of this thesis, yet still provide relevant information.

\section{C.1 $I_{b}-I_{s}$ Figures for All Phonon Channels}

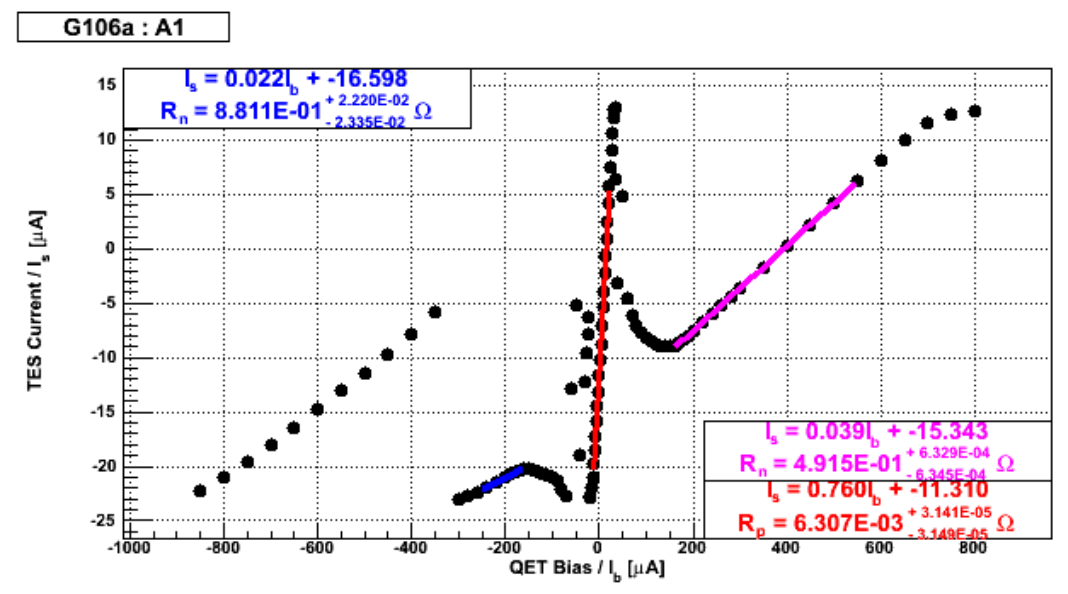

Figure C.1: $I_{b}-I_{s}$ measurement for G106a. Continued on next page. 

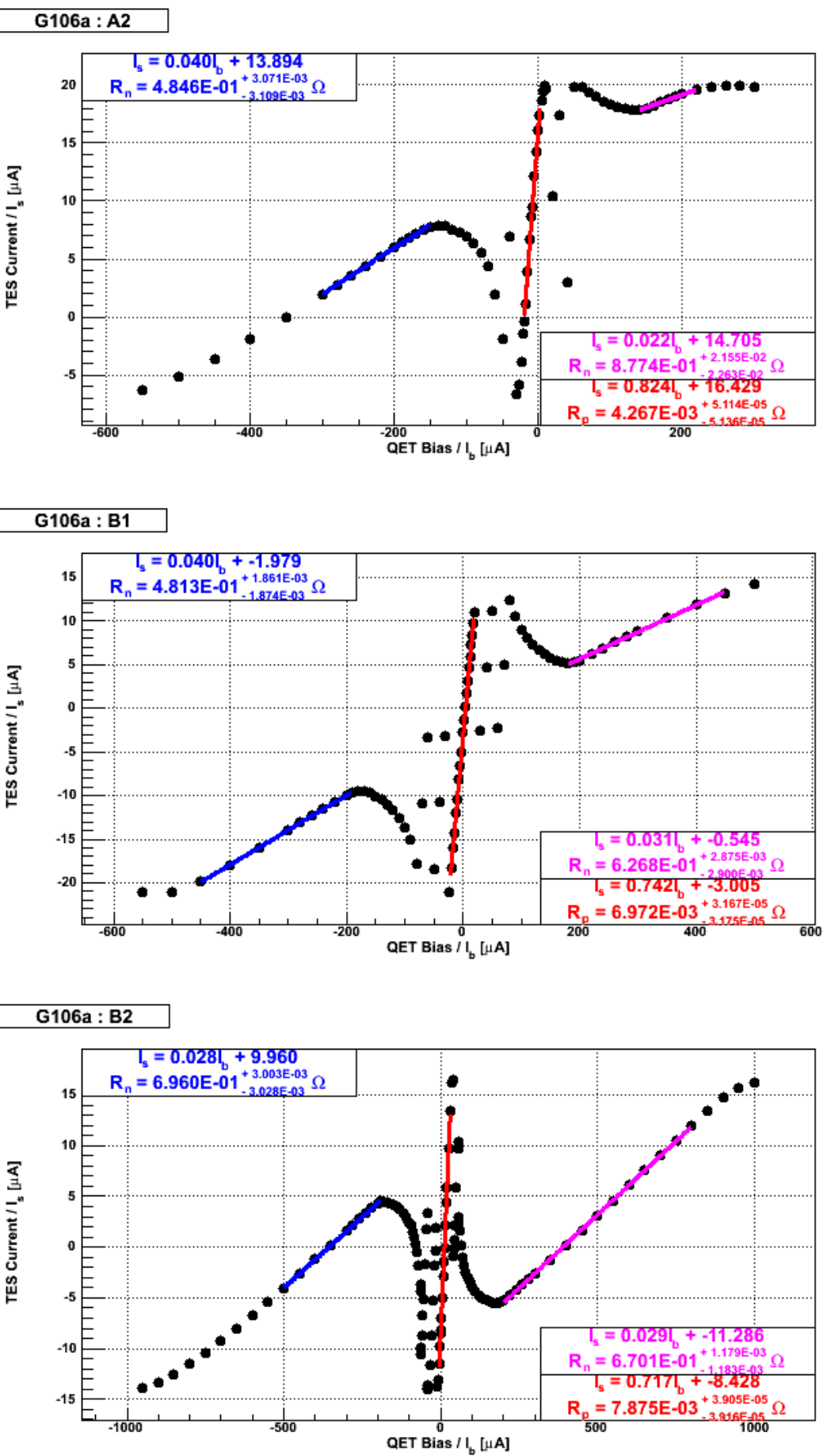

Figure C.1: Continued from previous page. $I_{b}-I_{s}$ measurement for G106a. Continued on next page. 
G106a : C1

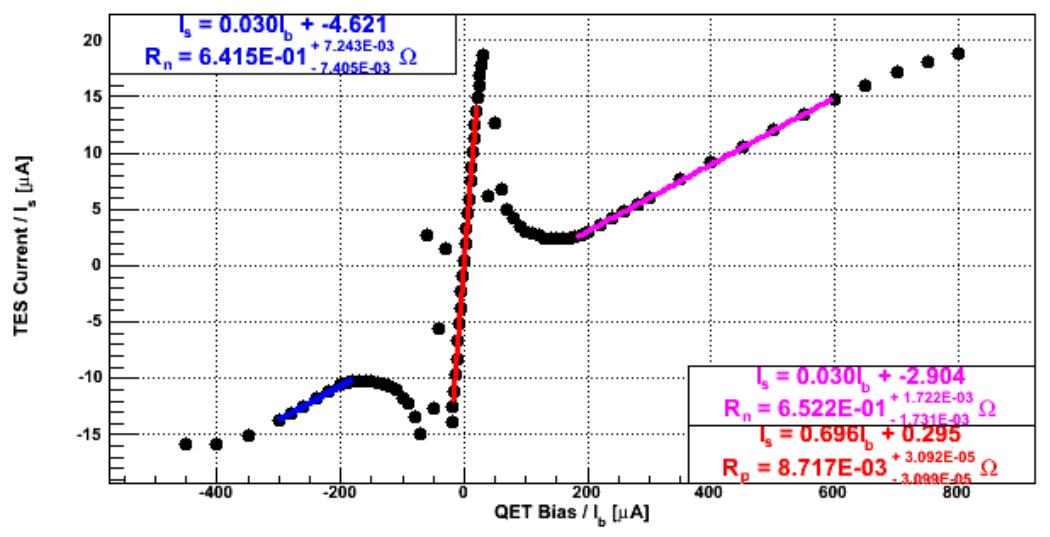

G106a : C2

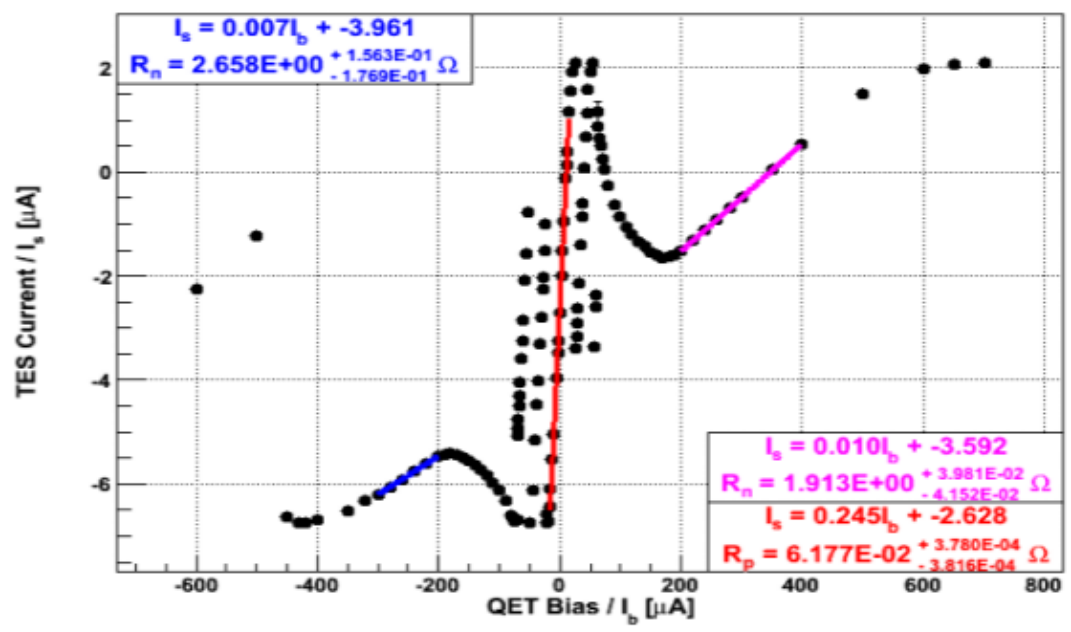

G106a : D1

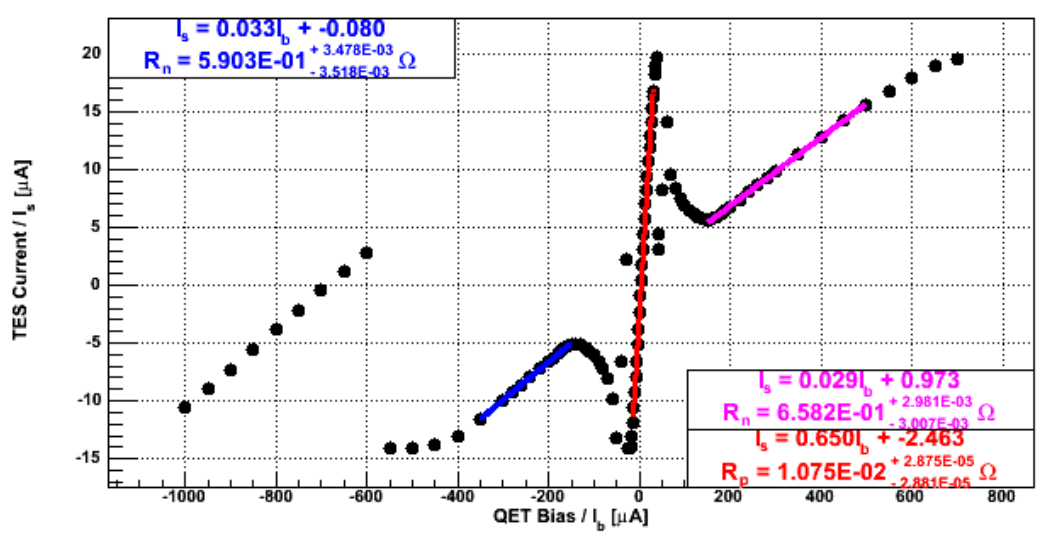

Figure C.1: Continued from previous page. $I_{b}-I_{s}$ measurement for G106a. Continued on next page. 


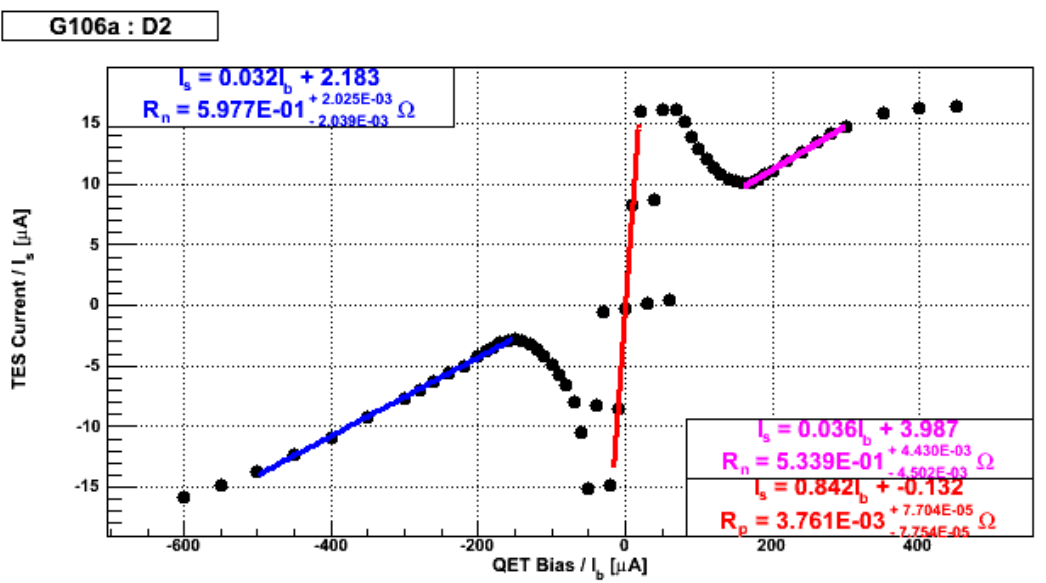

\section{G106a : A3}

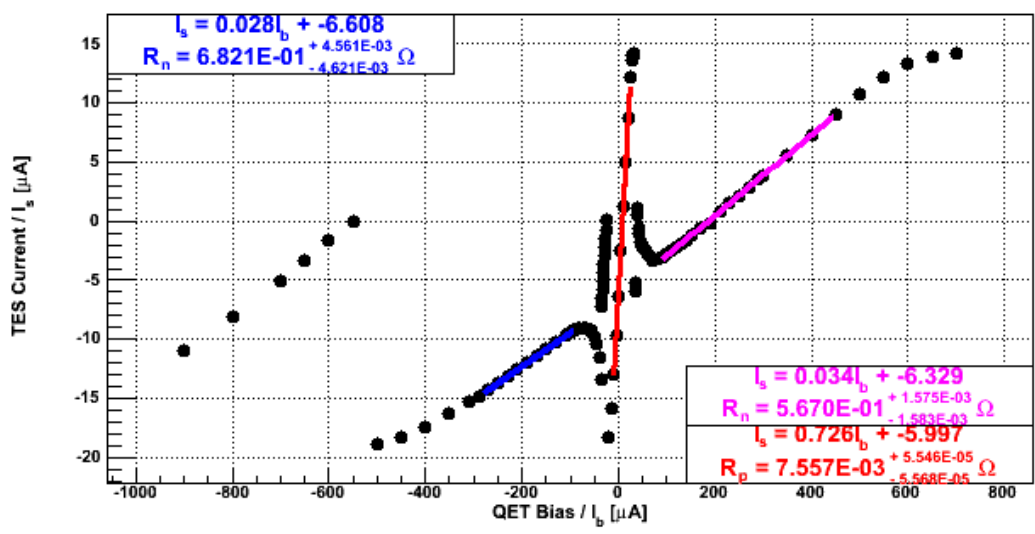

\section{G106a : B3}

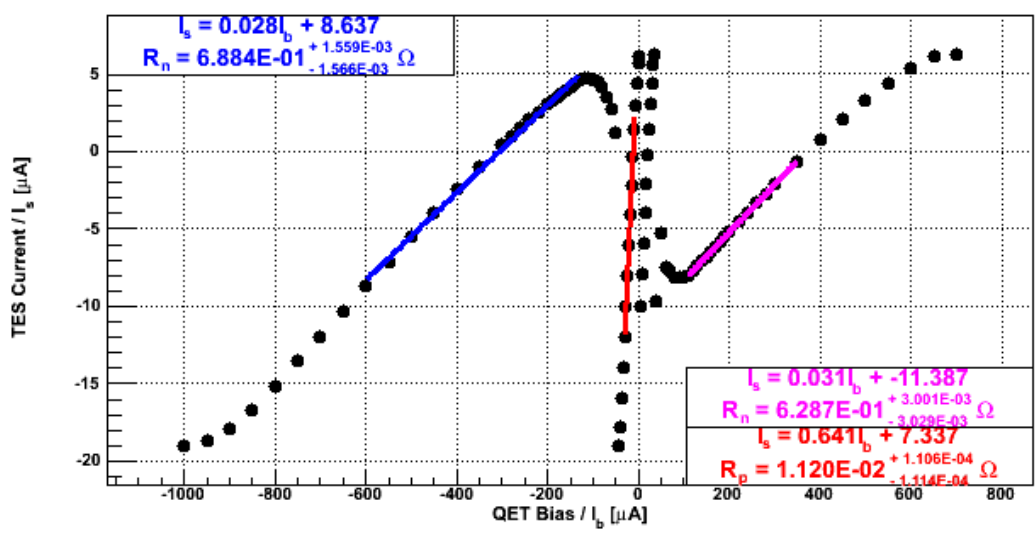

Figure C.1: Continued from previous page. $I_{b}-I_{s}$ measurement for G106a. Continued on next page. 


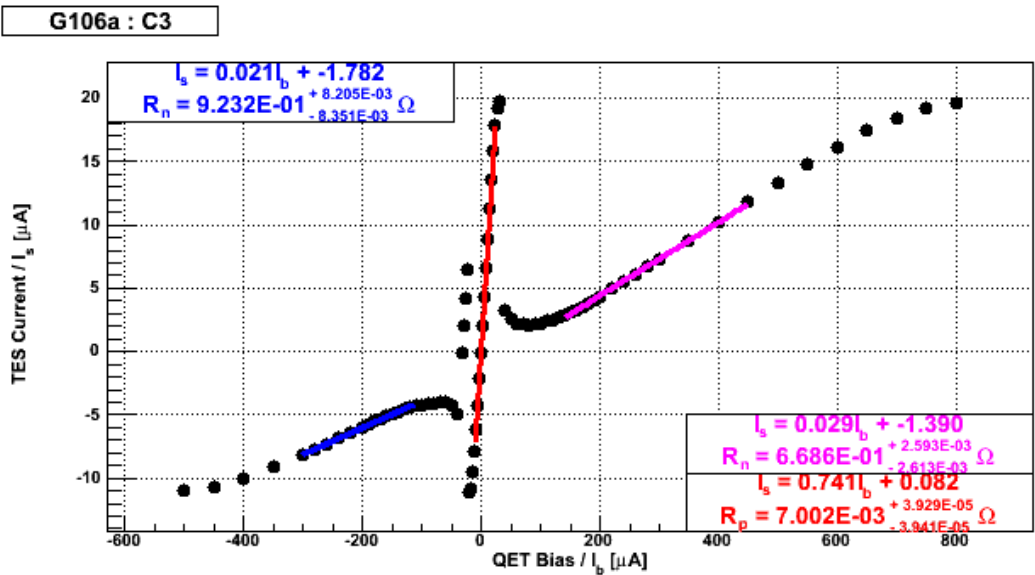

\section{G106a : D3}

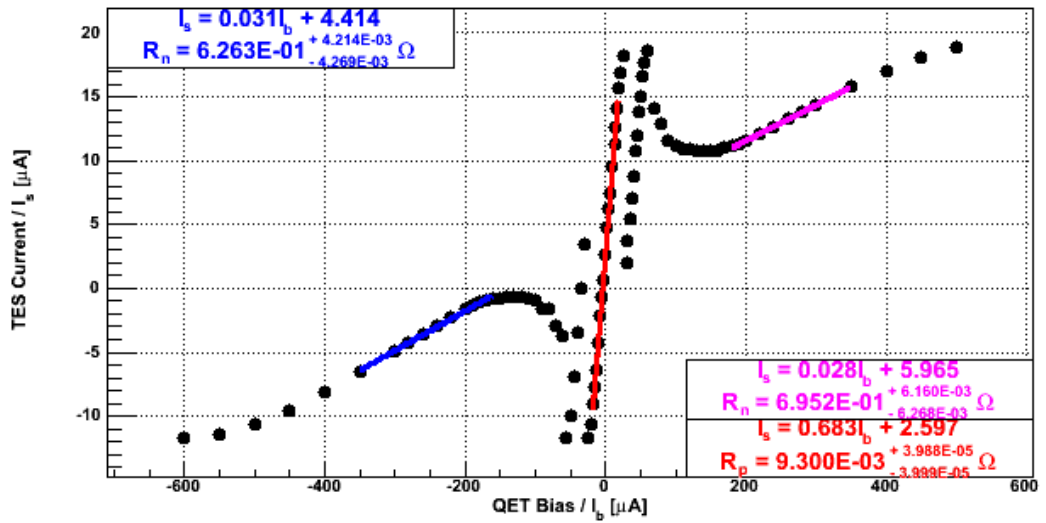

Figure C.1: Continued from previous page. $I_{b}-I_{s}$ measurement for $100 \mathrm{~mm}$ iZIP G106a, with superconducting region highlighted in red, and normal regions highlighted in blue (negative $I_{b}$ ) and magenta (positive $I_{b}$ ). Fitting the linear superconducting and normal regions allows for estimation of the parasitic and normal resistances of the sensors. The fits and estimated resistance values are shown in the upper left and lower right corners of each channel's plot. Figures courtesy of H. Chagani. 


\section{G106a : A1}

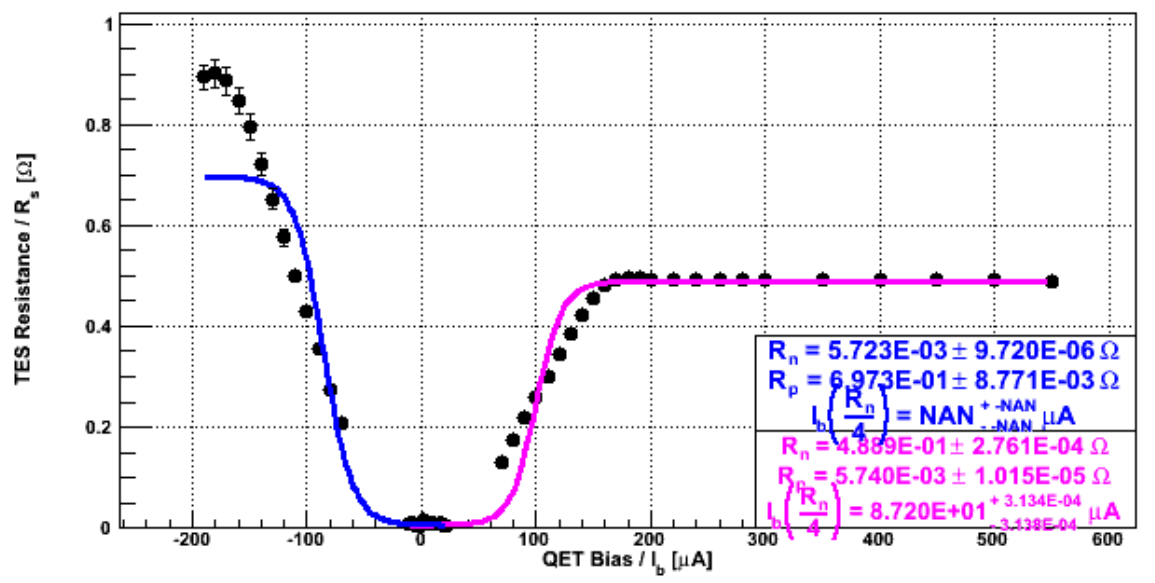

\section{G106a : A2}

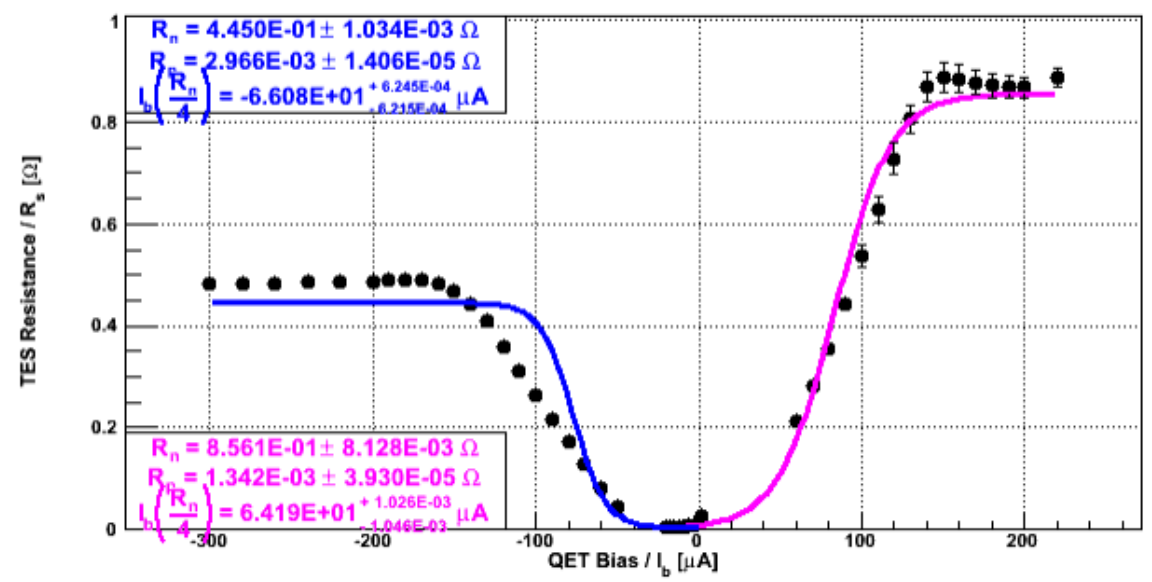

\section{G106a : B1}

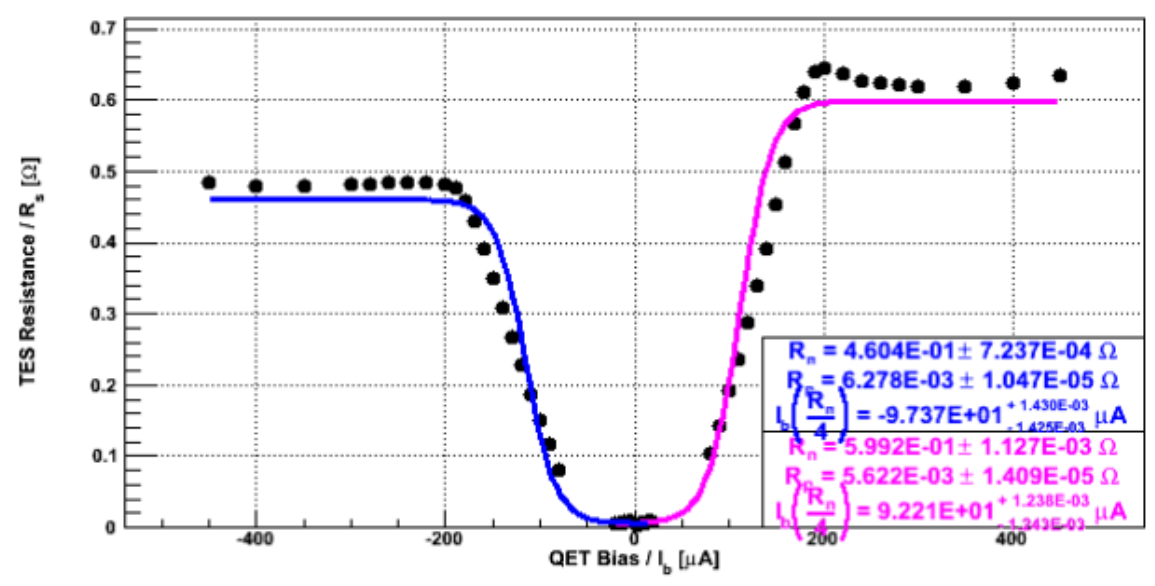

Figure C.2: $R_{s}$ plotted versus $I_{b}$ for G106a. Continued on next page. 


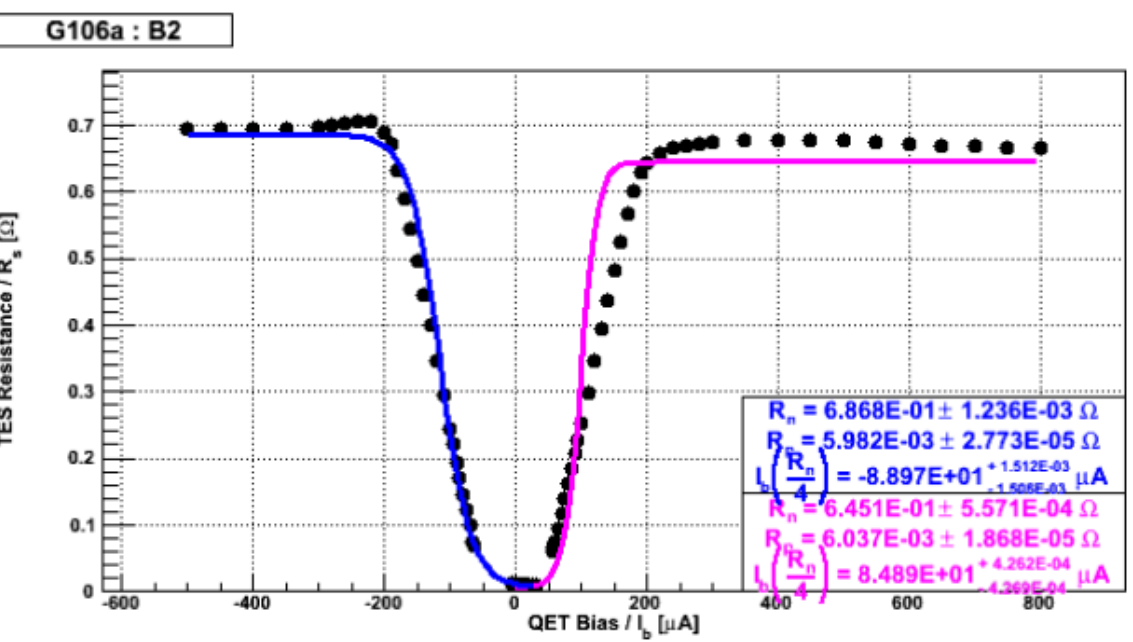

\section{G106a : C1}

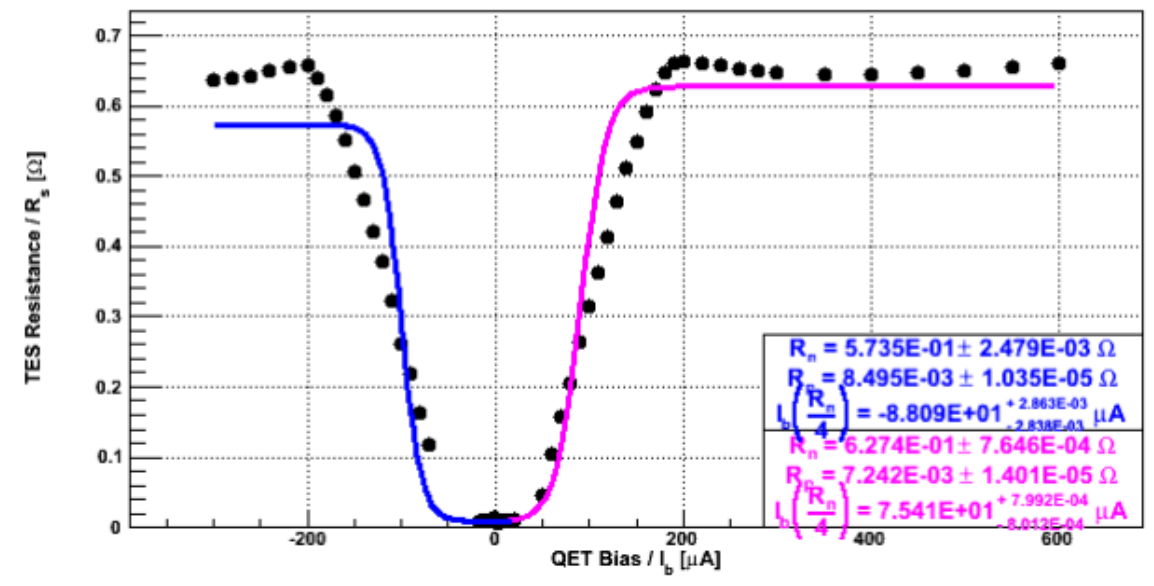

\section{G106a : D1}

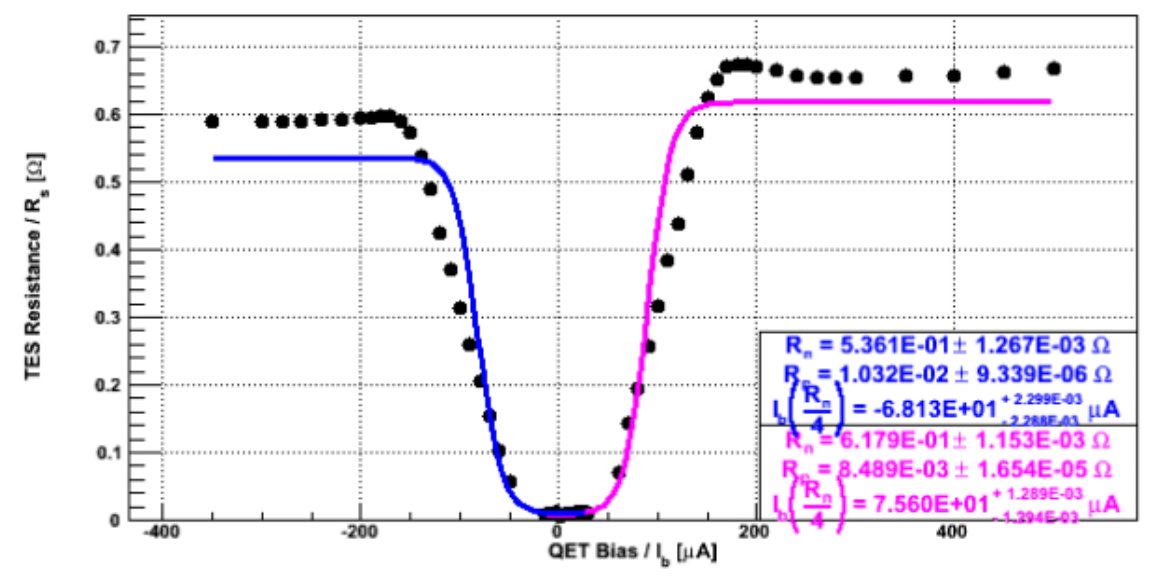

Figure C.2: Continued from previous page. $R_{s}$ plotted versus $I_{b}$ for G106a. Continued on next page. 


\section{G106a : D2}

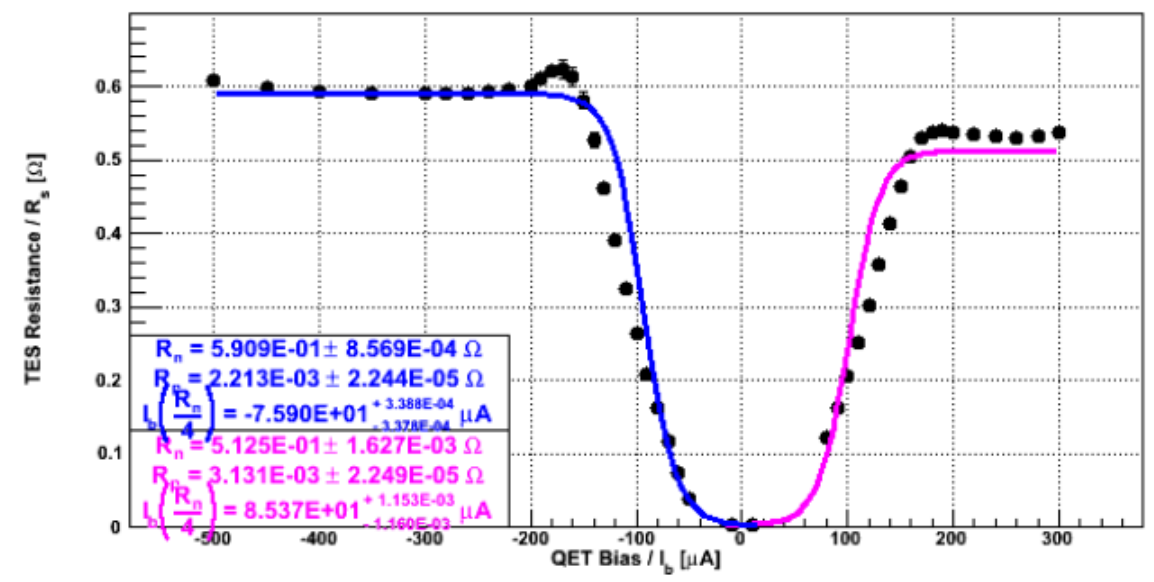

\section{G106a : A3}

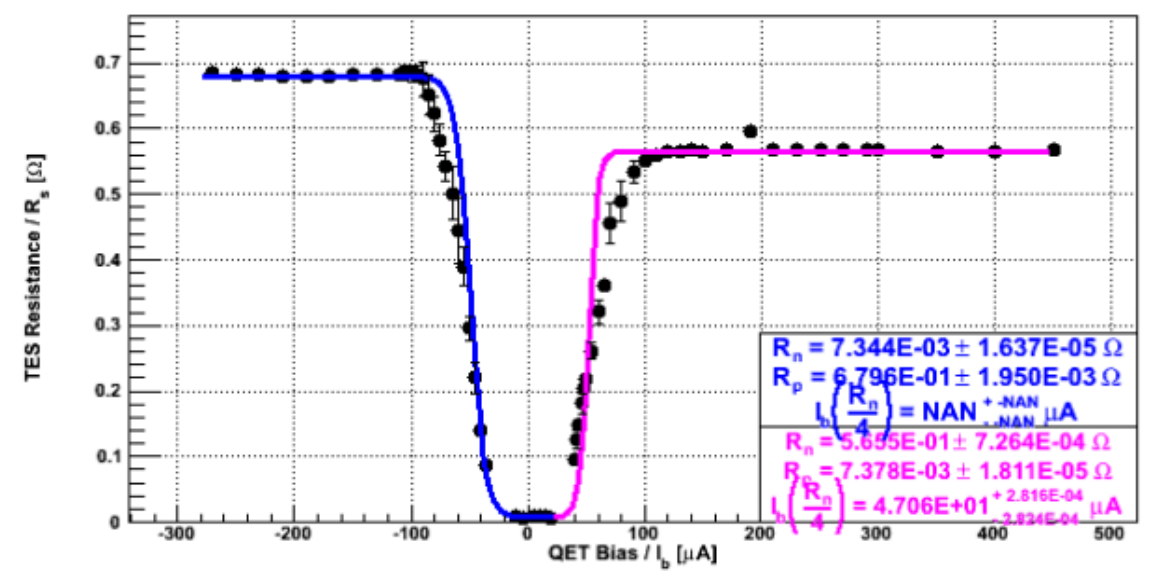

\section{G106a : B3}

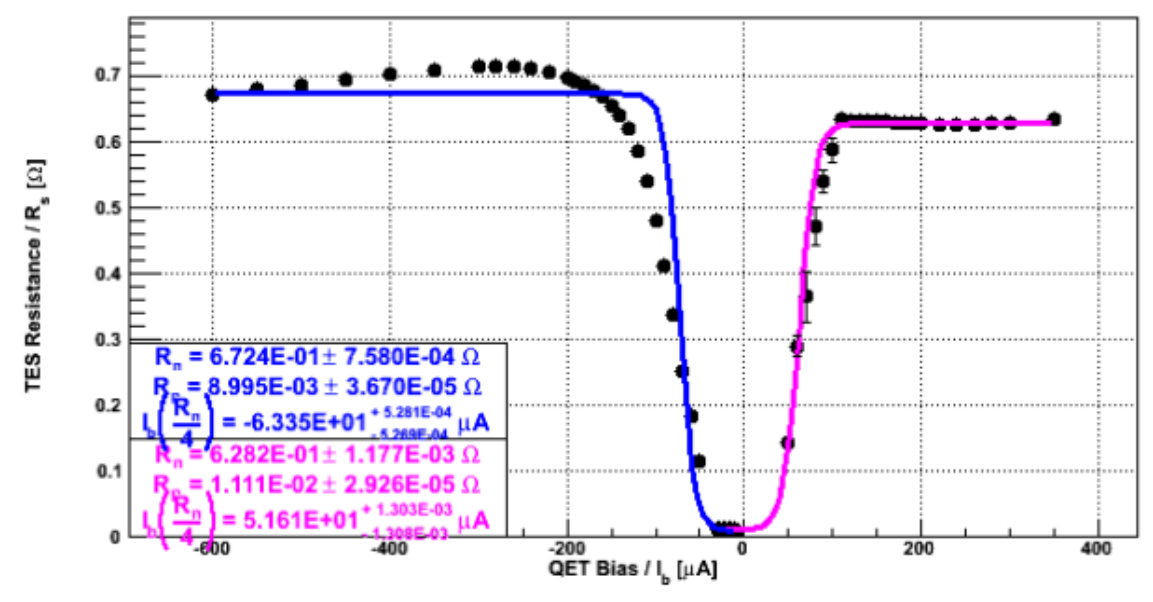

Figure C.2: Continued from previous page. $R_{s}$ plotted versus $I_{b}$ for G106a. Continued on next page. 

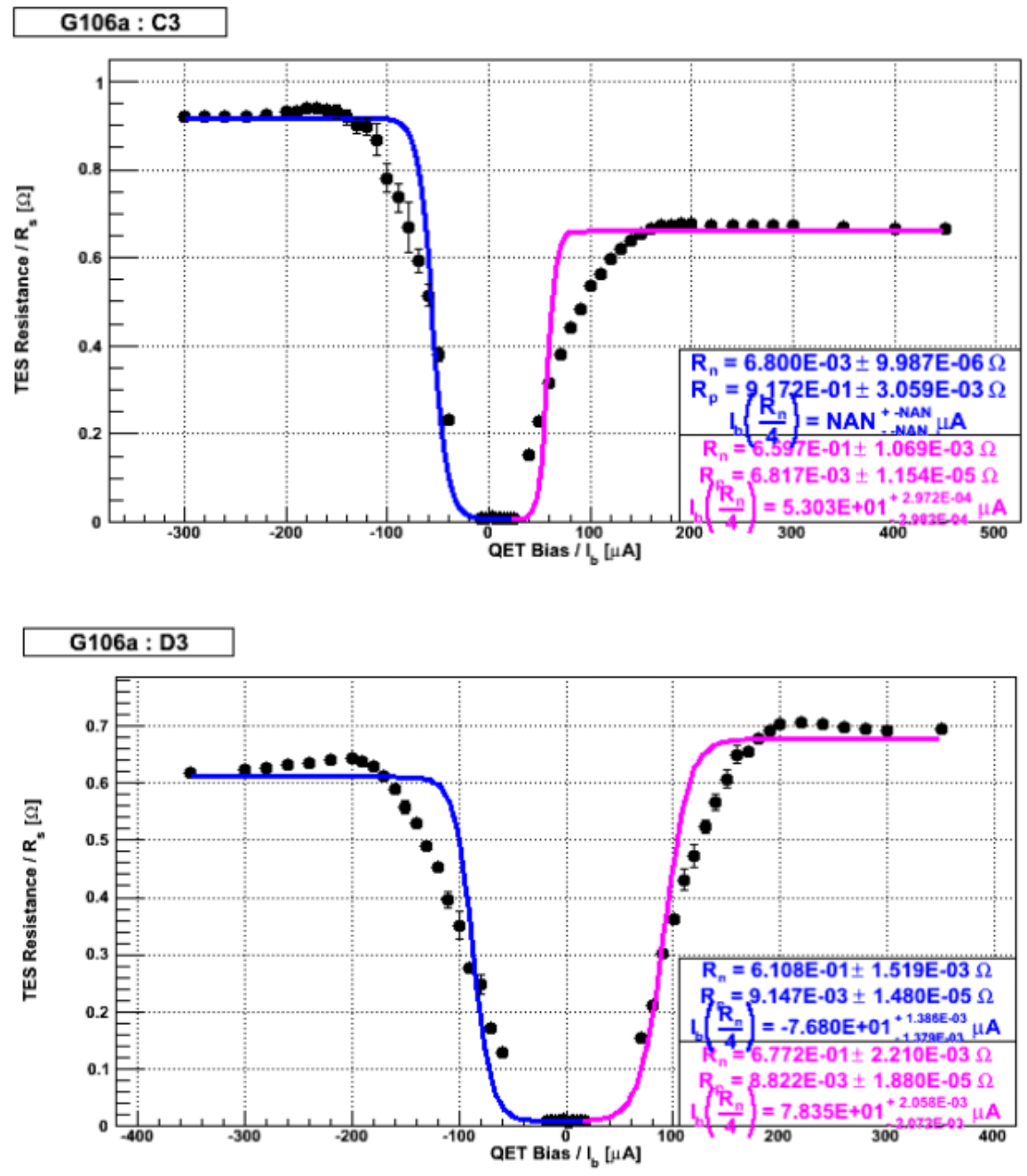

Figure C.2: Continued from previous page. $R_{s}$ plotted versus $I_{b}$ for each G106a phonon channel, excluding channel $\mathrm{C} 2$, which had inconsistent results. Plots show sensor resistance increasing from zero in the superconducting region and plateauing towards the positive and negative QET bias $R_{n}$ values. Positive and negative QET bias curves are fit to the data, shown in blue and pink. From the fit curve, $R_{p}$ and $R_{n}$ are found, along with the optimized QET bias corresponding to the start of the transition region, where $I_{b}=R_{n} / 4$. Estimated resistance values and optimized QET bias are shown in corners of each channel's plot. Figures courtesy of H. Chagani. 


\section{C.2 Stability of Relative Calibration for Phonon Channels}

Fig. C.3 monitors the change in calibration values, and the value deviations from mean, of each series with respect to each base channel, for a 15 hour data taking period of Run 54 .
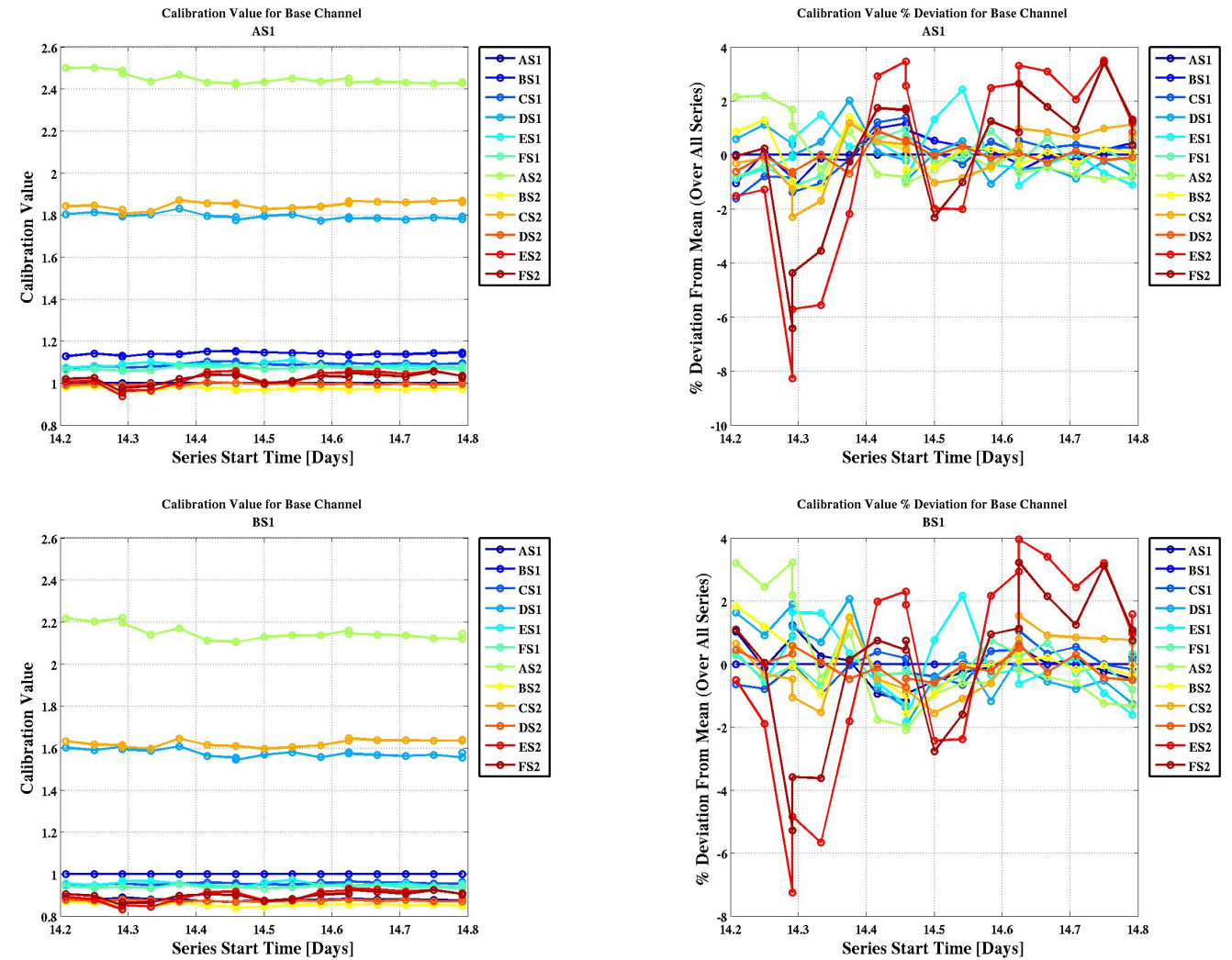

Figure C.3: (LEFT) Calibration values and (RIGHT) calibration value deviation from mean over time for all channels with respect to the given base channel. Continued on next page. 

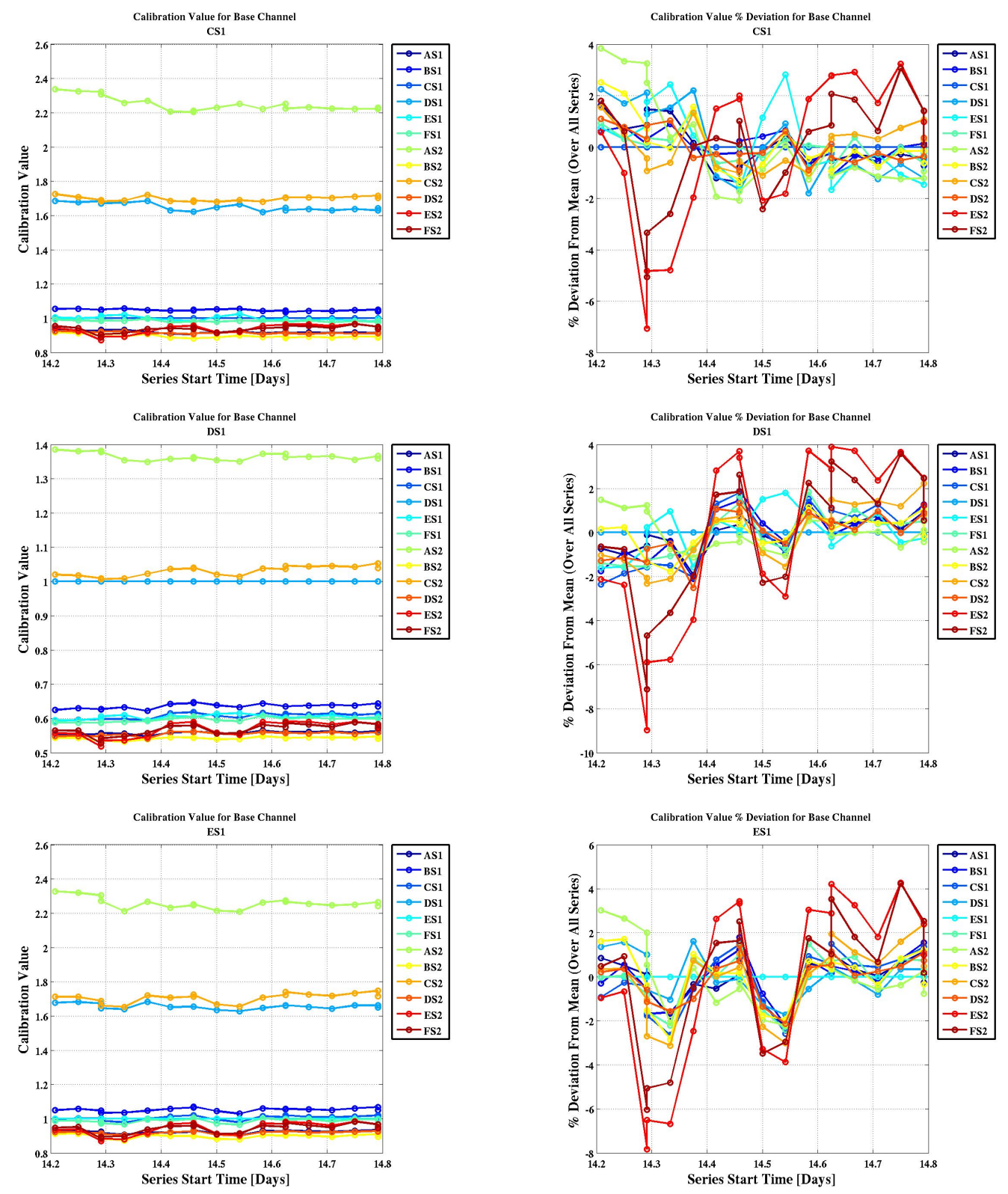

Figure C.3: Continued from previous page. (LEFT) Calibration values and (RIGHT) calibration value deviation from mean over time for all channels with respect to the given base channel. Continued on next page. 

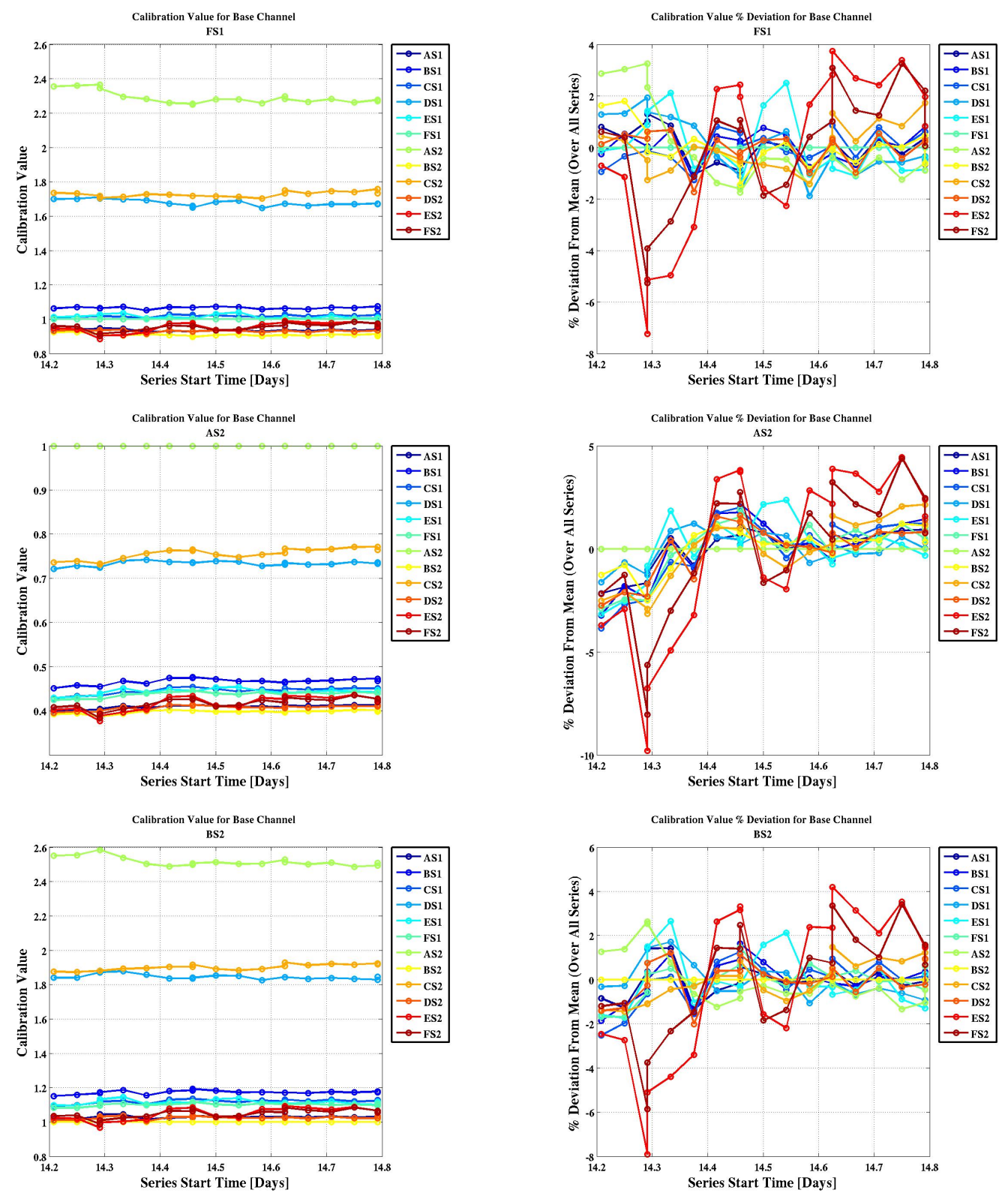

Figure C.3: Continued from previous page. (LEFT) Calibration values and (RIGHT) calibration value deviation from mean over time for all channels with respect to the given base channel. Continued on next page. 

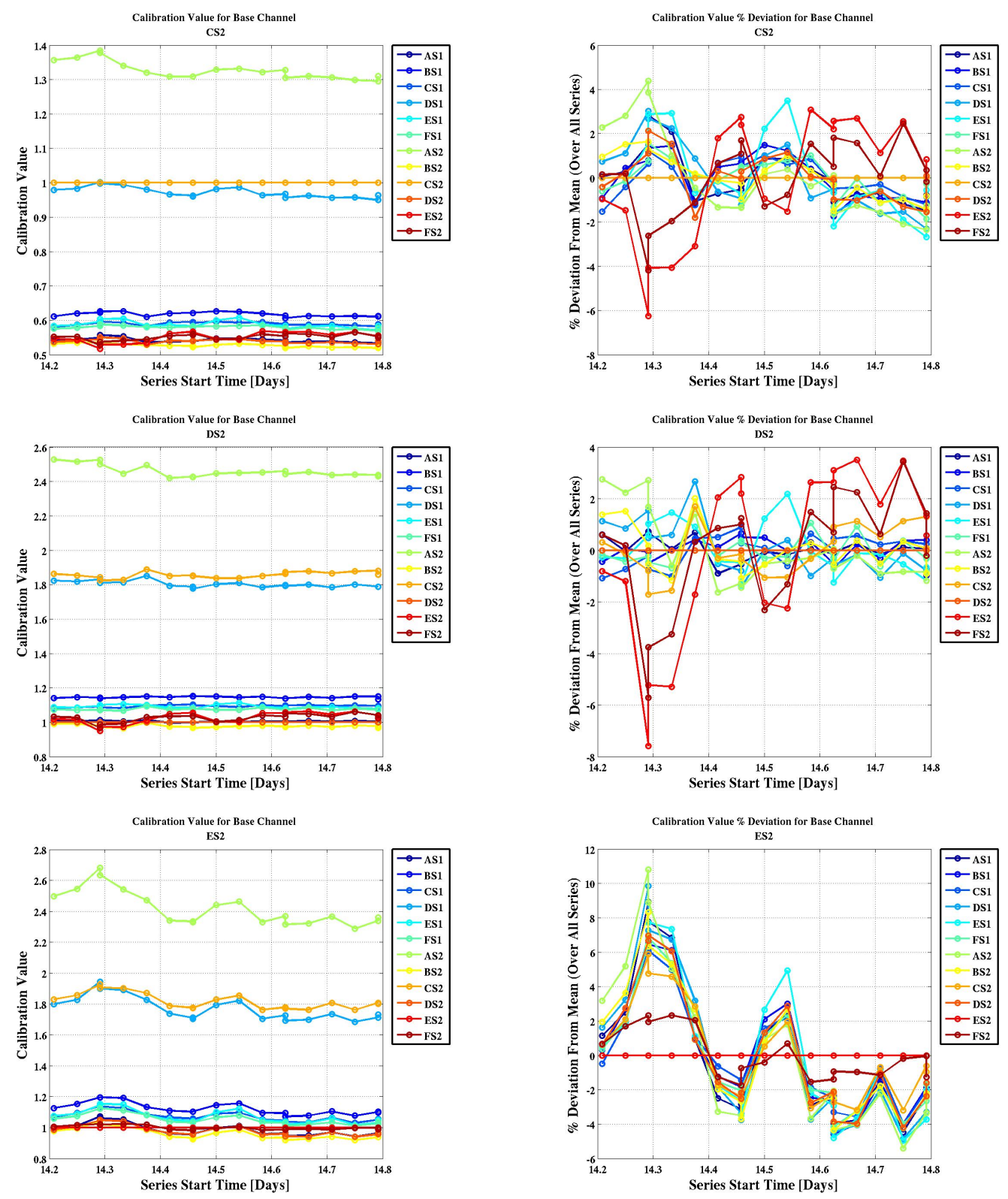

Figure C.3: Continued from previous page. (LEFT) Calibration values and (RIGHT) calibration value deviation from mean over time for all channels with respect to the given base channel. Continued on next page. 

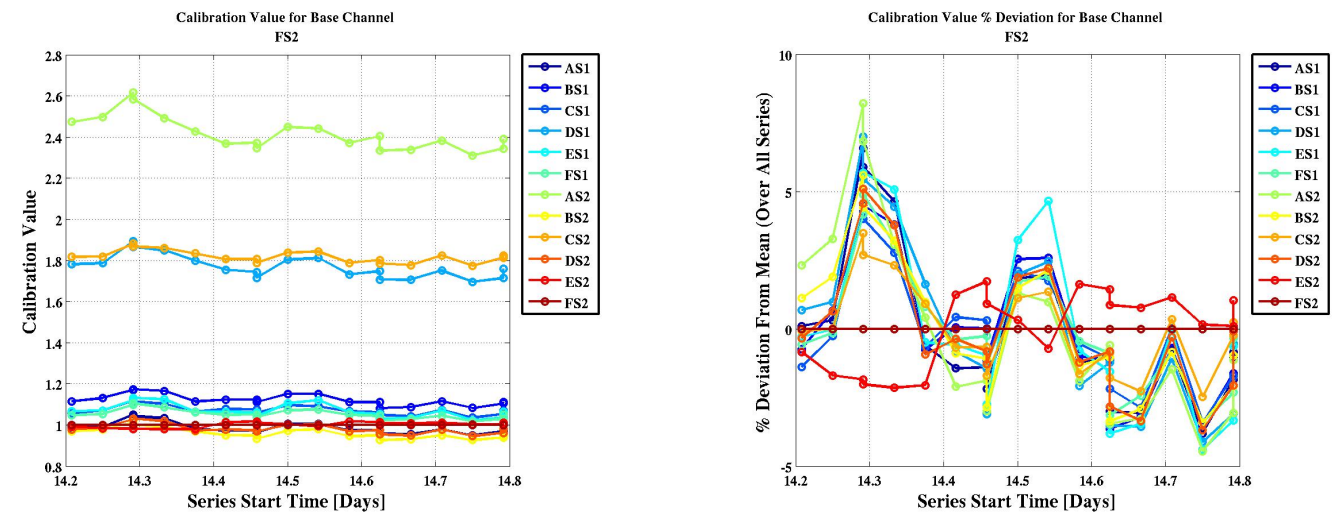

Figure C.3: Continued from previous page. (LEFT) Calibration values and (RIGHT) calibration value deviation from mean over time for all channels with respect to the given base channel. 